\title{
Monotonic Response of RC Exterior Beam-Column Joints Reinforced with Filler-Modules and FRP Composite Wraps/ Gussets
}

Praveen Kumar Reddy Majjigapu

pmajjiga@mix.wvu.edu

Follow this and additional works at: https://researchrepository.wvu.edu/etd

Part of the Structural Engineering Commons

\section{Recommended Citation \\ Majjigapu, Praveen Kumar Reddy, "Monotonic Response of RC Exterior Beam-Column Joints Reinforced with Filler-Modules and FRP Composite Wraps/Gussets" (2020). Graduate Theses, Dissertations, and Problem Reports. 7526.}

https://researchrepository.wvu.edu/etd/7526

This Dissertation is protected by copyright and/or related rights. It has been brought to you by the The Research Repository @ WVU with permission from the rights-holder(s). You are free to use this Dissertation in any way that is permitted by the copyright and related rights legislation that applies to your use. For other uses you must obtain permission from the rights-holder(s) directly, unless additional rights are indicated by a Creative Commons license in the record and/ or on the work itself. This Dissertation has been accepted for inclusion in WVU Graduate Theses, Dissertations, and Problem Reports collection by an authorized administrator of The Research Repository @ WVU.

For more information, please contact researchrepository@mail.wvu.edu. 


\title{
Monotonic Response of RC Exterior Beam-Column Joints Reinforced with Filler-Modules and FRP Composite Wraps/Gussets
}

\author{
Praveen Kumar Reddy Majjigapu
}

\author{
Dissertation submitted \\ to the Benjamin M. Statler College of Engineering and Mineral Resources \\ at West Virginia University \\ in partial fulfillment of the requirements for the degree of \\ Doctor of Philosophy in \\ Civil and Environmental Engineering
}

Hota V.S. GangaRao, Ph.D., P.E., F.ASCE, F.SEI, Chair

Udaya B. Halabe, Ph.D., P.E., F.ASCE, F.SEI, F.ASNT

P.V. Vijay, Ph.D., P.E.

Ruifeng R. Liang, Ph.D.

Karthik N. Ramanathan, Ph.D.

Wadsworth Department of Civil and Environmental Engineering

\author{
Morgantown, West Virginia
}

2020

Keywords: RC Exterior Beam-Column Joints; Filler-Modules; FRP Wraps; FRP Gussets;

Joint Shear; Joint Principal Strain; Strength; Stiffness; Ductility; Energy Dissipation

Copyright 2020 Praveen Kumar Reddy Majjigapu 


\title{
ABSTRACT \\ Monotonic Response of RC Exterior Beam-Column Joints Reinforced with Filler-Modules and FRP Composite Wraps/Gussets
}

\author{
Praveen Kumar Reddy Majjigapu
}

A review of post-earthquake reconnaissance studies revealed that reinforced concrete (RC) structures, designed and built before the development of Uniform Building Code (UBC) seismic design guidelines in 1976, have suffered complete collapse or severe damages due to the brittle failure of exterior beam-column joints. Over the past 50 years, several studies were conducted to strengthen exterior joints of in-service structures, with limited emphasis on developing simple, economic and durable repair strategies to improve energy absorption through large inelastic joint deformations. Even less emphasis was devoted to developing repair procedures that minimize stress-concentrations at joint corners and enhancing the strength, ductility, and energy dissipation capabilities of concrete structures with an emphasis on joint resistance improvements.

To address the above limitations, a novel approach of reinforcing vulnerable joints with fillermodules and fiber-reinforced polymer (FRP) composite wraps/gussets have been proposed and evaluated, herein. The proposed approach involved bonding filler-modules at the reentrant corners of a joint and securing them with reinforcing dowels to minimize corner stress-concentrations through smoother stress transfer in and around a joint. Additionally, bonding of FRP composite wraps or gussets on to the exposed beam-column faces was done to reinforce the joint core, thus enhancing the strength and energy absorption through joint confinement and reducing joint shear demand so that the plastic hinge could form away from the joint core.

To investigate the efficacy of the proposed approach in enhancing the joint structural capacities, twenty 2D RC exterior (T) joints were designed as per pre-1976 construction deficiencies and experimentally evaluated in control (as-built) and reinforced conditions through the variations in (i) filler-module geometric shape; (ii) filler-module material properties, (iii) FRP material, (iv) FRP wrap/gusset configurations, (v) confinement due to partial (U-anchors) versus complete $\left(360^{\circ}\right.$-anchors) diagonal wraps, and (vi) shear transfer through reinforcing dowels. The performance of the test specimens was recorded through numerical values of loads versus deformation and strains at the rupture of concrete, de-bond of FRP wrap from the concrete surface, yielding of steel rebar, shear failure of column or joint panel through diagonal tension and beam flexural failure phenomena. Test data evaluations measured up to the peak loads revealed that the proposed approach of reinforcing joints with filler-modules and FRP wraps/gussets is immensely useful in enhancing the strength and ductility by $\sim 300 \%$, and energy dissipation by about $1200 \%$. Depending upon the reinforcing scheme(s), the magnitude of failure- loads and patterns varied in a controlled manner. Joints tested in "control" condition exhibited shear failure through diagonal tension and diagonal compression while the strengthened specimens failed in beam flexure or column shear, but in a ductile manner through yielding of the column- or beam- rebars. The strains measured on rebar surfaces at different locations of joint- and beam- sections revealed a significant 
reduction in strain progression towards the joint panel (beam-column overlap). It was also noted that the use of low-stiffness filler-modules such as syntactic foam and engineered wood coupled with FRP wraps has tremendously enhanced the structural response of reinforced joints. It was also observed that joints reinforced with filler-modules and FRP wraps or gussets exhibiting beam flexural failure had more energy dissipation capacity when compared to specimens that failed in column shear. Based on the experimental results of reinforced joint specimens, joint behavior is characterized into three zones, i.e., A (onset of filler-module cracking), B (idealized yield - defined as the point beyond which a truss mechanism primarily resists the forces), and C (Peak load referred to as a highest numerical value of load recorded during the testing). Furthermore, limit states (principal tension and shear) for joint at the onset of filler-module cracking (i.e., point A) and idealized yield (i.e., point B) have been established as a function of the concrete tensile strength $\left(k \sqrt{f_{c}^{\prime}}\right)$.

The outcomes of this research have proven the ability of the proposed approach in strengthening concrete joints cost-effectively; thus, the overall structural integrity. Although the scope of this dissertation is limited to the evaluation of exterior beam-column joints designed before 1976, the concepts can be extended to other joint configurations, including timber and steel construction. Future research on the proposed approach must be directed towards evaluating the performance of joints with additional stiffness contributions from the slab, and transverse beams to establish joint curvature limit states. Furthermore, machine learning tools must be employed to train, evaluate, and develop strength prediction models after generating additional test data in a strategic sense with an understanding developed based on the current research. Besides, finite element analysis studies on joint inelastic behavior must be performed by incorporating material nonlinearity (postcracking behavior of concrete joint or element) to arrive at the optimized shape of filler-modules and optimized fiber orientation of FRP wraps/gussets as a function of the substrate strength versus stiffness and bond strengths of glue lines of the substrate, filler-modules and FRP wrap or gusset. 


\section{Dedication}

This dissertation is dedicated

To my

Father: Dr. Koti Reddy Majjigapu

Mother: Smt. Padma Majjigapu

Brother: Mr. Naveen Majjigapu 


\section{Acknowledgements}

I would like to express my sincere gratitude to my advisor, Dr. Hota V.S. GangaRao, for allowing me to develop this research project and for providing technical expertise, constant feedback and guidance, and funding that made this research possible. The amount of technical knowledge and recognition gained from this research would not have been possible without his mentorship.

I would also like to thank my dissertation committee members: Dr. Udaya B. Halabe, Dr. P.V. Vijay, Dr. Ruifeng R. Liang, and Dr. Karthik N. Ramanathan for spending their time to evaluate my research and provide constructive feedback. Their support at every stage of this research was invaluable.

This research would not have been possible without the outstanding help received from facility manager - Jerry Nestor; engineering scientist - Mark Skidmore; fellow graduate and undergraduate students - Andrew Pacifico, Shane Moore, Sebastian Bravo, Brian Walker and numerous other students during the experimental evaluations. The time spent and efforts made by all these folks are sincerely appreciated.

I am also grateful to the following organizations for their materials and services provided: Sika Corporation, Weyerhaeuser Buckhannon Plant, Engineered Syntactic Systems, and Precision Concrete. Special thanks are extended to Dr. Gregory Dhale for allowing me to use his Digital Image Correlation equipment.

I am greatly indebted to Aneesh Bethi and Prof. Sreenivasa Sarma for all the support, encouragement, and guidance that I received at every stage of my graduate studies.

This research was funded in part by the Maurice A. and JoAnn Wadsworth Professorship and in part by the National Science Foundation I/UCRC Center for Integration of Composites into Infrastructure. Besides, this research was also supported through WVU Foundation Distinguished Doctoral Scholarship, WVU Outstanding Merit Provost Doctoral Fellowship, Maurice A. and JoAnn Wadsworth Doctoral Fellowship, and NACE Foundation AUCSC Scholarship. 
Technical discussions and assistance from my colleagues and friends Dr. Piyush Soti, Dr. Jonas Kavi, Amir Houshmandyar, Guadalupe Leon, Seyednavid Mardmomen, Abhishek Regalla, Rajneesh Bharil, Shabnam Khanal, Ruben Joshi, John Harper, Ben Imes, Maria Martinez De Lahidalga and is much appreciated.

I am very grateful to Dr. A. R. Chandra Sekaran, Dr. V.K. Raju, Ashutosh Surampalli, Smt. Padma Surampalli, Viswanath Rao Surampalli, Dr. Sudhir Reddy Thaduri, Dr. Sathi Reddy Vadlakonda, Dr. Ravindra Mohan, Prof. Balaji Rao, Dr. Ramasheshu, Dr. Laxminarayana Pappula, B. Venkat Reddy, Mary Dillon, Anudeep Paraitham, Dheeraj Gadireddy, Dr. Vidyani Suryadevara, Sai Krishna Ganapuram, Santosh Reddy Vangala, Daniel Estep, Viswanath Cheruku, Aron Gebreslase, Martin James Vellore, Vishal Chakravarthy, Sai Tejesh Jammula, Dr. Srikanth Bajaj, and Rajeev Neela for their unconditional love and support throughout the years.

Finally, I am incredibly grateful to my parents, teachers, family, and friends for their loving support, encouragement, and patience. This dissertation is dedicated to each one of them. 


\section{Motivation}

"When things go wrong, as they sometimes will;

When the road you're trudging seems all uphill;

When the funds are low and the debts are high;

And you want to smile but you have to sigh.

When all is pressing you down a bit-

Rest if you must, but don't you quit

Success is failure turned inside out;

The silver tint on the clouds of doubt;

And you can never tell how close you are;

It may be near when it seems far.

So stick to the fight when you're hardest hit-

It's when things go wrong that you must not quit."

-John Greenleaf Whittier 


\section{Table of Contents}

Table of Contents .......................................................................................................................... viii

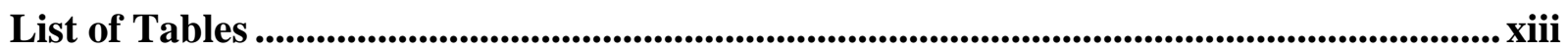

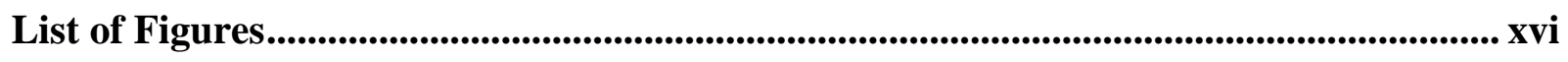

1. Introduction............................................................................................................................ 1

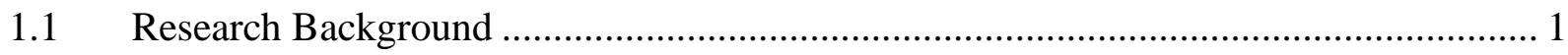

1.2 Joint Reinforcement with Filler-Modules and FRP Wraps or Gussets....................... 4

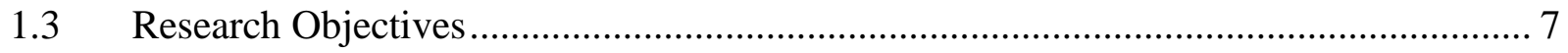

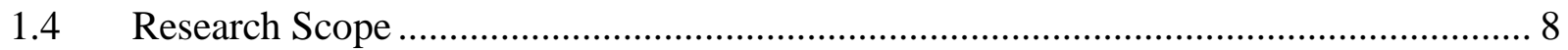

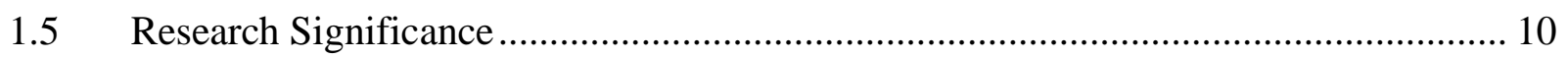

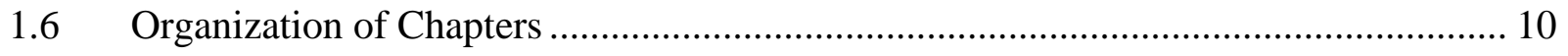

2. RC Exterior Joints Built Before 1976: A Brief Review .............................................. 12

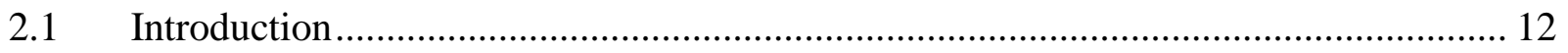

2.2 Vulnerability of Buildings with Deficient Joints under Extreme Events .................. 12

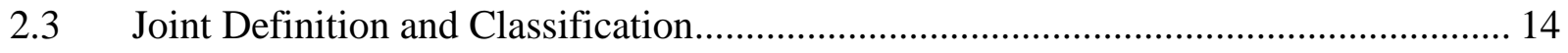

2.4 Force Transfer Mechanism in 2D RC Exterior Joints ......................................... 16

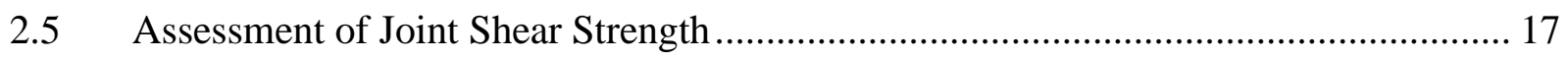

2.5.1 Computation of principal stresses in a joint panel ........................................... 19

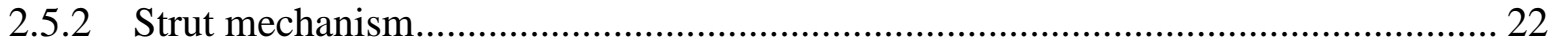

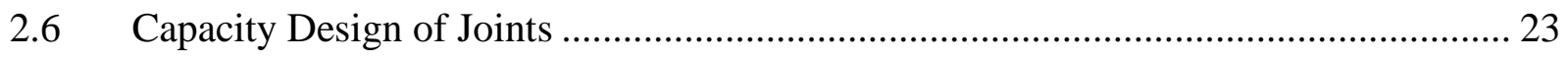

2.7 Parameters Influencing the Behavior of RC Exterior Joints................................. 24

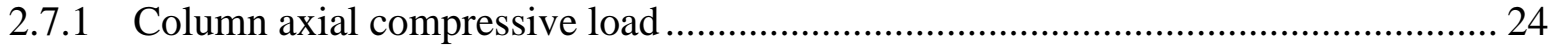

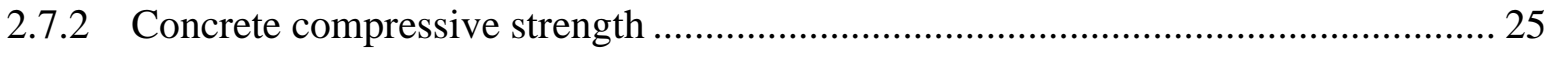

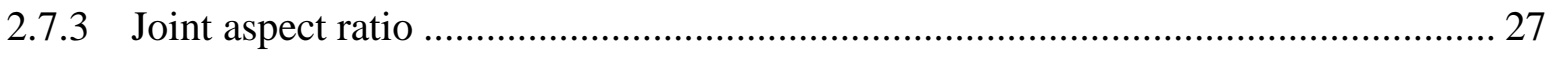

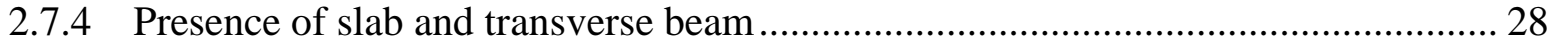




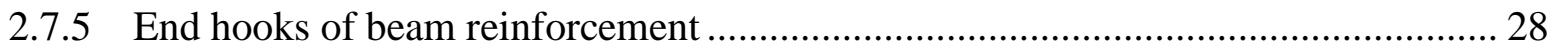

2.7.6 Joint transverse (shear) reinforcement ..................................................................... 29

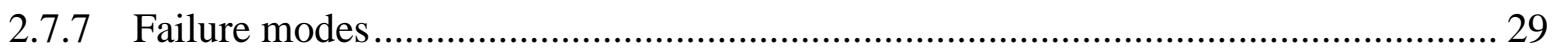

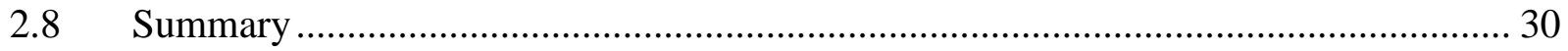

3. RC Exterior Joint Repair with FRP Composites: A Brief Review .................................. 32

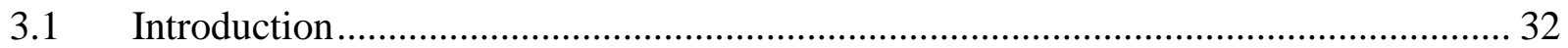

3.2 FRP Composites in Repair and Rehabilitation of Infrastructure ………….................. 32

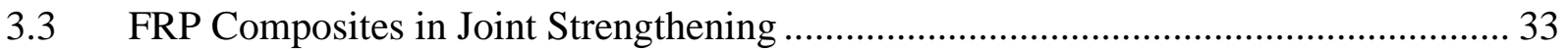

3.4 Previous Experimental Studies on FRP Composites in Joint Strengthening ................ 34

3.4.1 Effectiveness of FRP wraps on joint shear strengthening ……………………...... 34

3.4.2 Effectiveness of FRP anchors in preventing debond .............................................. 39

3.4.3 Influence of FRP confinement on bond strength of hooked bars ............................. 44

3.4.4 Influence of surface preparation on rehab effectiveness............................................ 49

3.4.5 Effectiveness of FRP wrap layup on joint strengthening .......................................... 53

3.4.6 Effectiveness of joint core confinement through FRP wrapping .............................. 58

3.4.7 Influence of FRP strengthening on joints with transverse beams ............................... 61

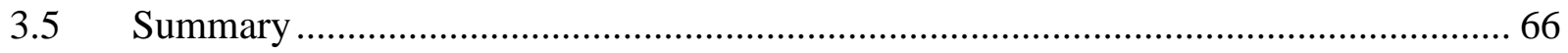

4. Experimental Program: Materials, Casting, Test Protocols, and Data Procurement.. 68

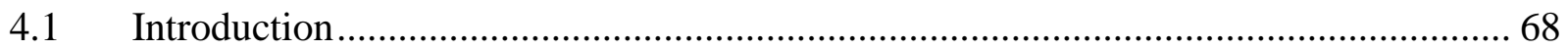

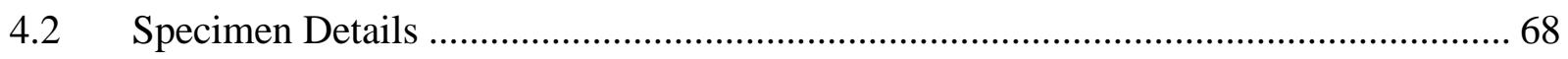

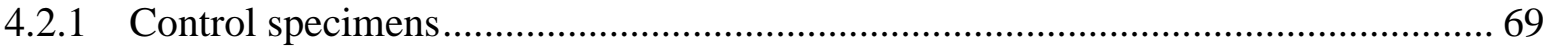

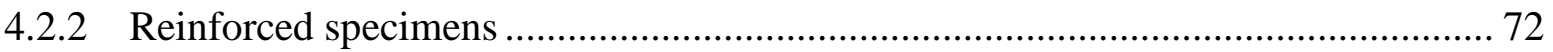

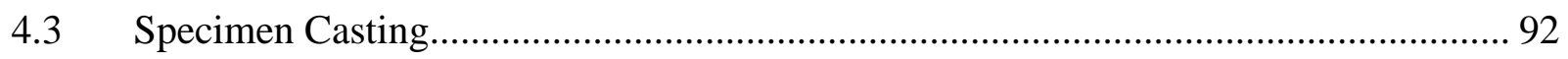

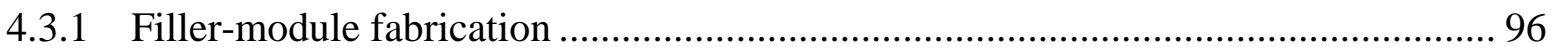

4.3.2 Installation of filler-modules at joint corners......................................................... 99

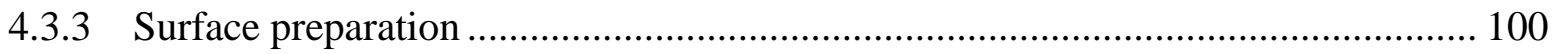

4.3.4 FRP wrap/gusset installation............................................................................... 101

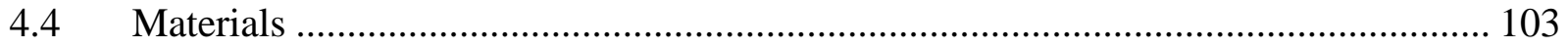




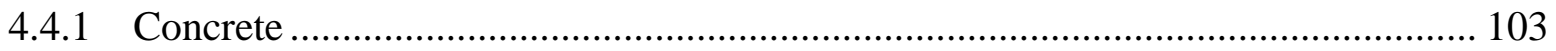

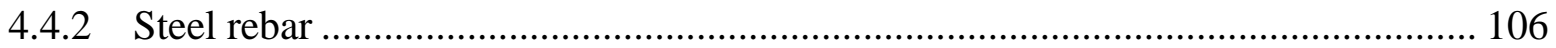

4.4.3 Engineered wood (PSL) .............................................................................. 109

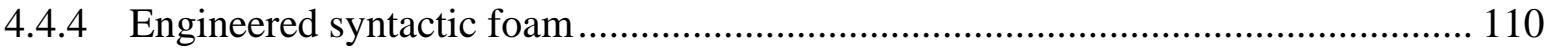

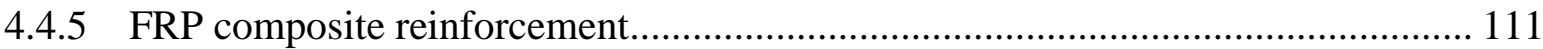

4.4.6 FRP composite confined concrete cylinders .......................................................... 111

$4.5 \quad$ Test Setup of 2D RC Exterior Joints....................................................................... 112

$4.6 \quad$ Loading Protocol.................................................................................................. 114

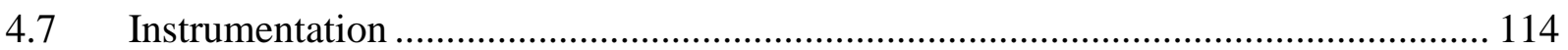

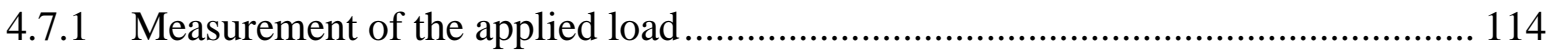

4.7.2 Measurement of displacement …………............................................................ 115

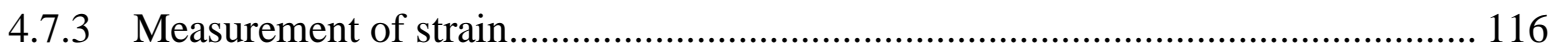

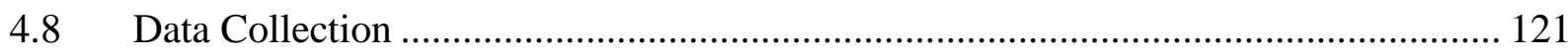

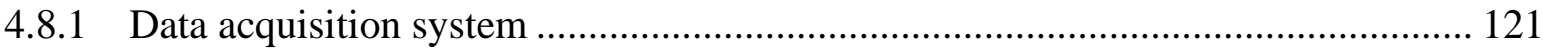

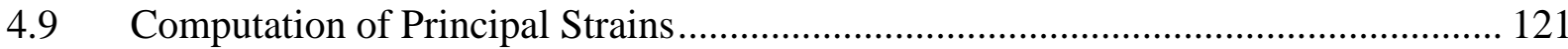

4.10 Computation of Shear Strain............................................................................ 122

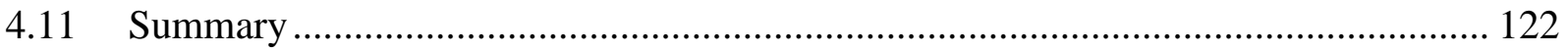

\section{Behavior of RC Joints Reinforced with Concrete/Syntactic Foam Filler-Modules and} FRP Wraps/Gussets ....................................................................................................................................... 123

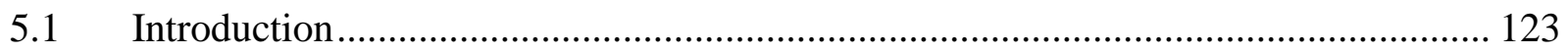

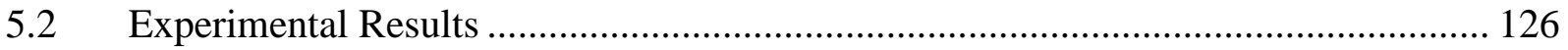

5.2.1 Behavior of control specimens (JI-1, JI-5, and JIII-1) .......................................... 126

5.2.2 Behavior of joint reinforced with concrete filler-modules (JI-2)........................... 134

5.2.3 Behavior of joints reinforced with filler-modules and FRP wraps/gussets ............ 138

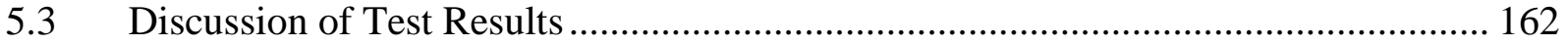

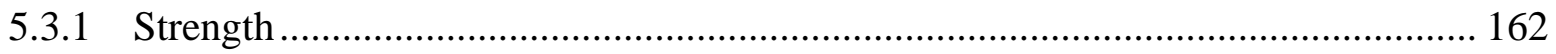

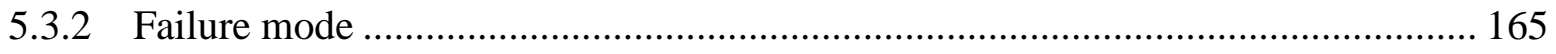

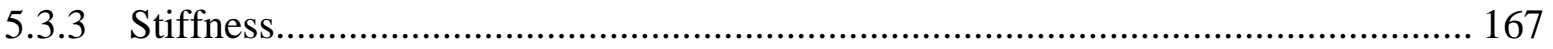




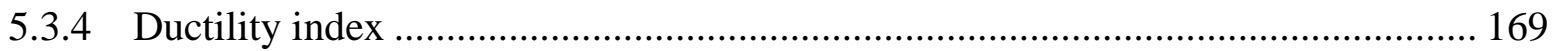

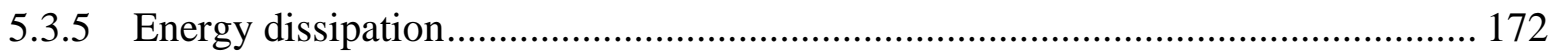

5.3.6 Strains in joint-, beam-, and column- rebars....................................................... 175

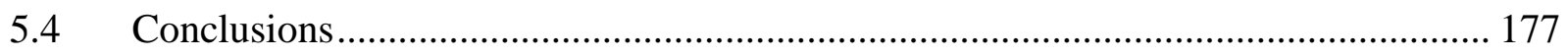

6. Behavior of RC Joints Reinforced with Engineered Wood (PSL) Filler-Modules and FRP Wrap....................................................................................................................................... 180

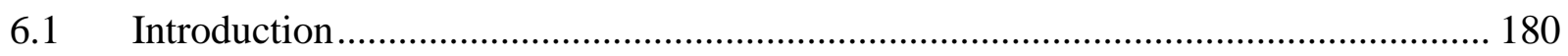

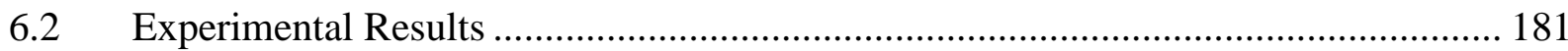

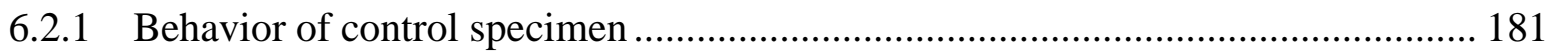

6.2.2 Behavior of joint reinforced with CFRP wrap .................................................... 183

6.2.3 Behavior of joints reinforced with PSL curve- and wedge- filler-modules............ 185

6.2.4 Behavior of joint reinforced with PSL curve filler-modules and CFRP wraps without

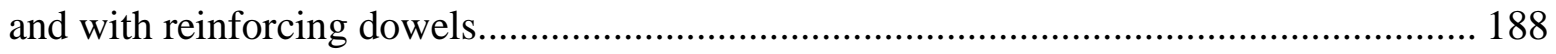

6.2.5 Behavior of joint reinforced with PSL wedge filler-modules and CFRP/GFRP wraps

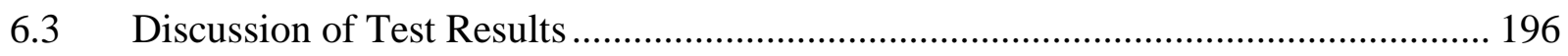

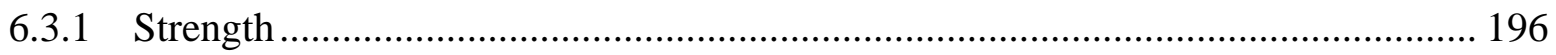

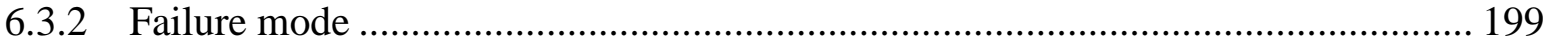

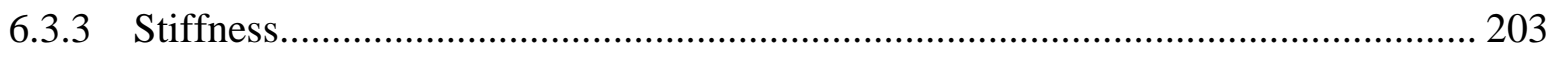

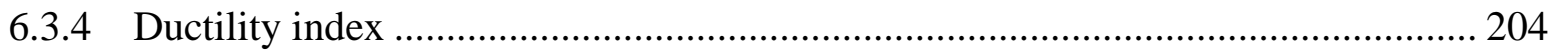

6.3.5 Energy dissipation ..................................................................................... 206

6.3.6 Strains in joint-, beam-, and column- rebars....................................................... 208

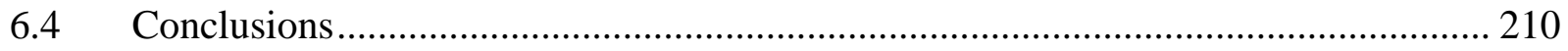

7. Summary, Conclusions, and Recommendations ..................................................................... 212

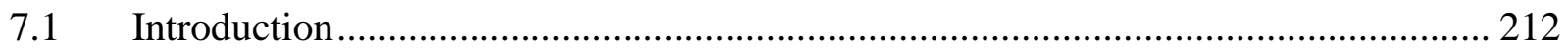

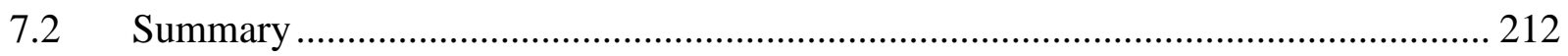

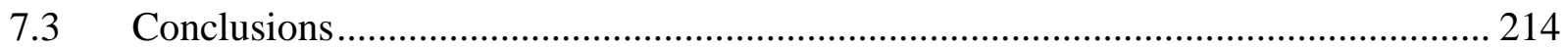

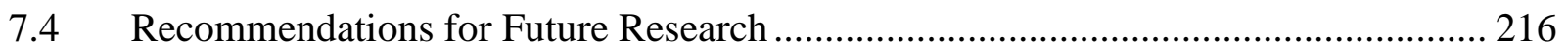


References

Appendix-A: Beam-Column Joints Reinforced with Concrete/Syntactic Foam FillerModules and CFRP Wraps..............................................................230

Appendix-B: Beam-Column Joints Reinforced with Concrete Filler-Modules and CFRP Gussets.

Appendix-C: Beam-Column Joints Reinforced with Concrete Filler-Modules and GFRP

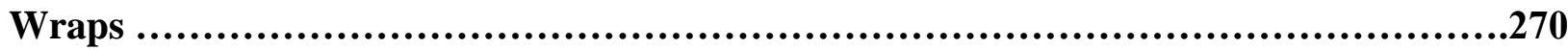

Appendix-D: Beam-Column Joints Reinforced with Engineered Wood Filler-Modules and

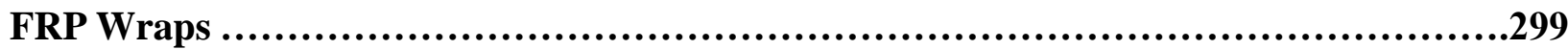

Appendix-E: FRP Wrapped Concrete Cylinders.........................................320 


\section{List of Tables}

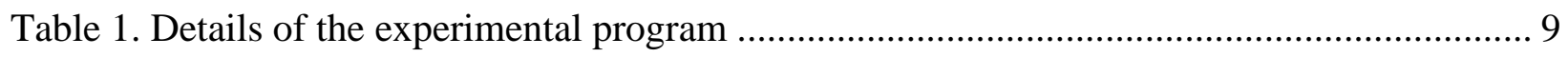

Table 2. Principal tensile stress limit states based on the available literature ......................... 21

Table 3. Summary of exterior RC joint failure modes [39] ............................................... 30

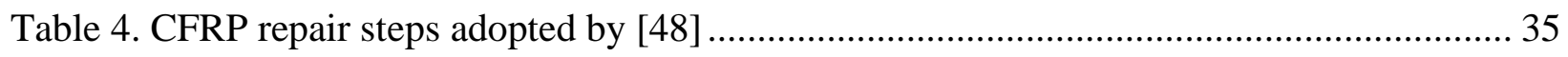

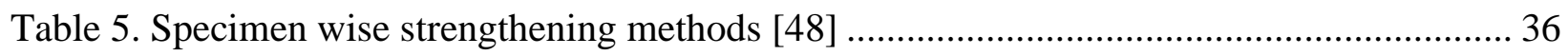

Table 6. Description of retrofit schemes [49] .................................................................. 41

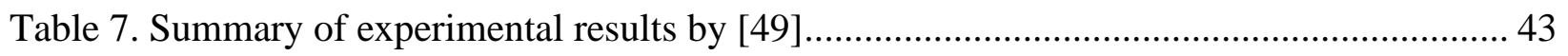

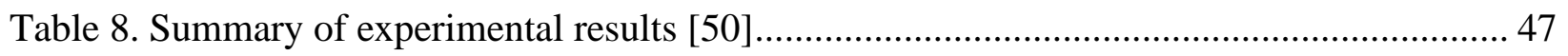

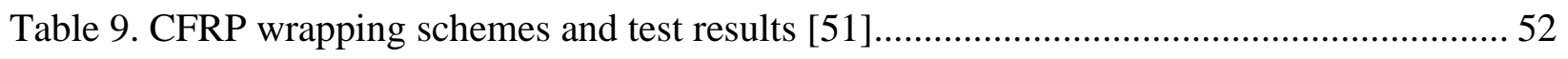

Table 10. Description of CFRP configurations [52] ........................................................ 55

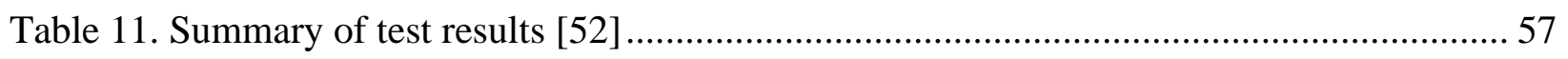

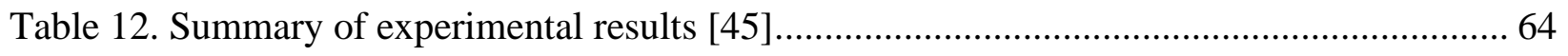

Table 13. Beam-column joint reinforcing scheme for JI-2 through JI-4 .............................. 75

Table 14. Beam-column joint reinforcing scheme for JI-6 through JI-8 ............................... 78

Table 15. Beam-column joint reinforcing scheme for JIII-2 through JIII-4 .......................... 82

Table 16. Beam-column joint reinforcing scheme for JII-2 …......................................... 86

Table 17. Beam-column joint reinforcing scheme for JII-3 and JII-4 _................................ 88

Table 18. Beam-column joint reinforcing scheme for JII-5 through JII-8 ............................. 89

Table 19. Properties of plain concrete (joint) specimens................................................ 104

Table 20. Properties of plain concrete (filler-modules) specimens ..................................... 105 
Table 21. Properties of steel rebar specimens 106

Table 22. Design properties of Parallam® PSL [54]..... 109

Table 23. Mechanical properties of SF grade syntactic foam [55] 110

Table 24. Properties of FRP composite materials [56] [57] 111

Table 25. Properties of FRP confined plain concrete (compression test)

Table 26. Properties of FRP confined plain concrete (split-tension test)

Table 27. Parameters tested to evaluate the performance of joints reinforced with concrete/syntactic foam filler-modules with and without FRP wraps/gussets 125

Table 28. Modulus of rupture for control joints (JI-1, JI-5, JIII-1) 127

Table 29. Principal strain limit state for control joints without axial load influence. 127

Table 30. Principal strain limit state for control joints with axial load influence. 127

Table 31. Summary of experimental results of joints reinforced with concrete filler-modules . 135

Table 32. Modulus of rupture of joints with filler-modules and CFRP wraps 140

Table 33. Summary of experimental results of joints with concrete/syntactic foam filler-modules with/out CFRP wraps 141

Table 34. Modulus of rupture of joints with filler-modules and CFRP gussets 146

Table 35. Summary of experimental results of joints with filler-modules and CFRP gussets ... 148

Table 36. Modulus of rupture of joints with filler-modules and GFRP wraps 155

Table 37. Summary of experimental results of joints with filler-modules and GFRP wraps ..... 156

Table 38. Load vs. deflection response of joints with filler-modules and FRP wraps/gussets... 164

Table 39. Stiffness of joints reinforced with filler-modules and FRP wraps/gussets ....... 167

Table 40. Ductility index of joints with filler-modules and FRP wraps/gussets 170 
Table 41. Energy dissipation capacity of joints with filler-modules and FRP wraps/gussets .... 174

Table 42. Rebar strain data of joints with filler-modules and FRP wraps/gussets 176

Table 43. Summary of test results: joints with filler-modules with/out FRP wraps or gussets .. 177

Table 44. Parameters tested to evaluate the performance of joints reinforced with PSL fillermodules and FRP wraps 180

Table 45. Modulus of rupture of joints reinforced with PSL filler-modules and FRP wraps..... 194

Table 46. Summary of experimental results of joints reinforced with PSL filler-modules and FRP wraps 195

Table 47. Load vs. deflection of joints reinforced with PSL filler-modules and FRP wraps ..... 196

Table 48. Failure modes of joints reinforced with PSL filler-modules and FRP wraps ............. 200

Table 49. Stiffness of joints reinforced with PSL filler-modules and FRP wraps................... 203

Table 50. Ductility index of joints reinforced with PSL filler-modules and FRP wraps........... 204

Table 51. Energy dissipation of joints reinforced with PSL filler-modules and FRP wraps...... 206

Table 52. Rebar strain data of joints reinforced with PSL filler-modules and FRP wraps........ 208

Table 53. Summary of test results: joints reinforced with PSL filler-modules and FRP wraps . 210 


\section{List of Figures}

Figure 1. Low-rise building collapse during 2016 Ecuador earthquake [9] .............................. 2

Figure 2. School building damage in Eastern Turkey due to exterior joint failure [10]............... 3

Figure 3. 2D exterior (T) joint reinforcement using filler-modules and FRP wraps [33]-[34] ...... 5

Figure 4. Beam-column-slab joint reinforcement using filler-modules and FRP gussets [35] ...... 6

Figure 5. 2016 Ecuador earthquake - building damage (Juan Cevallos/Getty Images) [17]....... 13

Figure 6. Joint classification based on the arrangement in a framed system [19] ..................... 15

Figure 7. Joint sub-classification based on the arrangement of beams and columns [20]........... 15

Figure 8. The force-transfer mechanism in a most practical case of RC exterior joint [19] ........ 16

Figure 9. Resultant forces on a 2D exterior joint under beam and column loads [21] ............... 17

Figure 10. Diagonal stress fields in an exterior joint [22] ............................................... 19

Figure 11. Strut mechanism of shear force transfer in an exterior joint [26].......................... 22

Figure 12. Joint shear/rebar bond deterioration failure in exterior joints [27]......................... 23

Figure 13. Effect of column axial load on joint shear strength [32] .................................... 25

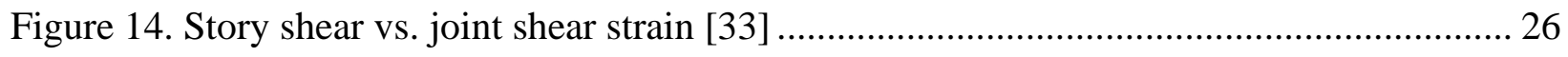

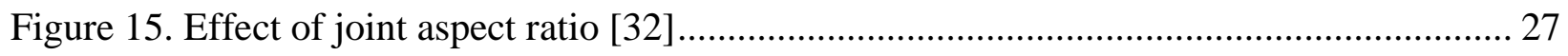

Figure 16. Steel reinforcement detailing of control and retrofitted test specimens [48] ............. 34

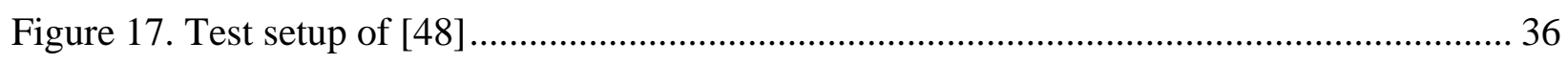

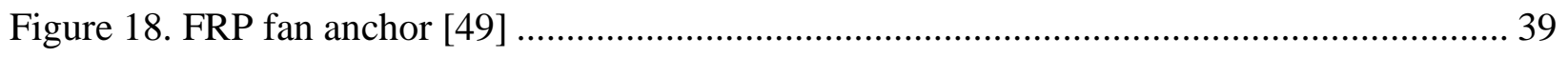

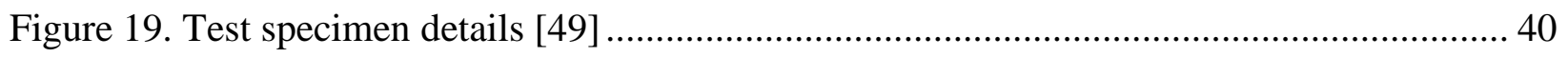

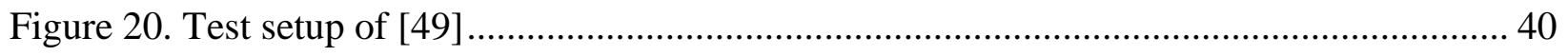




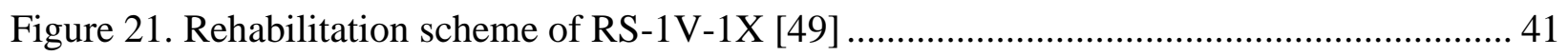

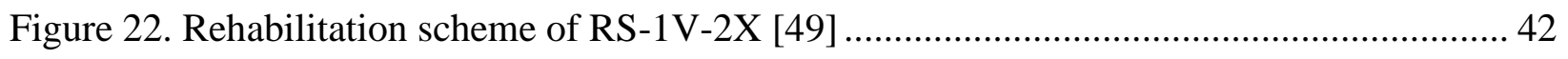

Figure 23. Rehabilitation scheme of RS-1V-2X-2H.I [49] ................................................ 42

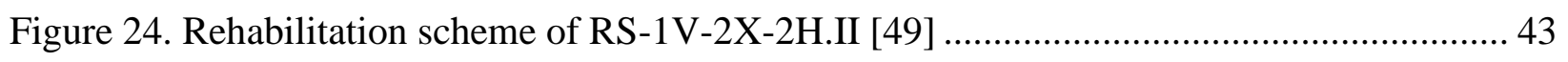

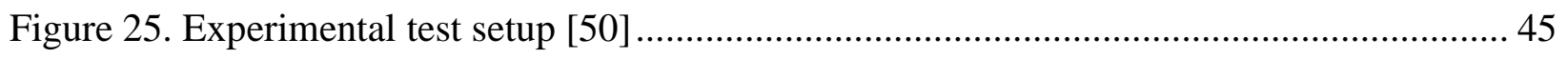

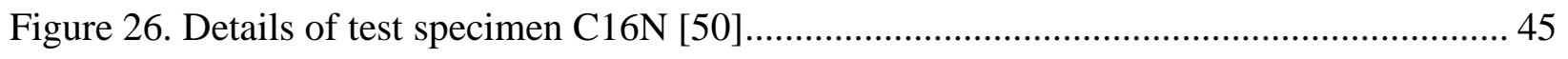

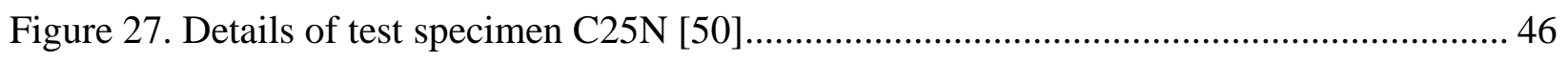

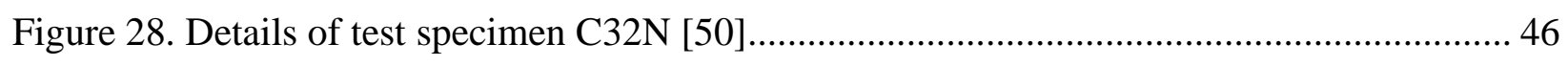

Figure 29. CFRP wrap details for C16N, C25N, and C32N [50] ...................................... 47

Figure 30. Test specimen reinforcement details and dimensions [51] .................................. 49

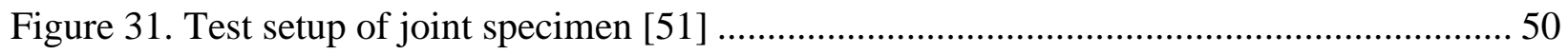

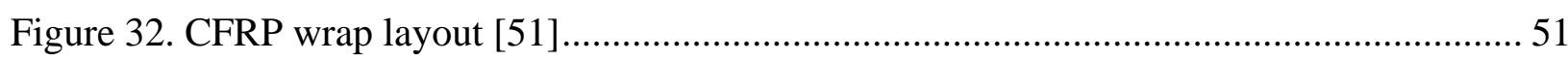

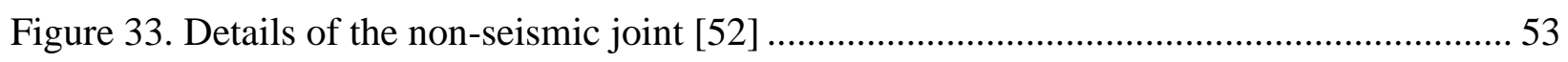

Figure 34. CFRP configurations for strengthening non-seismic joints [52] ........................... 54

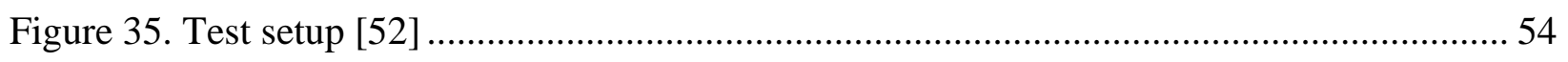

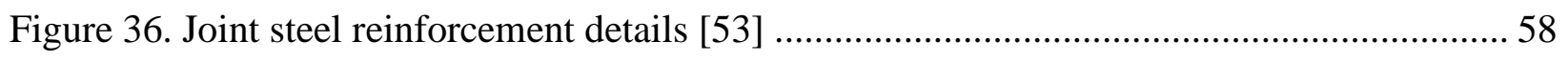

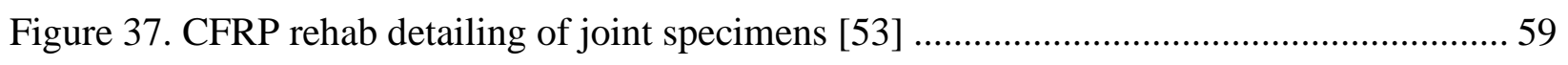

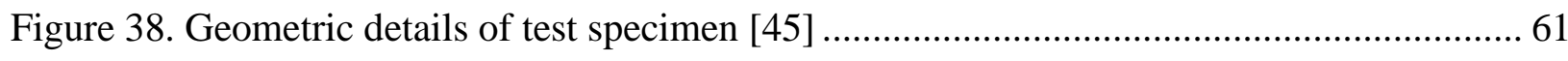

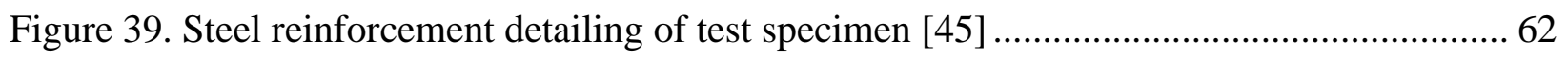

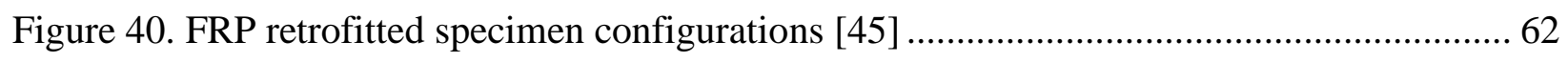

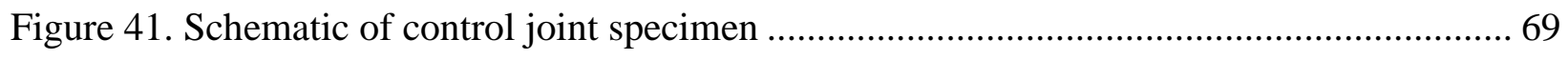


Figure 42. Geometric layout of control joint specimens

Figure 43. Steel rebar layout of control joint specimens .................................................. 71

Figure 44. Geometric layout of joint specimens reinforced with wedge filler-module.............. 72

Figure 45. Steel rebar layout of joint specimens reinforced with wedge filler-module ............. 73

Figure 46. Steel rebar layout of joint specimens reinforced with curve filler-module ............... 74

Figure 47. Plywood formwork for casting concrete joint specimens .................................. 92

Figure 48. Preparation of reinforcement cage for beam and column sections.......................... 93

Figure 49. Grinding at select locations of rebar cage for installation of strain gages................ 93

Figure 50. Closer view of the reinforcement cage after assembling in a mold ........................ 94

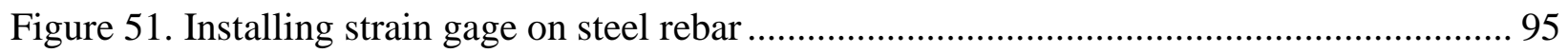

Figure 52. Placement of concrete during test specimen preparation ................................... 96

Figure 53. Casting of concrete filler-modules ................................................................ 97

Figure 54. Making of Parallel Strand Lumber (PSL) filler-modules ...................................... 98

Figure 55. Filler-module installation process at joint corners ........................................... 99

Figure 56. Surface preparation through water-jet washing .............................................. 100

Figure 57. Concrete surface before- and after- water-jet washing ...................................... 100

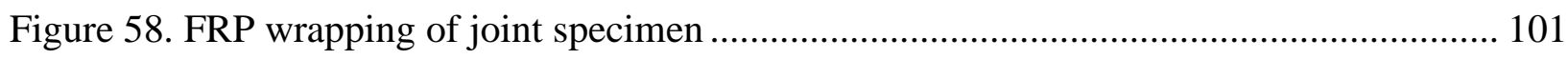

Figure 59. FRP gusset used in the current study for reinforcing joint specimens ................... 102

Figure 60. Load vs. position curves for plain concrete (joint) specimens ............................. 104

Figure 61. Load vs. position curves for plain concrete (filler-modules) specimens ................. 105

Figure 62. Failure modes of grade 60 \#6 rebar specimens ............................................... 107 
Figure 63. Failure modes of grade $60 \# 3$ rebar specimens 107

Figure 64. Stress-strain plots for grade 60 \#6 steel rebar specimens...................................... 108

Figure 65. Stress-strain plots for grade 60 \#3 steel rebar specimens......................................... 108

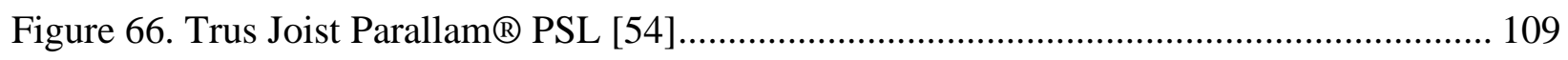

Figure 67. SF 3000 grade microsphere syntactic foam............................................................ 110

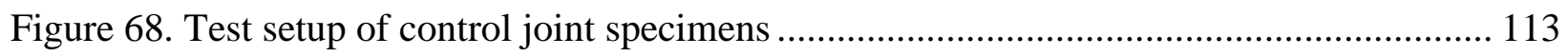

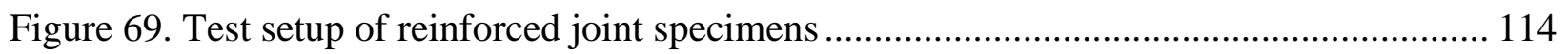

Figure 70. Linear variable displacement transducer (LVDT) (OMEGA Engineering) [58] ...... 115

Figure 71. Schematic view of strain gage arrangement on steel rebar cage …......................... 116

Figure 72. Strain gage arrangement on the concrete surface ................................................ 117

Figure 73. Strain gage arrangement on FRP wrap surface ………….................................. 118

Figure 74. Strain gage arrangement on FRP wrap surface ……………............................... 119

Figure 75. Strain gage arrangement on FRP wrap surface ……………………................... 120

Figure 76. Vishay 7000 data acquisition system [59] ............................................................ 121

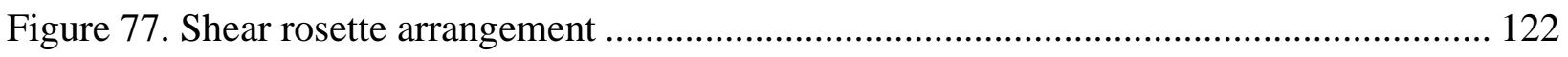

Figure 78. Load vs. column rebar strain response of a typical joint specimen in this study ...... 124

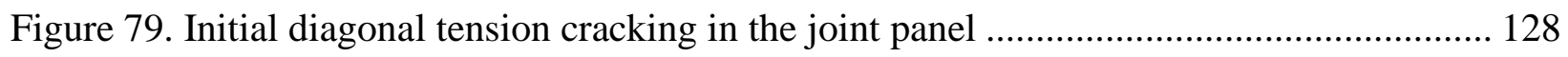

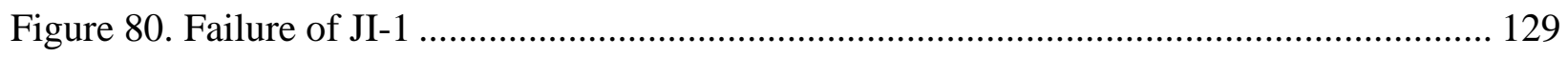

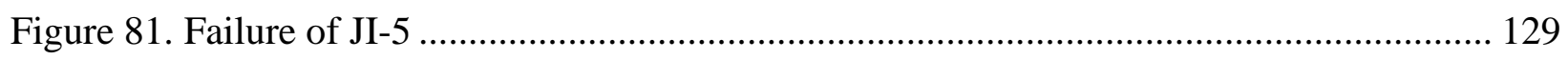

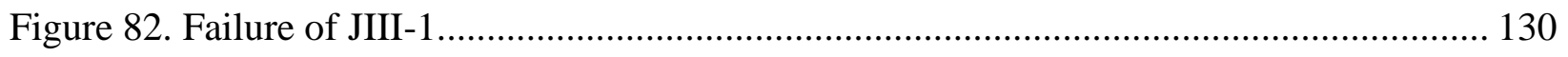

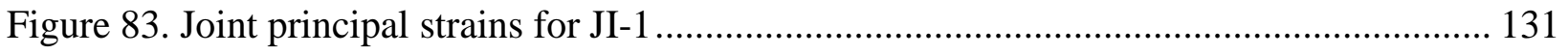

$$
\text { xix }
$$


Figure 84. Joint shear strain for JI-1

Figure 85. Joint principal strains for JI-5

Figure 86. Joint shear strain for JI-5

Figure 87. Joint principal strains for JIII-1

Figure 88. Joint shear strain for JIII-1 133

Figure 89. Failure of JI-2 (front view) 136

Figure 90. Failure of JI-2 (rear view) 136

Figure 91. Column principal strains for JI-2 137

Figure 92. Column shear strain for JI-2 .

Figure 93. Failure of JI-3 142

Figure 94. Failure of JI-4 142

Figure 95. Joint principal strains for JI-3 143

Figure 96. Joint shear strain for JI-3 143

Figure 97. Joint principal strains for JI-4 144

Figure 98. Joint shear strain for JI-4 144

Figure 99. Strain response along the longitudinal axis of filler-module (JI-7) 147

Figure 100. Strain response close to the filler-module tip on beam section (JI-8) 147

Figure 101. Failure of JI-6 149

Figure 102. Failure of JI-7 149

Figure 103. Failure of JI-8 150

Figure 104. Joint principal strains for JI-6 151 
Figure 105. Joint shear strain for JI-6

Figure 106. Joint principal strains for JI-7 152

Figure 107. Joint shear strain for JI-7 152

Figure 108. Joint principal strains for JI-8 153

Figure 109. Joint shear strain for JI-8 153

Figure 110. Failure of JIII-2 157

Figure 111. Failure of JIII-3 157

Figure 112. Failure of JIII-4 158

Figure 113. Joint principal strains for JIII-2 159

Figure 114. Joint shear strain for JIII-2 159

Figure 115. Joint principal strains for JIII-3 160

Figure 116. Joint shear strain for JIII-3 160

Figure 117. Joint principal strains for JIII-4 161

Figure 118. Joint shear strain for JIII-4 161

Figure 119. Load vs. deflection response of joints with filler-modules with/out CFRP wraps.. 163

Figure 120. Load vs. deflection response of joints with filler-modules and CFRP gussets ....... 163

Figure 121. Load vs. deflection response of joints with filler-modules and GFRP wraps ......... 164

Figure 122. Buckling of the column longitudinal rebars (JI-2) ......................................... 166

Figure 123. Cantilever beam with beam tip deflection .................................................... 167

Figure 124. Force-displacement relationship of a ductile structure [63] .............................. 169

Figure 125. Energy dissipation capacity of joints with filler-modules and CFRP wraps 172 
Figure 126. Energy dissipation capacity of joints with filler-modules and CFRP wraps 173

Figure 127. Energy dissipation capacity of joints with filler-modules and GFRP wraps. 173

Figure 128. Failure of specimen JII-1 181

Figure 129. Joint principal strains in JII-1 182

Figure 130. Joint shear strain in JII-1 182

Figure 131. Failure of specimen JII-2 183

Figure 132. Joint principal strains in JII-2 184

Figure 133. Joint shear strains in JII-2. 184

Figure 134. Failure of specimen JII-3 (left) and JII-4 (right) ...... 185

Figure 135. Column principal strain in JII-3 ..... 186

Figure 136. Column shear strain JII-3 186

Figure 137. Column principal strain in JII-4 187

Figure 138. Column shear strain in JII-4 187

Figure 139. Failure of specimen JII-5 (left) and JII-6 (right) 188

Figure 140. Joint principal strain in JII-5. 189

Figure 141. Joint shear strain in JII-5 189

Figure 142. Joint principal strain in JII-6. 190

Figure 143. Joint shear strain in JII-6 190

Figure 144. Failure of specimen JII-7 (left) and JII-8 (right) 191

Figure 145. Joint principal strain in JII-7. 192

Figure 146. Joint shear strain in JII-7 192 
Figure 147. Joint principal strain in JII-8. 193

Figure 148. Joint shear strain in JII-8 193

Figure 149. Load vs. deflection of joints reinforced with PSL filler-modules and FRP wraps.. 197 Figure 150. Energy dissipation of joints reinforced with PSL filler-modules and FRP wraps .. 206

Figure A.1. Beam load vs. column longitudinal rebar strain 230

Figure A.2. Beam load vs. beam longitudinal rebar strain 231

Figure A.3. Beam load vs. joint tail rebar strain 231

Figure A.4. Beam load vs. column longitudinal rebar strain 232

Figure A.5. Beam load vs. beam longitudinal rebar strain 232

Figure A.6. Beam load vs. joint tail rebar strain 233

Figure A.7. Beam load vs. concrete strain in joint panel 233

Figure A.8. Beam load vs. filler-module longitudinal strain 234

Figure A.9. Beam load vs. column longitudinal rebar strain 235

Figure A.10. Beam load vs. beam longitudinal rebar strain 235

Figure A.11. Beam load vs. beam stirrup strain 236

Figure A.12. Beam load vs. column principal strain 236

Figure A.13. Beam load vs. column shear strain 237

Figure A.14. Beam load vs. filler-module longitudinal strain 237

Figure A.15. Beam load vs. filler-module longitudinal strain . 238

Figure A.16. Beam load vs. column longitudinal rebar strain ....... 239

Figure A.17. Beam load vs. beam longitudinal rebar strain 239 
Figure A.18. Beam load vs. filler-module longitudinal strain

Figure A.19. Beam load vs. filler-module longitudinal strain 240

Figure A.20. Beam load vs. column principal strain 241

Figure A.21. Beam load vs. column shear strain 241

Figure B.1. Beam load vs. column longitudinal rebar strain 242

Figure B.2. Beam load vs. beam longitudinal rebar strain. 243

Figure B.3. Beam load vs. joint tail rebar strain 243

Figure B.4. Beam load vs. beam stirrup strain. 244

Figure B.5. Beam load vs. concrete strain in beam 244

Figure B.6. Beam load vs. concrete strain in beam 245

Figure B.7. Beam load vs. concrete strain in column 245

Figure B.8. Beam load vs. column longitudinal rebar strain 246

Figure B.9. Beam load vs. beam longitudinal rebar strain 246

Figure B.10. Beam load vs. beam stirrup strain.... 247

Figure B.11. Beam load vs. FRP strain in beam 248

Figure B.12. Beam load vs. beam principal strain . 248

Figure B.13. Beam load vs. beam shear strain. 249

Figure B.14. Beam load vs. filler-module longitudinal strain 249

Figure B.15. Beam load vs. filler-module longitudinal strain 250

Figure B.16. Beam load vs. column FRP strain..... 250

Figure B.17. Beam load vs. column rebar FRP strain 251 
Figure B.18. Beam load vs. FRP strain in joint panel............................................................ 251

Figure B.19. Beam load vs. filler-module FRP strain............................................................ 252

Figure B.20. Beam load vs. FRP strain in column and filler-module...................................... 252

Figure B.21. Beam load vs. FRP strain in column and filler-module ......................................... 253

Figure B.22. Beam load vs. column longitudinal rebar strain ................................................. 254

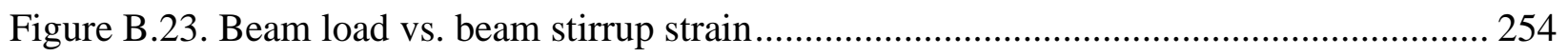

Figure B.24. Beam load vs. beam longitudinal rebar strain.................................................... 255

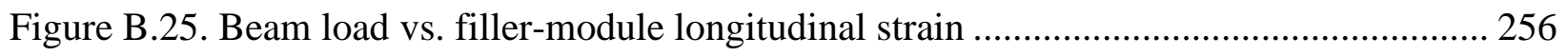

Figure B.26. Beam load vs. filler-module longitudinal strain ................................................ 256

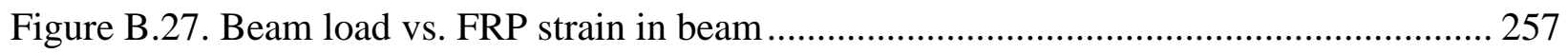

Figure B.28. Beam load vs. FRP strain in joint panel.......................................................... 257

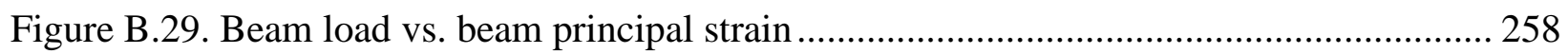

Figure B.30. Beam load vs. beam shear strain.................................................................. 258

Figure B.31. Beam load vs. filler-module FRP strain............................................................. 259

Figure B.32. Beam load vs. FRP strain in column and filler-module....................................... 259

Figure B.33. Beam load vs. FRP strain in column and filler-module......................................... 260

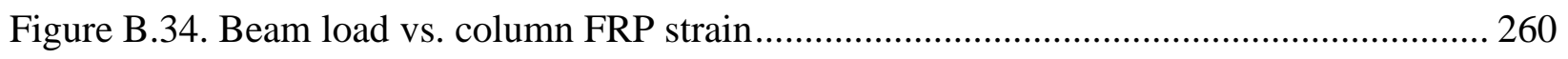

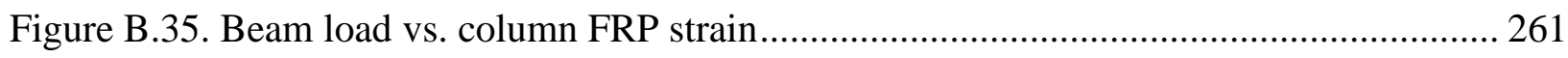

Figure B.36. Beam load vs. column longitudinal rebar strain ................................................... 262

Figure B.37. Beam load vs. beam longitudinal rebar strain.................................................... 262

Figure B.38. Beam load vs. beam stirrup strain ..................................................................... 263 
Figure B.39. Beam load vs. filler-module longitudinal strain 264

Figure B.40. Beam load vs. filler-module longitudinal strain 264

Figure B.41. Beam load vs. FRP strain in beam 265

Figure B.42. Beam load vs. FRP strain in joint panel. 265

Figure B.43. Beam load vs. beam principal strain 266

Figure B.44. Beam load vs. beam shear strain. 266

Figure B.45. Beam load vs. filler-module FRP strain. 267

Figure B.46. Beam load vs. FRP strain in column and filler-module. 267

Figure B.47. Beam load vs. FRP strain in column and filler-module. 267

Figure B.48. Beam load vs. column FRP strain. 268

Figure B.49. Beam load vs. column FRP strain. 269

Figure C.1. Beam load vs. column longitudinal rebar strain 270

Figure C.2. Beam load vs. beam longitudinal rebar strain 271

Figure C.3. Beam load vs. beam stirrup strain. 271

Figure C.4. Beam load vs. joint tail rebar strain 272

Figure C.5. Beam load vs. concrete strain in the joint panel 273

Figure C.6. Beam load vs. concrete strain in the joint panel 273

Figure C.7. Beam load vs. concrete strain in the joint panel 274

Figure C.8. Beam load vs. column strain in the joint panel. 274

Figure C.9. Beam load vs. column longitudinal rebar strain 275

Figure C.10. Beam load vs. beam longitudinal rebar strain 275 
Figure C.11. Beam load vs. beam stirrup strain.

Figure C.12. Beam load vs. filler-module longitudinal strain

Figure C.13. Beam load vs. filler-module longitudinal strain

Figure C.14. Beam load vs. FRP strain in the beam 278

Figure C.15. Beam load vs. FRP strain in the joint panel. 278

Figure C.16. Beam load vs. beam principal strain . 279

Figure C.17. Beam load vs. beam shear strain. 279

Figure C.18. Beam load vs. filler-module FRP strain. 280

Figure C.19. Beam load vs. FRP strain in column and filler-module. 280

Figure C.20. Beam load vs. FRP strain in column and filler-module. 281

Figure C.21. Beam load vs. column FRP strain. 281

Figure C.22. Beam load vs. column FRP strain. 282

Figure C.23. Beam load vs. column longitudinal rebar strain 283

Figure C.24. Beam load vs. beam longitudinal rebar strain..... 283

Figure C.25. Beam load vs. beam stirrup strain. 284

Figure C.26. Beam load vs. filler-module longitudinal strain 285

Figure C.27. Beam load vs. filler-module longitudinal strain 285

Figure C.28. Beam load vs. FRP strain in the beam.... 286

Figure C.29. Beam load vs. FRP strain in the joint panel. 286

Figure C.30. Beam load vs. beam principal strain 287

Figure C.31. Beam load vs. beam shear strain. 287 
Figure C.32. Beam load vs. filler-module FRP strain........................................................... 288

Figure C.33. Beam load vs. FRP strain in column and filler-module....................................... 288

Figure C.34. Beam load vs. FRP strain in column and filler-module....................................... 289

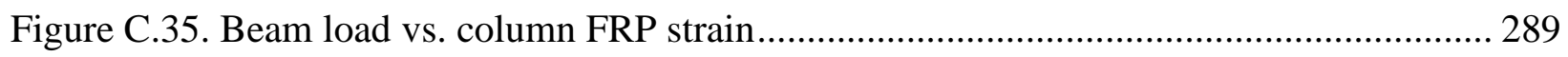

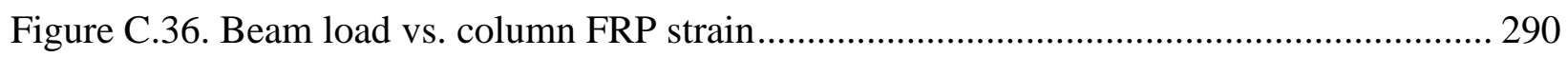

Figure C.37. Beam load vs. column longitudinal rebar strain ................................................ 291

Figure C.38. Beam load vs. beam longitudinal rebar strain...................................................... 291

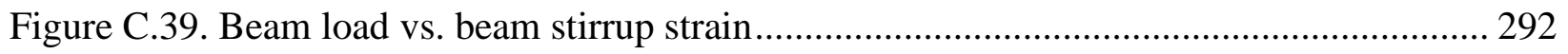

Figure C.40. Beam load vs. filler-module longitudinal strain ................................................. 293

Figure C.41. Beam load vs. filler-module longitudinal strain ................................................. 293

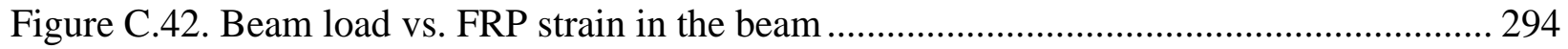

Figure C.43. Beam load vs. FRP strain in the joint panel....................................................... 294

Figure C.44. Beam load vs. beam principal strain ............................................................... 295

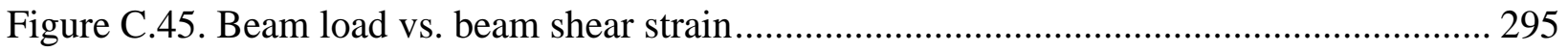

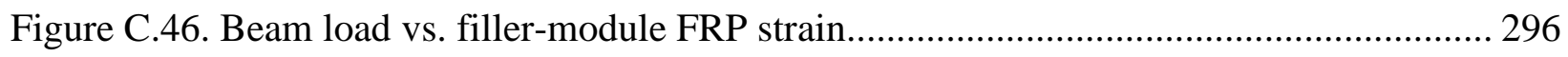

Figure C.47. Beam load vs. FRP strain in column and filler-module......................................... 296

Figure C.48. Beam load vs. FRP strain in column and filler-module........................................ 297

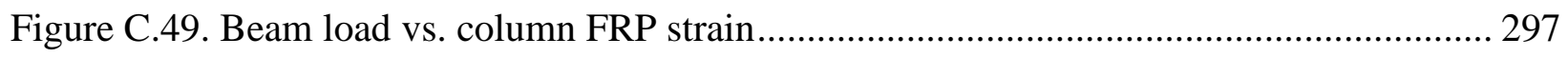

Figure C.50. Beam load vs. column FRP strain...................................................................... 298

Figure D.1. Beam load vs. column longitudinal rebar strain ................................................... 299

Figure D.2. Beam load vs. beam longitudinal rebar strain ......................................................... 300 
Figure D.3. Beam load vs. joint tail rebar strain 300

Figure D.4. Beam load vs. beam stirrup strain 301

Figure D.5. Beam load vs. joint principal strain 302

Figure D.6. Beam load vs. joint shear strain 302

Figure D.7. Beam load vs. column longitudinal rebar strain 303

Figure D.8. Beam load vs. beam longitudinal rebar strain 303

Figure D.9. Beam load vs. joint tail rebar strain 304

Figure D.10. Beam load vs. beam stirrup strain 304

Figure D.11. Beam load vs. column principal strain 305

Figure D.12. Beam load vs. column shear strain 305

Figure D.13. Beam load vs. column longitudinal rebar strain 306

Figure D.14. Beam load vs. beam longitudinal rebar strain ...... 306

Figure D.15. Beam load vs. joint tail rebar strain 307

Figure D.16. Beam load vs. beam stirrup strain 307

Figure D.17. Beam load vs. column principal strain 308

Figure D.18. Beam load vs. column shear strain 308

Figure D.19. Beam load vs. column longitudinal rebar strain 309

Figure D.20. Beam load vs. beam longitudinal rebar strain 309

Figure D.21. Beam load vs. beam stirrup strain 310

Figure D.22. Beam load vs. joint tail rebar strain 310

Figure D.23. Beam load vs. beam principal strain. 311 
Figure D.24. Beam load vs. beam shear strain 311

Figure D.25. Beam load vs. column longitudinal rebar strain 312

Figure D.26. Beam load vs. beam longitudinal rebar strain 312

Figure D.27. Beam load vs. joint tail rebar strain 313

Figure D.28. Beam load vs. beam stirrup strain 313

Figure D.29. Beam load vs. beam principal strain. 314

Figure D.30. Beam load vs. beam shear strain 314

Figure D.31. Beam load vs. column longitudinal rebar strain 315

Figure D.32. Beam load vs. beam longitudinal rebar strain ...... 315

Figure D.33. Beam load vs. beam stirrup strain 316

Figure D.34. Beam load vs. column longitudinal rebar strain 317

Figure D.35. Beam load vs. beam longitudinal rebar strain ...... 317

Figure D.36. Beam load vs. joint tail rebar strain 318

Figure D.37. Beam load vs. beam stirrup strain 318

Figure D.38. Beam load vs. beam principal strain. 319

Figure D.39. Beam load vs. beam shear strain 319

Figure E.1. Stress vs. strain behavior of JI-CS 320

Figure E.1. Stress vs. strain behavior of JI-S1 320

Figure E.2. Stress vs. strain behavior of JI-S2 321

Figure E.3. Stress vs. strain behavior of JII-CS 321

Figure E.5. Stress vs. strain behavior of JII-S1 322 
Figure E.6. Stress vs. strain behavior of JII-S2 ............................................................ 322

Figure E.7. Stress vs. strain behavior of JI-CS ........................................................... 323

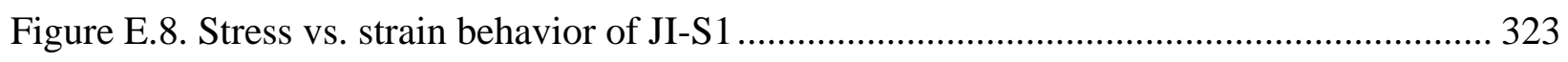

Figure E.9. Stress vs. strain behavior of JI-S2 ....................................................... 324

Figure E.10. Stress vs. strain behavior of JII-CS ...................................................... 324

Figure E.11. Stress vs. strain behavior of JII-S1 ..................................................... 325

Figure E.12. Stress vs. strain behavior of JII-S2 ......................................................... 325 


\section{Introduction}

\subsection{Research Background}

Natural disasters across the globe are growing at an alarming rate, causing severe damages to infrastructure and humankind while leading to severe economic imbalances for developing countries [1]. According to a study published by the World Bank Group in 2016, each year, natural disasters cause approximately $\$ 520$ billion in losses while pushing 26 million people into poverty [2]. A significant portion of losses incurred during natural disasters is attributed to infrastructure damages. For example, the 1906 San Francisco earthquake incurred \$42 billion (estimated in 2006 dollar value) in direct losses due to damage of 28,000 buildings while causing 3,000 fatalities [3]. Predications by seismologists reveal that, by 2036, there exists a $63 \%$ chance of occurrence of at least one major earthquake in the Bay area with a magnitude of 6.7 or higher [4]. If such a seismic event were to occur in the Bay area, nearly 127,000 buildings will either collapse or experience extensive damages in the Bay area that are beyond repair conditions, leading to 3,400 deaths and $\$ 120$ billion in losses [5].

One of the critical aspects leading to severe building damages is the inability of beam-column joints (hereafter referred to as "joints") to swiftly transfer forces (axial, shear, bending, torsional) between joints and adjoining members under extreme forces. The structural damages are even worse in pre-1976 buildings that are designed for gravity loads due to the lack of UBC seismic design codes [6]. The primary reasons for such failures are attributed to soft or weak story constructions, inadequate seismic design capacity, and poor reinforcement detailing, especially in the joint panel. The failure behavior of joints is often characterized by its non-optimal designing and detailing such as loss of rebar anchorage, insufficient development length of beam reinforcement extending into the column, insufficient lap splice length, bars with hook-end, smooth and rounded rebar, and little or no transverse reinforcement resulting in weak columnstrong beam combination [7]. This condition is even worse in exterior joints that are partially confined by the absence of structural elements (e.g., transverse beam, column, or slab) in one or more directions. Such joints in a framed system have been identified as more critical than the 
interior joints because of potential brittle failure at/near the joint, leading to the sudden loss of load-carrying capacity and resulting in catastrophic structural collapse [8], as shown in Figures 1 and 2. Even to date, many such structures still exist in seismically active zones around the world and particularly in the western United States. Based on several post-earthquake reconnaissance studies [9]-[12] it is well recognized that joints are the Achilles heel in maintaining the integrity of structural systems and hence must be strengthened to minimize the risk of catastrophic collapse.

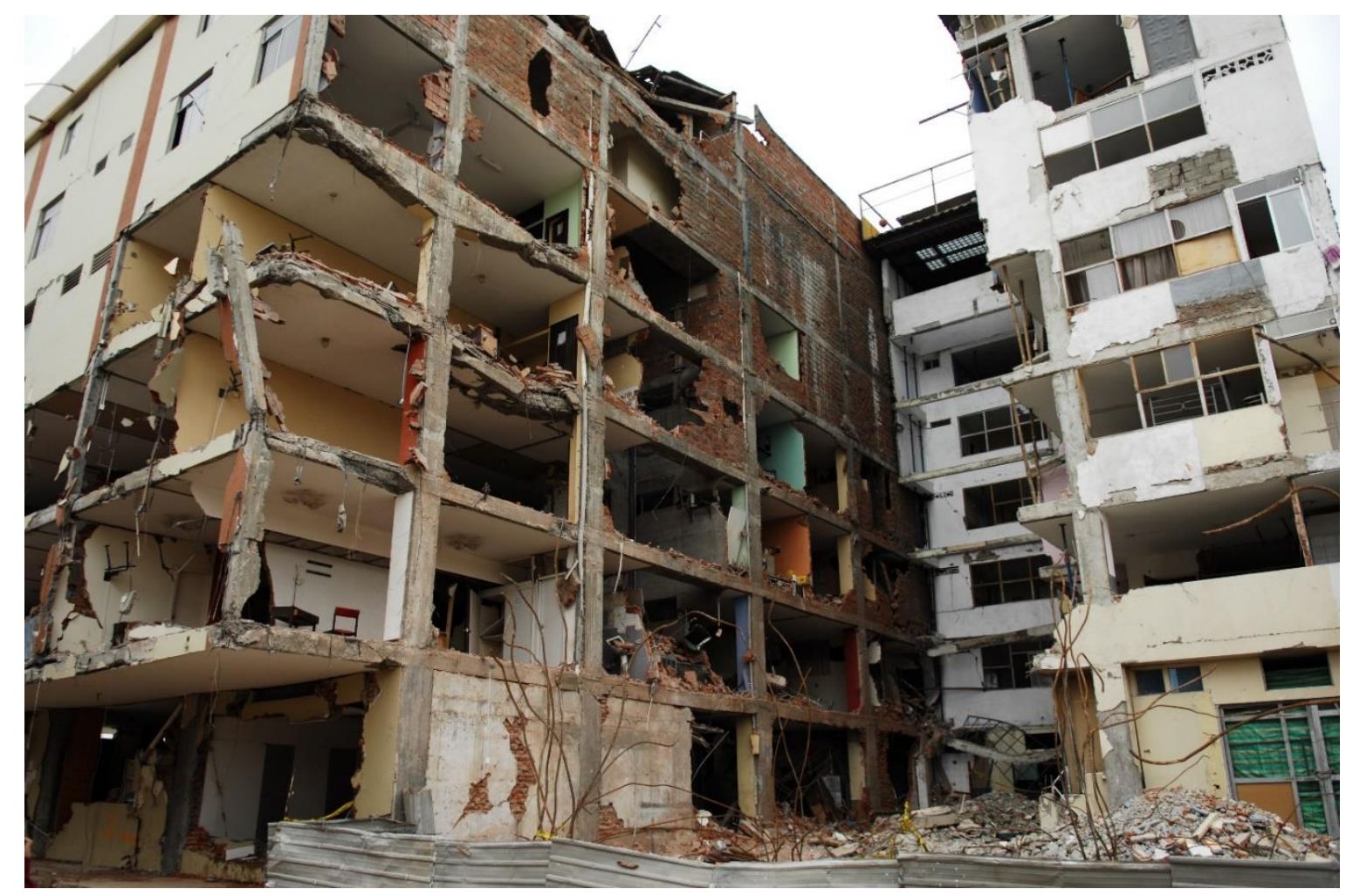

Figure 1. Low-rise building collapse during 2016 Ecuador earthquake [9] 

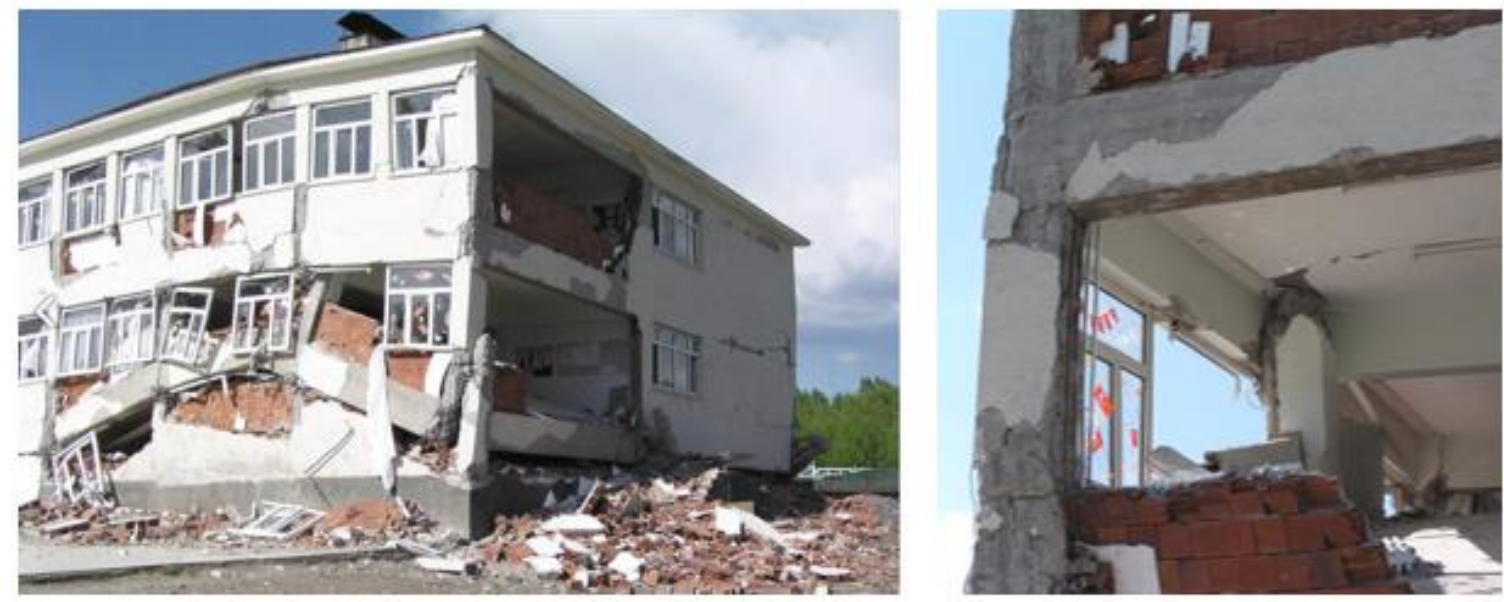

Figure 2. School building damage in Eastern Turkey due to exterior joint failure [10]

Over the past 50 years, several strengthening techniques have been proposed and evaluated to enhance the performance of joints. These techniques include: (i) externally bonded fiberreinforced polymer (FRP) wraps [15]-[19], (ii) addition of steel plates [20]-[21], (iii) steel haunch bolting at the interface of beam-column junction [22]-[24], (iv) concrete jacketing [11], (v) Lshaped polymer blocks bonded at the beam-column interface [12], (vi) planar joint enlargement [27]-[30], and (vii) concrete cover bonded together with CFRP jacket [31] and [32]. Despite the technical advancements and number of experimental studies conducted on the behavior of joints retrofitted under various schemes, relatively less effort has been placed on developing simple, yet economical and durable repair strategies to improve force transfer through joints. Even less emphasis was devoted to developing repair procedures that minimize stress-concentrations at joint corners, and enhancing the strength, ductility, and energy dissipation capacities. Hence, the current research using filler-modules and FRP composite wrap or gusset systems proposed by [33]-[35] has been undertaken to address some of the above concerns by experimentally evaluating twenty 2D exterior $(\mathrm{T})$ reinforced concrete $(\mathrm{RC})$ exterior joint specimens under monotonic loads. Although the research performed herein was focused on enhancing the behavior and integrity of exterior joints that are designed before 1976, the concepts can be extended to other joint configurations, including new construction. 


\subsection{Joint Reinforcement with Filler-Modules and FRP Wraps or Gussets}

Based on the understanding of joint behavior and shortcomings of existing repair procedures, a novel approach of reinforcing existing and newly built joints with filler-modules and FRP composite wraps or gussets proposed by [33]-[35] has been evaluated herein. The method's geometric intricacies are identified in Figures 3 and 4. The proposed approach involved bonding filler-modules at the reentrant corners of a joint and securing them with reinforcing dowels to minimize corner stress-concentrations through the smoother stress transfer in and around a joint. Additionally, it required bonding of FRP composite wraps or gussets on to the exposed faces to reinforce and confine the joint core, thus further enhancing the joint resistance. This approach can significantly reduce the shear induced in the joint panel with the relocation plastic hinge formation away from the joint core. The proposed schemes can enable notable improvements as a retrofit option for the in-service structures and new construction of structural systems under extreme events. Several advantages of retrofitting structures with the proposed approach are: (i) performance enhancement such as strength, energy absorption, and ductility, (ii) structural integrity improvements, avoiding catastrophic failure through better joint confinement and moment transfer, (iii) rebar development length increase extending into a joint, (iv) corner stressconcentration abatement, (v) joint durability and fire resistance enhancement, (vi) avoidance of joint rebar congestion for new construction, (vii) economic corrections in detailing errors in the designs, and (viii) others. 


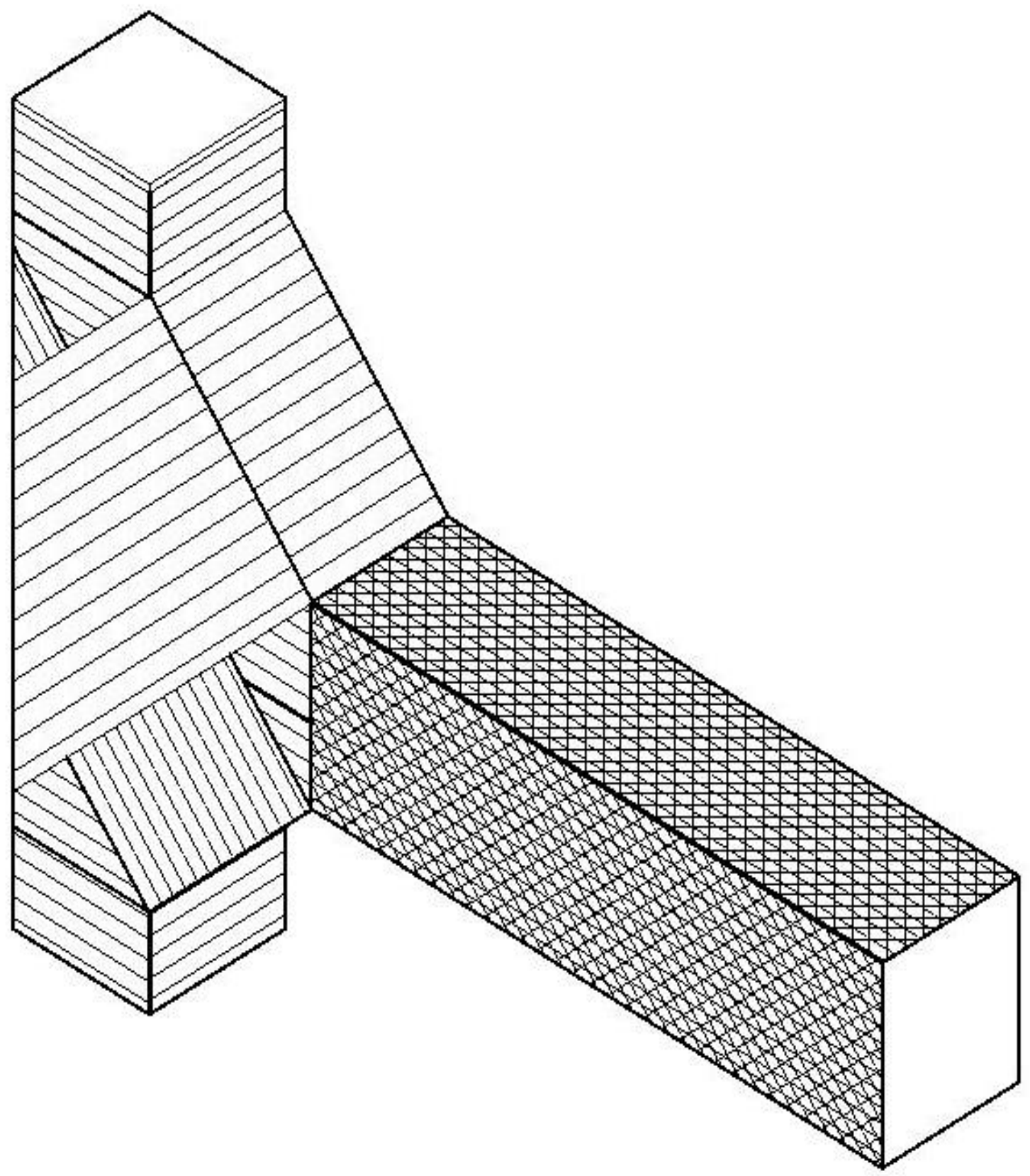

Figure 3. 2D exterior (T) joint reinforcement using filler-modules and FRP wraps [33]-[34] 


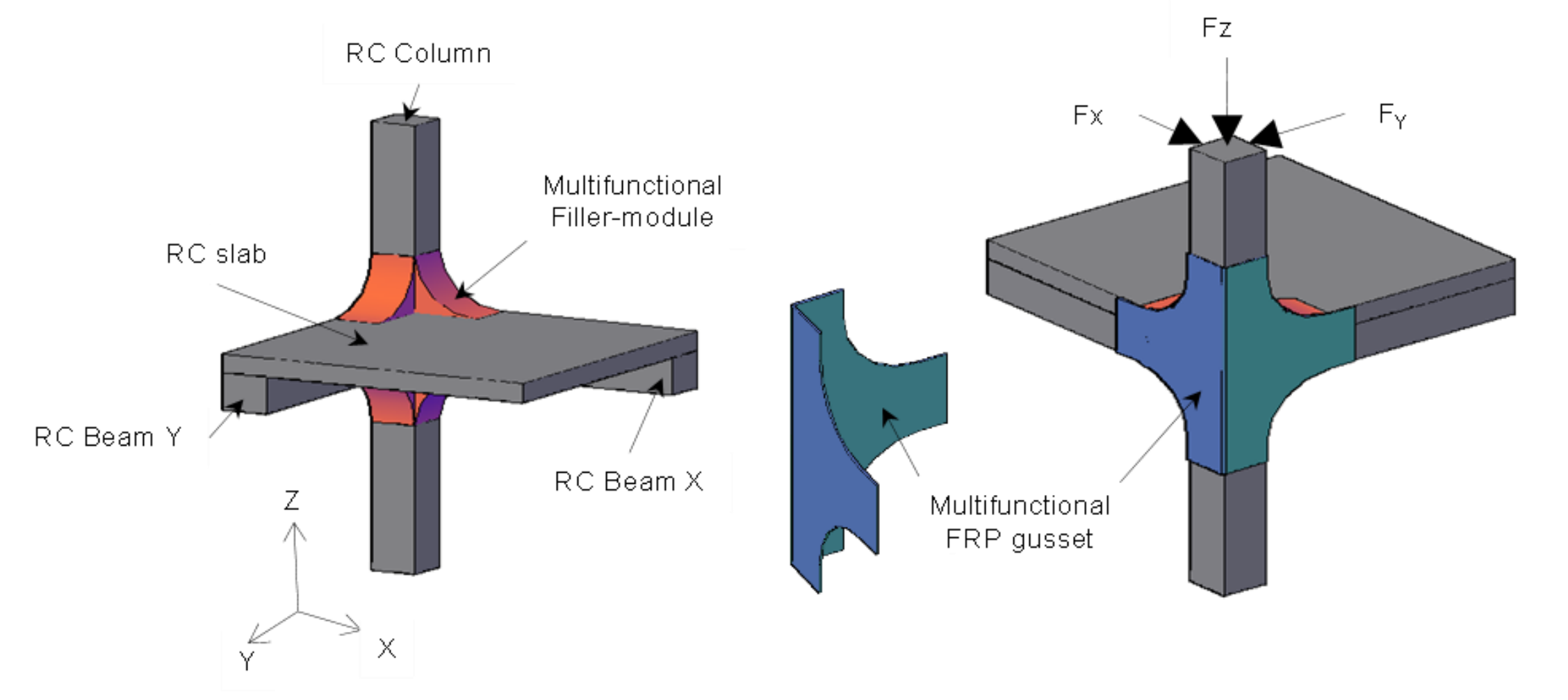

Figure 4. Beam-column-slab joint reinforcement using filler-modules and FRP gussets [35] 


\subsection{Research Objectives}

The primary objective of this research was to experimentally evaluate the effectiveness of fillermodules and FRP wraps or gussets for strengthening the reinforced concrete exterior joint specimens for improved structural performance. Emphasis was laid on evaluating 2D exterior (T) joints of non-ductile concrete frames constructed before the development of UBC seismic codes in 1976. To accomplish the research objective, the following tasks have been performed:

1. Understand the state-of-the-art behavior of reinforced concrete $2 \mathrm{D}$ exterior joints based on the published literature.

2. Review existing rehabilitation techniques from a phenomenological viewpoint.

3. Propose and develop a simple, reliable, and economical retrofit approach to strengthen reinforced concrete 2D joints based on the understanding developed from tasks 1 and 2 .

4. Design and construct reinforced concrete joints in control and reinforced conditions.

5. Test FRP wrapped concrete cylinders under axial compression and split-tension to establish the stress-strain relationship at the first crack and peak loads.

6. Investigate the efficacy of the proposed joint reinforcing schemes in improving structural responses such as strain distribution in rebars, strength, stiffness, ductility, energy dissipation, and failure modes under monotonic loads.

7. Provide recommendations for future research to advance the state-of-the-art of proposed research to $3 \mathrm{D}$ space frames with out-of-plane member attachments such as slab and transverse beams in two or more directions. 


\subsection{Research Scope}

The scope of this study is limited to experimental evaluation of 2D RC exterior (T) joint specimens exhibiting pre-1976 construction deficiencies: (i) low strength concrete $(\sim 2,639,3,309,3,866 \mathrm{psi})$, (ii) inadequate anchorage length of beam bottom bars extending into the joint, (iii) no transverse reinforcement (i.e., stirrups) in the joint panel, and (iv) weak column-strong beam design. A total of twenty specimens were tested in four batches under static axial (column) and bending (beam) loads to determine the efficiency of the proposed reinforcing system in improving the structural capacities such as strength, stiffness, ductility, and energy dissipation. The influence of the following parameters on the joint performance is evaluated as a part of the proposed scope:

(i) filler-module geometric shape: curve versus wedge.

(ii) filler-module material properties: low- (3,376 psi), medium- (5,472 psi), and high(9,545 psi) strength concrete; engineered syntactic foam microsphere (SF 3000); engineered wood, i.e., parallel strand lumber (PSL 2.0E).

(iii) shear transfer through reinforcing dowels: with and without dowels for filler-modules.

(iv) FRP material: glass (GFRP) versus carbon (CFRP).

(v) FRP application: wrap (hand layup) versus gussets (prefabricated laminates).

(vi) FRP anchors: no anchors, U-anchors, and $360^{\circ}$-anchors.

A summary of the experimental program is outlined in Table 1 . 
Table 1. Details of the experimental program

\begin{tabular}{|c|c|c|c|}
\hline Joint & $f_{c}^{\prime}$ & CL & Reinforcing scheme \\
\hline JI-1 & \multirow{4}{*}{ 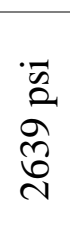 } & \multirow{4}{*}{$\begin{array}{l}\mathbb{i} \\
\underset{i}{\infty} \\
\stackrel{\infty}{0} \\
0\end{array}$} & Control \\
\hline JI-2 & & & $\mathrm{C}-\mathrm{W}(3.3 \mathrm{ksi})+\mathrm{D}$ \\
\hline JI-3 & & & C-W(3.3 ksi)+D+CFRP Wrap $+360^{\circ}$-anchor \\
\hline JI-4 & & & SF-W(4.3 ksi)+D+CFRP Wrap $+360^{\circ}$-anchor \\
\hline JI-5 & \multirow{4}{*}{$\begin{array}{l}\overrightarrow{D_{1}} \\
\text { aे } \\
\text { है }\end{array}$} & \multirow{4}{*}{$\begin{array}{l}\underset{i}{\infty} \\
\underset{0}{\infty} \\
\dot{0}\end{array}$} & Control \\
\hline JI-6 & & & C-W(5.5 ksi)+D+CFRP Gusset+U-anchor \\
\hline JI-7 & & & C-W(5.5 ksi)+D+CFRP Gusset+U-anchor* \\
\hline JI-8 & & & C-W(9.5 ksi)+D+CFRP Gusset+U-anchor* \\
\hline JIII-1 & \multirow{4}{*}{ 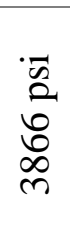 } & \multirow{4}{*}{$\frac{\mathbb{i}}{i}$} & Control \\
\hline JIII-2 & & & C-C(5.5 ksi)+D+GFRP Wrap+no anchor* \\
\hline JIII-3 & & & C-W(5.5 ksi)+D+GFRP Wrap+no anchor* \\
\hline JIII-4 & & & C-W(5.5 ksi)+D+GFRP Wrap+U-anchor* \\
\hline JII-1 & \multirow{8}{*}{ 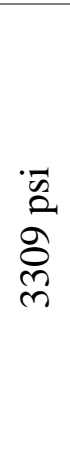 } & \multirow{8}{*}{$\frac{\sqrt{0}}{\stackrel{i}{\sigma}}$} & Control \\
\hline JII-2 & & & CFRP Wrap \\
\hline JII-3 & & & PSL-C+D \\
\hline JII-4 & & & PSL-W+D \\
\hline JII-5 & & & PSL-C+CFRP Wrap+360-anchor \\
\hline JII-6 & & & PSL-C+D+CFRP Wrap+360 -anchor \\
\hline JII-7 & & & PSL-W+D+CFRP Wrap+360'-anchor \\
\hline JII-8 & & & PSL-W+D+GFRP Wrap+360'-anchor \\
\hline
\end{tabular}

D: dowels; C-W: concrete wedge; C-C: concrete curve; SF-W: syntactic foam wedge; PSL-C: Parallel strand lumber curve; PSL-W: Parallel strand lumber wedge; CL: column axial load; $f_{c}^{\prime}$ : substrate concrete compressive strength; $A_{g}$ : gross cross-sectional area of concrete (column); *CFRP layer bonded between filler-module and substrate beam and column that receive fillermodules at the reentrant corners. 


\subsection{Research Significance}

The high vulnerability of existing structures built before 1976 is highlighted in a disaster resilience report [13]. For example, according to [14], if a 7.2 magnitude earthquake were to happen on the San Andreas Fault, nearly: (i) 25,000 buildings will be unsafe to occupy, (ii) 75,000 buildings will experience light to moderate damage, (iii) 3,500 buildings will have to be demolished and rebuilt, (iv) 200-300 people could be killed and (v) 7000 people could have injuries needing medical care. Also, 90\% of San Francisco's 120,000 buildings erected in mid-1970 are not compliant with current seismic requirements [15]. Replacement of such vulnerable structures with new designs following current seismic guidelines is not an economically sustainable solution. Hence, to avoid catastrophe under future earthquakes and ensure safe living conditions, there is an immediate need to develop alternative reinforcing schemes that are practically feasible, economically viable, and able to withstand extreme events structurally.

Therefore, the proposed study to reinforce RC joints with filler-modules and FRP composite wraps or gussets will not only enhance safety, service life, and cost-effectiveness through retrofitting, leading to near disaster-proof structures but also immensely useful in minimizing the high stressconcentration at corners. Experimental evaluations accomplished through this study will help create new knowledge of reinforcing joints, including a fundamental understanding of failure mechanisms and strain limits. The outcomes of this dissertation will underpin the future development of design procedures and guidelines to reinforce new and pre-existing joints and avoid economic hardship for those living in areas vulnerable to seismic or hurricane forces.

\subsection{Organization of Chapters}

Research carried out in this study is organized into the following chapters:

Chapter 1: This chapter provided the research- background, objectives, scope, and significance.

Chapter 2: This chapter provided a brief review on studies related to RC joints built before 1976 with emphasis on critical parameters that influence the joint behavior, such as column axial compressive load, concrete compressive strength, joint aspect ratio, presence of slab and transverse 
beam, end hooks $\left(180^{\circ}\right.$ or $\left.90^{\circ}\right)$ of beam reinforcement and their embedment length into joint, joint shear reinforcement (hereafter referred to as "transverse reinforcement"), and joint failure modes. Finally, this chapter provided conclusions based on the critical outcomes of these studies.

Chapter 3: This chapter provided a brief review of the repair and retrofit of RC exterior joints using FRP composites. This review was performed based on the influence of various parameters that govern the FRP composite repair of joints. Finally, this chapter was concluded based on the key findings from the previous experimental investigations.

Chapter 4: This chapter provided in-depth information about the experimental program conducted on 2D RC exterior joints under monotonic loads, including the types of materials employed, their properties, and the load strain/deformation response. More specifically, the experimental program described the specimen details, specimen casting process, reinforcing schemes, material properties, test setup, loading protocol, and instrumentation employed to monitor, record, and collect test data.

Chapter 5: This chapter dealt with the experimental investigations of 2D RC exterior joints reinforced with concrete/syntactic foam filler-modules and FRP wraps or gussets, performed as part of this research. The various sections in this chapter presented experimental findings, discussions of test results, and conclusions.

Chapter 6: This chapter dealt with the experimental investigations of $2 \mathrm{D} \mathrm{RC}$ exterior joints reinforced with PSL filler-modules and FRP wraps, performed as part of this research. The various sections in this chapter presented experimental findings, discussions of test results, and conclusions.

Chapter 7: A summary of conclusions based on the experimental investigations performed in chapters 5 and 6 was presented comprehensively. Besides, future recommendations for expanding the scope of this study are highlighted. 


\section{RC Exterior Joints Built Before 1976: A Brief Review}

\subsection{Introduction}

Structures built before the launch of seismic design codes by UBC in 1976 exhibited severe damages due to the lack of understanding of seismic capacity design principles. Several laboratory studies performed on joint specimens replicating pre-1976 construction deficiencies (identified in Section 2.2) also confirmed the vulnerability of such structures under extreme events. To enhance safe and economical design practices, fundamentals of joint behavior under the influence of various parameters governing the joint behavior must be fully understood. This chapter, therefore, provides a comprehensive review of studies performed on $2 \mathrm{D}$ exterior $(\mathrm{T})$ joints, as shown in Figure $7 \mathrm{~d}$, under the influence of critical parameters governing the joint behavior. The parameters reviewed in the following sections are:

i) column axial compressive stresses,

ii) concrete compressive strength,

iii) joint aspect ratio,

iv) presence of slab and transverse beam,

v) end hooks $\left(180^{\circ}\right.$ or $\left.90^{\circ}\right)$ of beam reinforcement and their embedment length into joint,

vi) joint shear reinforcement (hereafter referred to as "transverse reinforcement"), and vii) joint failure modes.

\subsection{Vulnerability of Buildings with Deficient Joints under Extreme Events}

Joints of a moment-resisting frame play a crucial role in distributing the induced forces between beam, column, and slab and maintaining structural integrity, especially in multistory structures. The vulnerability of such structures designed and built with an inadequate understanding of structural responses under extreme load events is often dependent on the joint performance. This behavior is evident from many of the published post-earthquake structural damage data (Mexico 1985, Loma Prieta 1989, Kobe 1995, Kocaeli 1999, Tehuacan 1999, Izmit 1999, Athens 1999, Chi-Chi, 1999, Boumerdes 2003, L'Aquila 2009) and analyses [39]-[42]. The primary role of 
joints is to maintain the structural integrity under combined forces (axial, shear, bending, and torsional) induced from seismic, wind, or blast loads. Under the above actions, higher joint shear forces and/or force gradient in the beam longitudinal rebars (due to moment reversals) are generated typically in a joint panel, resulting in either joint shear failure or rebar bond failure followed by the sudden loss of load resistance. This behavior ultimately may lead to the brittle collapse of the entire building frame, as shown in Figure 5, with low energy dissipation [16]. Therefore, to enhance safe and economical design practices, fundamentals of joint behavior under various loading scenarios must be fully understood.

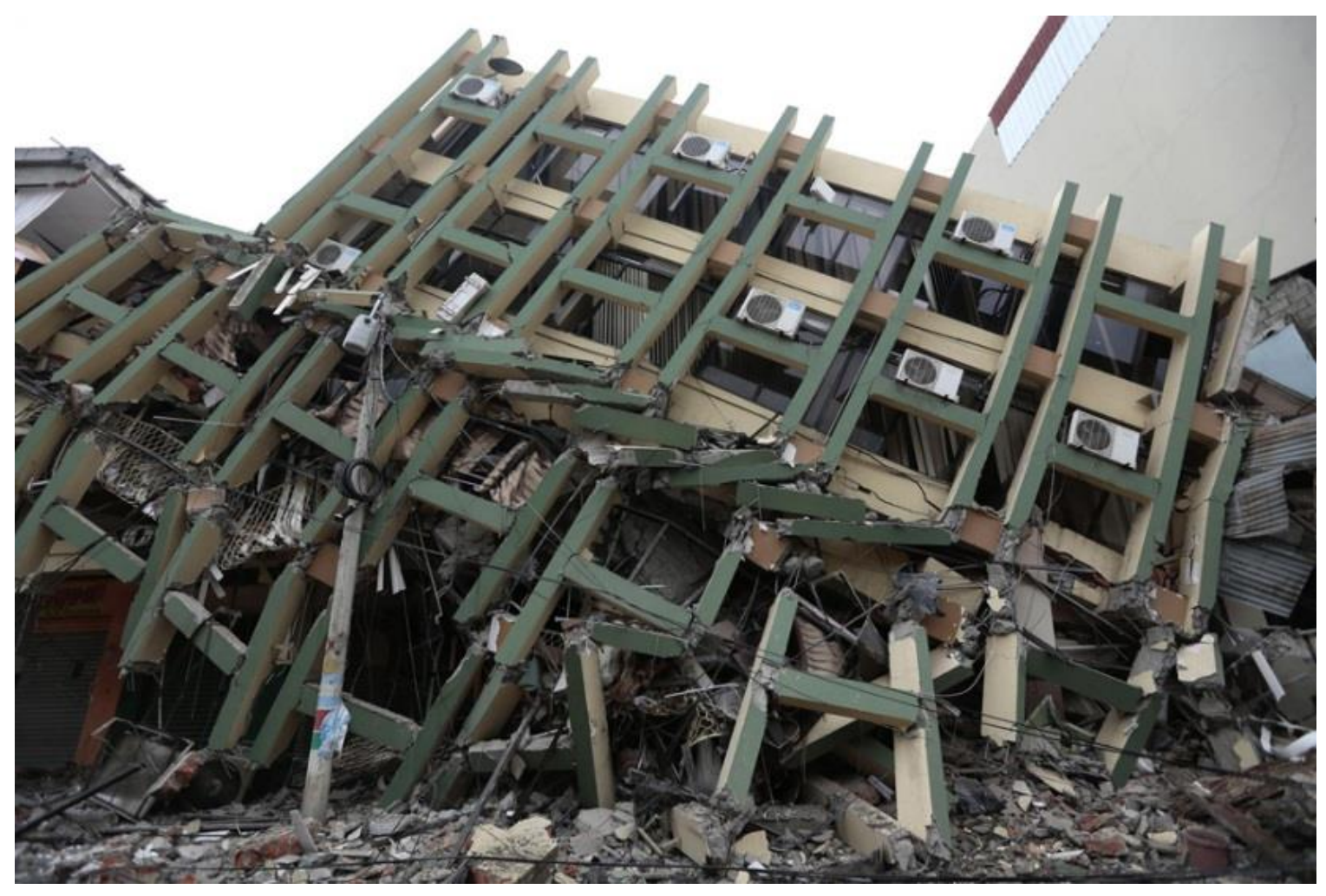

Figure 5. 2016 Ecuador earthquake - building damage (Juan Cevallos/Getty Images) [17]

Understanding of structural damages based on the post-earthquake reconnaissance studies by [6], [13], [20], [44], [45] have confirmed the innate weaknesses of pre-1976 structures due to the following deficiencies identified at or near the joint panels of buildings: inadequate shear (transverse) reinforcement in a joint panel, ineffective confinement of beam or column plastic hinge regions, poor anchorage detailing for beam reinforcement extending into the joint panel, 
inadequate column longitudinal and shear reinforcement near a joint, insufficient lap splice length of column rebars near the joint panels, and inferior quality construction materials when compared to existing standards. The highlighted deficiencies, especially in the joint region, have either led to partial damages (i.e., shear or rebar bond failure) or complete collapse (i.e., soft-story failures) of buildings.

\subsection{Joint Definition and Classification}

ACI 352R-02 defines a beam-column joint as "that portion of the column within the depth of the deepest beam that frames into the column." Based on the geometry and arrangement in a framed system, ACI 352R classifies joints in three categories, namely interior, exterior, or corner joints, as shown in Figure 6. This classification is essential because of the fundamental differences in reinforcement anchorage mechanisms of the interior, exterior, and corner joints. Depending on the number of transverse beams meeting at a joint and the presence of columns above and below the joint panel, joints are further classified, as shown in Figure 7. Joints can be considered elastic or inelastic depending on their structural behavior [18]. In a framed system, if plastic deformations occur either in a beam or column or both without cracking in the joint panel, then its behavior is considered as elastic. On the other hand, if a joint panel undergoes inelastic deformation (e.g., seismic event) without significant cracking while forming a plastic hinge at a distance from the beam or column face, then the joint is considered as inelastic. Based on the high vulnerability of exterior joint damages under seismic activities, the author chose to investigate 2D exterior joints without transverse (shear) reinforcement in the joint panel for carrying out this research. 


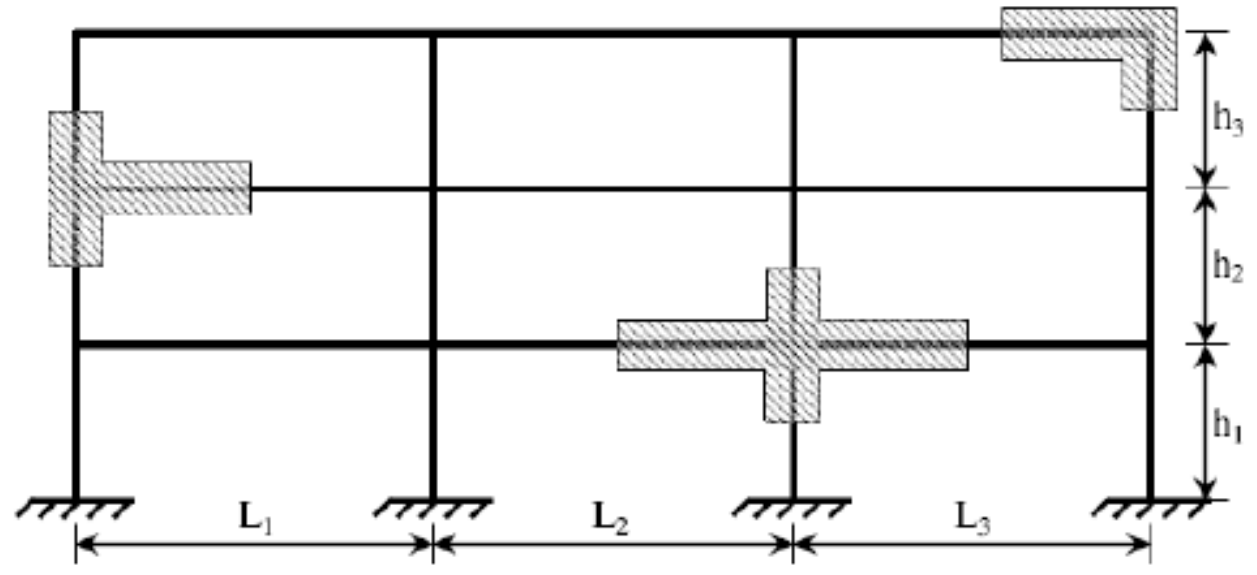

Figure 6. Joint classification based on the arrangement in a framed system [19]

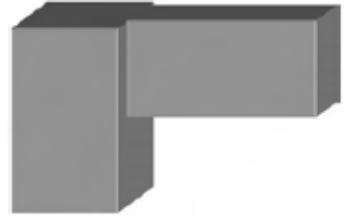

a) $2 \mathrm{D}$ roof corner joint

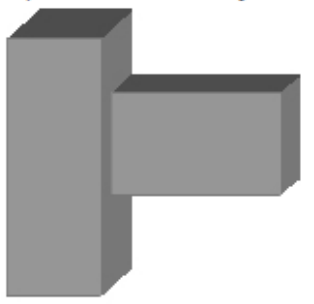

d) $2 \mathrm{D}$ exterior joint

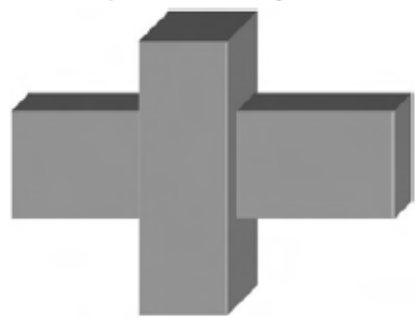

g) $2 \mathrm{D}$ interior joint

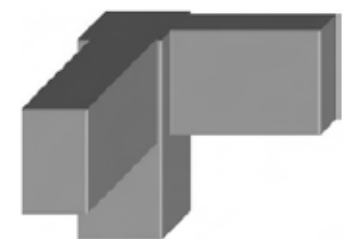

b) $3 \mathrm{D}$ roof corner joint

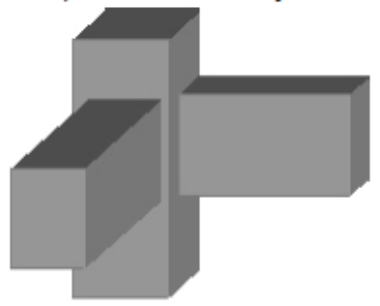

e) 3D corner joint

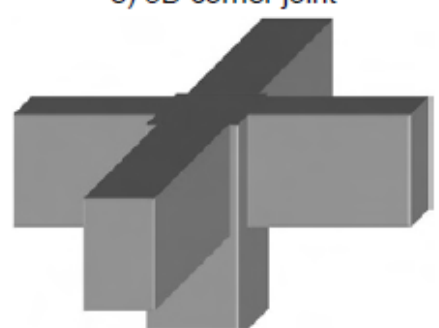

h) $3 \mathrm{D}$ roof interior joint

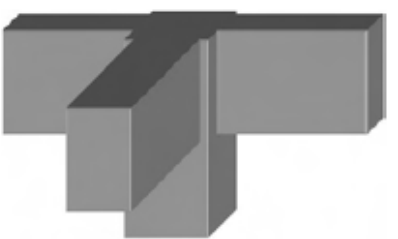

c) 3D roof edge joint
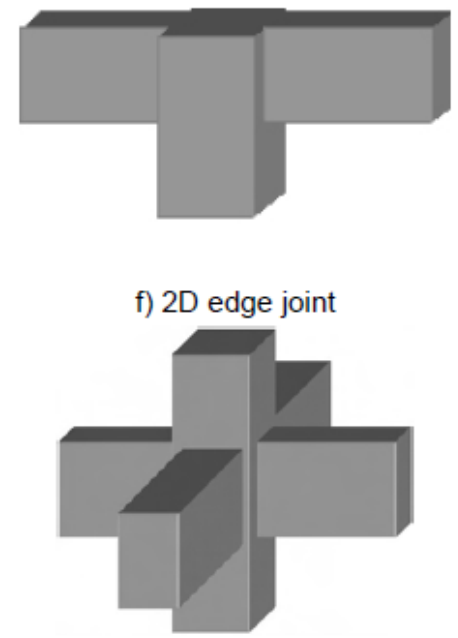

i) $3 \mathrm{D}$ interior joint

Figure 7. Joint sub-classification based on the arrangement of beams and columns [20]

Note: A typical building frame has a slab contributing to the joint performance. For simplicity, the slab was excluded in Figure 7. 


\subsection{Force Transfer Mechanism in 2D RC Exterior Joints}
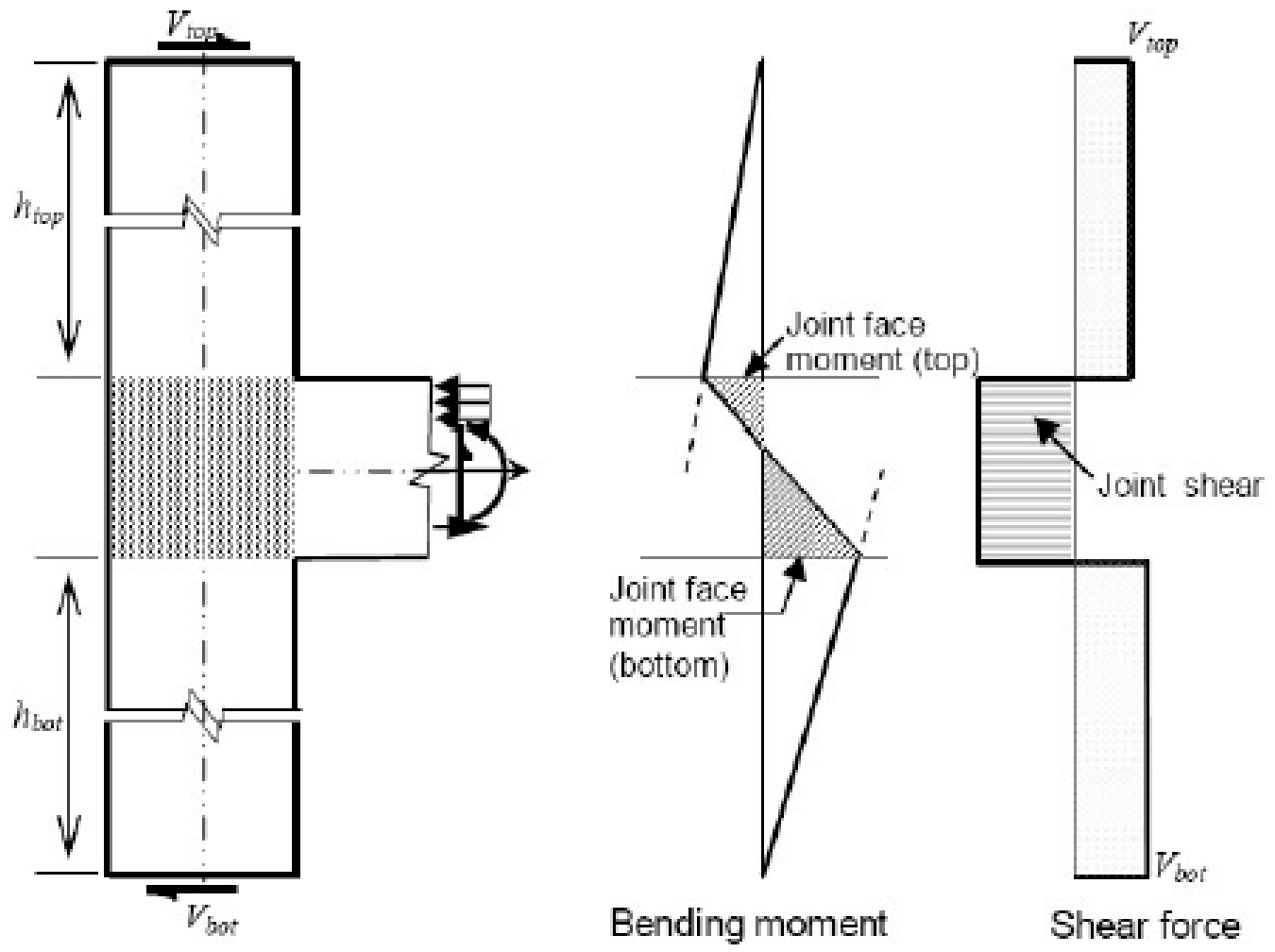

Figure 8. The force-transfer mechanism in a most practical case of RC exterior joint [19]

As shown in Figure 8, when an exterior joint is subjected to seismic forces, the column bending moments of high magnitudes having opposite signs are generated on either side of a joint panel, causing enormous joint shear forces at the interface. To satisfy the equilibrium of forces in a joint panel, the beam reinforcement has to be in compression on one face and tension on the other, causing force gradient, as shown in Figure 8. 


\subsection{Assessment of Joint Shear Strength}

The resultant forces acting on a joint panel as a consequence of applied beam and column loads are shown in Figure 9.

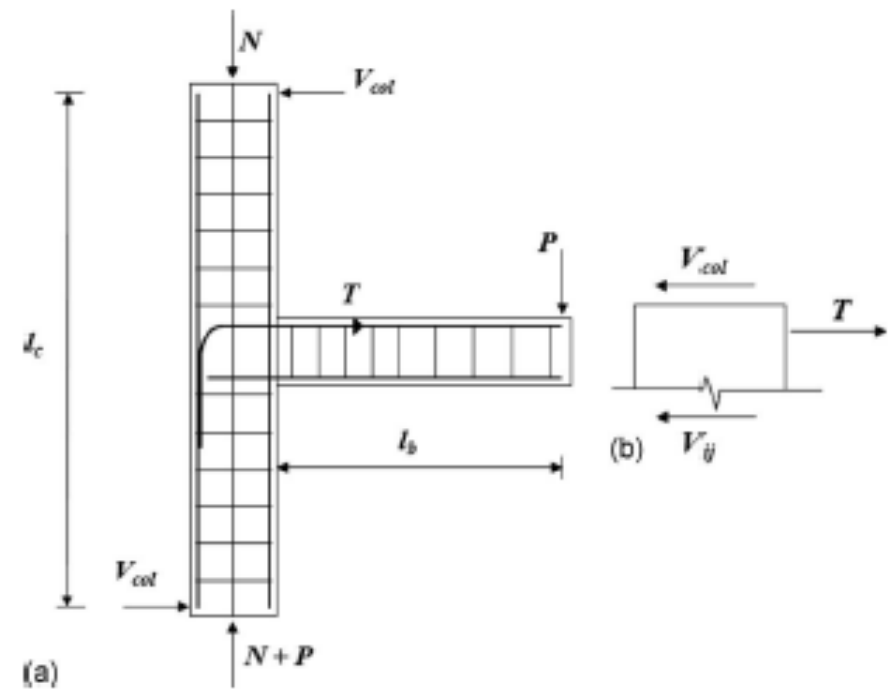

Figure 9. Resultant forces on a 2D exterior joint under beam and column loads [21]

Based on the equilibrium of forces, the horizontal shear force at the mid-height of a joint panel can be computed [21]:

$$
V_{j h}=T-V_{c o l}
$$

Where

$V_{c o l}$ : horizontal column shear force

$T$ : tensile force in the beam steel reinforcement can be computed as:

$$
T=\frac{P l_{b}}{j d}
$$

Where 
$j d$ : the distance between tensile and compression force resultants at the beam-joint interface The horizontal column shear force can be computed using:

$$
V_{c o l}=\frac{P\left(l_{b}+0.5 h_{c}\right)}{l_{c}}
$$

The nominal horizontal shear stress at the mid-height of a joint panel can be computed using:

$$
v_{j h}=V_{j h} / b_{j} h_{c}
$$

Where

$b_{j}$ : effective width of the joint panel,

$h_{c}$ : depth of the column. 


\subsubsection{Computation of principal stresses in a joint panel}

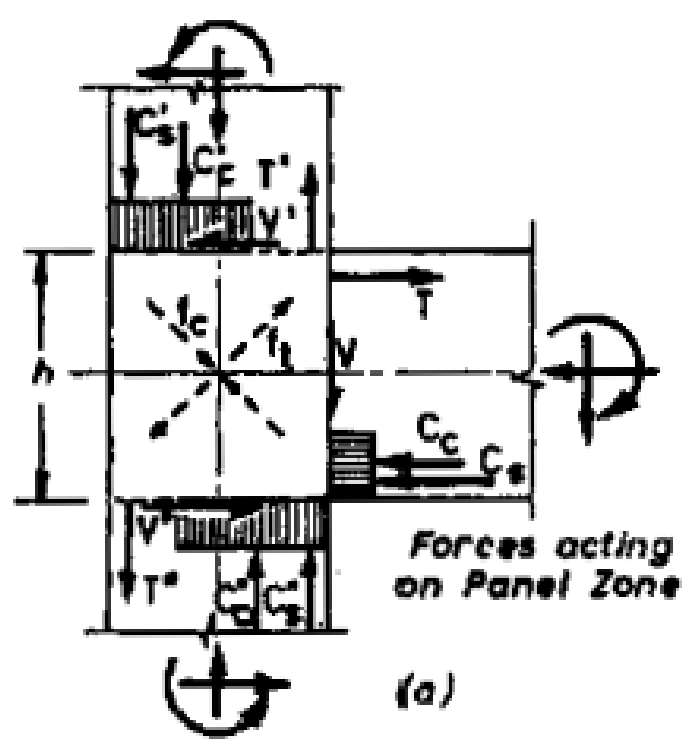

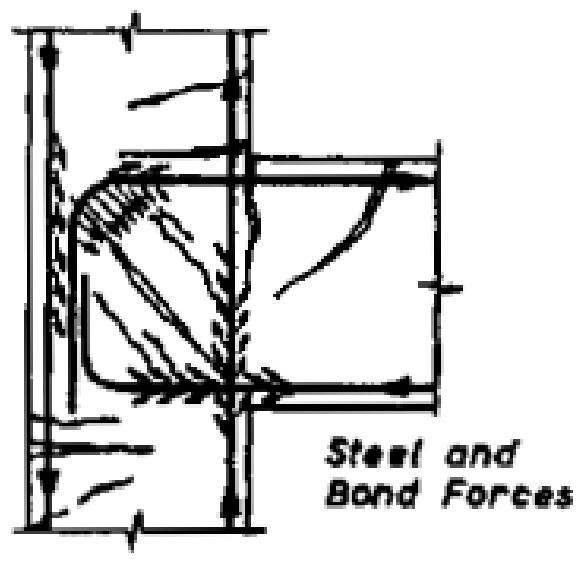

(b)

Figure 10. Diagonal stress fields in an exterior joint [22]

When an exterior joint is subjected to seismic excitation, two types of stress fields, namely diagonal tension and compression, are induced in a joint panel, as shown in Figure 10.

Using Mohr's circle approach as proposed, principal compressive and tensile stresses at the midheight of a joint panel can be computed [23]:

Principal compressive stress:

$$
p_{c}=\frac{-f_{a}}{2}-\sqrt{\left(\frac{f_{a}}{2}\right)^{2}+v_{j h}{ }^{2}}
$$

Principal tensile stress:

$$
p_{t}=\frac{-f_{a}}{2}+\sqrt{\left(\frac{f_{a}}{2}\right)^{2}+v_{j h}{ }^{2}}
$$

Joint shear stress: 


$$
v_{j h}=p_{t} \sqrt{1+\frac{f_{a}}{p_{t}}}
$$

The nominal (average) axial compressive stress on the column at the mid-height of a joint panel can be computed using:

$$
f_{a}=N / b_{j} h_{c}
$$

Where

$N$ : Axial compressive load on the column

The principal tension stress limit states proposed by various authors [51] and [52] based on experimental evaluations of 2D exterior joints without transverse reinforcement, are summarized in Table 2. However, [23] in their publication highlighted that the principal tensile stress approach could yield conservative results as additional shear resistance beyond diagonal tensile cracking is provided by the diagonal compression strut developed in the joint panel. 
Table 2. Principal tensile stress limit states based on the available literature

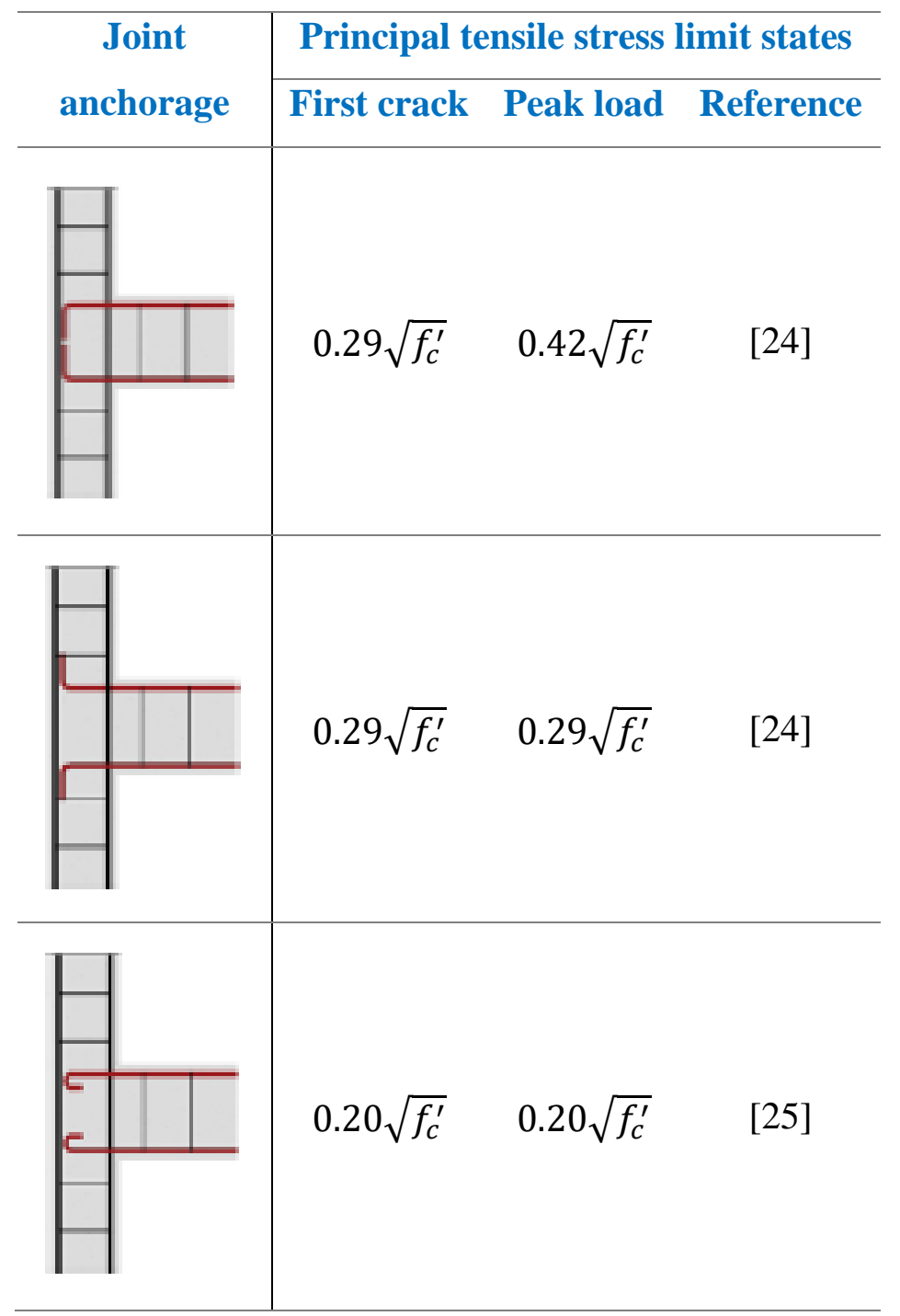




\subsubsection{Strut mechanism}

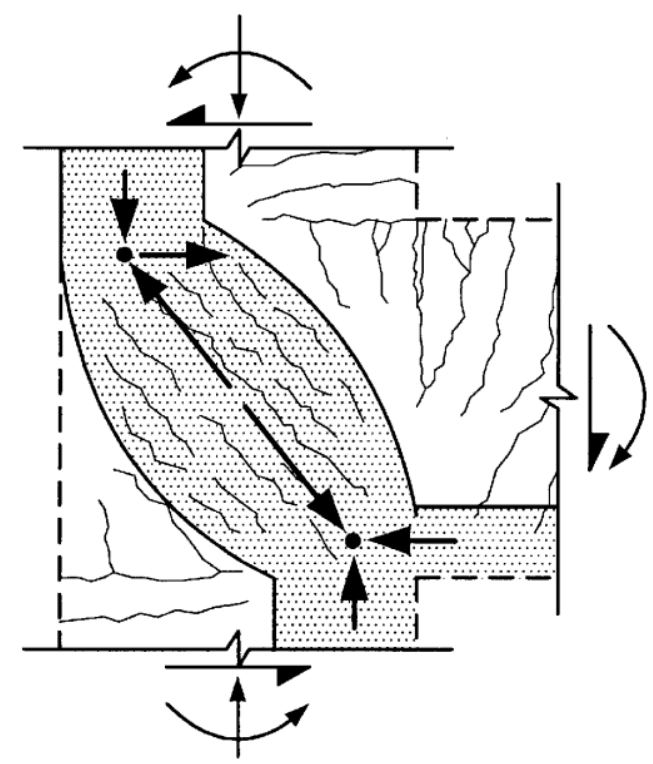

Figure 11. Strut mechanism of shear force transfer in an exterior joint [26]

While considering force transfer mechanisms, exterior joints without transverse (shear) reinforcement in the joint panel need particular focus because of the limited compressive force developed on the free end of the joint (exposed) face due to the absence of transverse beams in one or more directions. Therefore, a node formation is essential to develop a strut mechanism for resisting joint shear. The node formation can be achieved by providing a $90^{\circ}$ hook to the beam longitudinal reinforcement bent either into or away from the joint panel. In the case of beam reinforcement bent away from the joint panel, the strut will not be stable due to a lack of confinement from the bend radius, leading to premature failure.

On the other hand, joints with beam reinforcement bent towards the panel, exhibits diagonal strut formation between the node along the bent radius and other node formed due to compressive forces on the diagonally opposite end. The joint panel continues to resist shear until significant tensile stresses are developed, at which stage the strut loses its compressive strength, resulting in concrete crushing. Under specific span-to-depth ratios, the connection (beam + column + joint) behavior can also lead to shear compression failure. 


\subsection{Capacity Design of Joints}

Under extreme seismic forces, joints of a moment-resisting frame exhibit higher shear forces than the members framing into the joint, causing inelastic joint deformations (perhaps with higher ductility). Besides, due to load reversals, exterior joints with longitudinal beam bars having $180^{\circ}$ or no end hooks are required to develop higher rebar bond stresses to resist the force gradient in the joint panel. Inadequately designed joints structural capacities and anchorage can become weak links and cause catastrophic shear or rebar bond failure. To avoid catastrophe by maximizing the ductility and energy absorption of the overall structure, it is necessary to follow the capacity based design philosophy and make the joints stronger than the members framing into the joint. An indepth discussion on the deterministic capacity design sequence of structures is provided by [18] in their textbook for further review if needed.

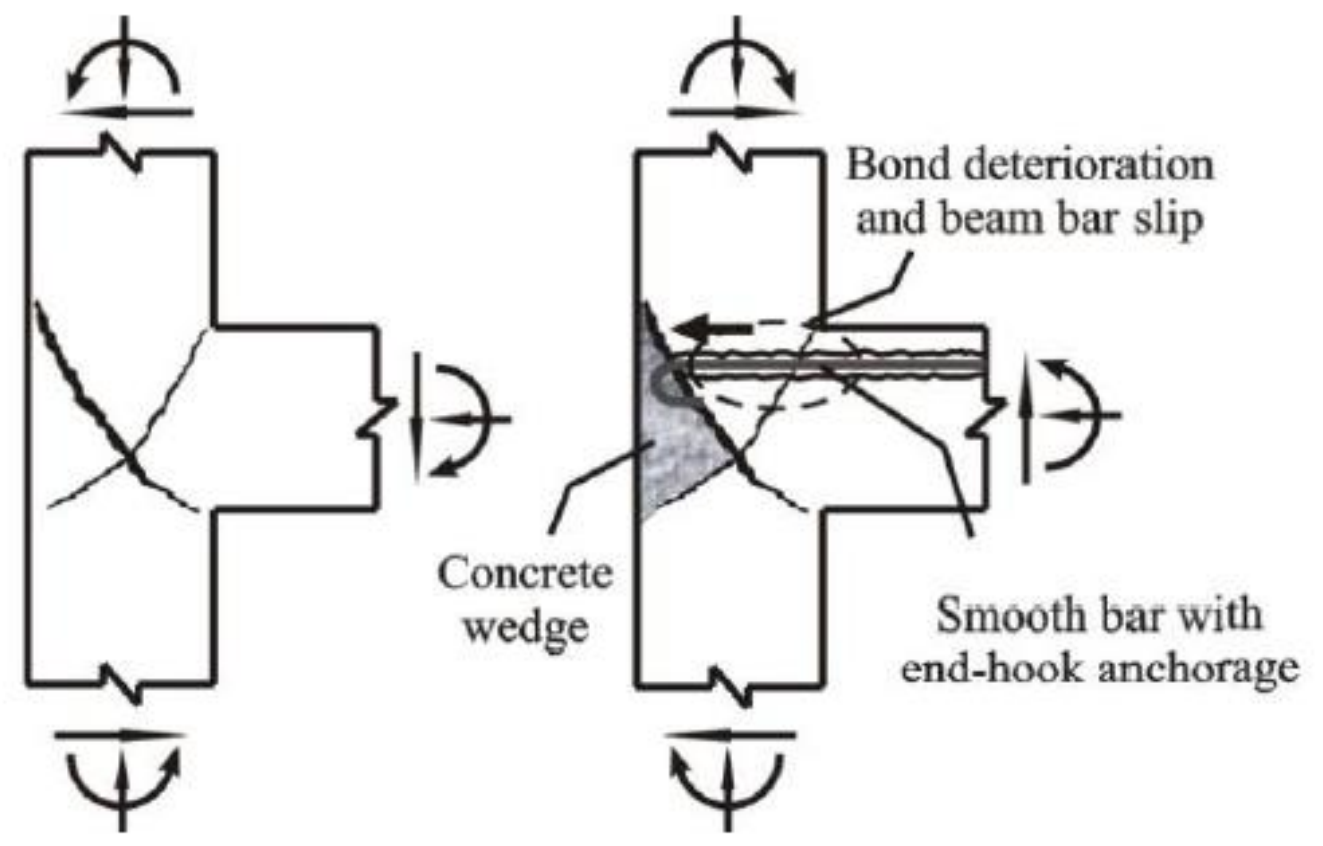

Figure 12. Joint shear/rebar bond deterioration failure in exterior joints [27] 


\subsection{Parameters Influencing the Behavior of RC Exterior Joints}

As identified in section 2.2, a broad spectrum of deficiencies can be found in non-ductile reinforced concrete structures built before 1976. However, the ones pertinent to exterior joints have been identified and discussed in this section. Based on several reconnaissance studies performed by researchers, following deficiencies were found in exterior joints leading to a sudden loss of structural integrity [28]: (i) minimum joint transverse reinforcement (stirrups), (ii) low strength concrete ( $\sim 3500$ psi or lower), (iii) beam longitudinal bars bent into the joint with inadequate development length for complete force transfer, and (iv) weak-column and strong-beam design. Hence, the review below highlights the behavior of such joints under the influence of critical parameters governing the failure behavior:

\subsubsection{Column axial compressive load}

[29] investigated the effect of low axial load by testing joints with hooked bars and found that the influence of axial column load is almost negligible. [30] made similar conclusions, but also identified that the axial column load influences the joint failure modes. On the contrary, [40], [58][60] showed that specimens with higher axial loads exhibited increased joint shear capacity due to improved diagonal tension resistance in a joint failure scenario. Further, [31] also reported that specimens with low axial load $\left(\leq 0.1 f_{c}^{\prime} A_{g}\right.$ ) exhibited 1.5 times more ductility in comparison to specimens with high axial load $\left(0.25 f_{c}^{\prime} A_{g}\right)$. As shown Figure 13, no substantial influence on joint shear strength can be noted when specimens are subjected to an axial load less than $(0.20-$ 0.25) $f_{c}^{\prime} A_{g}$. Moreover, the exterior joints under seismic loads are prone to load reversals, where the columns do not remain under constant axial loads. This behavior can alter the joint failure mechanism and can jeopardize structural integrity [27], especially in terms of energy absorption. 


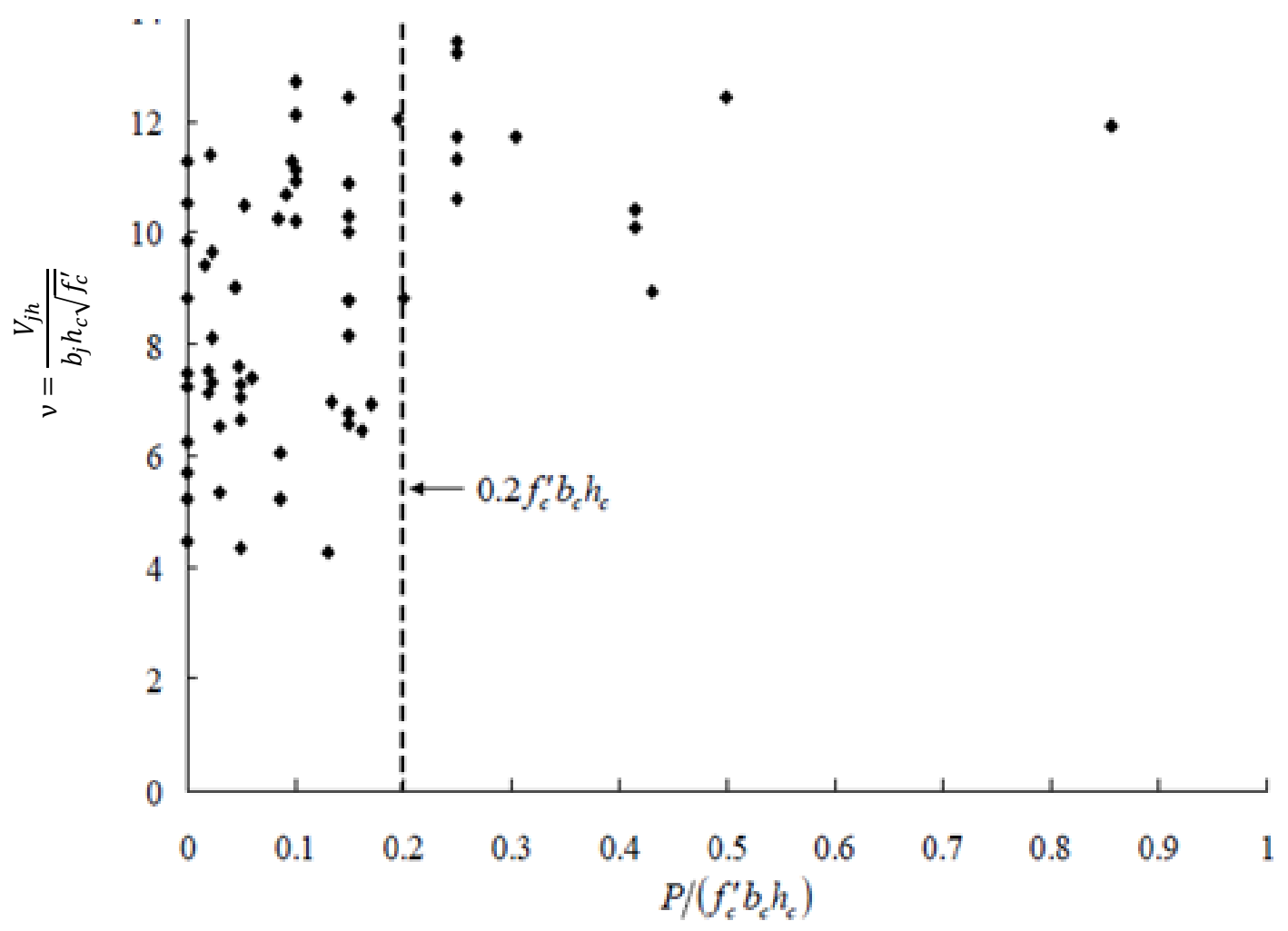

Figure 13. Effect of column axial load on joint shear strength [32]

\subsubsection{Concrete compressive strength}

[33] studied joint responses of 48 exterior joints under cyclic loading. Twenty-seven of their test specimens failed through yielding of beam rebar followed by joint shear failure (BJ) while the remaining 21 failed in joint shear failure without rebar yielding (J). The database was grouped using the following criteria: (i) no out-of-plane members (i.e., transverse beams and/or slabs), (ii) no eccentricity between beams and columns (i.e., the beam centroid coincides with the column centroid), (iii) conventional types of reinforcement anchorage with hooked bars (headed bars were excluded) and (iv) minimum amount of joint stirrups necessary for joint confinement. In general, the damage of a joint was caused by concrete cracking in or very close to a joint under diagonal tension, reinforcement yielding, and/or concrete crushing, which are identified as points $\mathrm{A}, \mathrm{B}$, and C, respectively, (see Figure 14). In case of exterior joints with "BJ" failure, the first stiffness change (Point A) occurred at the initiation of diagonal cracking [63] and [64] within the joint panel, while the second stiffness change (Point B) occurred at rebar yielding before concrete crushing 
(Point C). It was also reported that for specimen with "J" failure, no significant stiffness change was observed around Point B as there was no rebar yielding. The concrete compressive strength for the database collected by [33] varied from $\sim 3$ to $13 \mathrm{ksi}$, and the joint shear stress was found to be proportional to the square root of the concrete compressive strength $\left(\sqrt{f_{c}^{\prime}}\right)$, at points B and C. The New Zealand code [34], however, limits the compression (strut) stress in a joint panel to $0.2 f_{c}^{\prime}$ which is induced from beam-column shear force transfer to avoid concrete crushing failure (see Figure 14). [66] and [67] suggested that principal compression stress be limited to $0.3 f_{c}^{\prime}$.

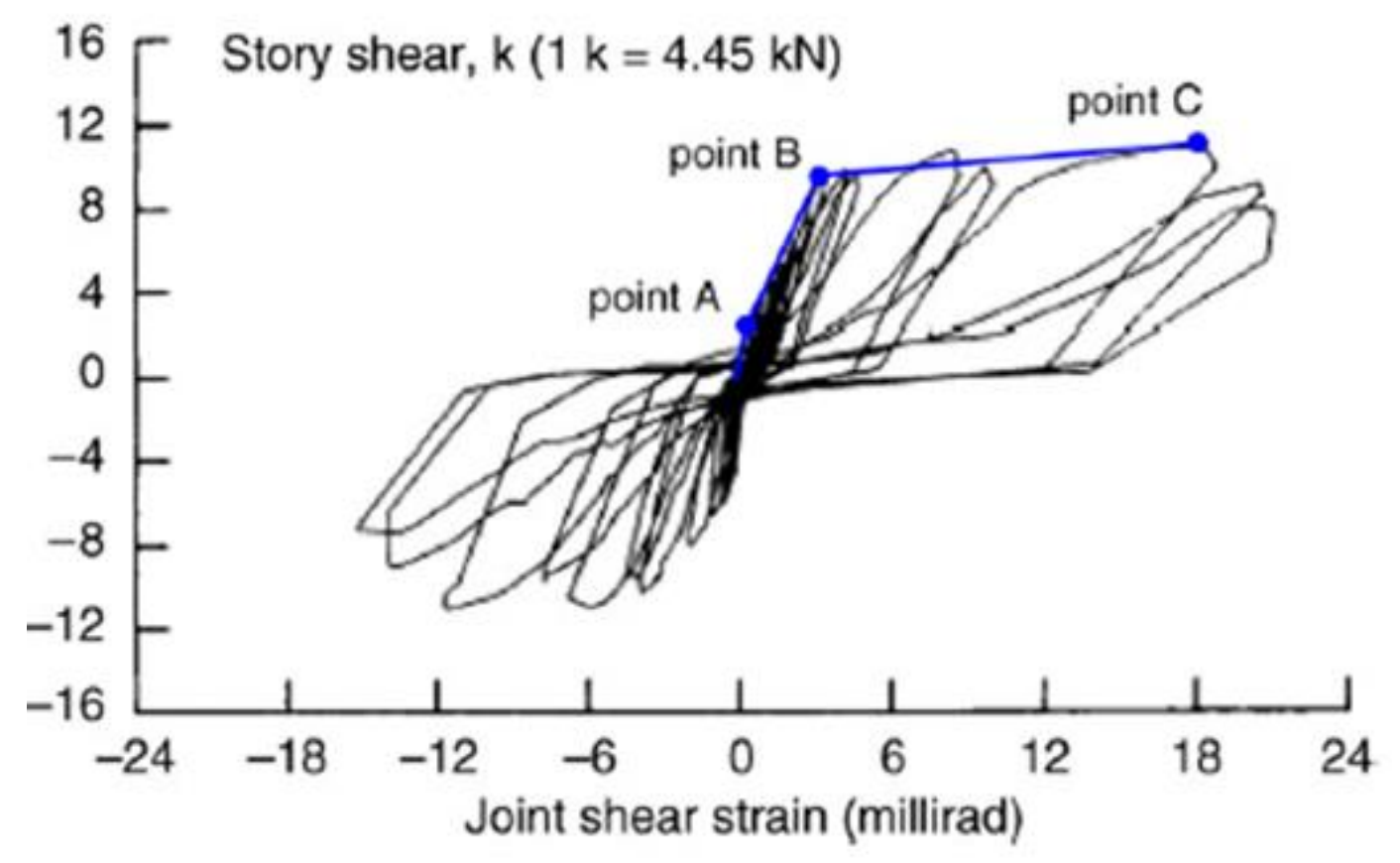

Figure 14. Story shear vs. joint shear strain [33] 


\subsubsection{Joint aspect ratio}

Three different variations of joint aspect ratios (1.0, 1.5, and 2.0) were evaluated by [35] on exterior joints without transverse reinforcement within a joint. As shown in Figure 15, joint shear strength was found to be inversely proportional to the joint aspect ratio [35]. [33] examined the influence of joint aspect ratio based on joint shear reinforcement with beam rebar yielding (BJ) and without beam rebar yielding $(\mathrm{J})$. By increasing the ratios of beam height to column depth $\left(h_{b} / h_{c}\right)$ from 1 to1.6, and beam width to column width $\left(b_{b} / b_{c}\right)$ from 0.56 to 1 , [33] reported: (i) slight reduction of joint shear stresses for $\mathrm{J}$ failure, and (ii) little influence on joint stresses and strains for BJ failure.

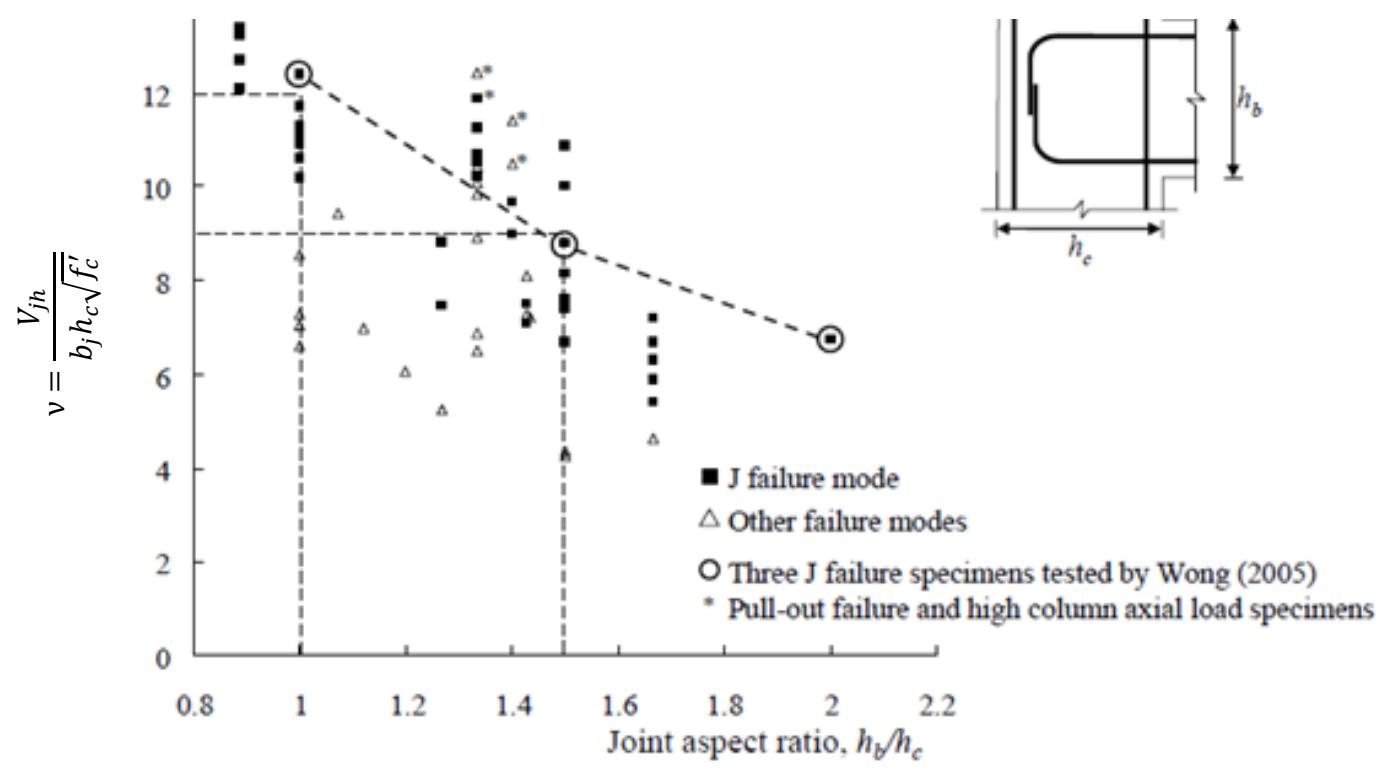

Figure 15. Effect of joint aspect ratio [32] 


\subsubsection{Presence of slab and transverse beam}

[36] tested 2D exterior joints to investigate the influence of a slab and traverse beam on joint shear strength under uni- and bi-directional quasi-static loads and varying axial load. Test specimens were created to replicate pre-1976 construction including (i) plain round steel bars, (ii) $180^{\circ}$ end hook anchorage for beam longitudinal reinforcement, and (iii) no joint transverse reinforcement. Their 2D study noted that the presence of slab at a joint significantly increased the beam yielding moment on the tension side while the confinement effect from the transverse beam (and slab) resulted in higher displacements before failure (i.e., better ductility). The additional ductility offered by the slab can be computed by arriving at an equivalent slab stiffness through finite element modeling. The equivalent stiffness thus obtained can be added to existing beam properties to arrive at a joint design procedure that is in-line with designs without a slab. Furthermore, it was also reported that for 3D specimens under bi-directional loading with axial load variations exhibited severe damage and rapid strength deterioration even with partial confinement induced by transverse beams [36].

\subsubsection{End hooks of beam reinforcement}

[29] performed tests on exterior joints with $90^{\circ}$ and $180^{\circ}$ hooks to evaluate their behavior under monotonic loading and concluded that the anchorage failure of hooked bars is primarily caused by concrete compression around the hook from stress concentration effect. [36] tested joints with slab and transverse beams and revealed that specimens with $180^{\circ}$ hook (small diameter) resulted in the loss of bond for beam reinforcement, exhibiting pinching of hysteresis loop with limited energy dissipation. It was observed that for specimens with bars bent into the joint, the forces were resisted primarily by the concrete compression strut (after initial diagonal cracking) until it is ultimately weekend by diagonal tension strains. Among the above-described beam end hook configurations, [52], [70]-[74] concluded that joints with $180^{\circ}$ hooks exhibited the worst performance with low energy dissipation due to the lack of concrete confinement. 


\subsubsection{Joint transverse (shear) reinforcement}

[37] found that the confinement effect from joint transverse reinforcement positively influenced the concrete shear resistance. [54] and [70] carried out experimental tests on 3D joints with plain round bars and $180^{\circ}$ hooks and no transverse reinforcement in the joint. Based on this study, it was concluded that the exterior joints with no transverse reinforcement exhibited lower shear strength with brittle behavior. Similarly, [38] experimentally evaluated four exterior joint specimens with transverse beams and floor slabs under quasi-static loading to evaluate non-ductile behavior in existing RC buildings, and found joint shear failure without beam-hinging.

\subsubsection{Failure modes}

Joint strength is often characterized based on the concrete compressive strength, and steel rebar (yield) strength. As reported in the literature, exterior joints typically exhibit two failure modes: (i) joint shear failure without beam reinforcement yielding, and (ii) joint shear failure after beam reinforcement yielding. [39] collected an extensive database of exterior reinforced (i.e., with joint stirrups) (189 tests - without joint transverse (tie) reinforcement, 14 tests - with one transverse beam, and 18 tests - with two transverse beams) and exterior unreinforced (i.e., no joint stirrups) (83 tests - without transverse beam, 29 tests - with one transverse beam, and nine tests - with two transverse beams) joint responses to develop a reliable joint shear strength determination model statistically. The database included: (i) concrete compressive strength, (ii) joint transverse reinforcement ratio, (iii) design joint shear stress, (iv) in-plane and out-plane geometry (i.e., presence of transverse beams in one or more directions), (v) joint panel geometry factors such as beam depth to column depth ratio and beam width to column width ratio, (vi) joint eccentricity (i.e., the beam centroid does not coincide with the column centroid), (vii) axial column load, (viii) ratio of "provided" to "required" column depth (or anchorage length) to beam rebar diameter, (ix) column-to-beam nominal moment ratio, and (x) ratio of intermediate column reinforcement strength to design joint shear demand. Based on [39] study, it was concluded that joint shear failure without rebar yielding was the predominant failure for both 110 of reinforced (with stirrups) and 57 of unreinforced (without stirrups) exterior joints. Joint shear failures without rebar yielding exhibit brittle failure due to diagonal tension, compromising the overall structural integrity [28]. 
Table 3. Summary of exterior RC joint failure modes [39]

\begin{tabular}{lcccc}
\hline \multirow{2}{*}{ Joint Type } & \multirow{2}{*}{ Failure mode } & \multicolumn{3}{c}{ Number of transverse beams } \\
\cline { 3 - 5 } & & None & One & Two \\
\hline Reinforced (with joint stirrups) & JS & 110 & 6 & - \\
\hline Reinforced (with joint stirrups) & BJS & 79 & 8 & 18 \\
\hline Unreinforced (without joint stirrups) & JS & 57 & 20 & - \\
\hline Unreinforced (without joint stirrups) & BJS & 26 & 9 & 9 \\
\hline
\end{tabular}

\subsection{Summary}

This chapter reviewed the performance of pre-1976 non-ductile concrete frame joints and outlined the potentially catastrophic failure mechanisms. Besides, crucial parameters influencing the joint behavior were highlighted. The key learning points from this review are as follows:

1. Under seismic actions, due to moment reversals, higher joint shear forces are developed with significant force gradient across the joint panel, resulting in either shear or rebar bond failure.

2. Depending upon the detailing of beam longitudinal rebar extending into the joint panel (i.e., either bent away or towards the joint core), exterior joints typically exhibit two failure modes: (i) joint shear failure without beam reinforcement yielding, and (ii) joint shear failure after beam reinforcement yielding. Typically joint shear failures without rebar yielding are brittle and lead to catastrophic failure (which is collapse).

3. Joints with beam longitudinal rebar having $180^{\circ}$ hooks did not form a compression strut in the joint panel due to inadequate confinement effect from beam longitudinal reinforcement, resulting in a premature rebar bond failure with low energy dissipation.

4. Under cyclic loading, the stress concentration effect around the $90^{\circ}$ bend radii significantly deteriorated the bond between concrete and rebar surface, thus causing anchorage failure.

5. For specimens with longitudinal beam bars bent into the joint, the forces were resisted primarily by the concrete compression strut (after initial diagonal cracking) until it is ultimately weekend by diagonal tension strains. 
6. Column axial loads up to $(0.20-0.25) f_{c}^{\prime} A_{g}$ exhibited no substantial influence on joint shear strength.

7. The presence of transverse beams and slab at a joint significantly increased the beam yielding moment while resulting in higher displacements before failure.

8. The joint aspect ratio $\left(h_{b} / h_{c}\right)$ varied between 1.0 and 1.6 had no significant effect on joint strength.

9. Higher $f_{c}^{\prime}$ coupled with specific span-to-depth ratios, can lead to shear compression failure. 


\section{RC Exterior Joint Repair with FRP Composites: A Brief Review}

\subsection{Introduction}

Over the past 25+ years, experimental research has been focused on strengthening deficient joints (identified in section 2.2 of the previous chapter) through the use of fiber-reinforced polymer (FRP) composites. This chapter provides a review of such experimental studies based on the influence of parameters as identified and elaborated herein. The following sections of this chapter provide a brief introduction on FRP composite applications in repair and rehabilitation of infrastructure systems, leading to discussions on FRP research in joint strengthening. The scope of this chapter is limited to $2 \mathrm{D}$ exterior joints.

\subsection{FRP Composites in Repair and Rehabilitation of Infrastructure}

FRPs were first introduced in the 1940s for applications in defense and aerospace industries [40] and now emerging with numerous opportunities in the infrastructure industry because of their high strength to weight ratio, non-corrosiveness, and excellent durability. FRPs are regarded as disruptive materials to reinforce existing structures that have either deteriorated due to environmental influences or under-designed for external forces. In particular, FRPs are well known for strengthening structural components of seismically deficient buildings and bridges [79]-[81] due to their cost-effectiveness and ease of installation. In general, FRP composites have been employed to repair or rehabilitate:

1. Beams: [82]-[84]

2. Columns: [85]-[87]

3. Joints: [88]-[90]

4. Slabs: [91]-[93]

5. Corroded members: [94]-[96]

6. Fire/heat damaged members: [97]-[99]

7. Vehicular impact damaged members: [100]-[102] 


\subsection{FRP Composites in Joint Strengthening}

One of the critical aspects of structural strengthening to resist extreme forces is to improve joint performance. Joint performance can be improved by bonding multiple sheets/strips of FRPs using epoxy or other adhesives across and around joints of in-service concrete structures. Many researchers adopted this technique to repair deficient joints and assess the performance under cyclic loads [17], [54], and [55]. A research study by [41] on repair of damaged RC joints using FRP laminates concluded that the repair scheme increases the strength of joints beyond its original design capacity. Another study by [42] on retrofit of beam-column joints using FRP overlays revealed significant improvements in strength and moderate increase in ductility. [43] conducted their work by experimenting with FRP retrofitted joints and concluded the increase in moment capacity of the joints. [44] evaluated RC joints rehabilitation with different FRP composite configurations, including U- and X- wraps. This study by [44] proved that FRP repairs can change the non-ductile joint shear failure to a ductile flexural failure mode. [45] experimented by strengthening several scaled joints using various FRP configurations and confirmed the increases in the strength, stiffness, and energy dissipation by up to $100 \%$. In addition, [45] concluded that the failure of joints is governed by the partial or complete debonding of FRPs from the substrate. Rehabilitation of beam-column joints by [46] proved the potential of FRPs in restoring the strength of a damaged joint and the ability to substantially increase the structural capacities by preventing the failure in the joint panel. Web-bonded FRP application to repair RC beam-column joints evaluated by [47] was found to be successful in restoring the strength, stiffness, and ductility of joints by relocating the plastic hinge away from the joint panel into the beam. 


\subsection{Previous Experimental Studies on FRP Composites in Joint Strengthening}

The following section provides a review of experimental work on FRP strengthening of RC exterior joints conducted by various researchers based on the parameters investigated through their research.

\subsubsection{Effectiveness of FRP wraps on joint shear strengthening}

[48] conducted an experimental study to evaluate the effectiveness of carbon fiber-reinforced polymer (CFRP) composite wrapping to strengthen joints that exhibit deficiencies typically encountered in Turkish construction. These deficiencies include low strength concrete (3.6 ksi or lower), structural type I steel (yield strength of $31.9 \mathrm{ksi}$ ), plain reinforcements (no lugs over steel bar surfaces), insufficient transverse reinforcement, lap-splicing lengths and beam sections higher than the column sections meeting at a joint (i.e., moment capacity of beams is higher than that of columns). Identified deficiencies were based on a comprehensive survey conducted on more than 50 buildings built between the 1950s and 1990s. Four 2D exterior joints were experimentally investigated by subsequently developing CFRP wrapping schemes based on the failure mode exhibited by the previous specimen. The control test specimen, including reinforcement details, are shown in Figure 16, and the CFRP repair methods are identified in Tables 4 and 5.

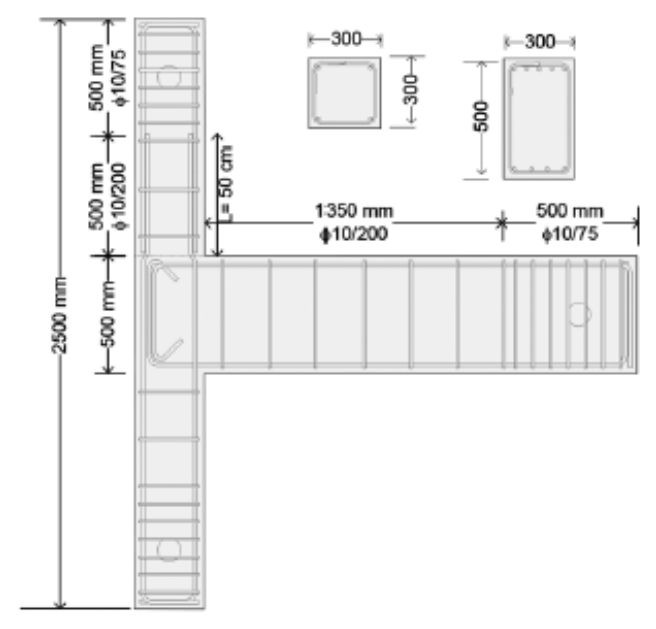

Figure 16. Steel reinforcement detailing of control and retrofitted test specimens [48] 
Table 4. CFRP repair steps adopted by [48]

\begin{tabular}{|c|c|c|}
\hline Step No. & CFRP application & Description of strengthening procedure \\
\hline Step 1 & & $\begin{array}{l}\text { Four horizontal holes of } 12 \mathrm{~mm} \text { in diameter were drilled across the beam to } \\
\text { insert the anchorages. }\end{array}$ \\
\hline Step 2 & & $\begin{array}{l}\text { Longitudinal CFRP sheet was applied outside the column surface for elimi- } \\
\text { nating lap splice defect and increasing moment capacities of column members. }\end{array}$ \\
\hline Step 3 & & $\begin{array}{l}\text { Top and bottom L-shaped CFRP sheets were applied to inside of the column } \\
\text { face extending toward the beam surface for eliminating lap splice defect and } \\
\text { increasing the resistance of joint region. }\end{array}$ \\
\hline Step 4 & & $\begin{array}{l}\text { One layer of diagonal CFRP fibers were placed on joint region to prevent } \\
\text { diagonal crack propagation along the joint surface. Same application was done } \\
\text { for opposite direction, resulting X-shaped CFRP orientation. }\end{array}$ \\
\hline Step 5 & & $\begin{array}{c}\text { Three layers of } 150 \mathrm{~mm} \text { wide CFRP patches were used for eliminating the } \\
\text { rupture of CFRP materials in the joint region. }\end{array}$ \\
\hline Step 6 & & $\begin{array}{l}\text { Top and bottom portion of the column was wrapped for eliminating early } \\
\text { debonding of already-applied L-shaped and longitudinal CFRP on column } \\
\text { surface. }\end{array}$ \\
\hline Step 7 & & $\begin{array}{l}\text { Beam member is wrapped to prevent buckling of beam longitudinal } \\
\text { reinforcement. }\end{array}$ \\
\hline Step 8 & & $\begin{array}{l}\text { To prevent the debonding, CFRP sheets were anchored to the already-applied } \\
\text { beam wrapping by fan-shaped CFRP anchors. }\end{array}$ \\
\hline
\end{tabular}


Table 5. Specimen wise strengthening methods [48]

\begin{tabular}{c|c|c|c|c|c|c|c|c}
\hline & Step 1 & Step 2 & Step 3 & Step 4 & Step 5 & Step 6 & Step 7 & Step 8 \\
\cline { 2 - 9 } Strengthening method & Holes & Longitudinal & L-shape & Diagonal & Patches & Column wrap & Beam wrap & Anchorage \\
\hline $\begin{array}{c}\text { Method 1 } \\
\text { (TR-5-FRP-1) }\end{array}$ & - & - & - & 1 layer & - & - & $\begin{array}{c}1 \text { layer } \\
500 \mathrm{~mm}\end{array}$ & - \\
\hline $\begin{array}{c}\text { Method 2 } \\
\text { (TR-5-FRP-2) }\end{array}$ & - & 1 layer & 1 layer & 1 layer & - & 2 layers & $\begin{array}{c}3 \text { layers } \\
300 \mathrm{~mm}\end{array}$ & - \\
\hline $\begin{array}{c}\text { Method 3 } \\
\text { (TR-5-FRP-3) }\end{array}$ & 4 holes & 1 layer & 1 layer & 1 layer & 3 layers & 2 layers & $\begin{array}{c}3 \text { layers } \\
300 \mathrm{~mm}\end{array}$ & $150 \mathrm{~mm}$ \\
\hline
\end{tabular}

The experimental specimens were tested as per the schematic shown in Figure 17 by applying a constant $\left(0.4 f_{c}^{\prime} A_{g}\right)$ axial load and reversed-cyclic lateral load on the column section.

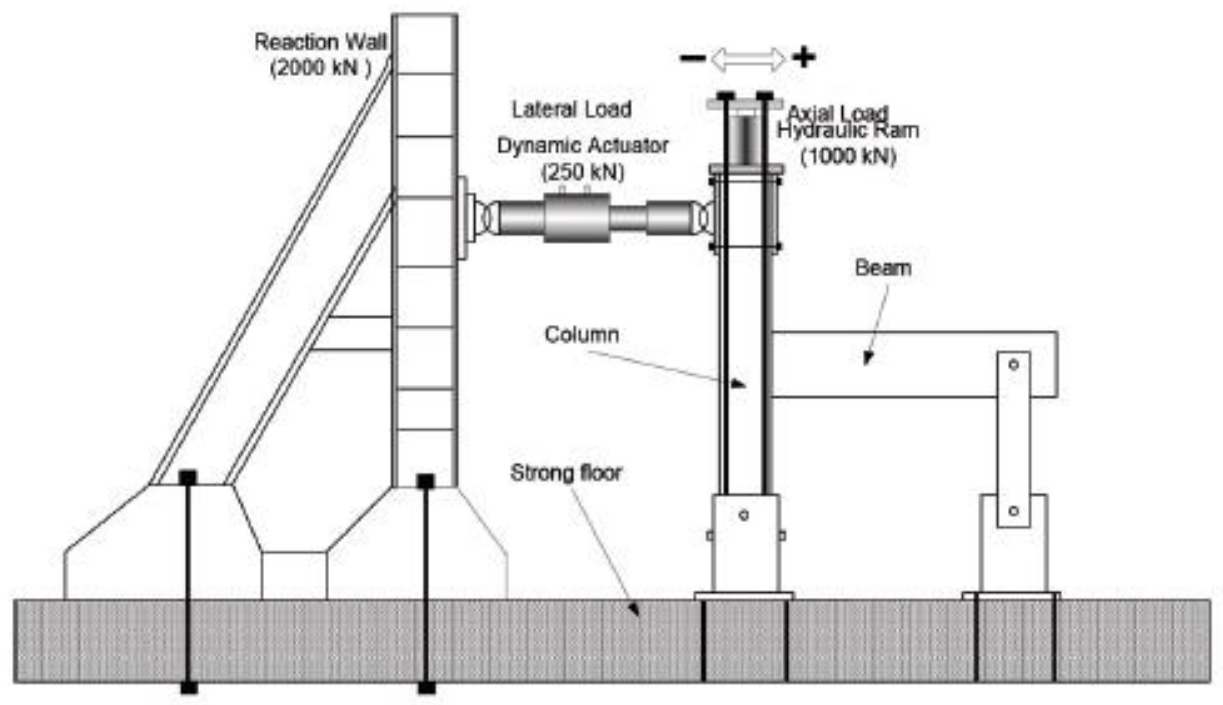

Figure 17. Test setup of [48]

The experimental results and failure behavior of test specimens evaluated by [48] are summarized as follows:

Specimen TR-5-Control:

- The first crack was identified at $0.35 \%$ (of specimen column height) drift, initiating at the top corner of the beam-column joint region. 
- The maximum load levels of 7.2 kip in the push and $10.6 \mathrm{kip}$ in pull directions were reached at $0.75 \%$ drift.

- $\quad$ Test specimen failed by diagonal shear cracking in the joint $(\mathrm{a} / \mathrm{d} \sim 3.7)$.

Specimen TR-5-FRP-1:

- At $1.4 \%$ drift, buckling of CFRP sheets was observed near the top and bottom faces of the beam towards the back face of the column.

- At $2.75 \%$ drift, CFRP sheets ruptured at the corner of beam-column joints.

- Upon removal of CFRP sheets, buckling of column longitudinal rebars was noticed.

Specimen TR-5-FRP-2:

- At $1.4 \%$ drift, initiation of rupture was observed in diagonal CFRP sheets at the interface of the beam-column joints. Cracks, however, did not penetrate to the center of the joint core as their growth was arrested by the additional three layers of CFRP sheets, as illustrated in step 5.

- The maximum load levels of $10.3 \mathrm{kip}$ in the push and $13.6 \mathrm{kip}$ in pull directions were reached at $2.75 \%$ drift. At this stage, in addition to de-bonding and buckling of CFRP sheets on the back face of the column, cracks on the beam widened, leading to concrete crushing.

- At $3.5 \%$ drift, beam hinging occurred.

Specimen TR-5-FRP-3:

- At $2.2 \%$ drift, initiation of rupture was observed in diagonal CFRP sheets at the interface of the beam-column joints. As in the case of specimen TR-5-FRP-2, the crack growth was arrested by an additional three layers of CFRP sheets. In this case, the spalling of concrete cover occurred at the beam bottom location.

- The initiation of de-bond at the tip of CFRP diagonal fibers was observed at $2.75 \%$ drift. However, due to the anchorage of CFRP diagonal fibers, the de-bond did not progress towards the joint. 
- At $3.5 \%$ drift, beam hinging occurred, followed by a buckling of beam bottom reinforcement.

Following are the key learning points based on the experimental evaluations performed by [48]:

- The absence of joint transverse (shear stirrups) reinforcement in the control specimen (TR5-Control) has led to joint shear failure at a low lateral load level.

- Lack of joint confinement in the control specimen has led to faster stiffness degradation.

- Diagonal CFRP sheets bonded to the joint panel orienting fibers parallel to the principal planes and perpendicular to the anticipated crack direction prevented the formation of joint shear cracks. This arrangement of fibers resulted in a ductile behavior with decreased stiffness degradation at the end of each loading cycle.

- In the case of CFRP strengthened specimens, the lateral load resisting capacity was enhanced by $50-100 \%$.

- In all the strengthened specimens, CFRP wrapping could not prevent the initiation of rupture at the beam-column interface, indicating the concentration of stresses around the corner.

- Beam anchor has prevented the de-bonding of sheets from the substrate, thus forcing plastic hinge formation away from the joint core. 


\subsubsection{Effectiveness of FRP anchors in preventing debond}

[49] tested RC strong beam-weak column joints to assess the viability of externally bonded reinforcement on grooves (EBROG) method coupled with the CFRP fan anchorage system, as shown in Figure 18, to resist seismic loads without surface de-bonding of FRP. To accomplish this task, five half-scale 2D exterior beam-column joints with inadequate joint transverse reinforcement were tested under constant axial and reversal-cyclic lateral loads. The test specimen configuration and setup are shown in Figures 19 and 20.
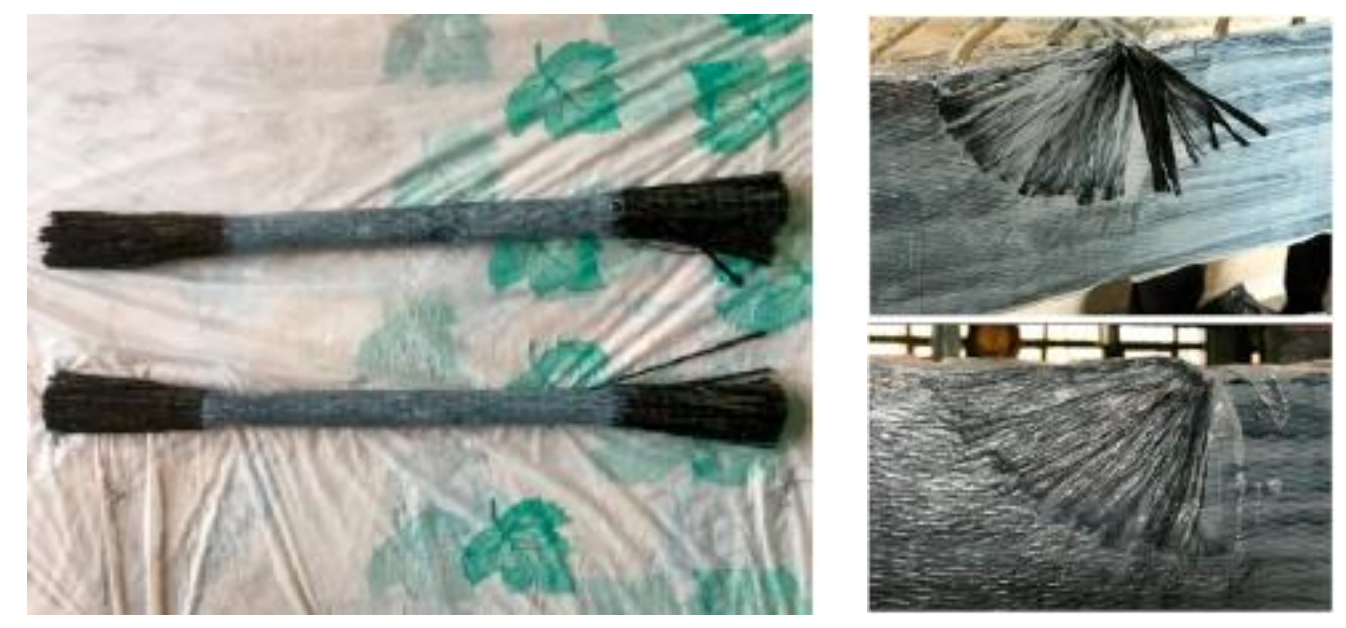

Figure 18. FRP fan anchor [49] 


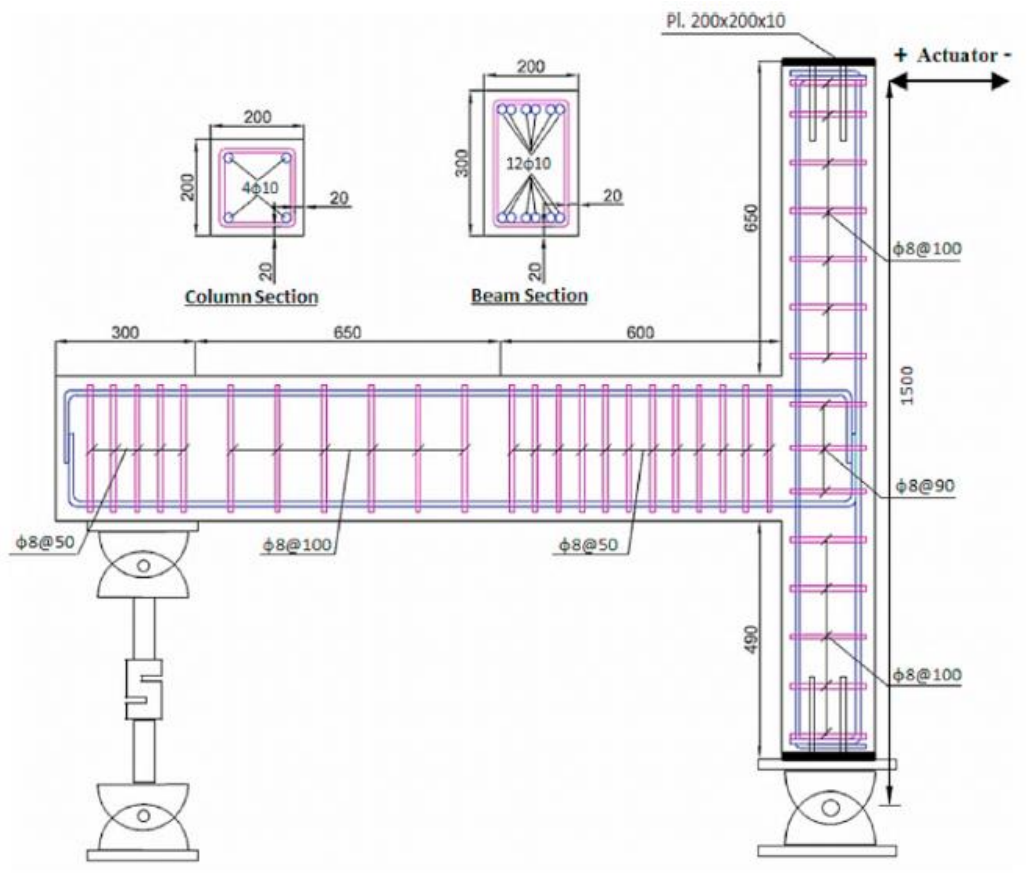

Figure 19. Test specimen details [49]

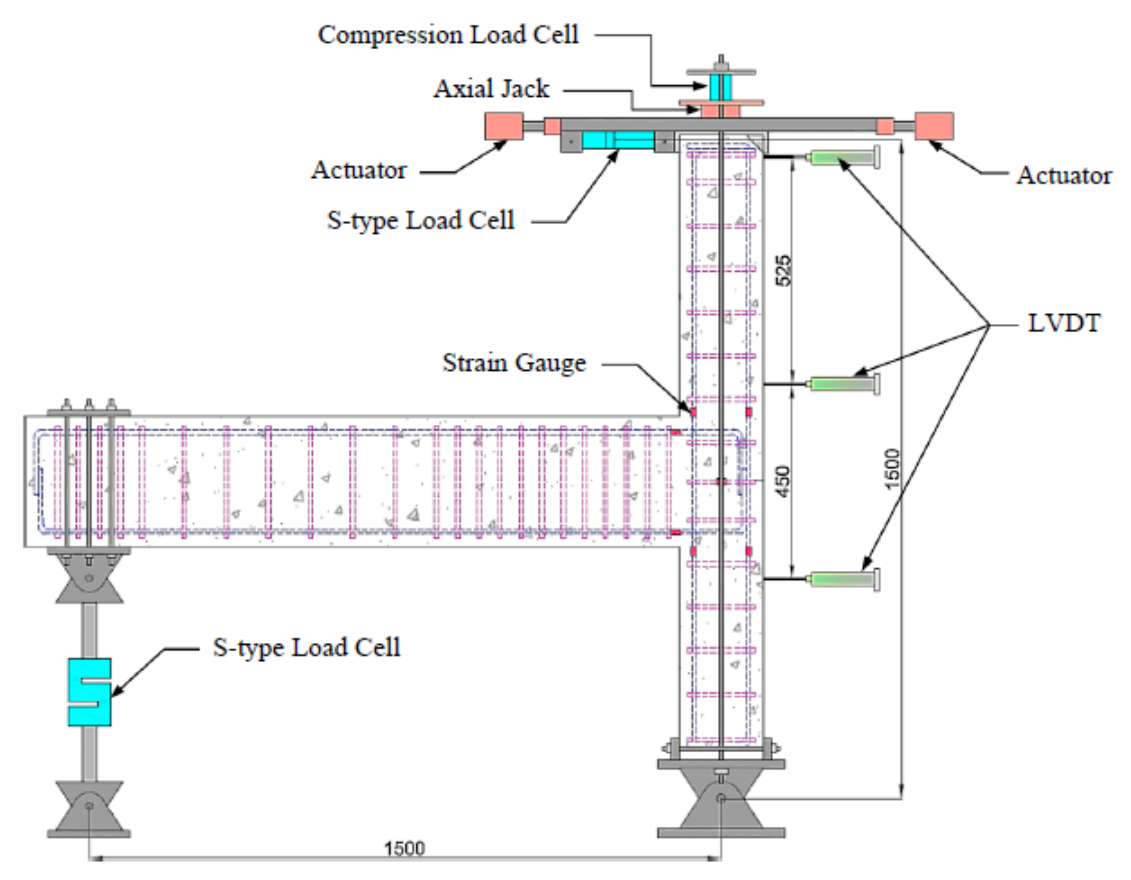

Figure 20. Test setup of [49] 
Descriptions of four specimens retrofitted using the EBROG method coupled with CFRP fan anchors are provided in Table 6 and shown in Figures 21 through 24.

Table 6. Description of retrofit schemes [49]

\begin{tabular}{|c|c|c|c|c|c|c|c|c|c|}
\hline \multirow[t]{2}{*}{ No } & \multirow[t]{2}{*}{ Specimen } & \multirow[t]{2}{*}{$\mathrm{f}^{\prime} \mathrm{c}(\mathrm{MPa})$} & \multicolumn{2}{|c|}{ Column strengthening } & \multicolumn{2}{|c|}{ Joint strengthening } & \multicolumn{3}{|c|}{ Beam strengthening } \\
\hline & & & ply No. & width $(\mathrm{mm})$ & ply No. & width $(\mathrm{mm})$ & ply No. & width $(\mathrm{mm})$ & Length $(\mathrm{mm})$ \\
\hline 1 & CS & 35 & - & - & - & - & - & - & - \\
\hline 2 & RS-1V-1X & 35 & 1 & 180 & 1 & 200 & - & - & - \\
\hline 3 & RS-1V-2X & 34 & 1 & 180 & 2 & 360 & - & - & - \\
\hline 4 & RS-1V-2X-2H.I & 34 & 1 & 180 & 2 & 360 & 2 & 180 & 150 \\
\hline 5 & RS-1V-2X-2H.II & 37 & 1 & 180 & 2 & 360 & 2 & 180 & 300 \\
\hline
\end{tabular}

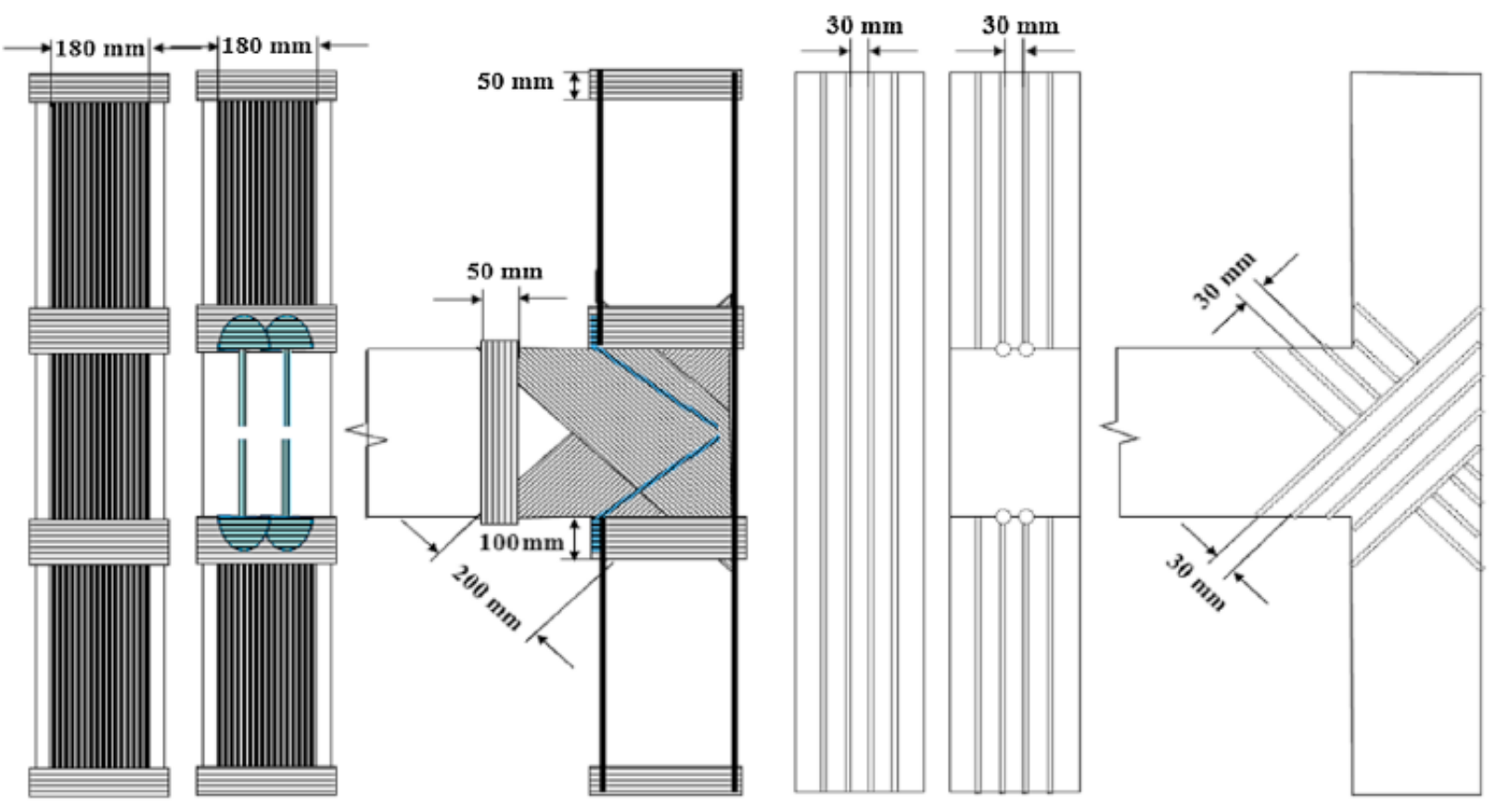

Figure 21. Rehabilitation scheme of RS-1V-1X [49] 


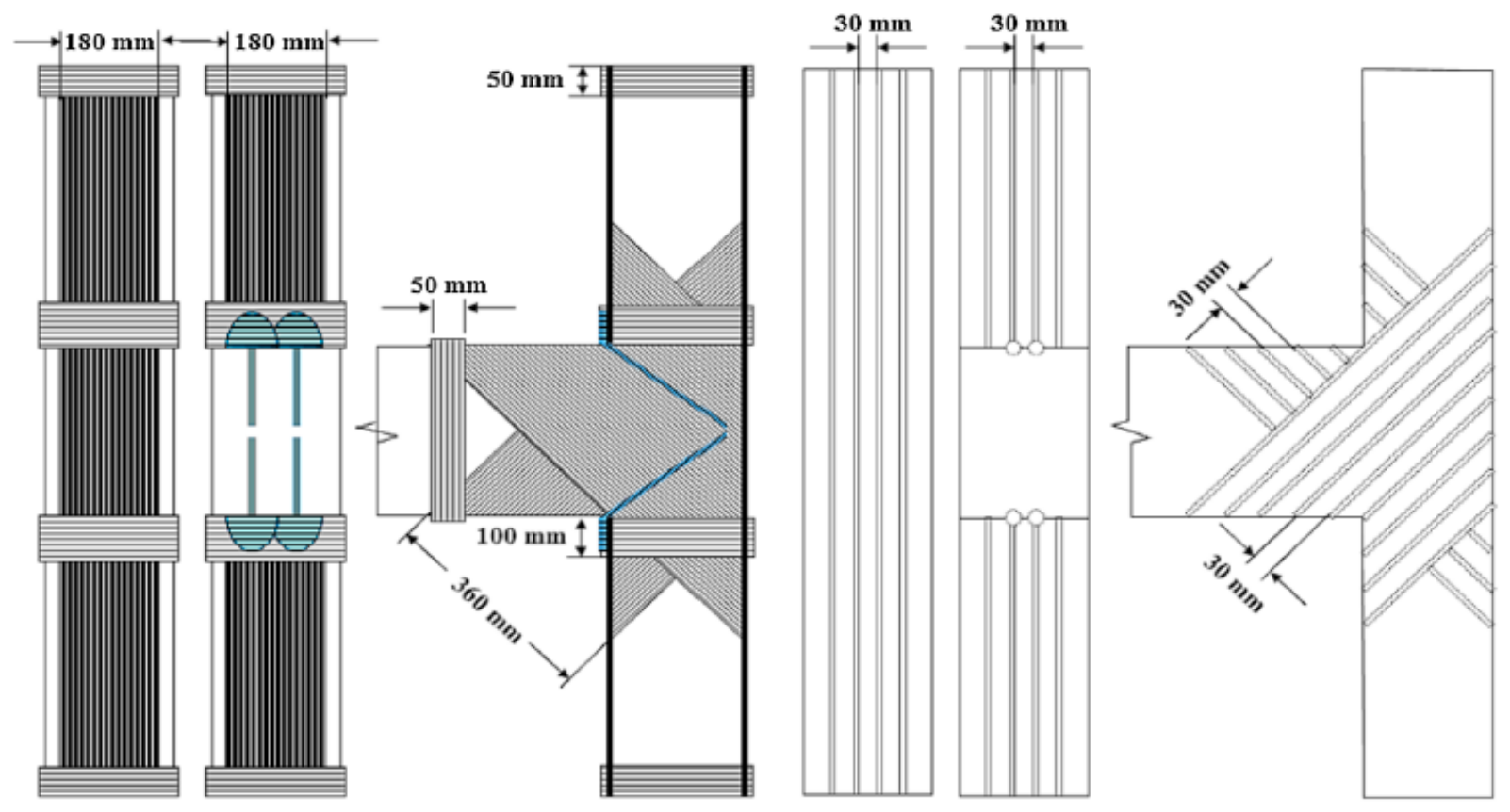

Figure 22. Rehabilitation scheme of RS-1V-2X [49]

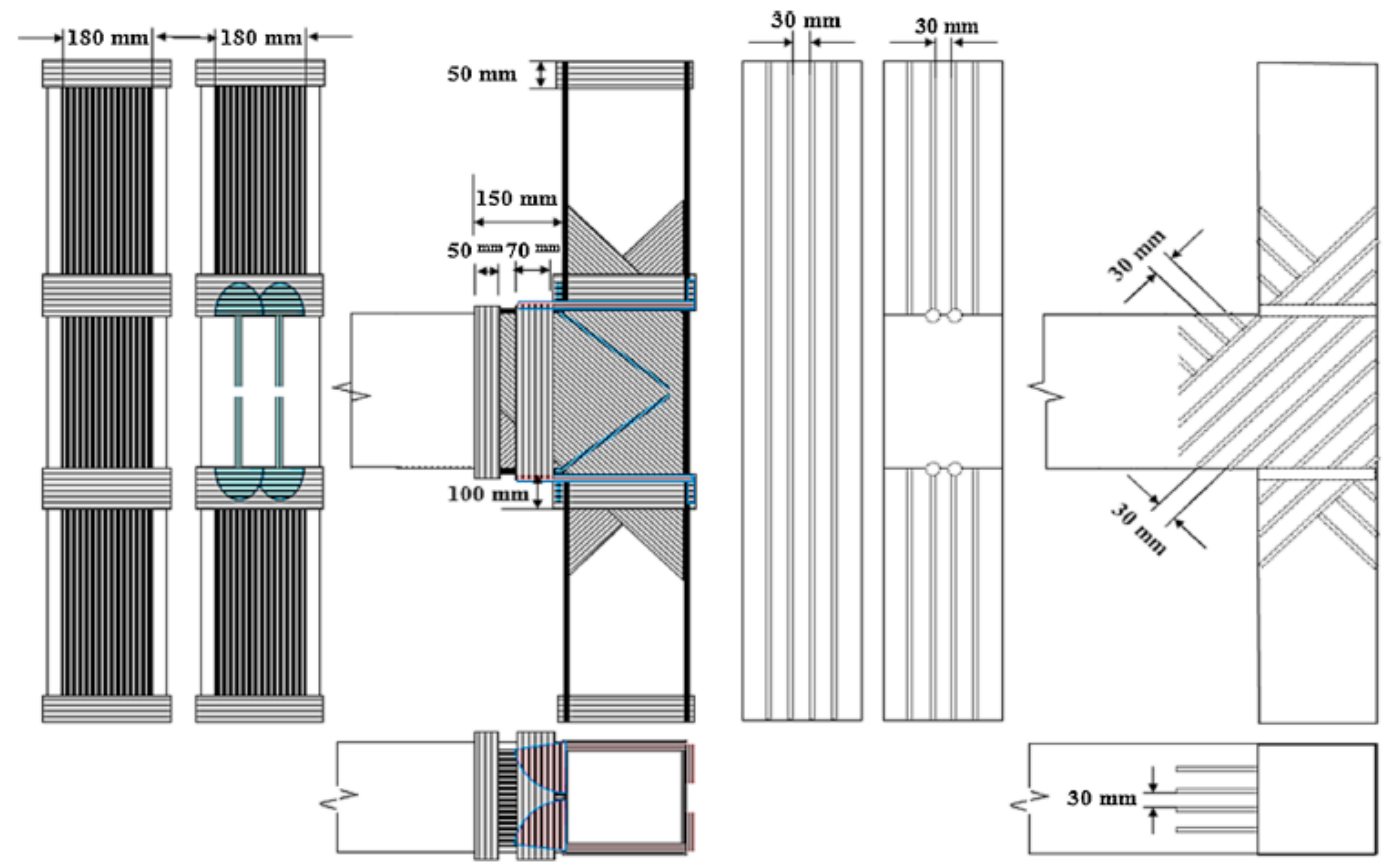

Figure 23. Rehabilitation scheme of RS-1V-2X-2H.I [49] 


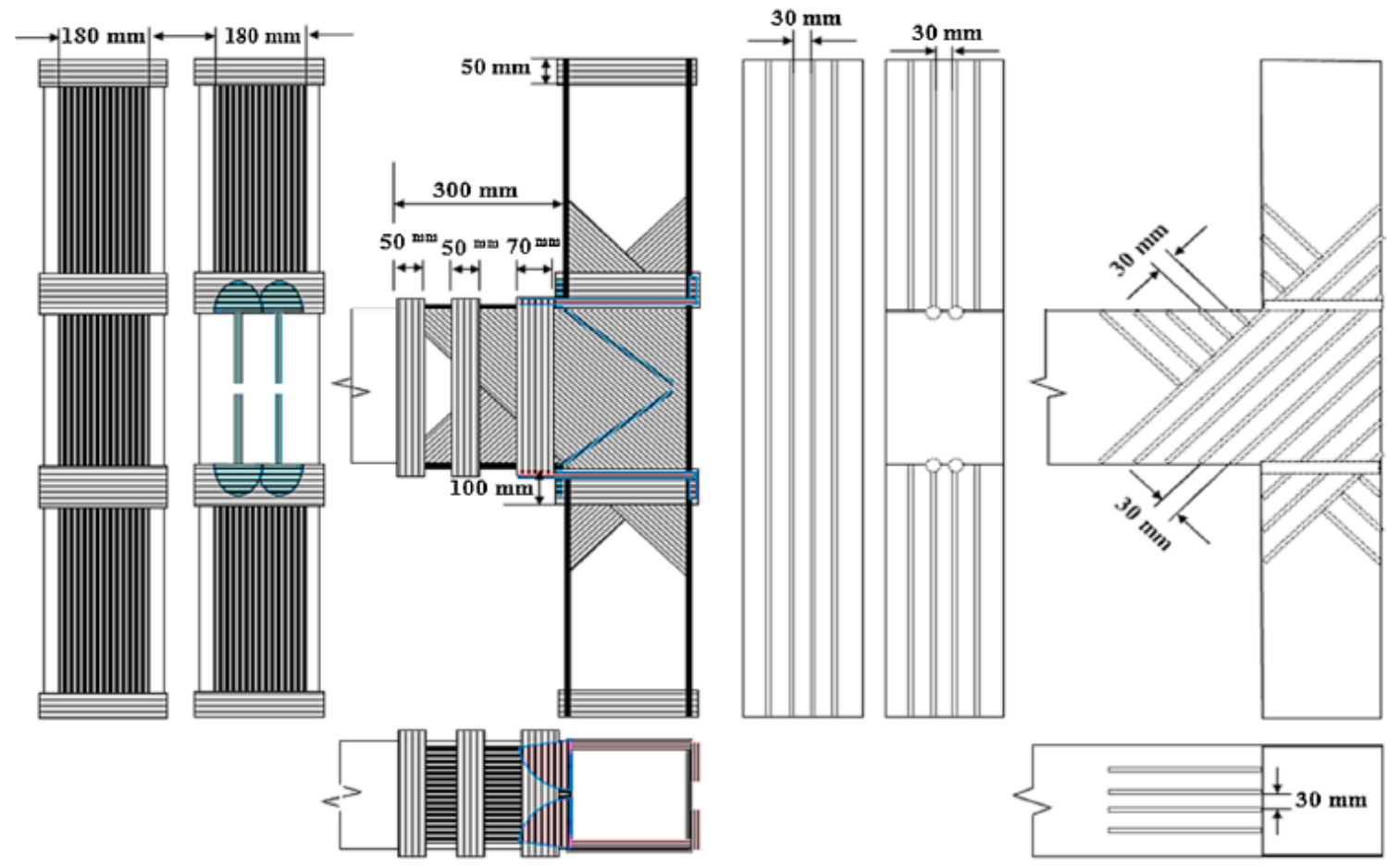

Figure 24. Rehabilitation scheme of RS-1V-2X-2H.II [49]

Test results on the experimental evaluations of [49] are summarized in Table 7.

Table 7. Summary of experimental results by [49]

\begin{tabular}{|c|c|c|c|c|c|c|c|c|c|}
\hline \multirow[t]{2}{*}{ Specimen } & \multicolumn{2}{|c|}{ Peak load $(\mathrm{kN})$} & \multirow[t]{2}{*}{ Average $(\mathrm{kN})$} & \multirow[t]{2}{*}{ Increase (\%) } & \multicolumn{2}{|c|}{ Drift ratio at peak load $(\%)$} & \multicolumn{2}{|c|}{ Ultimate load $(\mathrm{kN})$} & \multirow[t]{2}{*}{ Failure mode } \\
\hline & Push (+) & Pull (-) & & & Push (+) & Pull (-) & Push $(+)$ & Pull (-) & \\
\hline CS & 28.90 & 27.96 & 28.43 & - & 1.75 & 1.40 & 24.53 & 22.18 & $\begin{array}{l}\text { column flexural hinging alongside joint shear } \\
\text { failure }\end{array}$ \\
\hline RS-1V-1X & 42.27 & 43.52 & 42.89 & 51 & 2.75 & 2.75 & 28.06 & 31.84 & FRP rupture followed by joint shear failure \\
\hline RS-1V-2X & 47.08 & 46.25 & 46.67 & 64 & 2.20 & 2.20 & 32.85 & 29.27 & FRP rupture followed by joint shear failure \\
\hline RS-1V-2X-2H.I & 45.32 & 44.00 & 44.66 & 57 & 5.20 & 4.50 & 45.92 & 42.89 & beam flexural hinging \\
\hline RS-1V-2X-2H.II & 48.91 & 49.63 & 49.27 & 73 & 5.20 & 5.20 & 48.31 & 50.23 & beam flexural hinging \\
\hline
\end{tabular}

Following are the key learning points based on the experimental evaluations performed by [49]:

- EBROG method coupled with CFRP fan anchors was found to be efficient in preventing the de-bonding of CFRP sheets from its substrate. Based on the average values of peak loads in the push and pull directions, it was found that specimens RS-1V-1X, RS-1V-2X, RS-1V-2X-2H.I and RS-1V-2X-2H.II exhibited 51, 64, 57, and 73\% increase in peak load, 
respectively. This increase in peak loads is attributed to the amount of FRP bonded to the joint face and the effectiveness of EBROG in preventing debond failure.

- The maximum increase in ductility and energy absorption capacities of specimens strengthened using EBROG and anchor method was found to be $139 \%$ and $144 \%$, respectively. This improvement in ductility and energy absorption was attributed to the flexural hinge formation in the beams away from the column face.

- This method of reinforcing joints is heavily dependent on the amount and orientation of CFRP sheets bonded directly to the joint faces. Therefore, it is challenging to employ this technique and rely on similar capacity enhancements in actual building joints with transverse beams and slab.

\subsubsection{Influence of FRP confinement on bond strength of hooked bars}

Twelve normal-strength concrete (28 and 31.4 MPa) beam-column joints wrapped with carbon fiber reinforced polymer (CFRP) sheets were tested by [50] to evaluate the effectiveness of wrap confinement on bond strength of hooked bars. Of these twelve specimens, only six specimens (28 $\mathrm{MPa}$ ) with beam bars enclosed between column longitudinal bars are considered for review as they closely represent the arrangement in RC Joints. As shown in Figures 26 through 28, joints of three different reinforcement bar sizes with corresponding anchorage lengths (shorter than $l_{d h}$ specified by ACI 318-05) are considered for evaluations under control and CFRP bonded (see Figure 29) conditions. The specimens were monotonically loaded in increments of $10 \mathrm{kN}$ perpendicular to the longitudinal axis of the beam. 


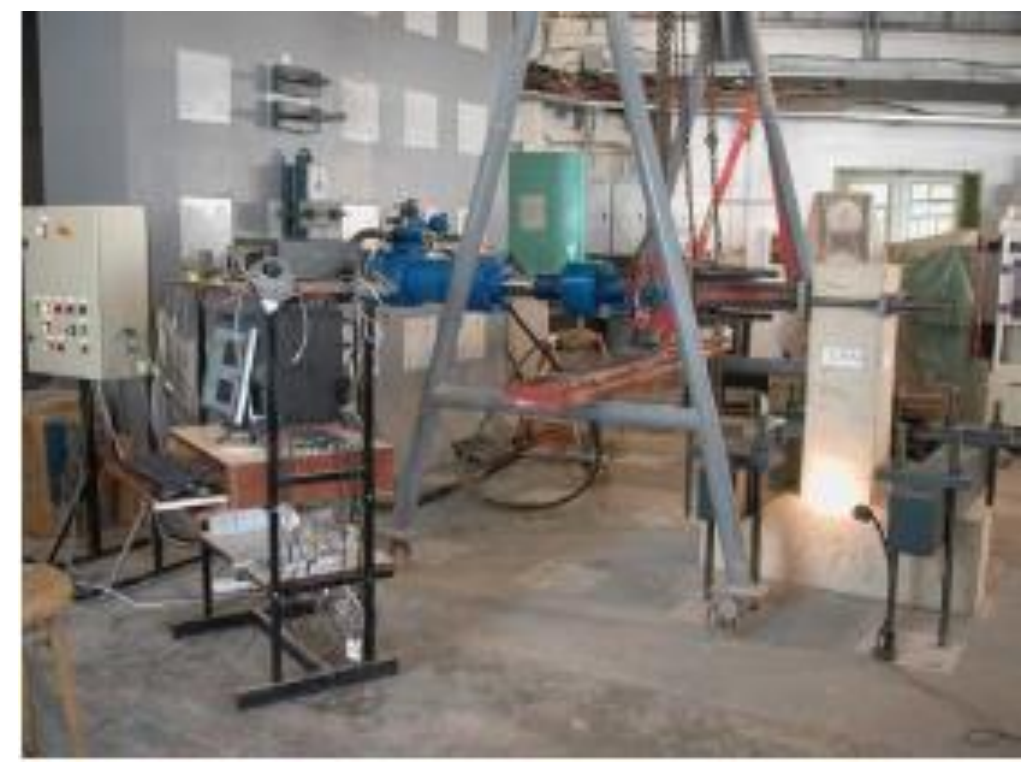

Figure 25. Experimental test setup [50]
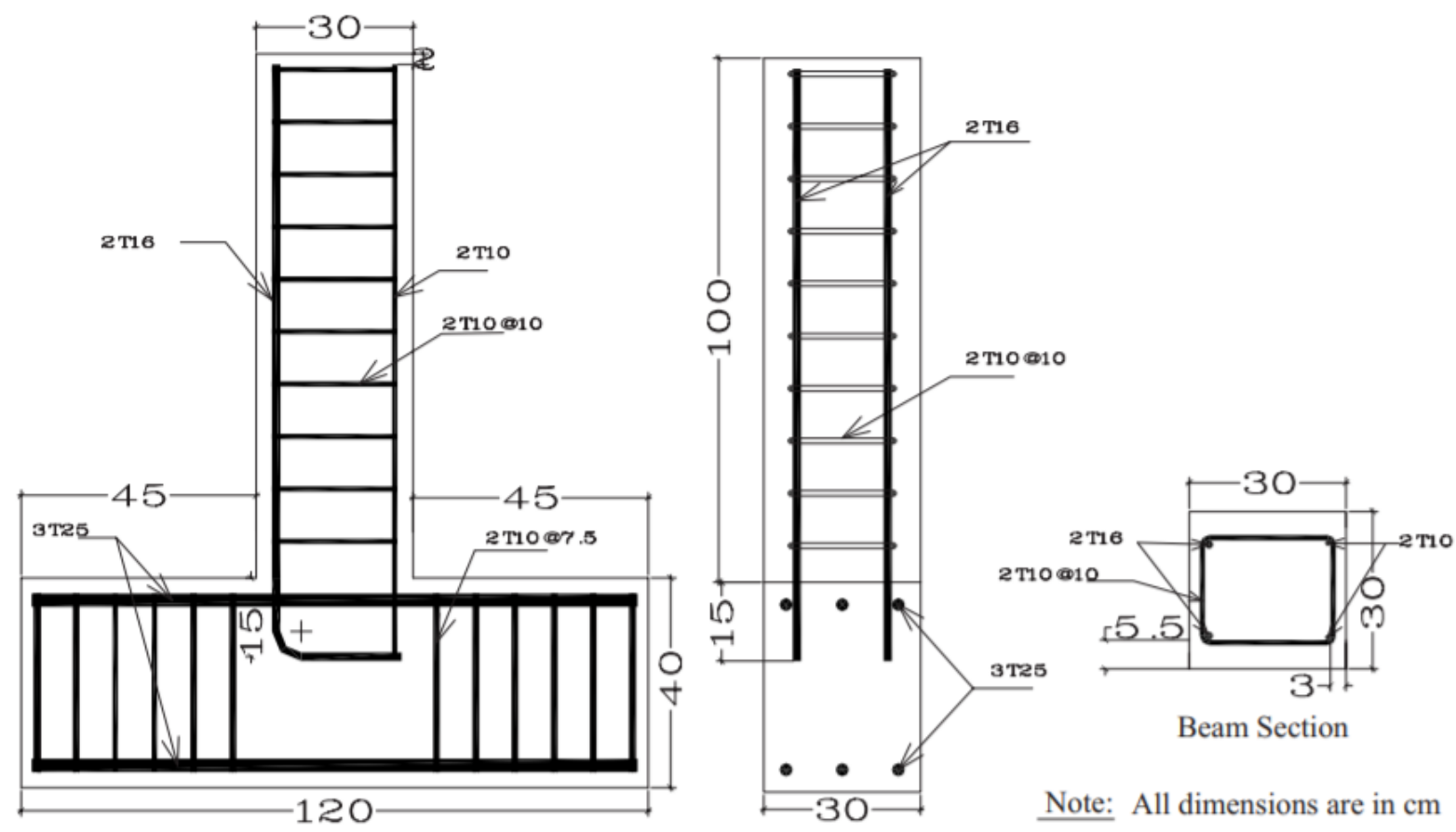

Side View

Figure 26. Details of test specimen C16N [50] 

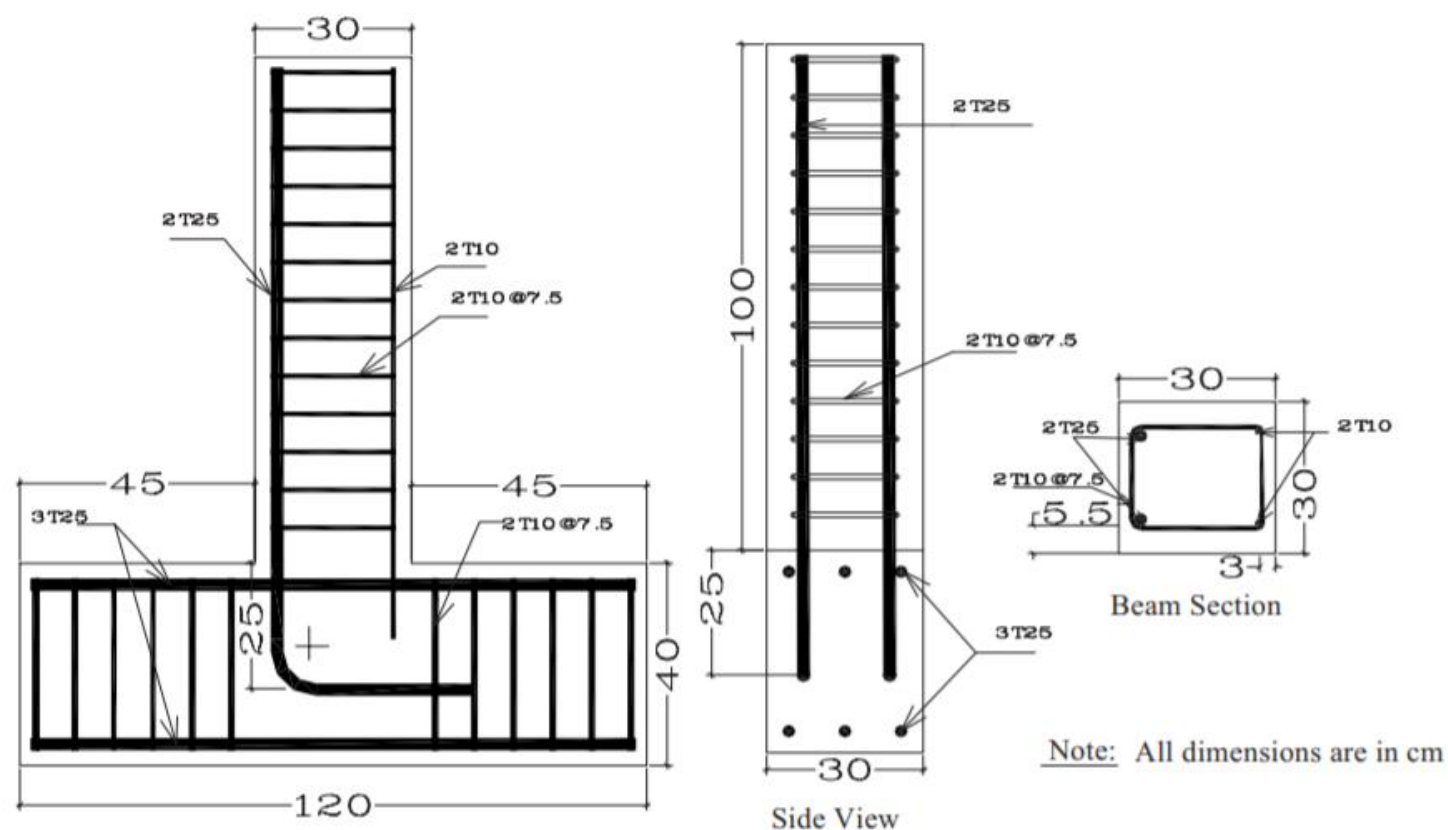

Side View

Figure 27. Details of test specimen C25N [50]
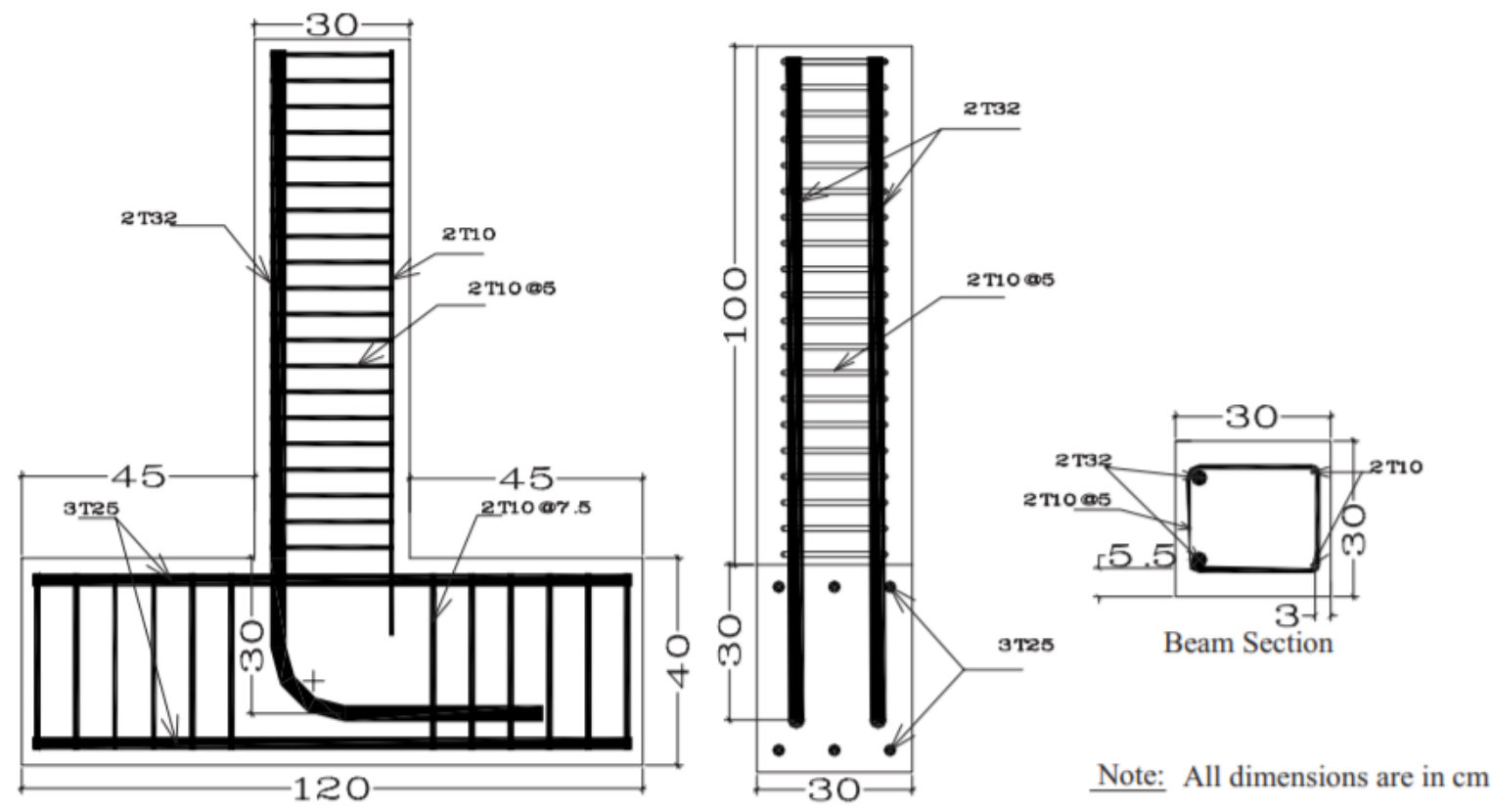

Side View

Figure 28. Details of test specimen C32N [50] 


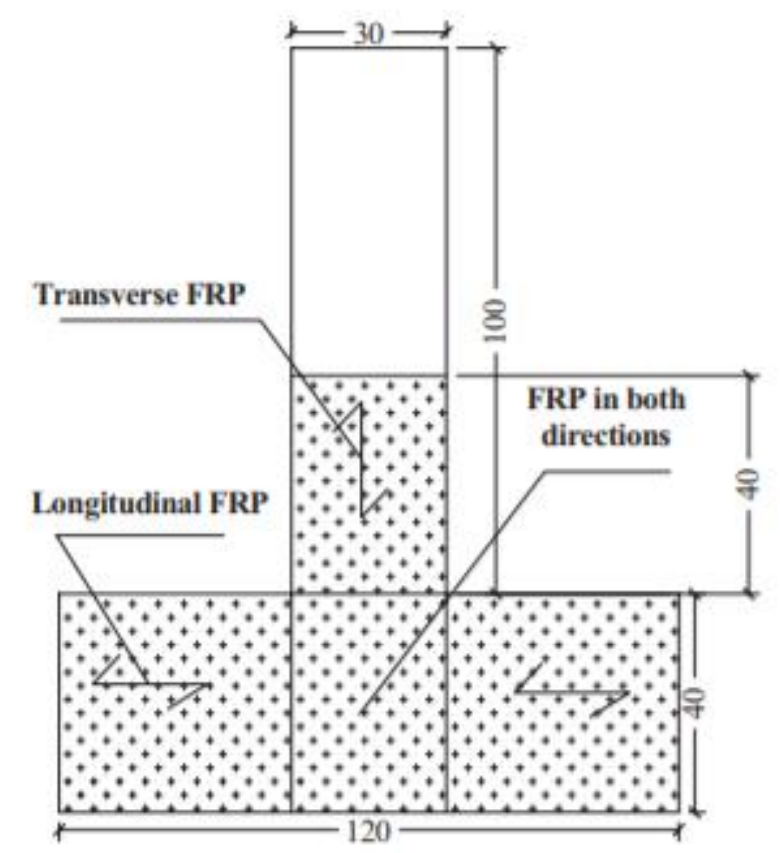

Figure 29. CFRP wrap details for C16N, C25N, and C32N [50]

Summary of experimental results by [50] is provided in Table 8.

Table 8. Summary of experimental results [50]

\begin{tabular}{lcccc}
\hline Specimen & $\begin{array}{c}\text { Tensile } \\
\text { bar }\end{array}$ & $\begin{array}{c}\text { CFRP } \\
\text { sheets }\end{array}$ & $\begin{array}{c}\text { Maximum load } \\
(\mathbf{k N})\end{array}$ & $\begin{array}{c}\text { Displacement measured under } \\
\text { beam loading point }(\mathbf{m m})\end{array}$ \\
\hline C16N & 16 & No & 60.5 & 12.6 \\
\hline C16N-F & 16 & Yes & 67.2 & 15.8 \\
\hline C25N & 25 & No & 112.8 & 19.8 \\
\hline C25N-F & 25 & Yes & 144.5 & 30.2 \\
\hline C32N & 32 & No & 146.5 & 20.0 \\
\hline C32N-F & 32 & Yes & 166.1 & 27.7 \\
\hline
\end{tabular}


The experimental results and failure behavior of test specimens evaluated by [50] are summarized as follows:

- In the case of control specimens, the first crack originated at the joint corner on the tension face having an inclination of $20^{\circ}-30^{\circ}$ with the horizontal. As the loading progressed, crack extended to the opposite corner of the joint interface. Following this, diagonal cracks appeared on the column at $10 \mathrm{~cm}$ below the tension side joint corner and propagated along the tail anchorage. The final failure of specimens occurred due to the high compressive stress concentrations at the inner radius of the bend resulting in the crushing of joint core concrete.

- Specimens strengthened with CFRP exhibited two different failure modes. (i) de-bonding or peeling off of CFRP sheets from the vertical face of the beam and (ii) tearing or shearing failure of CFRP sheets at the joint interface.

Following are the key learning points based on the experimental evaluations performed by:

- Improvement in load-carrying capacity of joints strengthened with CFRP sheets was found to be approximately $11 \%, 28 \%$, and $11 \%$ for $\mathrm{C} 16 \mathrm{~N}-\mathrm{F}, \mathrm{C} 25 \mathrm{~N}-\mathrm{F}$, and $\mathrm{C} 32 \mathrm{~N}-\mathrm{F}$ specimens, respectively when compared to their corresponding un-strengthened specimens.

- Both control and CFRP strengthened cases exhibited tension crack initiation at the interface of beam-column joints indicating the influence of stress concentrations.

- In the case of CFRP strengthened specimens, failure was either by CFRP de-bonding or tearing off of the CFRP sheets parallel to the fiber direction. The first case signifies the necessity of providing anchors for preventing such de-bonds, and the latter case reveals the need for using bi-directional fabric to avoid tearing off failure.

- FRP confinement effect on the bond strength of hooked bars attained by bonding CFRP sheets in a discontinuous manner (i.e., the beam or column sheets when bonded only to the vertical faces without the continuity of fabric between those two faces as a U-wrap or $360^{\circ}$ wrap) is insignificant.

- Resting the column base directly on the strong floor, as shown in Figure 25, will generate reactions throughout the length of the column base, causing additional concrete 
confinement that may delay the crack formations or avoid side-splitting failure of cover concrete along the tail portion of anchorage reinforcement. Furthermore, this behavior is not reflective of actual beam-column joints in a building.

\subsubsection{Influence of surface preparation on rehab effectiveness}

To assess the influence of CFRP composites in improving the joint strength, [51] tested 1/3-scale concrete joints by considering two types of surface preparation methods, namely wire brush and water jet (276 MPa up to $3 \mathrm{~mm}$ deep) coupled with structural adhesive. Dimensions of test specimen along with reinforcement details considered for evaluation and test setup are shown in Figures 30 and 31, respectively. Specimens were tested in an inverted position as opposed to their orientation in a typical building frame.

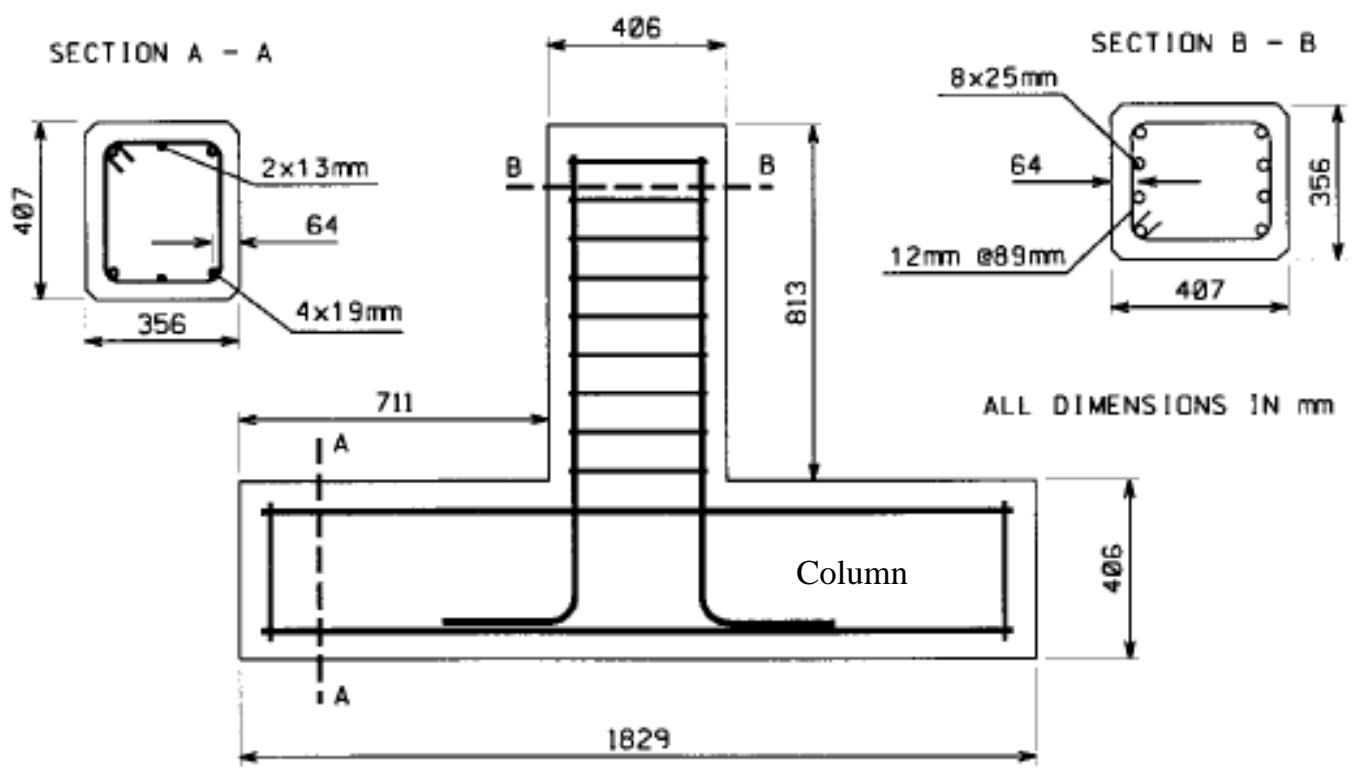

Figure 30. Test specimen reinforcement details and dimensions [51] 


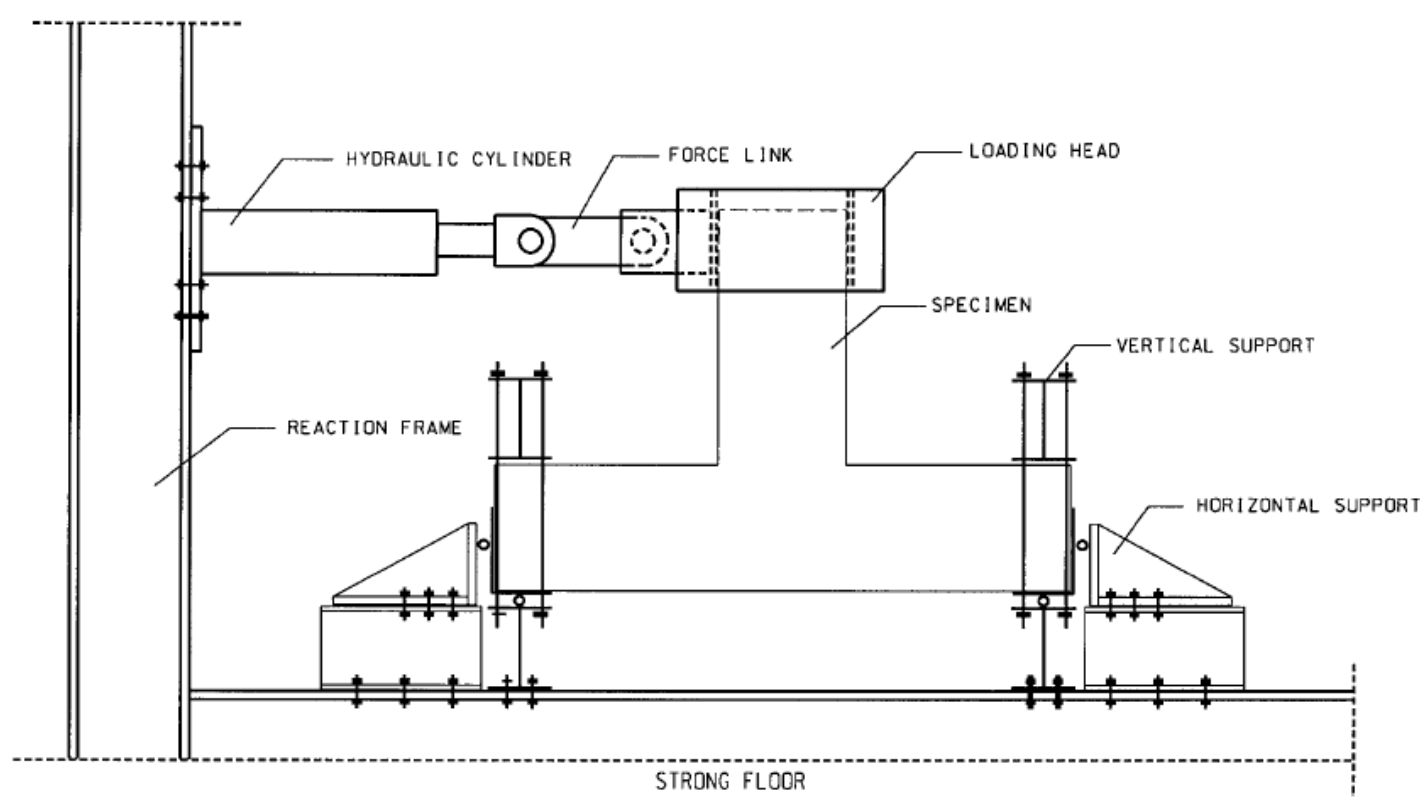

Figure 31. Test setup of joint specimen [51]

CFRP sheets were used to reinforce the deficient joints with the configurations shown in Figure 32 and listed in Table 9. The specimens were tested by applying a quasi-static cyclic lateral load on the column. At the end of testing, control specimens failed by shear damage (diagonal cracking initiated when the diagonal tensile stresses attained the concrete's tensile strength) in the joint region at low lateral loads. In the case of strengthened specimens, progressive delamination of CFRP sheets occurred starting at the face of the beam and progressing towards the joint. The strains measured on CFRP at the time of delamination varied from $0.1 \%$ to $0.35 \%$, indicating the premature separation of CFRP from the concrete surface way before reaching its ultimate strain of $1.0 \%$. The lower strain percent observed at delamination can be attributed to the use of inferior quality construction materials or inadequate surface preparation. The CFRP wrapped specimens exhibited failures similar to control specimens but at significantly higher load levels, as listed in Table 9, proving the effectiveness of CFRP wrapping in improving the joint shear strength. 

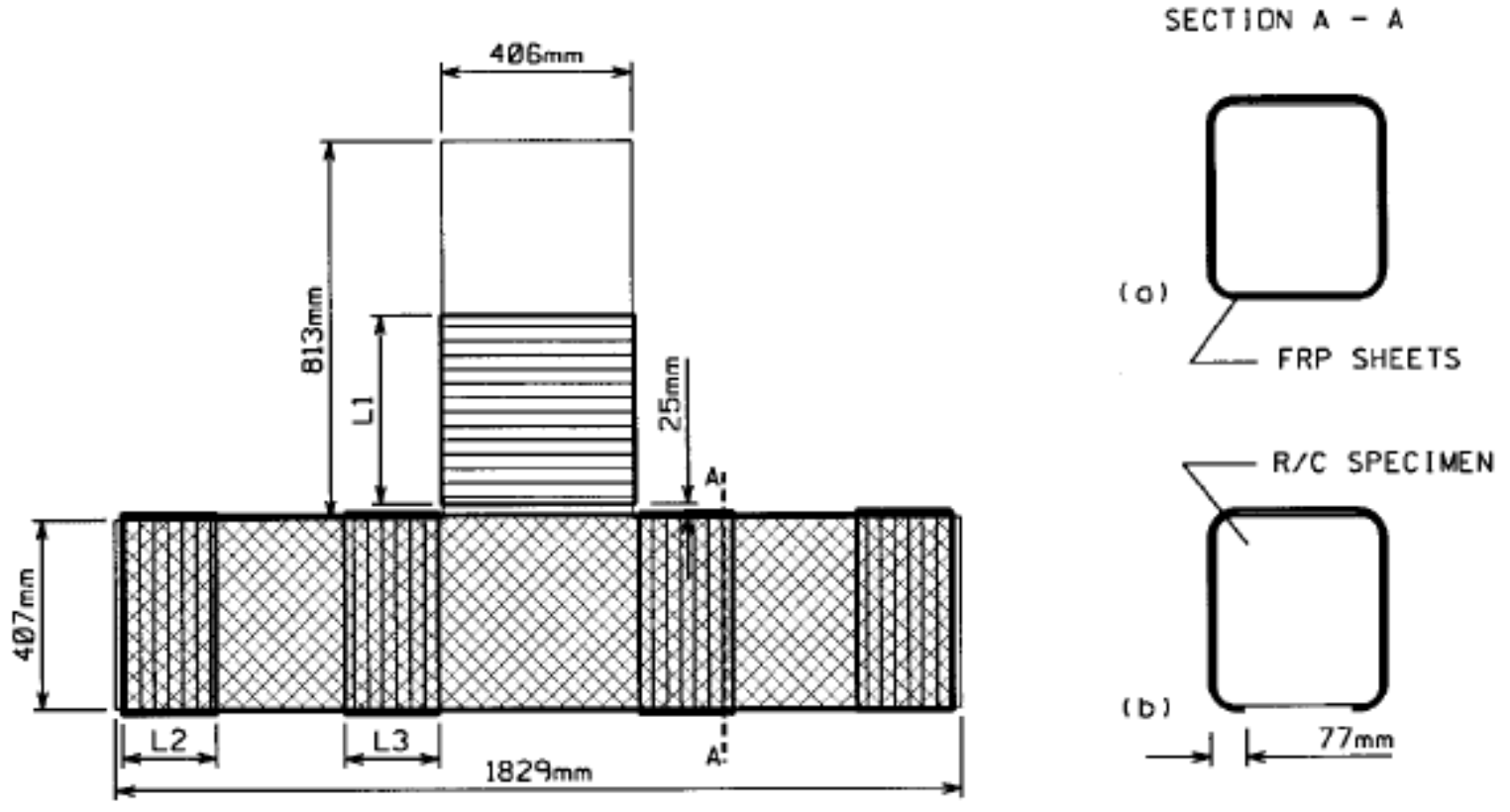

Figure 32. CFRP wrap layout [51] 
Table 9. CFRP wrapping schemes and test results [51]

\begin{tabular}{|c|c|c|c|c|c|c|c|c|c|}
\hline$f_{c}^{\prime}$ & Specimen & $\begin{array}{c}\text { Surface } \\
\text { preparation }\end{array}$ & $\begin{array}{c}\text { Cure } \\
\text { temperature }\end{array}$ & $\begin{array}{c}\text { CFRP } \\
\text { Sheets }\end{array}$ & $\mathbf{L}_{1}$ & $\mathbf{L}_{2}$ & $\mathbf{L}_{3}$ & $\begin{array}{r}\text { Push } \\
(\mathbf{k N})\end{array}$ & $\begin{array}{l}\text { Pull } \\
(\mathbf{k N})\end{array}$ \\
\hline \multirow{9}{*}{ 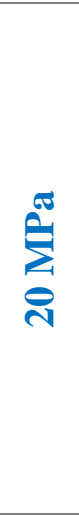 } & $1 \mathrm{~b}$ & N.A. & N.A. & No & N.A. & N.A. & N.A. & $\sim 129$ & $\sim 129$ \\
\hline & $2 \mathrm{~b}$ & N.A. & N.A. & No & N.A. & N.A. & N.A. & 129 & 142 \\
\hline & 3 & Wire brush & $350^{\circ} \mathrm{F}$ & $45^{\circ}$ & 0 & 178 & 0 & 182 & 142 \\
\hline & 4 & Wire brush & $350^{\circ} \mathrm{F}$ & $\pm 45^{\circ}$ & 0 & 178 & 0 & 187 & 187 \\
\hline & 5 & Wire brush & Room & $45^{\circ}$ & 0 & 229 & 0 & 161 & 154 \\
\hline & 6 & Wire brush & Room & $45^{\circ}$ & 432 & 229 & 406 & 158 & 140 \\
\hline & 7 & Wire brush & Room & $45^{\circ}$ & 432 & 229 & 406 & 156 & 143 \\
\hline & 8 & Wire brush & Room & $\pm 45^{\circ}$ & 0 & 203 & 0 & 187 & 187 \\
\hline & 9 & Wire brush & Room & $\pm 45^{\circ}$ & 406 & 229 & 203 & 216 & 200 \\
\hline \multirow{5}{*}{$\sum_{\vec{m}}^{\infty}$} & $10 \mathrm{~b}$ & N.A. & N.A. & No & N.A. & N.A. & N.A. & 126 & 116 \\
\hline & $11 \mathrm{~b}$ & N.A. & N.A. & No & N.A. & N.A. & N.A. & 148 & 98 \\
\hline & 12 & Water jet & Room & $\pm 45^{\circ}$ & 406 & 203 & 406 & 184 & 217 \\
\hline & 13 & Water jet & Room & $\pm 45^{\circ}$ & 406 & 203 & $406 c$ & 204 & 196 \\
\hline & 14 & Water jet & Room & $\pm 45^{\circ}$ & 406 & 203 & $406 c$ & 229 & 224 \\
\hline
\end{tabular}

${ }^{a}$ CFRP layout on each vertical face of beam, baseline specimens, ${ }^{c}$ only on three sides.

Following are the key learning points based on the experimental evaluations performed by [51]:

- Surface preparation of test specimens using water jet coupled with structural adhesive was found to be more efficient in enhancing the shear strength of joints with no shear reinforcement and longitudinal bars bent away from the core. However, in practice, it is unlikely to have a beam without any shear reinforcement.

- Although CFRP strengthened specimens exhibited joint shear failure patterns similar to control specimens, the failure was contained.

- Capacity enhancements in specimens strengthened with a single layer of CFRP having $45^{\circ}$ orientation were somewhat inconsistent compared to specimens strengthened with balanced $\pm 45^{\circ}$ orientation. Specimen with balanced $\pm 45^{\circ}$ orientation exhibited $31 \%$ higher load carrying capacity in compassion to a single layer of CFRP having $45^{\circ}$ orientation.

- Delamination of CFRP initiated from the corners of the joint panel, indicating the high importance of minimizing stress concentrations and the need for additional anchorage through confinement or other mechanisms. 
- The increase in nominal principal tensile stress developed in the joint panel was found to be in the range of $(0.17-0.33) \mathrm{MPa}$ for $45^{\circ}$ unsymmetrical orientation and $(0.62-1.21)$ $\mathrm{MPa}$ for symmetrical $\pm 45^{\circ}$ orientation parallel to the principal planes.

- Elevated temperature cure did not play a role in improving the shear strength of joints implying that the resin was adequately cured at room temperature before testing.

\subsubsection{Effectiveness of FRP wrap layup on joint strengthening}

[52] focused their research on enhancing the shear capacity of non-seismically designed joints and identifying the efficient CFRP wrapping configuration. Towards this goal, the authors cast seven specimens with no stirrup in the joint region, of which one specimen was evaluated in control (control) condition (see Figure 33) and the remaining six joints under varying CFRP configurations (see Figure 34). The description of CFRP configurations is provided in Table 10.
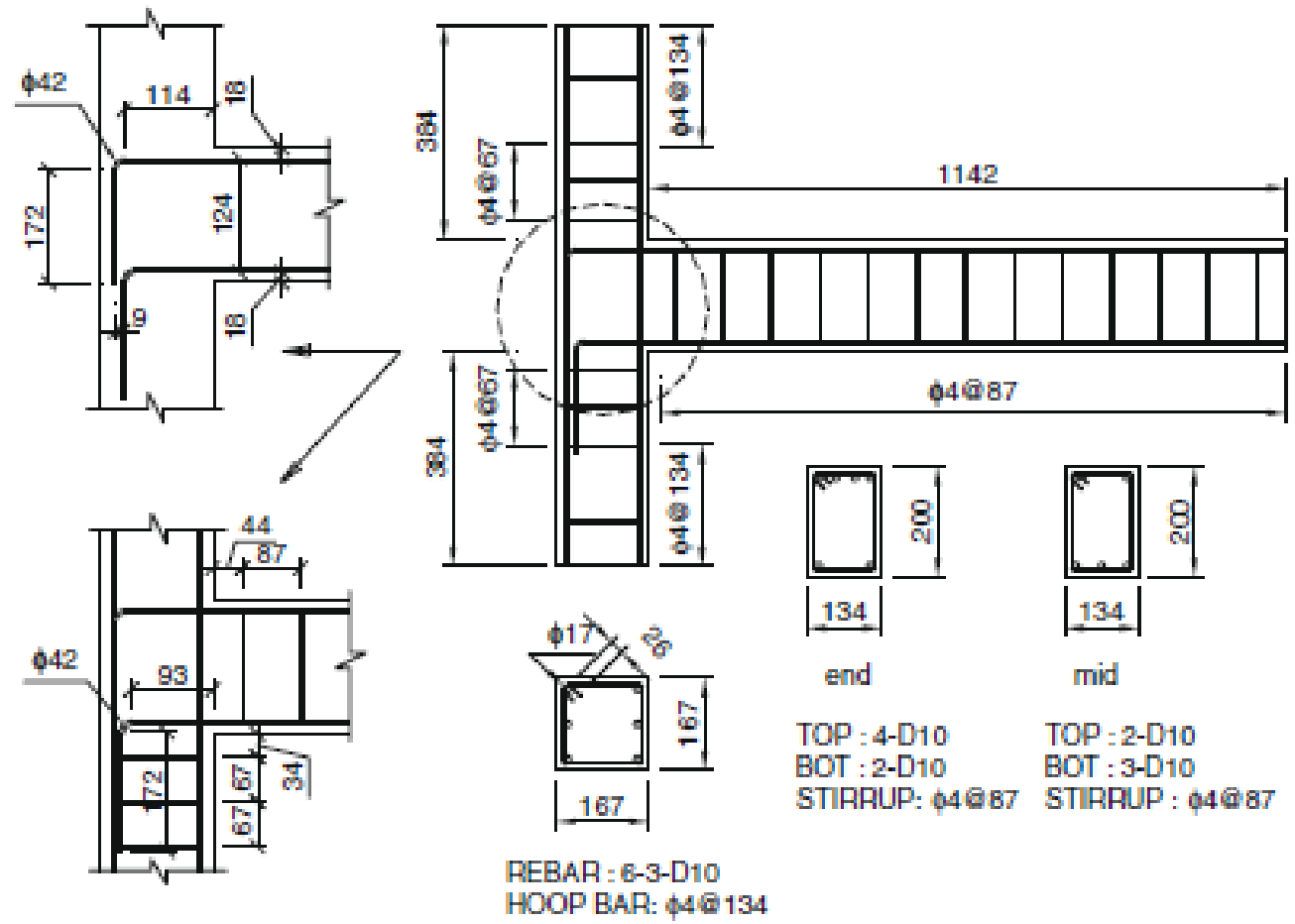

Figure 33. Details of the non-seismic joint [52] 


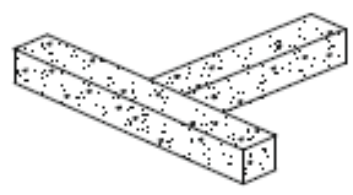

a. NS and SD

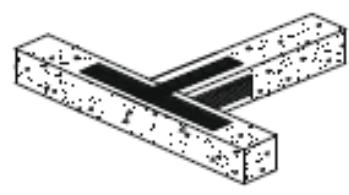

b. RNS-1

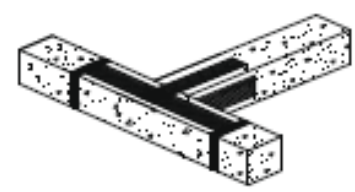

c. RNS-2

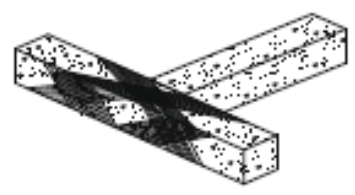

d. RNS-3

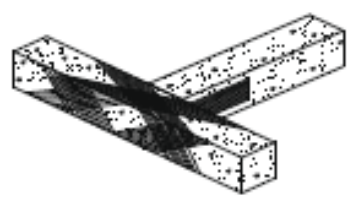

e. RNS-4

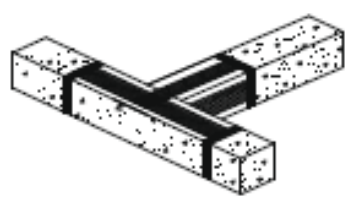

f. RNS-5

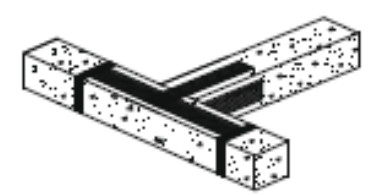

g. RNS-6

Figure 34. CFRP configurations for strengthening non-seismic joints [52]

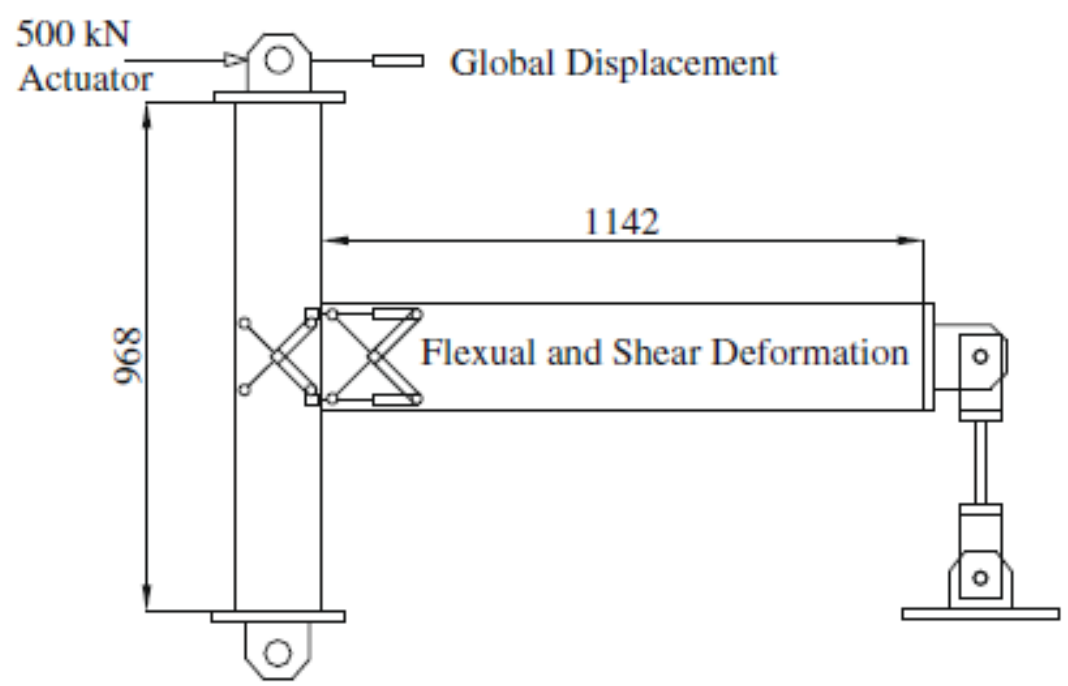

Figure 35. Test setup [52] 
Table 10. Description of CFRP configurations [52]

\begin{tabular}{llllll}
\hline No. & Specimen & T-shape & L-shape & X-shape \\
\hline 1 & NS & None & None & None & None \\
2 & SD & None & None & None & None \\
3 & RNS-1 & Both sides & On top and bottom of beam & None & None \\
4 & RNS-2 & Both sides & On top and bottom of beam & On three surfaces & None \\
5 & RNS-3 & None & None & On three surfaces & None \\
6 & RNS-4 & None & On top and Bottom of beam & None & Strip on column and beam \\
7 & RNS-5 & Both sides & On top and bottom of beam & Strip on column \\
8 & RNS-6 & Both sides (two layers) & On top and bottom of beam & None \\
\hline
\end{tabular}

Specimens were tested in setup, as shown in Figure 35, without applying column axial load.

The experimental results and failure behavior of test specimens evaluated by [52] are summarized as follows:

Specimen NS:

- The first crack (flexural) occurred at the beam-joint interface.

- At $12 \mathrm{~mm}$ displacement, joint shear cracks initiated and led to ultimate failure.

Specimen RNS-1:

- At $18 \mathrm{~mm}$ displacement, the L-shape CFRP attached to the column inner surface extending on to the beam de-bonded close to the joint region.

- At $24 \mathrm{~mm}$ displacement, T-shaped sheets bonded to the face of the joint with portions extending on to the beam, top and bottom column stubs, de-bonded towards the nonretrofitted face of the column causing slippage of the top longitudinal beam bars.

Specimen RNS-2:

- The damage was caused due to de-bonding of CFRP sheets similar to that of specimen RNS-1 but at a higher displacement. The addition of U-strip anchors delayed the debonding of CFRP sheets.

Specimen RNS-3:

- At $18 \mathrm{~mm}$ displacement, multiple flexural cracks appeared on the beam, especially close to the joint region. These cracks grew as the testing progressed. 
- At $36 \mathrm{~mm}$ displacement, the spalling of concrete was noticed on the beam surfaces.

- Specimen experienced beam flexural failure with insignificant cracks in the joint region.

Specimen RNS-4:

- The failure behavior was similar to that of RNS-3.

- The addition of L-shaped CFRP sheets delayed the formation of flexural cracks to $24 \mathrm{~mm}$ displacement.

- As the test progressed, L-shaped sheets de-bonded close to the joint region but did not progress farther.

- At the time of failure, more cracks were seen in the joint region in comparison to specimen RNS-3, which was attributed to the beam strength and stiffness enhancement due to the addition of L-shaped sheets.

Specimen RNS-5:

- At $24 \mathrm{~mm}$ displacement, the L-shape CFRP sheets de-bonded close to the joint region.

- U-shape strips bonded to the beam prevented the de-bonding of CFRP sheets from its surfaces, causing complete detachment of L- and T- shape close to the joint region on the column surfaces.

Specimen RNS-6:

- At $24 \mathrm{~mm}$ displacement, the CFRP sheets de-bonded, causing cracks on the non-retrofitted side of the column.

- Although an increase in the number of CFRP layers prevented joint shear failure, it weakend the non-retrofitted face of the column resulting in the spalling of large portions of cover concrete.

Results based on the experimental evaluations performed are summarized in Table 11. 
Table 11. Summary of test results [52]

\begin{tabular}{cccccccc}
\hline Specimen & NS & RNS-1 & RNS-2 & RNS-3 & RNS-4 & RNS-5 & RNS-6 \\
\hline Max. positive lateral load, kN & 8.56 & 10.10 & 9.87 & 10.06 & 9.90 & 9.52 & 11.27 \\
\hline \% increase comparing to NS & - & 17.99 & 15.30 & 17.52 & 15.65 & 11.21 & 31.66 \\
\hline
\end{tabular}

Following are the key learning points based on the experimental evaluations performed by [52]:

- Joint shear failure in the control specimen occurred due to the lack of transverse reinforcement.

- Bottom anchorage bars bent away from the joint panel did not provide adequate confinement to the joint core, causing brittle failure.

- Bonding discrete layers of L-and T-shape CFRP sheets without anchors has led to premature de-bonding from its substrate.

- The addition of U-strips enhanced the bond performance between underlying sheets and concrete substrate, thereby delaying the initiation of- or preventing- de-bond.

- $45^{\circ}$ orientation of fibers (continuous U-fabric covering three surfaces) in the joint region with respect to the beam longitudinal axis, has provided the best performance. This is because the fibers are more or less oriented parallel to the principal planes reinforcing the concrete strut.

- Specimens strengthened with one layer of discrete T- + L-shape sheets and X-pattern exhibited similar strength improvement. However, close to the optimal orientation of fibers and improved confinement in the latter case exhibited more ductile behavior due to beam flexural failure.

- Reinforcing the joint with CFRP sheets in continuous X-pattern has improved the confinement of joint core resulting in 5.3 times ductility over the control specimen.

- Although strengthening with two layers of discrete T- and L- CFRP sheets on either side of a joint improved its strength by $31.7 \%$, it did not provide adequate ductility as compared to specimen with continuous $\mathrm{X}$ - pattern. 


\subsubsection{Effectiveness of joint core confinement through FRP wrapping}

To improve the FRP confinement effect on a joint core, [53] proposed a method of bonding concrete covers and wrapping with FRP composites. To evaluate the efficiency of proposed method, four joints without transverse reinforcement were tested under reversed cyclic loading. Reinforcement details of test specimens in control and rehab conditions are shown in Figures 36 and 37 , respectively.

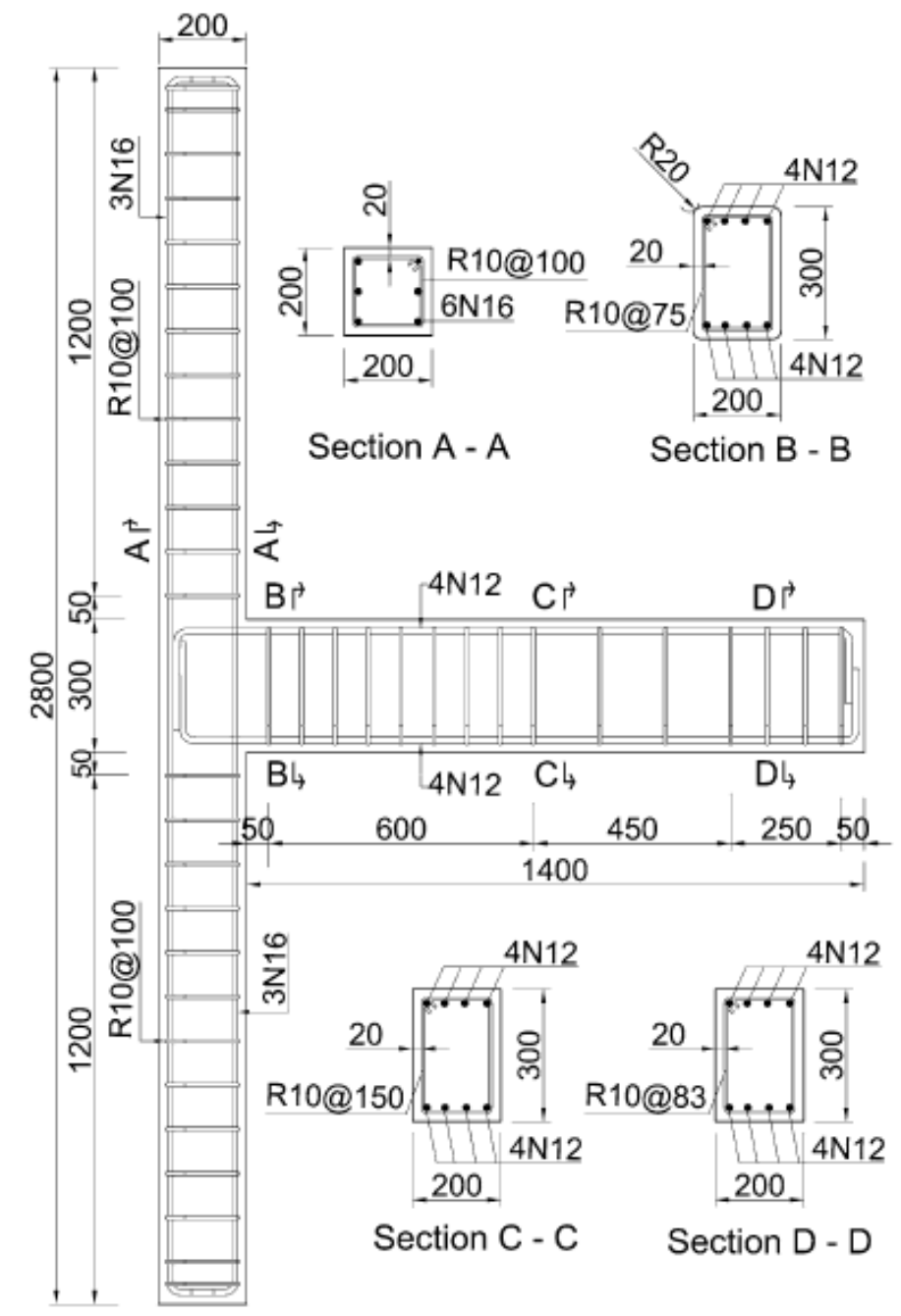

Figure 36. Joint steel reinforcement details [53] 


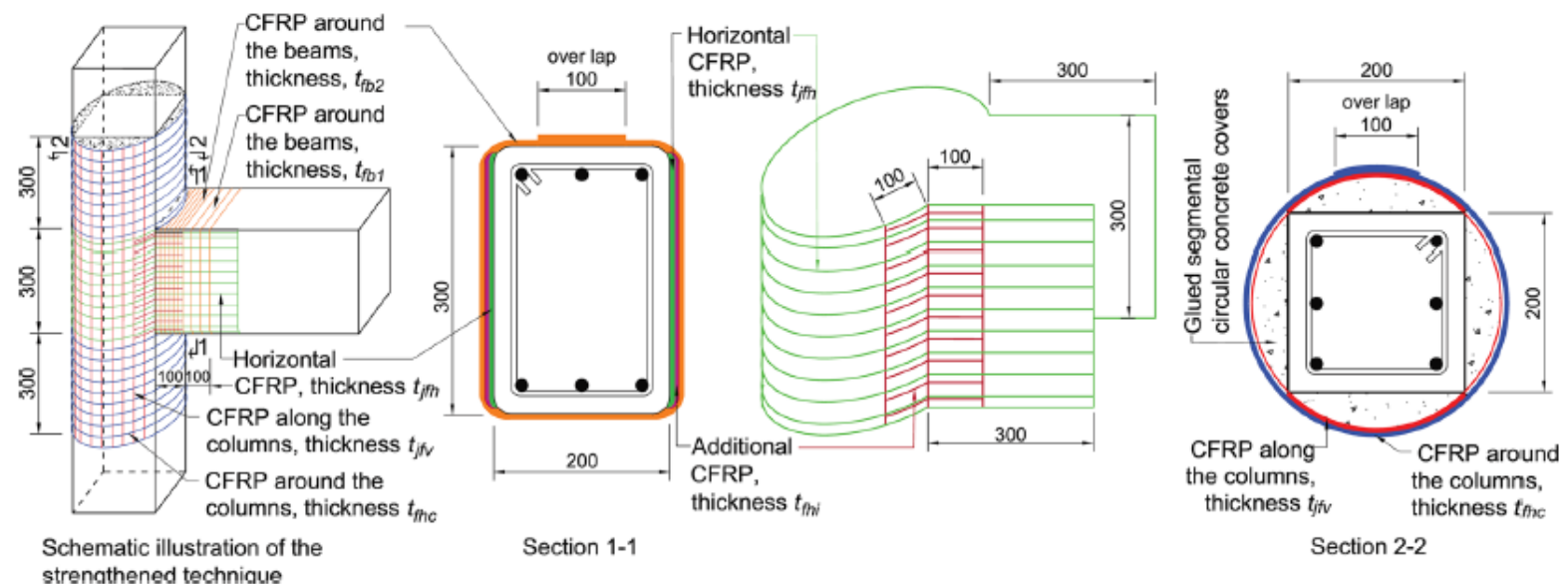

Figure 37. CFRP rehab detailing of joint specimens [53]

The experimental results and failure behavior of test specimens evaluated by [53] are summarized as follows:

Specimen T0:

- Diagonal shear cracks developed at the joint center when the specimen reached a peak load of $35.8 \mathrm{kN}$.

- As the test progressed, bond cracks developed along the column reinforcement. Diagonal cracks grew in size and merged with bond split cracks leading to joint shear failure.

- At the end of the test, concrete was crushed entirely in the joint core.

\section{Specimen TS:}

- When the specimen reached a peak load of $77.2 \mathrm{kN}$, horizontal CFRP around the joint at the beam-column interface ruptured due to the formation of the flexural hinge at the column face.

- As the specimen was further loaded, CFRP at the joint de-bonded causing joint shear failure.

- At the end of the test, large flexural cracks were seen at the beam-column interface along with diagonal shear cracks in the joint area. 
Specimen TS-1:

- Specimen TS-1 failed in joint shear due to the rupture of horizontal and vertical CFRP sheets in the joint area.

- When the specimen reached a peak load of $65.8 \mathrm{kN}$, initiation of CFRP rupture at the top and bottom face of the beam-column interface was noticed.

- As the specimen was loaded further, CFRP sheets started to de-bond in the joint region, following which CFRP sheets around the column ruptured.

- The specimen finally failed due to the complete de-bonding of CFRP sheet, resulting in joint shear failure.

Specimen TS-2:

- As the specimen reached a peak load of $140 \mathrm{kN}$, no de-bonding or rupture of CFRP was noticed in the joint region. Instead, this wrap configuration led to the formation of the flexural hinge in the beam outside the strengthened portion.

- Finally, specimen TS-2 failed in beam flexure with hinge formation at $250 \mathrm{~mm}$ away from the beam-column interface.

Following are the key learning points based on the experimental evaluations performed by:

- Specimen TO, TS, and TS-1 failed in joint shear with an increase in the load-carrying capacity of $84-115 \%$ depending on the thickness of CFRP wrap in and around the joint region. Specimen TS-2 failed through the beam hinging with an increase of $140 \%$ in load capacity.

- The maximum improvement in displacement ductility was found to be $87 \%$ for the TS-2 specimen that exhibited beam flexural failure.

- CFRP strengthened (TS-1) specimen with joint shear failure exhibited only a $33 \%$ improvement in displacement ductility.

- Improvement in energy dissipation capacity of TS, TS-1, and TS-2 was found to be 299, 99 , and $407 \%$, respectively. It should be noted that hinge formation in the beam irrespective 
of whether the final failure was concentrated in the joint or beam has led to significant improvement in energy dissipation.

- Although bonding of concrete covers coupled with CFRP wraps has improved the performance of 2D joint specimens due to confinement of joint core, it is highly challenging to reinforce actual building joints with transverse beams and slab using this technique.

\subsubsection{Influence of FRP strengthening on joints with transverse beams}

To investigate the influence of FRP strengthening on joints with transverse beams, [45] evaluated 2/3- scale exterior joints under simulated seismic loads. Test specimens were created without stirrups in the joint to replicate poorly detailed RC frame. The geometric dimensions and reinforcement details of test specimens are shown in Figures 38 through 40. shows the FRP strengthening configurations adopted for the test specimens.

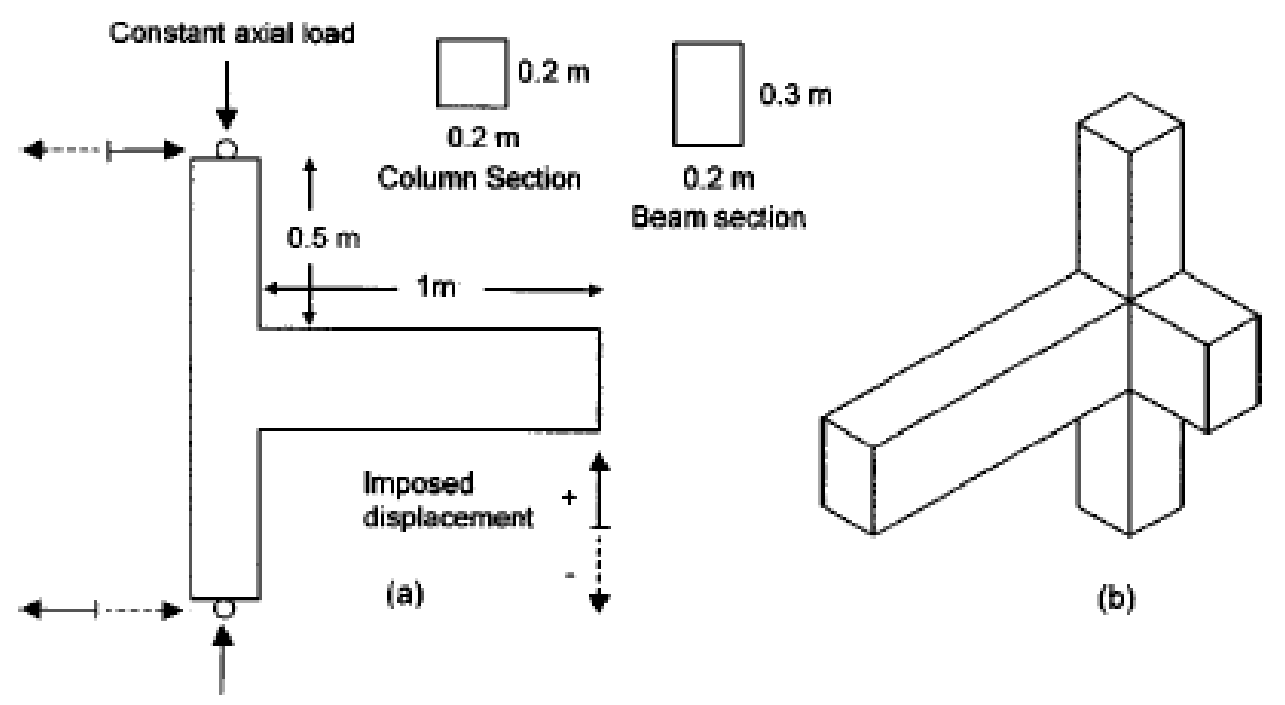

Figure 38. Geometric details of test specimen [45] 


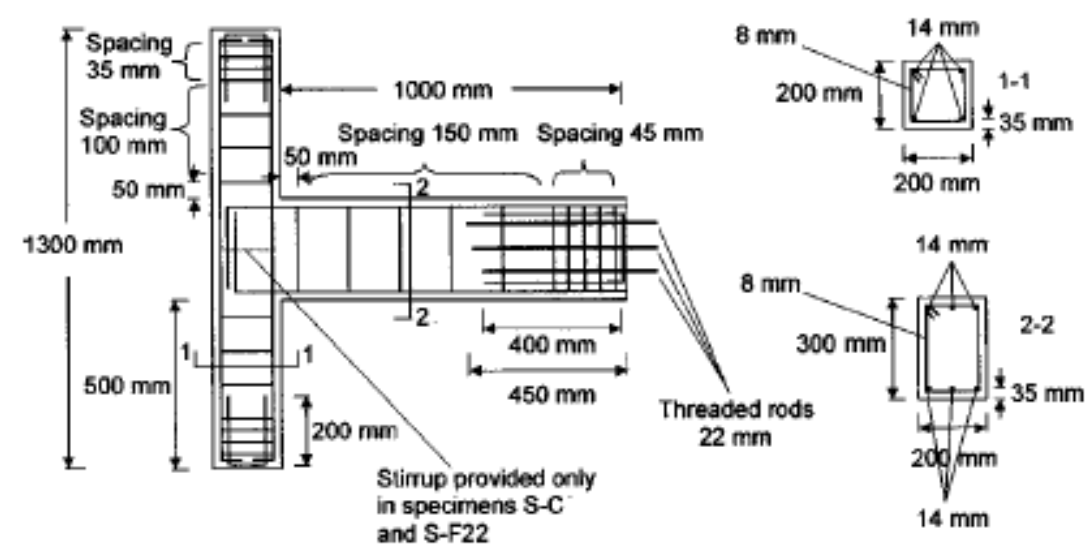

Figure 39. Steel reinforcement detailing of test specimen [45]
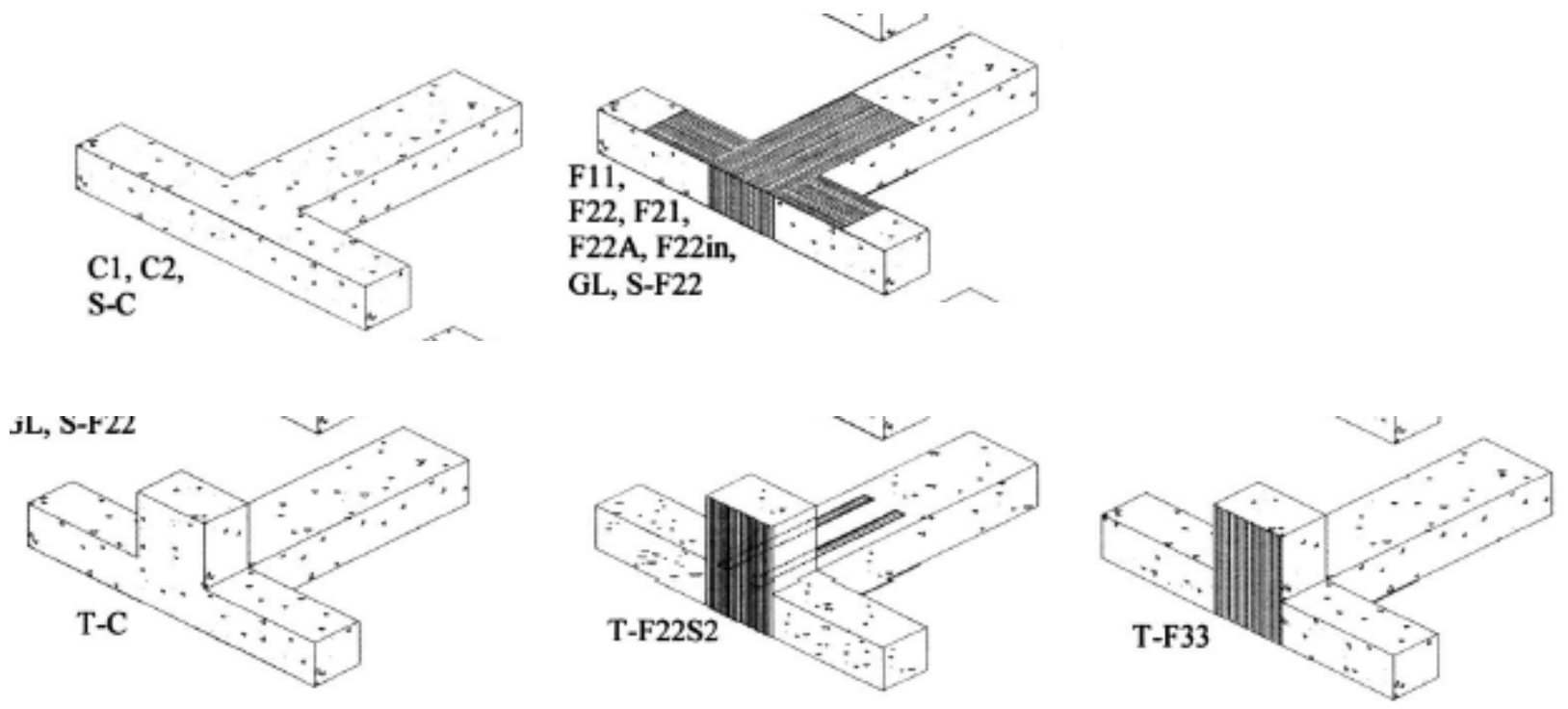

Figure 40. FRP retrofitted specimen configurations [45]

The experimental results and failure behavior of test specimens evaluated by [45] are summarized as follows:

Control specimens $C 1, C 2$, and $T-C$ :

- All the control specimens exhibited diagonal cracking of joint panel at a displacement of 10-15 mm, followed by joint shear failure. 
- Since the mode of failure is joint shear, bonding of FRP wraps perpendicular to the crack direction will increase the shear capacity of joints.

Specimen F11:

- At $20 \mathrm{~mm}$ displacement, the de-bonding of FRP sheets near the joint corners was noticed.

- At $35 \mathrm{~mm}$, a horizontal crack was noticed propagating over the entire length of beam FRP. As a result of the horizontal crack in beam FRP, the tension was generated perpendicular to the fibers in column FRP causing a partial fracture.

- At $40 \mathrm{~mm}$ displacement, one side of the beam experienced complete debonding of FRP sheets while other face of the beam experienced de-bonding followed by tensile fracture perpendicular to the fiber orientation in FRP sheets.

- Ultimately, the specimen failed in joint shear.

Specimen F22:

- Similar to F11, the de-bonding of FRP sheets initiated near the joint corners leading to complete detachment of FRP. However, fracture of FRP was not observed in F22.

- Ultimately, the specimen failed in joint shear.

Specimen T-F33:

- At $25 \mathrm{~mm}$ displacement, the de-bonding of FRP sheets initiated near the joint corners.

- Between 30-35 mm displacements, a transverse beam experienced splitting crack due to lack of beam stirrups and the presence of a duct to accommodate prestressing rods. The splitting crack progressed towards the joint panel, causing it to fail in shear.

Specimen T-F22S2:

- At -20 mm displacement, partial debonding of FRP was noticed on the tension face and at the joint corners.

- Tensile fracture of beam FRP sheets was noticed near the corners resulting in a horizontal crack initiation at 20-25 mm displacement. 
- At $35 \mathrm{~mm}$ displacement, the de-bonding of two FRP strips was noticed.

- The test stopped when the displacement reached $55 \mathrm{~mm}$.

Table 12. Summary of experimental results [45]

\begin{tabular}{lcccc}
\hline \multirow{2}{*}{ Specimen } & \multirow{2}{*}{$\boldsymbol{f}_{c}^{\prime}(\mathbf{M} \boldsymbol{P})$} & \multicolumn{2}{c}{ Maximum load $(\mathbf{k N})$} & \multirow{2}{*}{ Energy dissipated (kN.mm) } \\
\cline { 3 - 4 } C1 & 19.5 & 31.32 & 27.13 & - \\
\hline C2 & 23.7 & 30.82 & 31.08 & - \\
\hline Average (C1,C2) & $\mathbf{2 1 . 6}$ & $\mathbf{3 1 . 0 7}$ & $\mathbf{2 9 . 1 0}$ & $\mathbf{4 , 9 9 5}$ \\
\hline F11 & 22.8 & 42.76 & 42.44 & 6,395 \\
\hline F22 & 27.2 & 50.04 & 49.14 & 7,477 \\
\hline Average (F11,F22) & $\mathbf{2 5 . 0}$ & $\mathbf{4 6 . 4}$ & $\mathbf{4 5 . 7 9}$ & $\mathbf{6 , 9 3 6}$ \\
\hline T-C & 24.6 & 36.02 & 33.86 & 5,873 \\
\hline T-F33 & 26.0 & 44.26 & 44.45 & 7,695 \\
\hline T-F22S2 & 22.0 & 40.07 & 39.75 & 6,207 \\
\hline
\end{tabular}

Following are the key learning points based on the experimental evaluations performed by:

- Control joint specimen with a transverse beam has shown a maximum of $16 \%$ and $18 \%$ improvements in strength and energy dissipation, respectively, over joint without transverse beam.

- Specimens without transverse beam (with a slight difference in concrete strength):

- Maximum enhancements in strength and energy dissipation for the specimen with a single layer of FRP was found to be approximately $46 \%$ and $28 \%$, respectively.

- Maximum enhancements in strength and energy dissipation for the specimen with two layers of FRP was found to be approximately $69 \%$ and $50 \%$, respectively.

- Specimens with a transverse beam (with a slight difference in concrete strength):

- Maximum enhancements in strength and energy dissipation for the specimen with two layers of FRP and two FRP strips were found to be approximately $17 \%$ and $6 \%$, respectively.

- Maximum enhancements in strength and energy dissipation for the specimen with three layers of FRP was found to be approximately $31 \%$ and $31 \%$, respectively. 
- Comparing the FRP strength enhancements for joints with transverse beam (T-C Vs. TF33 or T-F22S2) against the joints without transverse beam (Average(C1, C2) Vs. Average (F11, F22)), following conclusions are drawn:

$\circ$ The presence of transverse beam reduced the effectiveness of CFRP sheet strengthening by $54 \%$ and $20 \%$ in terms of strength and energy absorption capabilities, respectively. In the case of specimen strengthened with CFRP sheets and strips, this reduction was even higher, i.e., $77 \%$ reduction in strength and $85 \%$ reduction in energy absorption.

- In the case of FRP strengthened specimens, de-bonding always initiated at the high-stress concentration joint corners highlighting the need to redistribute stresses away from the joint corners or provide adequate anchorage.

- Due to the premature de-bonding of FRP sheets from its substrate, especially at joint corners, an increase in the number of FRP layers did not proportionately increase the joint capacities.

- Rupture of FRP sheets perpendicular to the fiber directions signifies the need to provide a more balanced layup. 


\subsection{Summary}

FRPs, when used to strengthen joints, multiple sheets/strips are wrapped around a joint, using epoxy or other adhesives; these sheets are typically applied haphazardly, without maximizing the rupture stress resistance of the substrate materials through confinement. Therefore, there remains a serious concern of long-term integrity and the likelihood of cyclic loading on joints and components bonded in this manner. Furthermore, previous research was performed by randomly applying FRP composite sheets about a joint without focusing on minimizing stress concentration effects at joints. The stress concentration effects lead to de-bonding or delamination of FRP sheets from concrete substrate and even rupture prematurely at reentrant corners. Although small increases in mechanical properties, including energy absorption, were observed through random application of FRP sheets, the above-identified limitations lead to even more dramatic failures under seismic forces.

Current retrofit techniques are focused primarily on joint strengthening with composite wraps by wrapping columns and beams independently and then the beam-column joint area separately without providing uniform resistance to force transfer between structural elements (beam-columnslab). Furthermore, the majority of the research and evaluation work was focused on unidirectional carbon fiber composite layers that are placed orthogonal to each other on a layer-by-layer basis to build requisite composite thickness in and around a joint, as opposed to optimizing fabric architecture, which would provide higher strain to failure.

Key learning points based on the current review of concrete joints retrofitted with FRP composites are as follows:

- FRP repair can address several deficiencies identified in non-seismically designed RC joints and improve their performance.

- $45^{\circ}$ orientation of fibers in the joint region with respect to the beam longitudinal axis has provided the best performance. This is because the fibers are more or less oriented parallel to the principal planes reinforcing the concrete strut.

- Increasing the number of FRP layers did not proportionally increase the joint capacities. 
- Hinge formation in the beam section, irrespective of whether the final failure was concentrated in the joint or beam, has led to significant improvement in energy dissipation.

- Improvements in structural capacities of FRP strengthened 3D (with transverse beams) joints was lower than that of $2 \mathrm{D}$ (without transverse beams).

- In the case of FRP strengthened specimens, de-bonding always initiated at the high-stress concentration areas (joint corners), highlighting the need to redistribute stresses away from the corners or provide adequate anchorage to FRP wraps.

- The addition of U-strips enhanced the bond performance between underlying sheets and concrete substrate, thereby delaying the initiation of- or prevention of de-bond.

- Bonding discrete layers of L-and T-shape CFRP sheets without anchors has led to premature de-bonding from its substrate.

- Reinforcing the joint with CFRP sheets in continuous X-pattern has significantly improved the confinement of joint core resulting in higher ductility over the control specimen.

- Rupture of uni-directional FRP sheets perpendicular to the fiber directions signifies the need to provide balanced layup.

- Surface preparation of test specimens using a water jet coupled with structural adhesive was found to be more efficient in improving the shear capacity of joints when compared to the wire brush method.

- Although CFRP strengthened specimens exhibited joint shear failure patterns similar to that of control specimens, the failure was contained.

- Capacity enhancements in specimens strengthened with a single layer of CFRP having $45^{\circ}$ orientation were rather inconsistent when compared to specimens strengthened with balanced $\pm 45^{\circ}$ orientation.

- EBROG (externally bonded reinforcement on groves) method coupled with CFRP fan anchors was found to be efficient in preventing the de-bonding of CFRP sheets from its substrate. 


\section{Experimental Program: Materials, Casting, Test Protocols, and Data Procurement}

\subsection{Introduction}

This chapter provides in-depth information about the experimental program conducted on 2D reinforced concrete $(\mathrm{RC})$ exterior beam-column joints under monotonic loads, including the types of materials employed, their mechanical properties, and the load strain/deformation response. More specifically, the experimental program described herein includes specimen details, specimen casting process, material properties, test setup, loading protocol, and instrumentation employed to collect test data. Additionally, test data collection procedures and the type of data gathered from the test samples are provided so that readers can appreciate the need for meticulous care in data collection to perform experimental analysis (Chapters 5 and 6) utilizing fundamental principles of mechanics of reinforced concrete structures with FRP wraps and filler-modules.

\subsection{Specimen Details}

Twenty full-scale test specimens were cast according to pre-1976 construction details to evaluate the behavior of joint specimens under monotonic loads. As identified in section 2.2, the pre-1976 joint deficiencies considered for this study focused on: (i) low strength concrete ( 2600, 3300, 3800 psi), (ii) inadequate anchorage length of beam bottom bars extending into the joint, (iii) no transverse (stirrup) reinforcement in the joint panel, and (iv) weak column-strong beam design. All the test specimens were designed to ensure joint shear (diagonal tension) failure without yielding of rebars in beam/column by (i) providing span-to-depth ratios in the range of 2.5-5.5, (ii) limiting axial loads on the column to $0.28 f_{c}^{\prime} A_{g}$ or lower, and (iii) designing beams to exhibit higher moment and shear capacity than columns. Joint specimens were tested in both the control and reinforced conditions. The test specimen details are given in sections 4.2.1 and 4.2.2, respectively. 


\subsubsection{Control specimens}

Four of the twenty joint specimens were tested in control condition to evaluate the joint shear capacity and identify the underlying failure mechanisms. The test results of control (control) specimens (Figure 42) were then compared with those of reinforced specimens to assess the structural capacity enhancements and note the changes in failure mode. The specimen casting details of control joints, including the steel rebar layout, cover, and geometric properties, are shown in Figures 41 through 43.

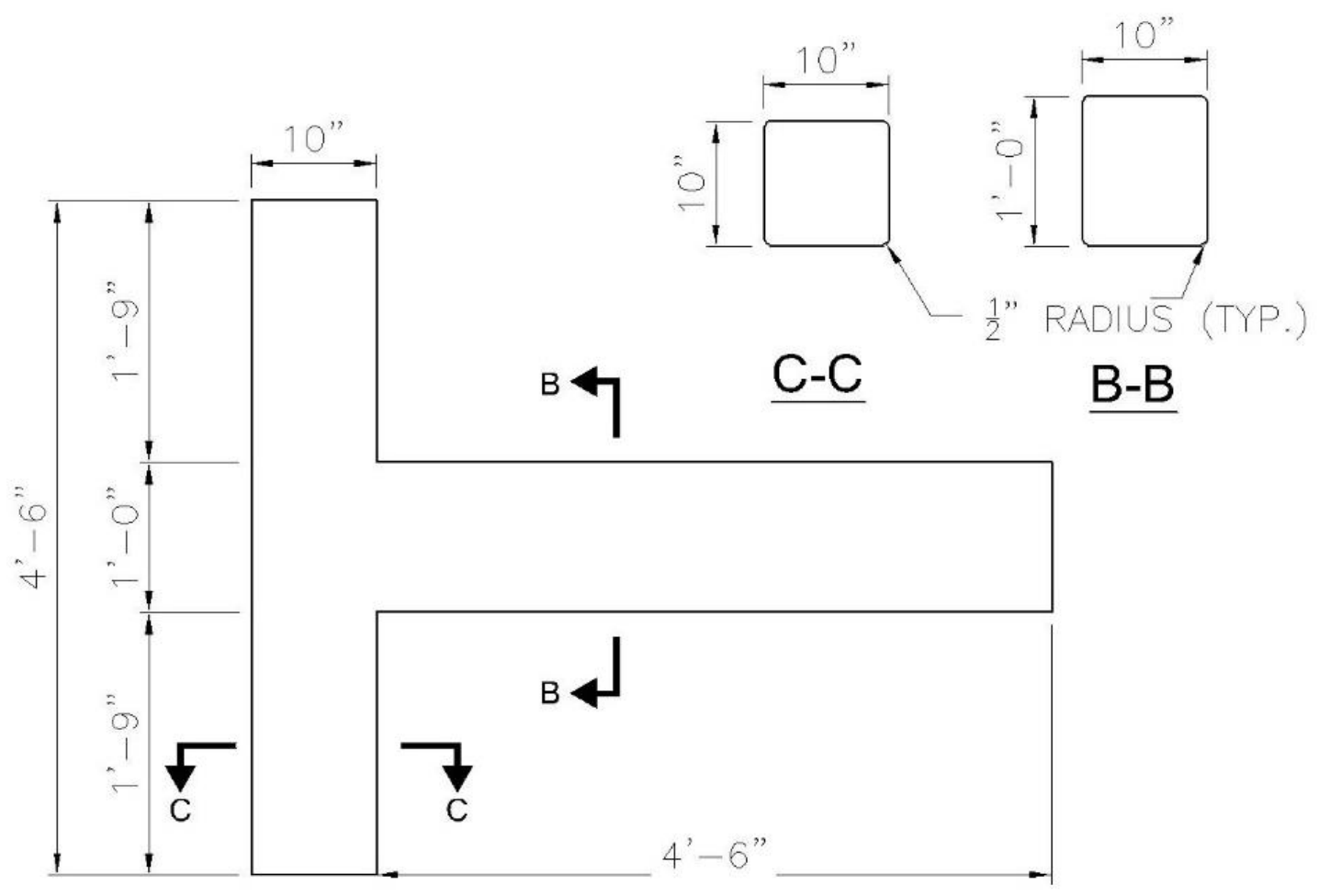

Figure 41. Schematic of control joint specimen 


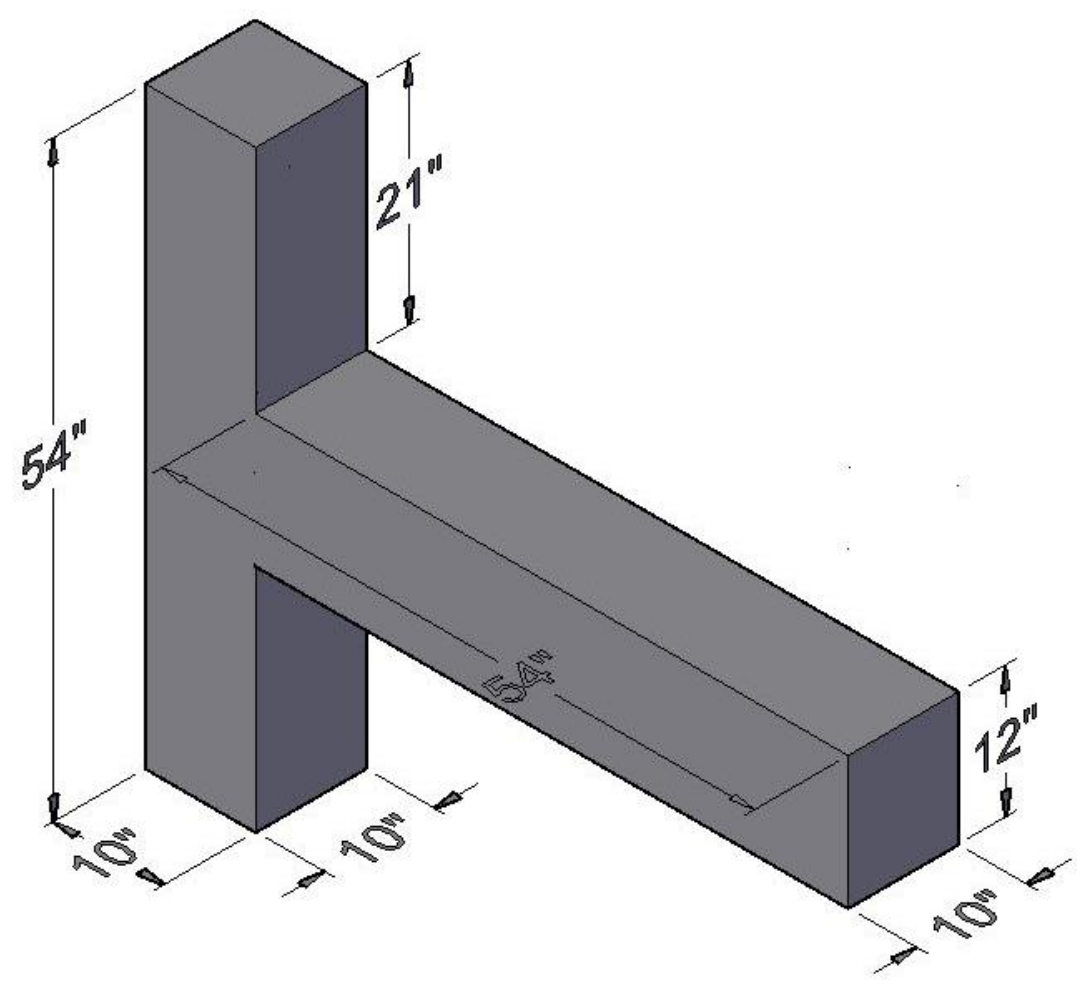

Figure 42. Geometric layout of control joint specimens 


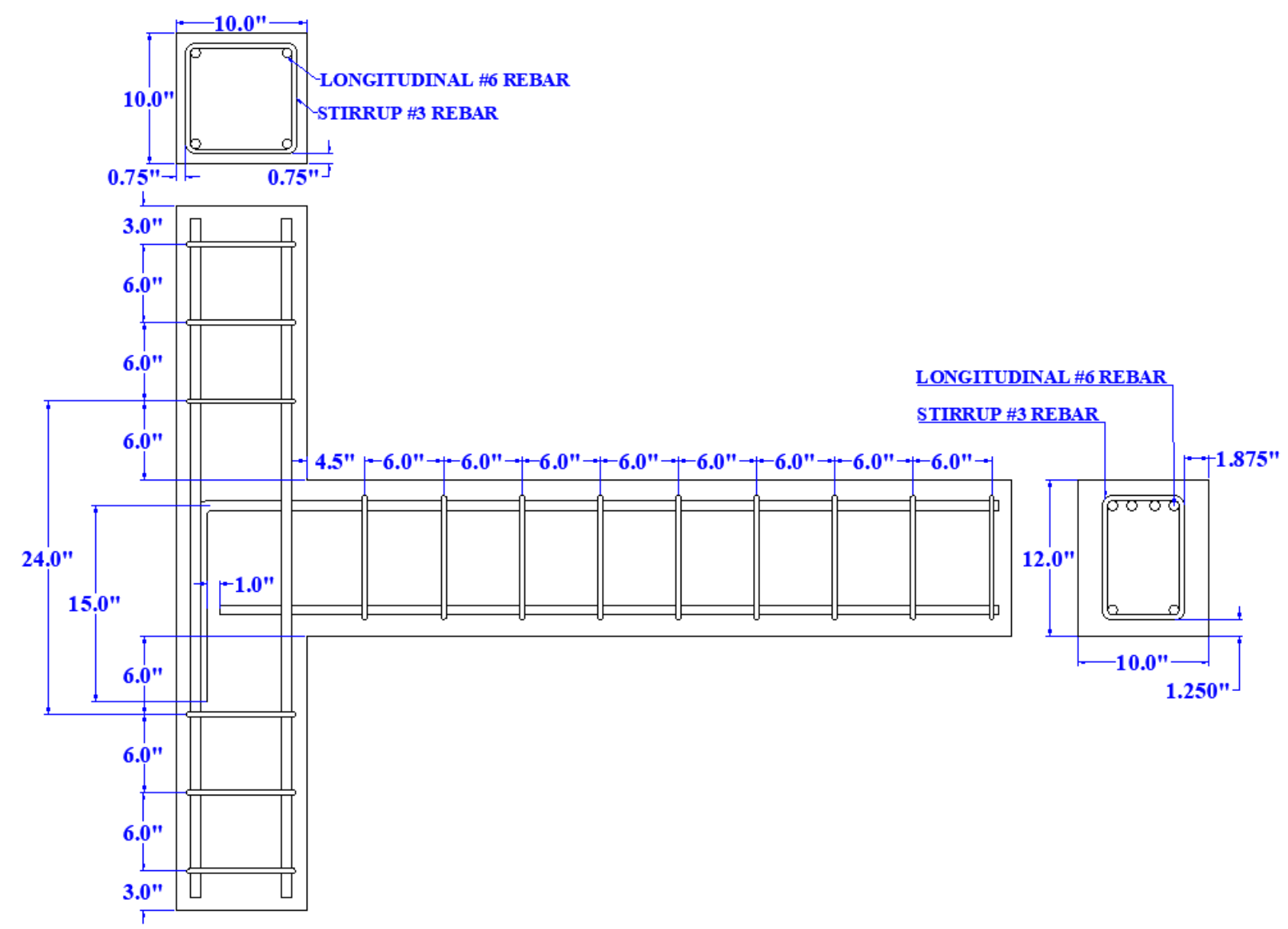

Figure 43. Steel rebar layout of control joint specimens 


\subsubsection{Reinforced specimens}

A total of sixteen specimens were reinforced with filler-modules with/out FRP wrap/gusset to evaluate the strength, energy absorption and ductility enhancement of reinforced joints over control specimens. The construction details of reinforced joint specimens, including the steel rebar layout, cover, and geometric properties, are shown in Figures 44 through 46. A step-by-step procedure of reinforcing schemes including FRP wrap/gusset configurations for each specimen is provided in Tables 13 to 18.

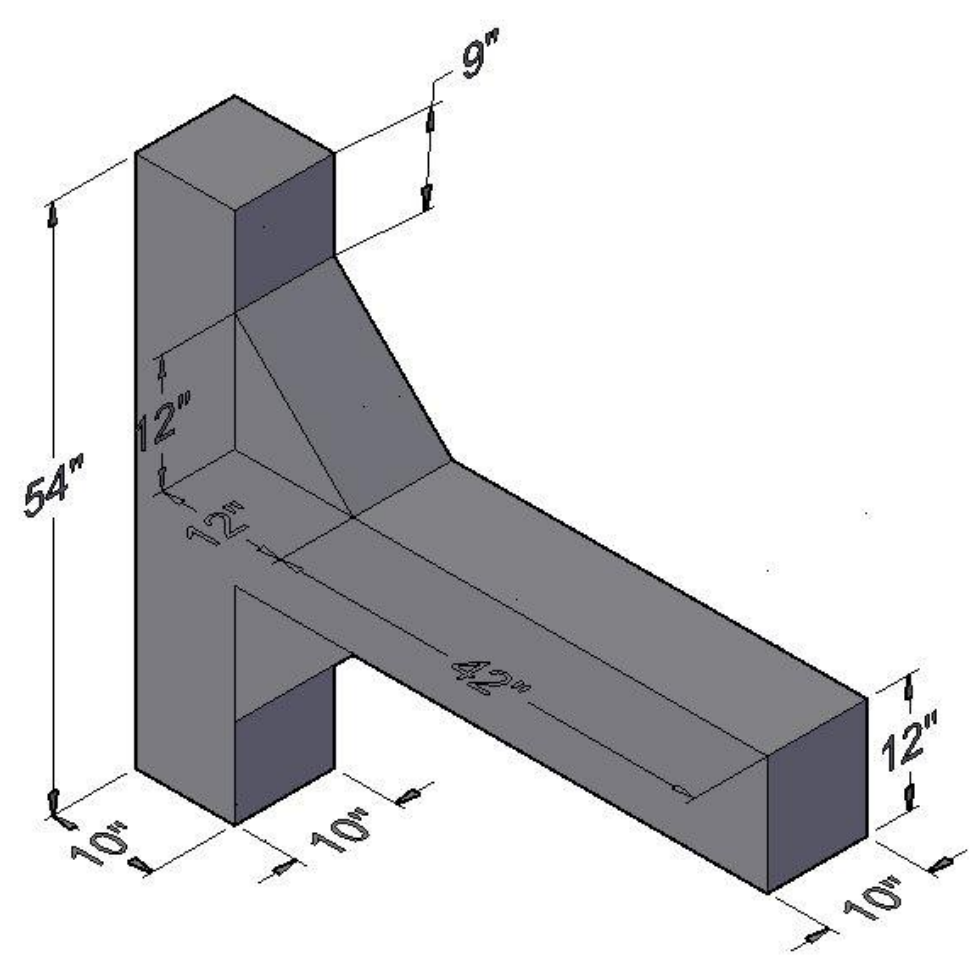

Figure 44. Geometric layout of joint specimens reinforced with wedge filler-module 

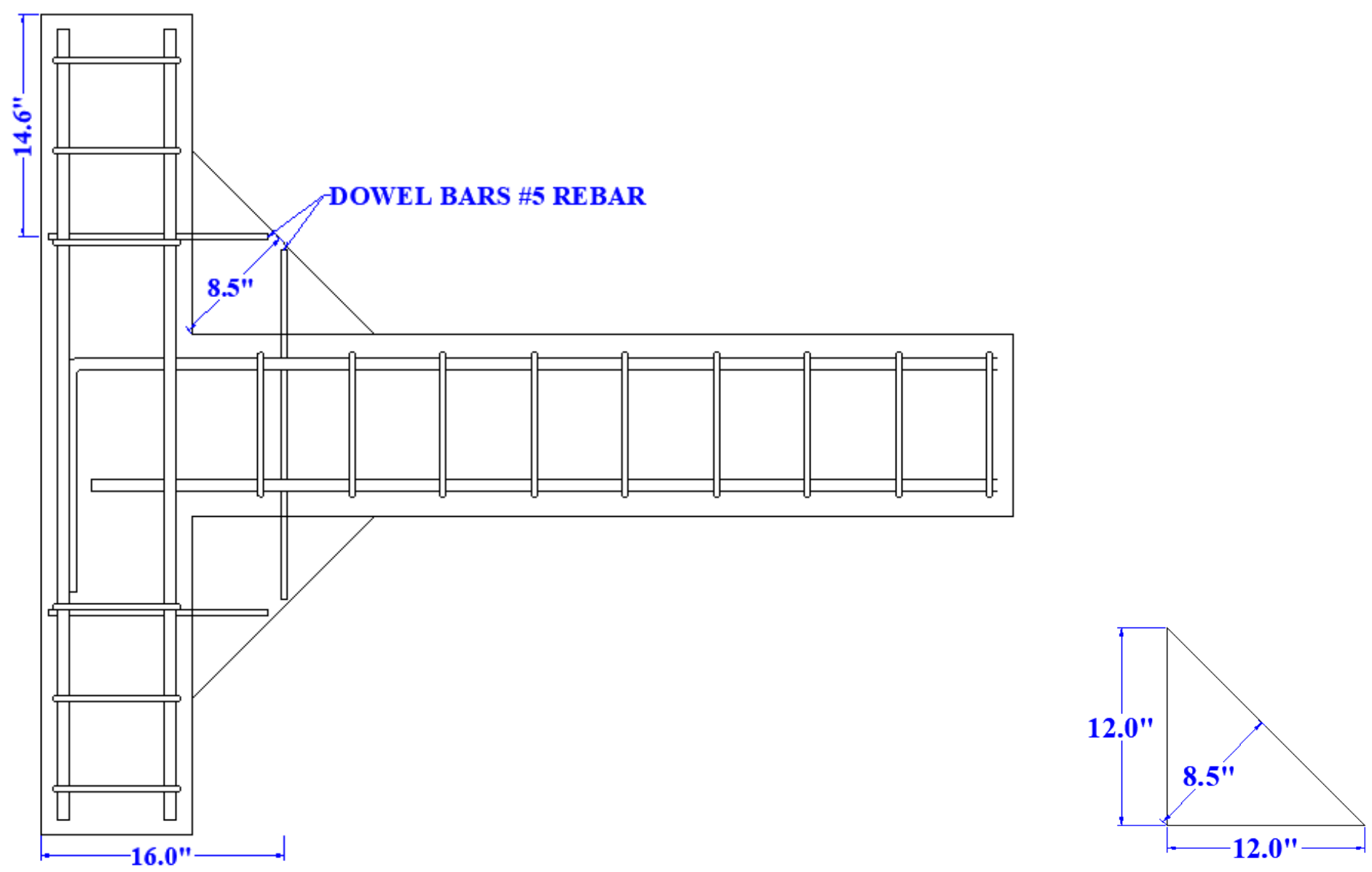

Figure 45. Steel rebar layout of joint specimens reinforced with wedge filler-module 

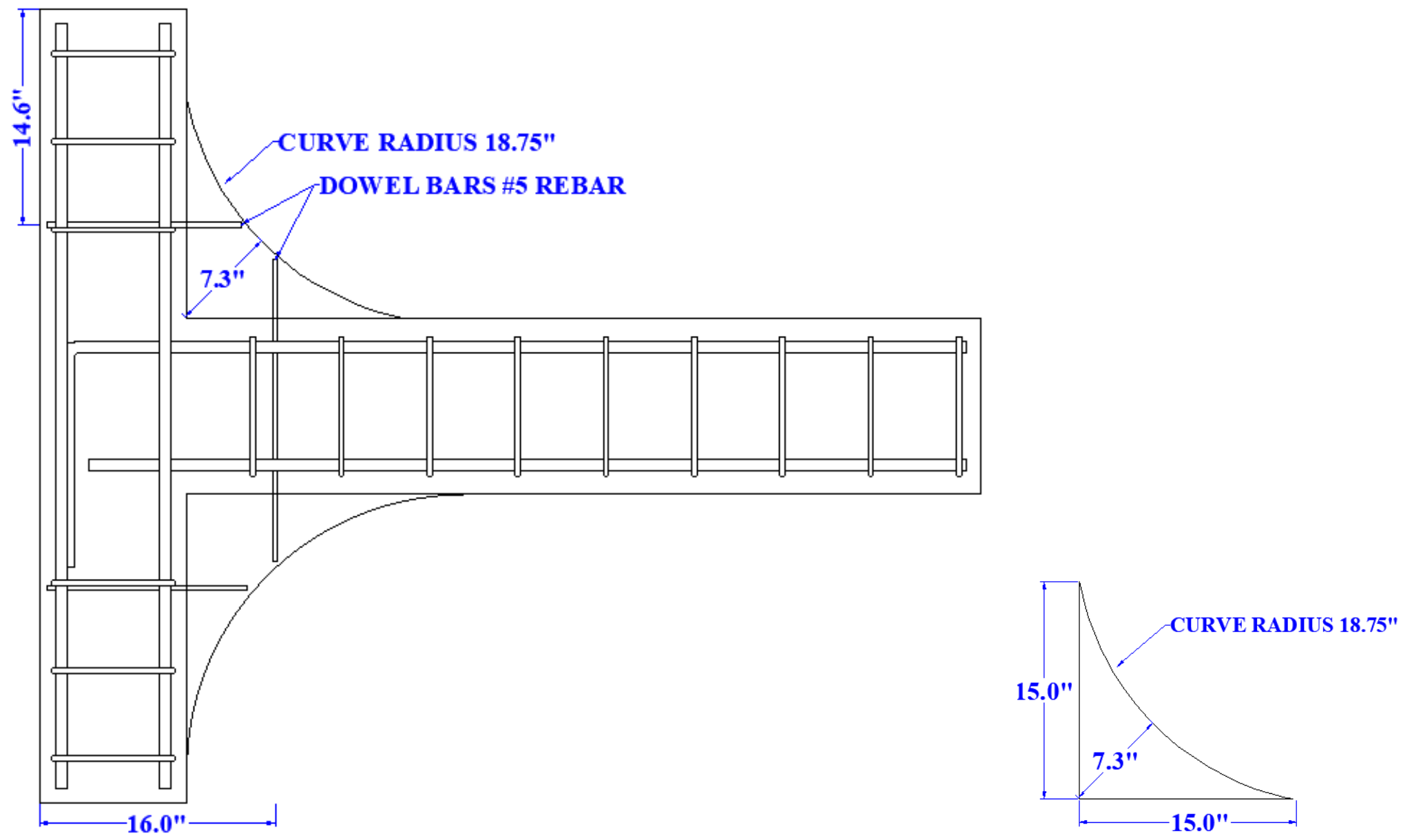

Figure 46. Steel rebar layout of joint specimens reinforced with curve filler-module 
Table 13. Beam-column joint reinforcing scheme for JI-2 through JI-4

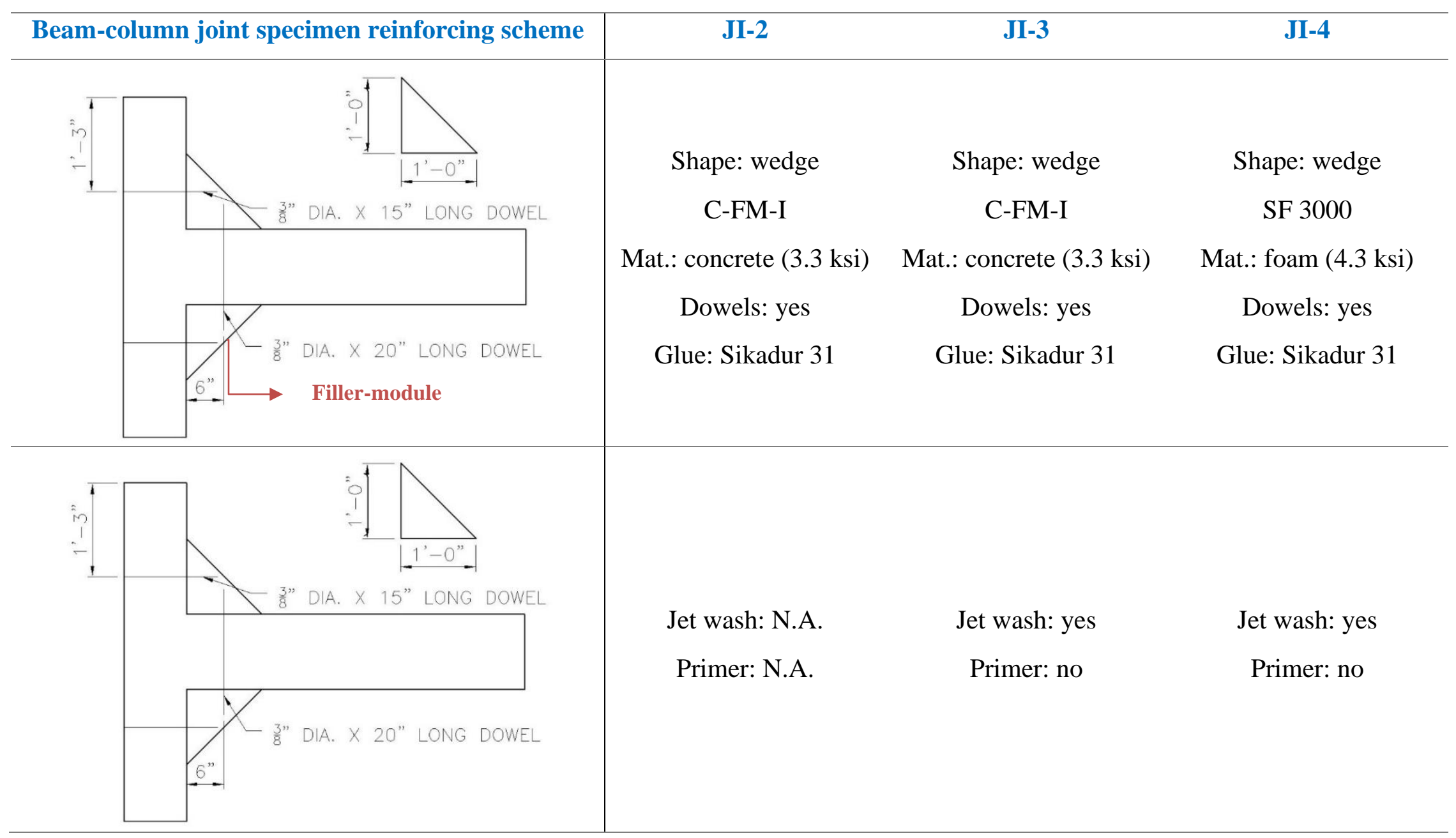




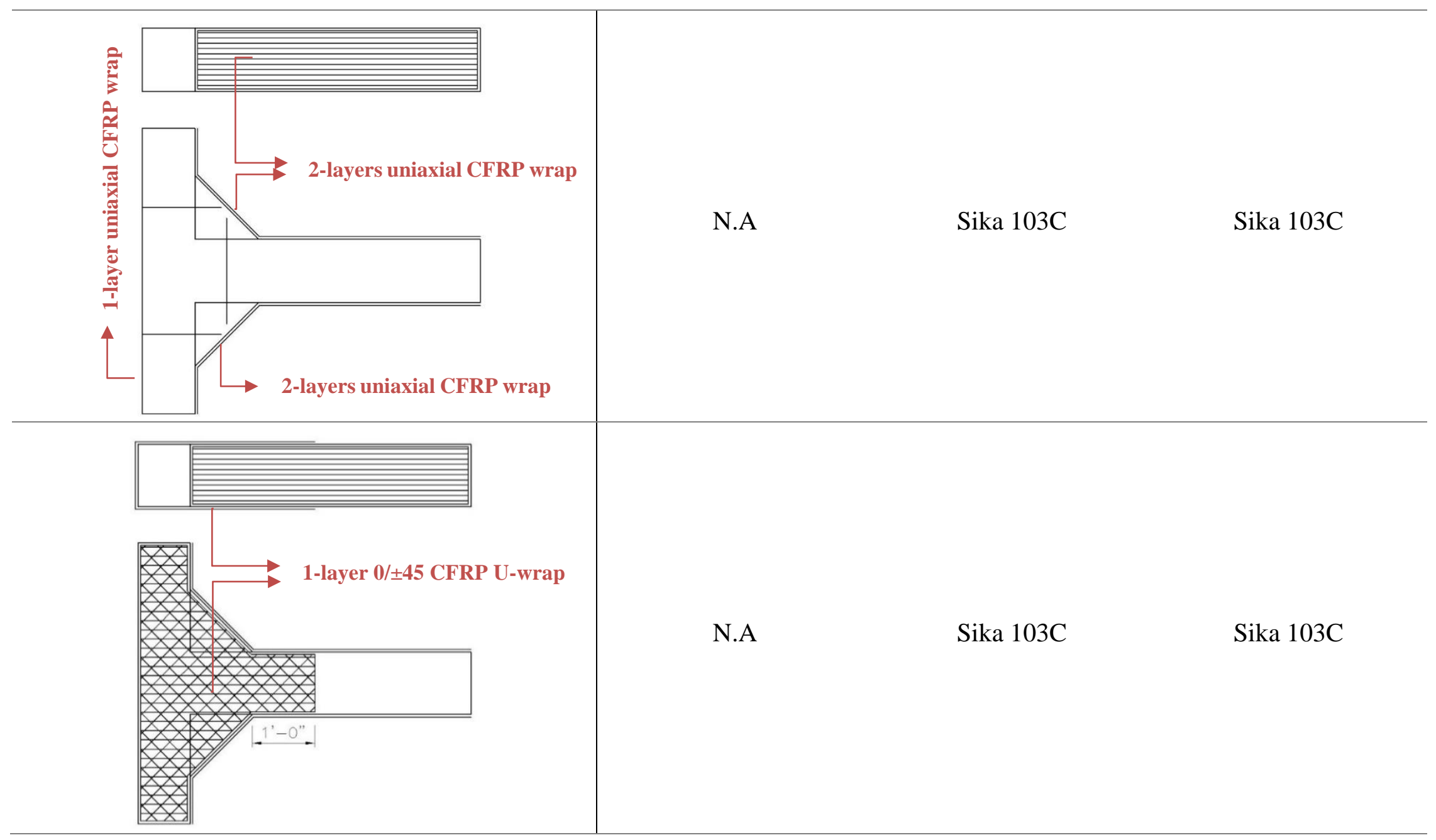




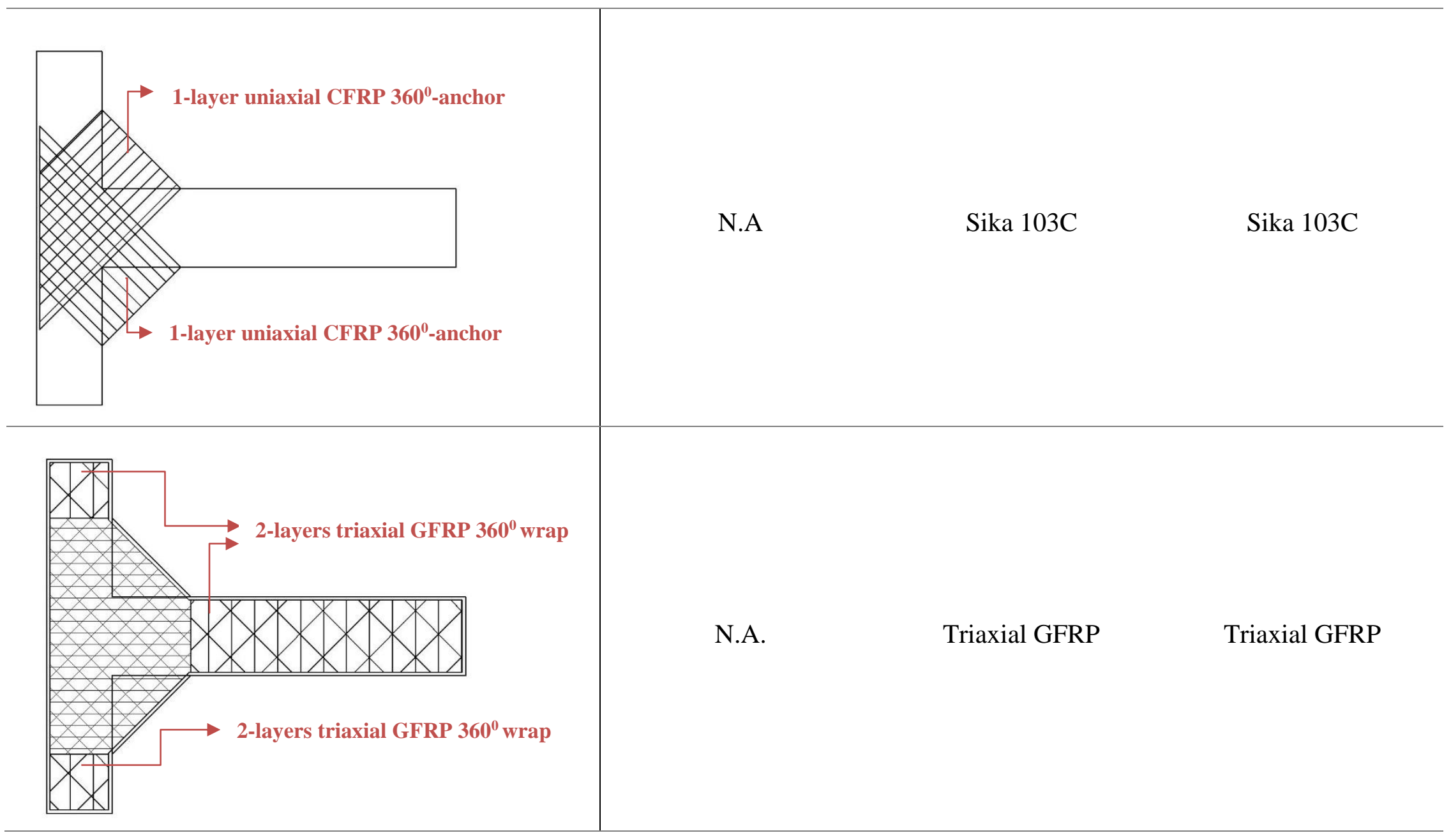


Table 14. Beam-column joint reinforcing scheme for JI-6 through JI-8

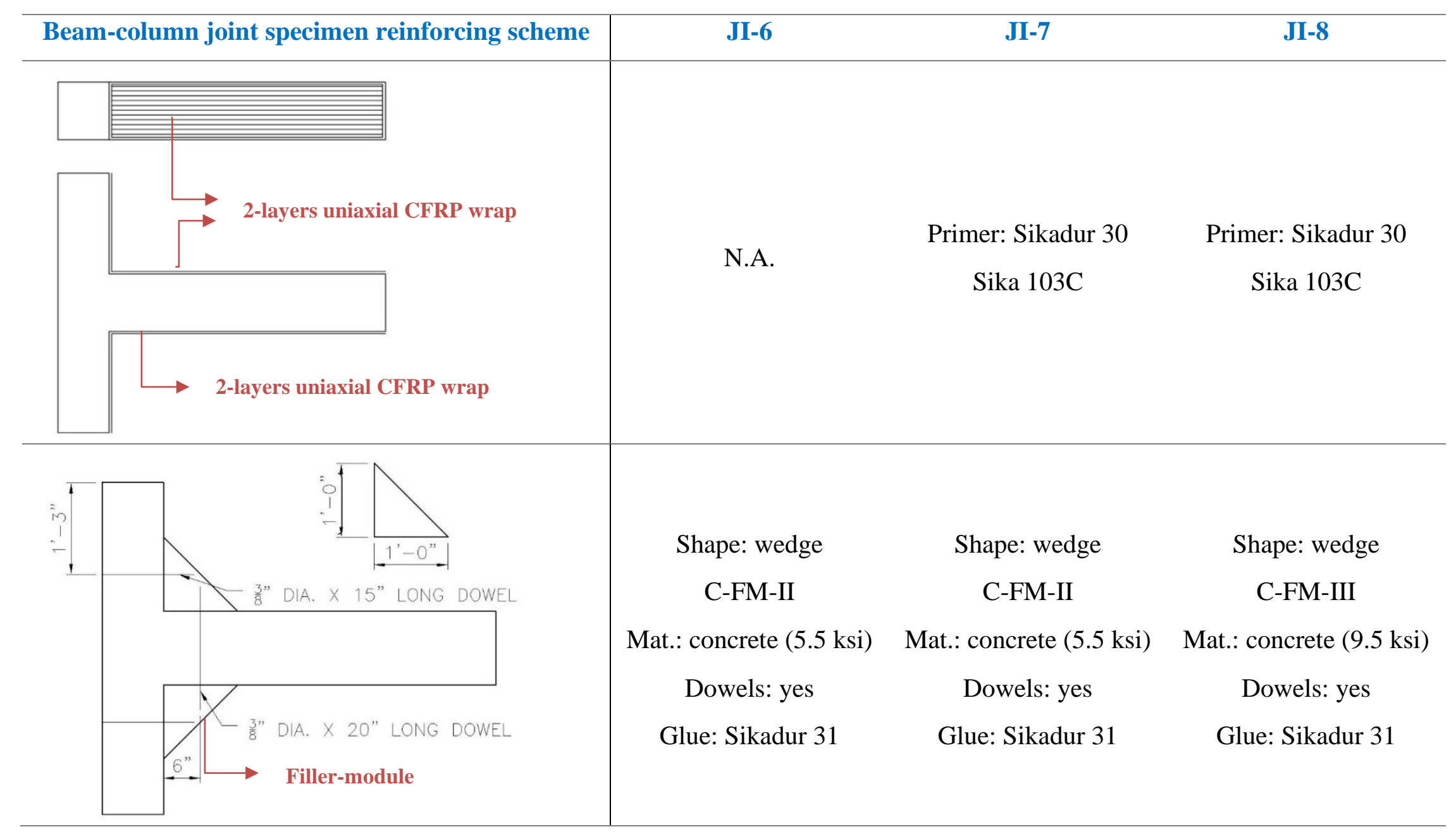




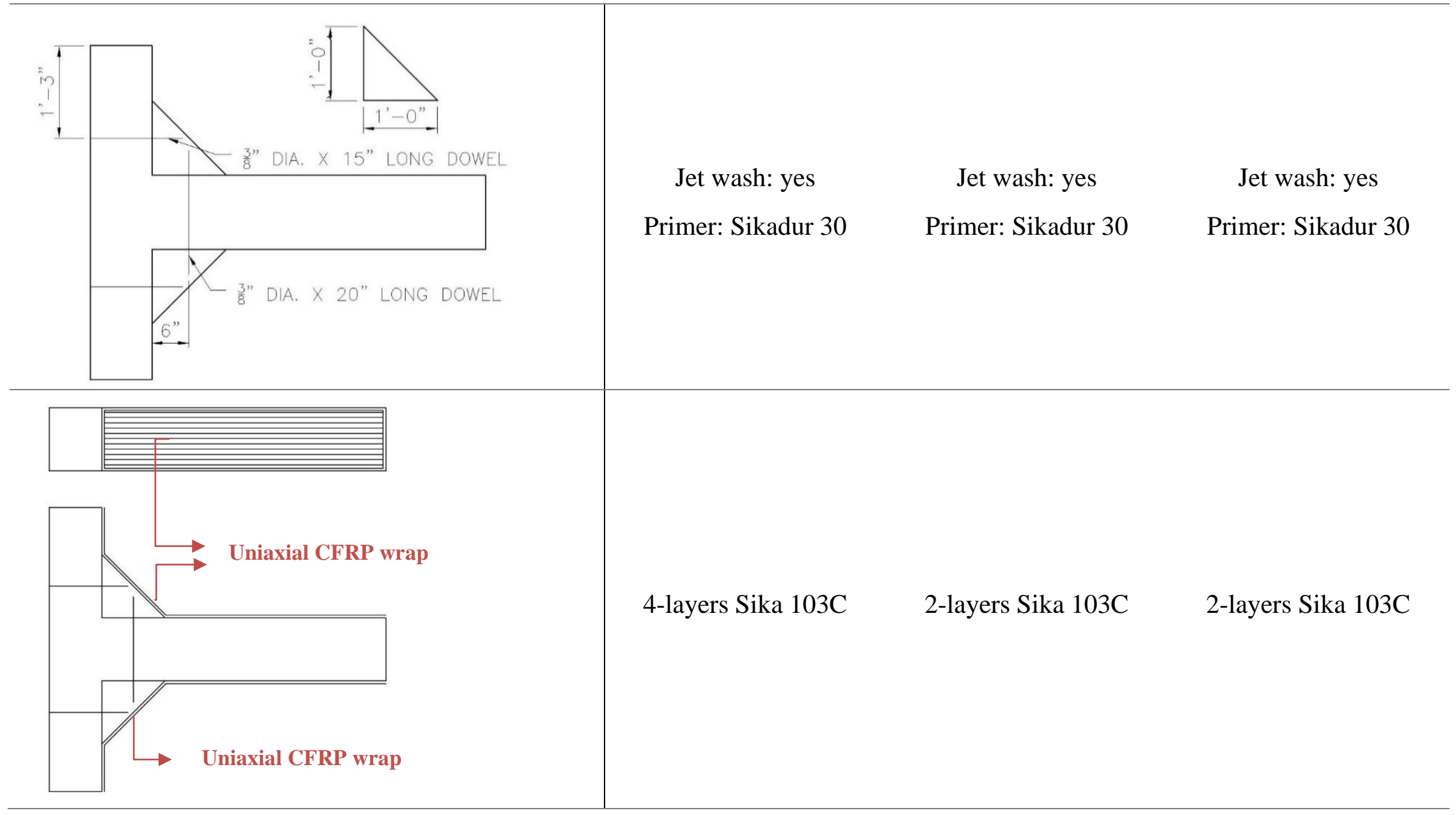




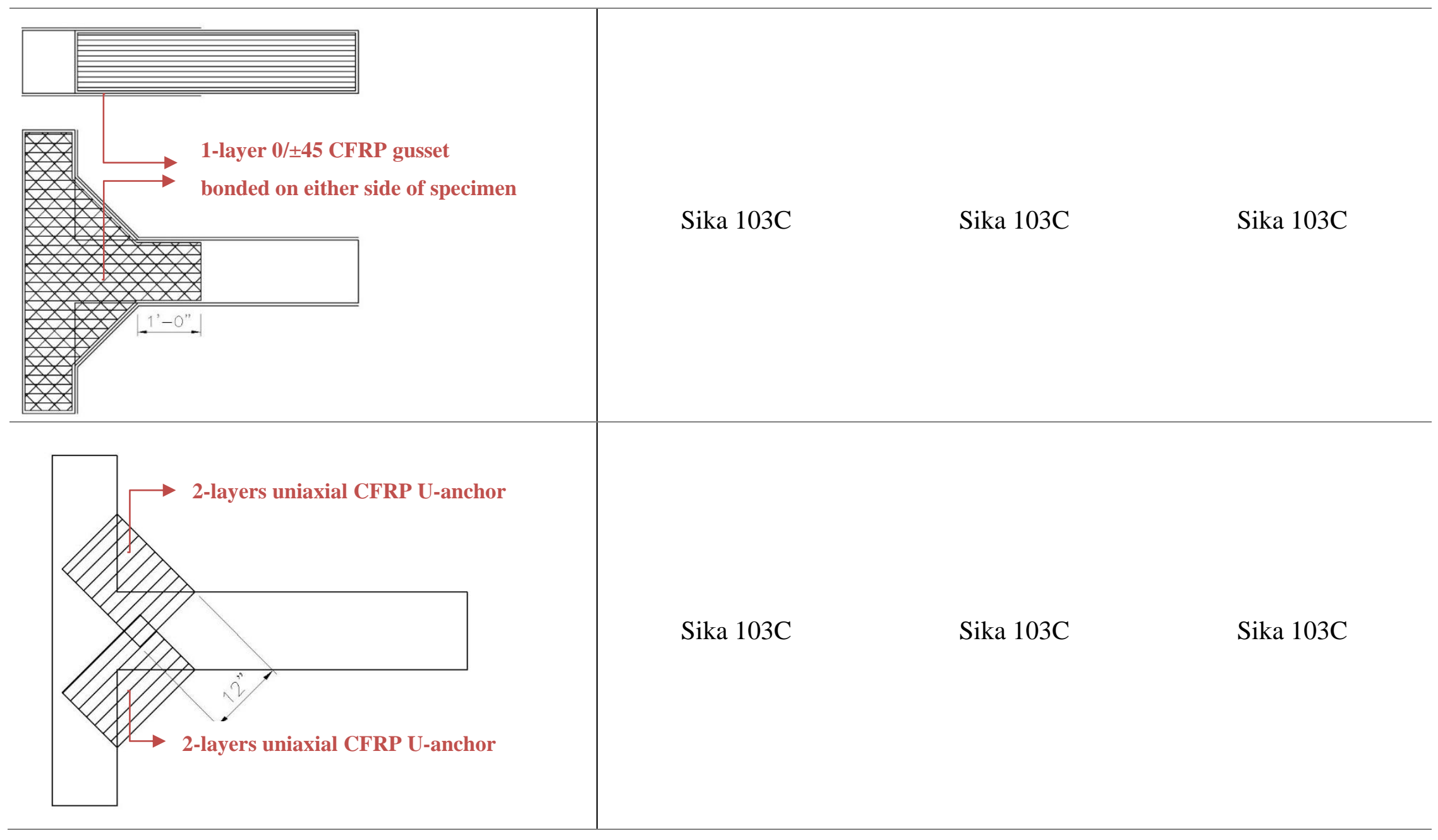




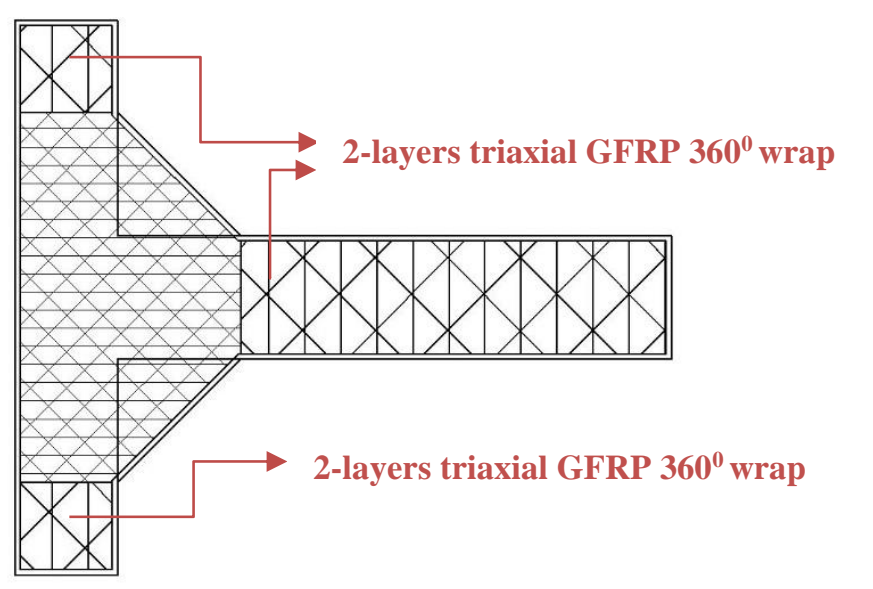

Triaxial GFRP

Triaxial GFRP

Triaxial GFRP 
Table 15. Beam-column joint reinforcing scheme for JIII-2 through JIII-4

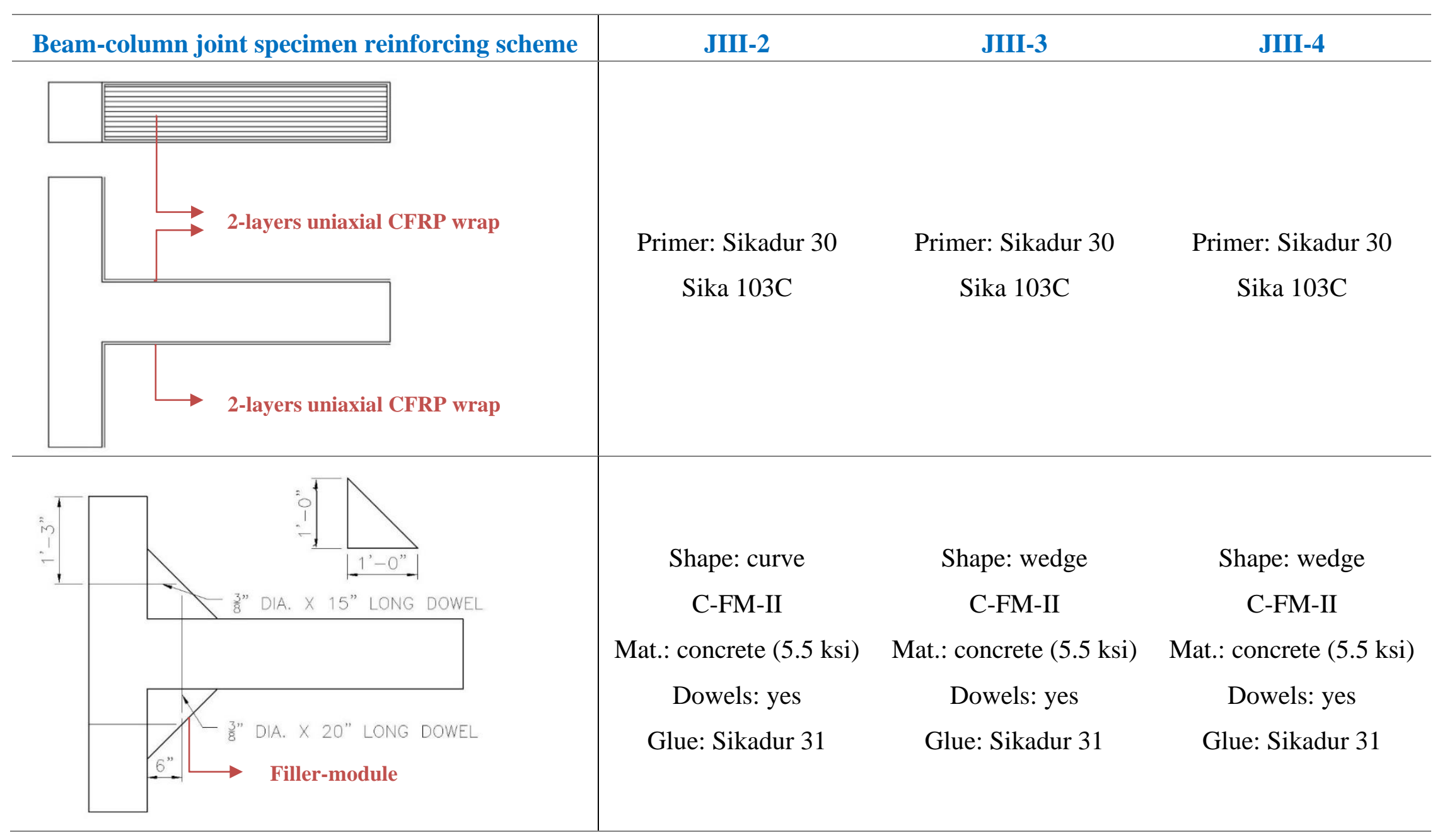




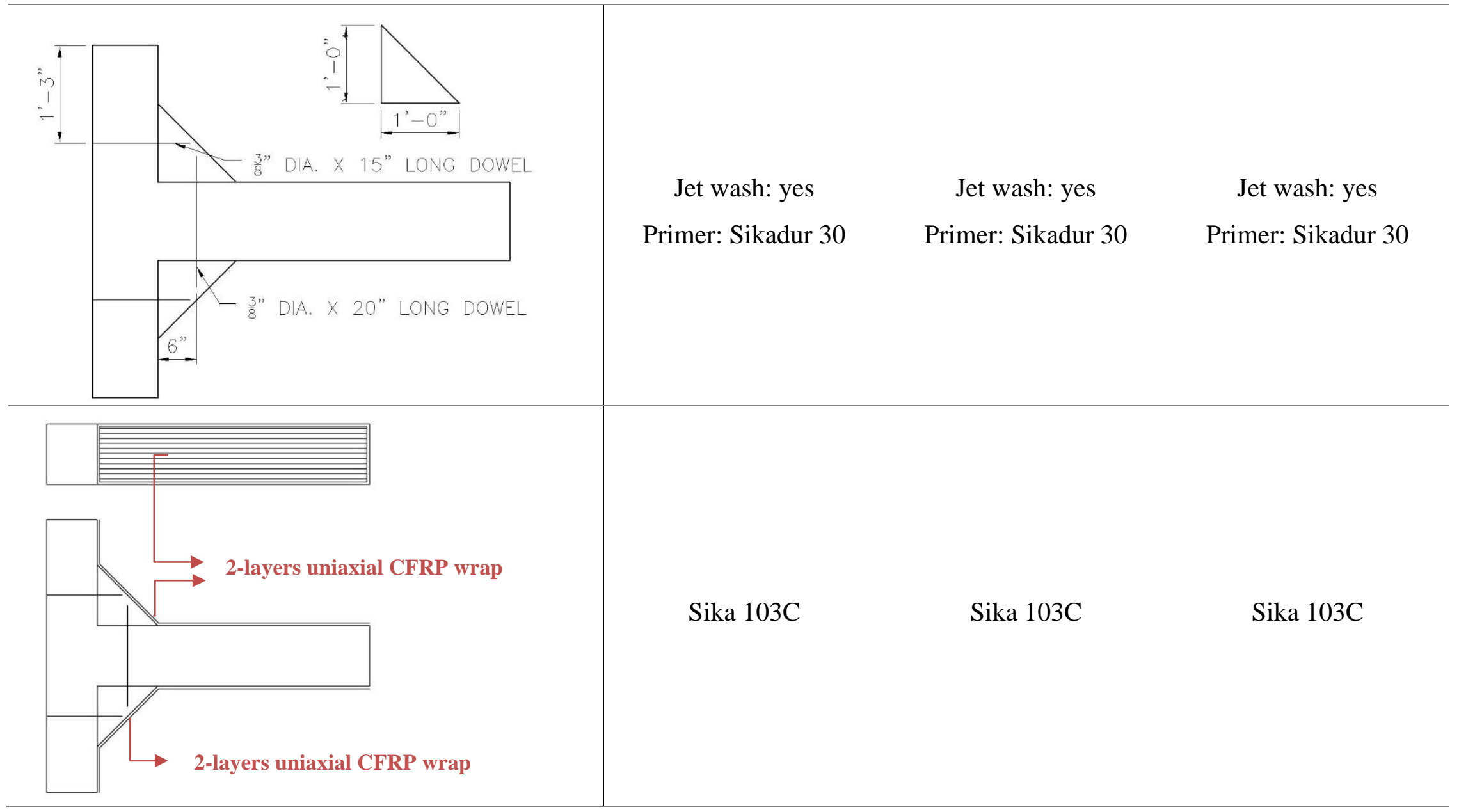




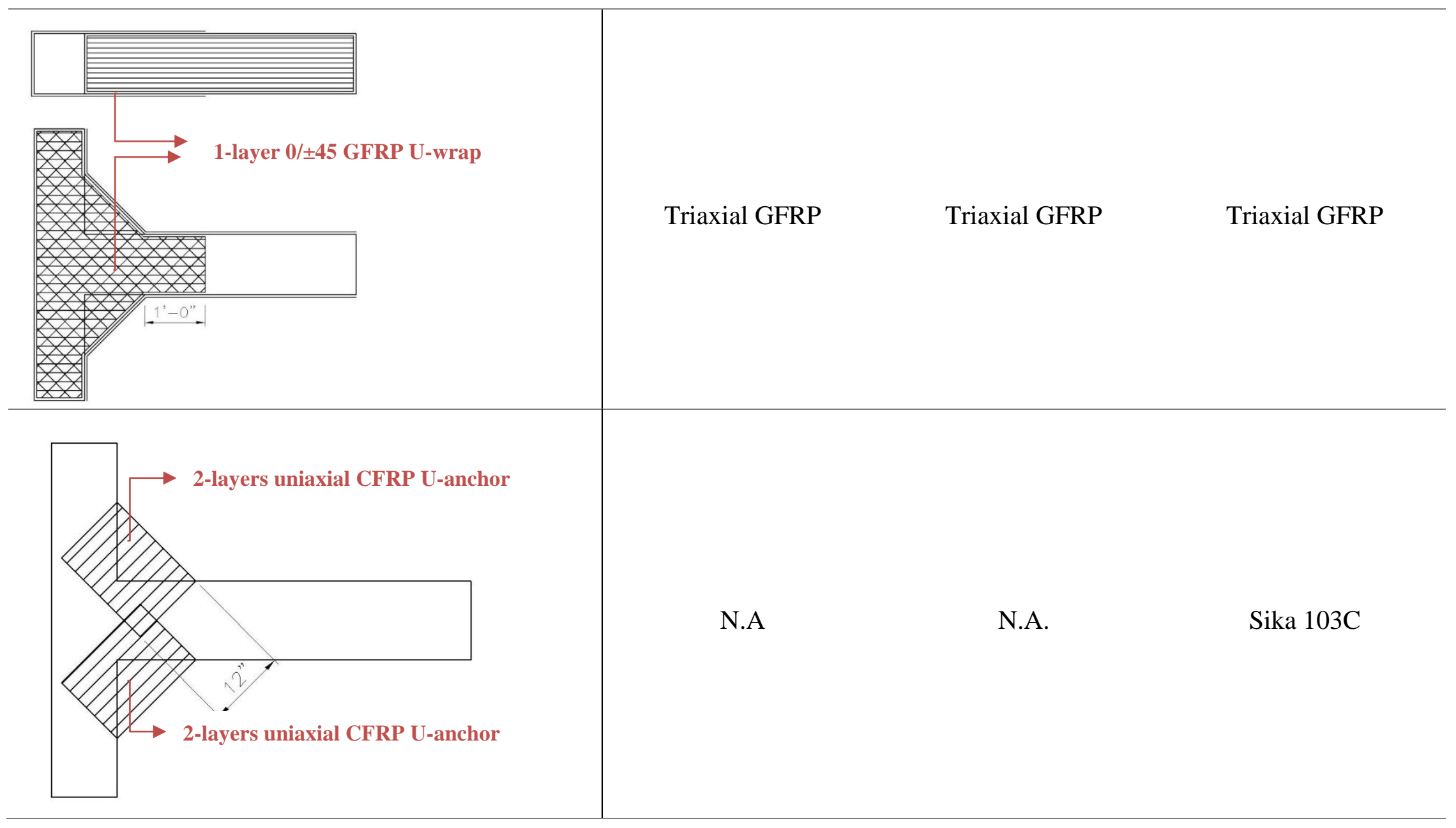




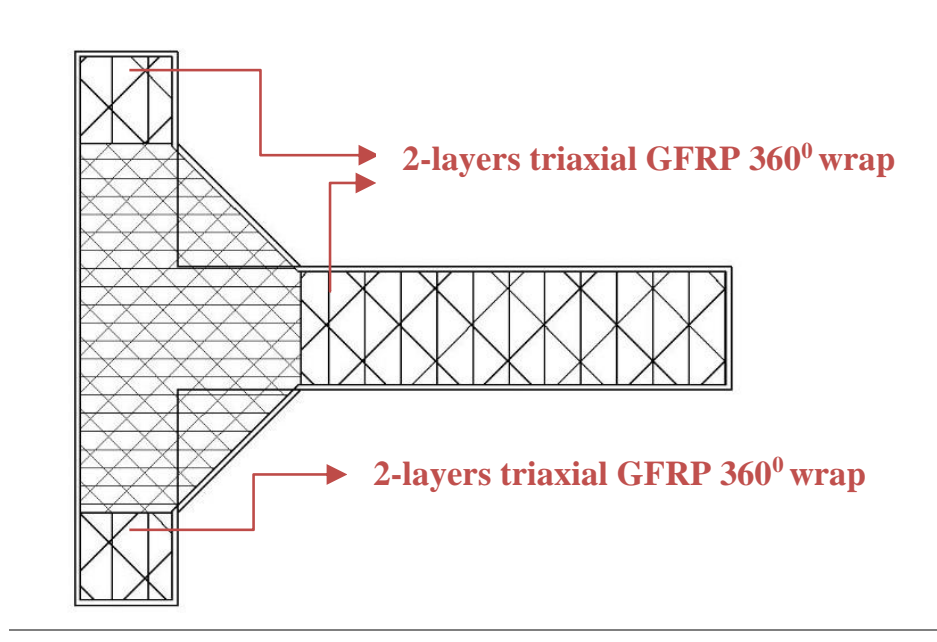

Triaxial GFRP

Triaxial GFRP

Triaxial GFRP 
Table 16. Beam-column joint reinforcing scheme for JII-2

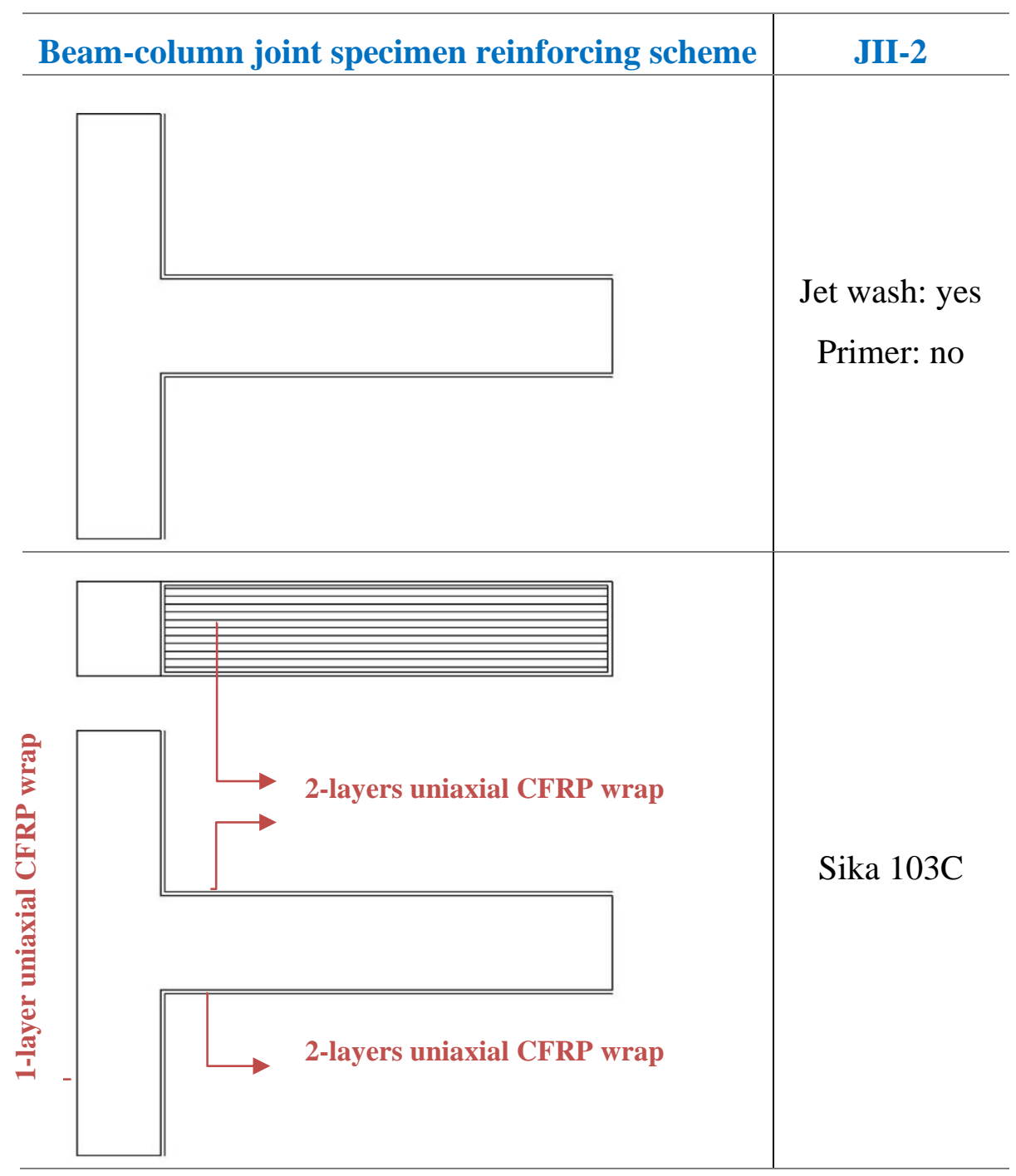




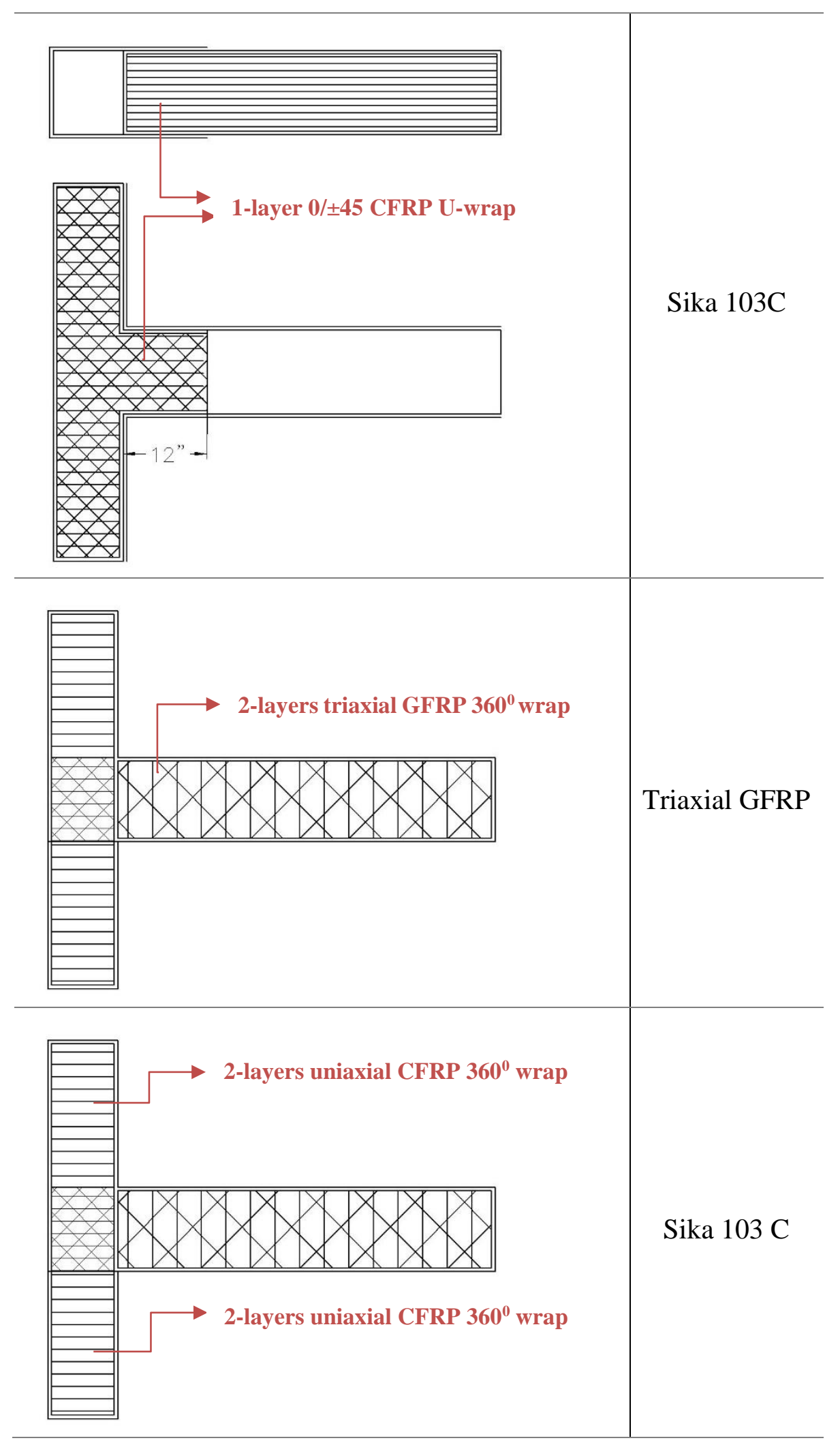


Table 17. Beam-column joint reinforcing scheme for JII-3 and JII-4

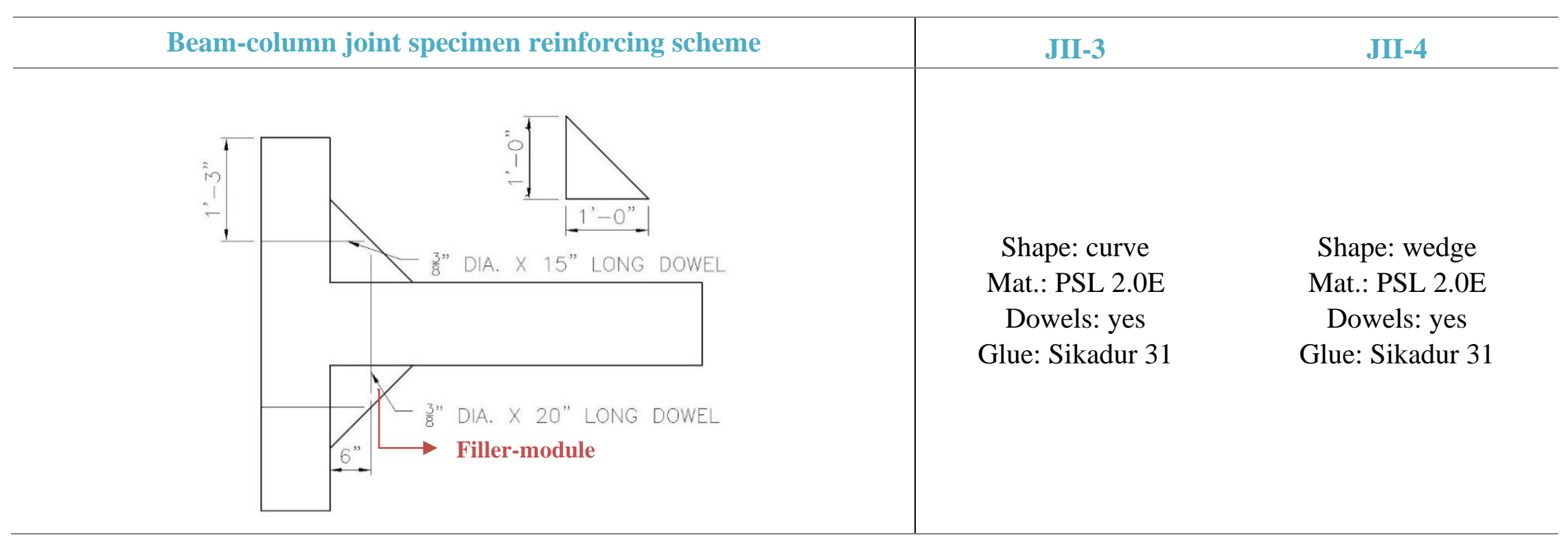


Table 18. Beam-column joint reinforcing scheme for JII-5 through JII-8

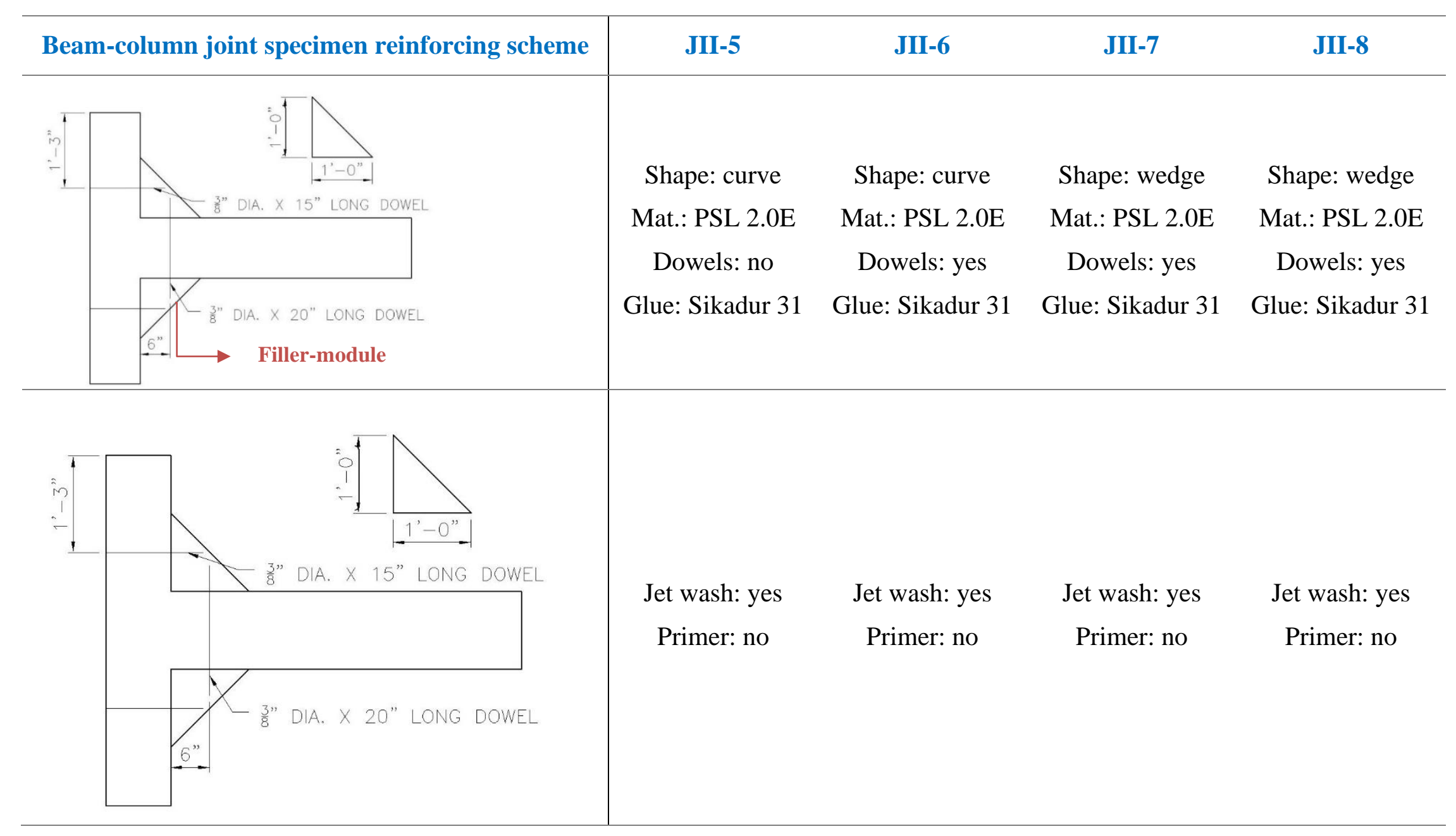




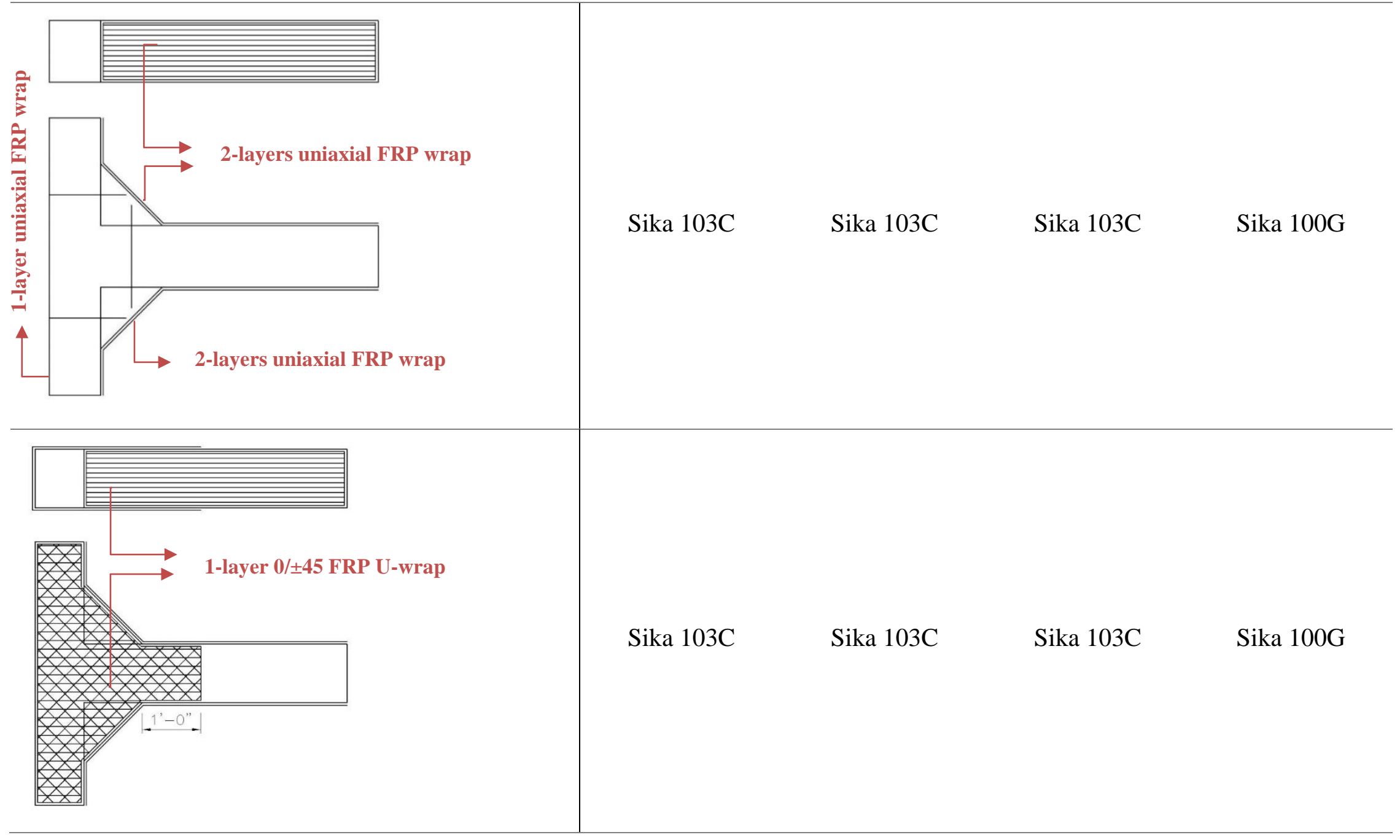




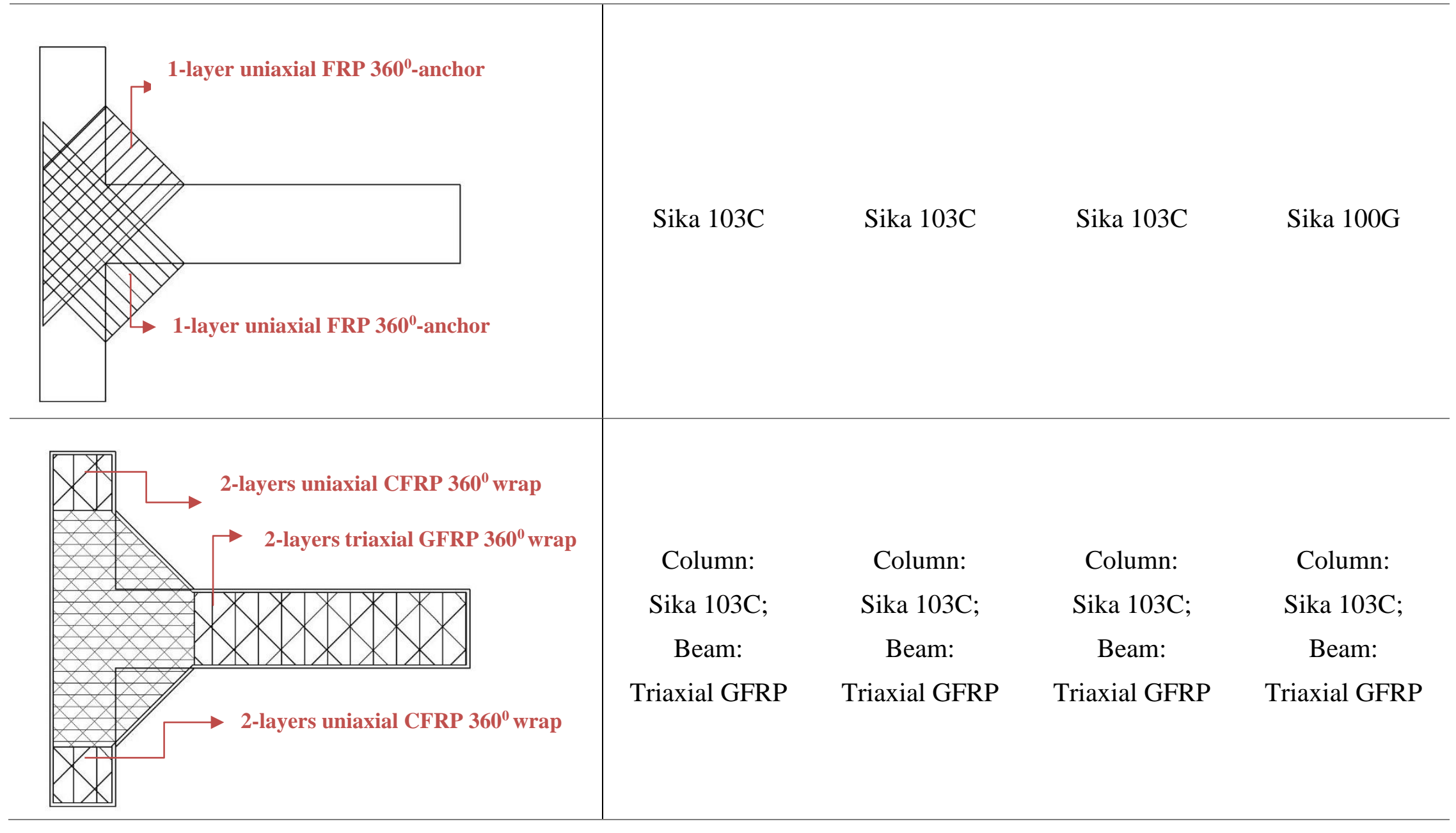




\subsection{Specimen Casting}

The joint test specimens were cast in T-shaped plywood molds resting horizontally on the floor over a plywood sheeting, as shown in Figure 47. To avoid bulging, the walls of the formwork were supported from outside using wooden blocks that are screwed to the plywood sheeting. The gaps along the edges between the plywood sheet and formwork were sealed with silicone material to prevent any leakage of concrete slurry. The inner walls of the formwork and floor of the plywood sheeting were made free of debris by vacuuming and then coated with an oil layer for easy release of the cured test specimen. The reinforcement cages for column and beam sections were constructed separately, as shown in Figure 48. Grade 60 deformed steel rebar of two different sizes (\#6 and \#3) were used for the construction of the reinforcement cage. The mechanical properties of steel bar specimens are provided in section 4.4.2. The \#6 bars were used as longitudinal reinforcement for beam and column sections (Figure 48), while the \#3 bars were used for stirrups. The reinforcement cages were ground at select locations as identified using a painter's tape (Figure 49) to allow for the installation of strain gages at a later stage.

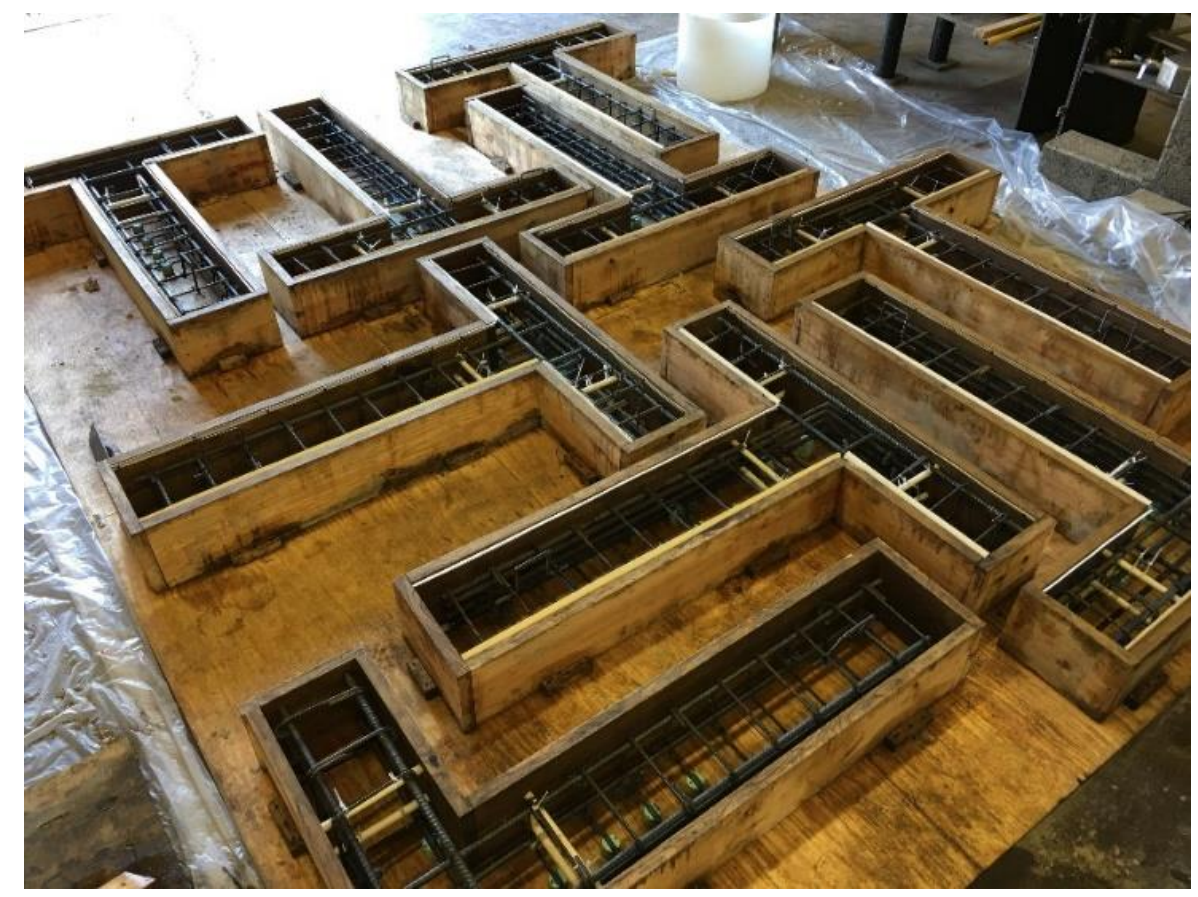

Figure 47. Plywood formwork for casting concrete joint specimens 


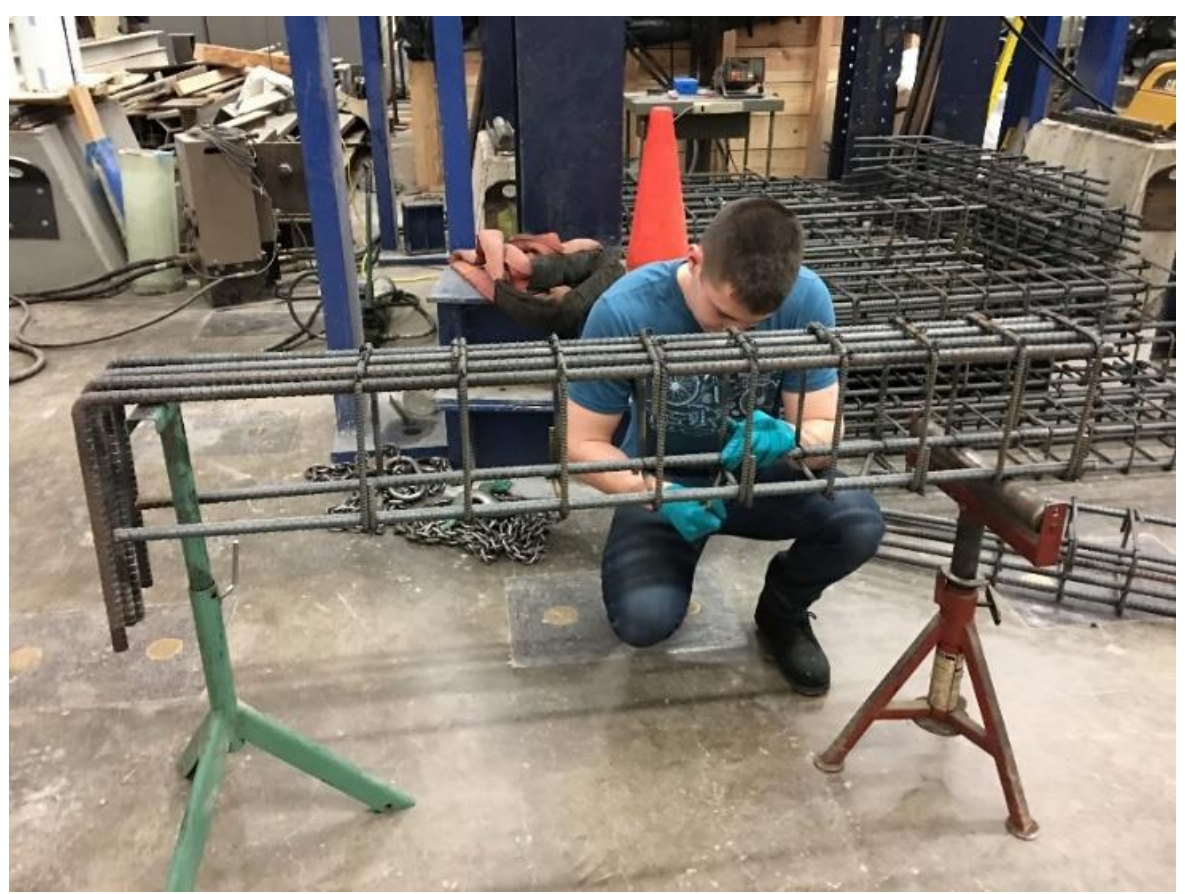

Figure 48. Preparation of reinforcement cage for beam and column sections

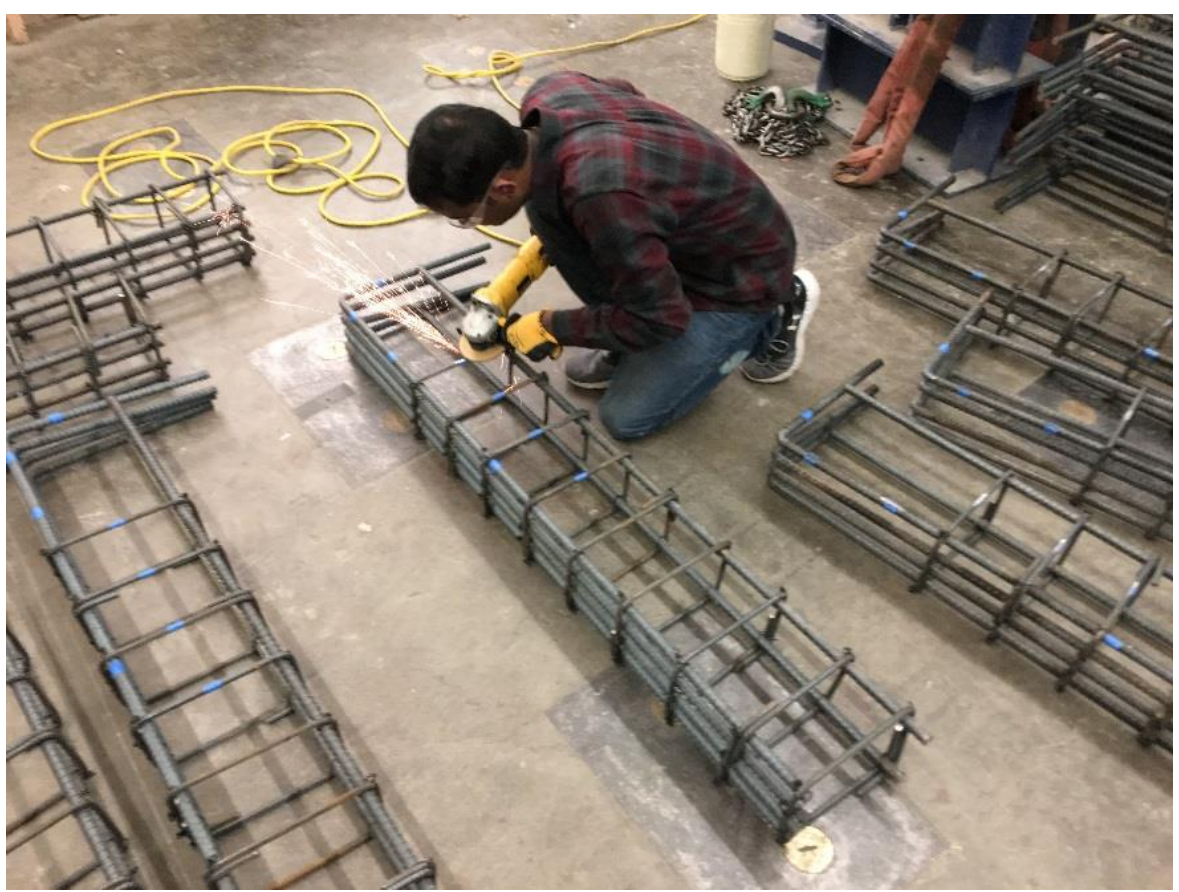

Figure 49. Grinding at select locations of rebar cage for installation of strain gages 
As shown in Figure 50, the beam and column reinforcement cages were assembled in molds by separating the rebar cage from touching the plywood using appropriate size concrete chairs. This arrangement ensures adequate concrete cover thickness. Besides, 2 in. diameter cylindrical foam plugs were placed underneath the locations where the rebar was ground. To prevent sliding of foam plugs during concrete placement and vibration, they were secured to the floor of plywood using screws. Once the joint specimens are cured, these plugs were removed to access the rebar surface for the installation of strain gages, as shown in Figure 51. To avoid drilling through the thickness of beam and column sections for dowel insertion, PVC pipes were tied to the reinforcement cage that served as placeholders.

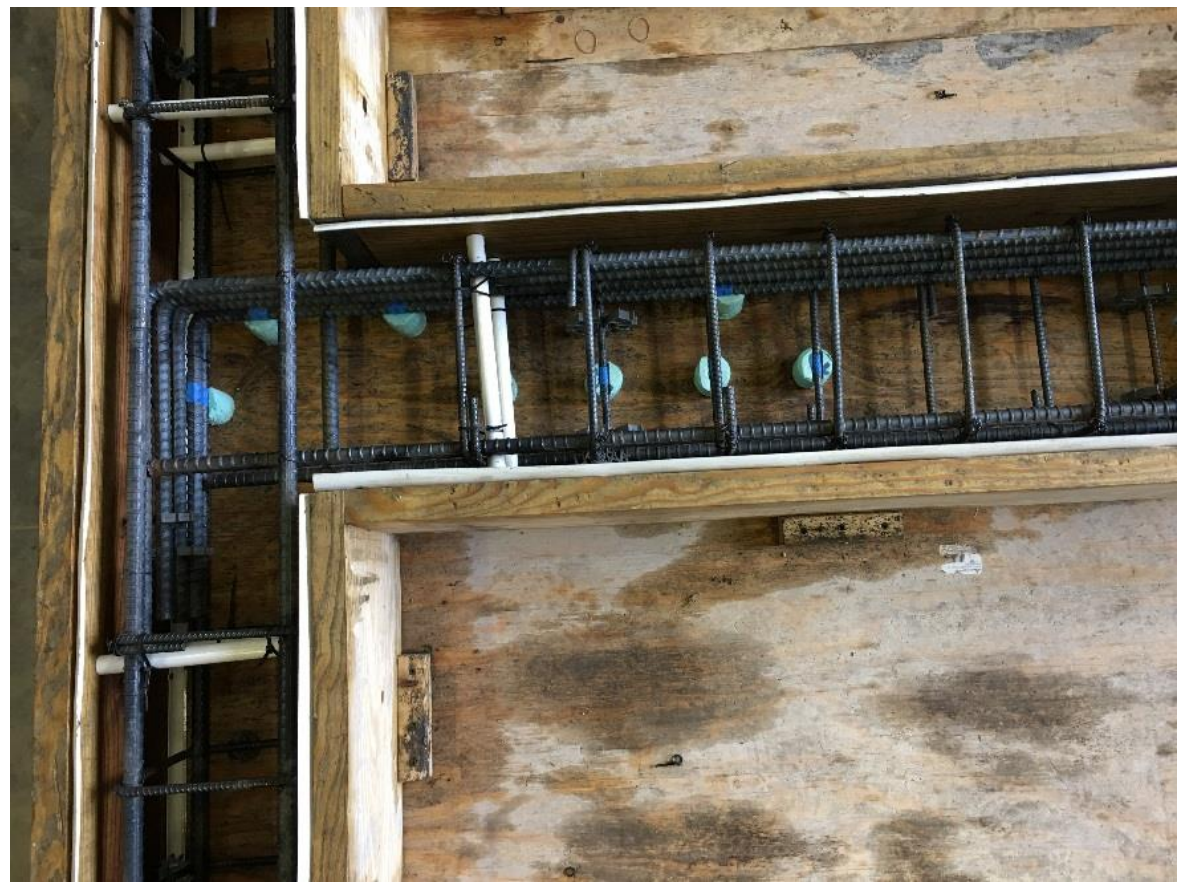

Figure 50. Closer view of the reinforcement cage after assembling in a mold 


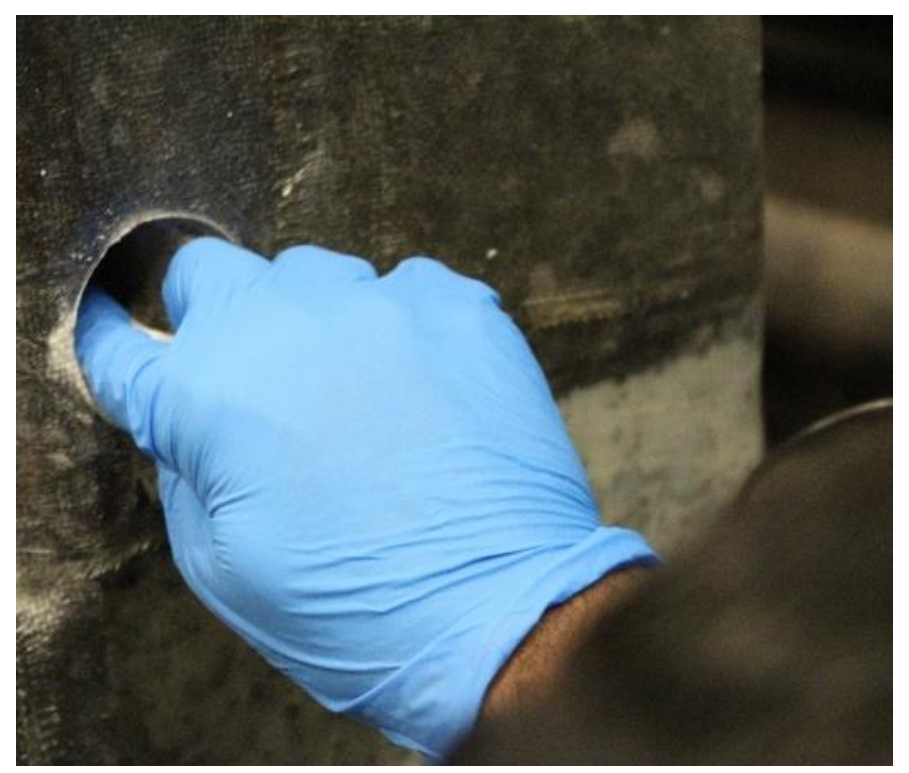

Figure 51. Installing strain gage on steel rebar

The joint test specimens were cast in three batches. Concrete for all three batches was supplied by a local manufacturer and transported to the lab facility using a transit mixer truck. As shown in Figure 52, concrete was placed in the forms while simultaneously vibrating with a pin-vibrator to minimize potential air voids and honeycombing. The test specimens were cured for seven days by sprinkling water after covering with wet burlap rolls. The formwork was stripped within 48 hours after the concrete pour. 


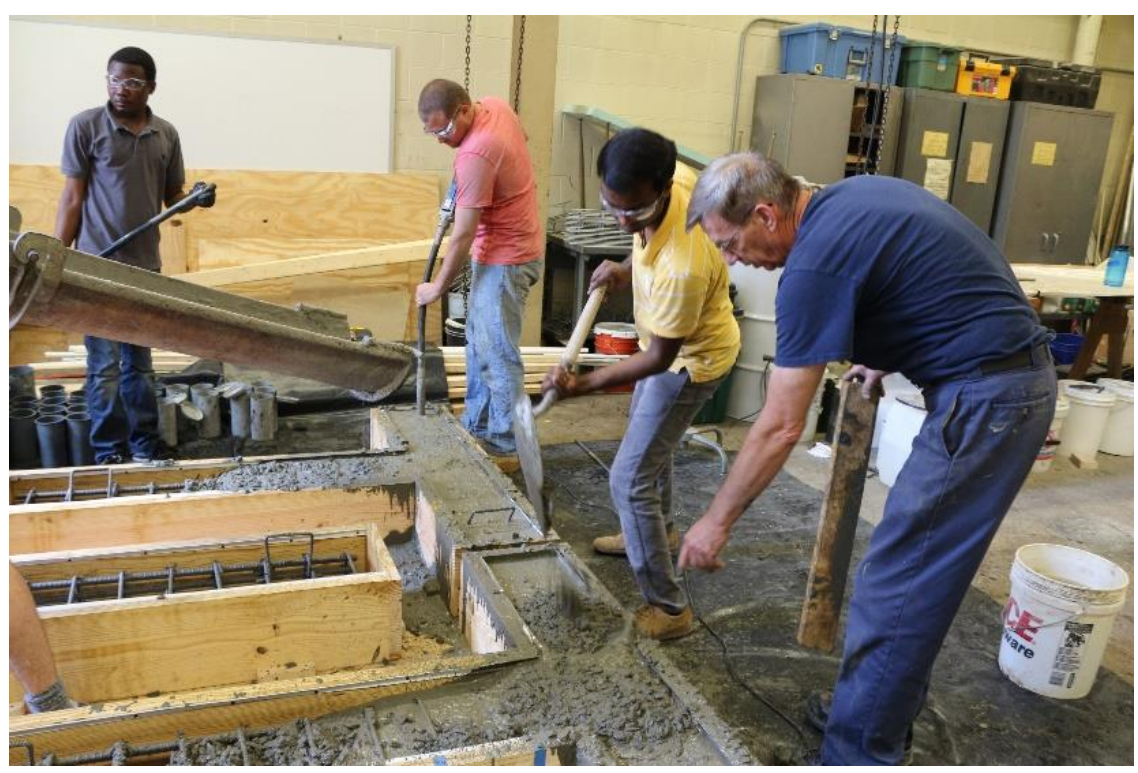

Figure 52. Placement of concrete during test specimen preparation

\subsubsection{Filler-module fabrication}

Prefabricated filler-modules made of three different materials (concrete, parallel strand lumber (PSL), and syntactic foam) were investigated in the present study. The mechanical properties of each filler-module material are provided in section 4.4 .

\subsubsection{Concrete filler-modules}

Concrete filler-modules were pre-cast by placing formwork against the joint specimens at their corners, as shown in Figure 53. Before creating the formwork for filler-modules, the aluminum tape is bonded to the joint specimen at its corners that receive filler-module. The aluminum tape acts as a barrier and alleviates the release of filler-modules from joint specimens. To ensure the alignment of dowel holes in filler-modules and joint specimen, PVC pipes were inserted as placeholders. The PVC pipes are removed within 24 hours after the concrete pour by gently tapping at their ends. The concrete filler-modules are cured in the same way as the joint specimens. 


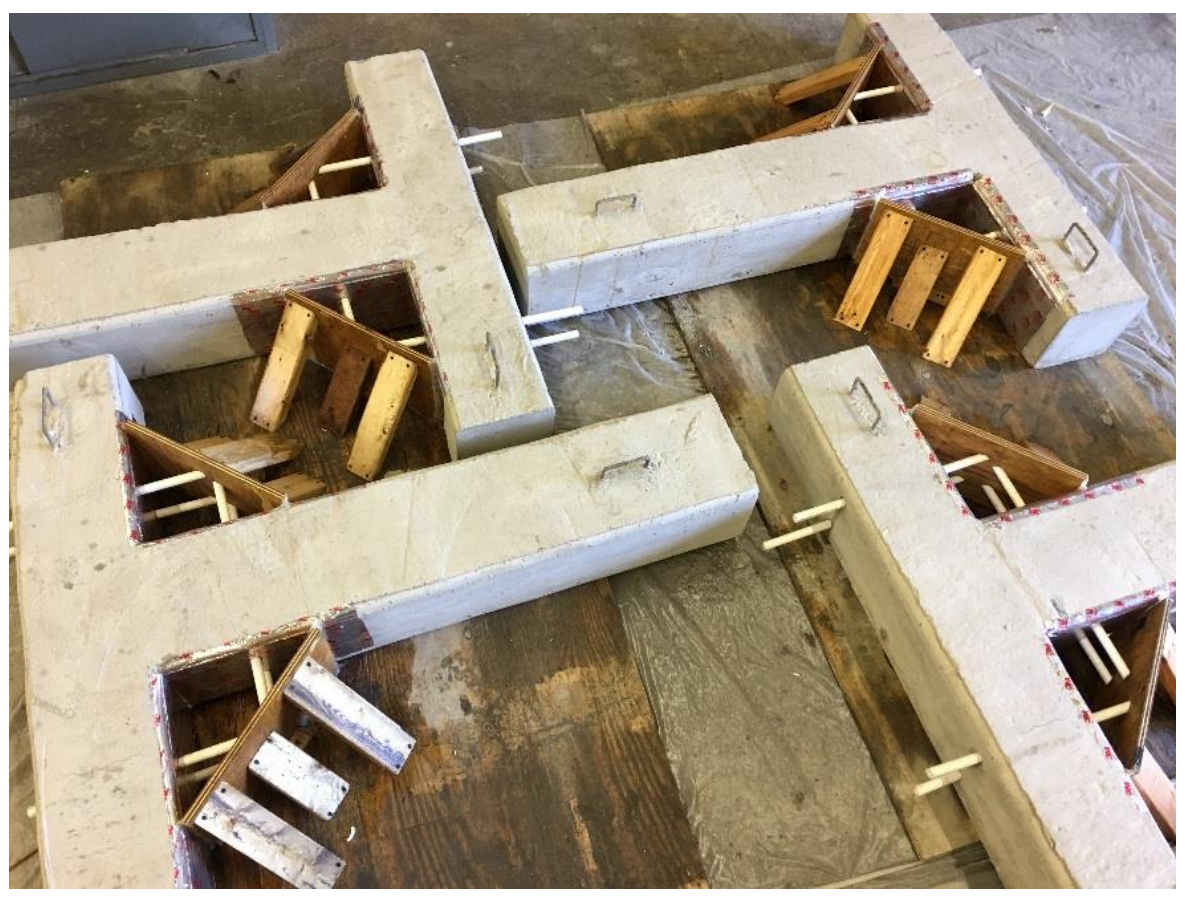

Figure 53. Casting of concrete filler-modules 


\subsubsection{Parellel strand lumber (PSL) filler-modules}

Parallel Strand Lumber (PSL) filler-modules were created by gluing together two 5 in. thick triangular blocks using the Titebond III. Quick-release clamps were used to hold the blocks together until cured, as shown in Figure 54. The compressive force from the clamps squeezed out excess glue, thereby minimizing air voids. Dowel holes were drilled through the filler-modules by orienting them against the joint corners that receive the modules.

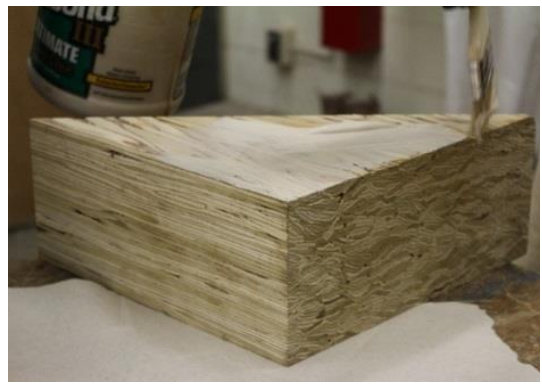

(a) Coating Titebond

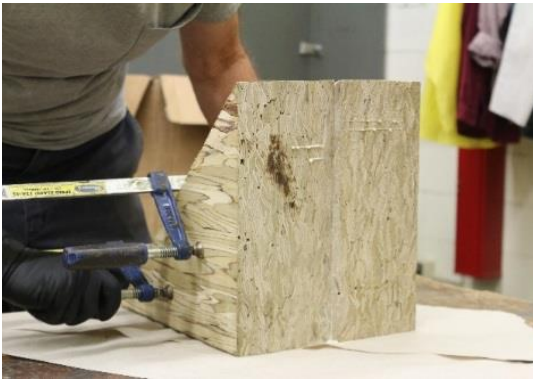

(b) Clamping until cured

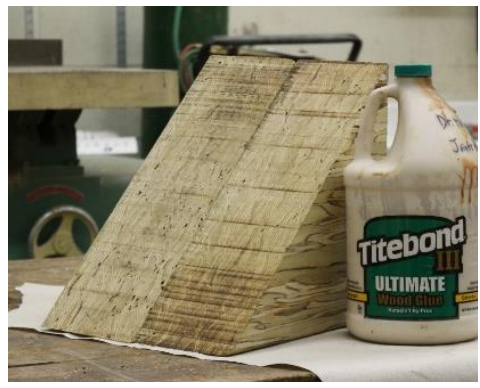

(c) PSL filler-module

Figure 54. Making of Parallel Strand Lumber (PSL) filler-modules

\subsubsection{Syntactic foam (SF) filler-modules}

Syntactic foam filler-modules were cast in a similar process to PSL filler-modules. 
4.3.2 Installation of filler-modules at joint corners

The steps illustrated in Figure 55 were followed for installation of prefabricated filler-modules at joint corners:

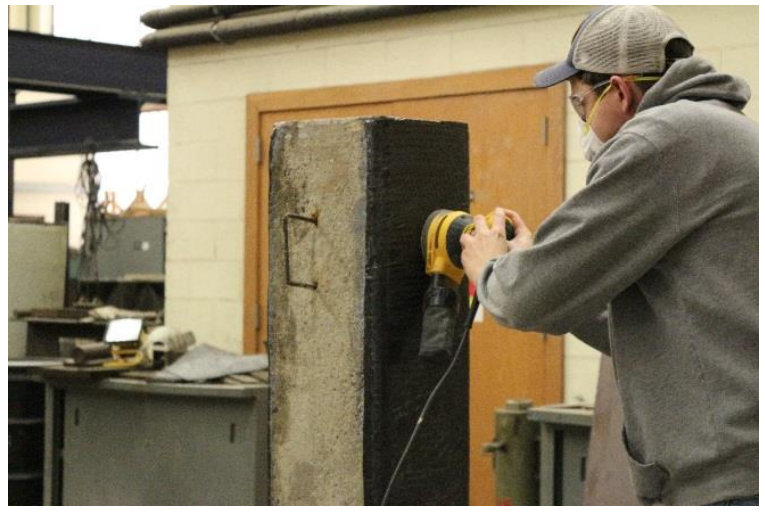

(a) Light sanding of FRP/concrete surface

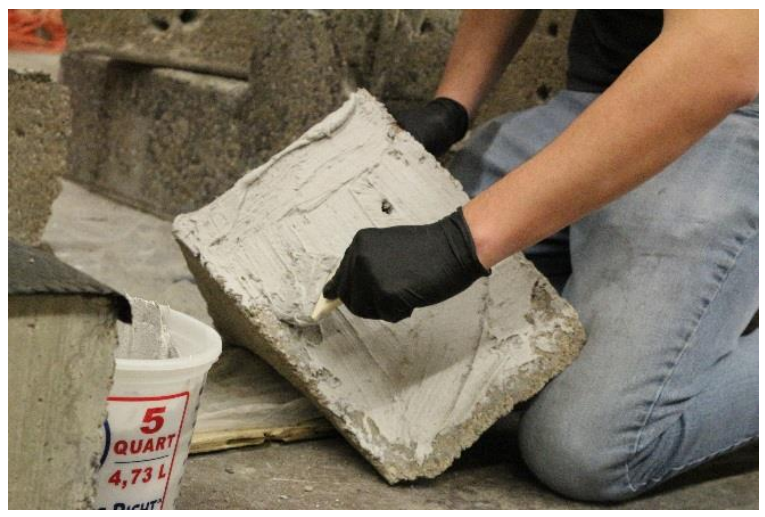

(c) Coating Sikadur 31 on filler-module(s)

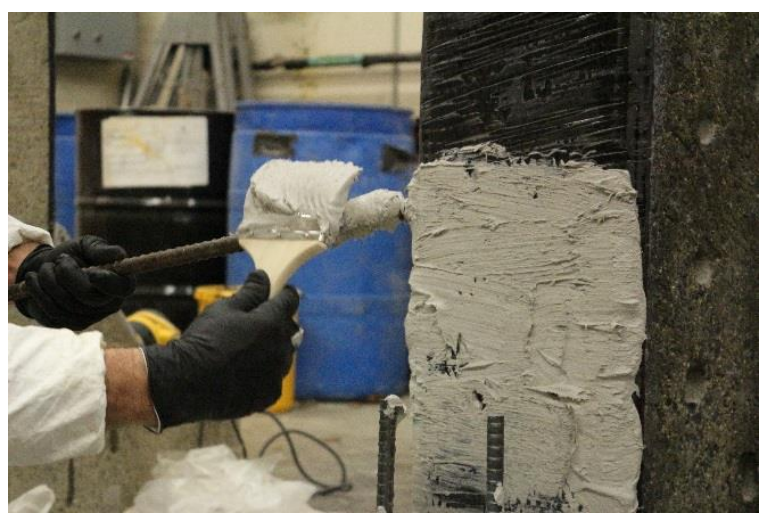

(e) Securing Filler-modules with dowels bars

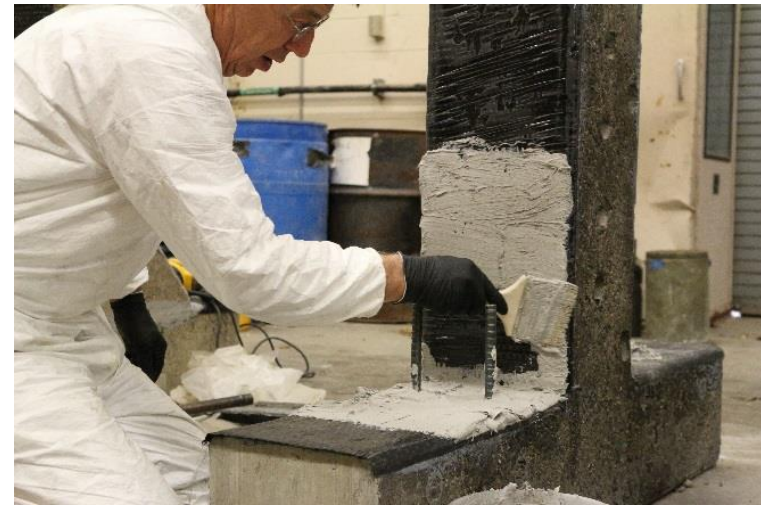

(b) Coating Sikadur 31 on substrate

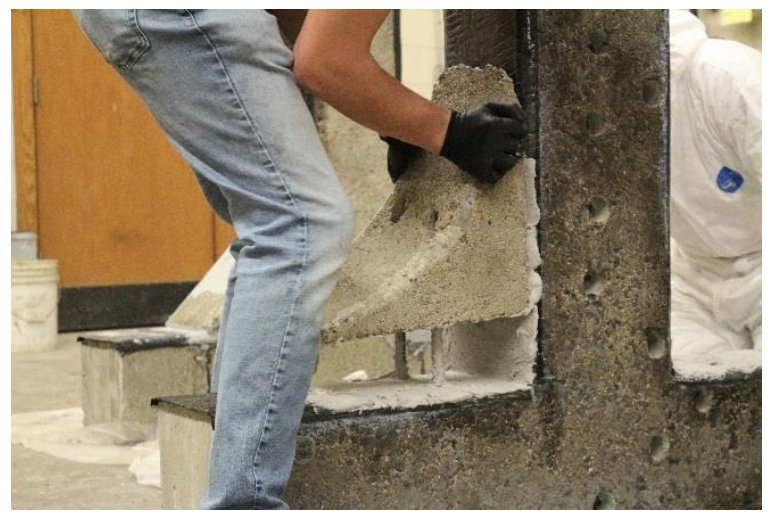

(d) Orienting filler-module(s) at joint corner

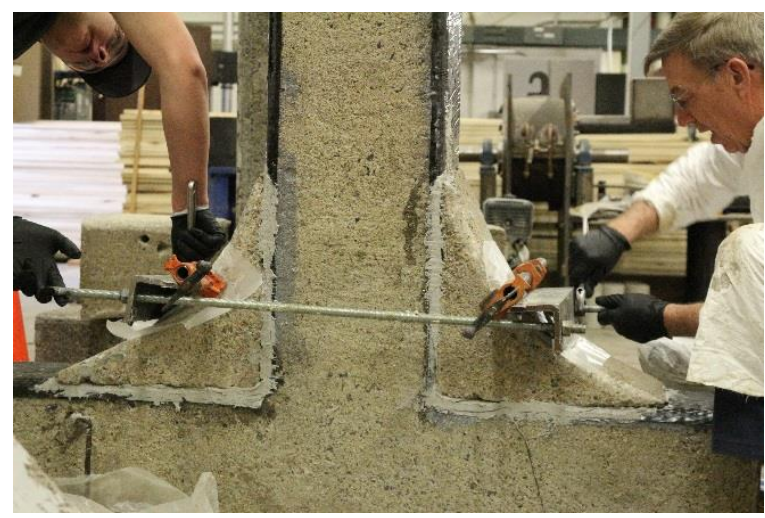

(f) Clamping of filler-modules until cured

Figure 55. Filler-module installation process at joint corners 


\subsubsection{Surface preparation}

The efficiency of an FRP strengthened concrete structure in resisting applied loads is heavily dependent on the bond (force-transfer) between the underlying substrate and FRP. Therefore, for creating efficient force-transfer between the substrate and FRP, surface preparation is essential. Hence, all the joint specimens in the current study were jet washed with water, as shown in Figure 56. Figure 57 shows a closer view of the concrete substrate surface before and after jet washing. The jet washed specimens were dried at room temperature for at least seven days before the installation of filler-modules or externally bonded FRP wraps.

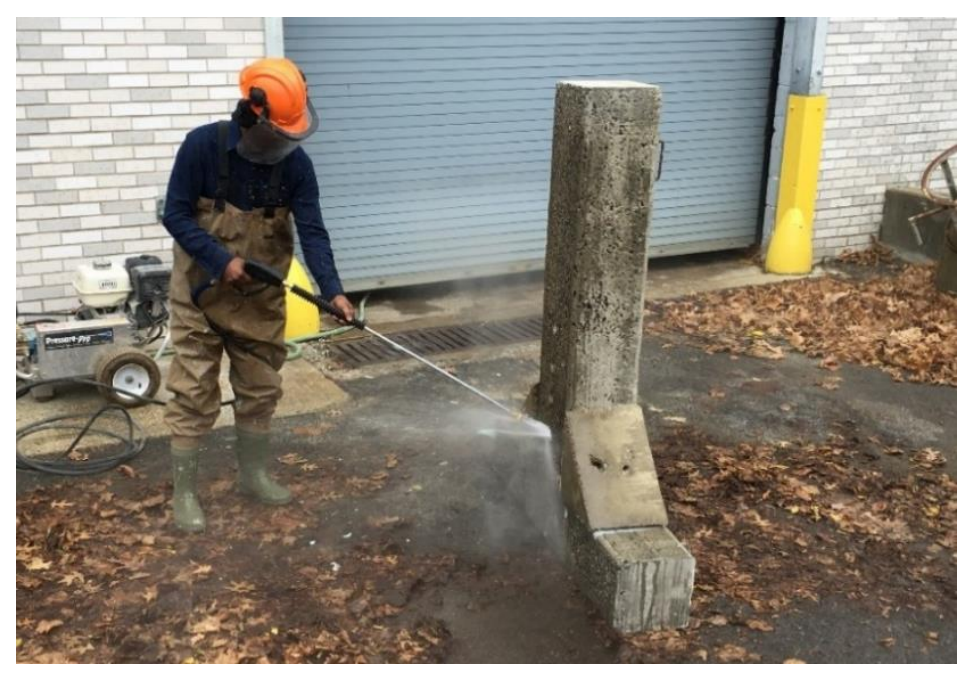

Figure 56. Surface preparation through water-jet washing

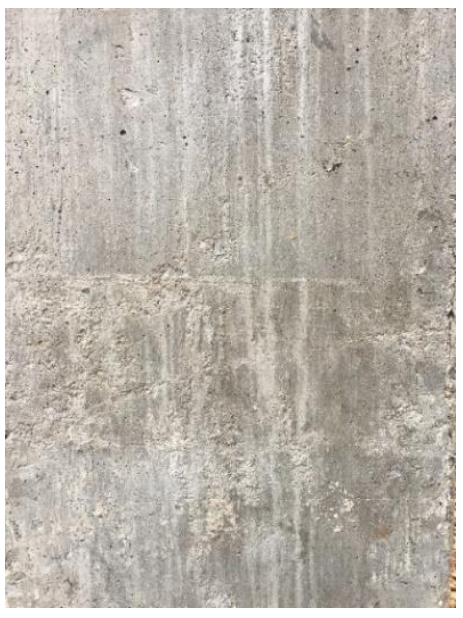

(a) Before jet wash

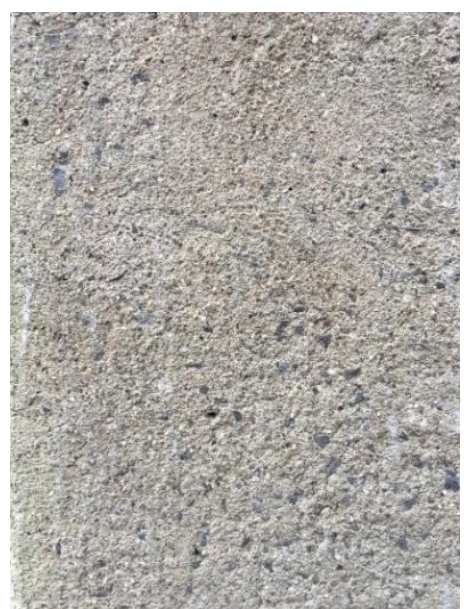

(b) After jet wash

Figure 57. Concrete surface before- and after- water-jet washing 


\subsubsection{FRP wrap/gusset installation}

Before bonding FRP wraps, Sikadur 30 was applied to the concrete substrate as a primer to fill any bug holes and create a uniform surface. The FRP wraps, cut to required shape and dimensions, were impregnated with Sika Hex 300 resin and bonded to the concrete substrate. Figure 58 illustrates the FRP wrap application process. After the application of each FRP layer, the wrap surface was smoothened using a hand-held paint roller by pressing against the specimen to squeeze out any excess resin, thus minimizing air voids.

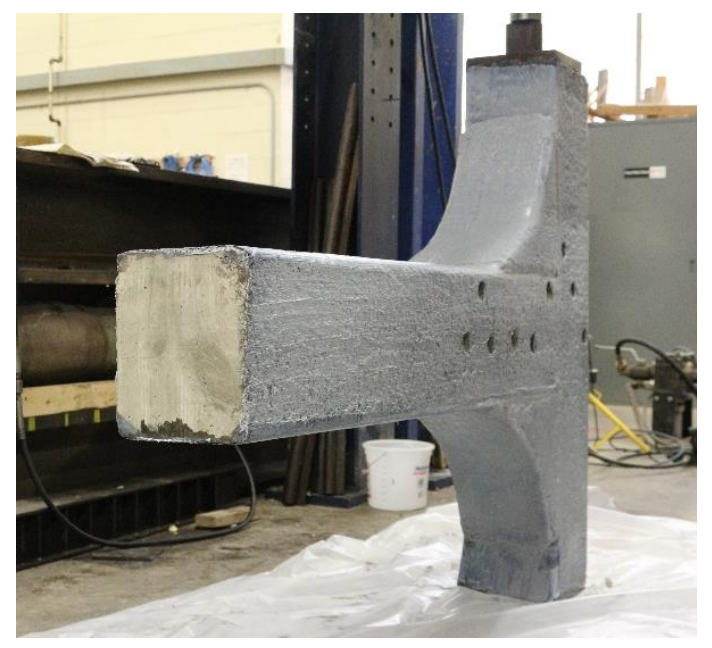

(a) Primer coated joint specimen

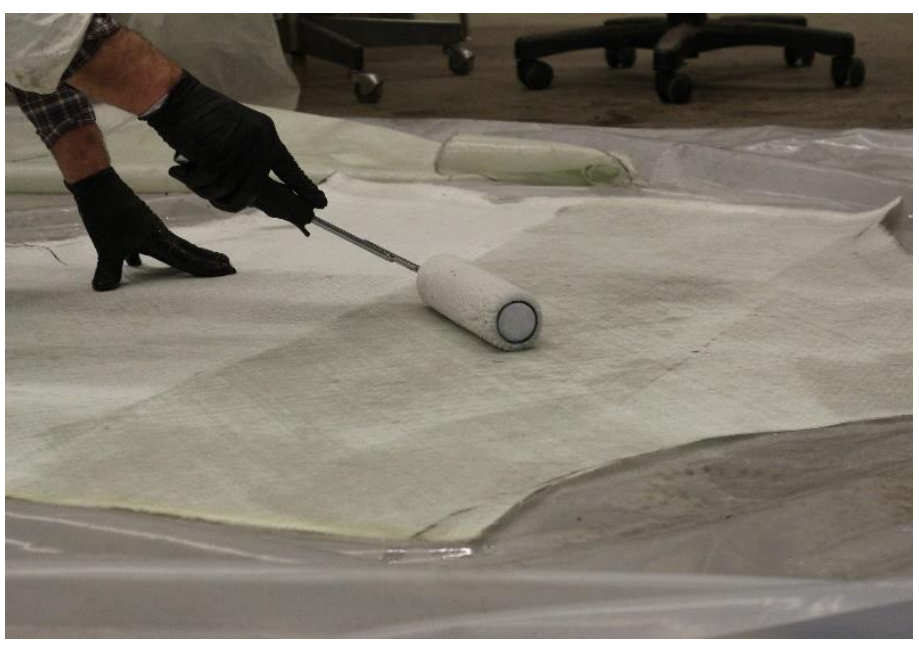

(b) Saturation of glass fabric with epoxy resin

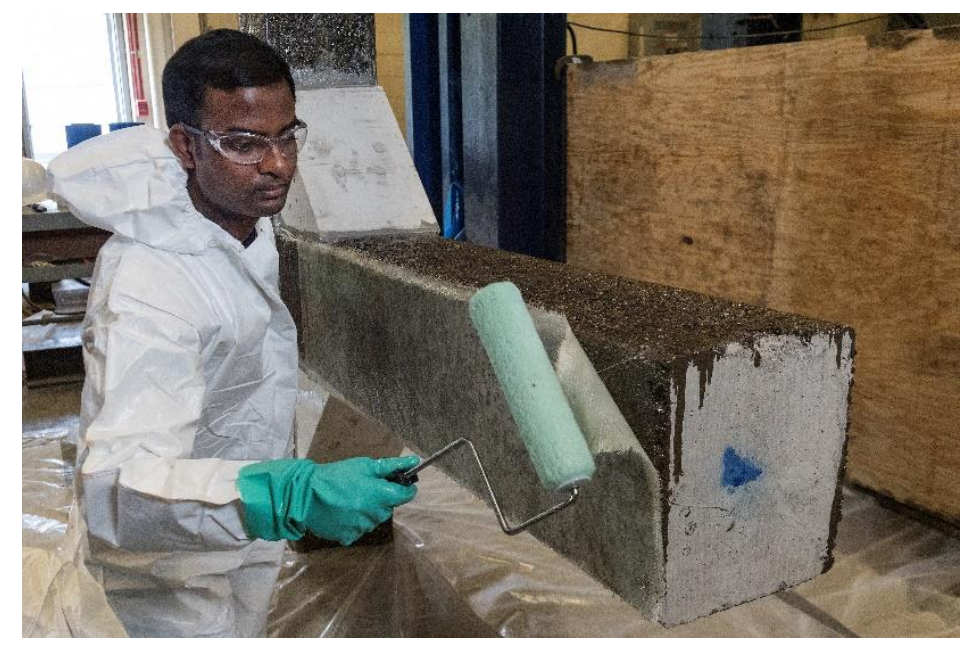

(c) Bonding FRP wrap to the concrete substrate

Figure 58. FRP wrapping of joint specimen 
FRP gusset is a pre-cured composite laminate manufactured using hand lay-up process by gluing together a stack of carbon fabric layers after saturating with Sika Hex 300 resin. Figure 59 shows the FRP gusset configuration used in the current study. The gussets were bonded to the primed concrete substrate using Sikadur 30.

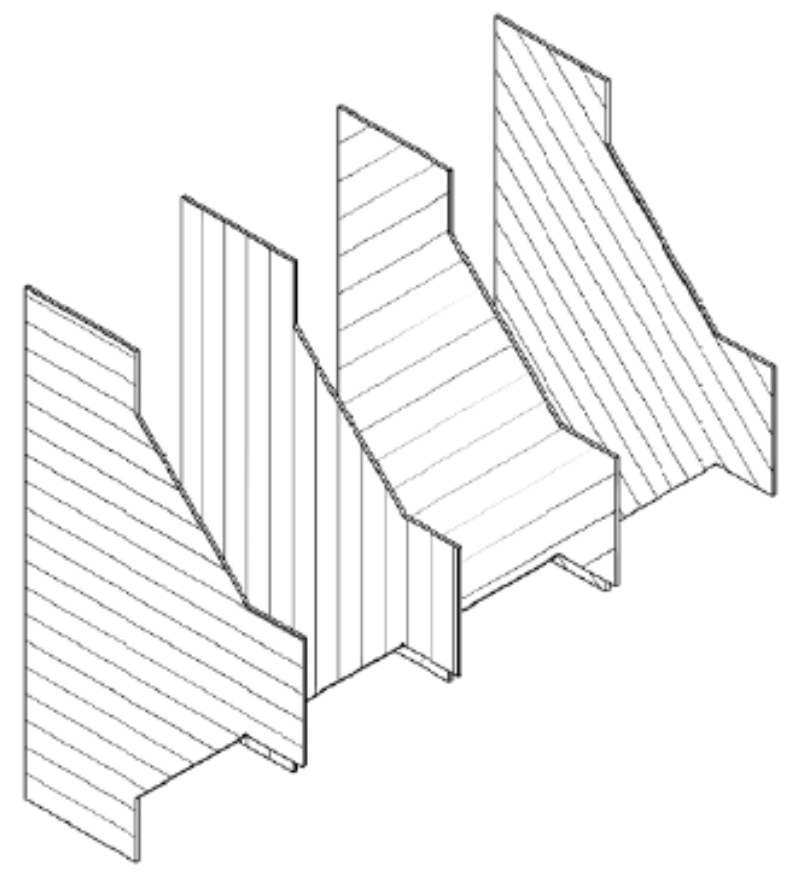

Figure 59. FRP gusset used in the current study for reinforcing joint specimens

Both the FRP wrapped and gusset reinforced joint specimens were cured for at least three days before testing the specimen to failure.

Note: Primer was applied only for specimens JI-5 through 8 and JIII-2 through 4. 


\subsection{Materials}

The following section provides details of various materials employed for this study, including their mechanical properties.

\subsubsection{Concrete}

Twelve (6 in. $x 12$ in.) and eight (4 in. $x 8$ in.) plain concrete cylinder specimens were tested to establish the mechanical properties of concrete used for casting joint and filler-module specimens, respectively. The cylinder specimens were tested within seven days after joint specimen testing. Before stripping the plastic molds, all the cylinder specimens were cured for 28 days by immersing in a water bath. Procedures listed in ASTM C39 and ASTM C496 were followed for evaluating the concrete compressive and tensile strengths, respectively. A summary of concrete material properties used in casting joint specimens and filler-modules is provided in Tables 19 and 20, respectively. 
Table 19. Properties of plain concrete (joint) specimens

\begin{tabular}{|c|c|c|c|}
\hline \multirow{2}{*}{\multicolumn{2}{|c|}{ Specimen ID }} & \multicolumn{2}{|c|}{ Unconfined concrete properties } \\
\hline & & $f_{c}^{\prime}(p s i)$ & $f_{c t}(p s i)$ \\
\hline \multirow{2}{*}{ J-I } & C6-S1 & 2491 & 237 \\
\hline & C6-S2 & 2788 & 233 \\
\hline \multicolumn{2}{|c|}{ J-I Average: } & 2639 & 235 \\
\hline \multirow{2}{*}{ J-II } & C6-S1 & 3363 & 305 \\
\hline & C6-S2 & 3254 & 286 \\
\hline \multicolumn{2}{|c|}{ J-II Average: } & 3309 & 296 \\
\hline \multirow{2}{*}{ J-III } & C6-S1 & 3817 & N.A. \\
\hline & C6-S2 & 3916 & N.A. \\
\hline \multicolumn{2}{|c|}{ J-III Average: } & 3866 & N.A \\
\hline
\end{tabular}

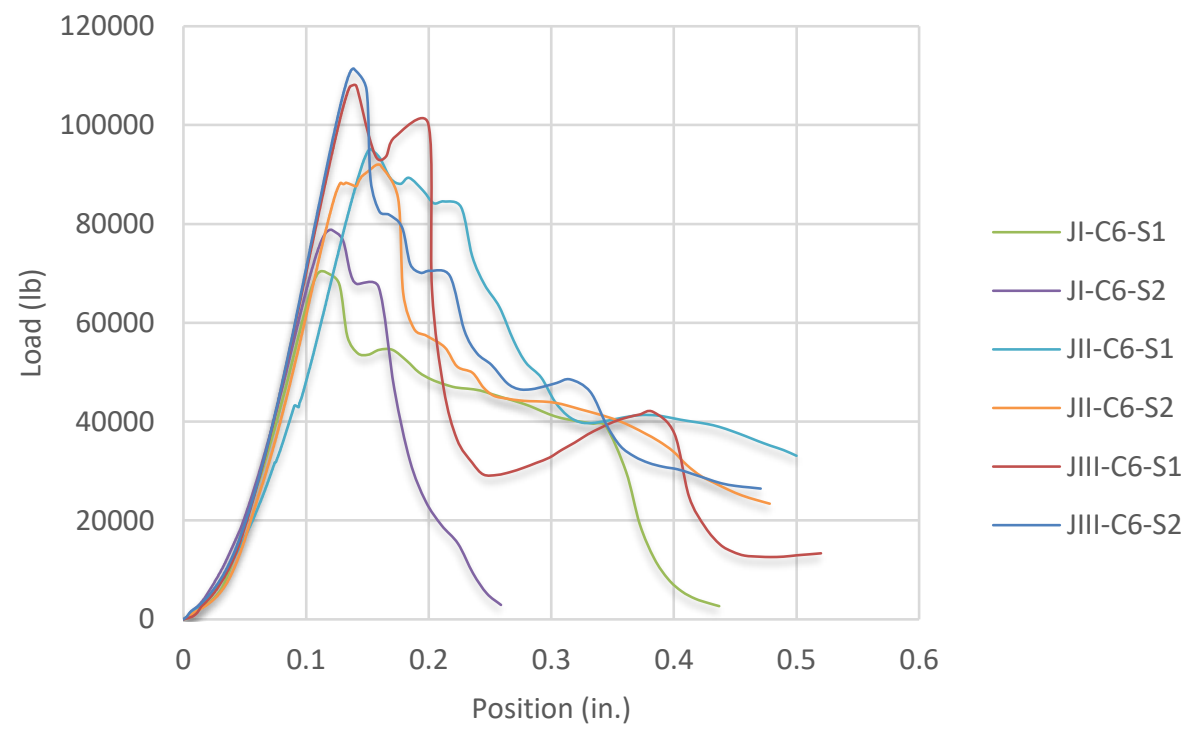

Figure 60. Load vs. position curves for plain concrete (joint) specimens 
Table 20. Properties of plain concrete (filler-modules) specimens

\begin{tabular}{lccc}
\hline \multirow{2}{*}{ Specimen ID } & \multicolumn{2}{c}{ Unconfined concrete properties } \\
\cline { 3 - 4 } & & $\boldsymbol{f}_{c}^{\prime}(\boldsymbol{p s i})$ & $\boldsymbol{f}_{\boldsymbol{c}}$ (psi) \\
\hline \multirow{2}{*}{ C-FM-I } & C4-S1 & 3374 & N.A. \\
\cline { 2 - 4 } & $\mathrm{C} 4-\mathrm{S} 2$ & 3377 & N.A. \\
\hline \multirow{2}{*}{ C-FM-I Average: } & $\mathbf{3 3 7 6}$ & N.A. \\
\hline \multirow{2}{*}{ C-FM-II } & C4-S1 & 5109 & 552 \\
\cline { 2 - 4 } & C4-S2 & 5836 & N.A. \\
\hline C-FM-II Average: & $\mathbf{5 4 7 2}$ & $\mathbf{5 5 2}$ \\
\hline \multirow{2}{*}{ C-FM-III } & C4-S1 & 9691 & 419 \\
\cline { 2 - 4 } & C4-S2 & 9400 & N.A. \\
\hline \multicolumn{2}{l}{ C-FM-III Average: } & $\mathbf{9 5 4 5}$ & $\mathbf{4 1 9}$ \\
\hline
\end{tabular}

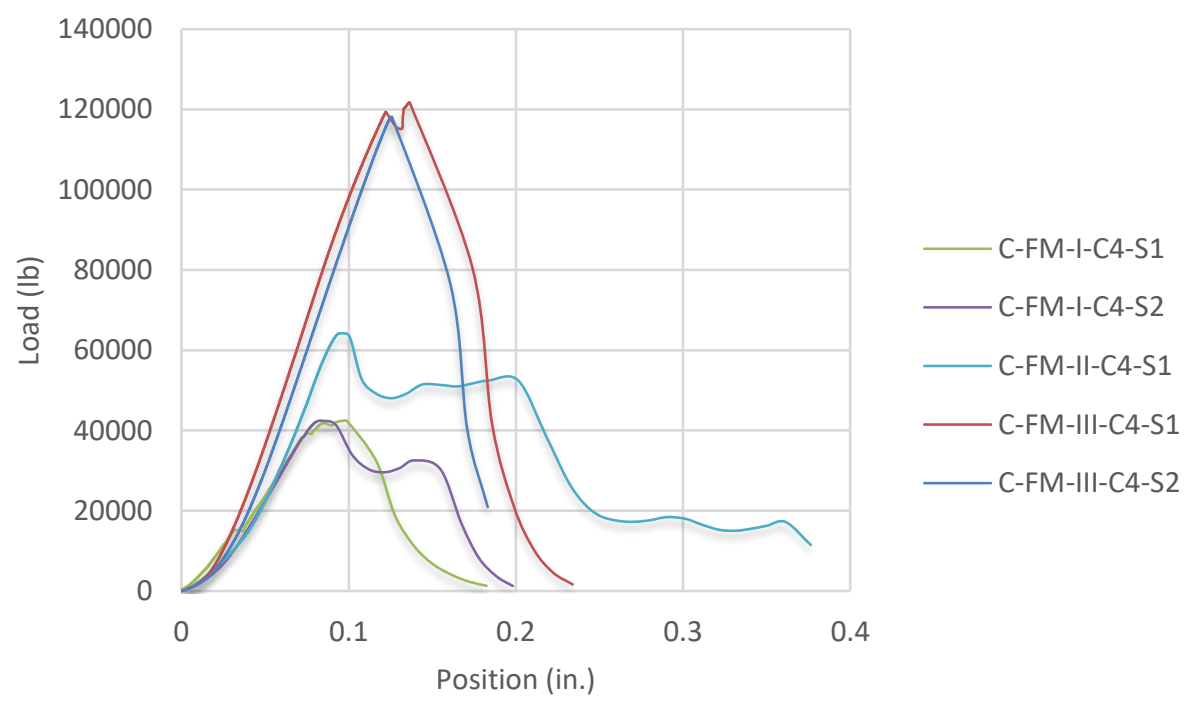

Figure 61. Load vs. position curves for plain concrete (filler-modules) specimens 


\subsubsection{Steel rebar}

Two different sizes (\#6 and \#3) of grade 60 deformed steel rebar specimens were evaluated to establish their tensile properties under monotonic loading. The steel rebar specimens were tested in an Instron machine by following ASTM A 370 standard. Two of the three \#6 rebar specimens failed near the grip ( 1.5 in. above the grip) while all other specimens fractured within the gage length. The final failure of each specimen is shown in Figures 62 and 63. Figures 64 and 65 show the stress-strain curves of \#6 and \#3 rebar specimens, respectively. The stress and strain values corresponding to the plateau are defined as yield numbers for all rebar specimens. A summary of the mechanical properties of \#6 and \#3 rebar specimens is provided in Table 21.

Table 21. Properties of steel rebar specimens

\begin{tabular}{|c|c|c|c|c|c|c|}
\hline \multirow{2}{*}{\multicolumn{2}{|c|}{ Specimen ID }} & \multicolumn{5}{|c|}{ Rebar tensile properties } \\
\hline & & $f_{u}(k s i)$ & $\varepsilon_{u}$ & $E_{s}(k s i)$ & $f_{y}(k s i)$ & $\varepsilon_{y}$ \\
\hline \multirow{3}{*}{ Bar \#6 } & $\mathrm{S} 1$ & 119.55 & 0.082 & 28260.50 & 73.34 & 0.0027 \\
\hline & $\mathrm{S} 2$ & 104.79 & 0.081 & 27948.16 & 72.72 & 0.0028 \\
\hline & S3 & 89.96 & 0.082 & 29778.16 & 73.76 & 0.0027 \\
\hline \multicolumn{2}{|c|}{ Bar \#6 Average: } & 104.77 & 0.082 & 28662.27 & 73.27 & 0.0027 \\
\hline \multirow{3}{*}{ Bar \#3 } & $\mathrm{S} 1 *$ & 101.75 & $0.033^{+}$ & 25577.13 & 76.98 & 0.0031 \\
\hline & $\mathrm{S} 2 *$ & 110.22 & $0.035^{+}$ & 23595.36 & 70.32 & 0.0030 \\
\hline & $\mathrm{S} 3 *$ & 109.67 & 0.082 & 23739.46 & 78.21 & 0.0034 \\
\hline \multicolumn{2}{|c|}{ Bar \#3 Average: } & 107.21 & 0.082 & 24303.98 & 75.17 & 0.0032 \\
\hline
\end{tabular}

Note: *Deviation in mechanical properties is attributed to the low carbon content in steel rebar. ${ }^{+}$Data ignored because of premature specimen failure. 


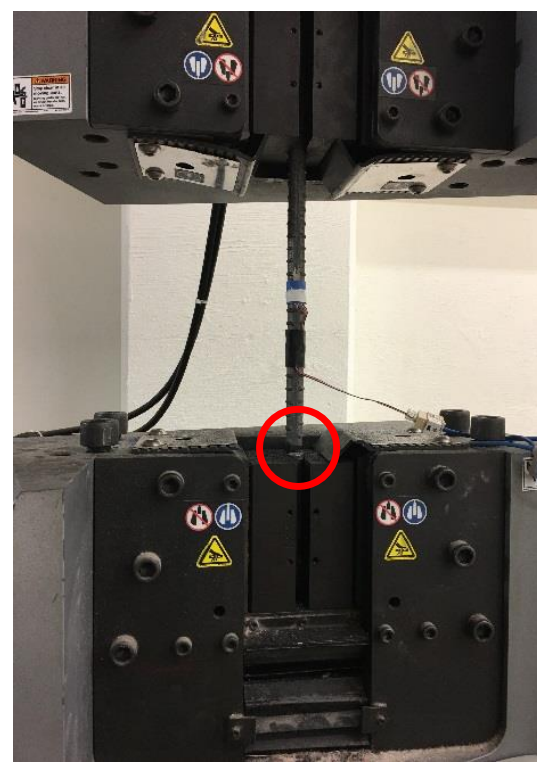

(a) Bar \#6 - S1

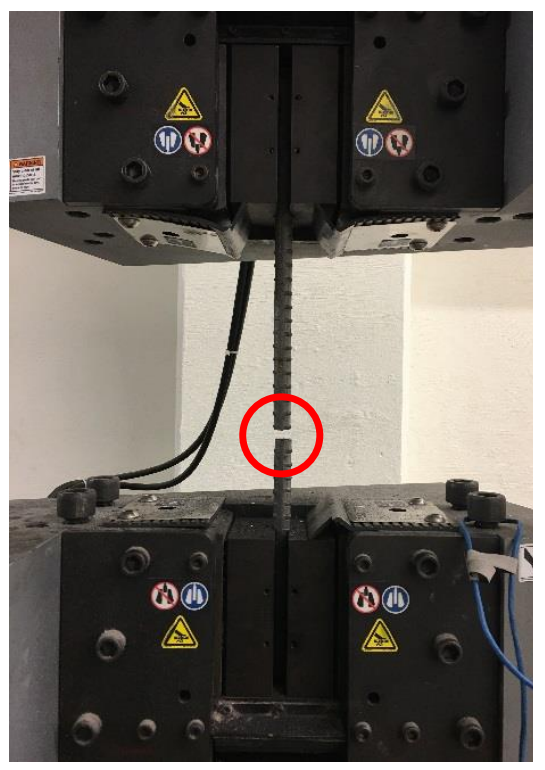

(b) Bar \#6 - S2

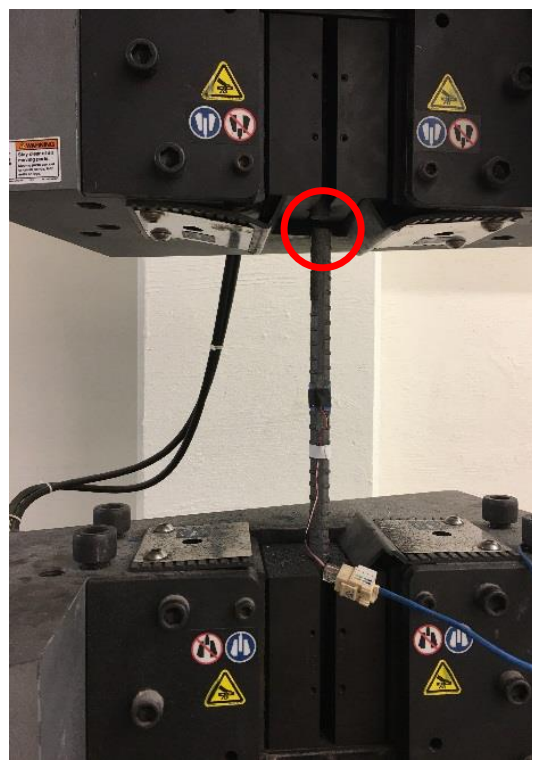

(c) Bar \#6 - S3

Figure 62. Failure modes of grade $60 \# 6$ rebar specimens

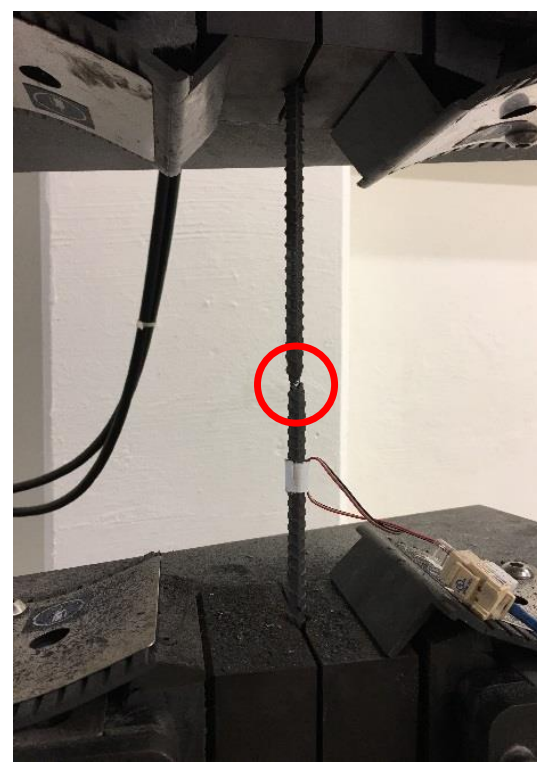

(a) Bar \#3-S1

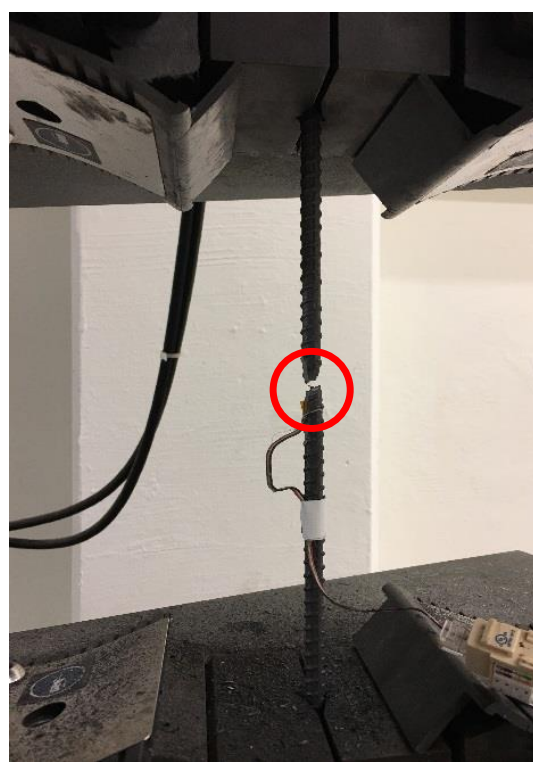

(b) Bar \#3 - S2

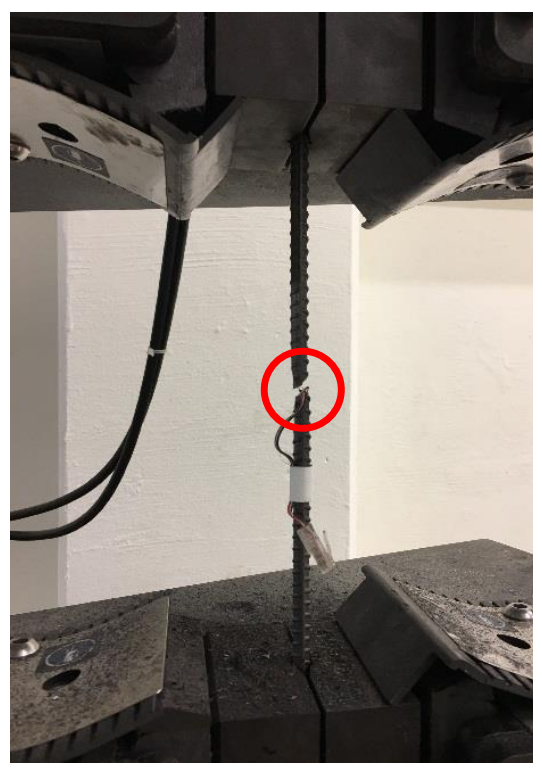

(c) Bar \#3-S3

Figure 63. Failure modes of grade $60 \# 3$ rebar specimens 


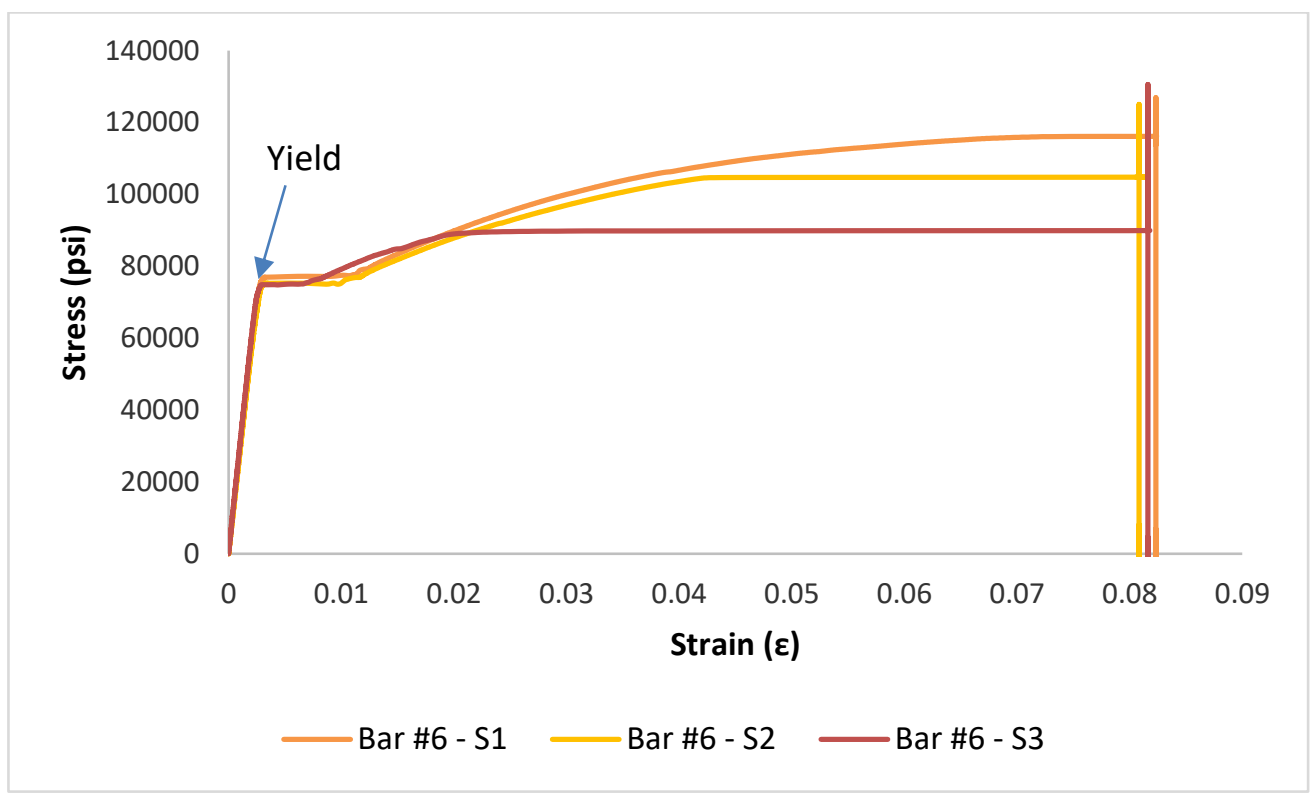

Figure 64. Stress-strain plots for grade 60 \#6 steel rebar specimens

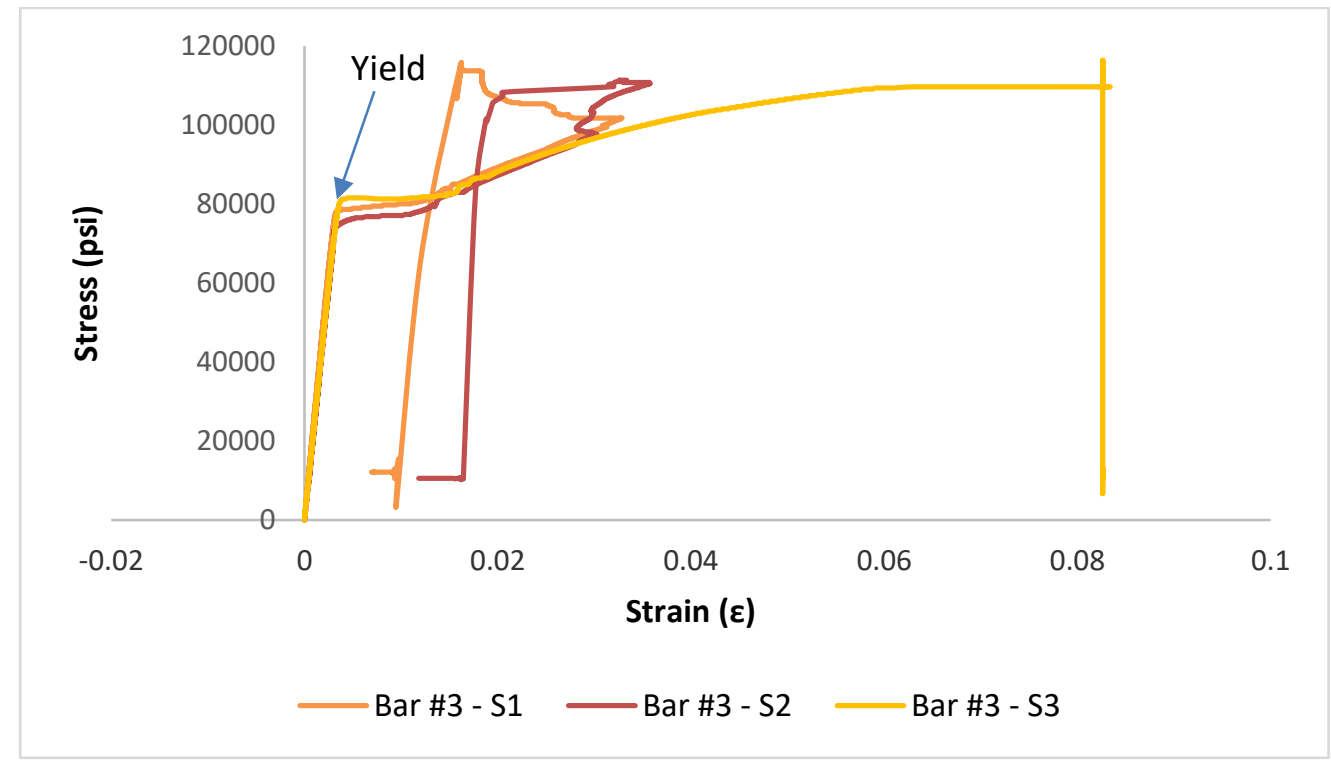

Figure 65. Stress-strain plots for grade $60 \# 3$ steel rebar specimens 


\subsubsection{Engineered wood (PSL)}

Parallel strand lumber (PSL), popularly known as engineered wood, is manufactured by orienting wood strands in a parallel direction and gluing them together under specific temperature and pressure conditions. PSL is a high-quality composite wood product with consistent mechanical properties that makes it an excellent choice for structural applications. Compared to traditional wood, PSL is less susceptible to bowing, cupping, shrinkage, warping, and even easier to treat; thus, making it suitable for outdoor applications. The light-weight characteristic of PSL allows for easy installation at job sites, primarily when used as filler-modules. Besides, PSL is ideally suited for seismic and blast-resistant applications due to its higher energy-absorption capacity under shock loads than concrete. The design properties of Trus Joist ${ }^{\circledR}$ 2.0E Parallam ${ }^{\circledR}$ PSL (Figure 66) used in this study are listed in Table 22. For additional information on this material, refer to Trus Joist specifier's guide.

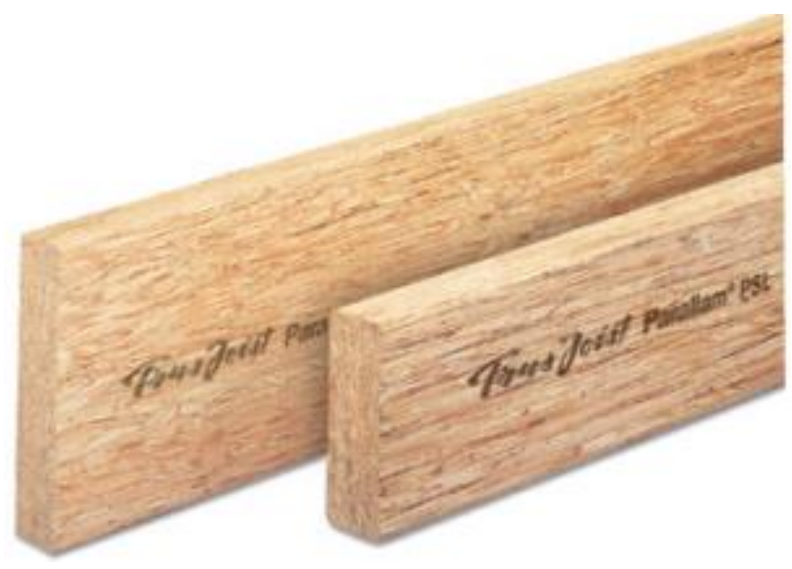

Figure 66. Trus Joist Parallam® PSL [54]

Table 22. Design properties of Parallam® PSL [54]

\begin{tabular}{ccccccccccc}
\hline \multirow{2}{*}{ Grade } & \multicolumn{3}{c}{ Modulus of Elasticity (msi) } & \multicolumn{7}{c}{ Stress (psi) } \\
\cline { 2 - 11 } & $\mathrm{G}$ & $\mathrm{E}$ & $\mathrm{E}_{\text {min }}$ & $\mathrm{F}_{\mathrm{b}}$ & $\mathrm{F}_{\mathrm{t}}$ & $\mathrm{F}_{\mathrm{c} \perp}$ & $\mathrm{F}_{\mathrm{c} \|}$ & $\mathrm{F}_{\mathrm{V}}(p s i)$ & $\mathrm{SG}$ \\
\hline $\mathbf{2 . 0 E}$ & 0.125 & 2.0 & 1.016 & 2900 & 2025 & 625 & 2900 & 290 & 0.50 \\
\hline
\end{tabular}




\subsubsection{Engineered syntactic foam}

Syntactic Foam (SF) is a low-density composite foam that offers excellent mechanical properties for use in structural applications. Depending on the grade, the compressive and flexural strengths of commercially available SF range up to 6700 psi and 5800 psi, respectively. The light-weight characteristic of SF allows for easy installation at job sites, primarily when used as joint reinforcement (as proposed in this study). Besides, non-degradable SFs also offer excellent longterm performance under outdoor exposures, which makes it suitable for reinforcing joints of parking garages and bridge bents that are exposed to de-icing salts. Also, SFs are ideally suited for seismic and blast-resistant applications due to their high energy-absorption capacity under shock loads. The mechanical properties of SF 3000 Grade Microsphere Syntactic Foam (Figure 67) used in this study are listed in Table 23. For additional information on this material, refer to SF Grade Microsphere Syntactic Foam technical datasheet.

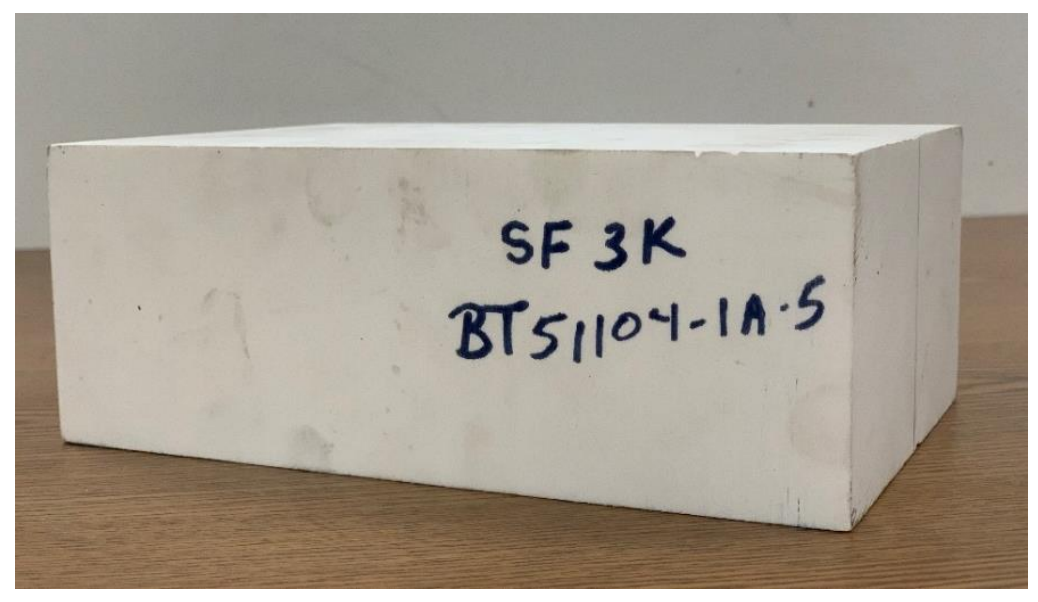

Figure 67. SF 3000 grade microsphere syntactic foam

Table 23. Mechanical properties of SF grade syntactic foam [55]

\begin{tabular}{|c|c|c|c|c|c|}
\hline \multirow{2}{*}{ Grade } & \multirow{2}{*}{$\begin{array}{c}\text { Density } \\
\left(\boldsymbol{l b} / \boldsymbol{f} t^{3}\right)\end{array}$} & \multicolumn{2}{|c|}{ Compression properties } & \multicolumn{2}{|c|}{ Flexural properties } \\
\hline & & Strength ( $p s i)$ & Modulus ( $k s i)$ & Strength $(p s i)$ & Modulus ( $k s i)$ \\
\hline SF 1500 & 35 & 2685 & 115 & 4390 & 412 \\
\hline SF 3000* & 39 & 4320 & 261 & 4920 & 441 \\
\hline SF 5000 & 46 & 6700 & 498 & 5860 & 490 \\
\hline
\end{tabular}

*Product used in the current study. 


\subsubsection{FRP composite reinforcement}

Properties of FRP composite reinforcements used in this study are obtained from manufactures technical datasheets, and a summary is presented in Table 24.

Table 24. Properties of FRP composite materials [56] [57]

\begin{tabular}{ccccc}
\hline \multirow{2}{*}{ Specimen ID } & FRP configuration & Ply thickness $(\boldsymbol{i}$..$)$ & \multicolumn{2}{c}{ FRP tensile properties } \\
\cline { 4 - 5 } & & & $f_{f u}(k s i)$ & $\varepsilon_{f u}(\mu \varepsilon)$ \\
\hline Sika 103C CFRP & Unidirectional fabric & 0.04 & 181 & 0.0175 \\
\hline Sika 100G GFRP & Unidirectional fabric & 0.04 & 88.6 & - \\
\hline
\end{tabular}

\subsubsection{FRP composite confined concrete cylinders}

Two different strengths ( 2.9 and $3.6 \mathrm{ksi}$ ) of plain concrete cylinder specimens were wrapped with FRP composite fabrics to evaluate the capacity enhancements through confinement under axial compression and split-tensile tests. Besides, the numerical values of load and strain at first crack and failure were recorded. A summary of the test results is presented in Tables 25 and 26.

Table 25. Properties of FRP confined plain concrete (compression test)

\begin{tabular}{lcccccc}
\hline \multirow{2}{*}{ Specimen IID } & \multicolumn{3}{c}{ J-I } & & J-II \\
\cline { 2 - 7 } & CS & S-1 & S-2 & CS & S-1 & S-2 \\
\hline Peak axial stress $($ psi) & 2925 & 14701 & 3239 & 3555 & 15827 & 12640 \\
\hline Hoop strain at peak stress $(\mu \varepsilon)$ & 0.012 & 0.009 & 0.0002 & -0.003 & 0.003 & 0.0015 \\
\hline Axial strain at peak stress $(\mu \varepsilon)$ & -0.003 & -0.03 & $-0.0008^{*}$ & -0.002 & -0.02 & -0.015 \\
\hline Axial stress at the $\mathbf{1}^{\text {st }}$ crack $(p s i)$ & 1440 & 2000 & 2200 & 1853 & 1703 & 2002 \\
\hline Axial strain at the $\mathbf{1}^{\text {st }}$ crack $(\mu \varepsilon)$ & 0.0006 & 0.0007 & 0.0005 & 0.0004 & 0.0003 & 0.0005 \\
\hline
\end{tabular}

*Experimental anomaly

Table 26. Properties of FRP confined plain concrete (split-tension test)

\begin{tabular}{lcccccc}
\hline \multirow{2}{*}{\multicolumn{1}{c}{ Property }} & \multicolumn{3}{c}{ J-I } & \multicolumn{3}{c}{ J-II } \\
\cline { 2 - 7 } & CS & S-1 & S- 2 & CS & S-1 & S-2 \\
\hline Peak tensile strength (psi) & 429 & 1234 & 1027 & 420 & 1691 & 1229 \\
\hline Tensile stress at $1^{\text {st }}$ crack (psi) & 200 & 200 & 200 & N.A. & 259 & 250 \\
\hline
\end{tabular}




\section{Note:}

J-I S-1: 2 layers of Sika103C unidirectional carbon wrap with 3 in. overlap

J-I S-2: 2 layers of Sika103C unidirectional carbon wrap with 0.75 in. gap between the ends

J-II S-1: 2 layers of Sika103C unidirectional carbon wrap with no overlap and no gap

J-II S-2: 2 layers of Sika100G unidirectional glass wrap with no overlap and no gap

Only one specimen was tested per category

\subsubsection{Summary of test results}

1. The first tensile crack occurred at $\sim(4-5) \sqrt{f_{c}^{\prime}}$.

2. Split tensile strength for fully confined $\left(360^{\circ}\right)$ FRP wrapped concrete cylinders ranged from $\sim(20-28) \sqrt{f_{c}^{\prime}}$.

3. Axial strain of FRP confined concrete cylinders was $~(8-10)$ times of unconfined cylinder.

4. Hoop strain of FRP confined cylinders was $\sim 3$ times of unconfined cylinder.

\subsection{Test Setup of 2D RC Exterior Joints}

A schematic of the test setup of control and reinforced joint specimens is shown in Figures 68 and 69 , respectively. The test specimens were designed such that the ends of the column and beam members represent the inflection points of a typical building frame. The specimen was set-up in a vertical position, i.e., the longitudinal axis of the column was oriented perpendicular to the strongfloor, and the longitudinal axis of the beam was oriented parallel to the strong-floor. The column was clamped to the loading frame at $6 \mathrm{in}$. from the column top and bottom ends using steel rollers and threaded rods. This arrangement provides lateral stability for the specimen against applied beam bending load. Besides, the column base was rested on roller support to allow for column rotation.

A pre-determined axial load was applied on the column through a hydraulic actuator using a manual pump. A load cell was placed between the column top surface and actuator to measure the applied axial load. The beam bending load was applied at 6 in. from the free end of the beam through a loading plate. A load cell situated between the hydraulic actuators and the loading plates 
measured the load applied on to the beam. The actuator was pinned at the top end to allow for rotation during the test. The beam loading actuator was controlled using MTS having a maximum capacity of 110-kip. Sensors such as strain gages, and LVDTs were placed at critical locations of the test specimen to record the data.

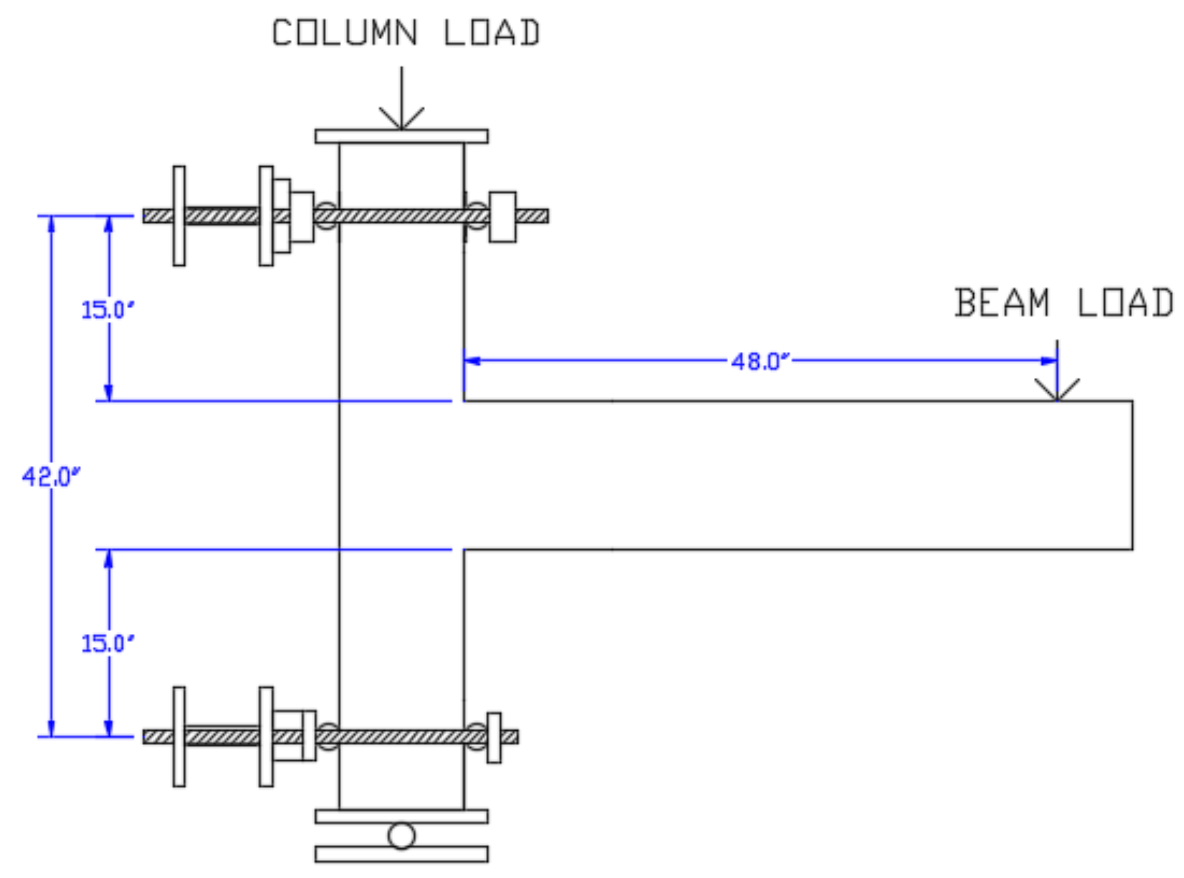

Figure 68. Test setup of control joint specimens 


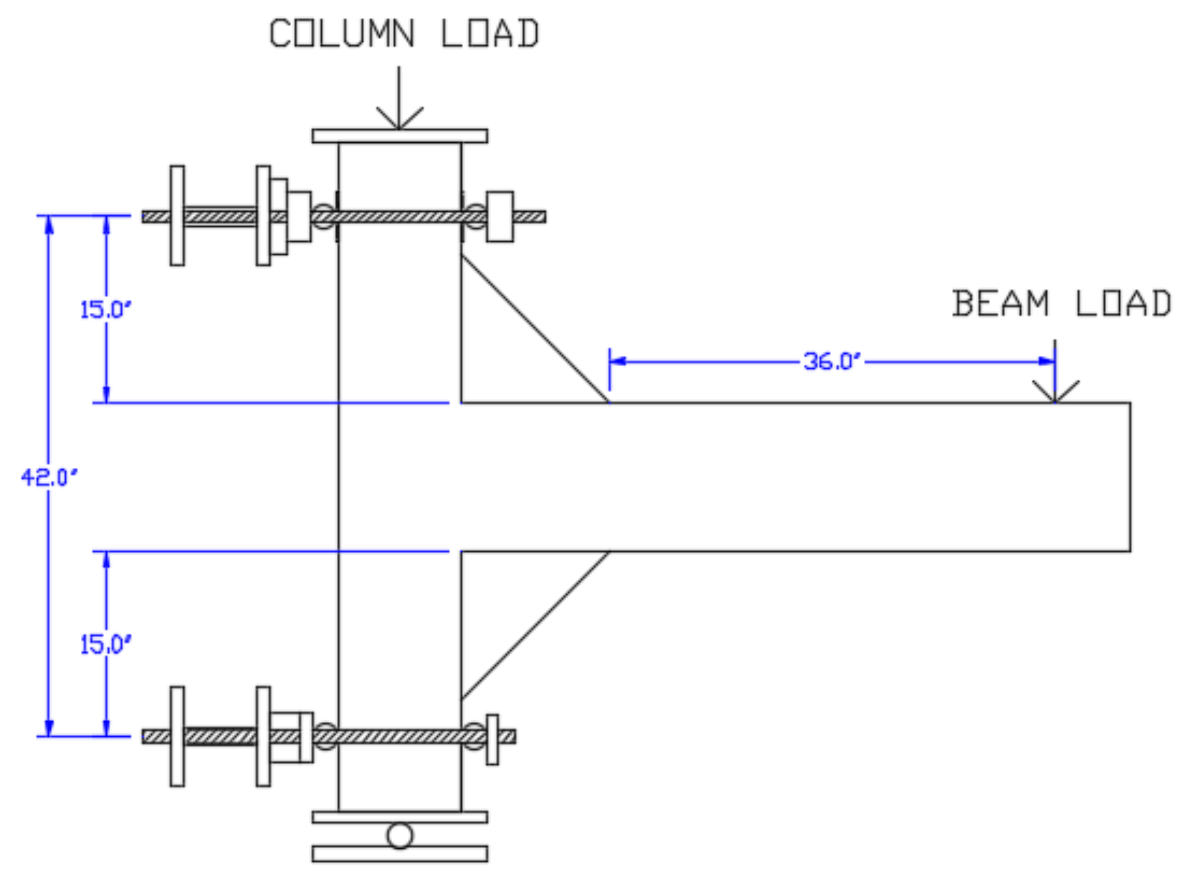

Figure 69. Test setup of reinforced joint specimens

\subsection{Loading Protocol}

Before beginning each test, the predetermined axial load was slowly applied to the column until the desired load level was achieved. Monotonic bending load was applied at six inches from the free end of the beam using a hydraulic actuator. The maximum loading capacity of the MTS controlled hydraulic actuator used in the current experimental testing was 110 kips. The desired axial compression load was maintained for the entire duration of the test by manually adjusting the actuator through a hand pump. The test specimens were loaded until a significant drop in the load-carrying capacity was observed, indicating the failure of the test specimen.

\subsection{Instrumentation}

\subsubsection{Measurement of the applied load}

Two load cells that are connected to Vishay 7000 data acquisition were placed one each on the column and beam surfaces under the actuators, to record the numerical values of applied loads. 
The load cells were calibrated before beginning the current experimental program. The axial load on the column was measured using a 200-kip load cell, while a 100-kip load cell was used to measure the beam bending load. The loads thus measured were plotted against the observed deflections (reported in Chapters 5 and 6) to compute the stiffness, ductility, and energy absorption capacities of joint test specimens.

\subsubsection{Measurement of displacement}

Linear variable displacement transducer (LVDT), shown in Figure 70, was placed at 6 in. from the free end of the beam to measure the vertical deflection under the load.

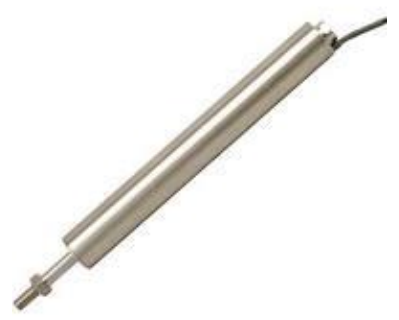

Figure 70. Linear variable displacement transducer (LVDT) (OMEGA Engineering) [58] 


\subsubsection{Measurement of strain}

\subsubsection{Strain measurement in steel rebar}

Strain gages with a resistance of $120 \Omega$ or $350 \Omega$ are bonded to the rebar surface at several critical locations of the beam, column, and joint sections of the test specimen. The critical sections were determined based on the projected yield and hinge formation locations. The strains measured at various load levels were used to evaluate the strain penetration from the beam and column sections into the joint, reported in Chapters 5 and 6 . A schematic view of the strain gage arrangement is shown in Figure 71.

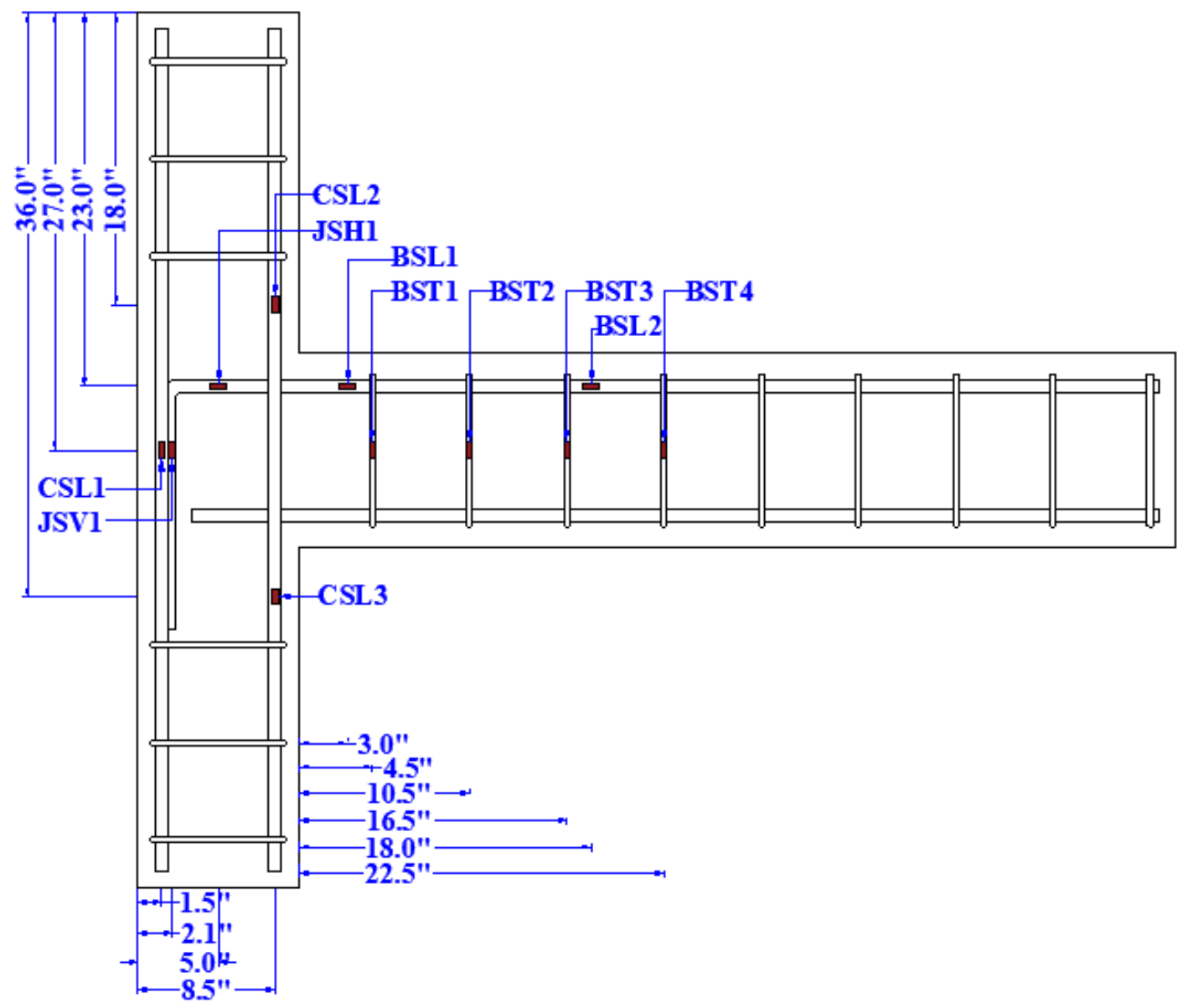

Figure 71. Schematic view of strain gage arrangement on steel rebar cage 


\subsubsection{Strain measurement on concrete surface}

As shown in Figure 72, a shear rosette was bonded to the concrete surface on the joint panel to determine the joint principal strains at first crack, rebar yielding, and peak load levels. The principal strains obtained were compared against the plain concrete cylinders tests to establish the limit states for potential shear or diagonal-tension failure modes, as reported in Chapters 5 and 6 .

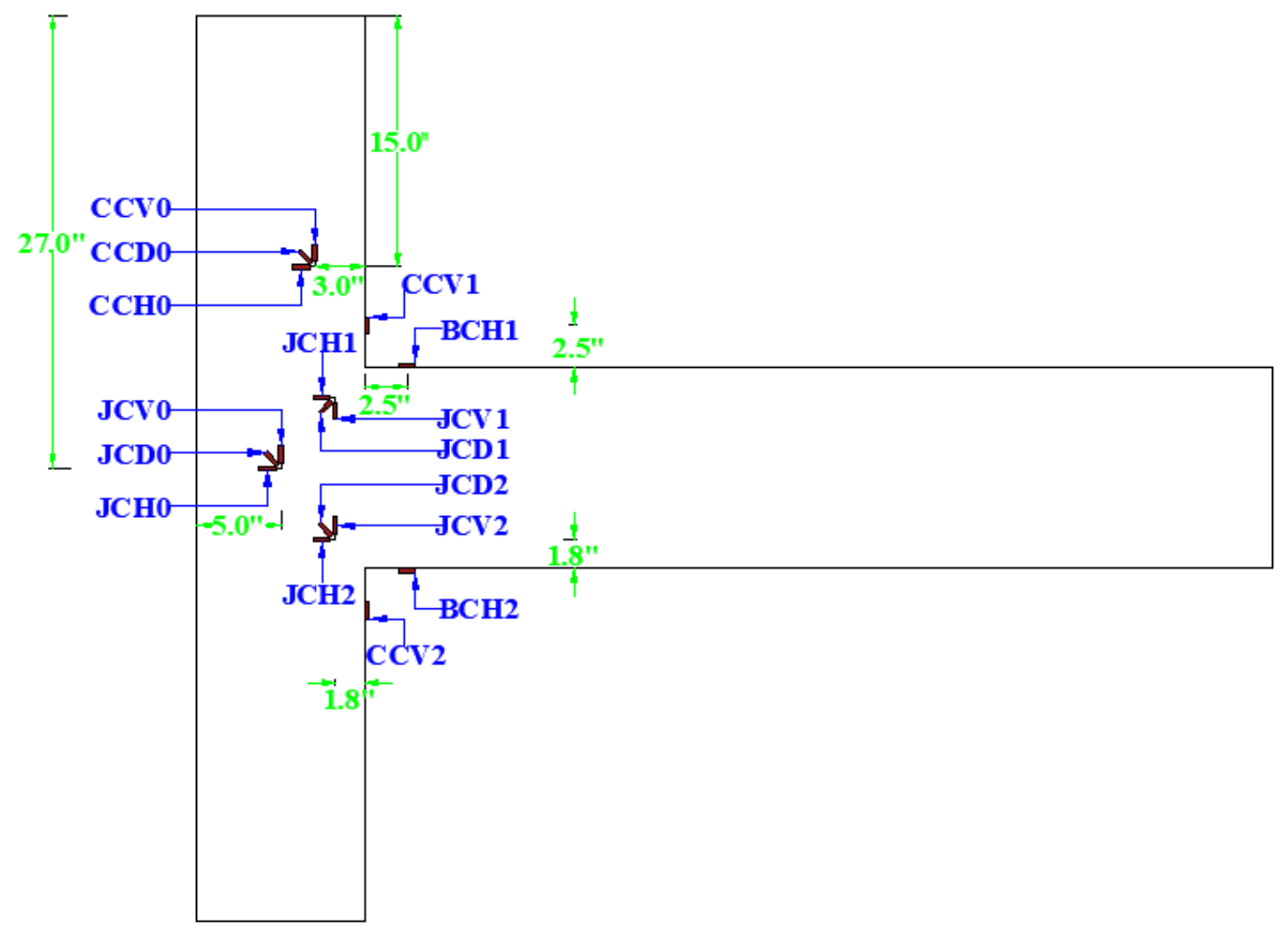

Figure 72. Strain gage arrangement on the concrete surface 


\subsubsection{Strain measurement on FRP composite wrap}

Reinforced joint specimens were extensively instrumented with strain gages on FRP composite wrap surface as shown in Figure 73. A total of twenty-six strain gages were installed to monitor the strain distribution at various load levels on beam, column and joint sections, reported in Chapters 5 and 6. Besides, shear rosette was installed on the joint panel to compute the joint principal strains at the first crack, rebar yielding, and peak load levels. Furthermore, the strain levels on the FRP wrap surface will establish the failure modes such as local de-bond, buckling or rupture of FRP composite wrap.

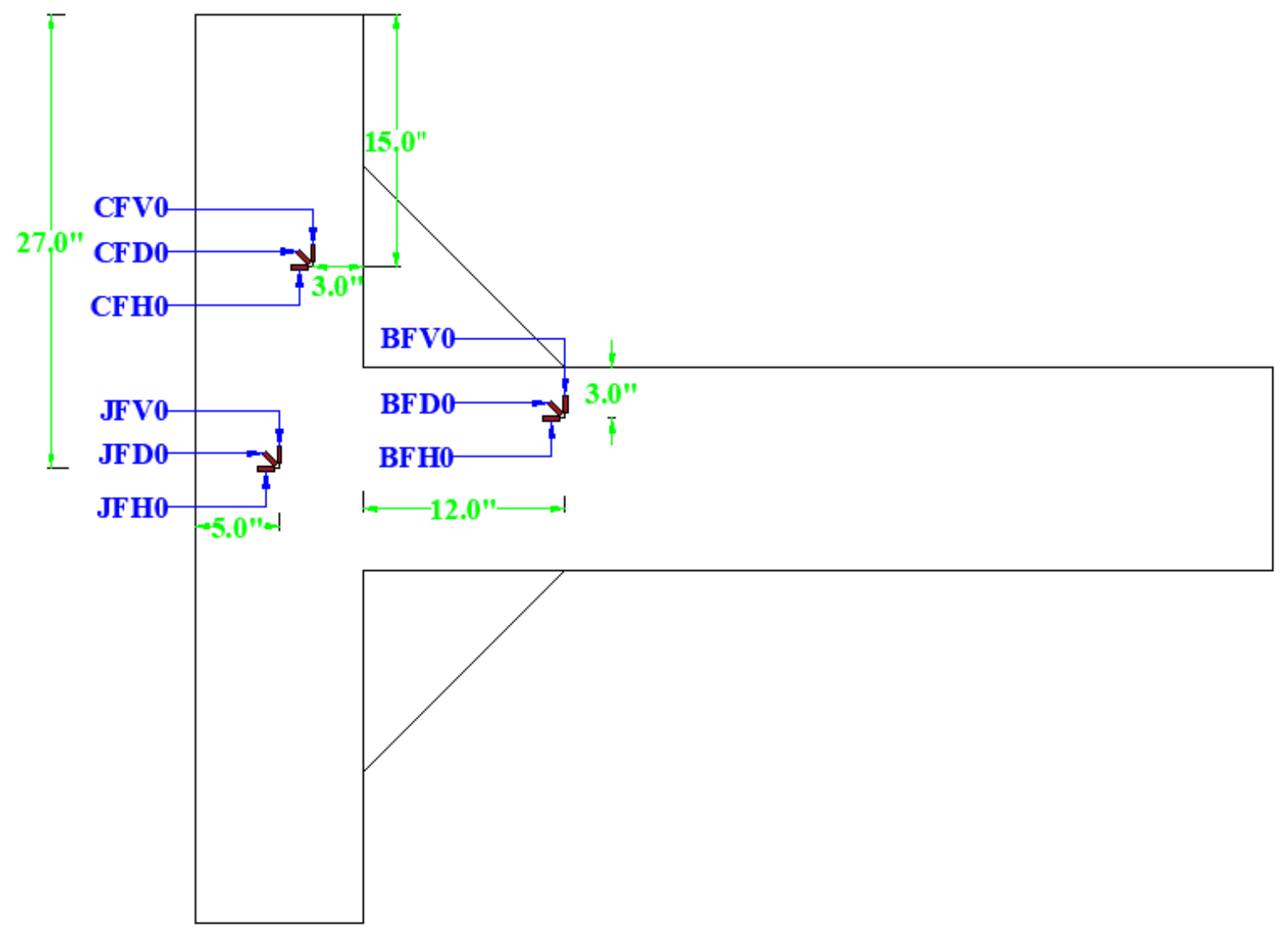

Figure 73. Strain gage arrangement on FRP wrap surface 


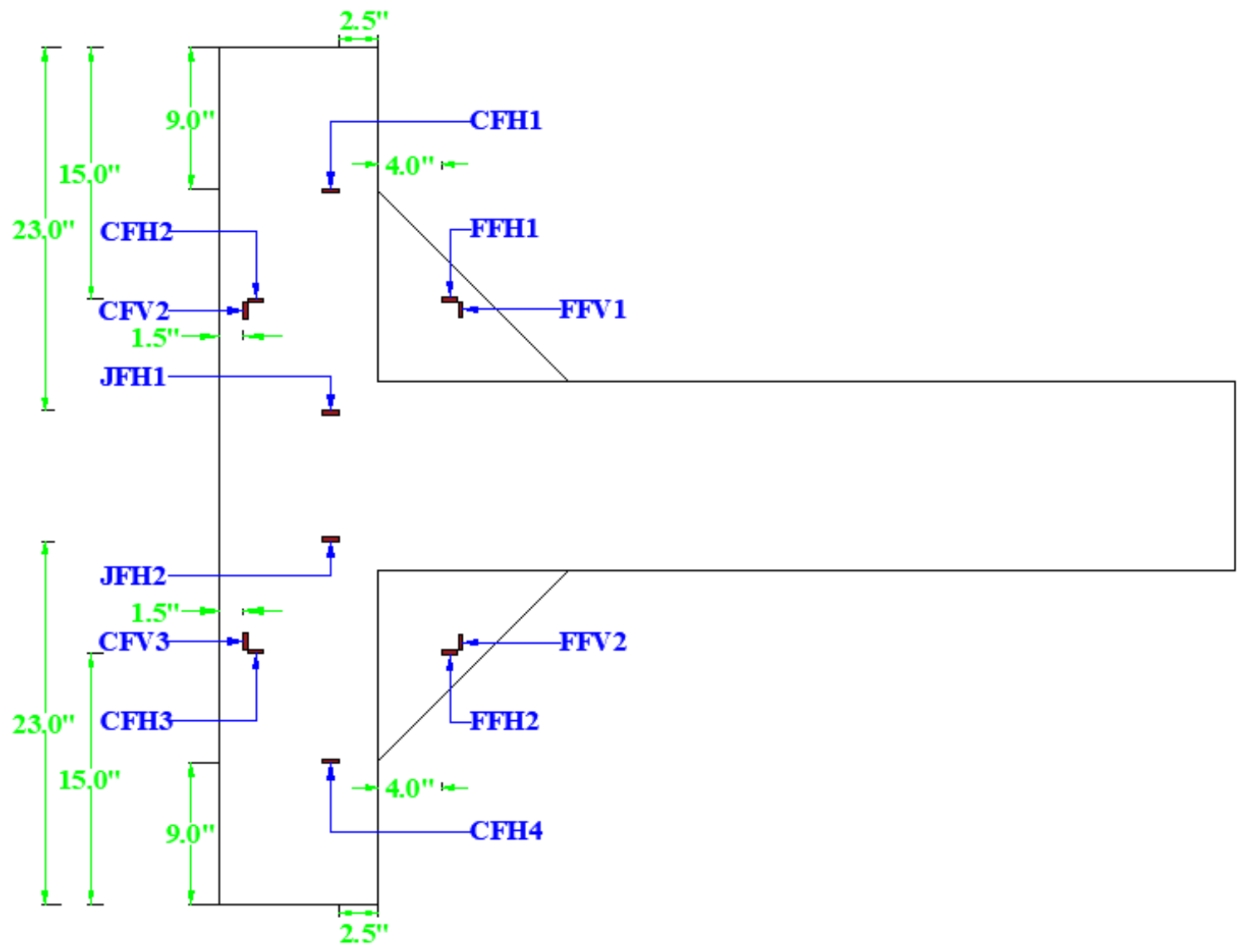

Figure 74. Strain gage arrangement on FRP wrap surface 


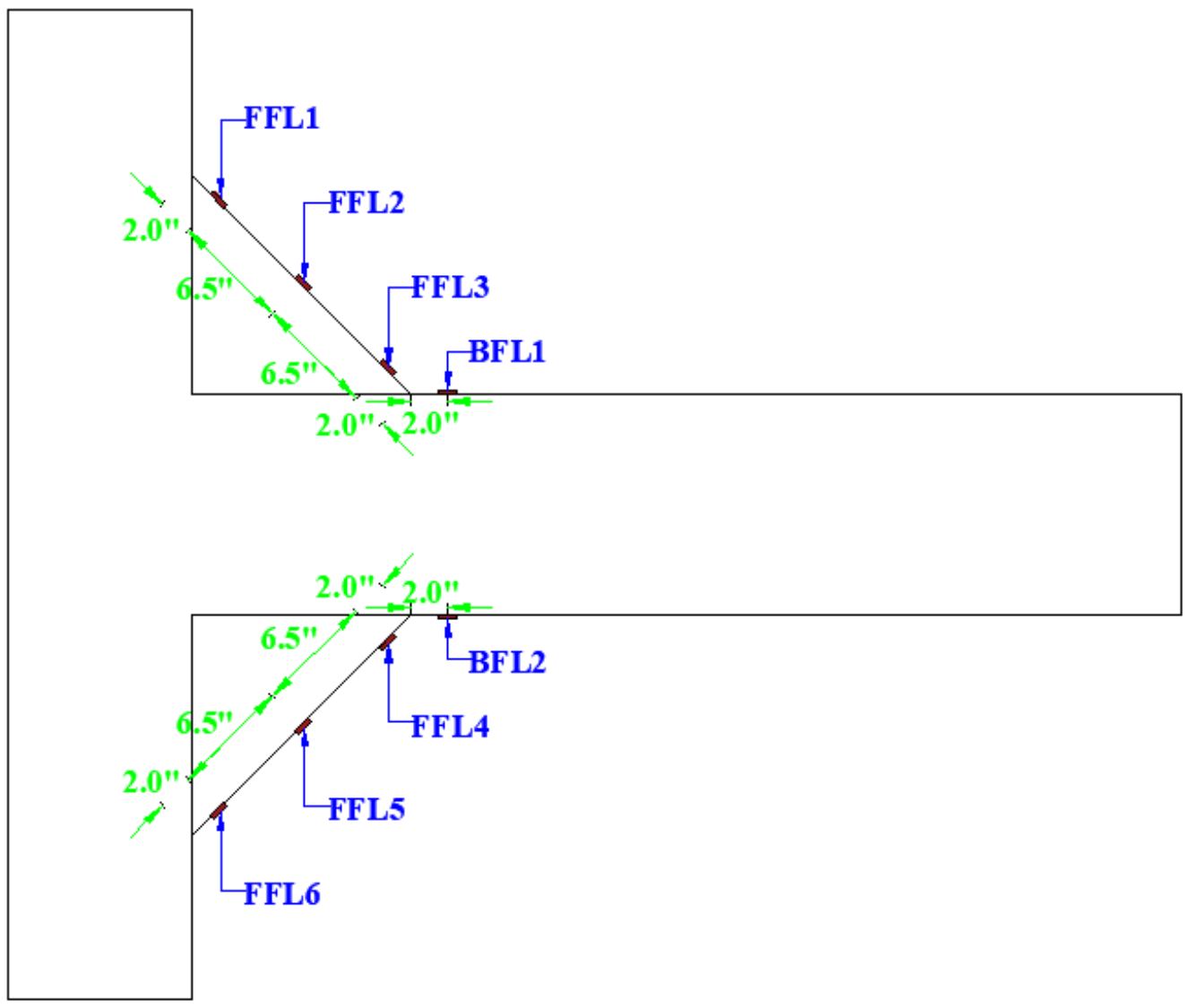

Figure 75. Strain gage arrangement on FRP wrap surface 


\subsection{Data Collection}

\subsubsection{Data acquisition system}

The test data from various measurement sensors, such as strain gages, load cells, and LVDTs, were collected through Vishay 7000 data acquisition system (shown in Figure 76) and processed using Strain Smart Software. The data thus processed is reduced and imported to a Microsoft Excel worksheet for further evaluations.

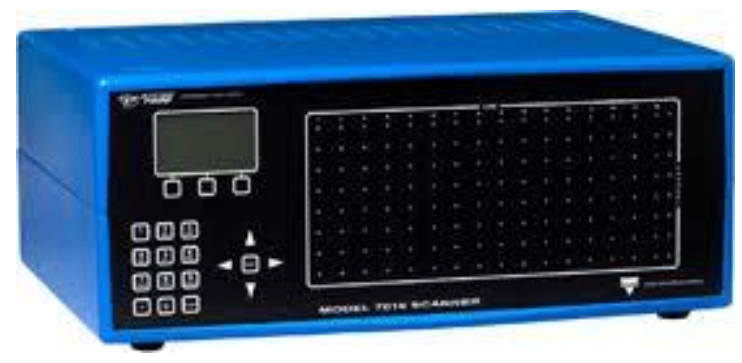

Figure 76. Vishay 7000 data acquisition system [59]

\subsection{Computation of Principal Strains}

Based on the strain data generated through the shear rosette (Figure 77), the numerical values of principal strain and the orientation of principal plane for each joint test specimen, as reported in Chapters 5 and 6, were computed using Equations 4.1 and 4.2. 


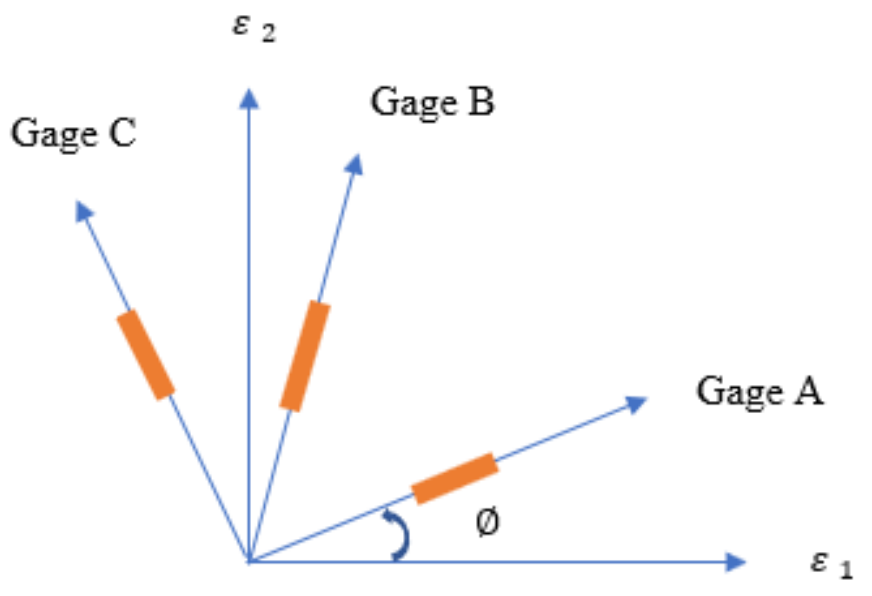

Figure 77. Shear rosette arrangement

$$
\begin{array}{r}
\varepsilon_{1,2}=\frac{\varepsilon_{A}+\varepsilon_{C}}{2} \pm \frac{1}{\sqrt{2}} \sqrt{\left(\varepsilon_{A}-\varepsilon_{B}\right)^{2}+\left(\varepsilon_{B}-\varepsilon_{C}\right)^{2}} \\
\varnothing=\frac{1}{2} \tan ^{-1}\left(\frac{\varepsilon_{A}-2 \varepsilon_{B}+\varepsilon_{C}}{\varepsilon_{A}-\varepsilon_{C}}\right)
\end{array}
$$

\subsection{Computation of Shear Strain}

Using the strain data generated through shear rosette, the joint shear strain for each joint specimen was computed using Equation 4.3.

$$
\gamma_{x y}=2 \varepsilon_{B}-\varepsilon_{A}-\varepsilon_{C}
$$

\subsection{Summary}

This chapter provided the details of the experimental program on 2D exterior joint specimens tested under monotonic loads. As identified in previous sections, all the test specimens were instrumented appropriately to capture the global, as well as local behavior. The experimental data collected through LVDTs, load cells, and strain gages were evaluated meticulously to study the improvements in joint behavior with filler-modules and FRP wraps or gussets, as reported in Chapters 5 and 6. 


\section{Behavior of RC Joints Reinforced with Concrete/Syntactic Foam Filler-Modules and FRP Wraps/Gussets}

\subsection{Introduction}

This chapter deals with the structural response evaluation of $\mathrm{RC}$ beam-column joints reinforced with concrete/syntactic foam filler-modules with/out fiber-reinforced polymer (FRP) composite wraps or gussets under monotonic loads. The joints without filler-modules are identified as control specimens, herein. The joint testing has been conducted in three phases to evaluate the influence of various parameters, as listed in Table 27:

(i) Phase-I: joints reinforced with concrete/syntactic foam filler-modules with/out CFRP composite wraps,

(ii) Phase II: joints reinforced with concrete filler-modules and CFRP gussets, and

(iii) Phase III: joints reinforced with concrete filler-modules and GFRP composite wraps.

When conceptualizing the reinforcing schemes using filler-modules and FRP wraps for joint specimens, it was projected that the joint load versus strain response could be classified into three distinct zones, i.e., initiation of filler-module cracking in the tension zone - refers to point A, transition of tensile forces to steel rebar cage after significant concrete cracking - refers to point B, and peak load the specimen can resist before complete collapse - refers to point $\mathrm{C}$. Based on the above hypothesis, the influence of the above parameters are studied by comparing the joint principal- and shear- strain responses at three salient points: A (onset of filler-module cracking), B (idealized yield - defined as the point beyond which a truss mechanism primarily resists the forces through internal rebar cage, FRP wrap, and rebar-concrete bond forces), and $\mathrm{C}$ (peak load - referred to as a highest numerical value of load recorded during the testing). Points A, B, and C were identified based on the load vs. strain response of the column rebar (CSL2, as identified in Figure 78). Based on the load vs. column rebar strain plot, "A" refers to the endpoint of the second slope change, and " $\mathrm{B}$ " refers to the endpoint of the third slope change. Point $\mathrm{C}$ is the peak load measured during laboratory testing. Figure 78 shows a generic load vs. column rebar strain of joint specimen reinforced with filler-modules and FRP wraps 
or gussets. Besides, one specimen from each group is tested as a control specimen to establish the baseline value for computation of structural capacity enhancements in joints reinforced with filler-modules with/out FRP wraps or gussets. The joint specimens were constructed, tested, and evaluated per the procedures described in Chapter 4 of this manuscript. The experimental results monitored through load cells, LVDTs, and strain gages and recorded using MTS and StrainSmart ${ }^{\circledR}$ data acquisition systems are presented and discussed in the following sections. Addition test data on load versus strain response of joint specimens is presented in Appendices A through D. Furthermore, discussion of experimental results in terms of strength, stiffness, ductility, and energy dissipation capacity enhancements is provided in sections 5.3.

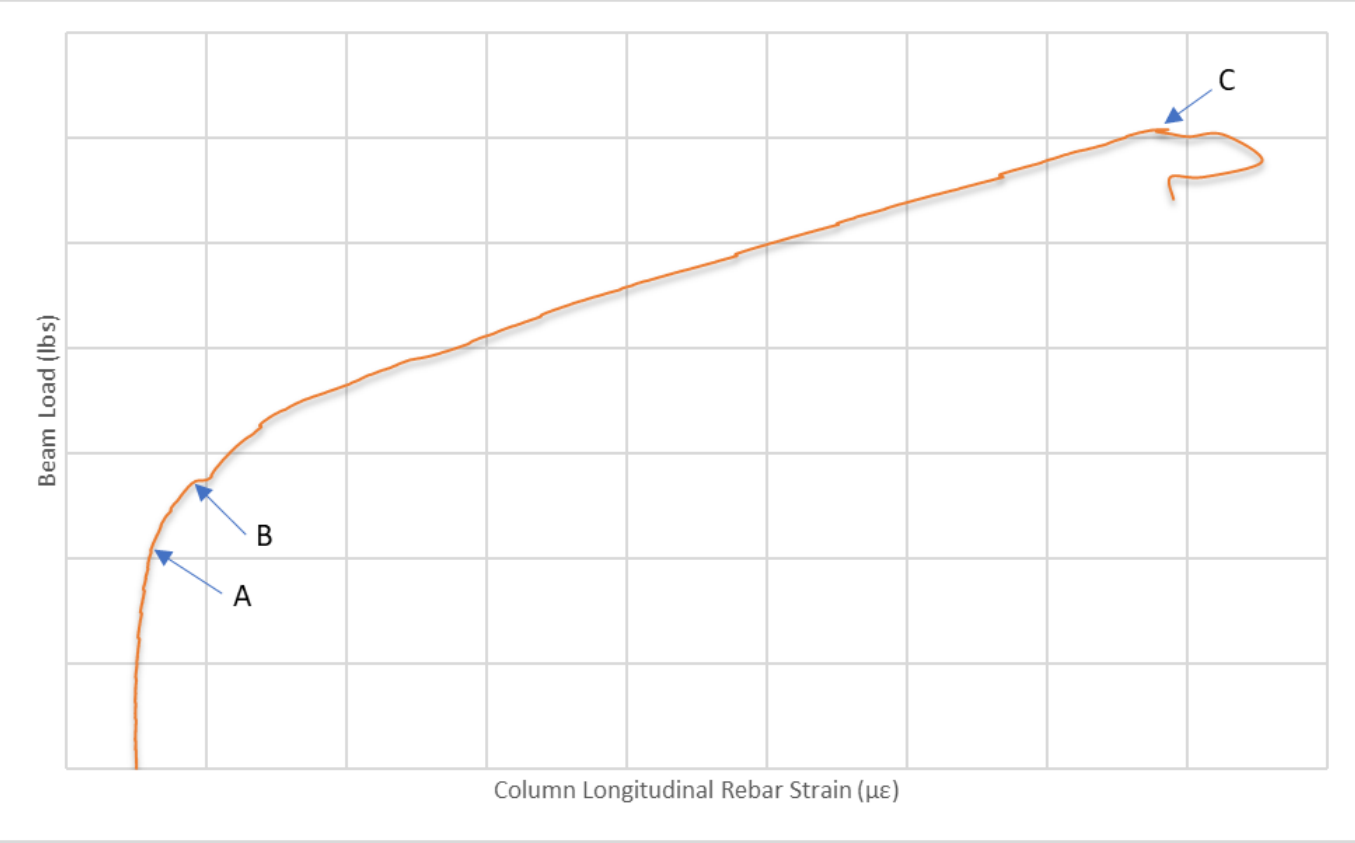

Figure 78. Load vs. column rebar strain response of a typical joint specimen in this study

Note:

1. The strains induced due to the axial load on the column were zeroed (before the application of beam bending load) and presented from the start of beam bending load, except for the specimens exhibiting compression failure. Based on the previous studies by [60], the influence of column axial load on the joint shear strength was found to be negligible and hence was not 
reported. However, in the case of specimens exhibiting compression failure mode, both results (with/out the influence of axial load) are presented.

2. Points $\mathrm{A}, \mathrm{B}$, and $\mathrm{C}$ were consistently identified with respect to the column rebar strain gage (CSL2) closer to the joint location. Based on the load vs. column rebar strain plot, "A" refers to the endpoint of the second slope change, and "B" refers to the endpoint of the third slope change. Point $\mathrm{C}$ is the peak load measured during laboratory testing.

Table 27. Parameters tested to evaluate the performance of joints reinforced with concrete/syntactic foam filler-modules with and without FRP wraps/gussets

\begin{tabular}{|c|c|c|c|c|}
\hline Phase \# & Joint & $\boldsymbol{f}_{c}^{\prime}$ & $\mathbf{C L}$ & Reinforcing scheme \\
\hline \multirow{4}{*}{$\underset{\mathscr{\Xi}}{\tilde{\Xi}}$} & JI-1 & \multirow{4}{*}{$\begin{array}{l}\bar{n} \\
\text { aे } \\
\text { ָे } \\
\text { dे }\end{array}$} & \multirow{4}{*}{ 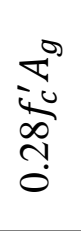 } & Control \\
\hline & JI-2 & & & $\mathrm{CW}(3.3 \mathrm{ksi})+\mathrm{D}$ \\
\hline & JI-3 & & & $\mathrm{CW}(3.3 \mathrm{ksi})+\mathrm{D}+\mathrm{CFRP}$ Wrap+360 -anchor \\
\hline & JI-4 & & & $\mathrm{SF}(4.3 \mathrm{ksi})+\mathrm{D}+\mathrm{CFRP}$ Wrap+360 ${ }^{\circ}$-anchor \\
\hline \multirow{4}{*}{ 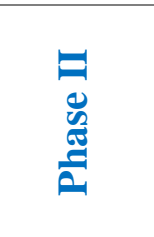 } & JI-5 & \multirow{4}{*}{$\begin{array}{l}\overline{\tilde{n}} \\
\text { aे } \\
\text { బે } \\
\text { dे }\end{array}$} & \multirow{4}{*}{$\underset{\infty}{\infty}$} & Control \\
\hline & JI-6 & & & $\mathrm{CW}(5.5 \mathrm{ksi})+\mathrm{D}+\mathrm{CFRP}$ Gusset+U-anchor \\
\hline & JI-7 & & & CW(5.5 ksi)+D+CFRP Gusset+U-anchor* \\
\hline & JI-8 & & & CW(9.5 ksi)+D+CFRP Gusset+U-anchor* \\
\hline \multirow{4}{*}{ 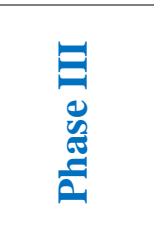 } & JIII-1 & \multirow{4}{*}{ 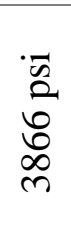 } & \multirow{4}{*}{$\underset{\infty}{\infty}$} & Control \\
\hline & JIII-2 & & & CC(5.5 ksi)+D+GFRP Wrap+no anchor* \\
\hline & JIII-3 & & & CW(5.5 ksi)+D+GFRP Wrap+no anchor* \\
\hline & JIII-4 & & & CW(5.5 ksi)+D+GFRP Wrap+U-anchor* \\
\hline
\end{tabular}

D: dowels; CW: concrete wedge; CC: concrete curve; SW: syntactic foam wedge; CL: column axial load; $f_{c}^{\prime}$ : substrate concrete compressive strength; $A_{g}$ : gross cross-sectional area of concrete (column); *CFRP layer bonded between filler-module and substrate beam and column that receive filler-modules at the reentrant corners. 


\subsection{Experimental Results}

This section describes the structural response of joint specimens tested in control and reinforced (joints with filler-modules with/out FRP wraps/gussets) conditions.

\subsubsection{Behavior of control specimens (JI-1, JI-5, and JIII-1)}

Three joint specimens (JI-1, JI-5, and JIII-1) were tested under control conditions to assess their performance under monotonic loading. The parameters varied herein include concrete compressive strength (2639 vs. $3866 \mathrm{psi}$ ) and induced column axial load (0.18 vs. $0.28 \%)$. A span-to-depth (a/d) ratio of 4.8 was adopted to ensure the diagonal tension failure of joints.

As the beam loading was applied, all control specimens exhibited corner cracking at the interface of a beam-column joint. The observed corner cracks are referred to as typical flexural-tension $\left(f_{r}\right)$ cracks associated with stress-concentrations effects. The strains measured on the surface of beam longitudinal rebar, close ( 2.5 in) to the interface of the beam-column joint, are in the range of $91-143 \mu \varepsilon$. Assuming a linear strain variation along the depth of the beam, the strains on the concrete surface were computed in the range of $137-214 \mu \varepsilon$. Translation of these strains as a function of tensile stresses $\left(k \sqrt{f_{c}^{\prime}}\right)$ revealed $k$ values in the range of $8-12$, which are typical numbers reported in [61]. A summary of the observed strains and computations corresponding to the corner cracking (modulus of rupture) for each control specimen is presented in Table 28. These corner cracks observed during the initial stages of testing did not progress beyond the concrete clear cover depth as they were restrained by the dowel action of beam longitudinal rebar. As the loading increased beyond $f_{r}$, diagonal tension cracks originated within the joint panel. Figure 79 shows a typical corner- and initial diagonal tension- cracking observed in all control test specimens. The joint principal tensile- and shear- strains at the formation of diagonal cracking were measured as $53-70 \mu \varepsilon$ and $86-147 \mu \varepsilon$, respectively. Tables 29 and 30 present the summary of principal- and shear- strains for control specimens without and with the influence of column axial loads, respectively. Figures 83 through 88 show the load vs. principal- and shear- strain plots of control joint specimens. 
Table 28. Modulus of rupture for control joints (JI-1, JI-5, JIII-1)

Joint Beam load (lb) Beam rebar strain $(\mu \varepsilon) \quad$ Concrete strain $(\mu \varepsilon) \quad k-f_{r}$

\begin{tabular}{ccccc}
\hline \multirow{2}{*}{ JI-1 } & 2,475 & 106 & 158 & 9 \\
\cline { 2 - 5 } & 2,889 & 143 & 214 & 12 \\
\hline \multirow{2}{*}{ JI-5 } & 1,625 & 92 & 138 & 8 \\
\cline { 2 - 5 } & 2,035 & 141 & 212 & 12 \\
\hline \multirow{2}{*}{ JIII-1 } & 2,014 & 91 & 137 & 8 \\
\cline { 2 - 5 } & 4,016 & 119 & 178 & 10 \\
\hline
\end{tabular}

Table 29. Principal strain limit state for control joints without axial load influence

\begin{tabular}{|c|c|c|c|c|c|c|c|}
\hline \multirow{2}{*}{ Joint } & \multirow{2}{*}{ PoI } & \multirow{2}{*}{ Beam load (lb) } & \multicolumn{3}{|c|}{ Joint principal strain $(\mu \varepsilon)$} & \multicolumn{2}{|c|}{ Joint limit state } \\
\hline & & & $\mathbf{P}(\mathbf{t})$ & $\mathbf{P}(\mathbf{c})$ & $\gamma$ & $k-P(t)$ & $\mathbf{k}-\gamma$ \\
\hline \multirow{3}{*}{ JI-1 } & A & 5,063 & 53 & -94 & -147 & 3.0 & 3.5 \\
\hline & B & 11,076 & 43 & -261 & -266 & 2.4 & 6.4 \\
\hline & $\mathrm{C}$ & 15,471 & Off-scale & Off-scale & Off-scale & N.A. & N.A. \\
\hline \multirow{3}{*}{ JII-5 } & A & 2,183 & 57 & -39 & 86 & 3.3 & 2.1 \\
\hline & B & 10,886 & Off-scale & Off-scale & Off-scale & N.A. & N.A. \\
\hline & $\mathrm{C}$ & 18,819 & Off-scale & Off-scale & Off-scale & N.A. & N.A. \\
\hline \multirow{3}{*}{ JIII-1 } & A & 7,076 & 70 & -16 & 86 & 4.0 & 2.1 \\
\hline & $\mathrm{B}$ & 15,658 & 91 & -142 & 72 & 5.2 & 1.8 \\
\hline & $\mathrm{C}$ & 24,740 & Off-scale & Off-scale & Off-scale & N.A. & N.A. \\
\hline
\end{tabular}

PoI: Point of interest; $\mathrm{P}(\mathrm{t})$ : principal tensile strain; $\mathrm{P}(\mathrm{c})$ : principal compressive strain; $\gamma$ : shear strain

Table 30. Principal strain limit state for control joints with axial load influence

\begin{tabular}{cccccccc}
\hline \multirow{2}{*}{ Joint } & \multirow{2}{*}{ PoI } & \multirow{2}{*}{ Beam load $(\mathbf{l b})$} & \multicolumn{2}{c}{ Joint principal strain $(\mu \varepsilon)$} & \multicolumn{2}{c}{ Joint limit state } \\
\cline { 3 - 8 } & & & $\mathbf{P}(\mathbf{t})$ & $\mathbf{P}(\mathbf{c})$ & $\gamma$ & $\mathbf{k}-\mathbf{P}(\mathbf{t})$ & $\mathbf{k}-\gamma$ \\
\hline \multirow{2}{*}{ JI-1 } & $\mathrm{A}$ & 5,063 & 29.18 & -160.48 & -123.08 & 3.01 & 3.54 \\
\cline { 2 - 8 } & $\mathrm{B}$ & 11,076 & 36.87 & -344.98 & -242.33 & 2.44 & 6.42 \\
\hline \multirow{2}{*}{ JIII-1 } & A & 7,076 & 79.70 & -101.40 & 113.73 & 4.54 & 2.75 \\
\cline { 2 - 8 } & B & 15,658 & -0.28 & -126.95 & 100.27 & -0.02 & 2.42 \\
\hline
\end{tabular}

PoI: Point of interest; $\mathrm{P}(\mathrm{t})$ : principal tensile strain; $\mathrm{P}(\mathrm{c})$ : principal compressive strain; $\gamma$ : shear strain 


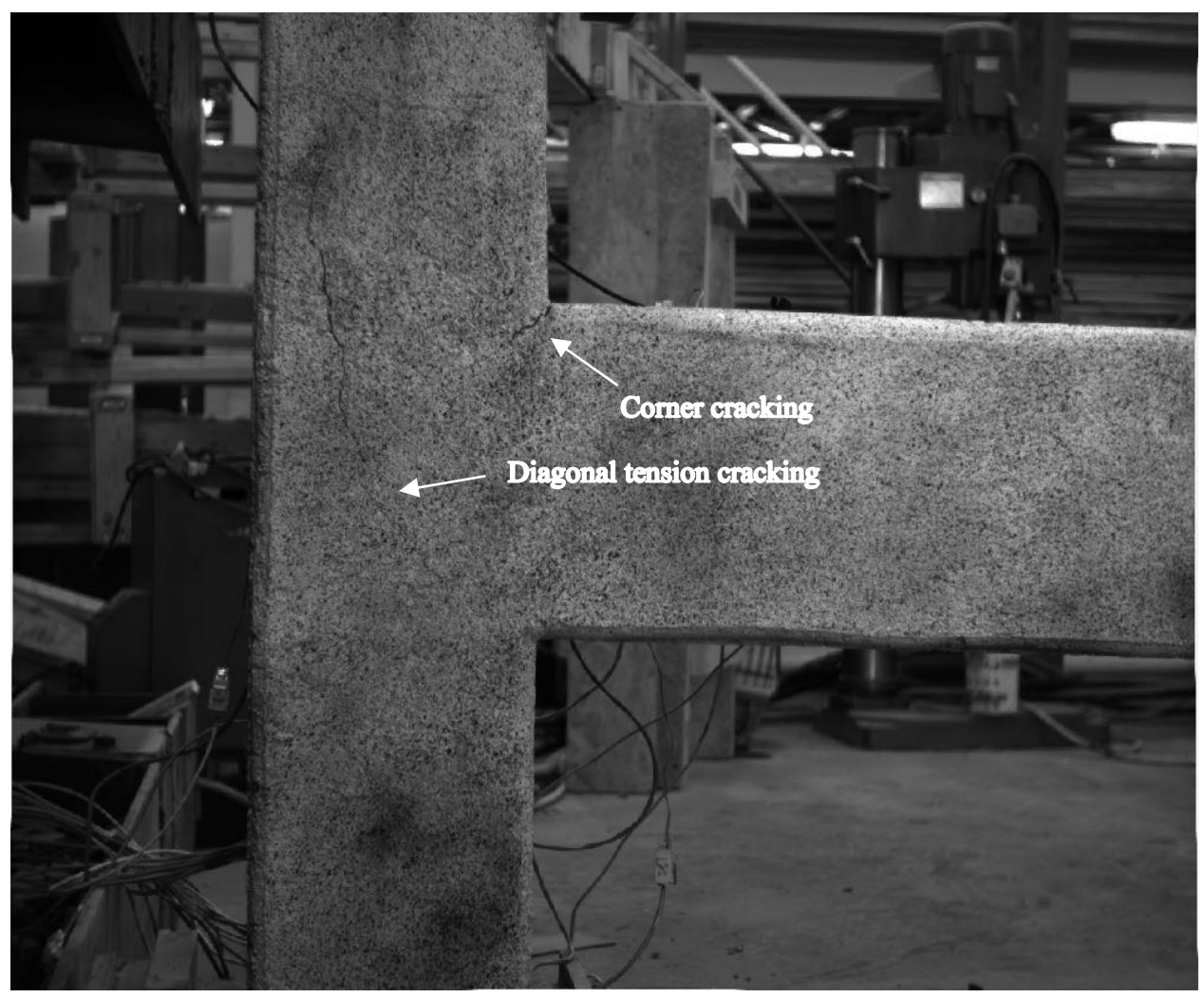

Figure 79. Initial diagonal tension cracking in the joint panel

Due to the sufficient anchorage length $\left(90^{\circ}\right.$ hook) provided for the beam longitudinal rebar extending into the joint panel, the tensile forces induced in rebar by the initial diagonal tension cracking did not result in bond failure or debond of rebar from concrete (at this stage); thus forming a concrete strut to resist additional joint shear forces. Any additional loading applied on the beam beyond this point was primarily resisted by the concrete strut and rebar-concrete bond forces. Upon bond failure, the additional shear forces induced in the joint panel were primarily resisted by concrete strut mechanism, shown in Figure 11, until it is significantly weakened by the concrete tensile strain. Ultimately, all control specimens failed in joint shear mode (Figure 82) followed by concrete cover delamination, except for specimen JI-1. Specimen JI-1 exhibited diagonal compression failure by crushing of concrete in the joint panel due to the presence of high axial compressive force $\left(0.28 f_{c}^{\prime} A_{g}\right)$ on the column. Figures 80 through 82 show the failure modes of control specimens tested under monotonic loading. 


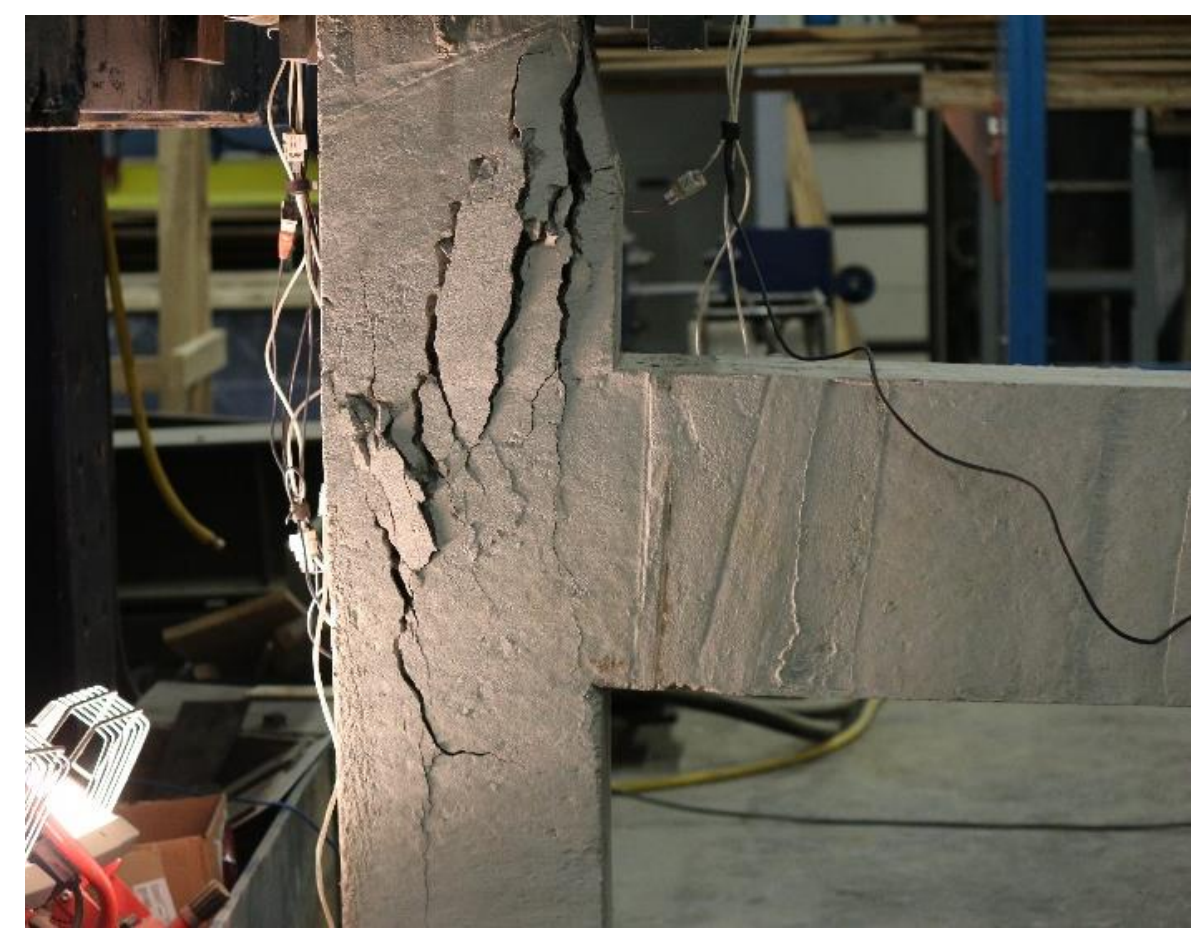

Figure 80. Failure of JI-1

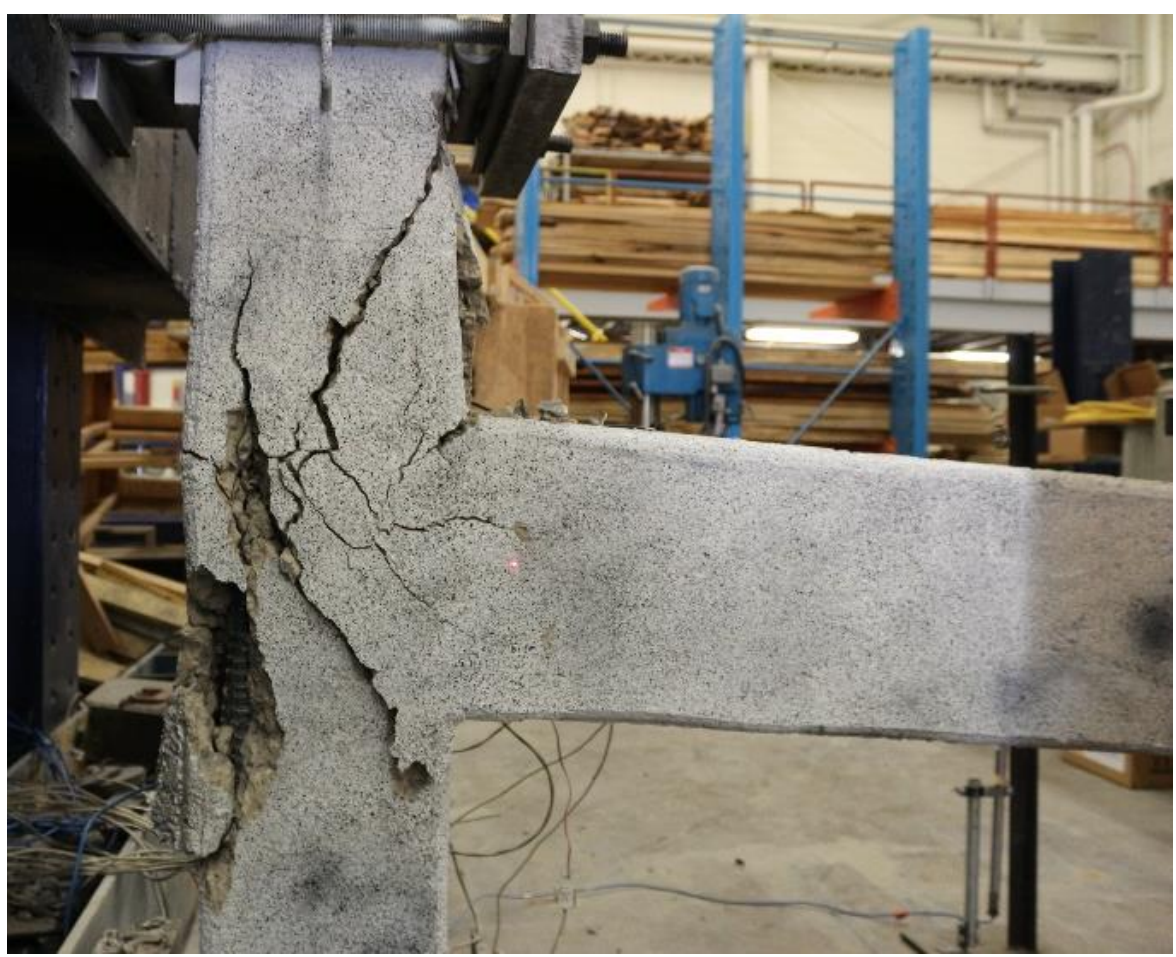

Figure 81. Failure of JI-5 


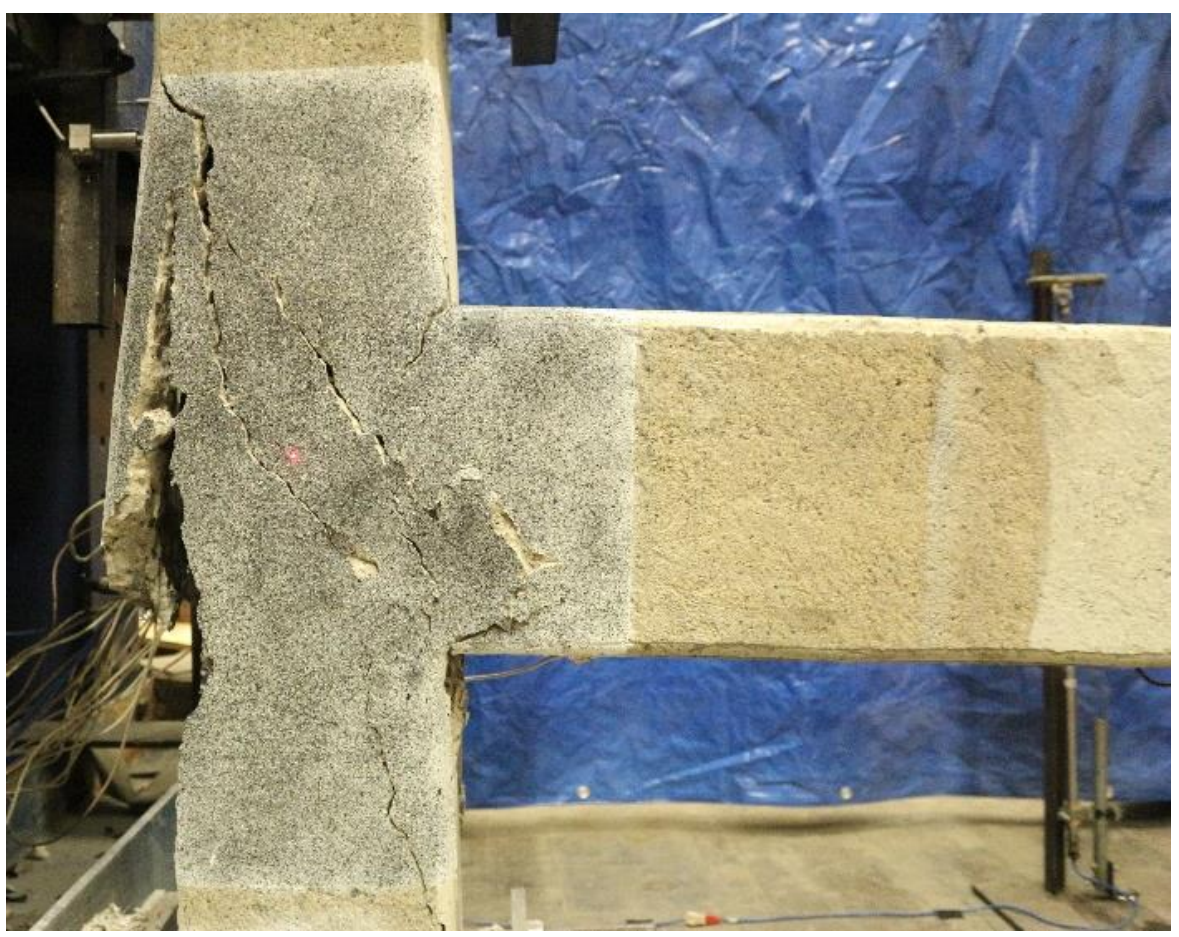

Figure 82. Failure of JIII-1 


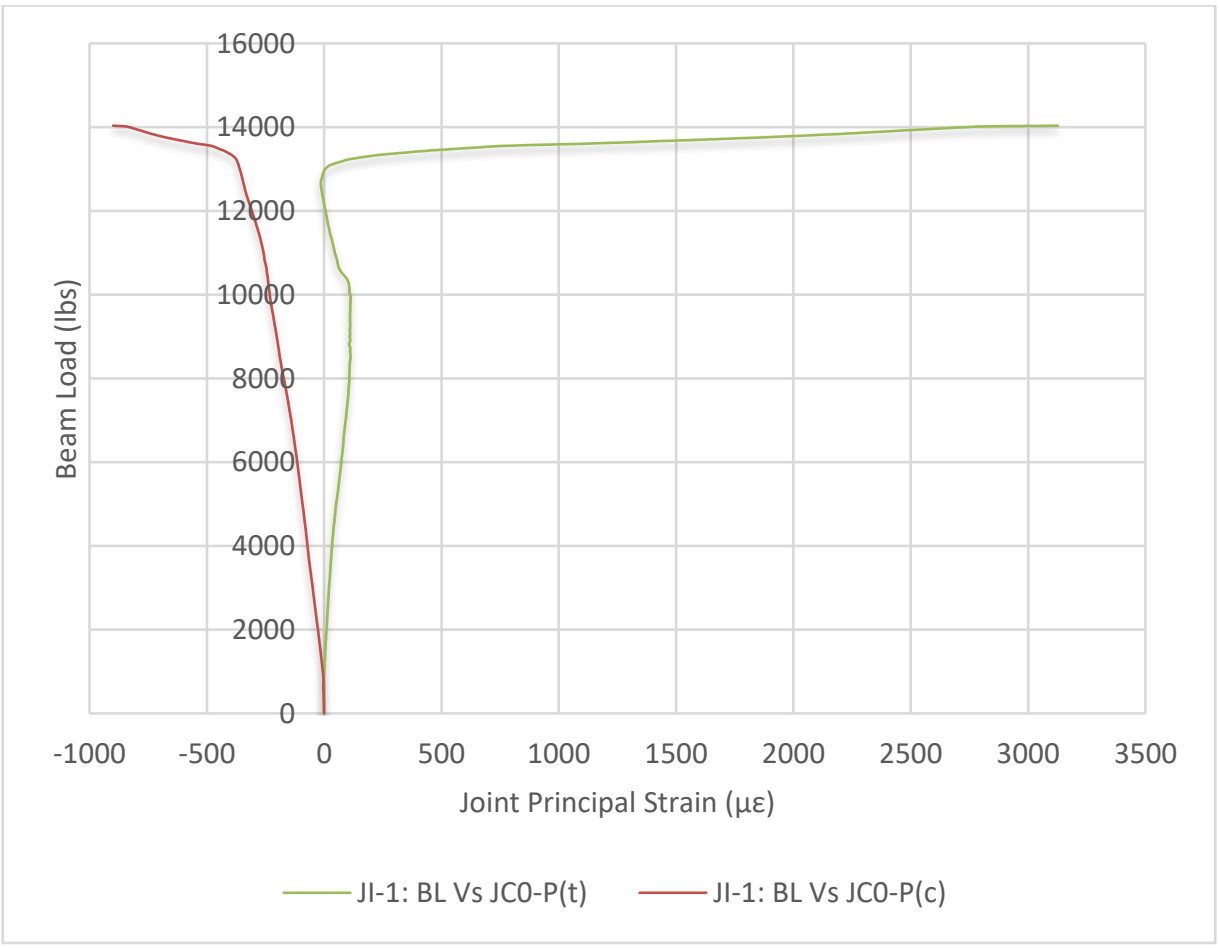

Figure 83. Joint principal strains for JI-1

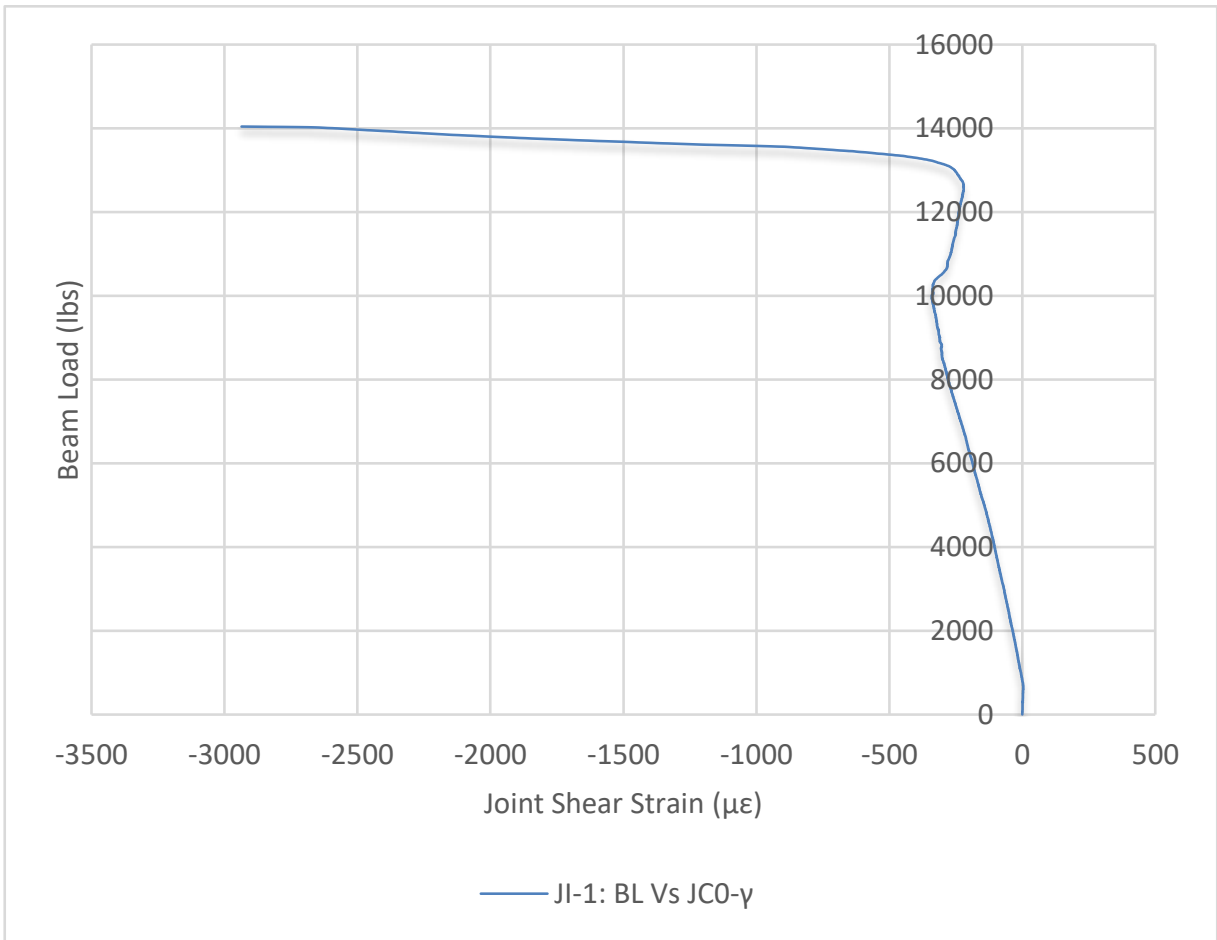

Figure 84. Joint shear strain for JI-1 


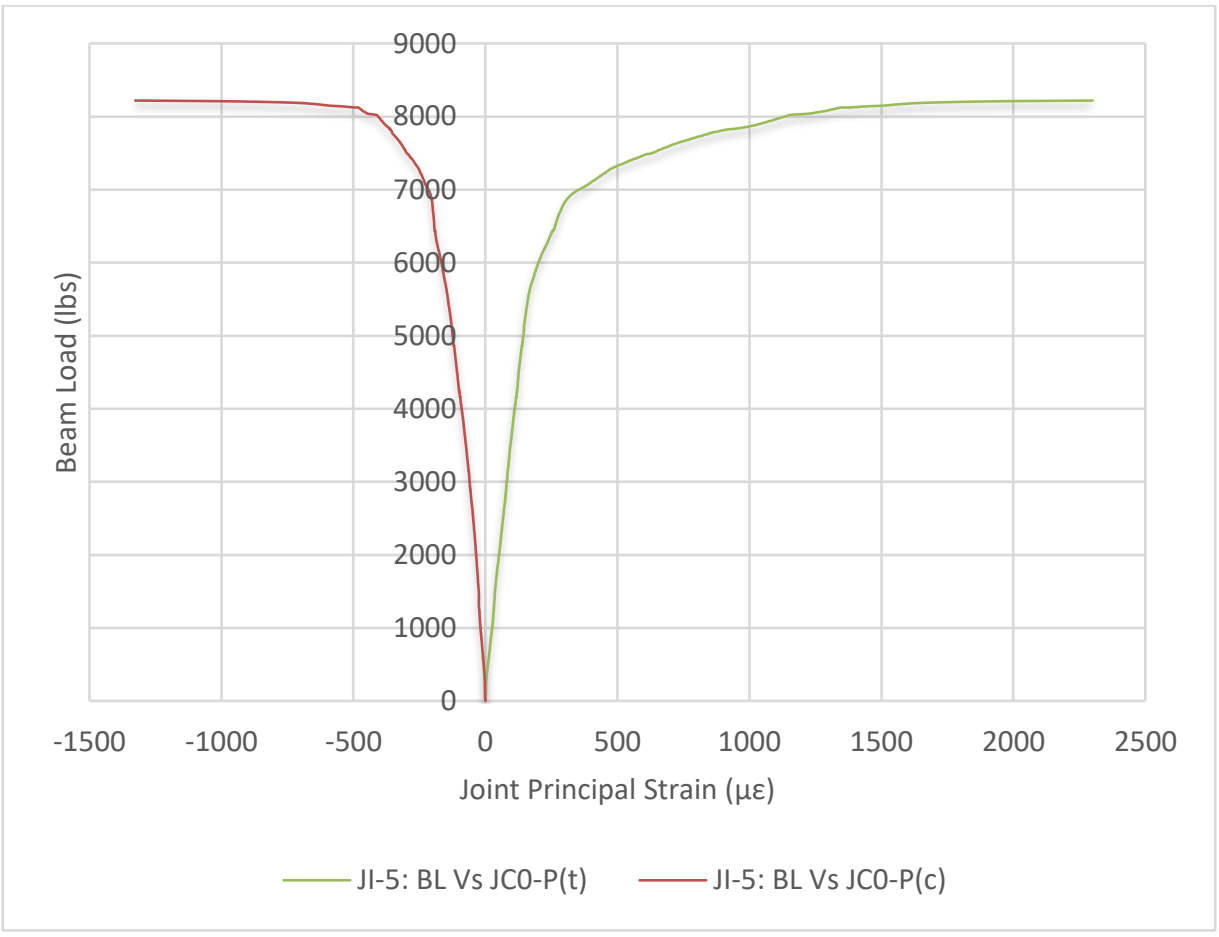

Figure 85. Joint principal strains for JI-5

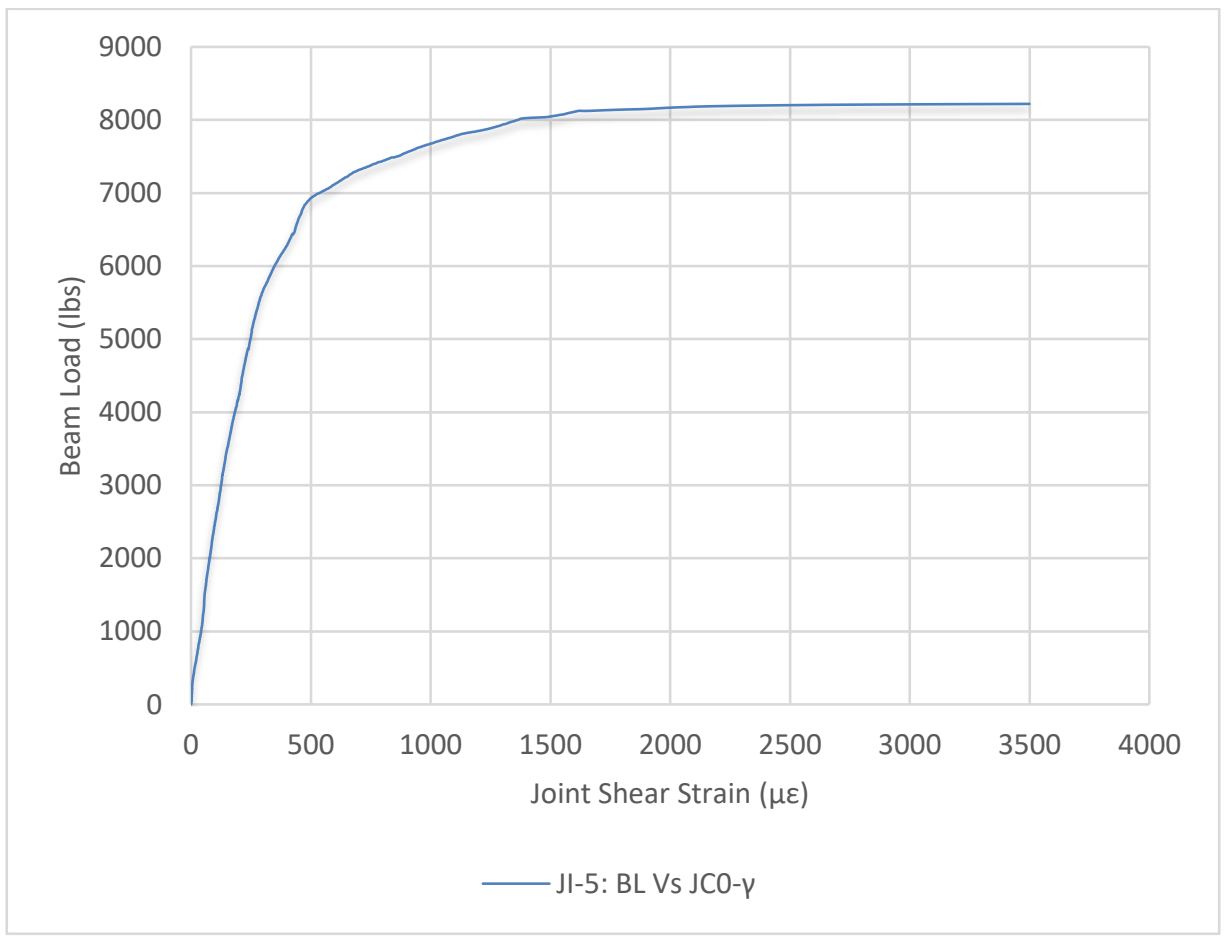

Figure 86. Joint shear strain for JI-5 


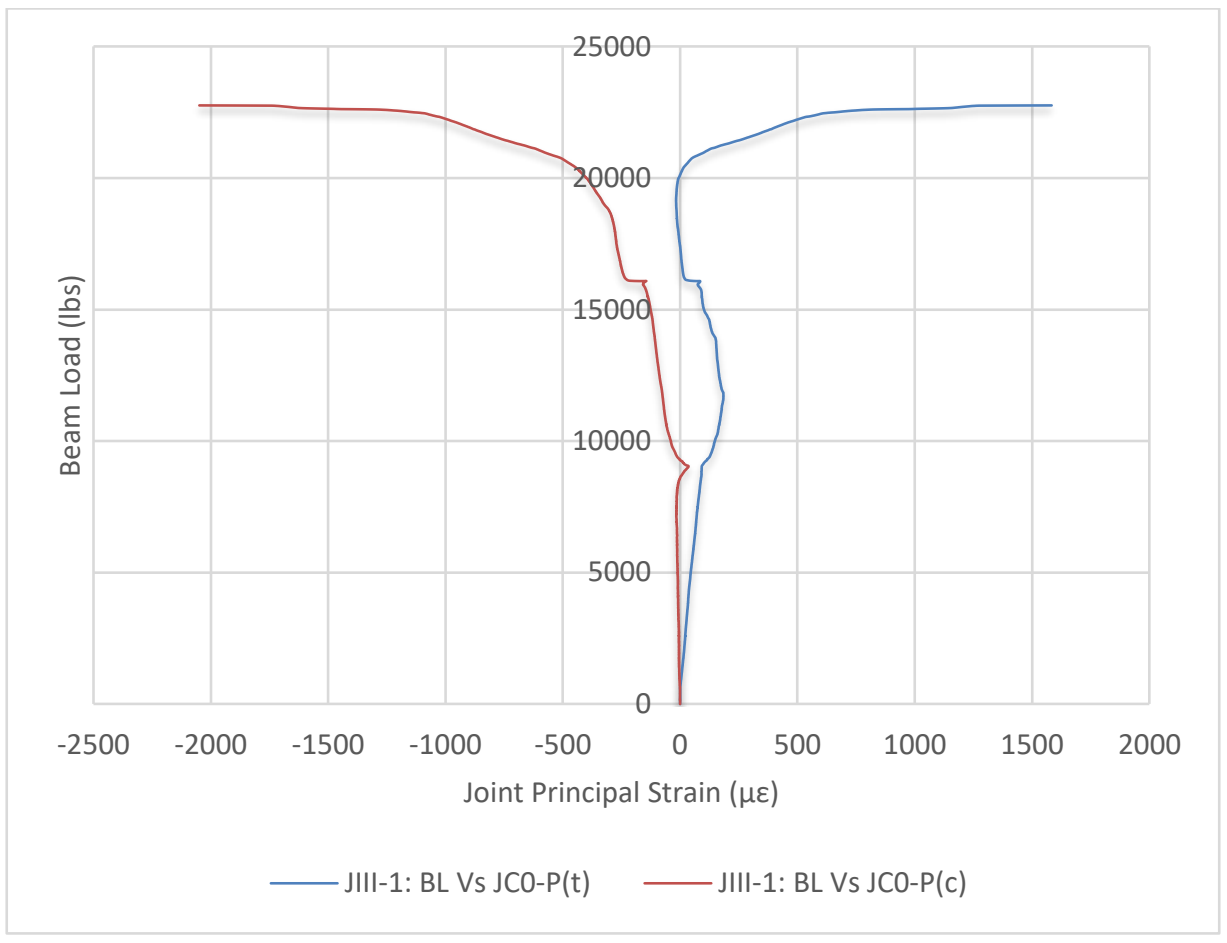

Figure 87. Joint principal strains for JIII-1

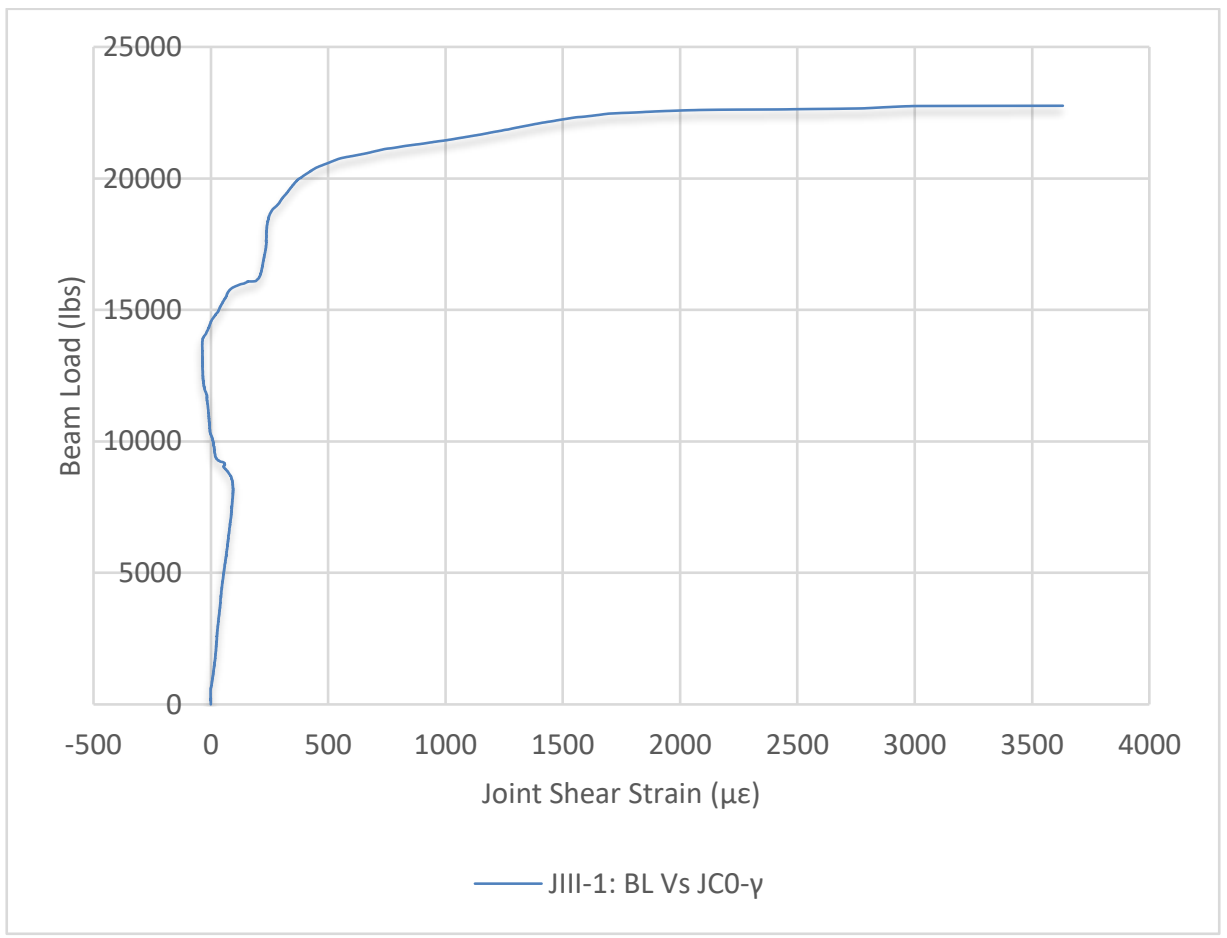

Figure 88. Joint shear strain for JIII-1 


\subsubsection{Behavior of joint reinforced with concrete filler-modules (JI-2)}

Specimen JI-2, shown in Figure 45, was tested to evaluate the influence of concrete filler-modules with reinforcing dowels. As the beam loading began, at 2,899 lb, a jump in the strain gage reading on the reinforcing dowel embedded inside the filler-module has revealed a potential bond loss with the surrounding concrete due to the presence of stress-concentration around the dowels. At 9,067 $\mathrm{lb}$, a crack in concrete close to the filler-module on the column face was observed. As the loading progressed to $\sim 18,649 \mathrm{lb}$, a maximum shear strain of $\sim 60 \mu \varepsilon$ (k-factor of 1.4) was recorded next to the crack location, as shown in Figure 90. Eventually, at 22,434 lb, the joint specimen failed in column shear, as shown in Figure 89. At the time of column failure, the strains in joint (JSH1)and column (CSL2)- rebar were measured to be $\sim 492$ and $\sim 241 \mu \varepsilon$, respectively. A summary of the experimental results is presented in Table 31. Compared to the control specimen JI-1, the improvements in load-carrying capacity and energy dissipation were noted to be $\sim 45$ and $35 \%$, respectively. However, no change in the ductility index (section 5.3.4) was observed. Similar joint reinforcing scheme was introduced by [27] and [28] as planar joint enlargement and further evaluated by [62] under different test conditions with maximum strength increase of about $28 \%$ [62].

Although reinforcing joint specimen with concrete filler-modules prevented joint shear failure, the enhancement in load-carrying capacity was limited to the strength of the weakest member meeting at the joint, i.e., column. Premature failure of the column is attributed to the influence of stress concentration effects at the tip of filler-module, as shown in Figure 90. This behavior can be further improved by the addition of FRP wraps or gussets that arrest the crack growth and offer additional force resistance through confinement while lowering the stress concentration effects. Hence, further research (section 5.2.3) was carried out by reinforcing joint specimens with FRP wraps or gussets in addition to bonding filler-modules at reentrant corners. 
Table 31. Summary of experimental results of joints reinforced with concrete filler-modules

\begin{tabular}{|c|c|c|c|c|c|c|c|c|c|c|c|c|}
\hline \multirow{2}{*}{ Joint } & \multirow{2}{*}{ PoI } & \multirow{2}{*}{$\begin{array}{c}\text { Beam load } \\
\text { (lb) }\end{array}$} & \multicolumn{3}{|c|}{ Column principal strain $(\mu \varepsilon)$} & \multicolumn{2}{|c|}{ Limit state } & \multicolumn{4}{|c|}{ Steel rebar strain $(\mu \varepsilon)$} & \multirow{2}{*}{$\begin{array}{c}\text { Observed } \\
\text { failure mode }\end{array}$} \\
\hline & & & $\mathrm{P}(\mathrm{t})$ & $\mathrm{P}(\mathrm{c})$ & $\gamma$ & $\mathrm{k}-\mathrm{P}(\mathrm{t})$ & $\mathrm{k}-\gamma$ & JSH1 & BSL1 & BSL2 & CSL2 & \\
\hline \multirow{3}{*}{ JI-2* } & A & 9,067 & 7 & -14 & 20 & 0.42 & 0.49 & 28 & 204 & - & 1 & \multirow{3}{*}{ Column shear } \\
\hline & B & 11,542 & 9 & -19 & 28 & 0.54 & 0.68 & 55 & 353 & - & 9 & \\
\hline & $\mathrm{C}$ & 22,434 & 366 & -23 & -15 & N.A. & N.A. & 492 & 1,216 & - & 241 & \\
\hline
\end{tabular}

PoI: Point of interest; $\mathrm{P}(\mathrm{t})$ : principal tensile strain; $\mathrm{P}(\mathrm{c})$ : principal compressive strain; $\gamma$ : shear strain; *principal- and shear- strains measured on the column next to the filler-module, identified in Figure 90.

Note:

1. Principal strains are computed based on Eq. 4.1

2. Shear strains are computed based on Eq. 4.3

3. Upon concrete cracking (i.e., after point B), the stress distribution becomes increasingly complex, and hence, the Mohr's circle concept is not adequate to determine the principal stresses beyond this point. 


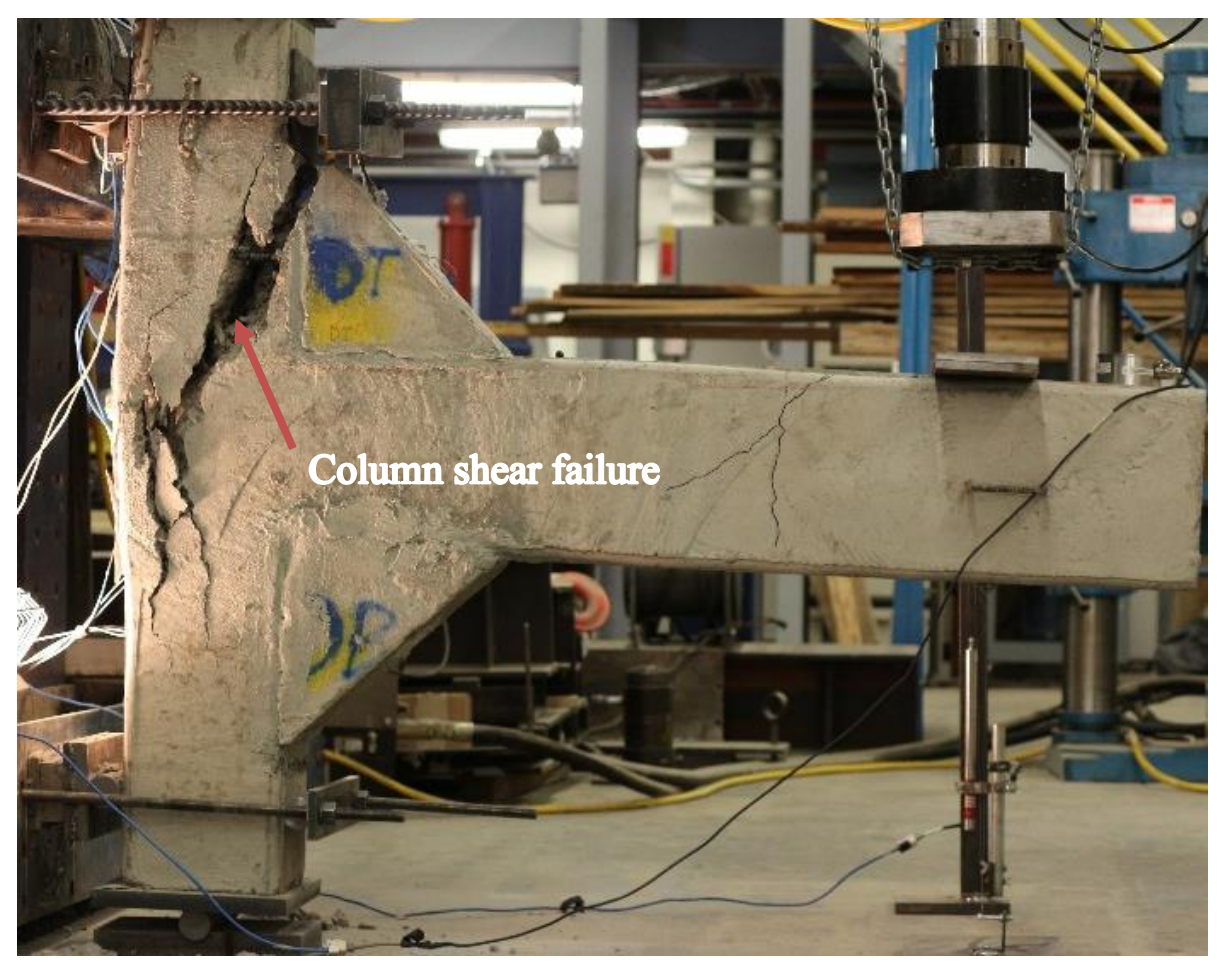

Figure 89. Failure of JI-2 (front view)

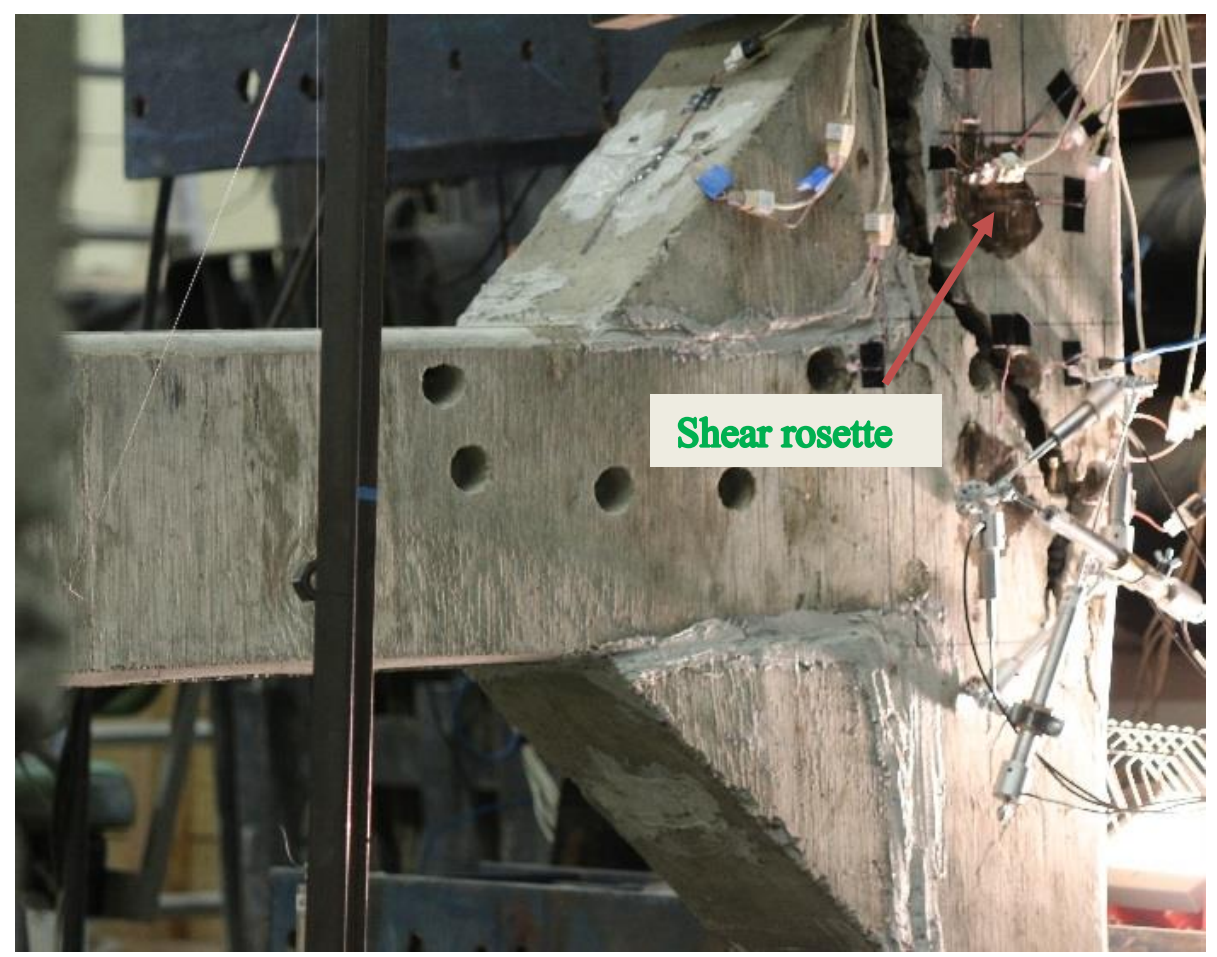

Figure 90. Failure of JI-2 (rear view) 


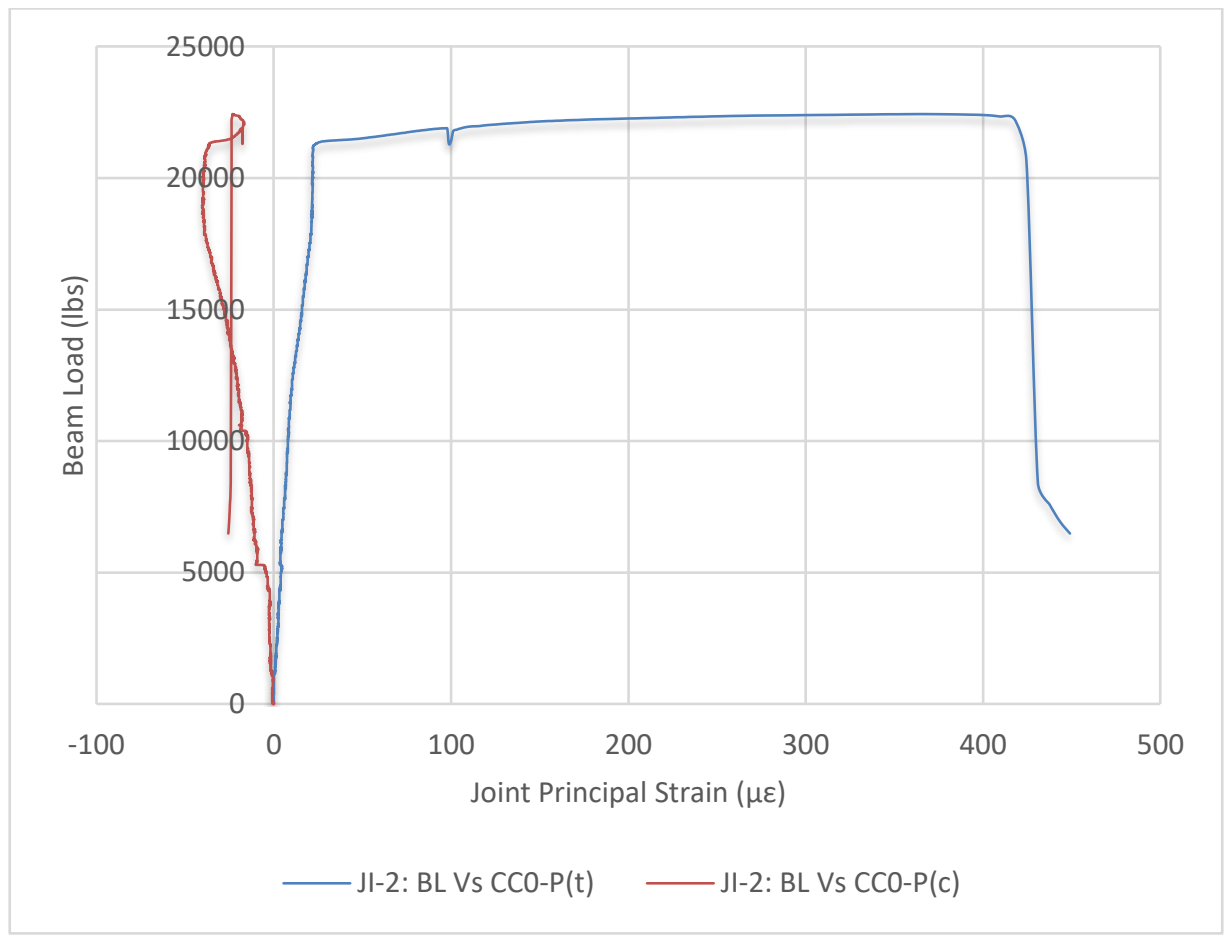

Figure 91. Column principal strains for JI-2

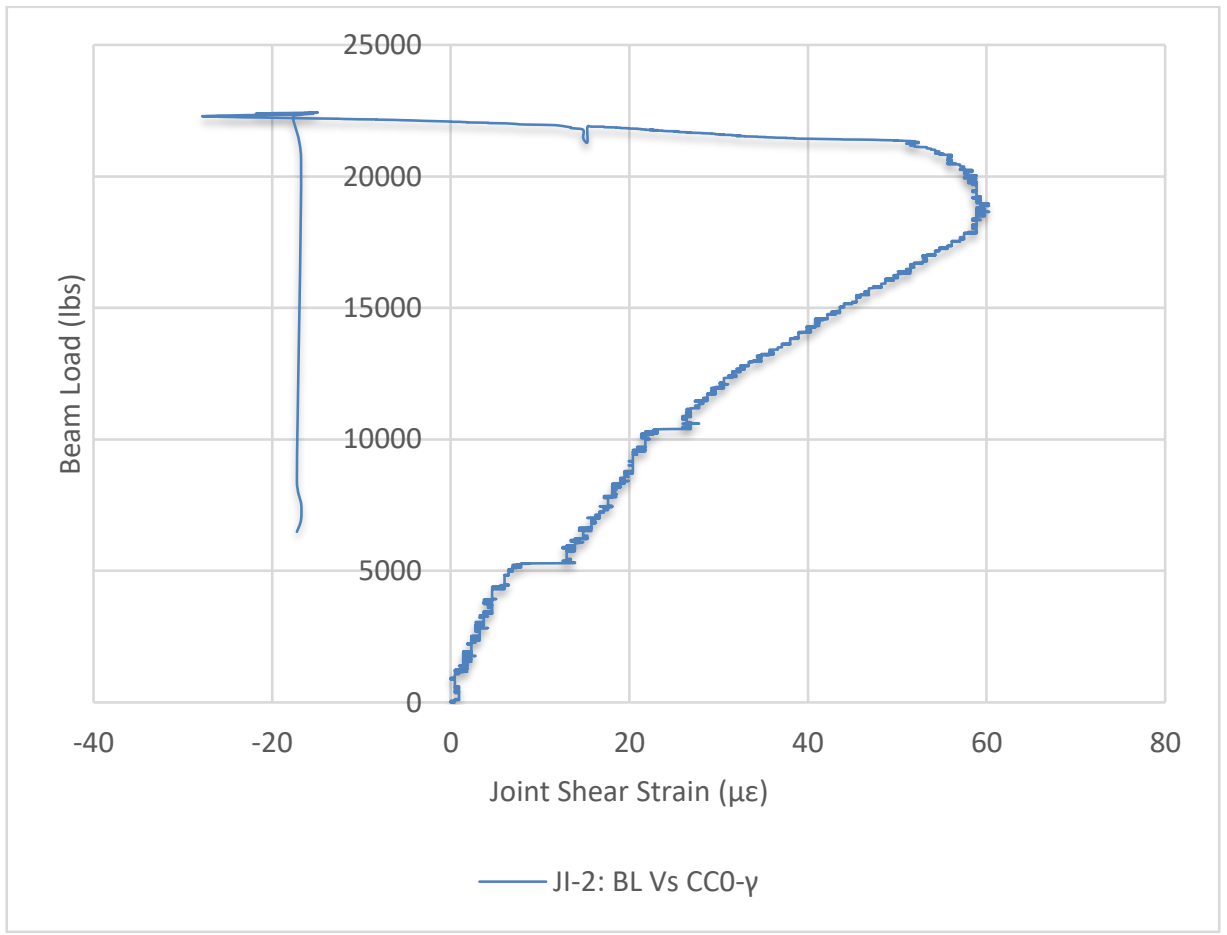

Figure 92. Column shear strain for JI-2 


\subsubsection{Behavior of joints reinforced with filler-modules and FRP wraps/gussets}

A total of eight joint specimens (JI-3 through 8, JIII-2 through 4) were tested to evaluate the response of beam-column joints reinforced with filler-modules and FRP wraps/gussets under different parameters listed in Table 27. The following section provides a general overview of the behavior of test specimens, followed by a summary of experimental results related to each phase.

As the load on the beam increased, joint specimens reinforced with filler-modules and FRP wraps/gussets exhibited flexural-tension cracks at the tip of the filler-module, in the vicinity of beam section. This was visually realized through a cloud formation at the tip of the filler-module due to the local de-bonding of FRP. The computed strains on the concrete surface by extrapolating the beam longitudinal rebar strains (as explained in earlier sections) were found to be in the range of $184-441 \mu \varepsilon$, with " $k$ " for flexural tension ranging from $10-25$. This range is higher than the control specimens due to the additional tensile resistance offered by the FRP wrap/gusset. Furthermore, the flexural-tension cracks did not progress into the core concrete due to the presence of compressive stresses induced by the bottom filler-module, in addition to the (tensile) resistance against tension crack formation offered by longitudinal beam rebar and confinement effects from FRP wrap/gusset. At this stage of loading, the resisting force is redistributed on to the fillermodules through FRP wrap/gusset until the top filler-module is cracked in tension. These cracks initiated when the joint rebar strain reached $58-209 \mu \varepsilon$ and grew significantly when the strains reached 217 - $495 \mu \varepsilon$, revealing a controlled crack growth (because of wrap) due to the contribution of FRP wrap/gusset. Data from strain gages oriented along the longitudinal axis of the top fillermodule revealed gradual cracking of filler-module at different stages of loading. The initial stage of filler-module cracking is referred to as point A and significant crack growth level (transition of force to column rebar) is referred to as point B. For design purposes, joints reinforced with fillermodules and FRP wrap/gusset are considered to behave in a linear elastic manner until point A. For operations under extreme event loading, point B is considered as idealized yield (defined as the point beyond which a truss mechanism primarily resists the forces through internal rebar cage, FRP wrap, and rebar-concrete bond forces) [63]. A truss mechanism primarily resists the induced force beyond point B. The test specimens finally failed either by beam flexure or column shear. 
Based on the observed behavior of joint specimens under monotonic loading, the author believes that the response of test specimens (beam-column-joint, together) in terms of peak load, ductility, and energy dissipation capacity can be further enhanced by confining (with $360^{\circ} \mathrm{FRP}$ wraps) the beam and column sections (up to plastic hinge length), and then bonding the filler-modules, to utilize the full potential of refurbished joint sections. This approach may also help in containing the failure within the filler-module (outside of joint core), which can be easily replaced in case of distress during extreme events.

\subsubsection{Behavior of joints reinforced with concrete/syntactic foam filler-modules and CFRP wraps (JI-3 and JI-4)}

Two joint specimens (JI-3 and JI-4) were tested in the current phase to evaluate the response of beam-column joints reinforced with concrete/foam filler-modules and CFRP wraps. A summary of the experimental observations is presented in the following section.

1. The flexure-tension cracks appeared at the tip of the filler-module when the concrete strains reached $228-441 \mu \varepsilon$. The k-factor for flexural-tension cracks was computed to be in the range of 13 - 25. Based on the author's evaluation of FRP wrapped cylinders under split tension test, reported in section 4.4.6, the tensile strength of FRP wrapped cylinders was noted to be $\sim(20-28) \sqrt{f_{c}^{\prime}}$. A summary of measured strains and computed k-factor is presented in Table 32.

2. As the loading increased resulting in internal stress redistribution, concrete cracking initiated in the top filler-module when the joint panel rebar strain reached $75-193 \mu \varepsilon$ and grew significantly when the rebar strains reached 217 - $329 \mu \varepsilon$ (i.e., at this strain level in the rebar, concrete reached its ultimate tensile strain).

3. The test specimens reached a peak load of $\sim 60,773-64,107 \mathrm{lb}$ and failed in beam-flexure. The measured rebar strains in the joint- and column- longitudinal reinforcement were found to be in the range of $1,175-2,263 \mu \varepsilon$ and $1,472-2,612 \mu \varepsilon$, respectively.

4. A summary of joint principal tensile- and shear- strains is presented in Table 33 and graphically shown in Figures 93 through 98. 
5. Comparison of the principal- and shear- strains at points A and B for JI-3 and JI-4 reveals that the foam filler-module is more efficient in redistributing the forces between the joint, beam-, and column- rebars due to its comparatively higher strength properties and lower stiffness (higher strain to failure response) than concrete filler-module. Furthermore, it can be concluded that the high tensile strength of the top filler-module is influential in pushing the limits of Point A and B (i.e., prolonged elastic zone).

6. As seen from Figures 93 and 94, both the test specimens JI-3 and JI-4 failed in beam flexure, closer to the tip of the filler-module.

Table 32. Modulus of rupture of joints with filler-modules and CFRP wraps

\section{Joint Beam load (lb) Beam rebar strain $(\mu \varepsilon)$ Concrete strain ( $\mu \varepsilon \varepsilon) \quad \mathrm{k}-f_{r}$}

\begin{tabular}{|c|c|c|c|c|}
\hline \multirow{2}{*}{ JI-1 } & 2,475 & 106 & 158 & 9 \\
\hline & 2,889 & 143 & 214 & 12 \\
\hline \multirow{2}{*}{ JII-3 } & 7,094 & 152 & 228 & 13 \\
\hline & 8,342 & 200 & 299 & 17 \\
\hline \multirow{2}{*}{ JI-4 } & 8,229 & 169 & 254 & 14 \\
\hline & 11,954 & 294 & 441 & 25 \\
\hline
\end{tabular}


Table 33. Summary of experimental results of joints with concrete/syntactic foam filler-modules with/out CFRP wraps

\begin{tabular}{|c|c|c|c|c|c|c|c|c|c|c|c|c|}
\hline \multirow{2}{*}{ Joint } & \multirow{2}{*}{ PoI } & \multirow{2}{*}{$\begin{array}{c}\text { Beam load } \\
\text { (lb) }\end{array}$} & \multicolumn{3}{|c|}{ Joint principal strain $(\mu \varepsilon)$} & \multicolumn{2}{|c|}{ Limit state } & \multicolumn{4}{|c|}{ Steel rebar strain $(\mu \varepsilon)$} & \multirow{2}{*}{$\begin{array}{c}\text { Observed } \\
\text { failure mode }\end{array}$} \\
\hline & & & $\mathrm{P}(\mathrm{t})$ & $\mathrm{P}(\mathrm{c})$ & $\gamma$ & $\mathrm{k}-\mathrm{P}(\mathrm{t})$ & $\mathrm{k}-\gamma$ & JSH1 & BSL1 & BSL2 & CSL2 & \\
\hline \multirow{3}{*}{ JI-1 } & A & 5,063 & 53 & -94 & -147 & 3.0 & 3.5 & 128 & 415 & - & 66 & \multirow{3}{*}{$\begin{array}{c}\text { Joint } \\
\text { Compression }\end{array}$} \\
\hline & $\mathrm{B}$ & 11,076 & 43 & -261 & -266 & 2.4 & 6.4 & 835 & 1,065 & - & 258 & \\
\hline & $\mathrm{C}$ & 15,471 & - & - & - & N.A. & N.A. & 1,362 & 1,457 & - & 868 & \\
\hline \multirow{3}{*}{$\mathrm{JI}-2 *$} & A & 9,067 & 7 & -14 & 20 & 0.42 & 0.49 & 28 & 204 & - & 1 & \multirow{3}{*}{ Column shear } \\
\hline & $\mathrm{B}$ & 11,542 & 9 & -19 & 28 & 0.54 & 0.68 & 55 & 353 & - & 9 & \\
\hline & $\mathrm{C}$ & 22,434 & 366 & -23 & -15 & N.A. & N.A. & 492 & 1,216 & - & 241 & \\
\hline \multirow{3}{*}{ JI-3 } & A & 21,976 & 75 & -94 & -166 & 4.27 & 4.00 & 75 & - & 855 & 28 & \multirow{3}{*}{ Beam flexure } \\
\hline & B & 27,308 & 107 & -118 & -221 & 6.08 & 5.33 & 217 & - & 1,116 & 84 & \\
\hline & $\mathrm{C}$ & 60,773 & 1,990 & -558 & $-2,447$ & N.A. & N.A. & 1,175 & - & 4,809 & 1,472 & \\
\hline \multirow{3}{*}{ JI-4 } & A & 21,574 & 22 & -91 & -110 & 1.23 & 2.65 & 193 & - & 664 & 114 & \multirow{3}{*}{ Beam flexure } \\
\hline & B & 23,970 & 40 & -103 & -137 & 2.25 & 3.32 & 329 & - & 773 & 135 & \\
\hline & $\mathrm{C}$ & 64,107 & 1,005 & -223 & $-1,182$ & N.A. & N.A. & 2,263 & - & 2,608 & 2,612 & \\
\hline
\end{tabular}

PoI: Point of interest; $\mathrm{P}(\mathrm{t})$ : principal tensile strain; $\mathrm{P}(\mathrm{c})$ : principal compressive strain; $\gamma$ : shear strain; *principal- and shear- strains measured on the column next to the filler-module.

Note:

1. Principal strains are computed based on Eq. 4.1

2. Shear strains are computed based on Eq. 4.3

3. Upon concrete cracking (i.e., after point B), the stress distribution becomes increasingly complex, and hence, the Mohr's circle concept is not adequate to determine the principal stresses beyond this point. 


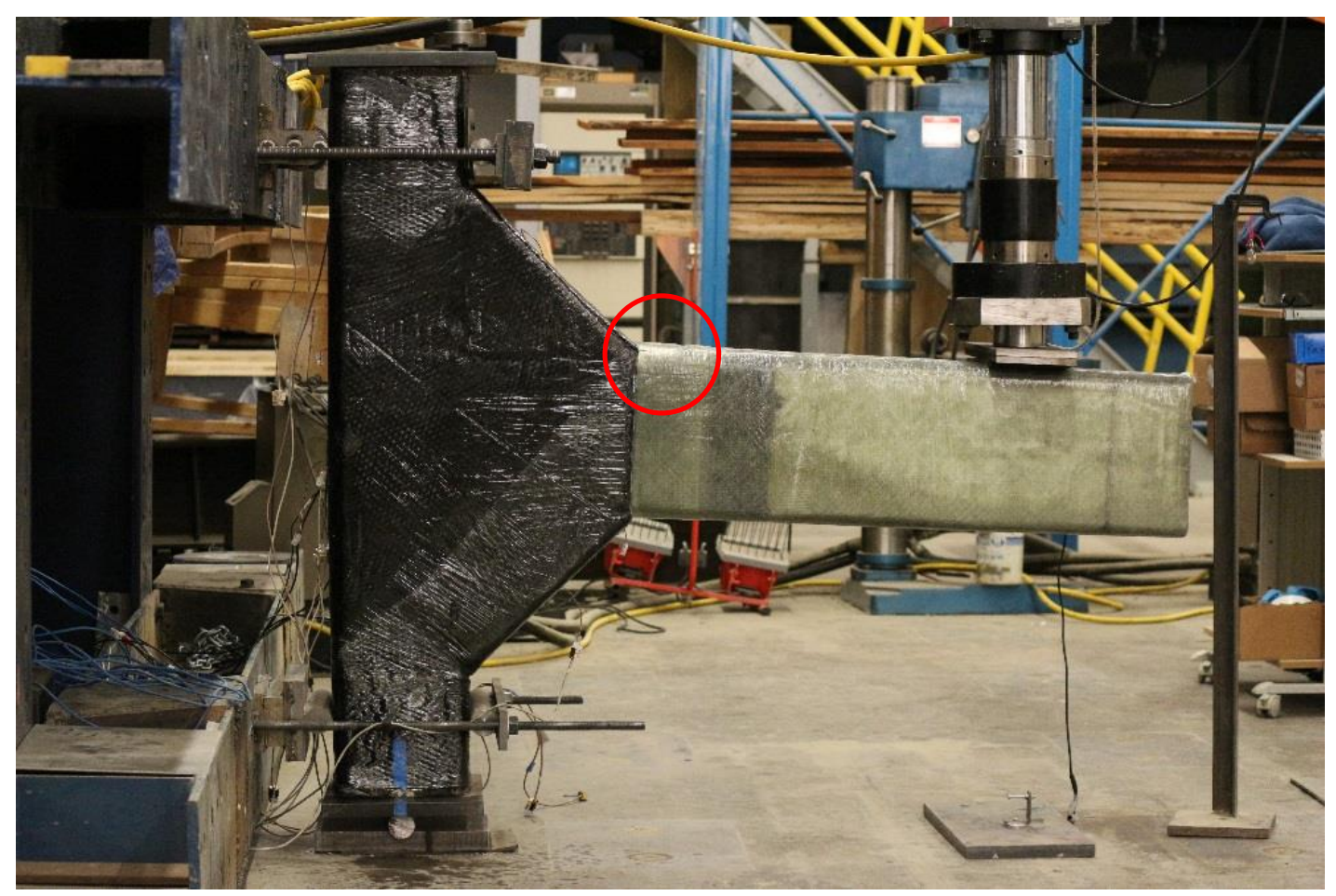

Figure 93. Failure of JI-3

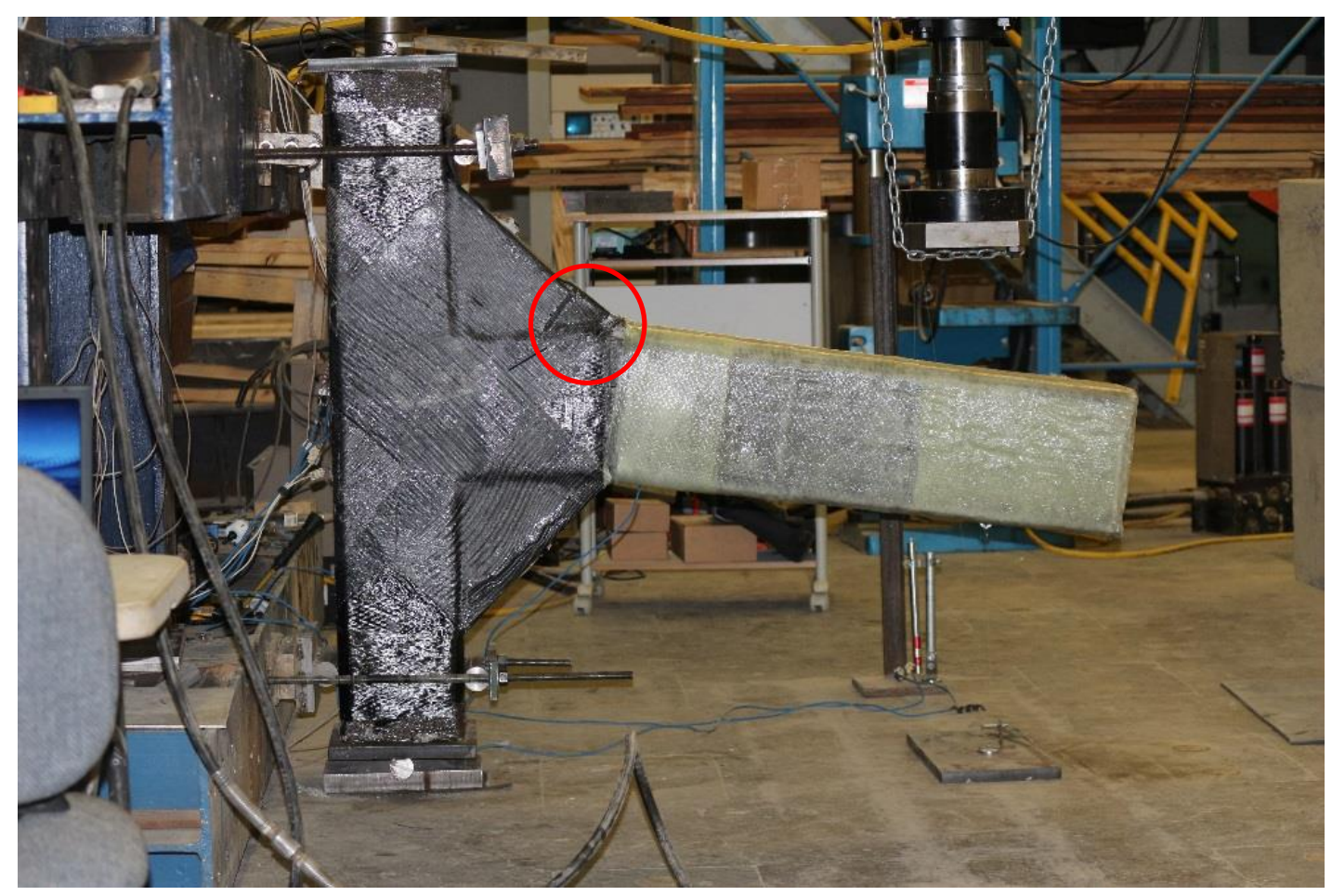

Figure 94 . Failure of JI-4 


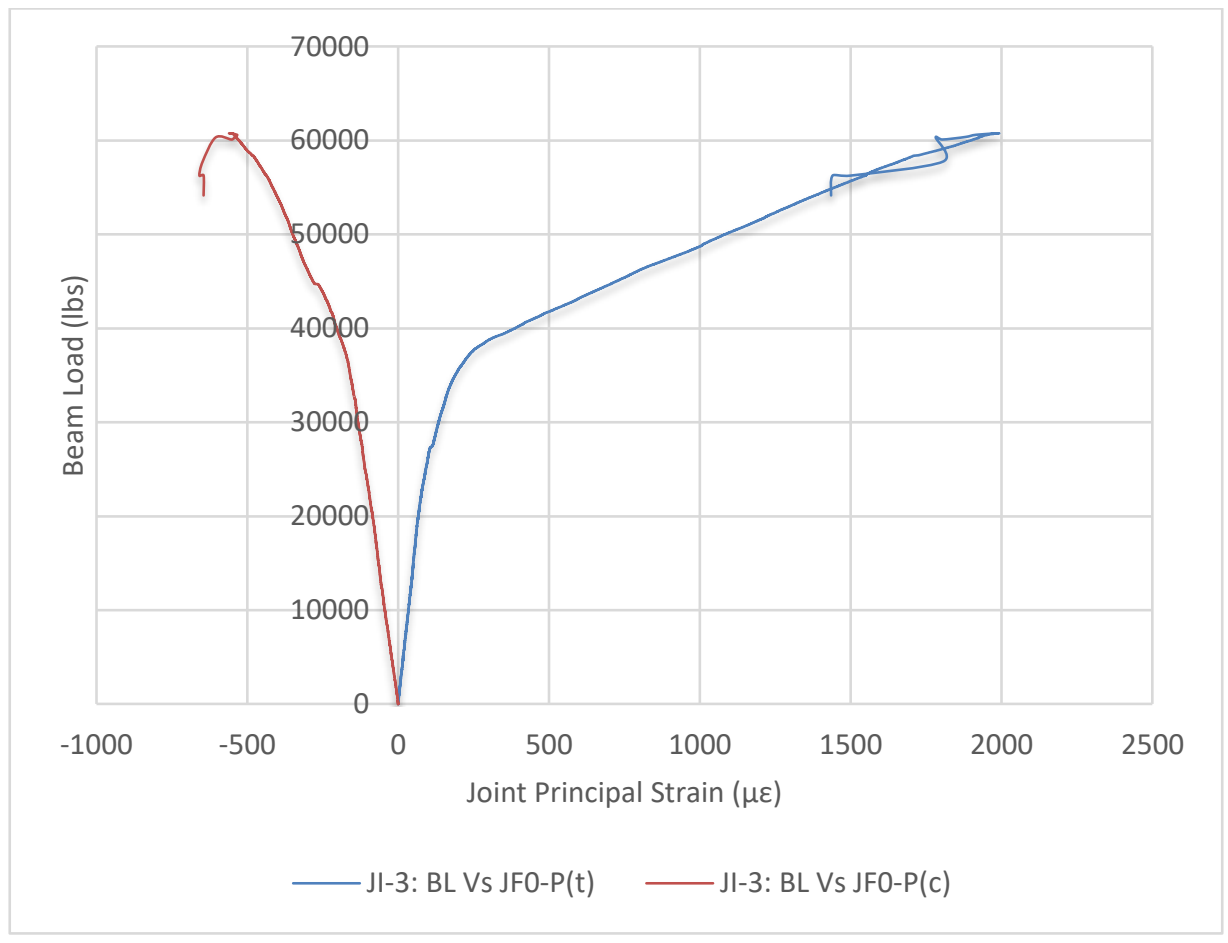

Figure 95. Joint principal strains for JI-3

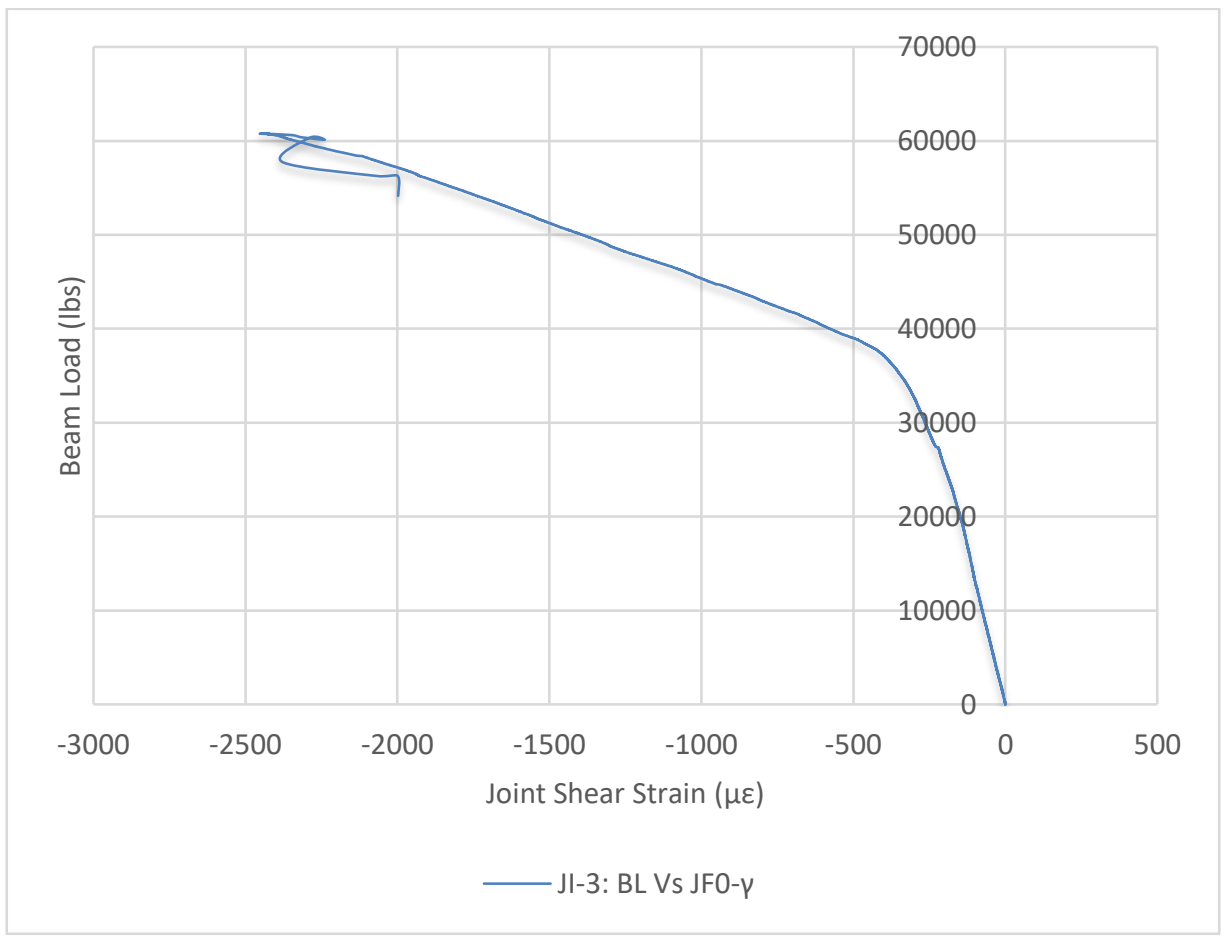

Figure 96. Joint shear strain for JI-3 


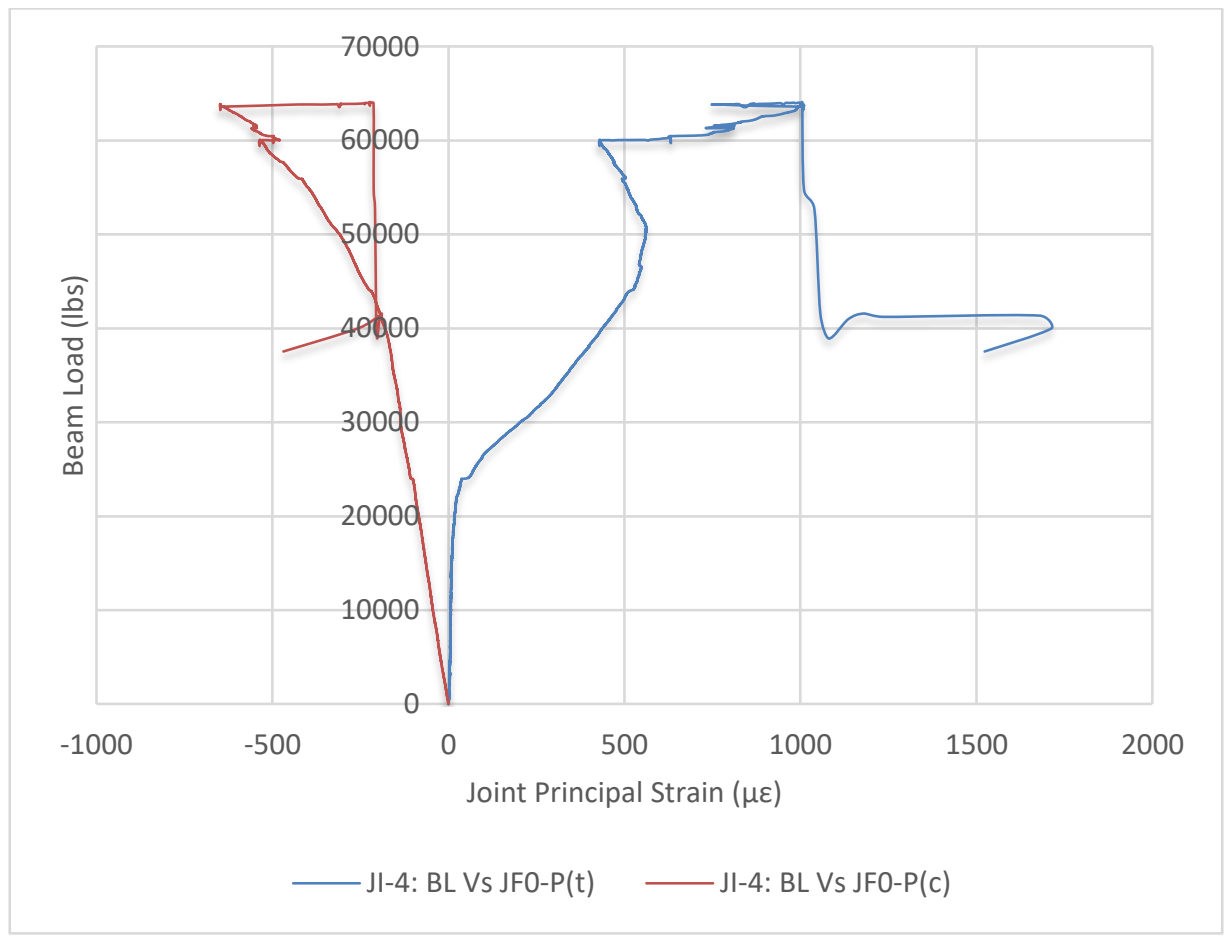

Figure 97. Joint principal strains for JI-4

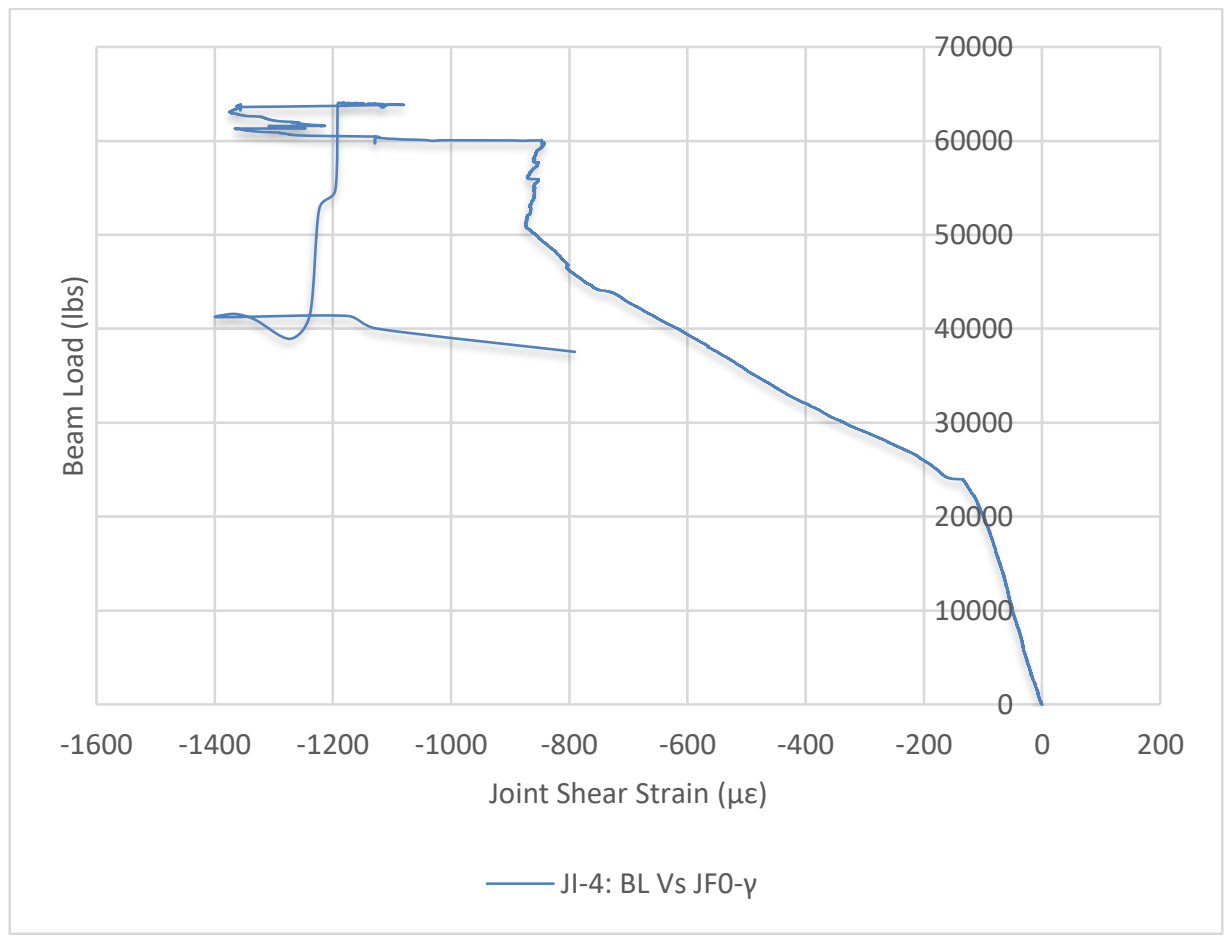

Figure 98. Joint shear strain for JI-4 


\subsubsection{Behavior of joints reinforced with concrete filler-modules and CFRP gussets (JI-6, $J I-7$, and JI-8)}

Three joint specimens (JI-6, JI-7, and JI-8) were tested in the current phase to evaluate the response of beam-column joints reinforced with concrete filler-modules and CFRP gussets. Use of composite FRP gussets instead of wraps was investigated primarily for four reasons: (i) to enhance the quality of composite end product by adopting consistent manufacturing process, (ii) to avoid construction errors and provide ease of installation, (iii) to minimize downtime incurred during the field implementation process, and (iv) to reduce the hassle involved with fabric cutting and disposal of waste material on-site, i.e., gussets can be prefabricated. A summary of the experimental observations is presented in the following section.

1. The flexure-tension cracks appeared at the tip of the filler-module when the concrete strains reached $261-425 \mu \varepsilon$. The k-factor for flexural-tension cracks was computed to be in the range of 15 - 24. Based on the author's evaluation of FRP wrapped cylinders under split tension test, reported in section 4.4.6, the tensile strength of FRP wrapped cylinders was noted to be $\sim(20-28) \sqrt{f_{c}^{\prime}}$. A summary of measured strains and computed k-factor is presented in Table 34.

2. As the loading increased resulting in internal stress redistribution, concrete cracking initiated in the top filler-module when the joint rebar strain reached $58-92 \mu \varepsilon$ and grew significantly when the strains reached $300-320 \mu \varepsilon$ (i.e., at this strain level in the rebar, concrete reached its ultimate tensile strain).

3. Based on the load vs. strain plots shown in Figures 99 and 100, some sort of bond-slip between the filler-module and underlying FRP was observed for specimens JI-7 and JI-8.

4. The test specimens reached a peak load of 51,018 - 72,040 lb and failed in column shear. The measured strains in the longitudinal joint- and column- reinforcement were found to be in the range of $1,126-1,427 \mu \varepsilon$ and $1,125-2,325 \mu \varepsilon$, respectively.

5. A summary of joint principal tensile- and shear- strains is presented in Table 35 and graphically shown in Figures 104 through 109. 
6. Comparison of the principal- and shear- strain data of JI-6 and JI-7 revealed that the FRP fabric layer bonded to the beam and column sections before gluing filler-modules had a detrimental effect on the joint structural response (strength, ductility, and energy dissipation) due to the bond-slip between FRP layer and glue line at early stages of loading $(\sim 17,000 \mathrm{lb})$.

7. Comparison of the principal- and shear- strain data of JI-7 and JI-8 revealed that the use of high strength concrete filler-module (5472 vs. 9545 psi) on the tension side has very little influence on the joint response after point A due to its brittle nature and/or bond-slip between filler-modules and underlying FRP layers. As the crack in the top filler-module progresses between points A and B, a gradual increase in terms of the influence of joint response is seen due to the formation of compression strut, resulting from the high strength filler-module in the compression zone. This influence has been more pronounced after point $B$, due to the full engagement of the compression strut mechanism, resulting in better ductility and energy dissipation capacity of the joint specimen.

8. As shown in Figures 101 through 103, all the test specimens failed in column shear through the debonding of CFRP gussets. Besides, buckling of the column longitudinal rebars was noticed.

Table 34. Modulus of rupture of joints with filler-modules and CFRP gussets

\section{Joint Beam load (lb) Beam rebar strain ( $\mu \varepsilon)$ Concrete strain $(\mu \varepsilon) \quad$ k- $f_{r}$}

\begin{tabular}{ccccc}
\hline \multirow{2}{*}{ JI-5 } & 1,625 & 92 & 138 & 8 \\
\cline { 2 - 5 } & 2,035 & 141 & 212 & 12 \\
\hline \multirow{2}{*}{ JI-6 } & 8,093 & 179 & 268 & 15 \\
\cline { 2 - 5 } & 11,030 & 283 & 425 & 24 \\
\hline \multirow{2}{*}{ JI-7 } & 7,255 & 201 & 301 & 17 \\
\cline { 2 - 5 } & 9,338 & 281 & 421 & 24 \\
\hline \multirow{2}{*}{ JI-8 } & 6,439 & 174 & 261 & 15 \\
\cline { 2 - 5 } & 9,008 & 277 & 416 & 24 \\
\hline
\end{tabular}




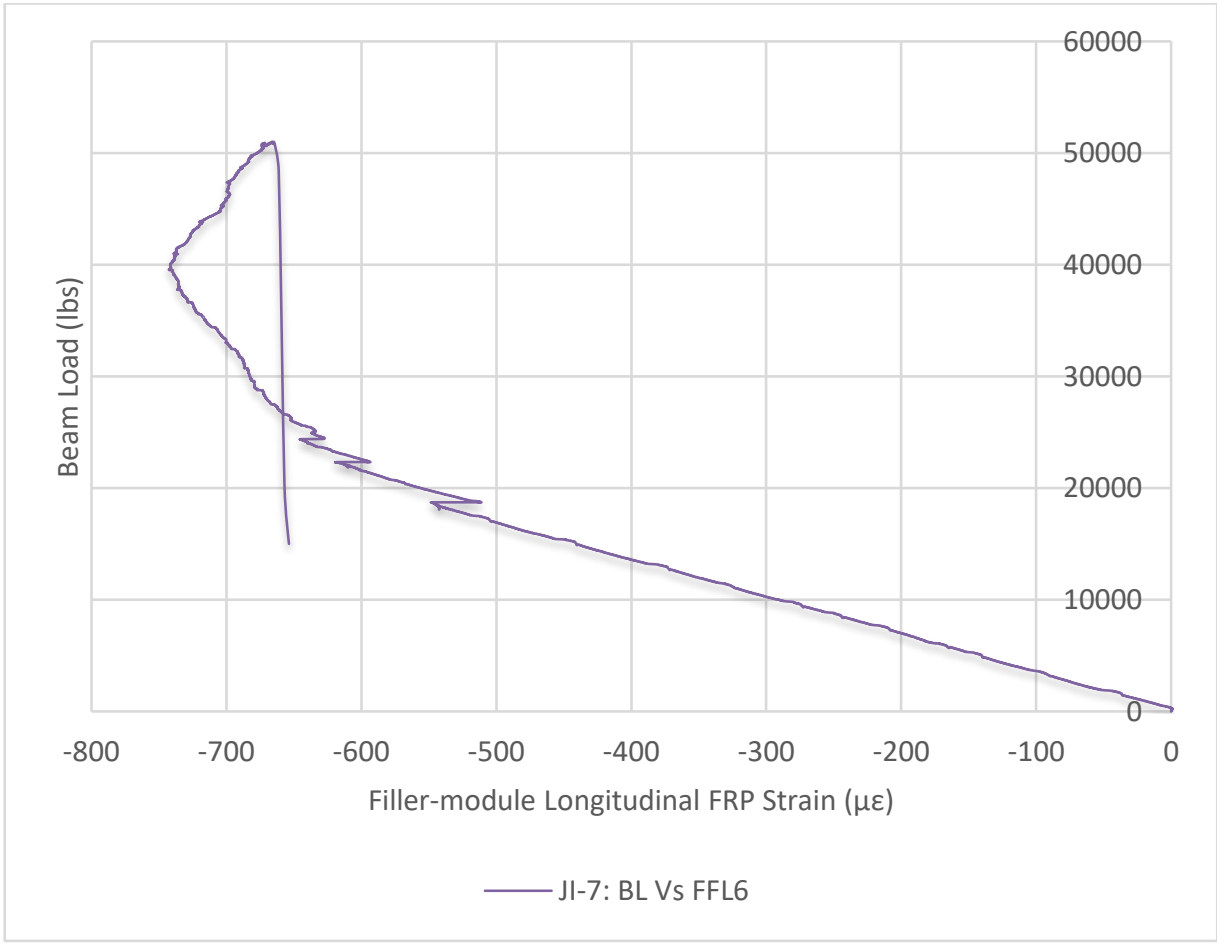

Figure 99. Strain response along the longitudinal axis of filler-module (JI-7)

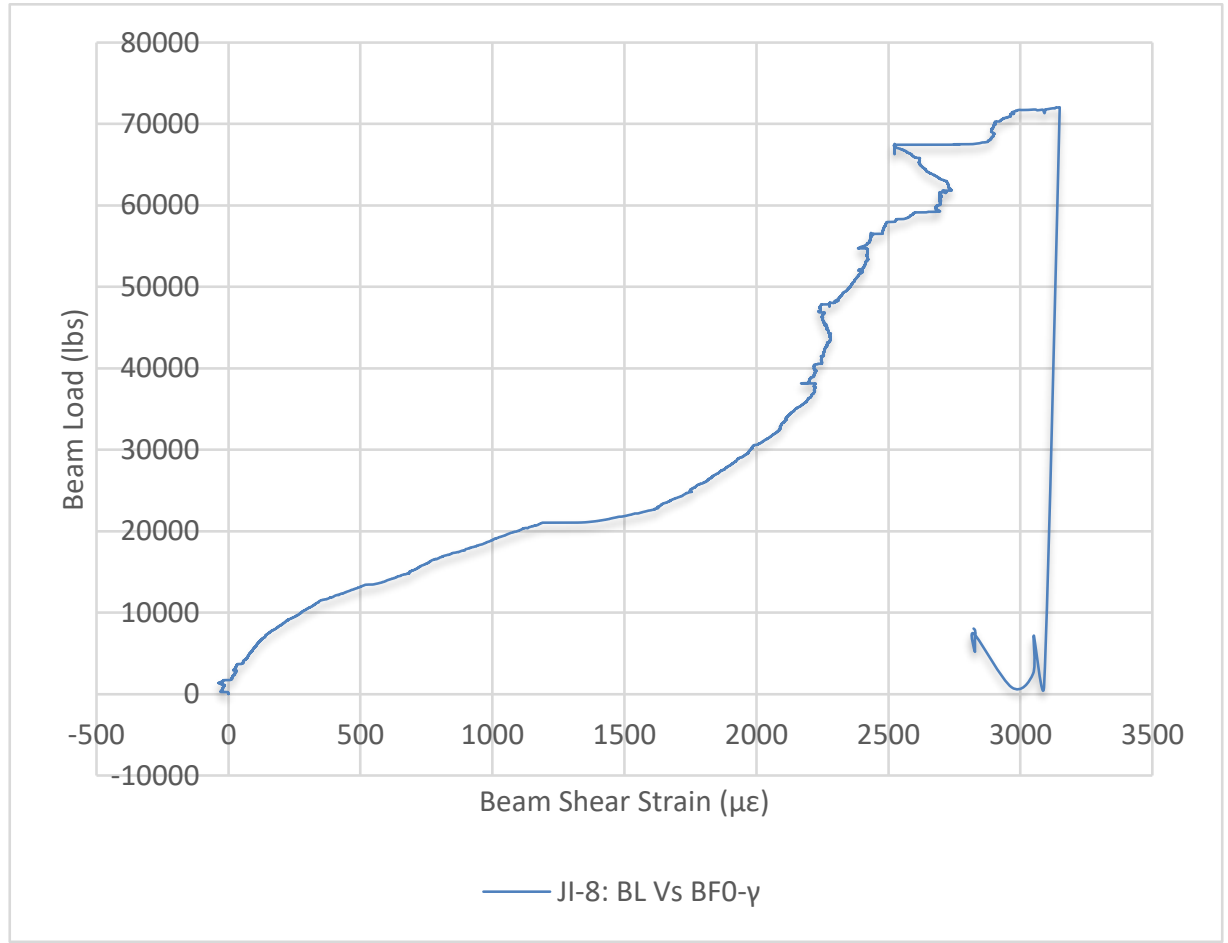

Figure 100. Strain response close to the filler-module tip on beam section (JI-8) 
Table 35. Summary of experimental results of joints with filler-modules and CFRP gussets

\begin{tabular}{|c|c|c|c|c|c|c|c|c|c|c|c|c|}
\hline \multirow{2}{*}{ Joint } & \multirow{2}{*}{ PoI } & \multirow{2}{*}{$\begin{array}{c}\text { Beam load } \\
\text { (lb) }\end{array}$} & \multicolumn{3}{|c|}{ Joint principal strain $(\mu \varepsilon)$} & \multicolumn{2}{|c|}{ Limit state } & \multicolumn{4}{|c|}{ Steel rebar strain $(\mu \varepsilon)$} & \multirow{2}{*}{$\begin{array}{c}\text { Observed } \\
\text { failure mode }\end{array}$} \\
\hline & & & $\mathrm{P}(\mathrm{t})$ & $\mathrm{P}(\mathrm{c})$ & $\gamma$ & $k-P(t)$ & $\mathrm{k}-\gamma$ & JSH1 & BSL1 & BSL2 & CSL2 & \\
\hline \multirow{3}{*}{ JI-5 } & $\mathrm{A}$ & 2,183 & 57 & -39 & 86 & 3.3 & 2.1 & - & 156 & 93 & 37 & \multirow{3}{*}{$\begin{array}{c}\text { Joint } \\
\text { diagonal } \\
\text { tension }\end{array}$} \\
\hline & B & 10,886 & - & - & - & N.A. & N.A. & - & 1,214 & 770 & 382 & \\
\hline & $\mathrm{C}$ & 18,819 & - & - & - & N.A. & N.A. & - & 2,044 & 1,486 & 1,351 & \\
\hline \multirow{3}{*}{ JI-6 } & A & 21,001 & 105 & -166 & 265 & 6.01 & 6.41 & 85 & 354 & 811 & -14 & \multirow{3}{*}{ Column shear } \\
\hline & $\mathrm{B}$ & 32,836 & 266 & -350 & 605 & 15.14 & 14.62 & 312 & 861 & 1,384 & 113 & \\
\hline & $\mathrm{C}$ & 58,961 & 1,958 & $-1,463$ & 3,394 & N.A. & N.A. & 1,126 & 1,917 & 3,307 & 2,325 & \\
\hline \multirow{3}{*}{ JI-7 } & $\mathrm{A}$ & 15,021 & 81 & -80 & 154 & 4.63 & 3.72 & 92 & 431 & 540 & 2 & \multirow{3}{*}{ Column shear } \\
\hline & B & 25,638 & 221 & -164 & 344 & 12.62 & 8.31 & 300 & 928 & 1,049 & 69 & \\
\hline & $\mathrm{C}$ & 51,018 & 1,580 & -943 & 2,473 & N.A. & N.A. & 1,242 & 1,997 & 2,833 & 1,392 & \\
\hline \multirow{3}{*}{ JI-8 } & $\mathrm{A}$ & 20,413 & 91 & -127 & 213 & 5.17 & 5.15 & 58 & 385 & 800 & -34 & \multirow{3}{*}{ Column shear } \\
\hline & B & 33,316 & 259 & -283 & 534 & 14.76 & 12.89 & 320 & 918 & 1,421 & 76 & \\
\hline & $\mathrm{C}$ & 72,040 & 1,797 & $-1,829$ & 3,597 & N.A. & N.A. & 1,427 & 2,396 & 11,426 & 1,125 & \\
\hline
\end{tabular}

PoI: Point of interest; $\mathrm{P}(\mathrm{t})$ : principal tensile strain; $\mathrm{P}(\mathrm{c})$ : principal compressive strain; $\gamma$ : shear strain

Note:

1. Principal strains are computed based on Eq. 4.1

2. Shear strains are computed based on Eq. 4.3

3. Upon concrete cracking (i.e., after point B), the stress distribution becomes increasingly complex, and hence, the Mohr's circle concept is not adequate to determine the principal stresses beyond this point. 


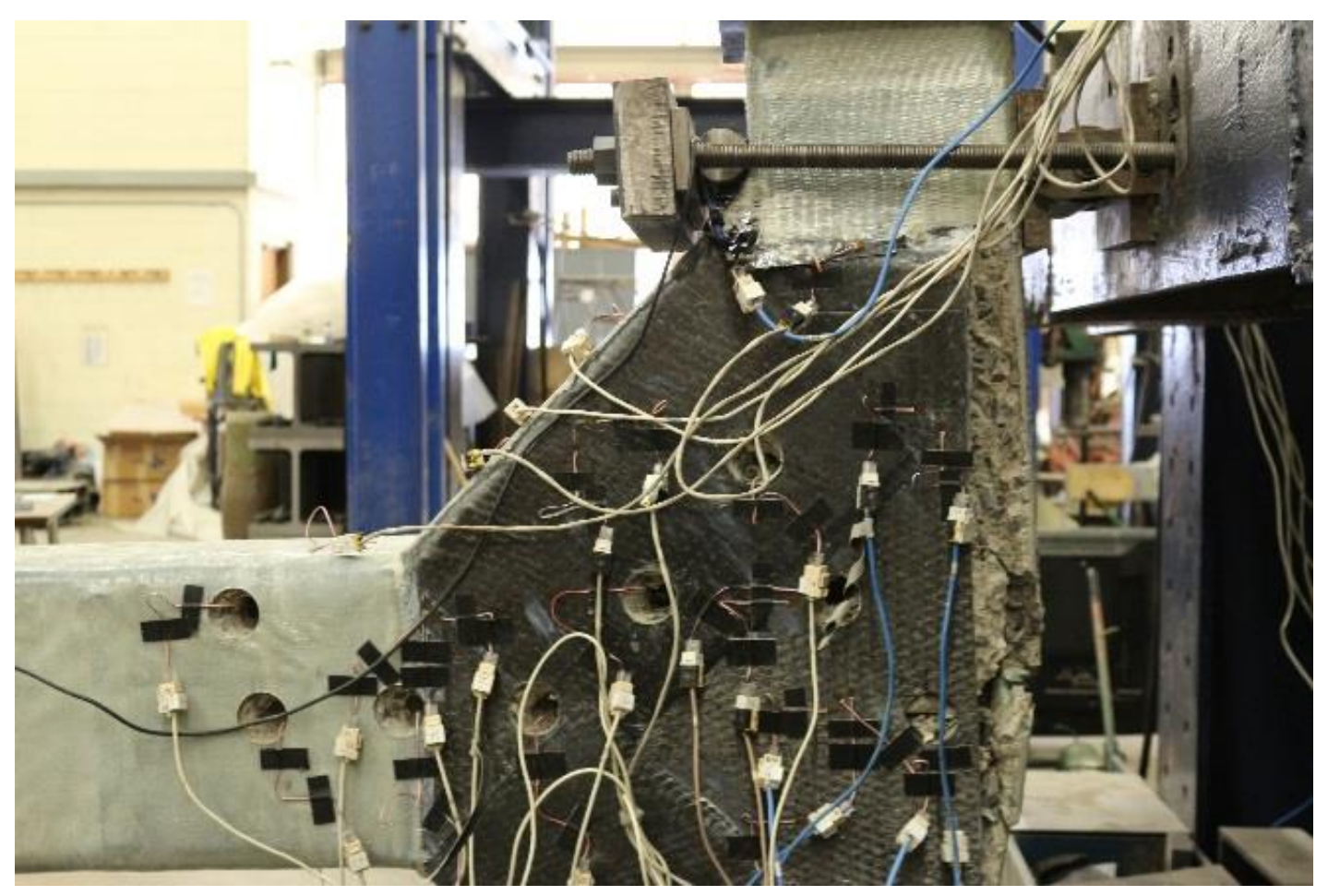

Figure 101. Failure of JI-6

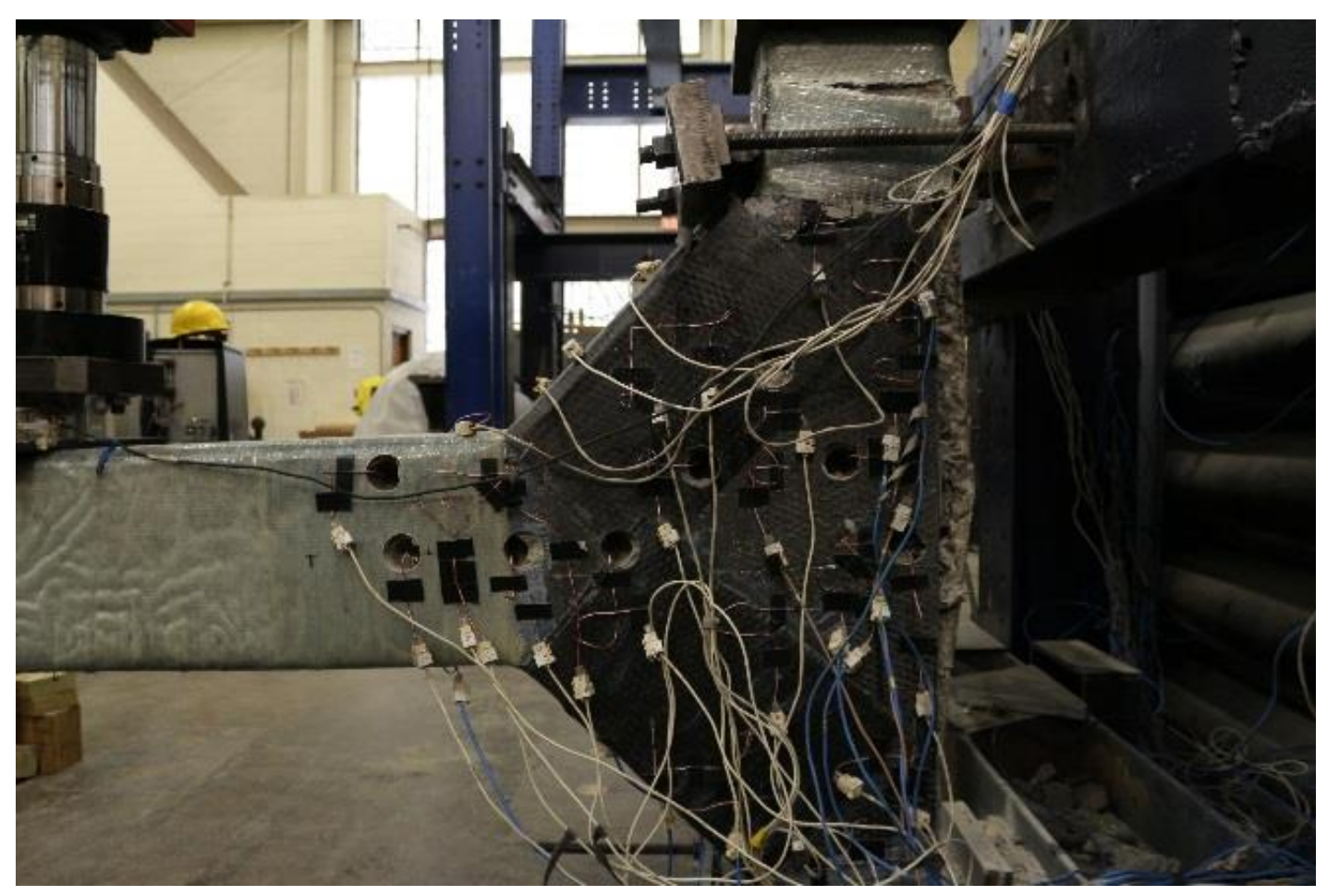

Figure 102. Failure of JI-7 


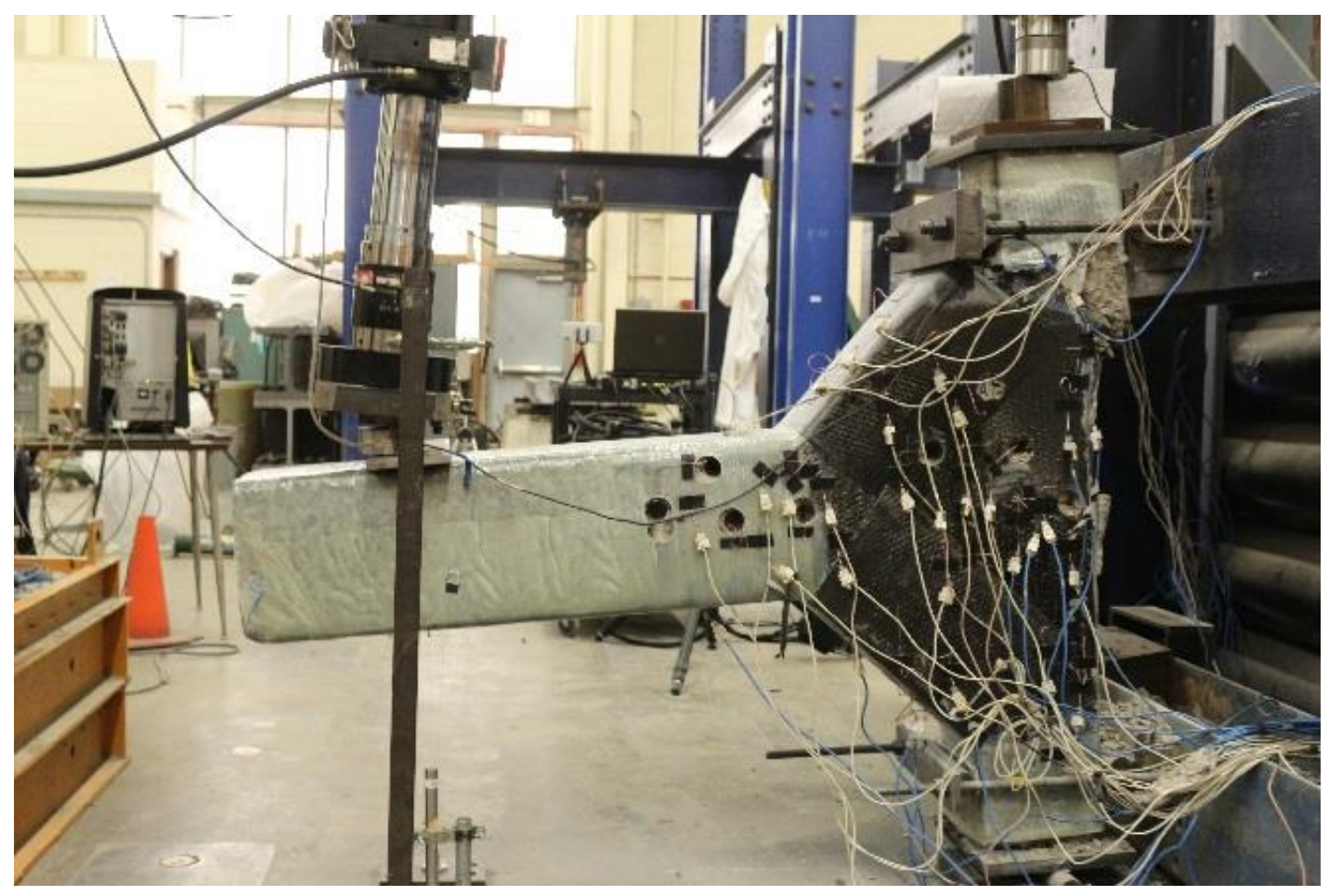

Figure 103. Failure of JI-8 


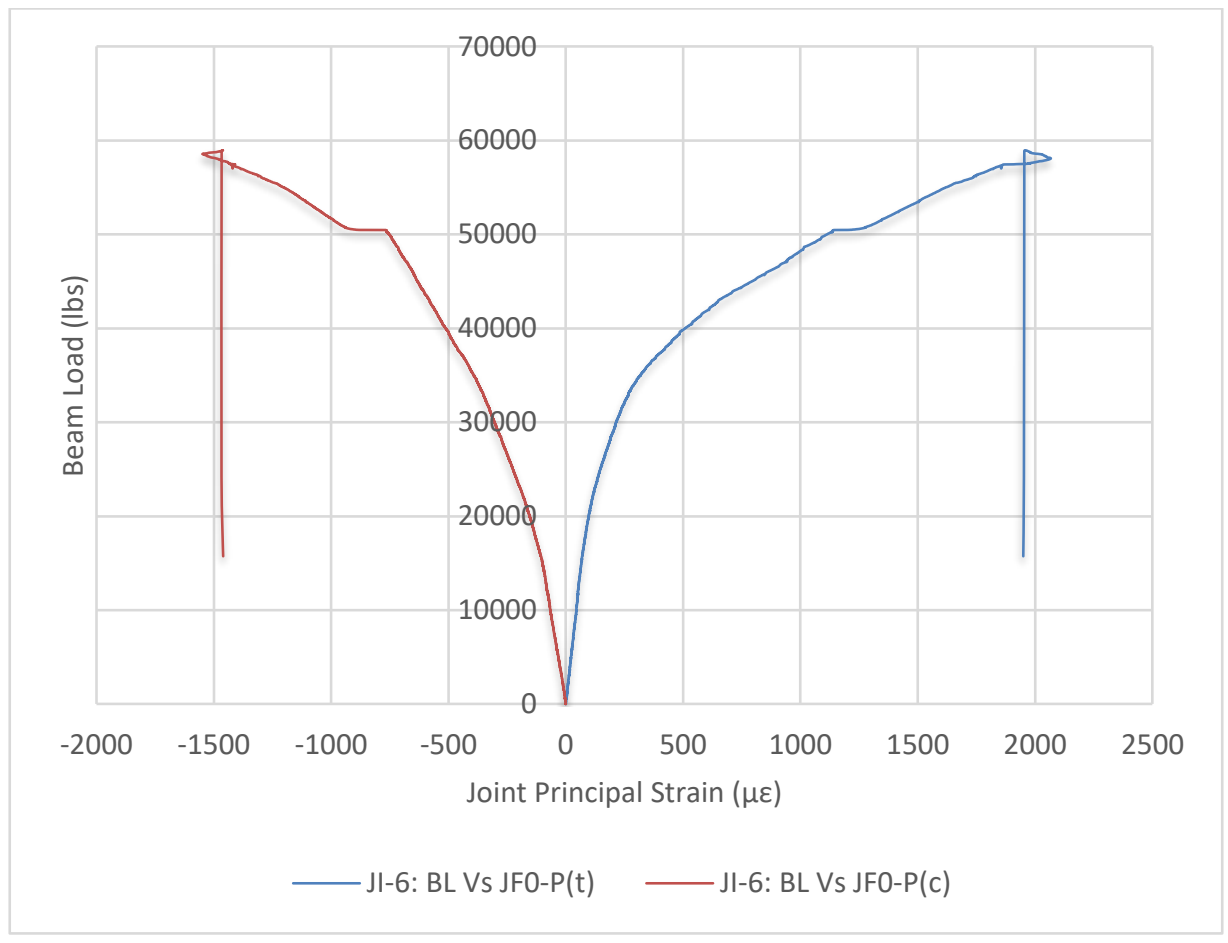

Figure 104. Joint principal strains for JI-6

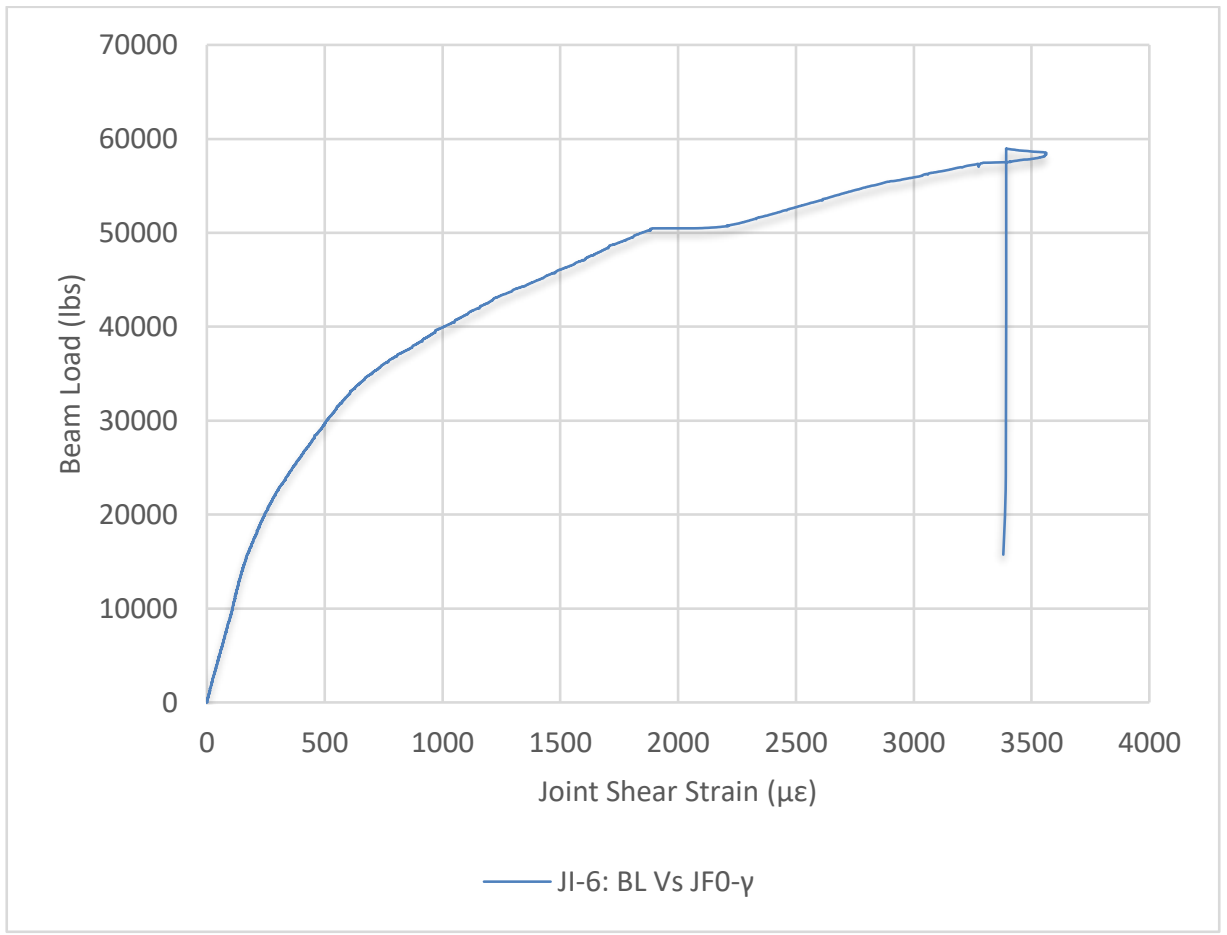

Figure 105. Joint shear strain for JI-6 


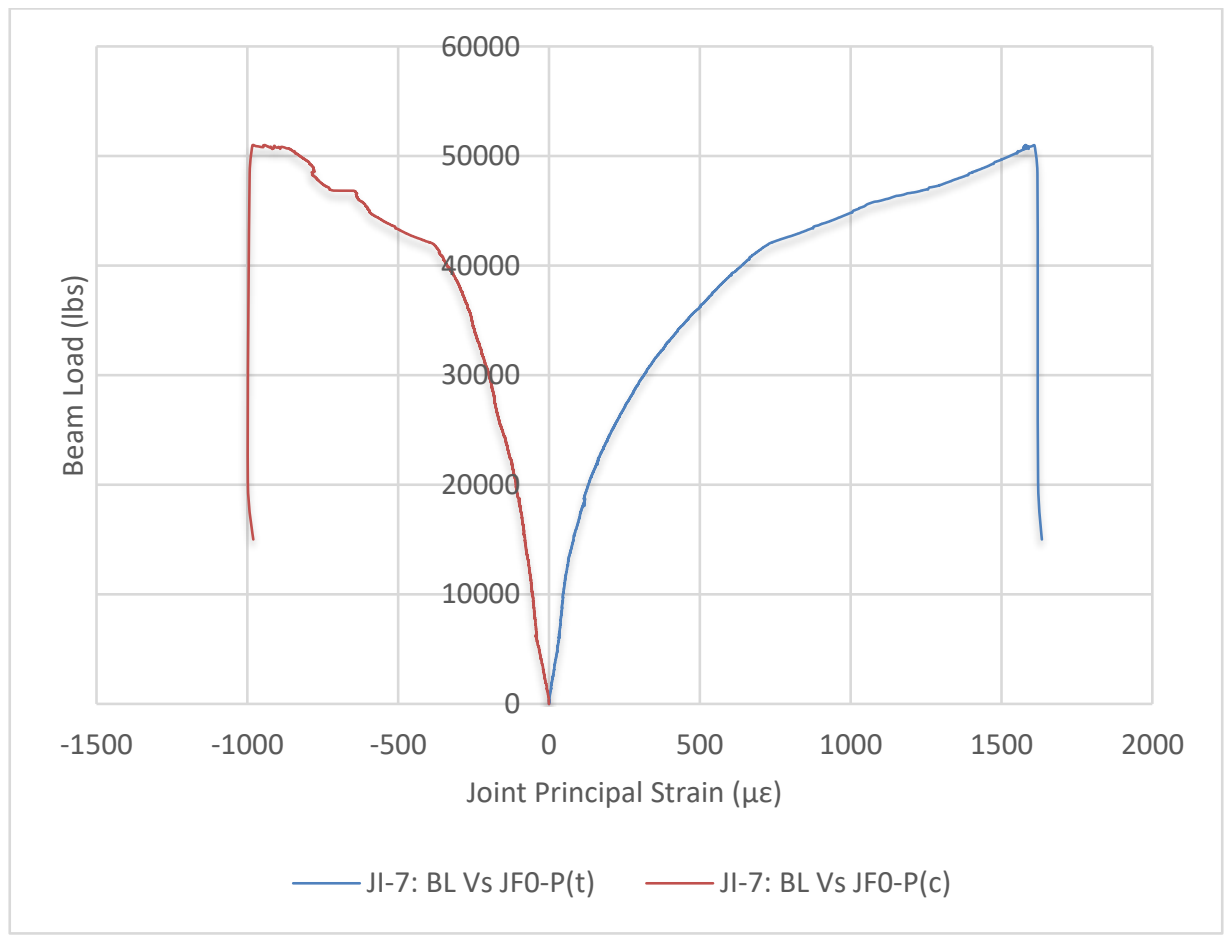

Figure 106. Joint principal strains for JI-7

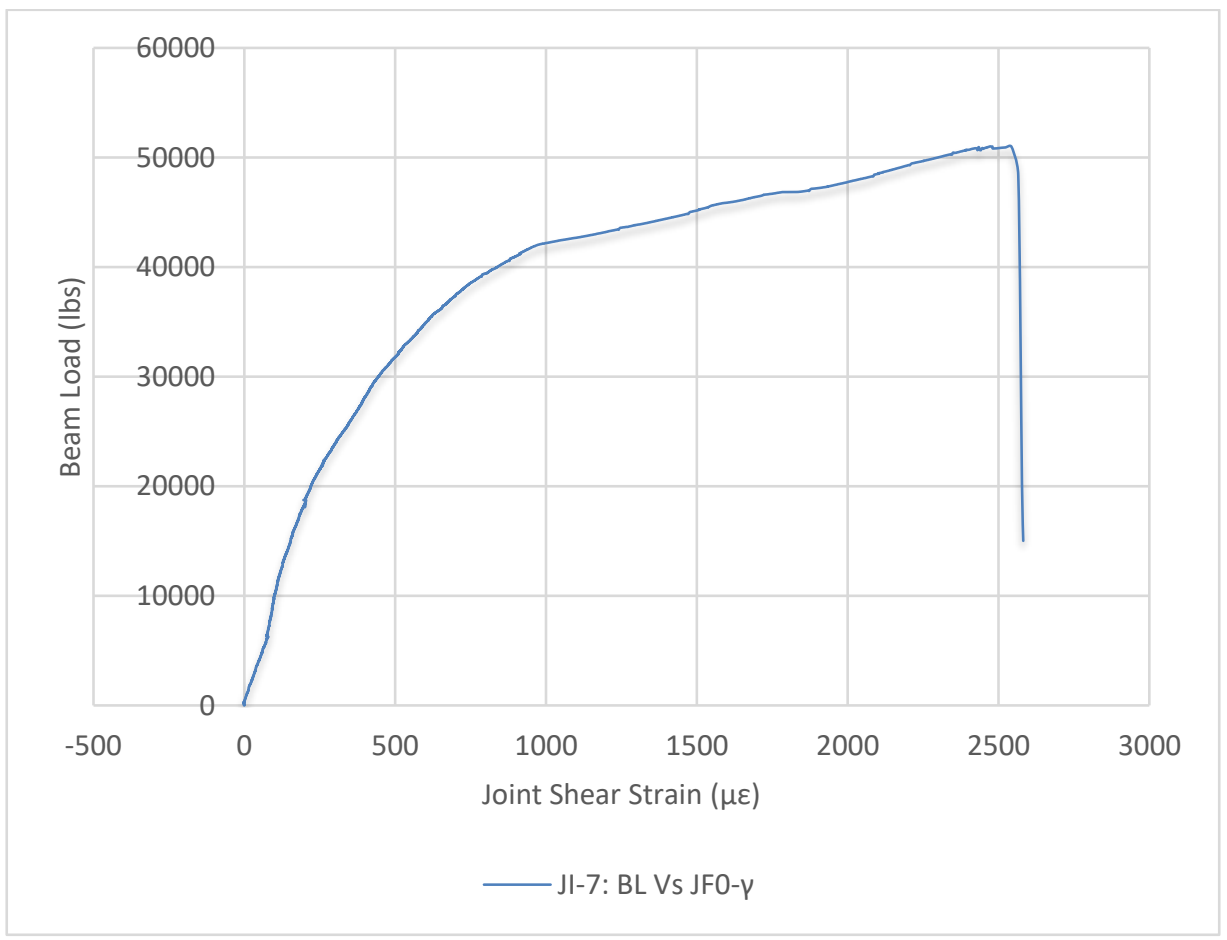

Figure 107. Joint shear strain for JI-7 


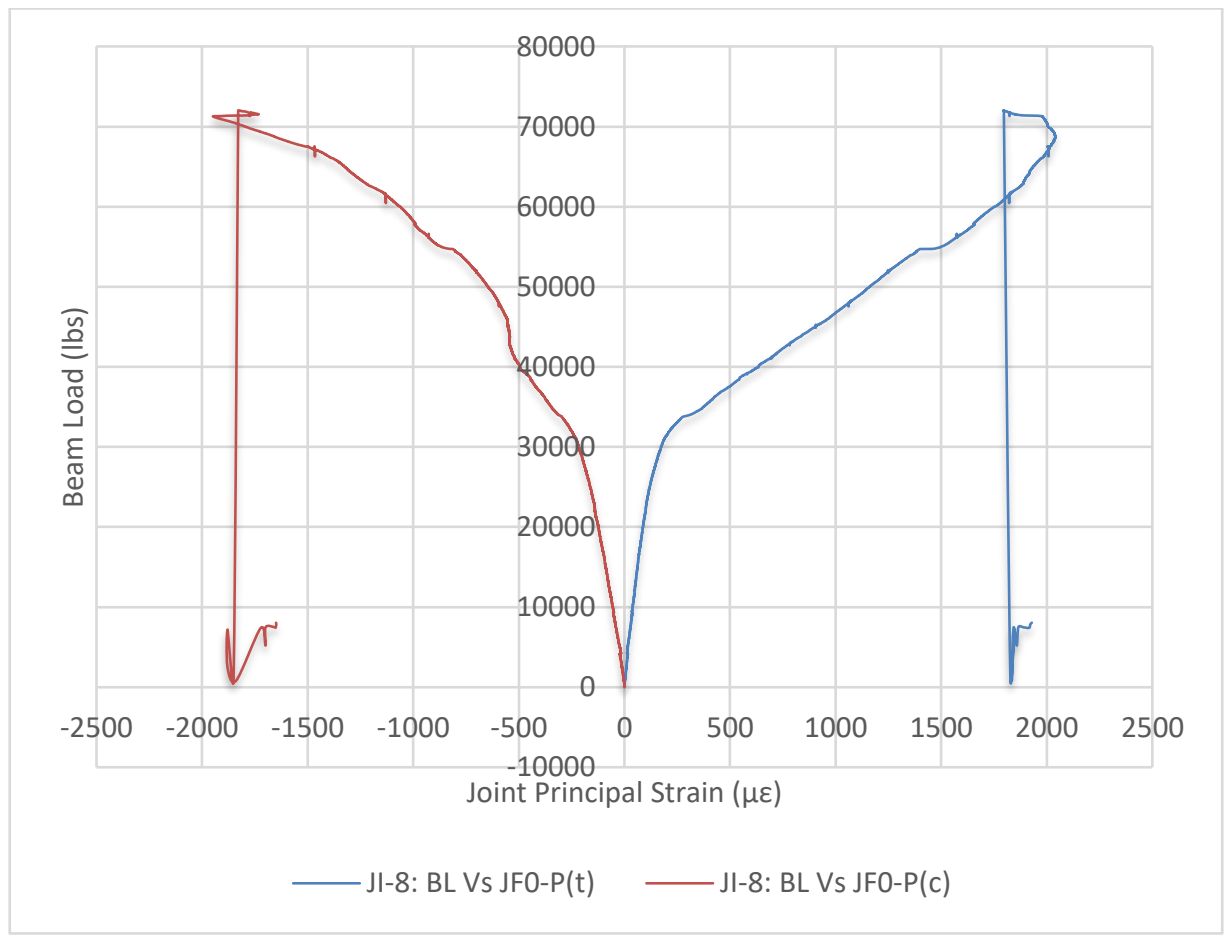

Figure 108. Joint principal strains for JI-8

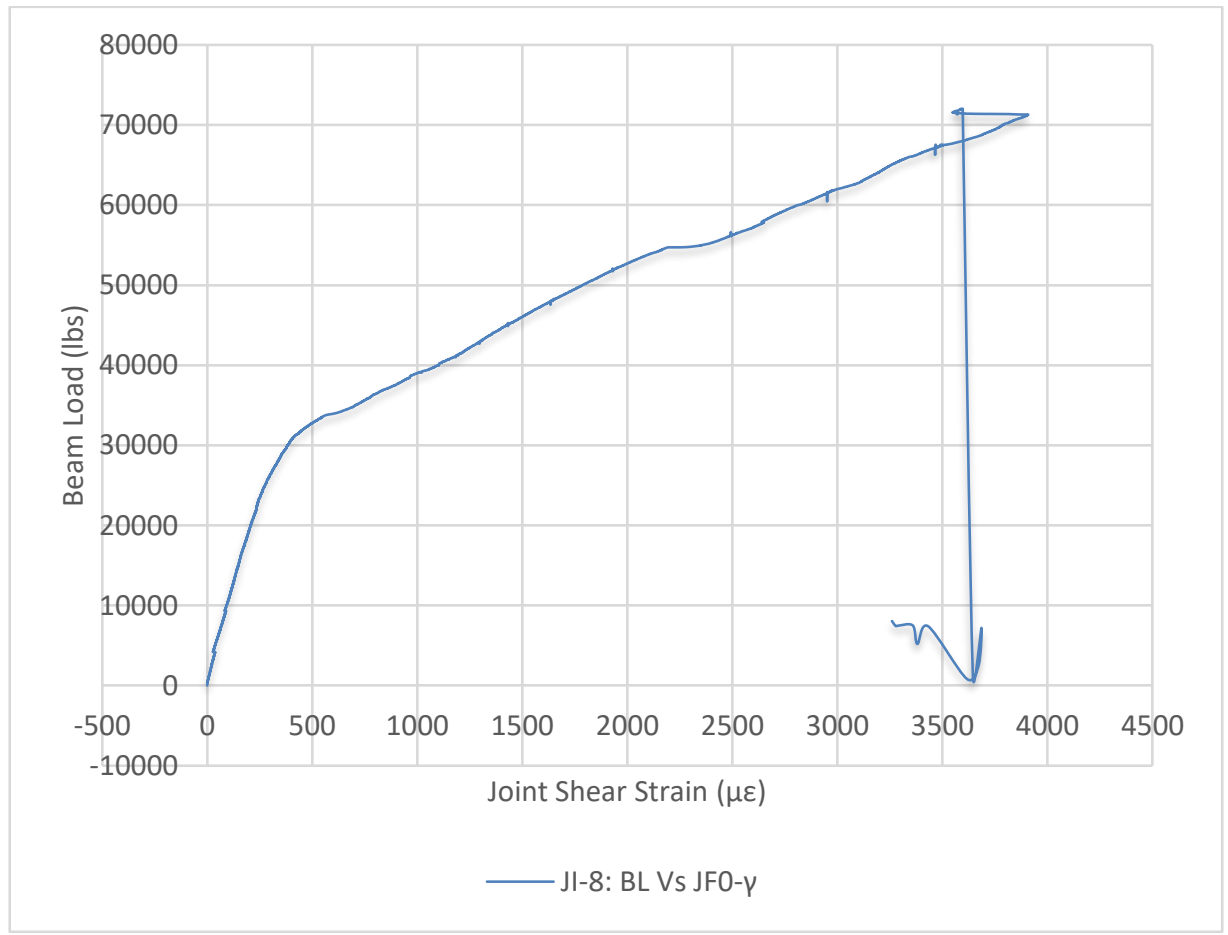

Figure 109. Joint shear strain for JI-8 


\subsubsection{Behavior of joints reinforced with concrete filler-modules and GFRP wraps (JIII-2, JIII-3, and JIII-4)}

Three joint specimens (JIII-2, JIII-3, and JIII-4) were tested in the current phase to evaluate the response of beam-column joints reinforced with concrete filler-modules and GFRP wraps. A summary of the experimental observations is presented in the following section.

1. The flexure-tension cracks appeared at the tip of filler-module when the concrete strains reached 184 - $374 \mu \varepsilon$. The k-factor for flexural-tension cracks was computed to be in the range of 10 - 21. Based on the author's evaluation of FRP wrapped cylinders under split tension test, reported in section 4.4.6, the tensile strength of FRP wrapped cylinders was noted to be $\sim(20-28) \sqrt{f_{c}^{\prime}}$. A summary of measured strains and computed k-factor is presented in Table 36.

2. As the loading increased resulting in internal stress redistribution, concrete cracking initiated in the top filler-module when the joint rebar strain reached $71-209 \mu \varepsilon$ and grew significantly when the strains ranged from 434 - $495 \mu \varepsilon$ (i.e., at this strain level in the rebar, concrete reached its ultimate tensile strain).

3. The test specimens reached peak loads of $\sim 53,072-77,391 \mathrm{lb}$ and failed in column shear. The measured strains in the longitudinal joint- and column- reinforcement were found to be in the range of $1,919-2,445 \mu \varepsilon$ and 1,623 - 2,573 $\mu \varepsilon$, respectively.

4. A summary of joint principal tensile- and shear- strains is presented in Table 37 and graphically shown in Figures 113 through 118.

5. Comparison of JIII-2 and JIII-3 revealed that the curvilinear filler-module exhibited better performance in redistributing the stresses around the joint panel, up to point $\mathrm{B}$. This is because the joint response up to point $\mathrm{A}$, and perhaps up to $\mathrm{B}$, is governed by the top fillermodule. Once the contribution of the top filler-module gets nearly ineffective beyond point $\mathrm{B}$, the compression force is resisted by the bottom filler-module until it helps form a strut mechanism. Since the curvilinear filler-module has reduced thickness at its legs, it does not form an efficient strut (reduced width at its throat). Such strut of ineffective force resistance results in a slightly lower strength and energy dissipation capacity compared to a specimen 
with wedge filler-module. The author believes that the performance of a joint reinforced with filler-modules and FRP wrap may be improved and/or optimized by providing a curvilinear filler-module on the tension side and wedge filler-module on the compression side.

6. Comparing JIII-3 with JIII-4 revealed that the specimen JIII-4 with U-anchors around the filler-modules certainly pushed point B to form at a higher load level (from 33,103 - 37,392 lb) by delaying the local de-bond of FRP wrap along the longitudinal axis of top fillermodule, upon concrete cracking (internally) at point A. The increase in peak load from $\sim 57,882-77,391 \mathrm{lb}$ (JIII-3 vs. JIII-4) is attributed to the enhanced load resistance through strut formation from the bottom filler-module due to FRP confinement through a U-anchor. The delay in peak load, i.e., a longer plateau between points $\mathrm{B}$ and $\mathrm{C}$, ensures superior ductility and energy dissipation capacity.

7. Figures 110 through 112 show the final failure of all the test specimens. Specimen JIII-2 and JIII-3 failed in a column shear after delamination of FRP wrap along the longitudinal axis of filler-module followed by crushing of concrete in the top filler-module. Specimen JIII-4 failed in column shear by the rupture of FRP wrapped around the column. Delamination of FRP from the surfaces of filler-module was prevented because of the Uanchors.

Table 36. Modulus of rupture of joints with filler-modules and GFRP wraps

Joint Beam load (lb) Beam rebar strain $(\mu \varepsilon) \quad$ Concrete strain $(\mu \varepsilon) \quad k-f_{r}$

\begin{tabular}{ccccc}
\hline \multirow{2}{*}{ JIII-1 } & 2,014 & 91 & 137 & 8 \\
\hline \multirow{2}{*}{ JIII-2 } & 4,016 & 119 & 178 & 10 \\
\hline \multirow{2}{*}{ JIII-3 } & 7,512 & 153 & 230 & 13 \\
\cline { 2 - 5 } & 10,454 & 235 & 352 & 20 \\
\hline \multirow{2}{*}{ JIII-4 } & 9,318 & 190 & 285 & 16 \\
\cline { 2 - 5 } & 5,363 & 232 & 349 & 20 \\
\hline 9,800 & 123 & 184 & $10^{+}$ \\
\hline
\end{tabular}

${ }^{+}$Poor result may be attributed to local de-bond of FRP at the tip of filler-module at low load levels. 
Table 37. Summary of experimental results of joints with filler-modules and GFRP wraps

\begin{tabular}{|c|c|c|c|c|c|c|c|c|c|c|c|c|}
\hline \multirow{2}{*}{ Joint } & \multirow{2}{*}{ PoI } & \multirow{2}{*}{$\begin{array}{c}\text { Beam load } \\
\text { (lb) }\end{array}$} & \multicolumn{3}{|c|}{ Joint principal strain $(\mu \varepsilon)$} & \multicolumn{2}{|c|}{ Limit state } & \multicolumn{4}{|c|}{ Steel rebar strain $(\mu \varepsilon)$} & \multirow{2}{*}{$\begin{array}{c}\text { Observed } \\
\text { failure mode }\end{array}$} \\
\hline & & & $\mathrm{P}(\mathrm{t})$ & $\mathrm{P}(\mathrm{c})$ & $\gamma$ & $\mathrm{k}-\mathrm{P}(\mathrm{t})$ & $\mathrm{k}-\gamma$ & JSH1 & BSL1 & BSL2 & CSL2 & \\
\hline \multirow{3}{*}{ JIII-1 } & $\mathrm{A}$ & 7,076 & 70 & -16 & 86 & 4.0 & 2.1 & 99 & 696 & 261 & 73 & \multirow{3}{*}{ Joint/shear } \\
\hline & B & 15,658 & 91 & -142 & 72 & 5.2 & 1.8 & 1,023 & 1,646 & 829 & 212 & \\
\hline & $\mathrm{C}$ & 24,740 & - & - & - & N.A. & N.A. & 1,573 & 2,535 & 1,535 & 1,171 & \\
\hline \multirow{3}{*}{ JIII-2 } & A & 21,479 & 49 & -116 & 151 & 2.78 & 3.64 & 209 & 938 & 654 & 31 & \multirow{3}{*}{ Column shear } \\
\hline & $\mathrm{B}$ & 27,364 & 100 & -174 & 260 & 5.68 & 6.27 & 487 & 1,264 & 874 & 153 & \\
\hline & $\mathrm{C}$ & 53,072 & 3,763 & 516 & 2,066 & N.A. & N.A. & 2,445 & 3,103 & 1,831 & 2,573 & \\
\hline \multirow{3}{*}{ JIII-3 } & $\mathrm{A}$ & 26,502 & 146 & -207 & 353 & 8.35 & 8.54 & 153 & 697 & 789 & 24 & \multirow{3}{*}{ Column shear } \\
\hline & B & 33,103 & 213 & -301 & 514 & 12.13 & 12.40 & 434 & 1103 & 1,016 & 92 & \\
\hline & $\mathrm{C}$ & 57,882 & 4,923 & $-1,576$ & 6,377 & N.A. & N.A. & 1,919 & 2,223 & 1,946 & 2,009 & \\
\hline \multirow{3}{*}{ JIII-4 } & A & 23,761 & 104 & -109 & 209 & 5.91 & 5.04 & 71 & 480 & 770 & -9 & \multirow{3}{*}{ Column shear } \\
\hline & B & 37,392 & 219 & -201 & 408 & 12.50 & 9.85 & 495 & 1,139 & 1,288 & 195 & \\
\hline & $\mathrm{C}$ & 77,391 & 6,003 & -315 & 6,046 & N.A. & N.A. & 2,087 & 9,091 & 6,930 & 1,623 & \\
\hline
\end{tabular}

PoI: Point of interest; $\mathrm{P}(\mathrm{t})$ : principal tensile strain; $\mathrm{P}(\mathrm{c})$ : principal compressive strain; $\gamma$ : shear strain

Note:

1. Principal strains are computed based on Eq. 4.1

2. Shear strains are computed based on Eq. 4.3

3. Upon concrete cracking (i.e., after point B), the stress distribution becomes increasingly complex, and hence, the Mohr's circle concept is not adequate to determine the principal stresses beyond this point. 


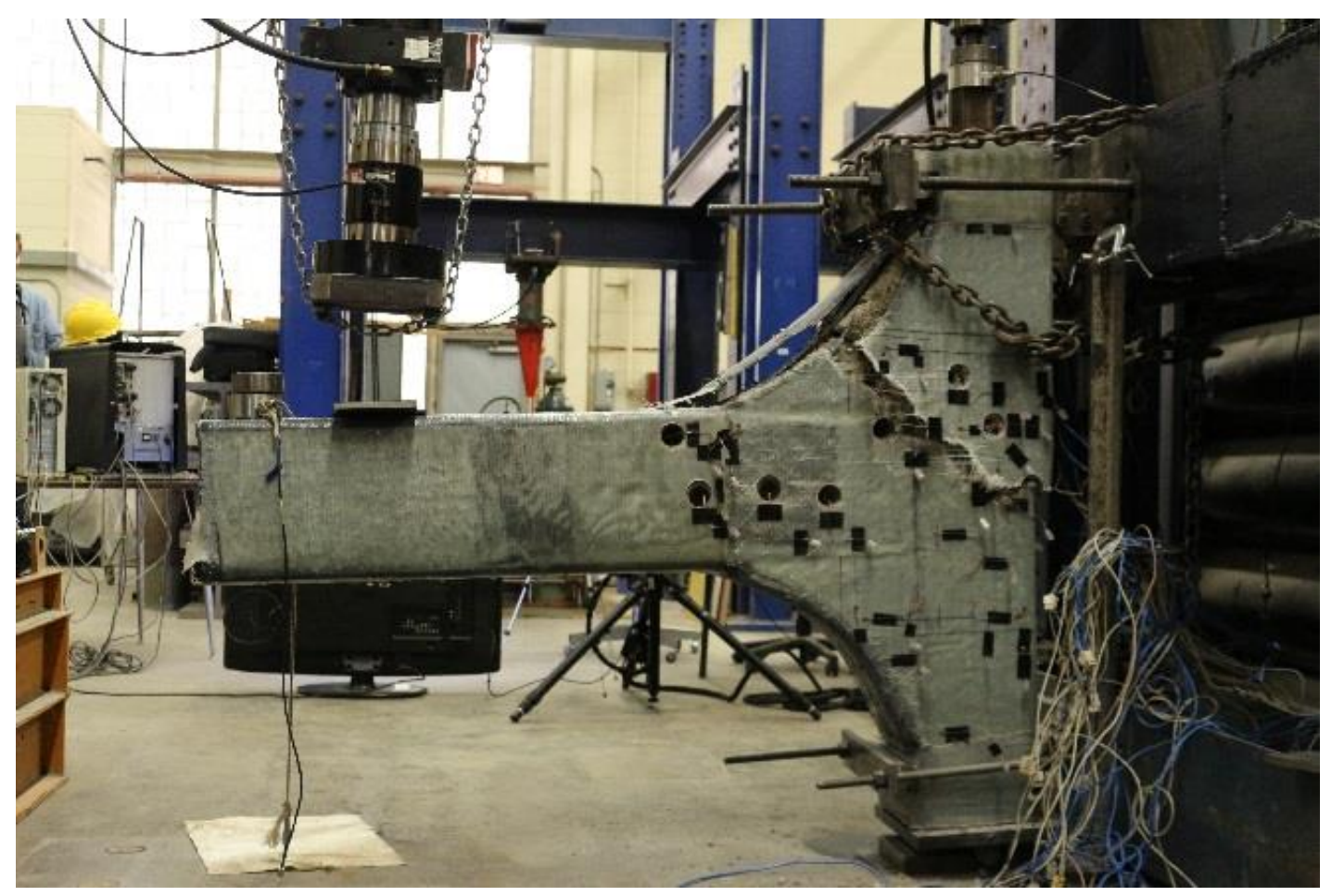

Figure 110. Failure of JIII-2

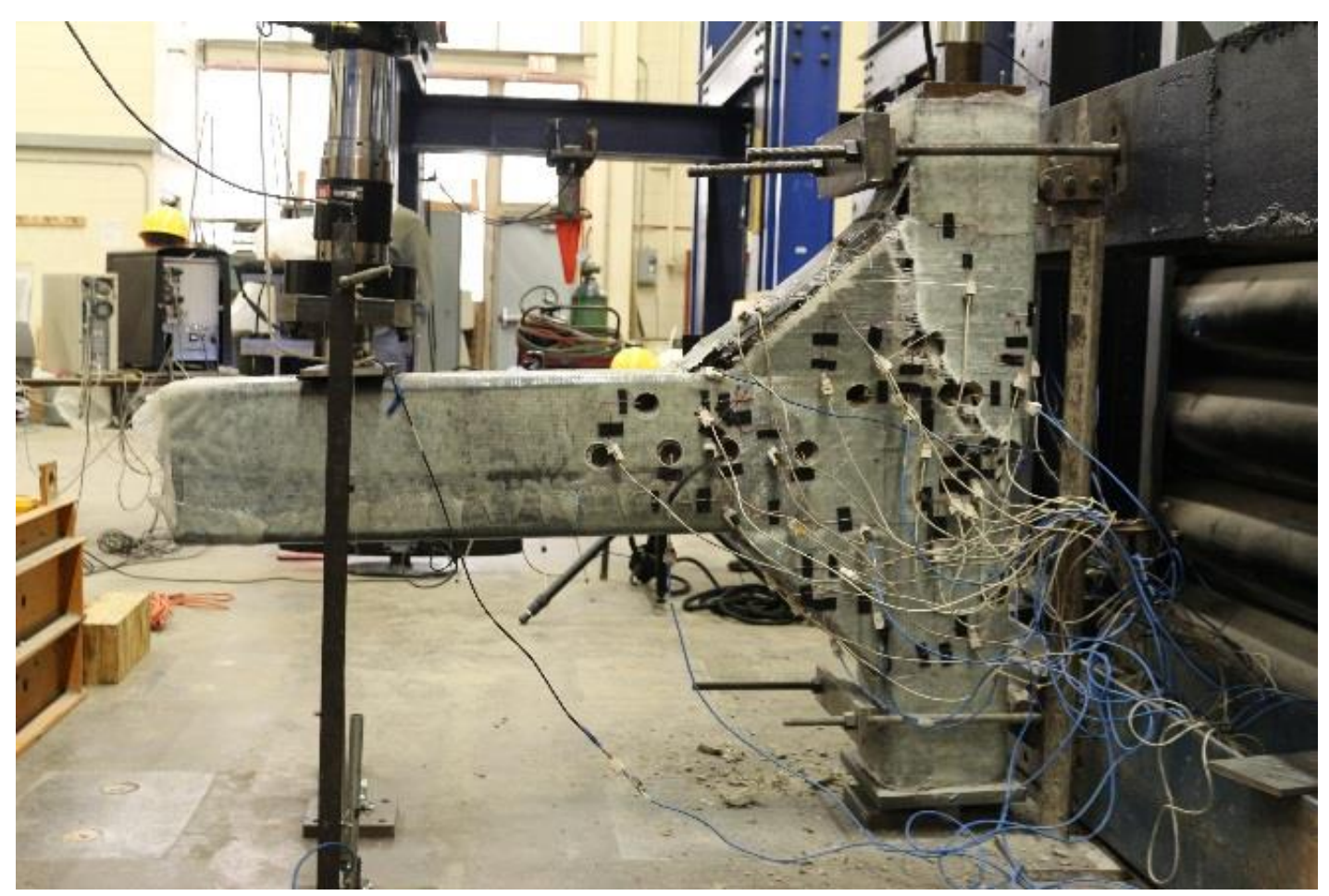

Figure 111. Failure of JIII-3 


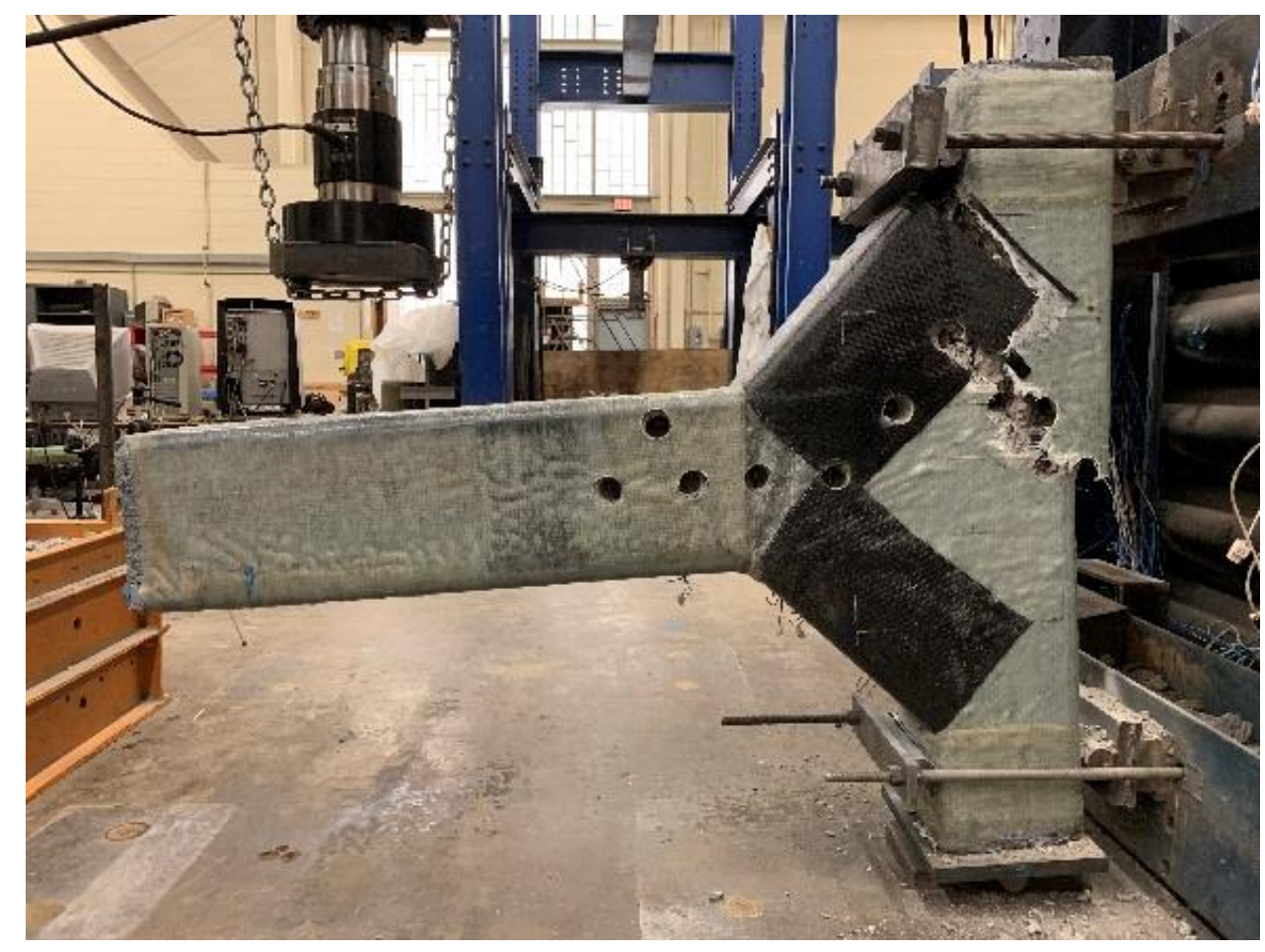

Figure 112. Failure of JIII-4 


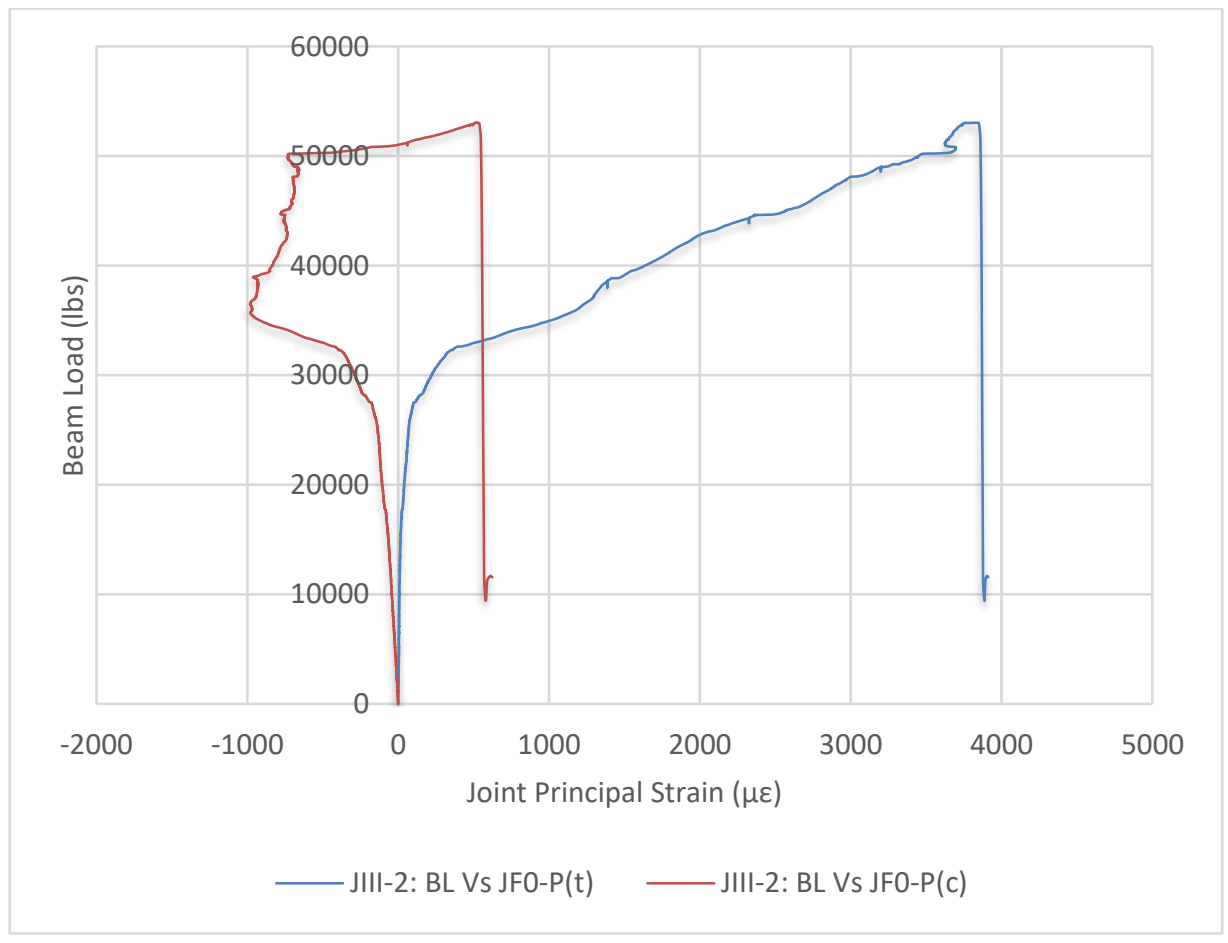

Figure 113. Joint principal strains for JIII-2

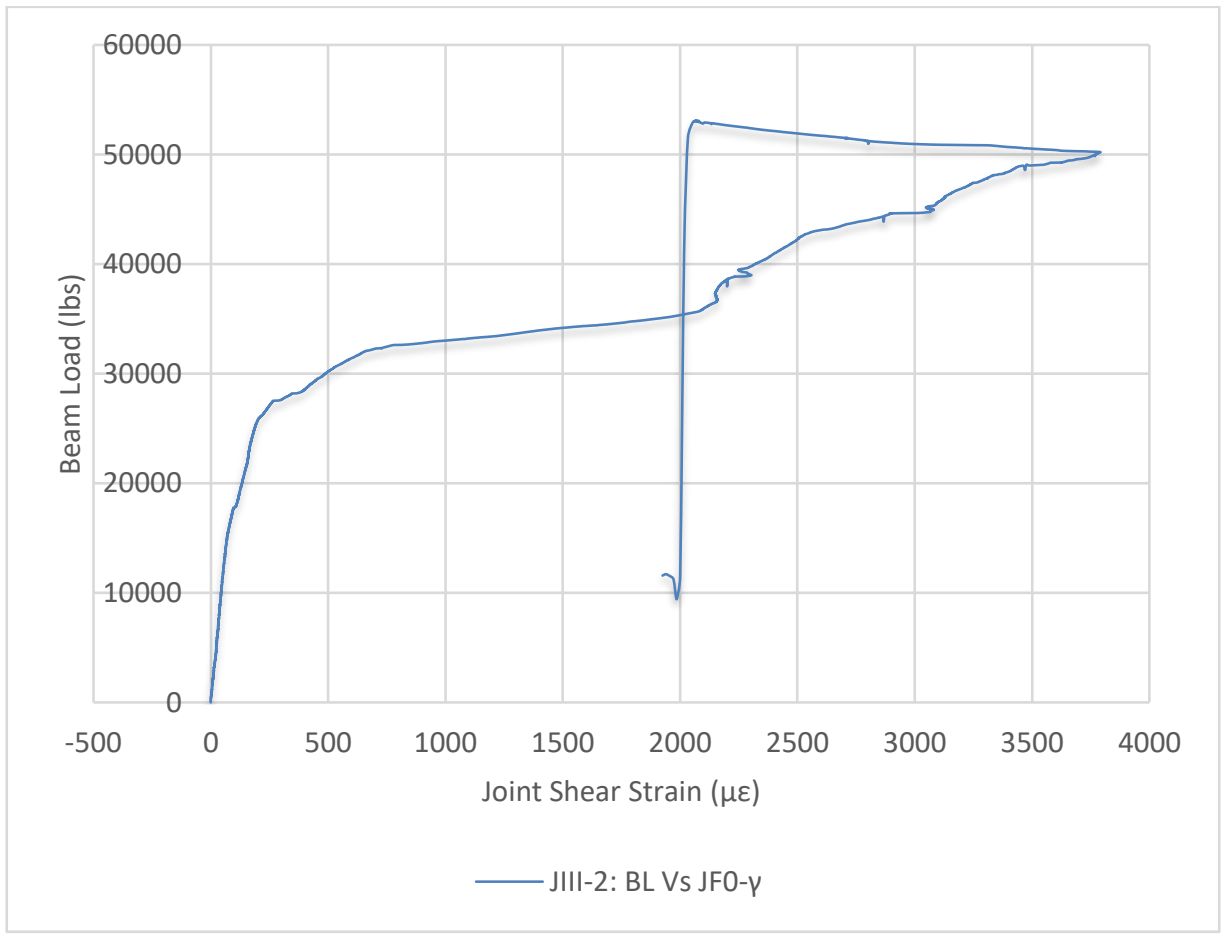

Figure 114. Joint shear strain for JIII-2 


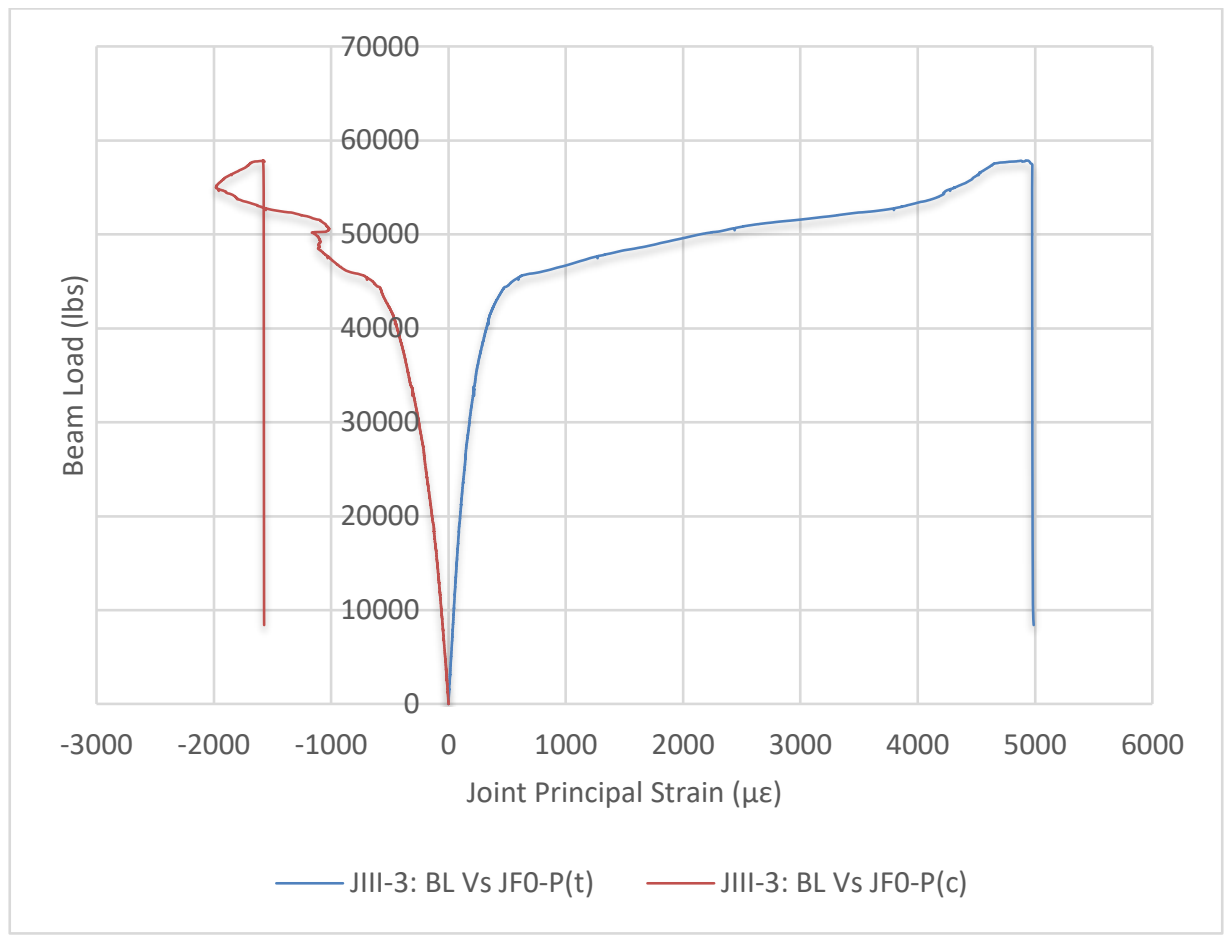

Figure 115. Joint principal strains for JIII-3

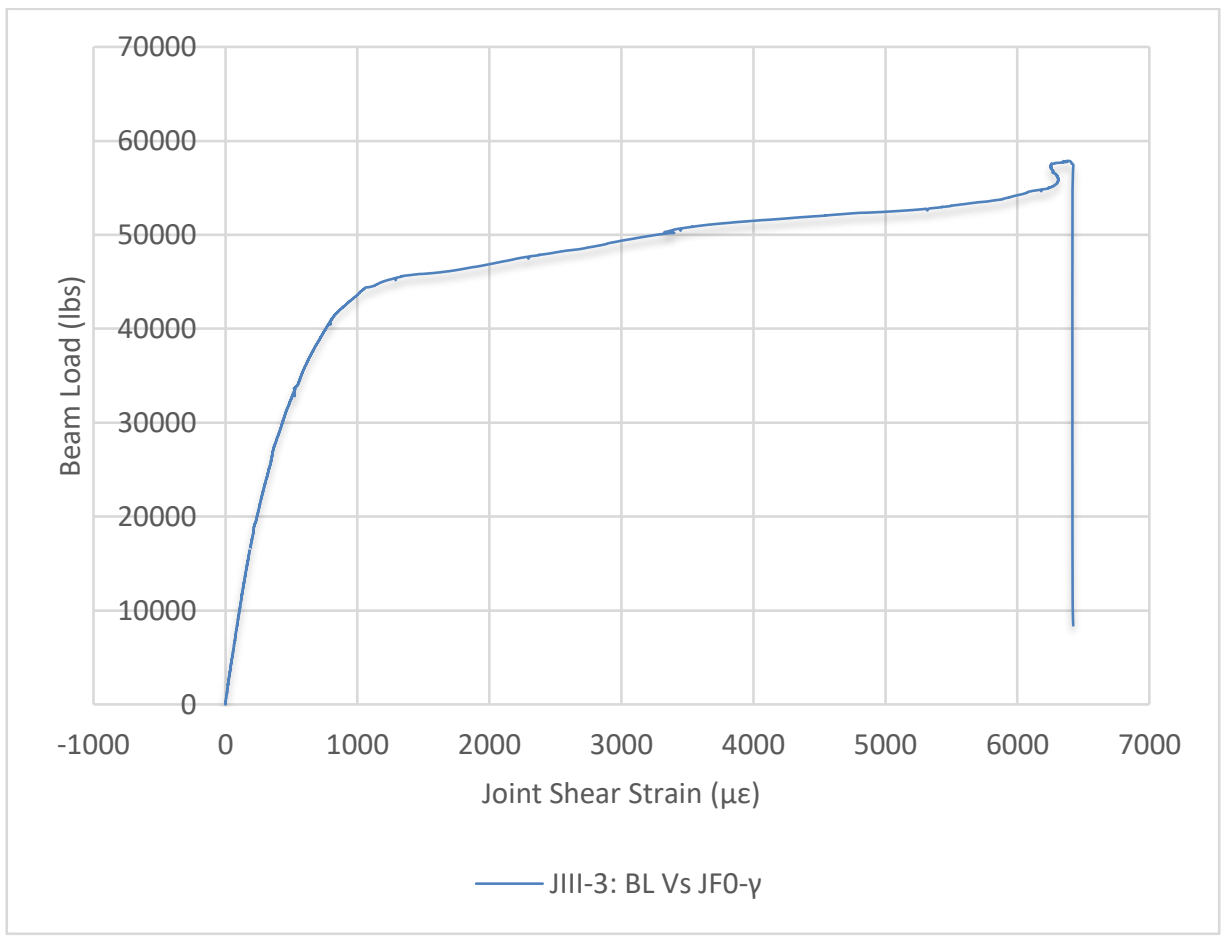

Figure 116. Joint shear strain for JIII-3 


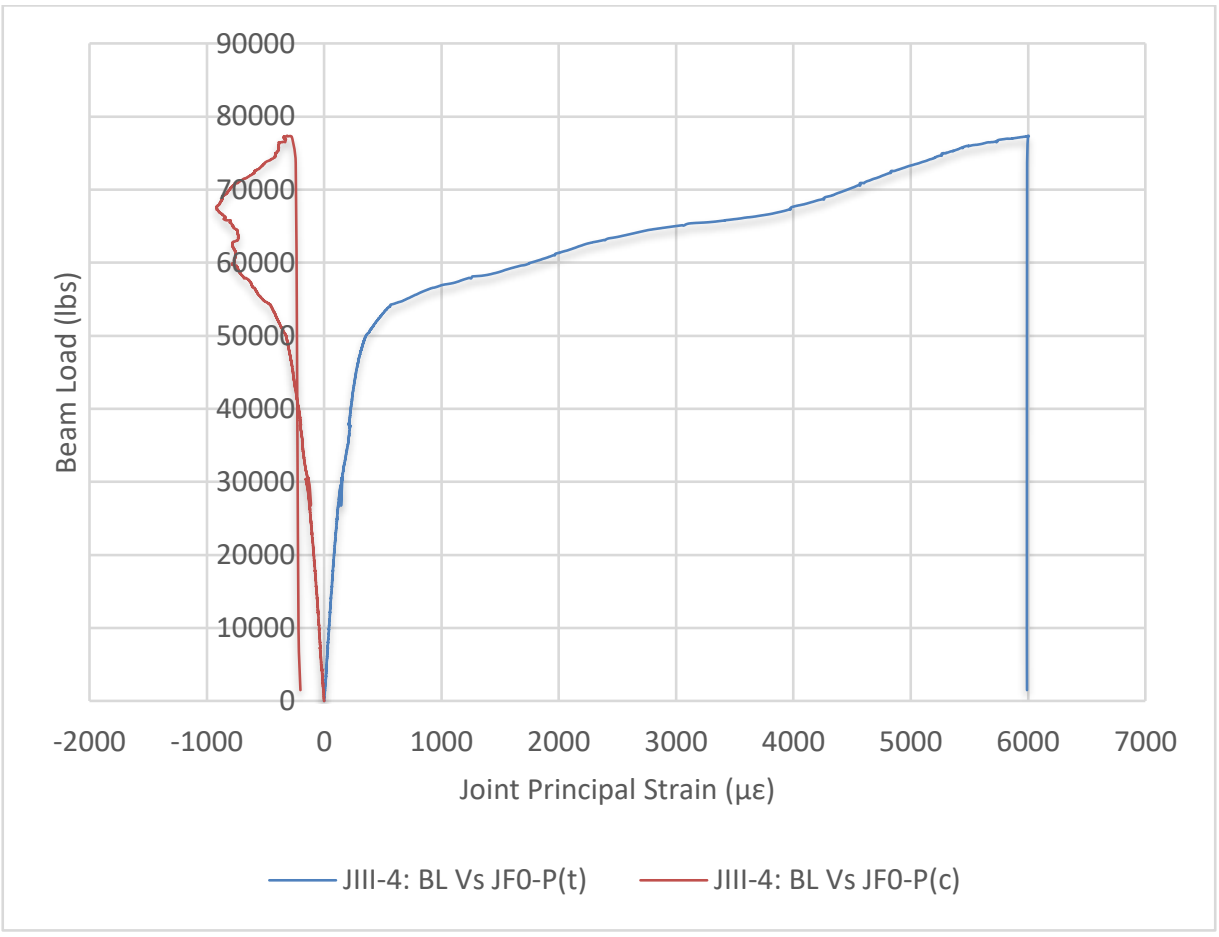

Figure 117. Joint principal strains for JIII-4

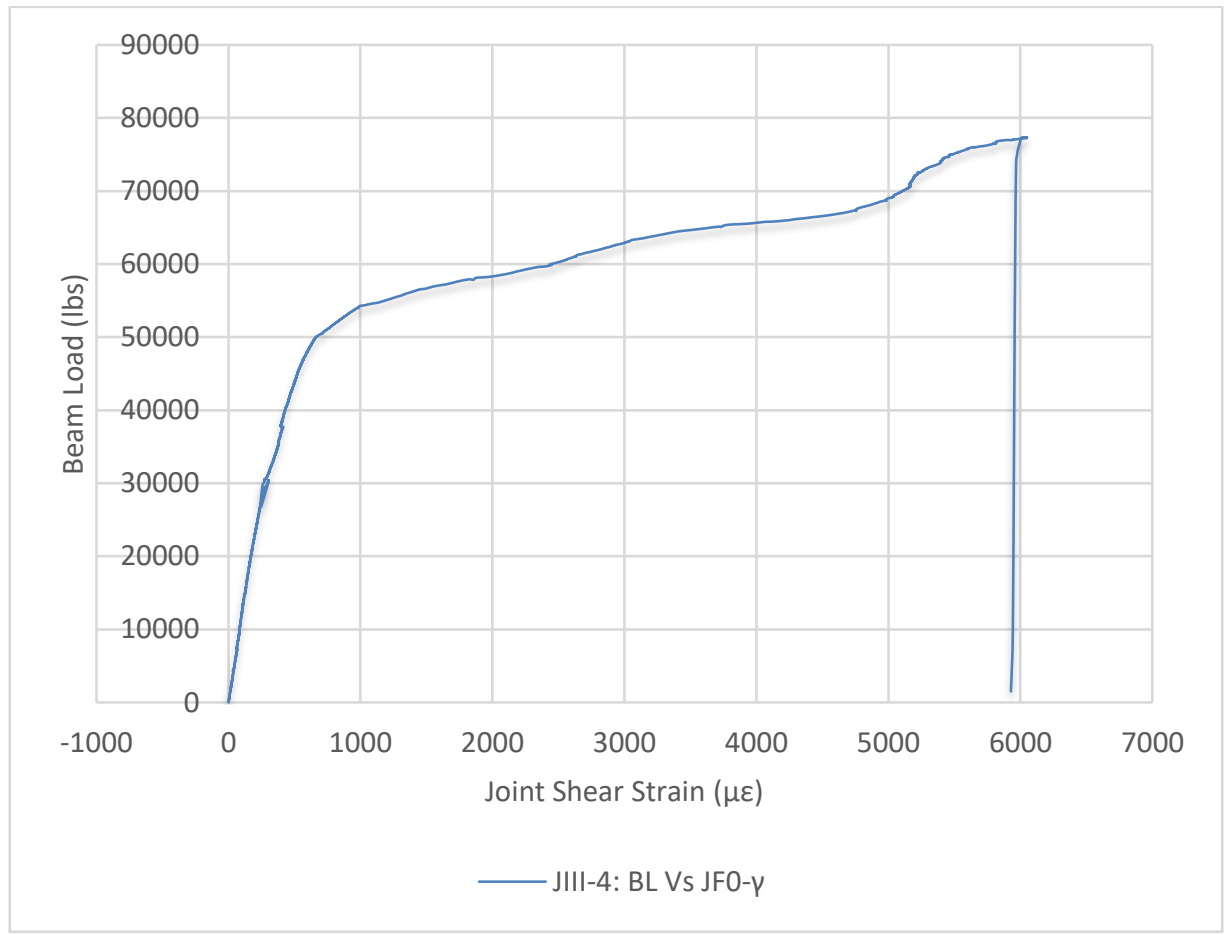

Figure 118. Joint shear strain for JIII-4 


\subsection{Discussion of Test Results}

The primary objective of the experimental program was to evaluate the structural response (strength, stiffness, strain distribution, ductility index, energy dissipation, and failure mode) of joints reinforced with filler-modules with/out FRP wraps or gussets. Among the above responses, ductility index and energy dissipation capabilities are especially important while designing structures to resist extreme events such as earthquakes, wind, blast, and other dynamic forces. Therefore, the following sections 5.3.1 through 5.3.6 discussed the structural responses of reinforced joint specimens by comparing their behavior with the corresponding control specimens.

\subsubsection{Strength}

The load versus deflection responses of twelve joint specimens evaluated in the current study are shown in Figures 119 through 121. The loading was terminated when the test specimens either exhibited a sudden failure through the crushing of concrete or complete debonding of FRP wraps/gussets from the substrate, leading a sudden drop in the load-carrying capacity. A summary of the experimental results, including percentage increases with respect to corresponding control specimen and the change of failure modes of joints with concrete/foam filler-modules coupled with FRP wraps/gussets, is noted in Table 38.

All the joint reinforcing schemes were effective in enhancing the load-carrying capacity to as high as $314 \%$ over the control specimen. Specimens reinforced with filler-modules and FRP wraps/gussets exhibited higher increases in load-carrying capacity ( 115-314\%), while specimens reinforced with filler-modules alone have resulted in a nominal increase $(\sim 45 \%)$. The increase in load-carrying capacity of reinforced joints was governed by the rupture strength of strengthened beam or column member and the ability of rebar to develop plastic strains under confined conditions, at the location of hinge formation. For example, beam rebar of specimens JI-8 and JIII4, presented in Table 42, exceeded the plastic strain limit and exhibited the highest strength increases in the corresponding batches. As shown in Figure 119, deflections measured at the ultimate/rupture of reinforced joints was as high as $5.6 \mathrm{in}$. compared to $1.8 \mathrm{in}$. for a control specimen, indicating an extended inelastic zone, resulting in superior strength, ductility and energy dissipation capacities. 


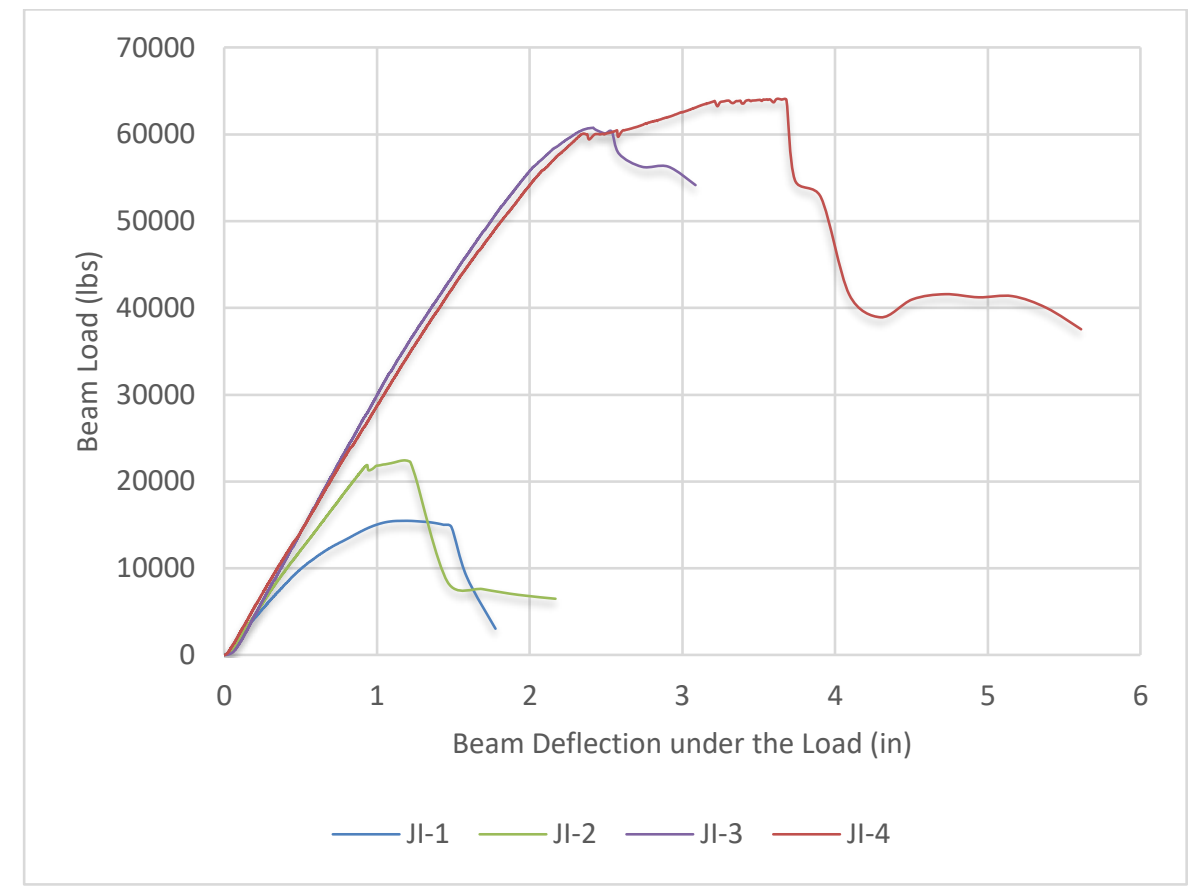

Figure 119. Load vs. deflection response of joints with filler-modules with/out CFRP wraps

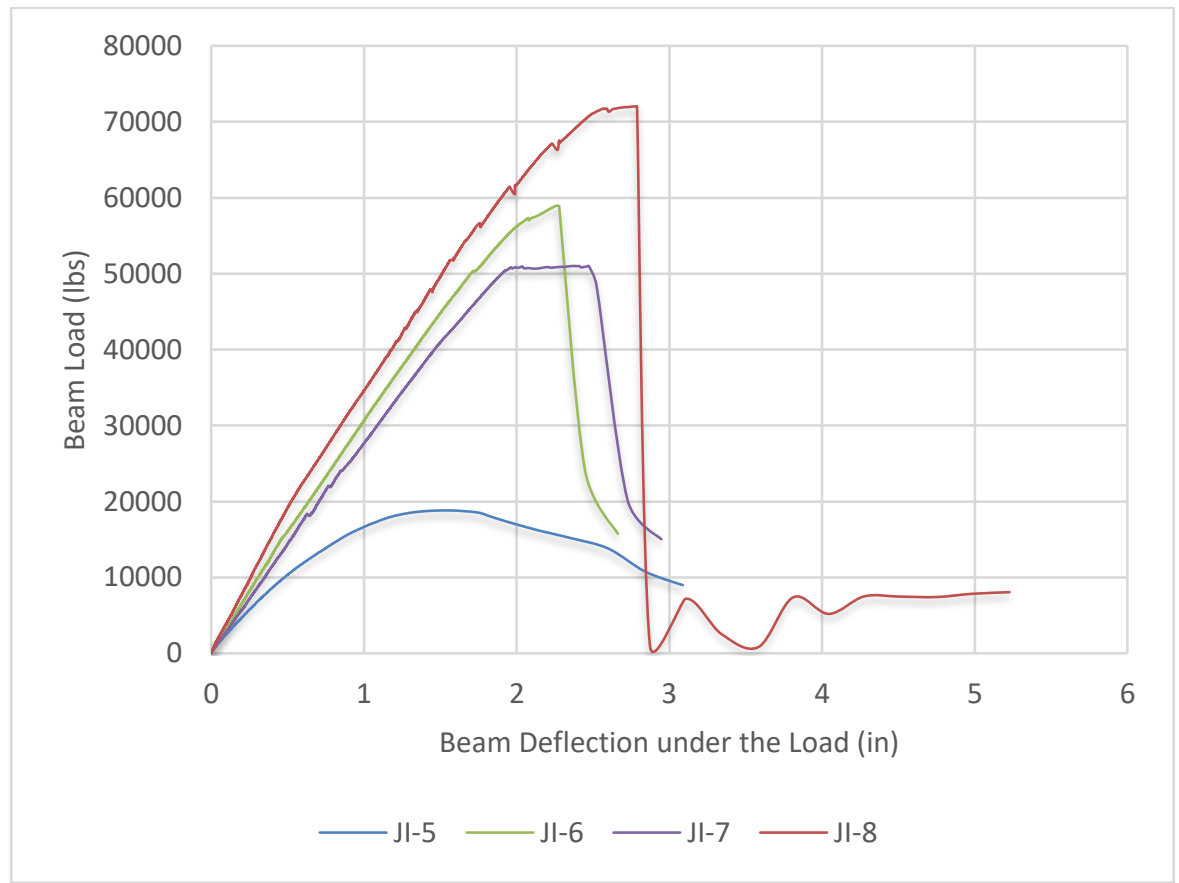

Figure 120. Load vs. deflection response of joints with filler-modules and CFRP gussets 


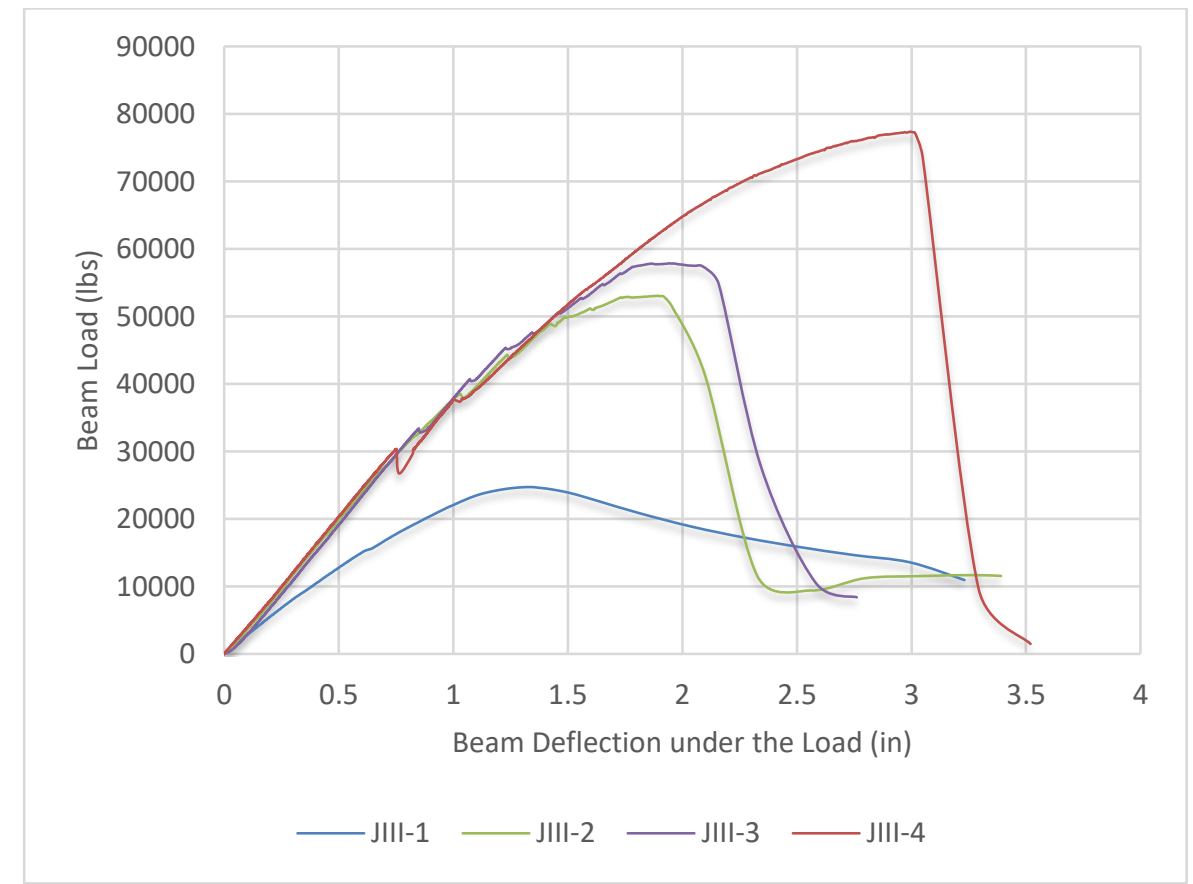

Figure 121. Load vs. deflection response of joints with filler-modules and GFRP wraps

Table 38. Load vs. deflection response of joints with filler-modules and FRP wraps/gussets

\begin{tabular}{ccccc}
\hline \multirow{2}{*}{ Joint } & \multicolumn{3}{c}{ Measured at Peak load } & \multirow{2}{*}{ Observed failure mode } \\
\cline { 2 - 5 } & Beam load (lb) & \% increase & $\Delta$ (in) & \\
\hline JI-1 & 15,471 & Control & 1.2 & Joint compression \\
\hline JI-2 & 22,434 & 45 & 1.2 & Column shear \\
\hline JI-3 & 60,773 & 293 & 2.4 & Beam flexure \\
\hline JI-4 & 64,107 & 314 & 3.6 & Beam flexure \\
\hline JI-5 & 18,819 & Control & 1.5 & Joint diagonal tension \\
\hline JI-6 & 58,961 & 213 & 2.3 & Column shear \\
\hline JII-7 & 51,018 & 171 & 2.4 & Column shear \\
\hline JII-8 & 72,040 & 283 & 2.8 & Column shear \\
\hline JIII-1 & 24,740 & Control & 1.3 & Joint diagonal tension \\
\hline JIII-2 & 53,072 & 115 & 1.9 & Column shear \\
\hline JIII-3 & 57,882 & 134 & 1.9 & Column shear \\
\hline JIII-4 & 77,391 & 213 & 3.0 & Column shear \\
\hline
\end{tabular}




\subsubsection{Failure mode}

As identified in Table 38, all the control specimens (JI-1, JI-5, and JIII-1) exhibited brittle joint shear failure in concrete without the yielding of the beam or column rebars. Specimens JI-5 and JIII-1 exhibited diagonal tension failure while specimen JI-1 failed through the crushing of concrete (compression) in the joint core. The high axial load applied to the column of JI-1 did not allow the concrete to reach its full tensile capacity due to additional compressive (confinement) stresses induced on the joint panel, thus leading to the crushing of concrete. Although the failure of specimen JI-5 Figure 90 initiated through diagonal tension in the joint panel, it appears that the column portion above the joint panel (next to the top filler-module) has reached its shear capacity at the ultimate axial and bending load combinations. The newly developed joint reinforcement schemes with filler-modules with/out FRP wraps/gussets were effective in forcing the formation of plastic hinge away from the joint panel while ensuring the occurrence of the final failure in the column or beam sections. Furthermore, specimens (JI-3 and JI-4) reinforced with $360^{\circ}$-diagonal anchors around the joint exhibited beam flexural failure (Figure 93) as opposed to column shear failure (Figure 112) in specimens that are reinforced with no- or partial U-anchors. As shown in Figure 122, the buckling of longitudinal rebars in columns or beams was observed in all the reinforced joints depending upon the location of the final failure. Addition of filler-modules and FRP wraps/gussets enhanced the joint capacity well above the beam or column capacity; hence the failure was shifted to weakest member, i.e., column or beam meeting at a joint. To avoid catastrophic collapse, the optimal joint capacity with filler-modules and FRP wraps/gussets must be established to balance the capacities of column, beam, and joint. 


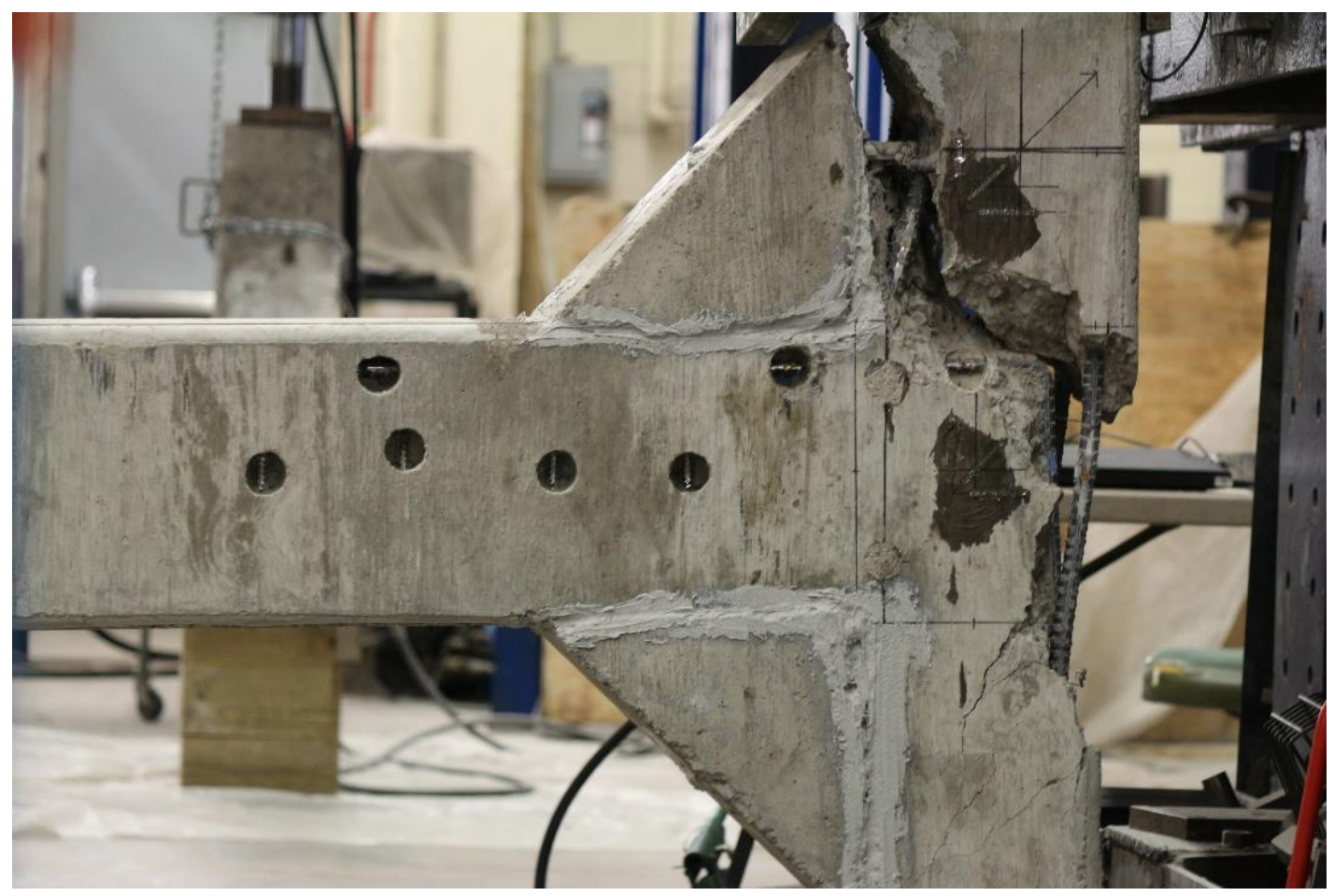

Figure 122. Buckling of the column longitudinal rebars (JI-2) 


\subsubsection{Stiffness}

Stiffness is defined as the ability of a structural member to resist deformation (translational, rotational, and shear) under applied loads. In the present study, the stiffness (tangent modulus) of beam-column joint specimens was determined by considering the slope of the initial load vs. deflection plot passing through the origin and connecting to the $30 \%$ of peak load value. It is generally accepted that concrete exhibits linear stress-strain response until $30 \%$ of the peak compressive stress. Following the definition adopted in the current study, a summary of the test results is presented in Table 39.

Table 39. Stiffness of joints reinforced with filler-modules and FRP wraps/gussets

Joint Tangent modulus \% increase

\begin{tabular}{ccc}
\hline JII-1 & 21,223 & Control \\
\hline JII-2 & 24,101 & 14 \\
\hline JII-3 & 29,027 & 37 \\
\hline JII-4 & 28,821 & 36 \\
\hline JI-5 & 23,025 & Control \\
\hline JII-6 & 31,737 & 38 \\
\hline JII-7 & 28,835 & 25 \\
\hline JII-8 & 37,803 & 64 \\
\hline JIIII-1 & 27,268 & Control \\
\hline JIII-2 & 39,782 & 46 \\
\hline JIII-3 & 37,975 & 39 \\
\hline JIII-4 & 40,811 & 50
\end{tabular}

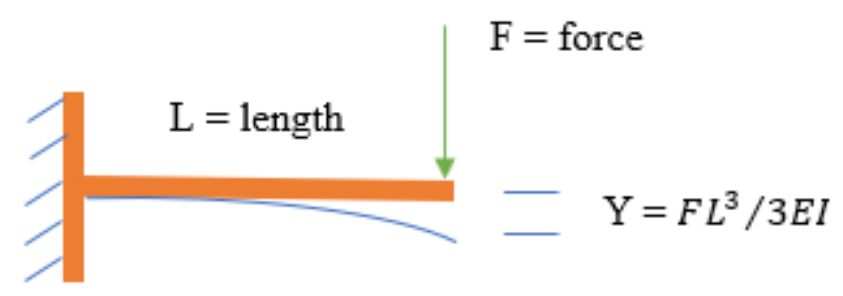

Figure 123. Cantilever beam with beam tip deflection 


$$
\begin{gathered}
k(\text { translational stiffness })=\frac{3 E I}{L^{3}} \\
k(\text { rotational stiffness })=\frac{E I}{L}
\end{gathered}
$$

As seen from Figure 123 and Eq. 5.1 and 5.2, the stiffness of a structural member depends on the modulus of elasticity (E) of a material, the moment of inertia (I) of a section under consideration, and the span length $(\mathrm{L})$. Based on the above understanding, the stiffness of a reinforced joint specimen is directly proportional to the material- and geometric- properties of the filler-module (depends on the $\mathrm{E}$ and I) and inversely proportional to the cube of the span length (depends on the location of hinge formation from the point of load application). Furthermore, the increase in stiffness also depends on the externally bonded FRP material properties and partially on the confinement effects from FRP and internal rebar cage.

As seen from Table 39, all the joint specimens reinforced with filler-modules with/out FRP wraps or gussets exhibited higher stiffness (up to 64\%) compared to the control specimen. The increase in stiffness is smaller ( $14 \%)$ for joints reinforced with filler modules alone, while it is higher in case of joints reinforced with high-strength concrete (9,545 psi) filler-modules and CFRP gussets ( 64\%). Comparing specimen JIII-3 (no anchor) and JIII-4 (with U-anchor) revealed that providing an anchor around the filler-module was influential in enhancing the stiffness of the joint specimen. Comparing specimen JI-6 with JI-7 revealed that bonding of FRP layers to the beam and column before gluing the filler-modules has a detrimental effect on the stiffness of joint specimen due to the potential slippage at the glue-line interface. Although an increase in the stiffness was noticed due to the addition of filler-modules, no consistent trend was noted in terms of material and geometric properties of filler-module because of the premature bond-slip at FRP/filler-module glue line. 


\subsubsection{Ductility index}

The ductility of a structural member is defined as its ability to undergo large inelastic deformation without significant loss in the load-carrying capacity. The ductility index is defined as the ratio of the ultimate displacement $\left(\Delta_{u}\right)$ to the yield displacement $\left(\Delta_{y}\right)$. Since concrete structures do not exhibit yield behavior, the ductility index based on the idealized yield concept, as illustrated by [63] has been adopted in this study. According to this concept, the ductility index is defined as the ratio of the anticipated level of displacement $\left(\Delta_{u}\right)$ to the displacement at idealized yield $\left(\Delta_{y i}\right)$.

$$
\mu=\Delta_{u} / \Delta_{y i}
$$

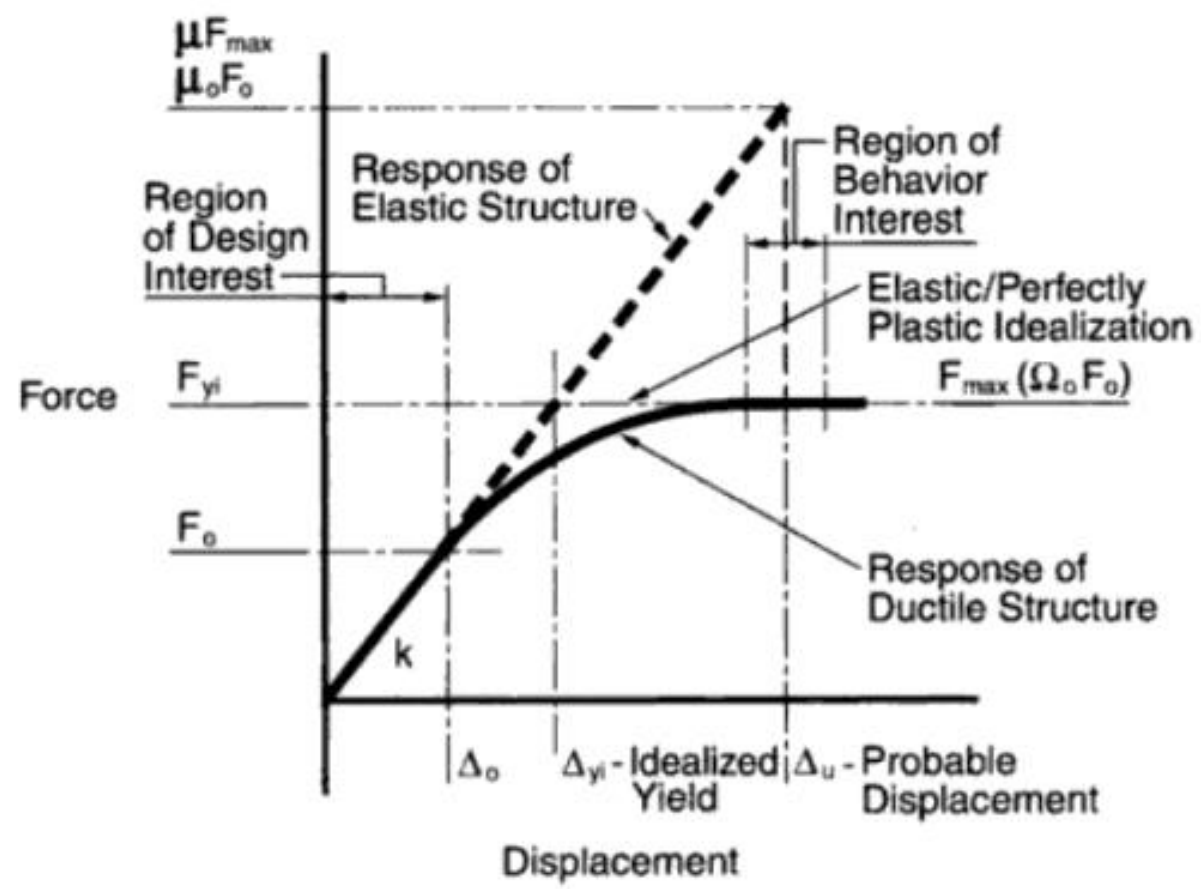

Figure 124. Force-displacement relationship of a ductile structure [63]

Based on the above concept, the ductility index in this study was computed as the ratio of the ultimate displacement (i.e., displacement at point C) to idealized displacement (i.e., displacement at point B). In order to evaluate the ductility enhancement of joints reinforced with filler-modules and FRP wraps or gussets, the idealized displacement of corresponding control specimen was 
considered as a reference point for all the joint specimens in a given batch. As per this definition, a summary of the ductility index for the twelve joint specimens is presented in Table 40.

Table 40. Ductility index of joints with filler-modules and FRP wraps/gussets

\begin{tabular}{ccccc}
\hline \multirow{2}{*}{ Joint } & \multicolumn{2}{c}{ Peak values } & Idealized yield & $\begin{array}{c}\text { Ductility index } \\
\end{array}$ \\
\cline { 2 - 4 } & Beam load (lb) & $\Delta_{u}$ (in) & $\Delta_{y i}($ in $)$ & $\left.\Delta_{u} / \Delta_{y i}\right)$ \\
\hline JI-1 & 15,471 & 1.2 & 0.6 & 2 \\
\hline JI-2 & 22,434 & 1.2 & 0.6 & 2 \\
\hline JI-3 & 60,773 & 2.4 & 0.6 & 4 \\
\hline JI-4 & 64,107 & 3.6 & 0.6 & 6 \\
\hline JI-5 & 18,819 & 1.5 & 0.5 & 3 \\
\hline JI-6 & 58,961 & 2.3 & 0.5 & 4 \\
\hline JI-7 & 51,018 & 2.4 & 0.5 & 4 \\
\hline JI-8 & 72,040 & 2.8 & 0.5 & 5 \\
\hline JIII-1 & 24,740 & 1.3 & 0.6 & 3 \\
\hline JIII-2 & 53,072 & 1.9 & 0.6 & 3 \\
\hline JIII-3 & 57,882 & 1.9 & 0.6 & 5 \\
\hline JIII-4 & 77,391 & 3.0 & 0.6 & \\
\hline
\end{tabular}

Note:

1. Typically, the ultimate displacement is taken at a $15 \%$ drop in the peak-load value. However, in this study, due to the lack of test data points beyond peak-load, the displacement at peak-load is considered as the ultimate displacement for computation of the ductility index.

The ductility index of reinforced joints was found to be as high as 6. It appears that reinforcing joint with filler-modules without FRP wraps (JI-2) did not offer any additional ductility beyond the control specimen (JI-1). The use of low-stiffness syntactic foam filler-module (JI-4) has profoundly influenced the enhancement of ductility index (6) compared to the concrete fillermodule of high stiffness (JI-3). The improvement in ductility index of JI-4 over JI-3 is due to the near-uniform strain distribution in and around the joint panel of JI-4, as identified in Table 42. Strengthening JI-8 with high-strength filler-modules (9,545 psi) exhibited a slightly higher ductility index (5) than JI-7 with medium-strength filler-modules (5,472 psi). The improved behavior of JI-8 over JI-7 is primarily attributed to the additional strut resistance offered by the 
higher strength filler-module (in the compression zone) in the inelastic region, i.e., between points $B$ and $C$. The shape of the filler-modules, i.e., curve vs. wedge did not influence the ductility response of joint specimens. The confinement effect from the $\mathrm{U}$-anchor on the compression fillermodule of JIII-4 has provided additional strut resistance between points B and C, thus resulting in a higher ductility index. 


\subsubsection{Energy dissipation}

Energy dissipation capability is an essential characteristic that a structure must exhibit when subjected to extreme events such as hurricanes and seismic forces. The energy dissipation capacity is computed as the area enclosed inside the load $(\mathrm{P})$ vs. deflection $(\Delta)$ plot. The summation of consecutive areas computed at regular intervals of 0.1 in. deflection, measured up to the $15 \%$ drop in the peak-load, is defined as cumulative energy dissipation. However, in this study, the cumulative energy dissipation capacity of joint specimens is computed only up to the peak load. Therefore, it must be highlighted that there is a significant amount of energy dissipation (essential to prevent catastrophic failures) even beyond the peak load, as seen from load vs. deflection plots. The cumulative energy dissipation curves of all the twelve specimens are shown in Figures 125 through 127, and a summary is presented in Table 41.

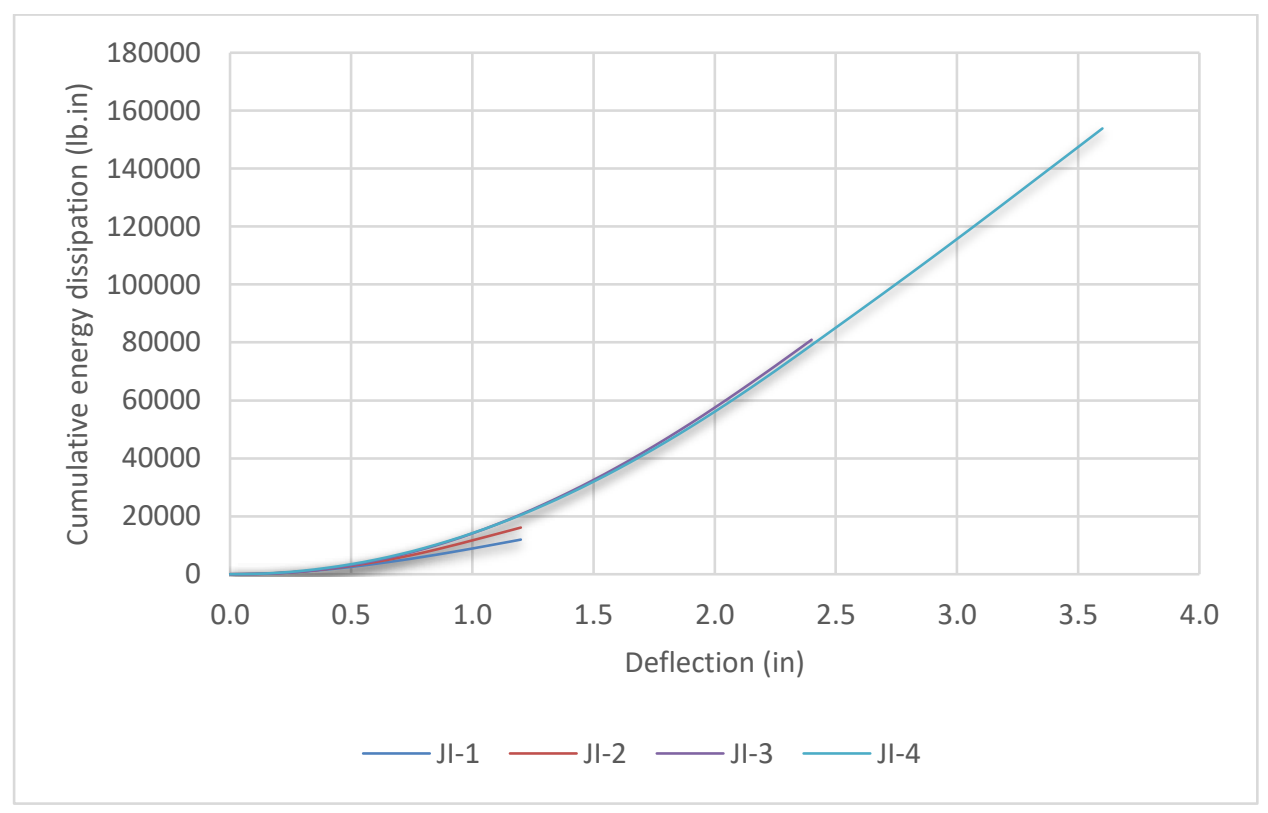

Figure 125. Energy dissipation capacity of joints with filler-modules and CFRP wraps 


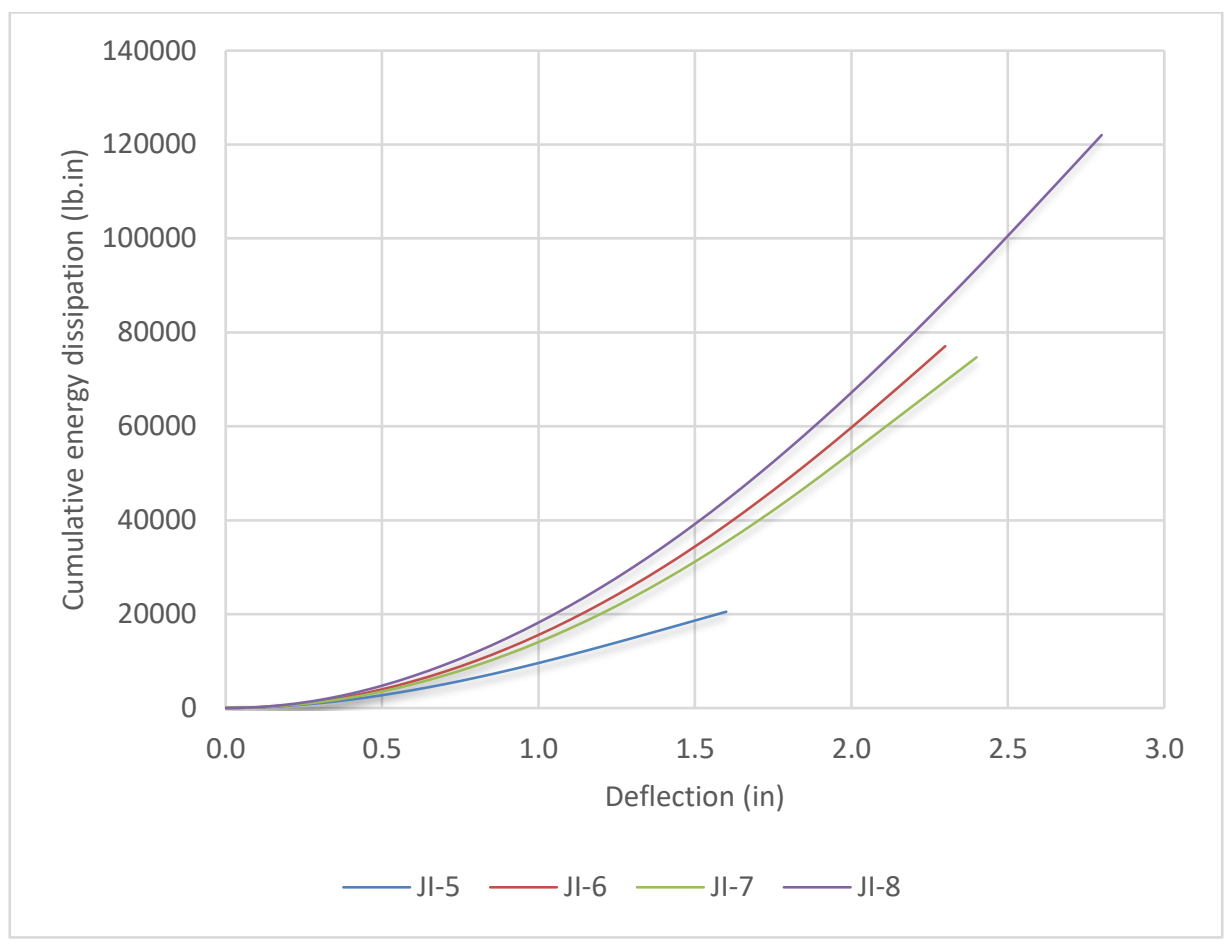

Figure 126. Energy dissipation capacity of joints with filler-modules and CFRP wraps

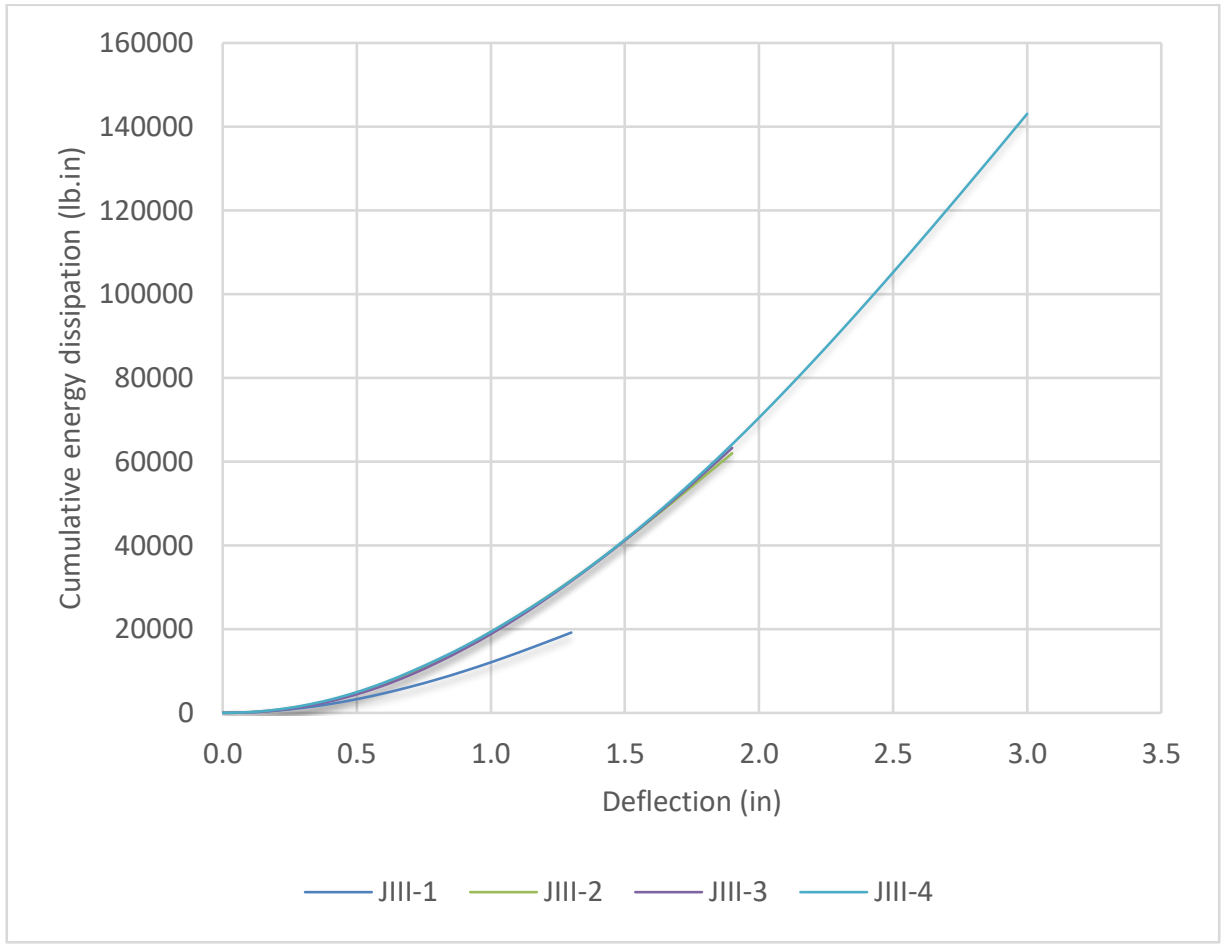

Figure 127. Energy dissipation capacity of joints with filler-modules and GFRP wraps 173 
Table 41. Energy dissipation capacity of joints with filler-modules and FRP wraps/gussets

\section{Joint Cumulative energy dissipated (lb.in) \% increase}

\begin{tabular}{ccc}
\hline JII-1 & 11,948 & Control \\
\hline JI-2 & 16,109 & 35 \\
\hline JI-3 & 80,930 & 577 \\
\hline JI-4 & 153,865 & 1,188 \\
\hline JII-5 & 20,513 & Control \\
\hline JI-6 & 77,062 & 276 \\
\hline JII-7 & 74,695 & 264 \\
\hline JII-8 & 122,018 & 495 \\
\hline JIII-1 & 19,184 & Control \\
\hline JIII-2 & 62,012 & 223 \\
\hline JIII-3 & 63,287 & 229 \\
\hline JIII-4 & 143,072 & 646
\end{tabular}

Specimen JI-2 reinforced with concrete filler-modules without FRP wraps/gussets dissipated 35\% more energy compared to the control specimen (JI-1). The cumulative energy dissipated by specimens JI-3 and JI-4 was $\sim 6$ and 12 times higher than the control specimen (JI-1), respectively. The low stiffness of the syntactic-foam filler-modules in JI-4 has allowed the beam/column to flex more and contribute to the near-uniform distribution of strains in and around the joint panel, resulting in the highest energy dissipation capacity. The use of high-strength concrete fillermodules has allowed the specimen JI-8 to dissipate more energy ( $\sim 5$ times) compared to the control specimen JI-5. This behavior is attributed to the enhanced force resistance (strut formation) offered by the high-strength filler-modules in resisting compression forces between points B and C. The shape of the filler-modules did not seem to influence the energy dissipation capacity of joint specimens. The increase in strut strength through the confinement of the bottom filler-module using U-anchors has resulted in dissipating more energy ( 6 times) compared to the control specimen JIII-1. 


\subsubsection{Strains in joint-, beam-, and column- rebars}

In order to assess the strain progression from the beam- and column- rebars into the joint panel, several gages were mounted on steel rebars at critical locations, as shown in Figure 71. Results deduced from these strain gages at locations JSH1, BSL1, BSL2, and CSL2 are studied carefully and presented in Table 42 to assess the influence of various reinforcing parameters.

As expected, all the control specimens (JI-1, JI-5, and JIII-1) failed in the joint panel without yielding of the beam or column rebars. In all the three specimens, the strain gage location (BSL1) that is 3 in. away from the reentrant corner on the beam section exhibited higher strains indicating the formation of hinge next to the column face. This behavior can lead to the sudden progression of crack into the joint panel, causing brittle joint failure.

For specimen JI-2, the strains at BSL1 were lower than the control specimen (JI-1), indicating the distribution of strain over a certain length of rebar, i.e., the formation of hinge further away from the face of the column. However, the reinforcing technique adopted for JI-2 was unable to develop yield strains in the rebar, even at failure. Although specimen JI-3 failed through yielding of beam longitudinal rebar, it attracted more strains at BSL2 due to the use of stiffer concrete filler-module. The use of low stiffness syntactic foam filler-module in JI-4 has resulted in a near-uniform distribution of strains at all the locations, i.e., JSH1, BSL2, and CSL2, leading to a higher ductility index and energy dissipation capacity.

Specimens JI-6, JI-7, and JI-8 failed through the yielding of beam longitudinal rebar with higher strain values at BSL2 location. However, the use of a high-strength concrete filler-module in JI-8 has allowed the rebar to reach the plastic strain limit, resulting in higher ductility and energy dissipation capacities.

The use of wedge shape filler-modules in JIII-3 induced lower strains in the rebar at all the four locations (JSH1, BSL1, BS12, and CSL2) compared to the curved filler-module in JIII-2. Confining the filler-modules of JIII-4 with U-anchors has resulted in superior performance by allowing the rebars to resist more forces by attaining the plastic strain limit. 
Table 42. Rebar strain data of joints with filler-modules and FRP wraps/gussets

\begin{tabular}{|c|c|c|c|c|c|c|}
\hline \multirow{2}{*}{ Joint } & \multirow{2}{*}{ PoI } & \multirow{2}{*}{ Beam load (lbs) } & \multicolumn{4}{|c|}{ Rebar strain $(\mu \varepsilon)$} \\
\hline & & & JSH1 & BSL1 & BSL2 & CSL2 \\
\hline \multirow{3}{*}{ JI-1 } & A & 5,063 & 128 & 415 & - & 66 \\
\hline & B & 11,076 & 835 & 1,065 & - & 258 \\
\hline & $\mathrm{C}$ & 15,471 & 1,362 & 1,457 & - & 868 \\
\hline \multirow{3}{*}{$\mathrm{JI}-2$} & A & 9,067 & 28 & 204 & - & 1 \\
\hline & B & 11,542 & 55 & 353 & - & 9 \\
\hline & $\mathrm{C}$ & 22,434 & 492 & 1,216 & - & 241 \\
\hline \multirow{3}{*}{$\mathrm{JI}-3$} & A & 21,976 & 75 & - & 855 & 28 \\
\hline & B & 27,308 & 217 & - & 1,116 & 84 \\
\hline & $\mathrm{C}$ & 60,773 & 1,175 & - & 4,809 & 1,472 \\
\hline \multirow{3}{*}{ JI-4 } & A & 21,574 & 193 & - & 664 & 114 \\
\hline & B & 23,970 & 329 & - & 773 & 135 \\
\hline & $\mathrm{C}$ & 64,107 & 2,263 & - & 2,608 & 2,612 \\
\hline \multirow{3}{*}{$\mathrm{JI}-5$} & A & 2,183 & - & 156 & 93 & 37 \\
\hline & B & 10,886 & - & 1,214 & 770 & 382 \\
\hline & $\mathrm{C}$ & 18,819 & - & 2,044 & 1,486 & 1,351 \\
\hline \multirow{3}{*}{ JI-6 } & A & 21,001 & 85 & 354 & 811 & -14 \\
\hline & $\mathrm{B}$ & 32,836 & 312 & 861 & 1,384 & 113 \\
\hline & $\mathrm{C}$ & 58,961 & 1,126 & 1,917 & 3,307 & 2,325 \\
\hline \multirow{3}{*}{ JI-7 } & A & 15,021 & 92 & 431 & 540 & 2 \\
\hline & B & 25,638 & 300 & 928 & 1,049 & 69 \\
\hline & $\mathrm{C}$ & 51,018 & 1,242 & 1,997 & 2,833 & 1,392 \\
\hline \multirow{3}{*}{ JI-8 } & A & 20,413 & 58 & 385 & 800 & -34 \\
\hline & B & 33,316 & 320 & 918 & 1,421 & 76 \\
\hline & $\mathrm{C}$ & 72,040 & 1,427 & 2,396 & 11,426 & 1,125 \\
\hline \multirow{3}{*}{ JIII-1 } & A & 7,076 & 99 & 696 & 261 & 73 \\
\hline & B & 15,658 & 1,023 & 1,646 & 829 & 212 \\
\hline & $\mathrm{C}$ & 24,740 & 1,573 & 2,535 & 1,535 & 1,171 \\
\hline \multirow{3}{*}{ JIII-2 } & A & 21,479 & 209 & 938 & 654 & 31 \\
\hline & B & 27,364 & 487 & 1,264 & 874 & 153 \\
\hline & $\mathrm{C}$ & 53,072 & 2,445 & 3,103 & 1,831 & 2,573 \\
\hline \multirow{3}{*}{ JIII-3 } & A & 26,502 & 153 & 697 & 789 & 24 \\
\hline & B & 33,103 & 434 & 1,103 & 1,016 & 92 \\
\hline & $\mathrm{C}$ & 57,882 & 1,919 & 2,223 & 1,946 & 2,009 \\
\hline \multirow{3}{*}{ JIII-4 } & A & 23,761 & 71 & 480 & 770 & -9 \\
\hline & B & 37,392 & 495 & 1,139 & 1,288 & 195 \\
\hline & C & 77,391 & 2,087 & 9,091 & 6,930 & 1,623 \\
\hline
\end{tabular}

Red color indicates rebar yielding and attainment of plastic strains in some cases. Green color indicates the near-uniform distribution of strains in rebars. 


\subsection{Conclusions}

A summary of the test results based on the experimental findings of joints reinforced with fillermodules and FRP wraps/gussets is presented in Table 43. Besides, the following sections (5.4.1.1 through 5.4.1.7) provide concluding remarks on various parameters evaluated in this study:

Table 43. Summary of test results: joints with filler-modules with/out FRP wraps or gussets

\begin{tabular}{ccccc}
\hline Joint & Peak load (lbs) & Ductility index & $\begin{array}{c}\text { Energy dissipated } \\
\text { (lb.in) }\end{array}$ & Failure mode \\
\hline JII-1 & 15,471 & 2 & 11,948 & Joint/compression \\
\hline JII-2 & $22,434(45)$ & 2 & $16,109(35)$ & Column shear \\
\hline JII-3 & $60,773(293)$ & 4 & $80,930(577)$ & Beam flexure \\
\hline JII-4 & $64,107(314)$ & 6 & $153,865(1,188)$ & Beam flexure \\
\hline JII-5 & 18,819 & 3 & 20,513 & Joint/diagonal tension \\
\hline JII-6 & $58,961(213)$ & 4 & $77,062(276)$ & Column shear \\
\hline JII-7 & $51,018(171)$ & 4 & $74,695(264)$ & Column shear \\
\hline JII-8 & $72,040(283)$ & 5 & $122,018(495)$ & Column shear \\
\hline JIII-1 & 24,740 & 2 & 19,184 & Joint/diagonal tension \\
\hline JIIII-2 & $53,072(115)$ & 3 & $62,013(223)$ & Column shear \\
\hline JIIII-3 & $57,882(134)$ & 3 & $63,287(230)$ & Column shear \\
\hline JIIII-4 & $77,391(213)$ & 5 & $143,073(646)$ & Column shear \\
\hline
\end{tabular}

Numbers inside ( ) indicate \% increases with respect to the control specimen of a given batch.

\subsubsection{Influence of concrete filler-modules without FRP wrap/gusset (JI-2)}

Although reinforcing joints with concrete filler-modules has prevented the joint shear failure, the increases in strength and energy dissipation were limited to $45 \%$ and $35 \%$, respectively, when compared to the control specimens. This method of joint reinforcement did not allow the rebars to attain yield strains due to the premature column failure resulting from stress concentrations at the tip of filler-module and glue lines. This approach, if used to reinforce pre-1976 joints, especially weak column strong-beam designs, may lead to catastrophic collapse under extreme events. 


\subsubsection{Influence of CFRP wrap coupled with filler-modules (JI-2 vs. JI-3)}

The addition of CFRP wrap to a joint specimen reinforced with concrete filler-modules has improved the strength, ductility, and energy dissipation by $\sim 170 \%, 100 \%$, and $402 \%$, respectively, over specimen JI-2. This improvement is primarily due to the additional tensile resistance offered by the CFRP wrap in addition to the concrete confinement effect. This approach of reinforcing joints with CFRP wrap and $360^{\circ}$-anchor around the filler-modules has shifted the non-ductile column failure mode to a more ductile beam flexural failure.

\subsubsection{Influence of concrete vs. foam filler-modules with CFRP wraps (JI-3 vs. JI-4)}

Comparing the structural responses of joints reinforced with concrete filler-modules (JI-3) and syntactic foam filler-modules (JI-4) coupled with CFRP wraps revealed that the specimen JI-4 has exhibited superior strength, ductility, and energy dissipation capabilities. The low-stiffness property of the syntactic foam filler-module has allowed the adjoining beam and column members to flex more in unison with the rotational values of the beam and column meeting at the joint (instead of creating stress risers) and contribute to the near-uniform distribution of strains in and around the joint panel, resulting in a superior behavior. The use of syntactic foam filler-modules coupled with CFRP wraps to reinforce deficient joints may be essential to withstand extreme events and prevent catastrophic collapses.

\subsubsection{Influence of medium-versus high-strength concrete filler-modules with CFRP gussets (JI-7 vs. JI-8)}

Both the medium- and high- strength concrete filler-modules significantly improved the structural response of joints. The higher the strength of filler-module, the longer the plateau between points $\mathrm{B}$ and $\mathrm{C}$, i.e., more prolonged inelastic zone. This behavior implies that the use of high-strength filler-modules will provide better ductility and energy dissipation capacity due to enhanced compressive force resistance (strut formation) offered by the bottom filler-module, while the steel cage tends to reach its maximum tensile force resistance. Besides, this will also delay the initial cracking of filler-module in the tension zone and push the occurrence of points A and B at higher load levels. 


\subsubsection{Influence of curve versus wedge filler-modules with GFRP wraps (JIII-2 vs. JIII-3)}

The shape of the filler-modules did not significantly alter the behavior of joints in terms of strength, ductility, and energy dissipation capacities ( $<9 \%$ variation). The low variation in test results is because the final failure was governed by the debonding of FRP wrap along the filler-module profile, followed by the crushing of concrete in the top filler-module.

\subsubsection{Influence of CFRP U-anchor for filler-modules (JIII-3 vs. JIII-4)}

The use of CFRP U-anchors around the filler-modules of JIII-4 significantly improved the strength, ductility, and energy dissipation capacities by $213 \%, 150 \%$, and $646 \%$, respectively, over the control specimen (JIII-1). The confinement effect from the U-anchor has prevented the fillermodules from crushing and allowed the rebar to attain plastic strains. This behavior has created a more prolonged inelastic zone, i.e., a longer plateau between points $\mathrm{B}$ and $\mathrm{C}$ thus, resulting in superior structural response over unconfined specimens.

\subsubsection{Influence of hinge formation within-versus outside- the filler module length}

Comparing the rebar strains in joints (JI-6 through JI-8) with (JIII-2 through JIII-4) reveals that the formation of hinge within the filler-module length is more beneficial in improving the strength, ductility, energy dissipation capacities. The formation of hinge within the filler-module length will offer additional resistance to induced forces because of the higher moment of inertia at that section. 


\section{Behavior of RC Joints Reinforced with Engineered Wood (PSL) Filler-Modules and FRP Wrap}

\subsection{Introduction}

This chapter dealt with the structural response evaluation of RC beam-column joints reinforced with PSL wood filler-modules and fiber-reinforced polymer (FRP) composite wraps under monotonic loads. The joint without filler-modules is identified as control specimens, herein. The joint testing has been conducted to evaluate the influence of various parameters, as listed in Table 44. The behavior of joints reinforced with PSL filler-modules and FRP wraps was studied in a similar manner as elaborated in section 5.1. Furthermore, this chapter presented the experimental results (section 6.2) followed by discussion on test results (section 6.3) with emphasis on strength, stiffness, ductility index, strain distribution in rebars, energy dissipation, and failure modes. Finally, the conclusions based on the experimental observations are presented in section 6.4.

Table 44. Parameters tested to evaluate the performance of joints reinforced with PSL fillermodules and FRP wraps

\begin{tabular}{|c|c|c|c|c|}
\hline Phase \# & Joint & $\boldsymbol{f}_{c}^{\prime}$ & CL & Reinforcing scheme \\
\hline \multirow{8}{*}{ 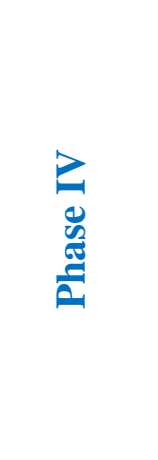 } & JII-1 & \multirow{8}{*}{$\begin{array}{l}\bar{n} \\
\text { مे } \\
\text { है } \\
\text { m. }\end{array}$} & \multirow{8}{*}{$\frac{\pi}{\stackrel{\pi}{L}}$} & Control \\
\hline & JII-2 & & & CFRP Wrap \\
\hline & JII-3 & & & PSL-C+D \\
\hline & JII-4 & & & PSL-W+D \\
\hline & JII-5 & & & PSL-C+CFRP Wrap $+360^{\circ}$-anchor \\
\hline & JII-6 & & & PSL-C+D+CFRP Wrap+360'-anchor \\
\hline & JII-7 & & & PSL-W+D+CFRP Wrap+360'-anchor \\
\hline & JII-8 & & & PSL-W+D+GFRP Wrap+360'-anchor \\
\hline
\end{tabular}

D: dowels; PSL: Parallel strand lumber; C: curve; W: wedge; CL: column axial load; $f_{c}^{\prime}$ : substrate concrete compressive strength; $A_{g}$ : gross cross-sectional area of concrete (column) 


\subsection{Experimental Results}

This section describes the structural response of joint specimens tested in control and reinforced (joints with PSL filler-modules and FRP wraps) conditions.

\subsubsection{Behavior of control specimen}

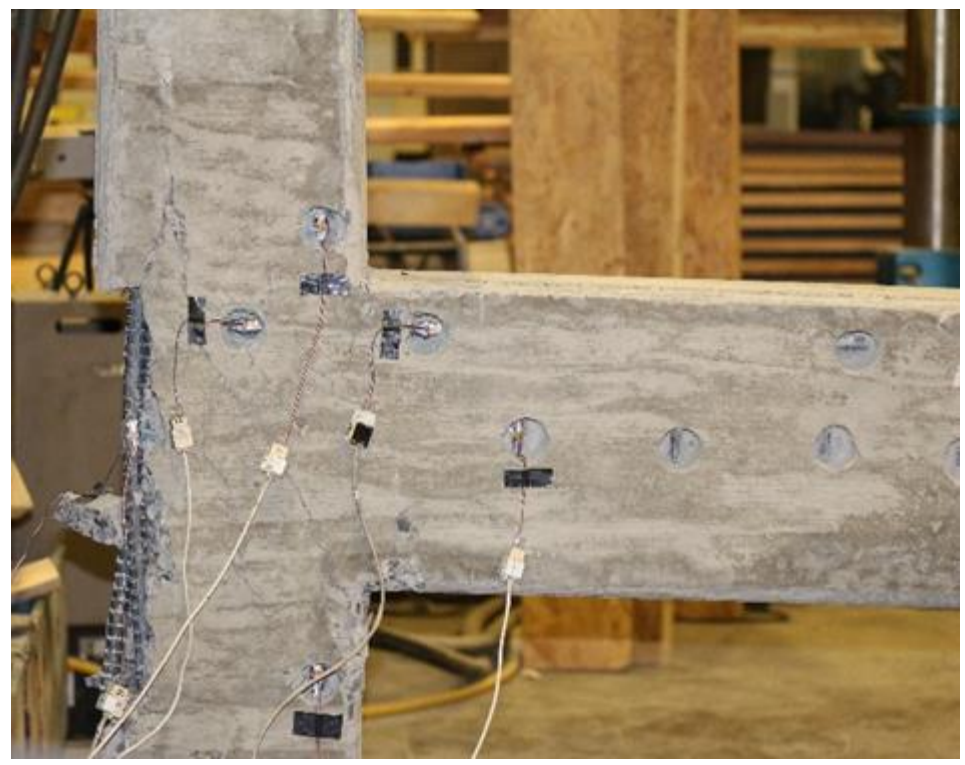

Figure 128. Failure of specimen JII-1

Specimen JII-1 was tested as a control specimen to establish the behavior of pre-1976 joints under monotonic loading and compare with reinforced joints, i.e., joints with filler-modules and FRP wraps. As the beam loading was applied, the specimen exhibited corner cracking at the interface of the beam and column. At the time of corner cracking, the strains on the rebar and concrete surface were noted to be 103-139 $\mu \varepsilon$ and 154-208 $\mu \varepsilon$, respectively. The load levels corresponding to these strains are provided in Table 45. As the loading on the beam continued, diagonal tension crack on the joint panel appeared and eventually led to joint shear failure with concrete cover delamination, as shown in Figure 128. A summary of the joint principal- and shear- strains measured during the testing are tabulated in Table 46. The beam load versus joint principal- and shear- strains are graphically shown in Figures 129 and 130, respectively. 


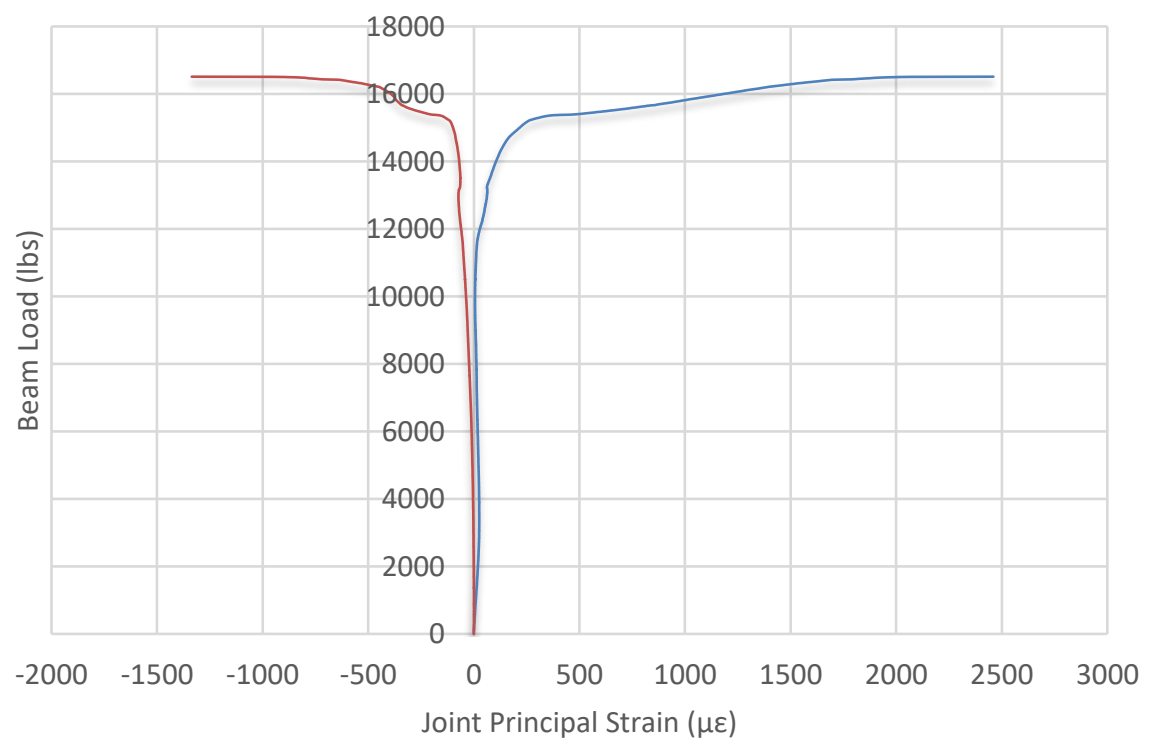

—JII-1: BL Vs JC1-P(t) — JII-1: BL Vs JC1-P(c)

Figure 129. Joint principal strains in JII-1

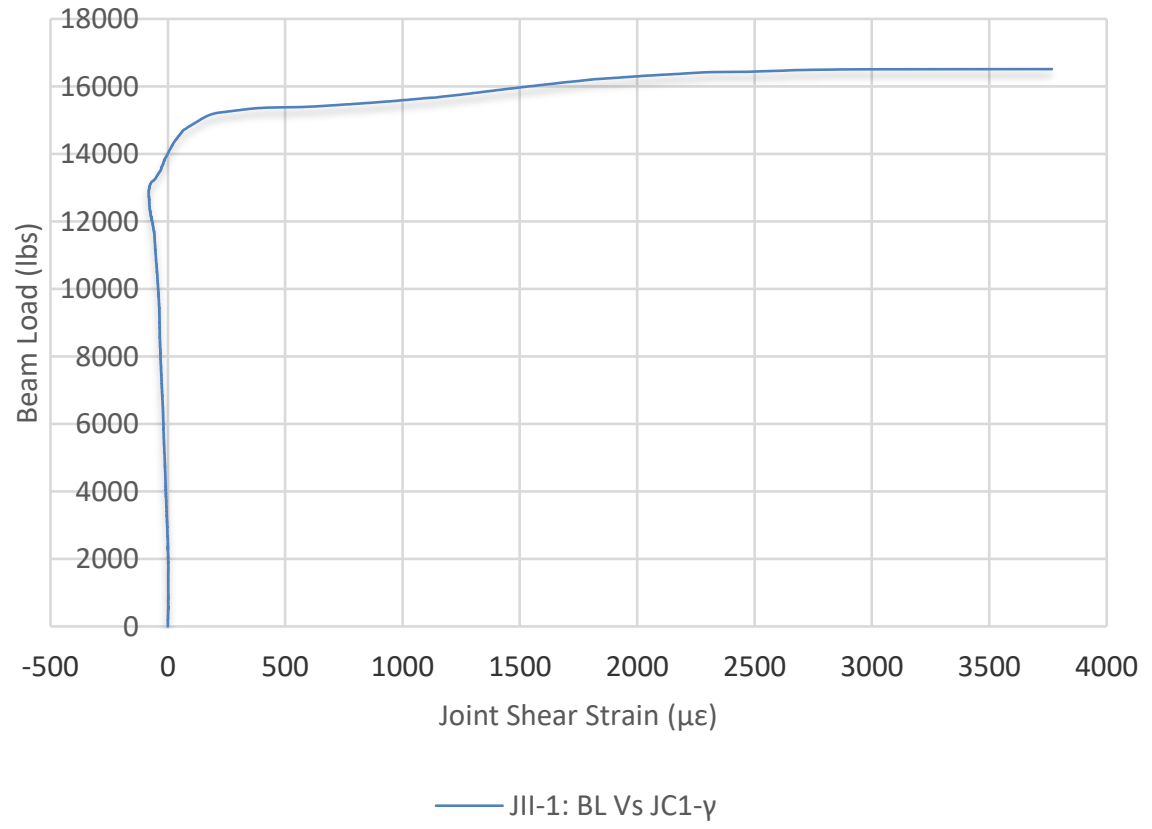

Figure 130. Joint shear strain in JII-1 


\subsubsection{Behavior of joint reinforced with CFRP wrap}

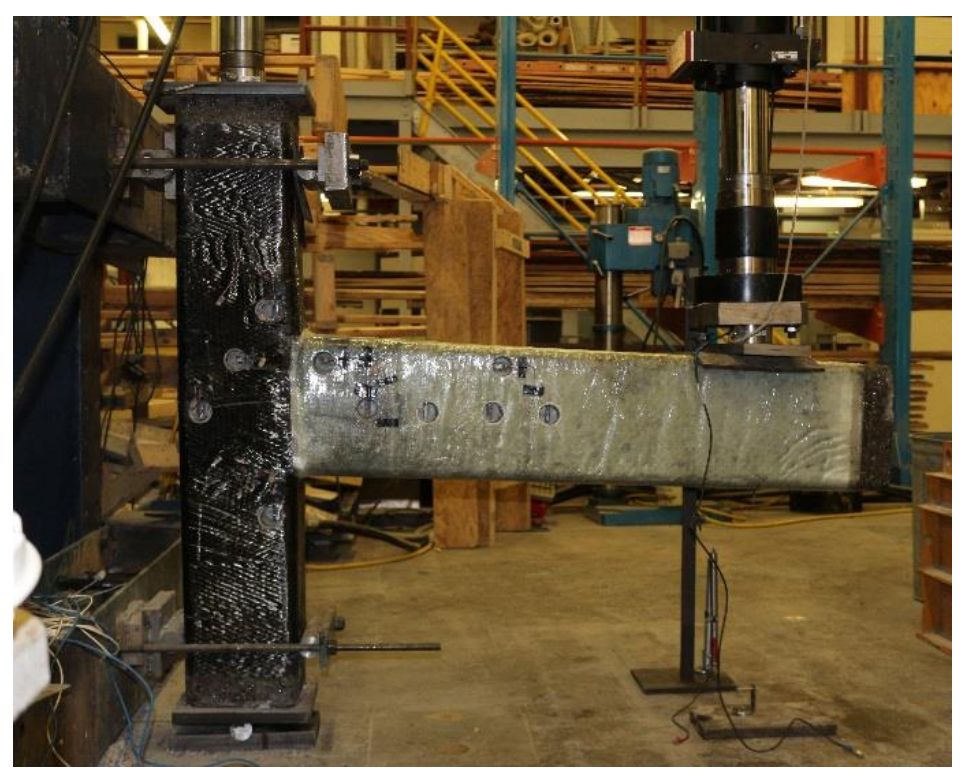

Figure 131. Failure of specimen JII-2

Specimen JII-2 was strengthened using CFRP wraps in the joint panel. The main objective of this reinforcing scheme was to evaluate the effectiveness of CFRP wraps in preventing joint failure and enhancing the strength, ductility, and energy dissipation over the control specimen.

As the beam loading was applied, the flexure-tension cracks appeared close to the tip of the fillermodule on the beam section when the concrete tensile strains reached 154-429 $\mu \varepsilon$. The k-factor for flexural-tension cracks was computed to be in the range of 9-24, as reported in Table 45. Based on the author's evaluation of FRP wrapped cylinders under split tension test, the tensile strength of FRP wrapped cylinders was found to be $\sim(20-28) \sqrt{f_{c}^{\prime}}$, as reported in section 4.4.6. As the loading continued, the crushing of concrete was delayed due to the confinement effect from CFRP wrap, thus allowing the rebar cage to resist additional tensile forces by yielding of rebars. Eventually, the specimen exhibited joint failure through the rupture of FRP at the joint corner, as shown in Figure 131. Although specimen JII-2 exhibited a similar failure to JII-1, the failure is contained due to the confinement effect from CFRP wraps, which resulted in plastic yielding $(\sim 14,330 \mu \varepsilon)$ of beam longitudinal rebar closer to the joint panel. However, this approach of FRP wrapping around a joint panel in a real building scenario is not practically feasible due to the 
presence of transverse beams and slab in two or more directions. A summary of the joint principaland shear- strains measured during the testing are tabulated in Table 46. The beam load versus joint principal- and shear- strains are graphically shown in Figures 132 and 133, respectively.

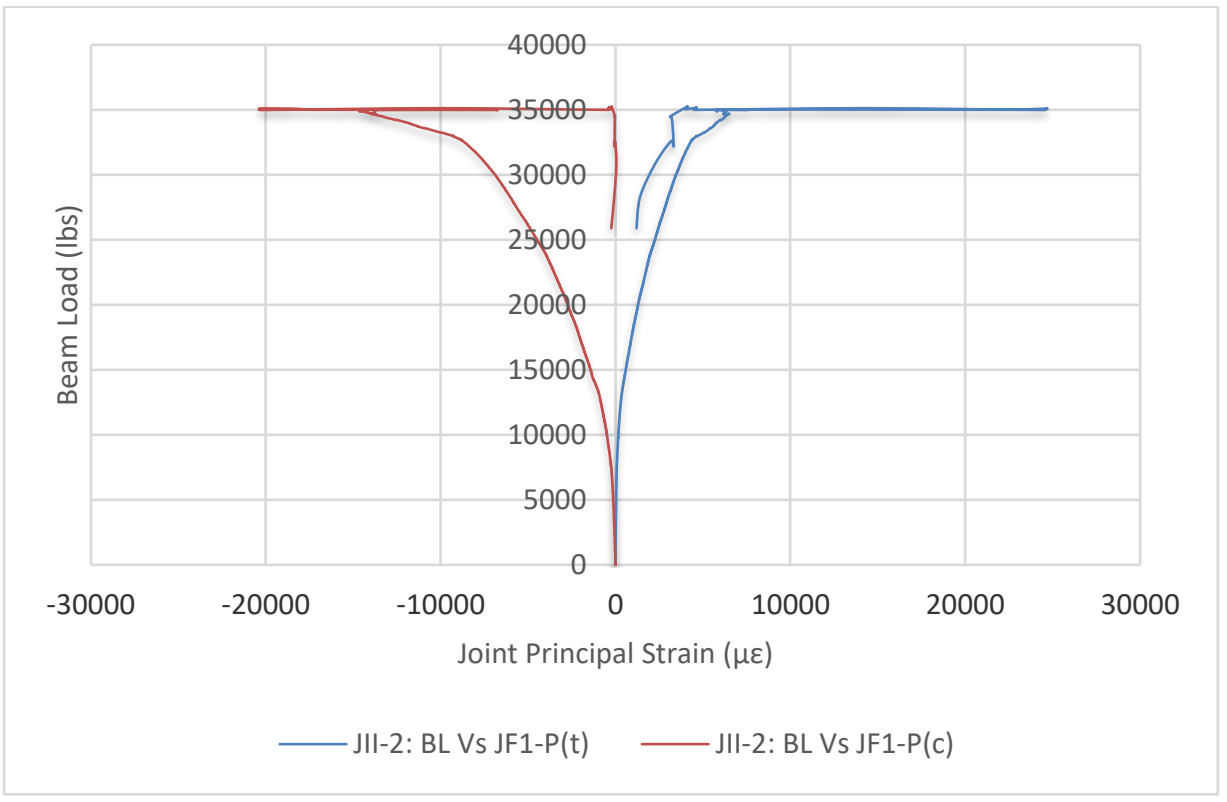

Figure 132. Joint principal strains in JII-2

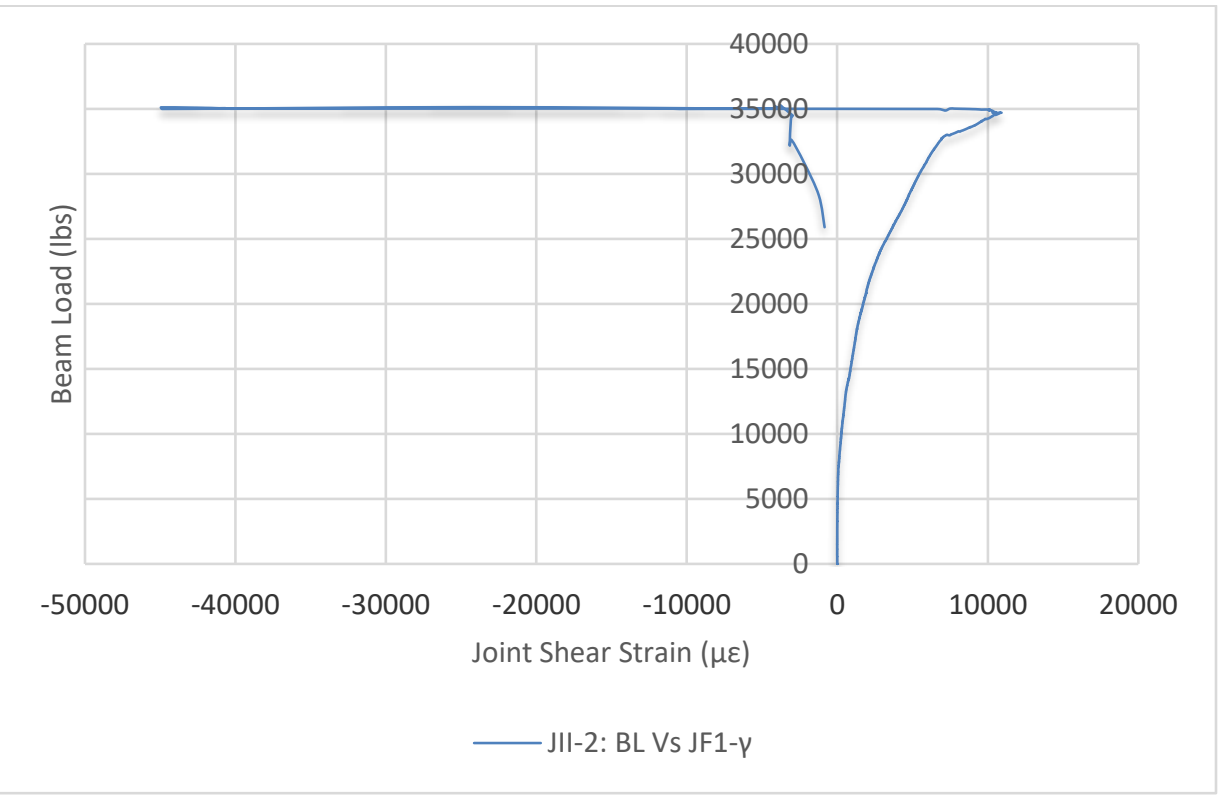

Figure 133. Joint shear strains in JII-2 


\subsubsection{Behavior of joints reinforced with PSL curve- and wedge- filler-modules}
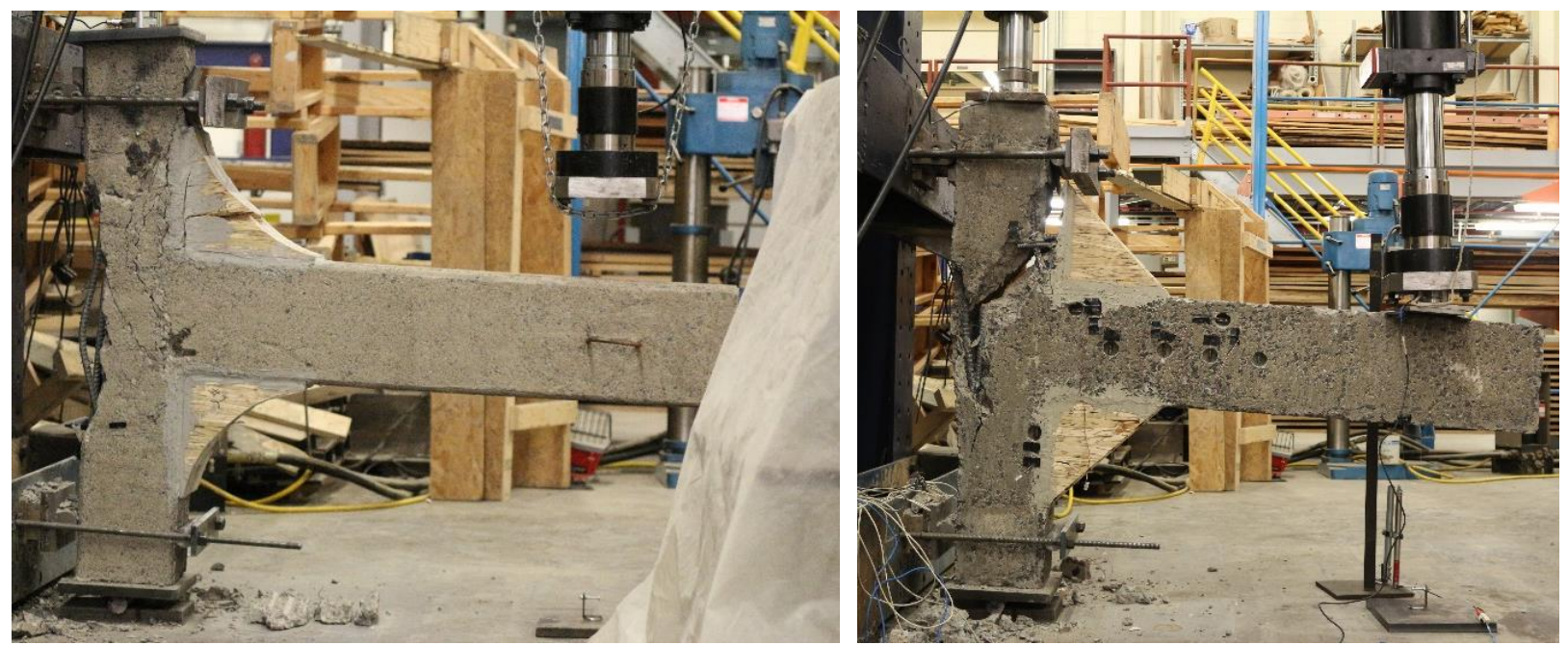

Figure 134. Failure of specimen JII-3 (left) and JII-4 (right)

Specimens JII-3 and JII-4 were tested to evaluate the influence of filler-module shape on the joint structural behavior. For this reason, specimen JII-3 was reinforced with PSL curve filler-modules and dowels, while specimen JII-4 was reinforced with wedge filler-modules and dowels.

Both the specimens JII-3 and JII-4 exhibited some distress at the tip of the filler-module during the initial stages of loading. The load values and corresponding strains in concrete and steel rebar at the time of distress are reported in Table 45. As the loading progressed further, the specimen JII-3 exhibited diagonal cracking in the joint panel and eventually merged with column shear crack, as shown in Figure 134. Finally, the specimen JII-3 failed through the spalling of concrete cover and buckling of longitudinal column rebars. At failure, the splitting of wood fibers on the top filler-module was noticed.

Specimen JII-4 failed in column shear next to the top filler-module, as shown in Figure 134. This crack originated at the tip of the filler-module due to the influence of stress-concentration and progressed into the column core. At failure, buckling of longitudinal rebars was observed along with side-splitting of column cover concrete. Comparing the failure modes of both specimens revealed that the wedge filler-module had attracted more stresses at its tip, unlike the curve fillermodules, which exhibited a better stress distribution between the joint and members meeting at a 
joint. A summary of the joint principal- and shear- strains measured during the testing are tabulated in Table 46. The beam load versus column principal- and shear- strains next to the crack location for both specimens JII-3 and JII-4 are graphically shown in Figures 135 through 138.

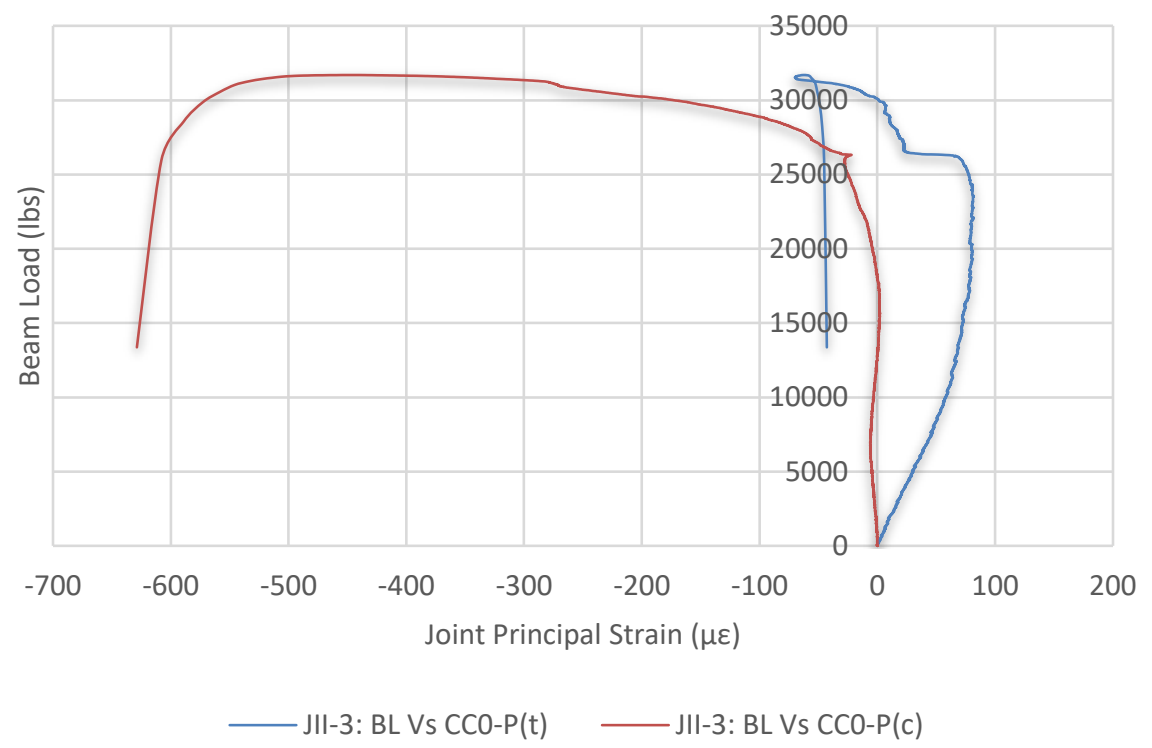

Figure 135. Column principal strain in JII-3

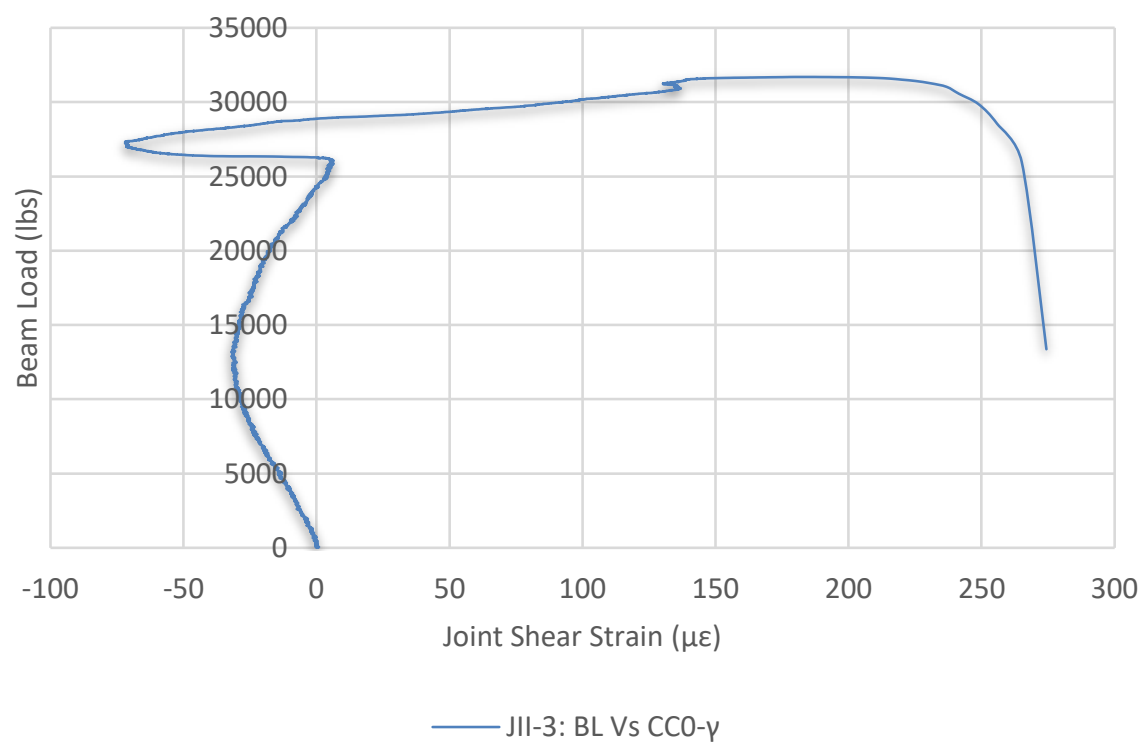

Figure 136. Column shear strain JII-3 


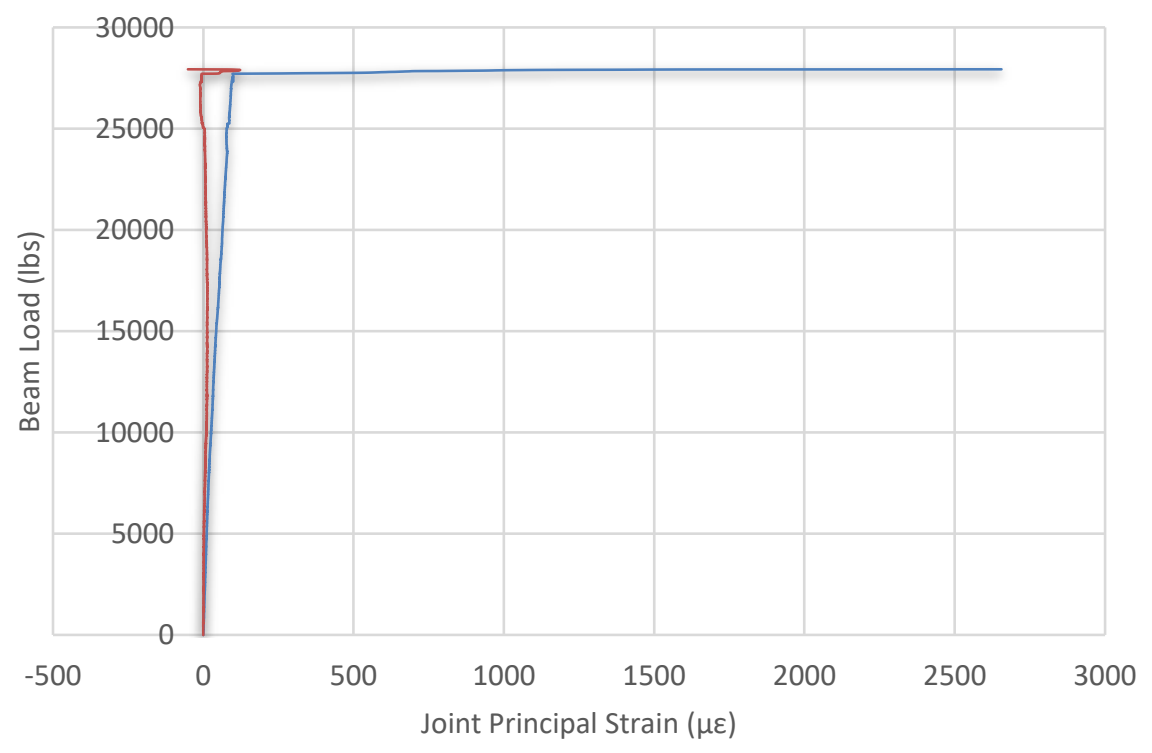

JII-4: BL Vs CCO-P(t) — JII-4: BL Vs CCO-P(c)

Figure 137. Column principal strain in JII-4

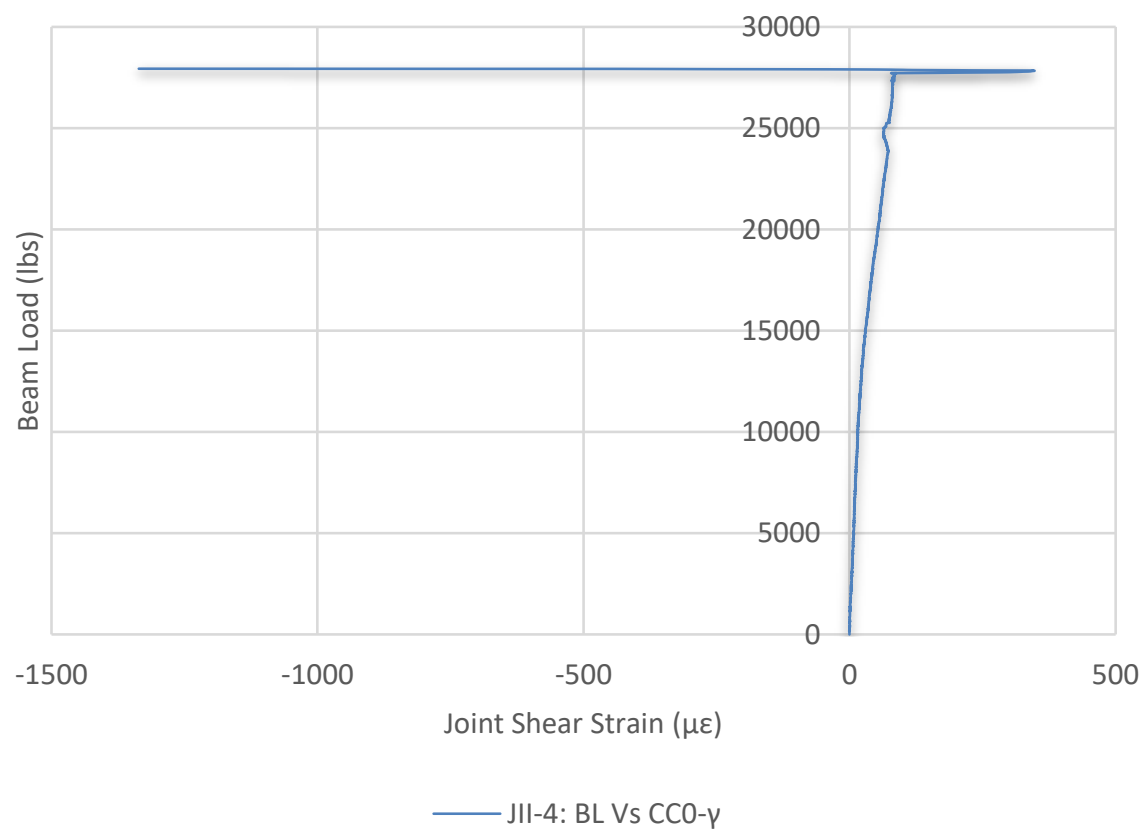

Figure 138. Column shear strain in JII-4 


\subsubsection{Behavior of joint reinforced with PSL curve filler-modules and CFRP wraps without and with reinforcing dowels}
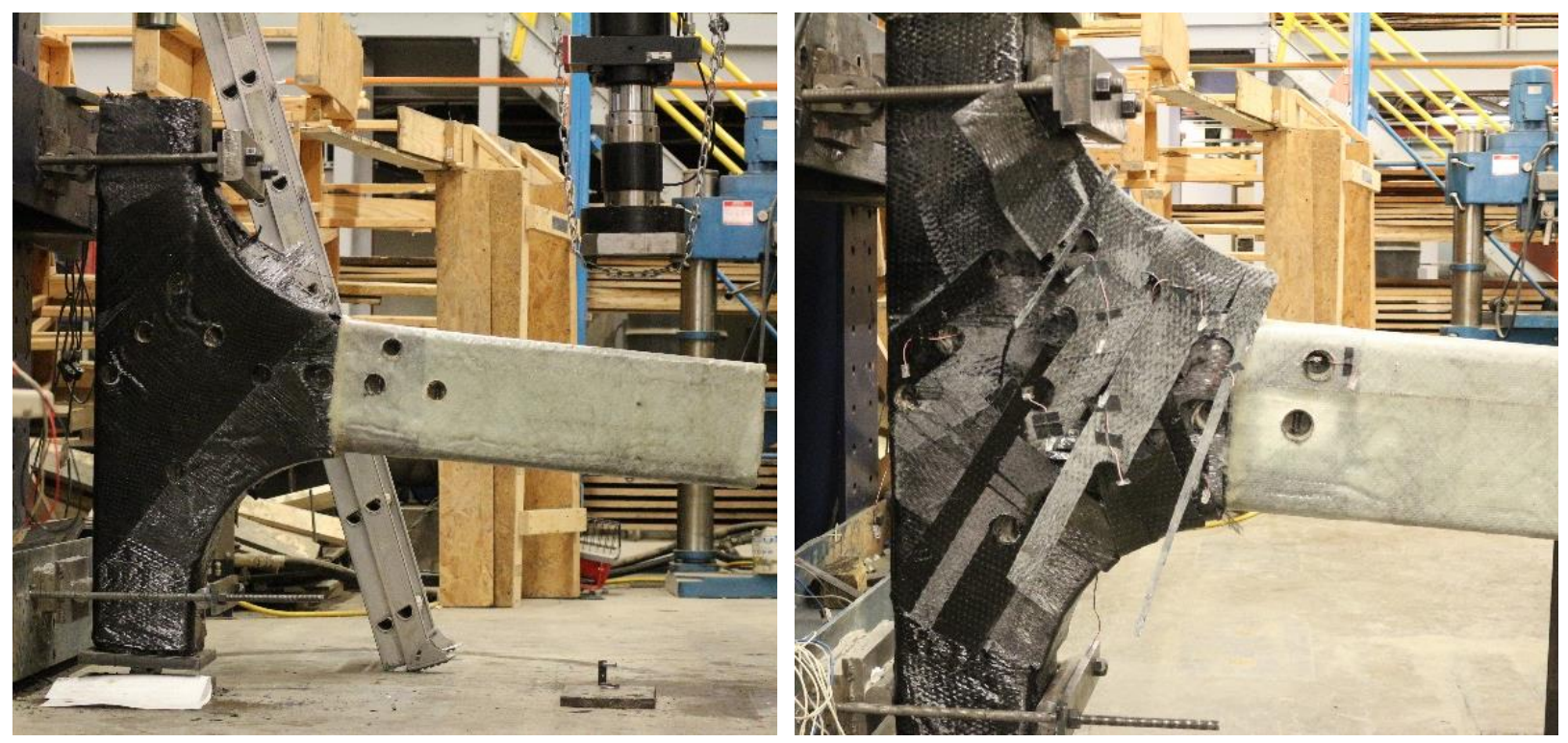

Figure 139. Failure of specimen JII-5 (left) and JII-6 (right)

Specimens JII-5 and JII-6 were tested to evaluate the influence of dowels on the structural behavior of joint reinforced with PSL filler-modules and CFRP wraps. For this reason, specimen JII-5 was reinforced with PSL curve filler-modules and CFRP wrap without dowels, while specimen JII-6 was reinforced with PSL curve filler-modules, CFRP wrap, and dowels.

Lack of dowels in specimen JII-5 has resulted in a joint shear failure due to the premature debonding of top filler-module, as opposed to a column shear failure in specimen JII- 6 with dowels. The presence of dowels in JII-6 provided better composite action between the fillermodules and the members meeting at a joint through the efficient transfer of shear forces. The composite action developed due to dowel bars has distributed the stresses over a large area and reduced the shear demand in the joint panel., thus avoiding joint failure. Although specimen JII-5 exhibited joint shear failure, the confinement from $360^{\circ} \mathrm{CFRP}$ wrap around the joint has resulted in better strength and energy dissipation compared to the control specimen. A summary of the joint principal- and shear- strains measured during the testing are tabulated in Table 46. The beam load 
versus joint principal- and shear- strains for both specimens JII-5 and JII-6 are graphically shown in Figures 140 through 143.

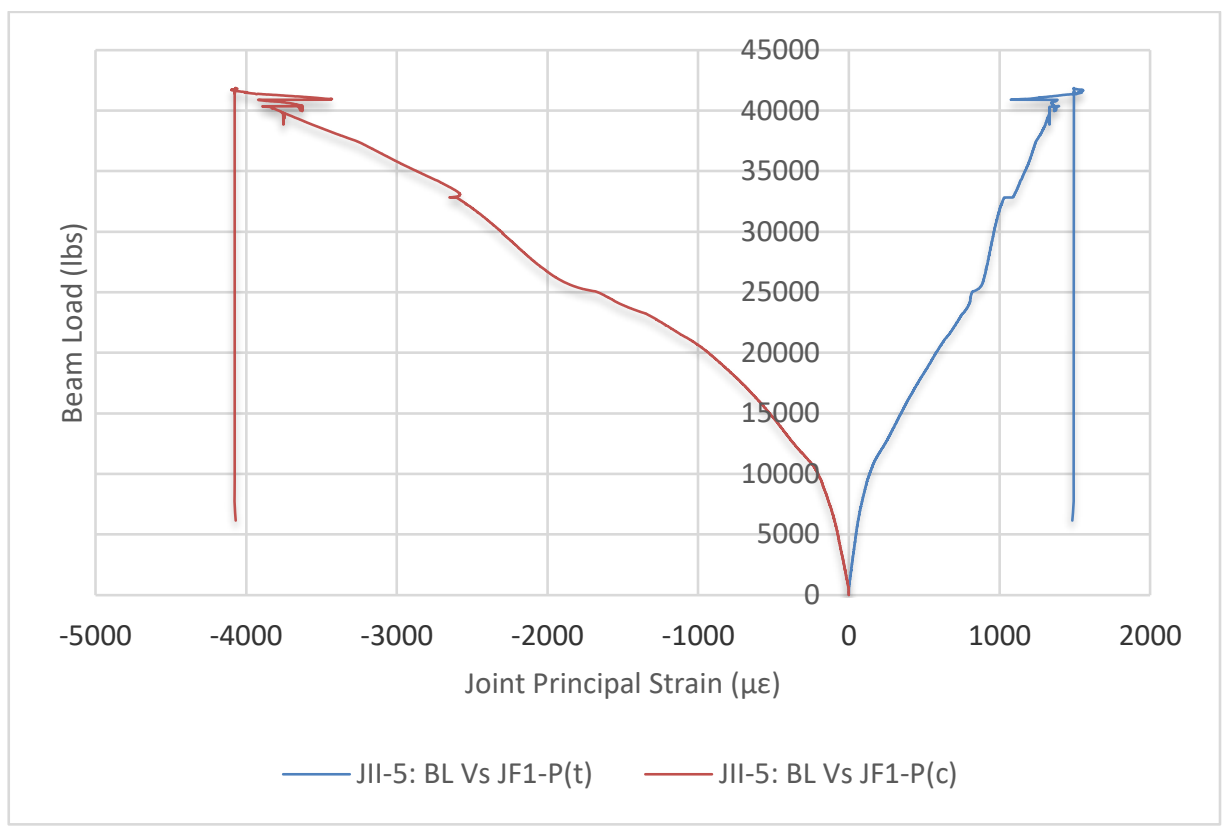

Figure 140. Joint principal strain in JII-5

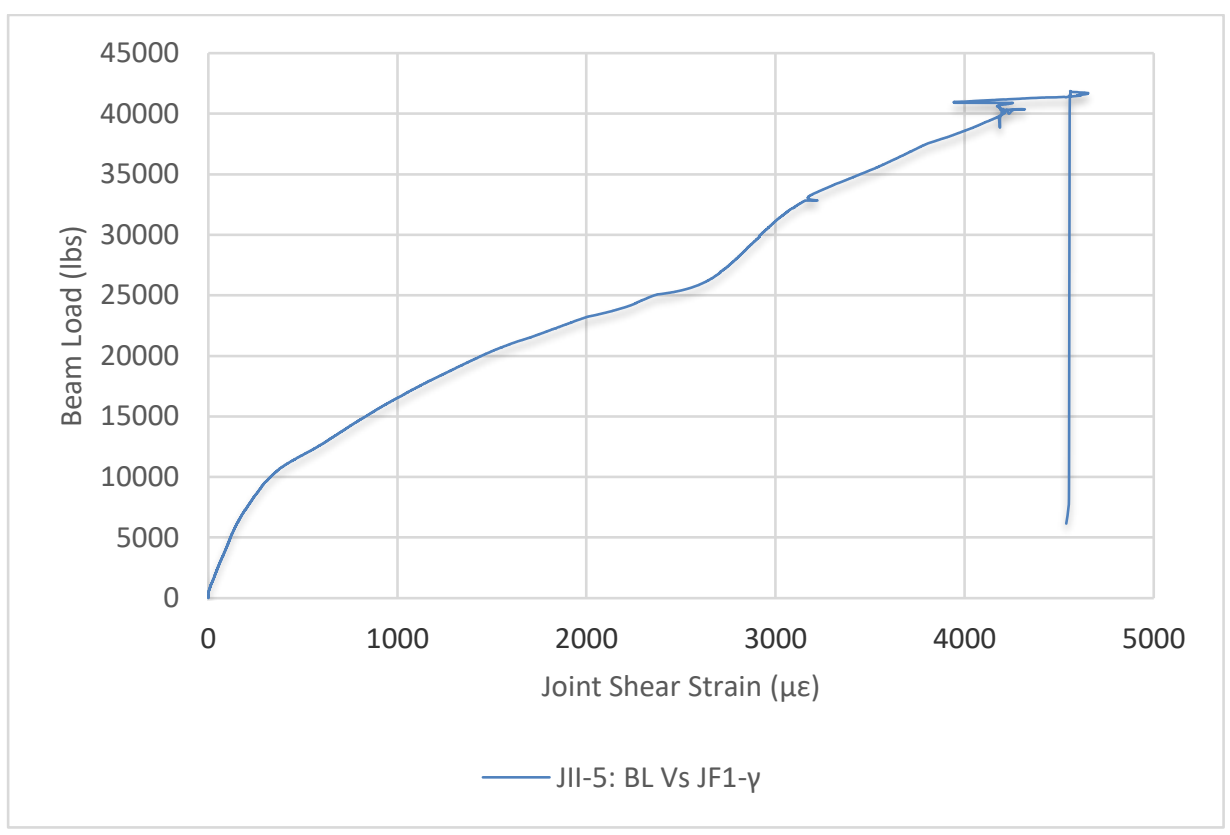

Figure 141. Joint shear strain in JII-5 


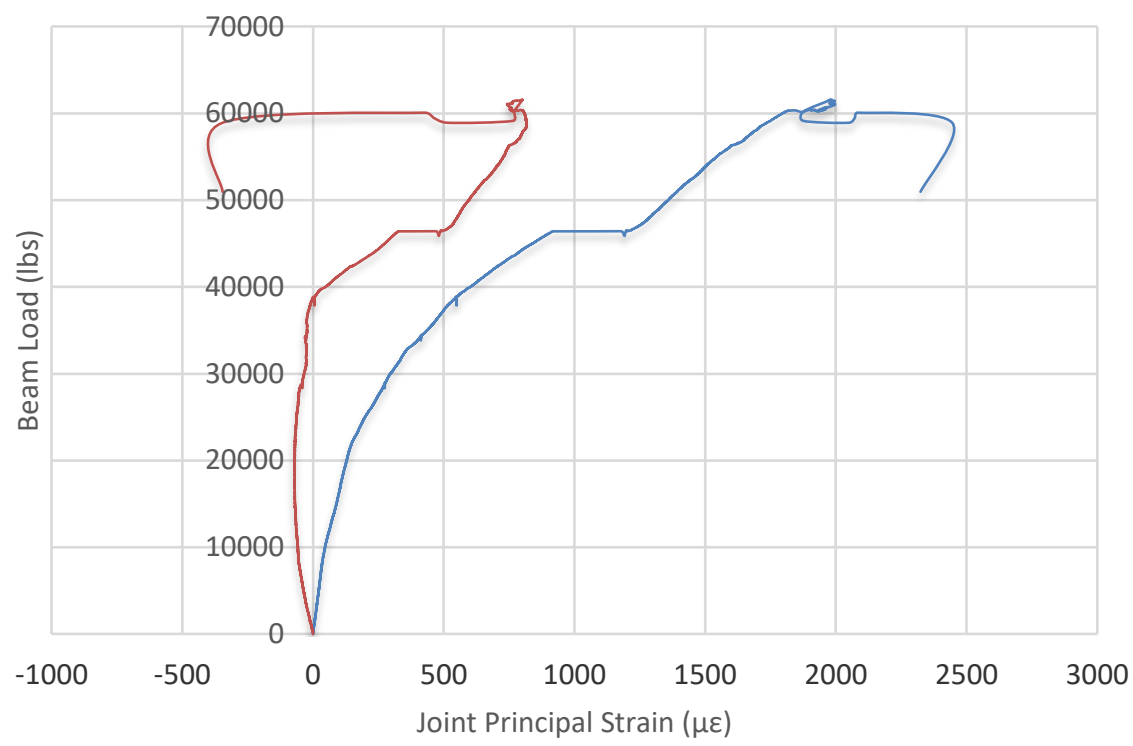

—JII-6: BL Vs JF1-P(t) — JII-6: BL Vs JF1-P(c)

Figure 142. Joint principal strain in JII-6

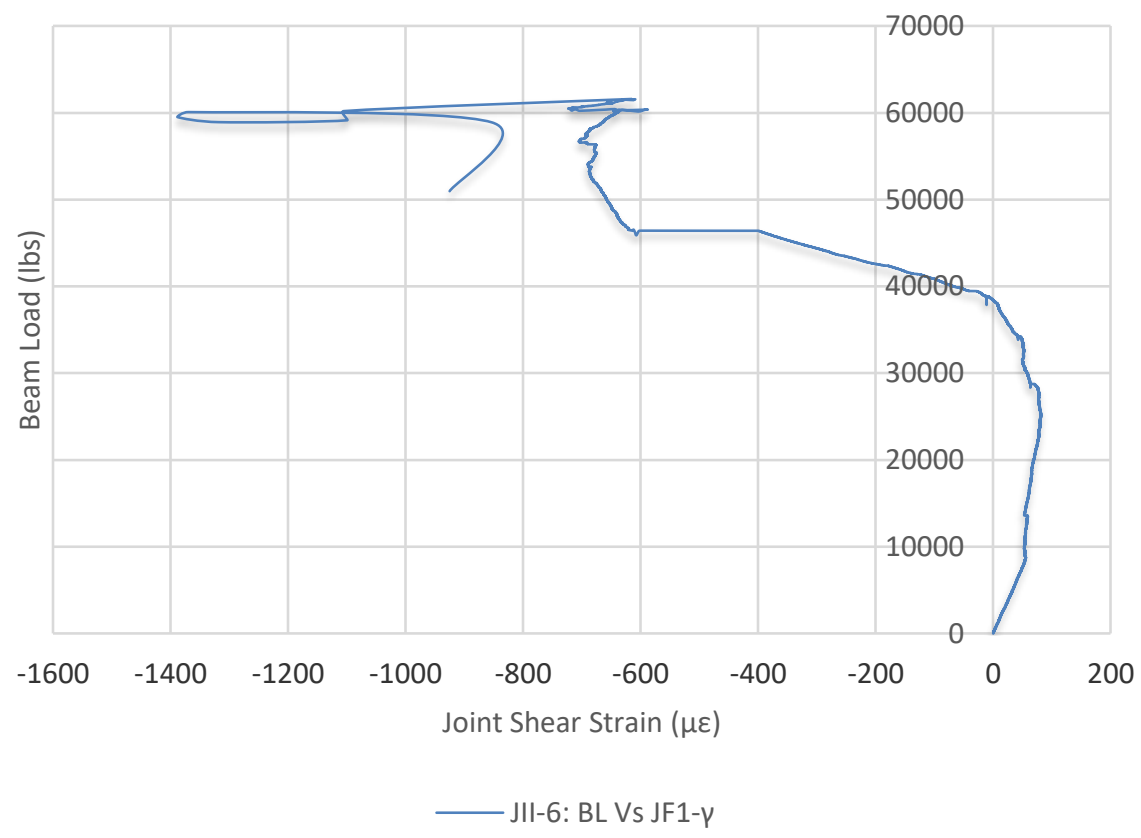

Figure 143. Joint shear strain in JII-6 


\subsubsection{Behavior of joint reinforced with PSL wedge filler-modules and CFRP/GFRP wraps}
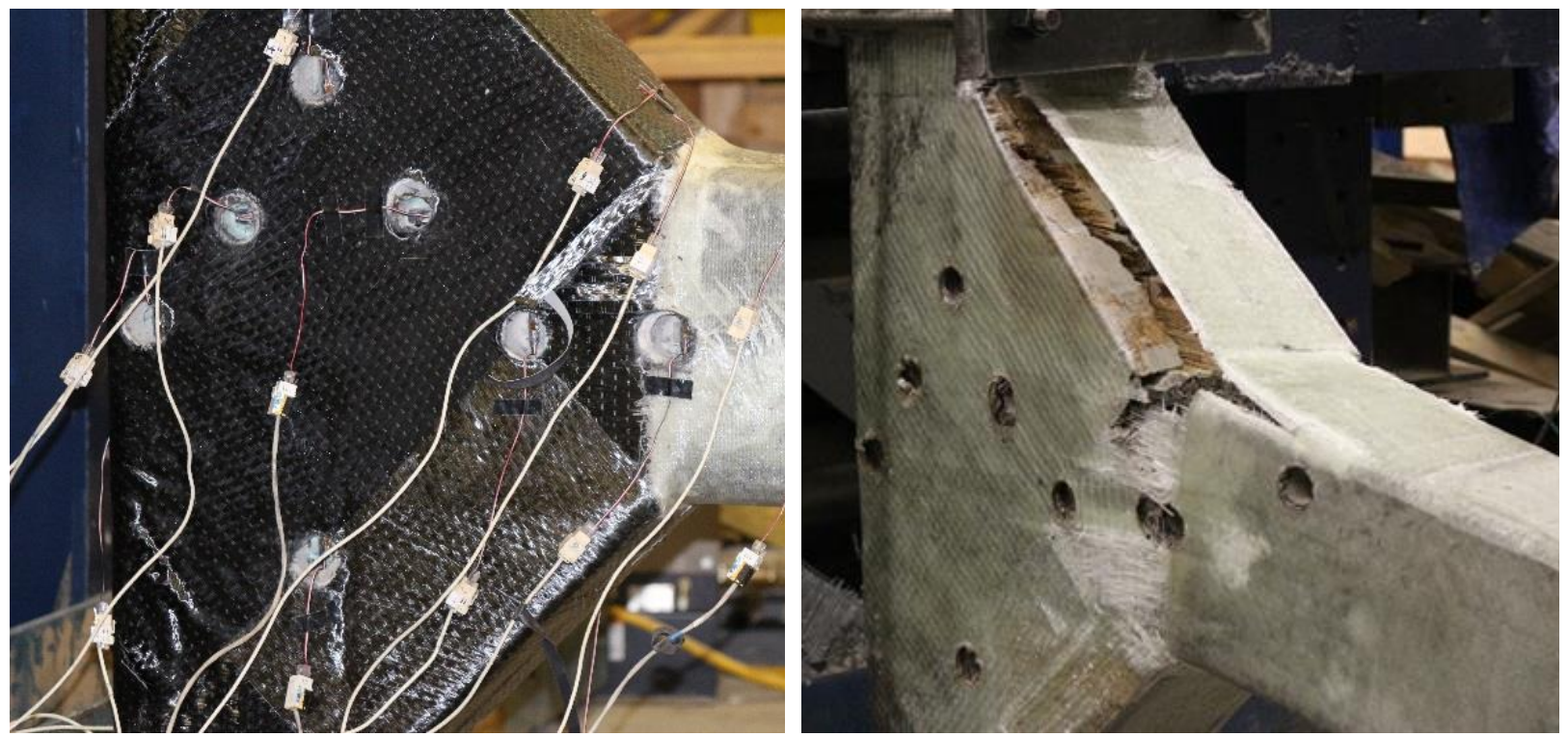

Figure 144. Failure of specimen JII-7 (left) and JII-8 (right)

Specimens JII-7 and JII-8 were tested to evaluate the influence of CFRP versus GFRP on the structural behavior of joints reinforced with PSL wedge filler-modules. For this reason, specimen JII-7 was reinforced with PSL wedge filler-modules, dowels, and CFRP wrap, while specimen JII8 was reinforced with PSL wedge filler-modules, dowels, and GFRP wrap.

As expected, the wedge filler-modules in both specimens attracted slightly higher strains close to the tip at every stage of beam loading. This response is evident from the rebar strain data reported in Table 52. It must be noted that both, CFRP and GFRP specimens exhibited similar strain response until the load on JII-7 was abruptly terminated due to the slippage of test specimen form the setup. As seen from Figure 144, both specimens JII-7 and JII-8 exhibited beam hinging. However, the use of GFRP wrap in JII-8 provided better stiffness compatibility with the underlying substrate resulting in additional force resistance until the beam longitudinal rebars at the $90^{\circ}$ hook in the joint panel were fractured. A summary of the joint principal- and shear- strains measured during the testing are tabulated in Table 46. The beam load versus joint principal- and shear- strains for both specimens JII-7 and JII-8 are graphically shown in Figures 145 through 148. 


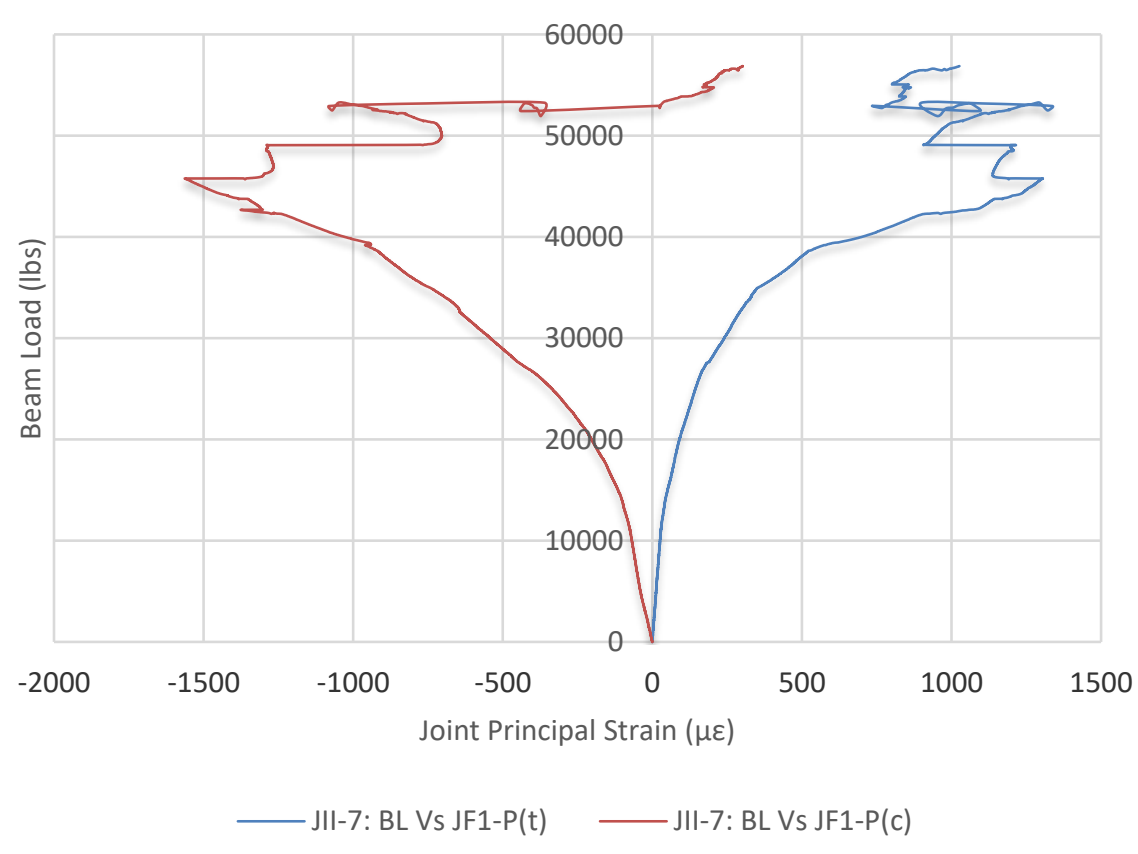

Figure 145. Joint principal strain in JII-7

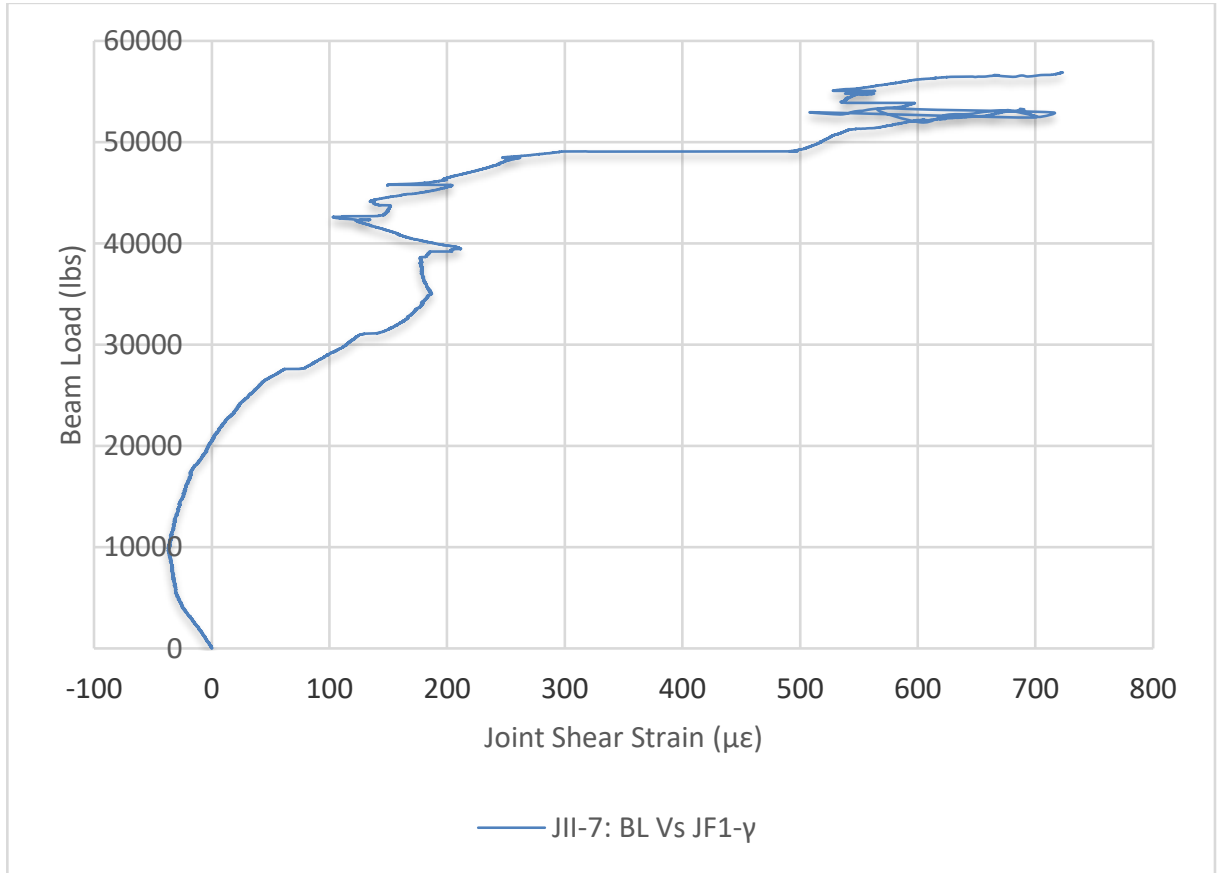

Figure 146. Joint shear strain in JII-7 


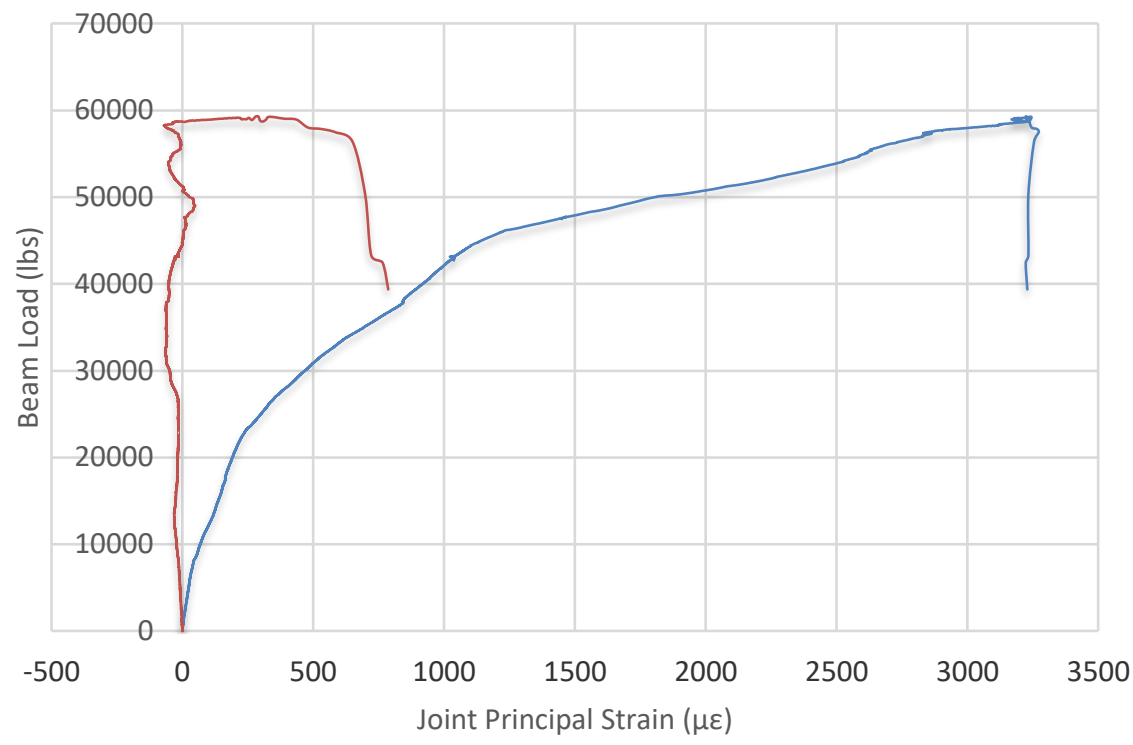

—JII-8: BL Vs JF1-P(t) — JII-8: BL Vs JF1-P(c)

Figure 147. Joint principal strain in JII-8

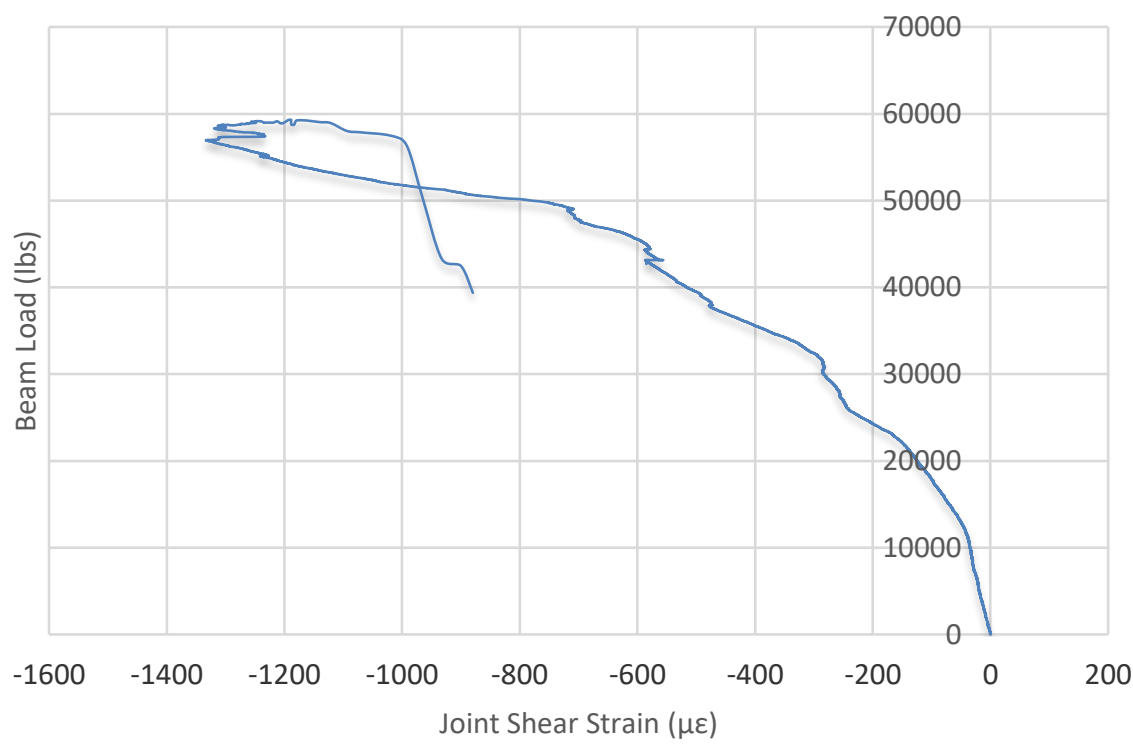

- JII-8: BL Vs JF1- $ү$

Figure 148. Joint shear strain in JII-8 
Table 45. Modulus of rupture of joints reinforced with PSL filler-modules and FRP wraps Joint Beam load (lb) Beam rebar strain ( $\mu \varepsilon)$ Concrete strain $(\mu \varepsilon) \quad \mathrm{k}-f_{r}$

\begin{tabular}{|c|c|c|c|c|}
\hline \multirow{2}{*}{ JII-1 } & 1,558 & 103 & 154 & 9 \\
\hline & 2,005 & 139 & 208 & 12 \\
\hline \multirow{2}{*}{ JII-2 } & 3,667 & 100 & 150 & 9 \\
\hline & 6,111 & 286 & 429 & 24 \\
\hline \multirow{2}{*}{ JII-3 } & 2,815 & 114 & 171 & 10 \\
\hline & 4,759 & 225 & 338 & 19 \\
\hline \multirow{2}{*}{ JII-4 } & 3,161 & 124 & 185 & 11 \\
\hline & 4,788 & 219 & 329 & 19 \\
\hline \multirow{2}{*}{ JII-5 } & 3,895 & 125 & 188 & 11 \\
\hline & 6,131 & 251 & 376 & 21 \\
\hline \multirow{2}{*}{ JII-6 } & 5,561 & 126 & 189 & 11 \\
\hline & 8,151 & 216 & 325 & 19 \\
\hline \multirow{2}{*}{ JII-7 } & 5,745 & 144 & 216 & 12 \\
\hline & 7,812 & 232 & 348 & 20 \\
\hline \multirow{2}{*}{ JII-8 } & 5,358 & 120 & 180 & 10 \\
\hline & 8,193 & 228 & 343 & 20 \\
\hline
\end{tabular}


Table 46. Summary of experimental results of joints reinforced with PSL filler-modules and FRP wraps

\begin{tabular}{|c|c|c|c|c|c|c|c|c|c|c|c|c|}
\hline \multirow{2}{*}{ Joint } & \multirow{2}{*}{ PoI } & \multirow{2}{*}{$\begin{array}{c}\text { Beam load } \\
\text { (lb) }\end{array}$} & \multicolumn{3}{|c|}{ Joint principal strain $(\mu \varepsilon)$} & \multicolumn{2}{|c|}{ Limit state } & \multicolumn{4}{|c|}{ Steel rebar strain $(\mu \varepsilon)$} & \multirow{2}{*}{$\begin{array}{c}\text { Observed } \\
\text { failure mode }\end{array}$} \\
\hline & & & $\mathrm{P}(\mathrm{t})$ & $\mathrm{P}(\mathrm{c})$ & $\gamma$ & $\mathrm{k}-\mathrm{P}(\mathrm{t})$ & $\mathrm{k}-\gamma$ & JSH1 & BSL1 & BSL2 & CSL2 & \\
\hline \multirow{3}{*}{ JII-1 } & A & 12,724 & 56 & -72 & -81 & 3.2 & 2.0 & 931 & 1,040 & N.A. & 410 & \multirow{3}{*}{$\begin{array}{c}\text { Joint shear/ } \\
\text { diagonal } \\
\text { tension }\end{array}$} \\
\hline & $\mathrm{B}$ & 13,295 & 64 & -65 & -51 & 3.6 & 1.2 & 1,021 & 1,110 & N.A. & 478 & \\
\hline & $\mathrm{C}$ & 20,900 & - & - & - & - & - & 1,947 & 1,750 & N.A. & 1,380 & \\
\hline \multirow{3}{*}{ JII-2 } & $\mathrm{A}$ & 10,808 & 202 & -587 & 342 & 11.5 & 8.3 & 416 & 704 & 551 & 176 & \multirow{3}{*}{$\begin{array}{l}\text { Joint shear } \\
\text { failure }\end{array}$} \\
\hline & $\mathrm{B}$ & 14,995 & 583 & -1414 & 875 & 33.2 & 21.1 & 820 & 1,060 & 812 & 350 & \\
\hline & $\mathrm{C}$ & 35,273 & 4,125 & -217 & $-3,788$ & N.A. & N.A. & 3,235 & 14,330 & 2,311 & 1,909 & \\
\hline \multirow{3}{*}{ JII-3 } & $\mathrm{A}$ & 11,289 & 64 & -2 & -31 & 3.7 & 0.7 & 132 & 462 & 712 & 34 & \multirow{3}{*}{$\begin{array}{l}\text { Joint shear } \\
\text { failure }\end{array}$} \\
\hline & B & 16,028 & 75 & 2 & -27 & 4.3 & 0.7 & 434 & 818 & 1,135 & 66 & \\
\hline & $\mathrm{C}$ & 31,695 & -61 & -454 & 187 & - & - & 2,142 & 1,858 & 2,533 & 1,877 & \\
\hline \multirow{3}{*}{ JII-4 } & A & 15,404 & 45 & 12 & 33 & 2.6 & 0.8 & 202 & 630 & 1,053 & 86 & \multirow{3}{*}{$\begin{array}{l}\text { Column shear } \\
\text { failure }\end{array}$} \\
\hline & $\mathrm{B}$ & 17,668 & 54 & 13 & 41 & 3.1 & 1.0 & 290 & 809 & 1,217 & 110 & \\
\hline & $\mathrm{C}$ & 34,989 & - & - & - & - & - & 1,595 & 2,014 & 2,776 & 2,651 & \\
\hline \multirow{3}{*}{ JII-5 } & A & 12,014 & 216 & -332 & 522 & 12.3 & 12.6 & 137 & 474 & 648 & 93 & \multirow{3}{*}{ Joint failure } \\
\hline & B & 23,219 & 753 & $-1,344$ & 2,003 & 42.9 & 48.4 & 1,061 & 1,289 & 1,459 & 355 & \\
\hline & $\mathrm{C}$ & 41,868 & 1,494 & $-4,071$ & 4,559 & 85.2 & 110.1 & 2,061 & 3,298 & 7,412 & 2,680 & \\
\hline \multirow{3}{*}{ JII-6 } & A & 29,512 & 286 & -38 & 60 & 16.3 & 1.5 & 370 & 825 & 1,223 & 81 & \multirow{3}{*}{$\begin{array}{l}\text { Column shear } \\
\text { failure }\end{array}$} \\
\hline & $\mathrm{B}$ & 34,063 & 407 & -30 & 48 & 23.2 & 1.2 & 522 & 1,048 & 1,534 & 106 & \\
\hline & $\mathrm{C}$ & 61,592 & 1,984 & 802 & -616 & 113.1 & 14.9 & 1,909 & 2,797 & 14,627 & 1,364 & \\
\hline \multirow{3}{*}{ JII-7 } & A & 28,390 & 205 & -478 & 89 & 11.7 & 2.2 & 445 & 1,044 & 1,516 & 90 & \multirow{3}{*}{$\begin{array}{l}\text { Beam flexural } \\
\text { failure }\end{array}$} \\
\hline & B & 38,946 & 550 & -940 & 184 & 31.3 & 4.4 & 816 & 1,612 & 2,188 & 232 & \\
\hline & $\mathrm{C}$ & 56,894 & 1,027 & 302 & 723 & 58.5 & 17.5 & 1,784 & 11,519 & 16,638 & 1,870 & \\
\hline \multirow{3}{*}{ JII-8 } & A & 30,612 & 491 & -58 & -284 & 28.0 & 6.8 & 550 & 1,218 & 1,605 & 222 & \multirow{3}{*}{$\begin{array}{l}\text { Beam flexural } \\
\text { failure }\end{array}$} \\
\hline & $\mathrm{B}$ & 36,706 & 785 & -62 & -440 & 44.7 & 10.6 & 797 & 1,549 & 2,007 & 359 & \\
\hline & $\mathrm{C}$ & 59,320 & 3,223 & 285 & $-1,190$ & 183.7 & 28.7 & 2,340 & 16,375 & 22,325 & 2,157 & \\
\hline
\end{tabular}

PoI: Point of interest; $\mathrm{P}(\mathrm{t})$ : principal tensile strain; $\mathrm{P}(\mathrm{c})$ : principal compressive strain; $\gamma$ : shear strain. 


\subsection{Discussion of Test Results}

To assess the effectiveness of joints reinforced with PSL filler-modules and FRP wraps in various configurations, the most critical parameters relevant for the structural response of joint specimens under monotonic loading are discussed in sections 6.3.1 through 6.3.6. The structural responses of joint specimens discussed herein include strength, stiffness, strain distribution, ductility index, energy dissipation, and failure modes. The assessment of the structural response of reinforced joint specimens was performed by comparing their response with the control specimen.

\subsubsection{Strength}

The load versus deflection of all the eight joint specimens is summarized in Table 47 and graphically shown in Figure 149. As seen from Table 47, all the joint reinforcement configurations evaluated in this study were proved to be effective in enhancing the load-carrying capacity to a maximum of $195 \%$ over the control specimen.

Table 47. Load vs. deflection of joints reinforced with PSL filler-modules and FRP wraps

\begin{tabular}{lcccc}
\hline \multirow{2}{*}{ Joint } & \multicolumn{3}{c}{ Measured at peak load } & \multirow{2}{*}{ Observed failure mode } \\
\cline { 2 - 4 } & Beam load (lb) & \% increase & $\Delta$ (in) & \\
\hline JII-1 & 20,900 & Control & 1.3 & Joint/diagonal tension \\
\hline JII-2 & 35,273 & 69 & 2.4 & Joint shear failure \\
\hline JII-3 & 31,695 & 52 & 1.8 & Joint/column shear failure \\
\hline JII-4 & 34,989 & 67 & 1.7 & Column shear failure \\
\hline JII-5 & 41,868 & 100 & 2.9 & Joint failure \\
\hline JII-6 & 61,592 & 195 & 3.2 & Column shear failure \\
\hline JII-7* & 56,894 & 172 & 3.4 & Beam flexural failure \\
\hline JII-8 & 59,320 & 184 & 4.6 & Beam flexural failure \\
\hline
\end{tabular}

* Loading was terminated abruptly before reaching the ultimate load due to the slippage of the specimen from the test setup.

Specimen JII-2, reinforced with CFRP wrap without filler-modules, exhibited a 69\% increase in load-carrying capacity over the control specimen. Although an increase in the load-carrying 
capacity was noted for JII-2, failure was still concentrated in the joint panel. This increase in the load-carrying capacity of JII-2 is because of the confinement from the CFRP wrap, which delayed the crushing of concrete and allowed the steel rebars to resist additional tensile forces.

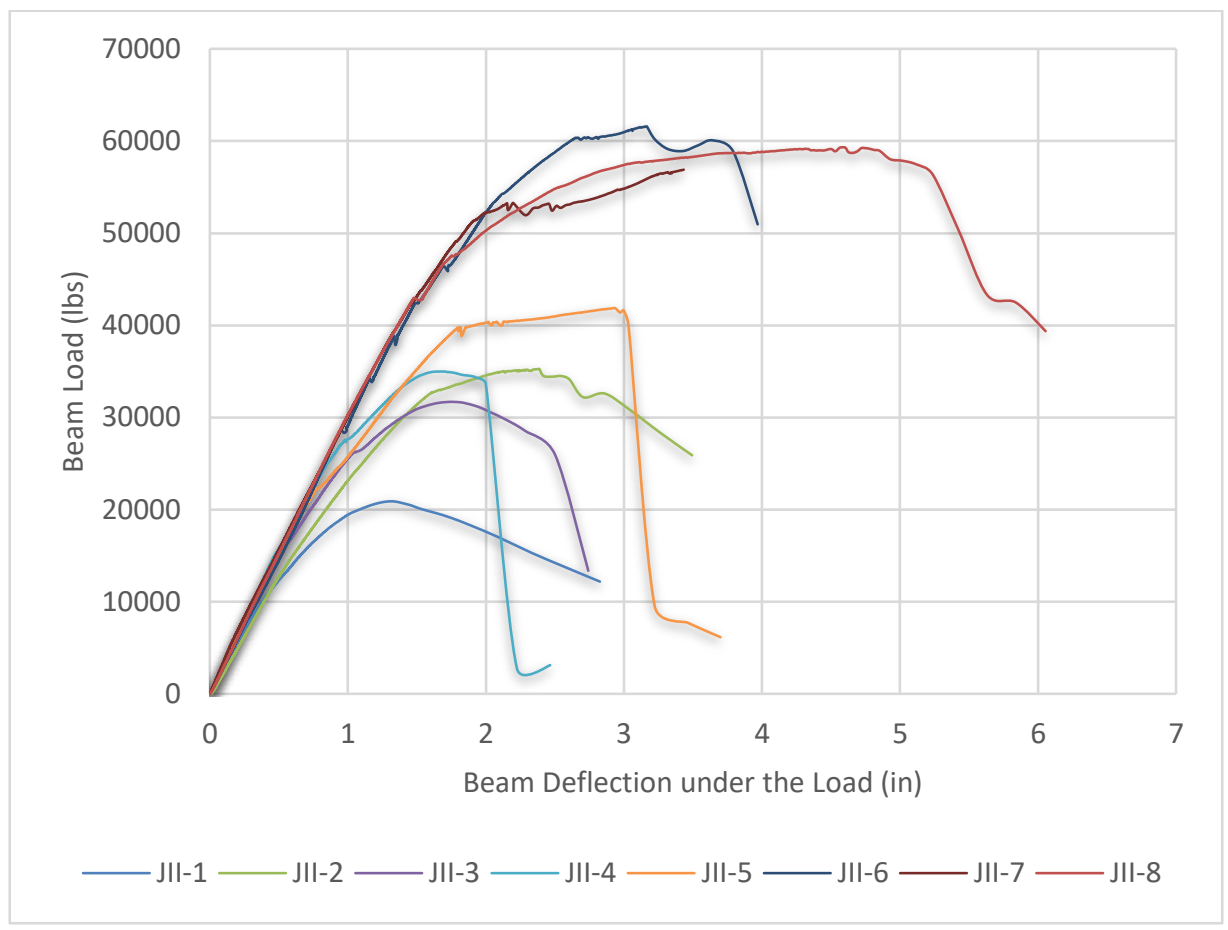

Figure 149. Load vs. deflection of joints reinforced with PSL filler-modules and FRP wraps

Among the specimens reinforced with filler-modules, the specimen JII-4 having wedge fillermodules exhibited a $67 \%$ increase in the load-carrying capacity as opposed to a $52 \%$ increase for specimen JII-3 with curved filler-modules. The additional increase in load-carrying capacity for JI-4 is attributed to the better compression (strut) resistance offered by the wedge filler-module when compared to a curved filler-module.

The use of dowels in specimen JII-6 significantly contributed to the increase in the load-carrying capacity ( $47 \%$ higher) when compared to the specimen JII-5 without dowels. This increase reveals the significance of dowels in providing composite action between the filler-modules and underlying substrate, offering additional resistance through the efficient transfer of shear forces over a larger area of the beam, column, and joint sections. 
Specimens reinforced with CFRP wraps (JII-7) and GFRP wraps (JII-8) exhibited $~ 184 \%$ and $172 \%$ increases in load-carrying capacity, respectively. No specific discussions were made comparing the contributions of CFRP vs. GFRP wraps because of the early termination of load for specimen JII-7. 


\subsubsection{Failure mode}

The failure modes of joint specimens observed during the lab testing are presented in Table 48. As expected, the control specimen without transverse (stirrup) reinforcement exhibited joint shear failure before rebar yielding, which is commonly seen in buildings collapsed under earthquakes.

Specimen JII-2, reinforced with CFRP wrap, exhibited joint shear failure through the rupture of FRP at the reentrant corner in the tension zone. This behavior reveals the need for installing fillermodules at joint corners to redistribute stresses and avoid such failures.

Failure in specimen JII-3 initiated as a diagonal cracking in the joint panel but ultimately merged with column shear cracks, resulting in early loss of load-carrying capacity. The initiation of cracking in the joint panel reveals the need for using a filler-module of high-strength with increased throat depth to redistribute forces over a larger area of the specimen. In the case of joint reinforced with wedge filler-modules (JII-4), the failure initiated at the tip of the filler-module next to the column and progressed as a shear crack into the column panel. The initiation of cracking at the tip of the filler-module can be delayed or prevented by bonding FRP wraps in a direction perpendicular to the orientation of the cracks and further confining with $360^{\circ}$ - or U- anchors. At failure, buckling of column longitudinal rebars was seen in both specimens JII-3 and JII-4.

Lack of dowels in specimen JII-5 has resulted in a joint shear failure due to the premature debonding of filler-module, as opposed to a column shear failure in specimen JII-6 with dowels. The joint shear failure in specimen JII-5 has resulted in a small increase of structural capacities compared to specimen JII-6.

Both specimens JII-7 and JII-8 reinforced with CFRP and GFRP wraps, respectively, exhibited beam flexural cracking. Besides, for specimen JII-8, splitting of wood fibers in the top fillermodule along with the fracture of beam longitudinal rebars at the $90^{\circ}$ hook was noticed. The superior performance of JII-8 is attributed to the better bond ability of GFRP to the underlying substrate (i.e., because of better stiffness compatibility between GFRP and substrate).

Note: Specimen JII-7 would have exhibited better response, provided the specimen was loaded until final failure. 
Table 48. Failure modes of joints reinforced with PSL filler-modules and FRP wraps
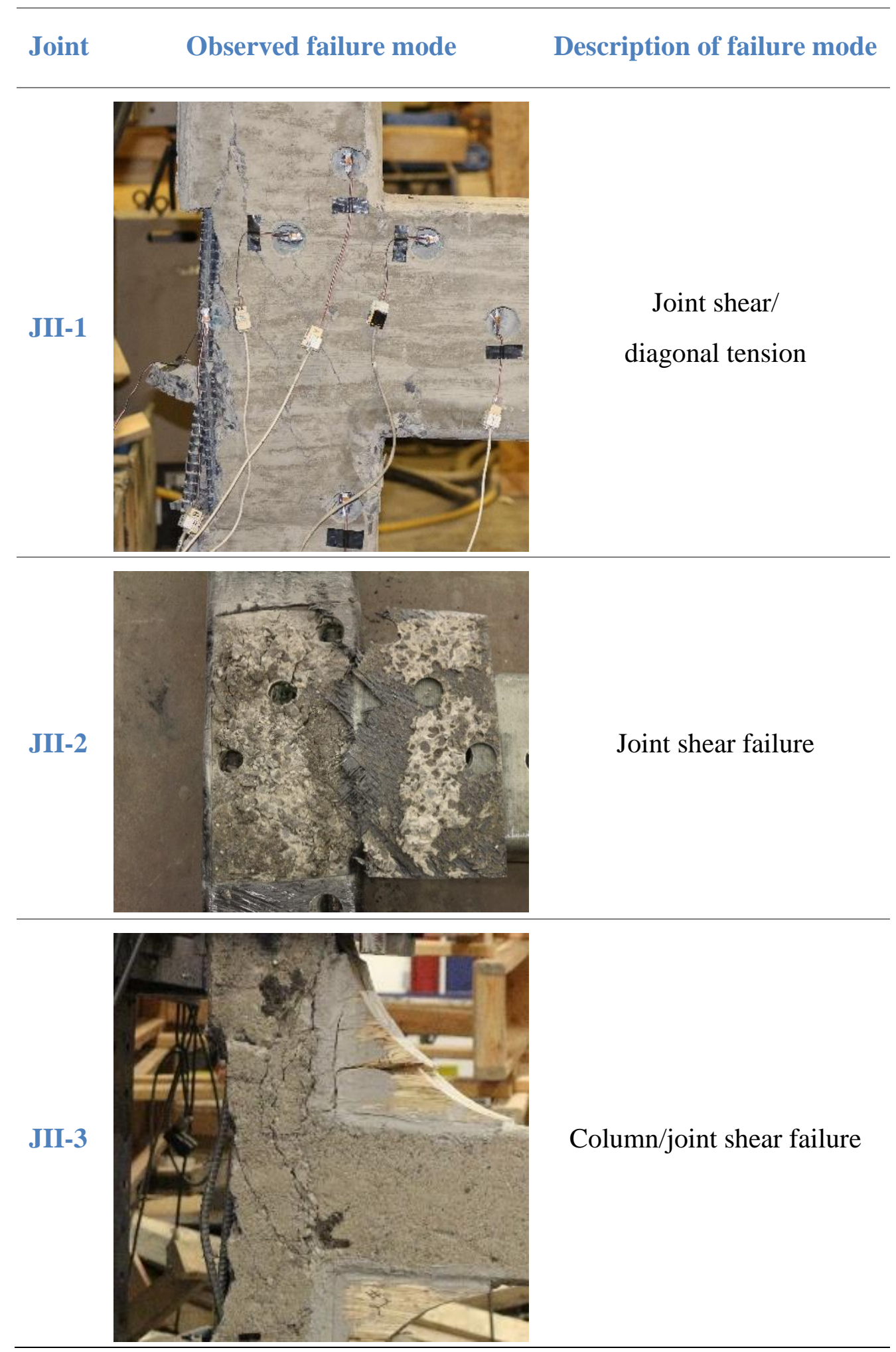

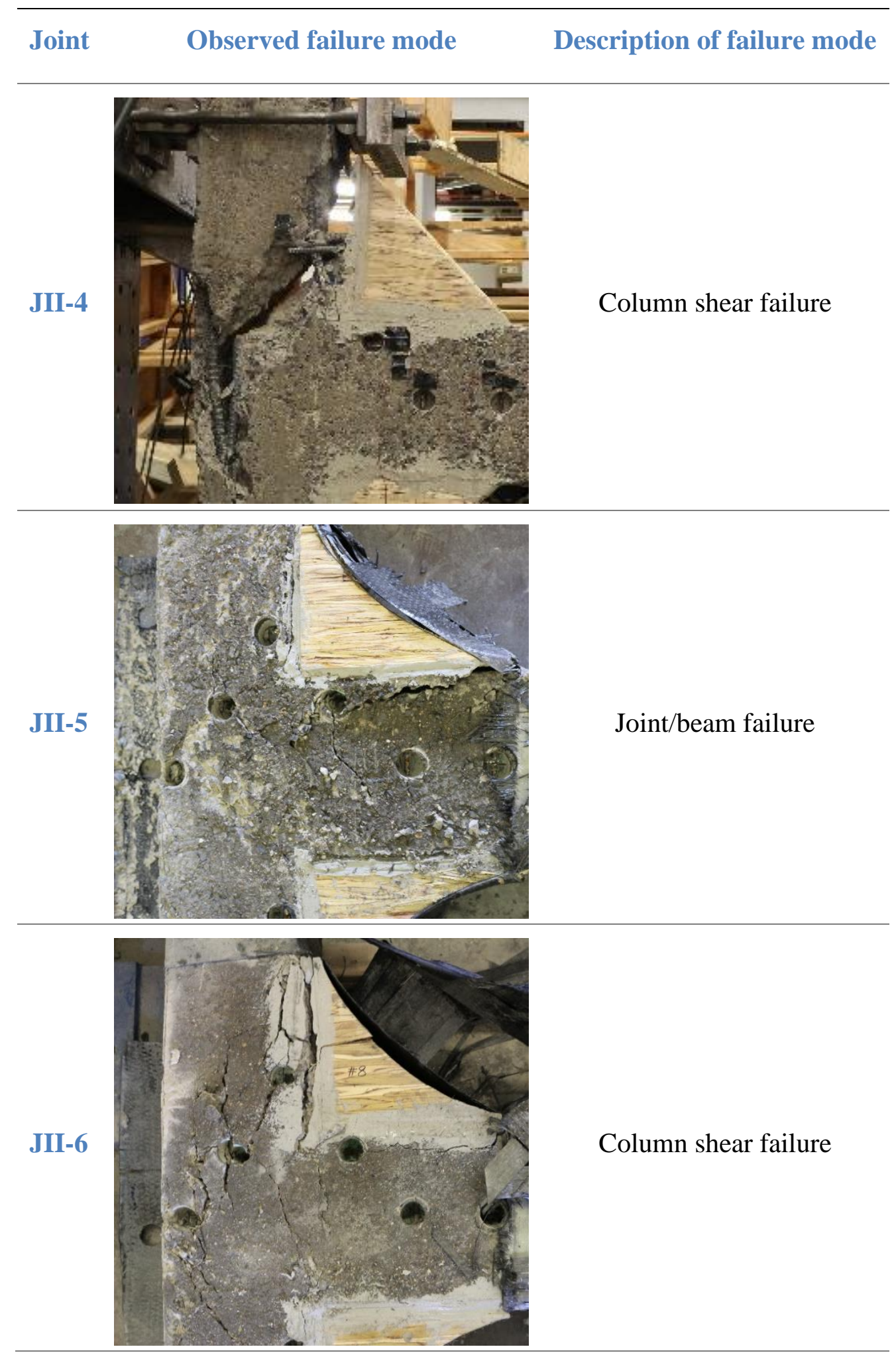

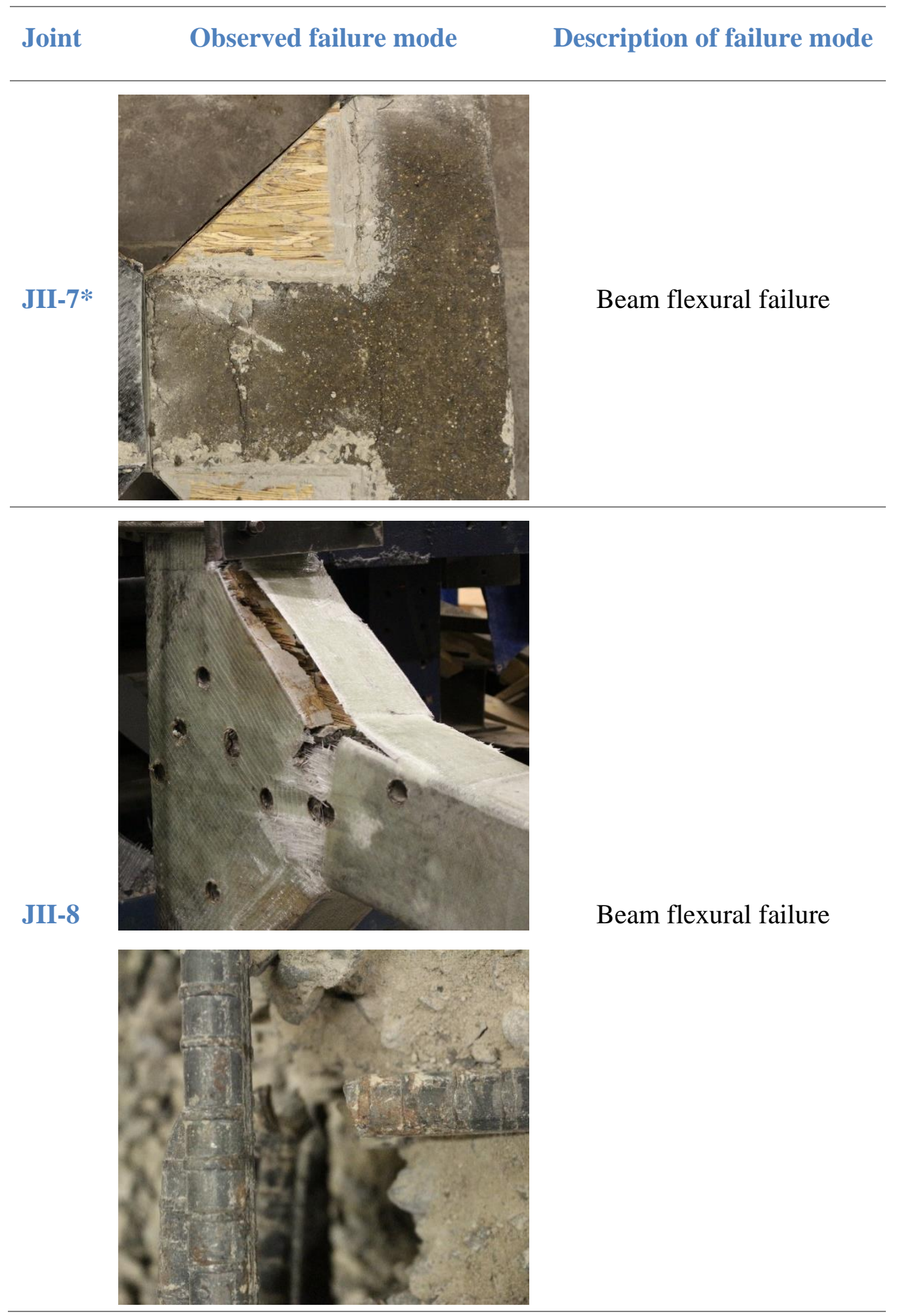

* Loading was terminated abruptly before reaching the ultimate load due to the slippage of the specimen from the test setup. 


\subsubsection{Stiffness}

The stiffness of each of the eight joint specimens was computed as defined in section 5.3.3. Furthermore, a summary of the computations is presented in Table 49.

Table 49. Stiffness of joints reinforced with PSL filler-modules and FRP wraps

\begin{tabular}{lcc}
\hline Joint & Tangent modulus & \% increase \\
\hline JII-1 & 28,176 & Control \\
\hline JII-2 & 25,517 & -9 \\
\hline JII-3 & 30,010 & 7 \\
\hline JII-4 & 32,036 & 14 \\
\hline JII-5 & 29,665 & 5 \\
\hline JII-6 & 29,250 & 4 \\
\hline JII-7* & 31,106 & 10 \\
\hline JII-8 & 30,401 & 8 \\
\hline
\end{tabular}

* Loading was terminated abruptly before reaching the ultimate load due to the slippage of the specimen from the test setup.

As seen in Table 49, the maximum increase in stiffness of joint specimens due to the addition of filler-modules with or without FRP wraps was found to be $\sim 14 \%$. The relatively small increase in stiffness is attributed to the low modulus of elasticity of PSL wood filler-modules parallel to the wood fiber orientation, which did not stiffen the beams, thus resulting in higher beam tip deflections. 


\subsubsection{Ductility index}

The ductility index of all the joint specimens was computed as defined in section 5.3.4. Furthermore, a summary of the computations is presented in Table 50.

Table 50. Ductility index of joints reinforced with PSL filler-modules and FRP wraps

\begin{tabular}{|c|c|c|c|c|}
\hline \multirow{2}{*}{ Joint } & \multicolumn{2}{|c|}{ Measured at peak load } & \multirow{2}{*}{$\frac{\text { Idealized yield }}{\Delta_{y i} \text { (in) }}$} & \multirow{2}{*}{$\begin{array}{l}\text { Ductility index } \\
\left(\mu=\Delta_{u} / \Delta_{y i}\right)\end{array}$} \\
\hline & Beam load (lb) & $\Delta_{u}($ in $)$ & & \\
\hline JII-1 & 20,900 & 1.3 & 0.6 & 2 \\
\hline JII-2 & 35,273 & 2.4 & 0.6 & 4 \\
\hline JII-3 & 31,695 & 1.8 & 0.6 & 3 \\
\hline JII-4 & 34,989 & 1.7 & 0.6 & 3 \\
\hline JII-5 & 41,868 & 2.9 & 0.6 & 5 \\
\hline JII-6 & 61,592 & 3.2 & 0.6 & 5 \\
\hline JII-7* & 56,894 & 3.4 & 0.6 & 6 \\
\hline JII-8 & 59,320 & 4.6 & 0.6 & 8 \\
\hline
\end{tabular}

* Loading was terminated abruptly before reaching the ultimate load due to the slippage of the specimen from the test setup.

All the reinforcing schemes adopted in this study for joint strengthening were proved to be efficient in enhancing the ductility index over the control specimen. For specimen JII-2, the confinement effect from CFRP wrap contained the concrete from crushing and allowed the rebar cage to offer more resistance against induced tensile forces, thus resulting in additional deformation before the collapse. This increase of deformation in the inelastic zone due to CFRP confinement has resulted in a better ductility index over the control specimen.

The use of low-stiffness PSL wood filler-modules for reinforcing joint specimens JII-3 and JII-4 resulted in a better ductility index over the control specimen. This improvement in the ductility index of specimens JII-3 and JII-4 is attributed to the additional compressive force resistance (strut formation) offered by the bottom filler-module between the points $\mathrm{B}$ and $\mathrm{C}$ before any sudden failure taking place due to weakness of oriented material like engineered wood. Although, slightly better strain distribution in the rebars was observed for joint specimen reinforced with curve fillermodules over wedge filler-modules, no appreciable difference in the ductility index was noticed. 
Comparing specimens JII-5 and JII-6 reveal that the use of dowels in JII-6 does not influence the ductility index. Although specimen JII-5 without dowels exhibited premature debonding of the top filler-module, the confinement effect from $360^{\circ}$-anchor around the filler-module and joint helped resist the forces in the inelastic zone without resulting in sudden collapse.

Among all the reinforcing schemes except for specimen JII-7, specimen JII-8 exhibited the highest ductility index of 8 . The enhanced compositeness between GFRP wraps and the substrate due to the better strain compatibility of constituent materials resulted in shifting the joint/column failure to a ductile beam flexural failure, thus leading to a superior response of JII- 8 over other reinforcing schemes.

Note: * Loading was terminated abruptly before reaching the ultimate load due to the slippage of the specimen from the test setup. 


\subsubsection{Energy dissipation}

The cumulative energy dissipation capacity of each of the eight joint specimens was computed as defined in section 5.3.5. Furthermore, a summary of the computations is presented in Table 51 and graphically shown in Figure 150.

Table 51. Energy dissipation of joints reinforced with PSL filler-modules and FRP wraps

\begin{tabular}{lcc}
\hline Joint & Cumulative energy dissipated (lb.in) & \% increase \\
\hline JII-1 & 17,458 & Control \\
\hline JII-2 & 56,373 & 223 \\
\hline JII-3 & 37,373 & 114 \\
\hline JII-4 & 37,488 & 115 \\
\hline JII-5 & 85,476 & 390 \\
\hline JII-6 & 126,654 & 625 \\
\hline JII-7* & 133,506 & 665 \\
\hline JII-8 & 204,972 & 1,074 \\
\hline
\end{tabular}

* Loading was terminated abruptly before the complete failure of the specimen.

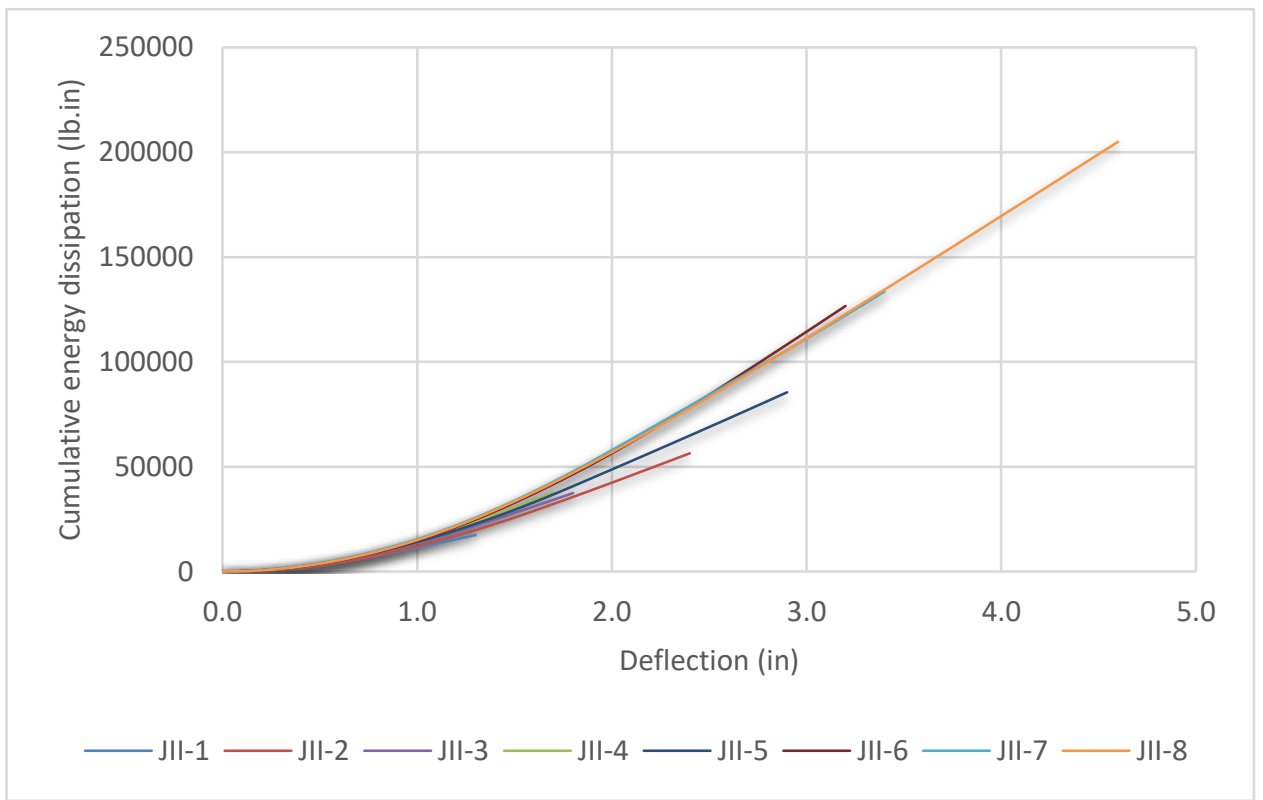

Figure 150. Energy dissipation of joints reinforced with PSL filler-modules and FRP wraps 
Specimen JII-2, reinforced with CFRP wraps without filler-modules, dissipated $223 \%$ more energy compared to the control specimen JII-1. The increase in energy dissipation capacity of JII-2 over JII-1 is attributed to the additional tensile resistance offered by the rebar cage due to the confined concrete effect from CFRP wrap between points B and C. No significant difference was observed in the energy dissipation capacity of specimens JII-3, and JII-4 reinforced with curve and wedge filler-modules, respectively. It must be highlighted that the presence of dowels alleviated the specimen JII-6 to dissipate $48 \%$ more energy compared to the specimen JII- 5 without dowels. This response reveals that the presence of dowels ensured efficient composite action between the beam, column, and joint through shear transfer, thus resulting in a superior structural capacity. Among the joint specimens reinforced with filler-modules and FRP wraps, specimen JII-8 with GFRP wrap exhibited the highest ( 11 times) energy dissipation capacity. The superior performance of JII-8 reveals that the strain/stiffness compatibility between FRP wraps and the underlying substrate is essential for the structures to dissipate more energy under earthquake forces, especially in the inelastic zone. 


\subsubsection{Strains in joint-, beam-, and column- rebars}

In order to assess the strain progression from the beam- and column- rebars into the joint panel, several gages were mounted on steel rebars at critical locations, as shown in Figure 71. Results deduced from these strain gages at locations JSH1, BSL1, BSL2, and CSL2 are studied carefully and presented in Table 52 to asses the influence of various reinforcing schemes.

Table 52. Rebar strain data of joints reinforced with PSL filler-modules and FRP wraps

\begin{tabular}{|c|c|c|c|c|c|c|}
\hline \multirow{2}{*}{ Joint } & \multirow{2}{*}{ PoI } & \multirow{2}{*}{ Beam load (lbs) } & \multicolumn{4}{|c|}{ Rebar strain $(\mu \varepsilon)$} \\
\hline & & & JSH1 & BSL1 & BSL2 & CSL2 \\
\hline \multirow{3}{*}{ JII-1 } & $\mathrm{A}$ & 12,724 & 931 & 1,040 & N.A. & 410 \\
\hline & B & 13,295 & 1,021 & 1,110 & N.A. & 478 \\
\hline & $\mathrm{C}$ & 20,900 & 1,947 & 1,750 & N.A. & 1,380 \\
\hline \multirow{3}{*}{ JII-2 } & $\mathrm{A}$ & 10,808 & 416 & 704 & 551 & 176 \\
\hline & B & 14,995 & 820 & 1,060 & 812 & 350 \\
\hline & $\mathrm{C}$ & 35,273 & 3,235 & 14,330 & 2,311 & 1,909 \\
\hline \multirow{3}{*}{ JII-3 } & A & 11,289 & 132 & 462 & 712 & 34 \\
\hline & $\mathrm{B}$ & 16,028 & 434 & 818 & 1,135 & 66 \\
\hline & $\mathrm{C}$ & 31,695 & 2,142 & 1,858 & 2,533 & 1,877 \\
\hline \multirow{3}{*}{ JII-4 } & A & 15,404 & 202 & 630 & 1,053 & 86 \\
\hline & $\mathrm{B}$ & 17,668 & 290 & 809 & 1,217 & 110 \\
\hline & $\mathrm{C}$ & 34,989 & 1,595 & 2,014 & 2,776 & 2,651 \\
\hline \multirow{3}{*}{ JII-5 } & A & 12,014 & 137 & 474 & 648 & 93 \\
\hline & $\mathrm{B}$ & 23,219 & 1,061 & 1,289 & 1,459 & 355 \\
\hline & $\mathrm{C}$ & 41,868 & 2,061 & 3,298 & 7,412 & 2,680 \\
\hline \multirow{3}{*}{ JII-6 } & A & 29,512 & 370 & 825 & 1,223 & 81 \\
\hline & B & 34,063 & 522 & 1,048 & 1,534 & 106 \\
\hline & $\mathrm{C}$ & 61,592 & 1,909 & 2,797 & 14,627 & 1,364 \\
\hline \multirow{3}{*}{ JII-7 } & A & 28,390 & 445 & 1,044 & 1,516 & 90 \\
\hline & B & 38,946 & 816 & 1,612 & 2,188 & 232 \\
\hline & $\mathrm{C}$ & 56,894 & 1,784 & 11,519 & 16,638 & 1,870 \\
\hline \multirow{3}{*}{ JII-8 } & A & 30,612 & 550 & 1,218 & 1,605 & 222 \\
\hline & $\mathrm{B}$ & 36,706 & 797 & 1,549 & 2,007 & 359 \\
\hline & $\mathrm{C}$ & 59,320 & 2,340 & 16,375 & 22,325 & 2,157 \\
\hline
\end{tabular}

Red color indicates rebar yielding and attainment of plastic strains in some cases. 
As expected, the control specimens (JII-1) failed in the joint panel without yielding of the beam or column rebar. The strain gage location (BSL1) that is $3 \mathrm{in}$. away from the reentrant corner on the beam section exhibited higher strains indicating the formation of hinge next to the column face. This behavior can lead to the sudden progression of crack into the joint panel, causing brittle joint failure.

Specimen JII-2, confined with CFRP wrap, failed in joint shear after yielding (reached plastic strain) of beam longitudinal rebar at BSL1. Yielding in rebar occurred before the attainment of peak-load.

Specimens JII-3 reinforced with curved PSL filler-modules exhibited near-uniform strain distribution in the rebars of the beam, column, and joint compared to specimen JII-4 reinforced with wedge filler-modules. The strain in rebar location BSL2 for specimen JII-4 yielded at the peak load.

In all the joint specimens reinforced with filler-modules and FRP wrap (JII-5 through 8), the rebar yielded at both locations BSL1 and BSL2. For all the specimens JII-5 through 8, yielding occurred first at BSL2 then followed by BSL1, indicating the gradual progression of yield strain towards the joint panel in a controlled manner. At ultimate load (i.e., collapse), all the four beam longitudinal rebars in JII- 8 fractured at the $90^{\circ}$ hook in the joint panel, as shown in Table 48 . 


\subsection{Conclusions}

A summary of the test results based on the experimental findings of joints reinforced with PSL filler-modules and FRP wraps is presented in Table 53. Besides, the following sections (6.4.1.1 through 6.4.1.4) provide concluding remarks on various parameters evaluated in this study:

Table 53. Summary of test results: joints reinforced with PSL filler-modules and FRP wraps

\begin{tabular}{ccccc}
\hline Joint & $\begin{array}{c}\text { Peak load } \\
\text { (lbs) }\end{array}$ & $\begin{array}{c}\text { Ductility } \\
\text { index }\end{array}$ & $\begin{array}{c}\text { Energy dissipated } \\
\text { (lb.in) }\end{array}$ & Failure mode \\
\hline JII-1 & 20,900 & 2 & 17,458 & Joint/diagonal tension \\
\hline JII-2 & $35,273(69)$ & $4(100)$ & $56,373(223)$ & Joint shear failure \\
\hline JII-3 & $31,695(52)$ & $3(50)$ & $37,373(114)$ & Joint/column shear failure \\
\hline JII-4 & $34,989(67)$ & $3(50)$ & $37,488(115)$ & Column shear failure \\
\hline JII-5 & $41,868(100)$ & $5(150)$ & $85,476(390)$ & Joint failure \\
\hline JII-6 & $61,592(195)$ & $5(150)$ & $126,654(625)$ & Column shear failure \\
\hline JII-7* & $56,894(172)$ & $6(200)$ & $133,506(665)$ & Beam flexural failure \\
\hline JII-8 & $59,320(184)$ & $8(300)$ & $204,972(1,074)$ & Beam flexural failure \\
\hline
\end{tabular}

Numbers inside ( ) indicate \% increases with respect to the control specimen of a given batch.

* Loading was terminated abruptly before the complete failure of the specimen.

\subsubsection{Influence of CFRP wrap without filler-modules (JII-2)}

Although reinforcing joints with CFRP wrap without filler-modules has increased the strength, ductility, and energy dissipation capacities by $69 \%, 100 \%$, and $223 \%$, respectively, over the control specimen, the failure was still concentrated in the joint panel. The increase in structural capacities is attributed to the confinement from the CFRP wrap, which delayed the crushing of concrete and allowed the steel rebars to resist additional tensile forces. This approach of reinforcing joints with CFRP wraps around a joint is challenging to implement on a real building frame due to the presence of transverse beam(s) and slab(s) in one or more directions. 


\subsubsection{Influence of curve- versus wedge- filler-module (JII-3 vs. JII-4)}

Both the curve- and wedge- filler-module reinforced joint specimens exhibited an increase in the strength, ductility, and energy dissipation by up to $67 \%, 50 \%$, and $115 \%$, respectively. Although the increase in ductility and energy absorption was similar between curve- and wedge- fillermodule reinforced joints, the additional $\sim 10 \%$ increase in the strength of wedge filler-module specimen is attributed to the higher compression (strut) resistance offered by the wedge fillermodule when compared to a curved filler-module.

\subsubsection{Influence of dowels (JII-5 vs. JII-6)}

Specimen JII-6 reinforced with dowels exhibited a 47\% increase in the strength and energy dissipation capacities over the specimen JII-5 reinforced without dowels. This behavior reveals that the presence of dowels ensured efficient composite action between the beam, column, and joint through shear transfer, thus resulting in a superior structural capacity.

\subsubsection{Influence of CFRP versus GFRP wrap (JII-7 vs. JII-8)}

Both the specimens reinforced with CFRP- and GFRP- wraps significantly improved the structural response of joints. No specific discussions were made comparing the contributions of CFRP vs. GFRP wraps because of the early termination of load for specimen JII-7. However, it should be highlighted that the trend in rebar strain distribution (shown in Table 52) of both specimens revealed a similar performance until the point of load termination.

Specimen JII-8 reinforced with GFRP wrap exhibited the highest ductility ( $\sim 3$ times) and energy dissipation capacity ( $\sim 11$ times) among all the reinforcing schemes evaluated in the current phase. The superior performance of JII- 8 reveals that the strain/stiffness compatibility between FRP wraps and the underlying substrate is essential for the structures to dissipate more energy under earthquake forces, especially in the inelastic zone. 


\section{Summary, Conclusions, and Recommendations}

\subsection{Introduction}

This chapter summarizes experimental findings and provides the key conclusions derived from the current study on "Monotonic response of RC exterior joints reinforced with filler-modules and FRP composite wraps/gussets." The conclusions for joints evaluated in control- and reinforced(with filler-modules and with/out FRP wraps/gussets) conditions are drawn primarily based on the strength, ductility, and energy dissipation corresponding to peak loads, because of their importance under extreme load events. The joint behavior is a function of many parameters, and their influence on the structural behavior has been exhaustively studied, with key conclusions reported herein. This chapter also provides recommendations for future research to expand the scope of the study to real structural scenarios for devising appropriate design methodologies.

\subsection{Summary}

Over the past 50 years, several studies were conducted to strengthen exterior joints of in-service structures, with limited emphasis on developing simple, economic and durable repair strategies to improve energy absorption through large inelastic joint deformations. Even less emphasis was devoted to developing repair procedures that minimize stress-concentrations at joint corners and enhancing the strength, ductility, and energy dissipation capabilities of concrete structures with an emphasis on joint resistance improvements.

To address the above limitations, a novel approach of reinforcing vulnerable joints with fillermodules and fiber-reinforced polymer (FRP) composite wraps/gussets have been proposed and evaluated, herein. A total of twenty 2D RC exterior (T) joints were designed as per pre-1976 construction deficiencies and experimentally evaluated in control (as-built) and reinforced conditions through the variations in (i) filler-module geometric shape; (ii) filler-module material properties, (iii) FRP material, (iv) FRP wrap/gusset configurations, (v) confinement due to partial (U-anchors) versus complete $\left(360^{\circ}\right.$-anchors) diagonal wraps, and (vi) shear transfer through reinforcing dowels. The performance of the test specimens was recorded through numerical values 
of loads versus deformation and strains at the rupture of concrete, de-bond of FRP wrap from the concrete surface, yielding of steel rebar, shear failure of column or joint panel through diagonal tension and beam flexural failure phenomena. Test data evaluations measured up to the peak loads revealed that the proposed approach of reinforcing joints with filler-modules and FRP wraps/gussets is immensely useful in enhancing the strength and ductility by $\sim 300 \%$, and energy dissipation by about $\sim 1200 \%$. Depending upon the reinforcing scheme(s), the magnitude of failure- loads and patterns varied in a controlled manner. Joints tested in "control" condition exhibited shear failure through diagonal tension and diagonal compression while the strengthened specimens failed in beam flexure or column shear, but in a ductile manner through yielding of the column- or beam- rebars. The strains measured on rebar surfaces at different locations of jointand beam- sections revealed a significant reduction in strain progression towards the joint panel (beam-column overlap). It was also noted that the use of low-stiffness filler-modules such as syntactic foam and engineered wood coupled with FRP wraps has tremendously enhanced the structural response of reinforced joints. It was also observed that joints reinforced with fillermodules and FRP wraps or gussets exhibiting beam flexural failure had more energy dissipation capacity when compared to specimens that failed in column shear. Based on the experimental results of reinforced joint specimens, joint behavior is characterized into three zones, i.e., A (onset of filler-module cracking), B (idealized yield - defined as the point beyond which a truss mechanism primarily resists the forces), and C (Peak load - referred to as a highest numerical value of load recorded during the testing). Furthermore, limit states (principal tension and shear) for joint at the onset of filler-module cracking (i.e., point A) and idealized yield (i.e., point B) have been established as a function of the concrete tensile strength $\left(k \sqrt{f_{c}^{\prime}}\right)$ and presented in Tables 33, 35, 37 , and 46.

The behavior of joints reinforced with filler-modules and FRP wraps/gussets up to the point A and perhaps point B is governed by the location of the plastic hinge away from the joint panel, which primarily depends on the strength and stiffness of bottom filler-module (i.e., in the compression zone) and to some extent on the tensile resistance offered by the top filler-module (i.e., in the tension zone). For example, if the hinge forms within the filler-module length, the specimen offers additional force resistance because of the higher moment of inertia (I) at that section. Besides, the 
shear deformations $(\gamma)$ induced in the panel are lower due to the shorter lever-arm (i.e, distance from C.G. of the joint panel to the hinge point). Furthermore, the resistance to induced stresses in the joint panel can be enhanced by simply bonding FRP reinforcement to the exposed joint section as a "gusset" or by confining through $360^{\circ}$ or U-wrap - depending on the accessibility of joint panel). On the other hand, if the hinge forms outside of the filler-module width (i.e., either in the beam or column section), the resistance offered is limited to the capacity of beam or column due to reduced "I". Although this approach may yield better results (i.e., increase in the peak load, ductility, and energy absorption) over the control specimen, it may not necessarily maximize the overall structural response in relation to the enhanced joint capacity. Latter approach has been proposed and followed by several researchers, i.e., merely pushing the hinge formation farther away from the column face, into the beam section by providing steel haunches [22]-[24] or FRP wrapping of beam and column stubs up to their plastic hinge locations [15]-[19] or planar joint enlargement [27]-[30], and [125] and other approaches. Unlike other approaches, the current study is unique in terms of maximizing the structural response using low-stiffness filler-modules with moderate- to high- compressive strength coupled with FRP wraps/gussets.

\subsection{Conclusions}

The use of filler-modules coupled with FRP wraps/gussets for reinforcing 2D RC exterior joints has been developed and investigated in this study. The experimental findings and observations from this study, presented in sections 5.3 and 6.3 of this manuscript, revealed the following key conclusions:

1. The tensile strength of FRP wrapped cylinders tested under split tension was found to be $\sim(20-28) \sqrt{f_{c}^{\prime}}$ as opposed to $\sim(6-8) \sqrt{f_{c}^{\prime}}$ for normal weight plain concrete cylinders (section 4.4.6).

2. The test results of joint (control) specimens indicated poor performance of pre-1976 designs, resulting in brittle joint shear failure. Besides, joint (control) specimens subjected to axial column loads in the range of 0.18 to $0.21 f_{c}^{\prime} A_{g}$ resulted in joint failure through 
diagonal tension cracking, while the specimen subjected to axial column load of $0.28 f_{c}^{\prime} A_{g}$ exhibited joint failure through the crushing of concrete (section 5.2.1, and 6.2.1).

3. Although FRP strengthening of joint panels (section 6.4.1.1) exhibited $\sim 69 \%, 100 \%$, $223 \%$ in strength, ductility, and energy dissipation capacities, respectively, the failure was still concentrated in the joint panel.

4. Reinforcing joints with filler-modules without FRP wraps/gussets exhibited a limited increase in strength, ductility, and energy dissipation capacities due to the premature column failure initiated at the tip of the filler-module (as shown in Table 43 and Table 53 and sections 5.4.1.1 and 6.4.1.2).

5. The use of filler-modules coupled with FRP wraps/gussets increased the strength, ductility, and energy dissipation by up to $\sim 314 \%, 300 \%, \sim 1,118 \%$, respectively, over the control specimen (Table 43). These increases are attributed to the relocation of plastic hinge away from the joint panel, resulting in either column shear or beam flexural failure. The joint specimens that failed in beam flexure exhibited superior structural response than joint specimens that failed in column shear.

6. Joint specimens reinforced with filler-modules, dowels, and FRP wraps/gussets were found to be effective in preventing the joint shear failure while forcing the plastic hinge to form in the beam or column sections (Tables 43 and 54).

7. The joint specimen reinforced with concrete filler-modules, CFRP wrap, and $360^{\circ}$-anchor, exhibited an increase of $\sim 170 \%, 100 \%$, and $402 \%$ in strength, ductility, and energy dissipation capacities over the joint specimen reinforced with concrete filler-modules without CFRP wrap and $360^{\circ}$-anchor (Table 43). This improvement is primarily due to the tensile resistance offered by the CFRP wrap in addition to the concrete confinement effect from $360^{\circ}$-anchor. This approach of reinforcing joints with CFRP wrap and $360^{\circ}$-anchor around the filler-modules has shifted the non-ductile column shear failure (which is not a preferred failure mode) to a more ductile beam flexural failure.

8. The low-stiffness syntactic foam filler-modules coupled with CFRP wraps facilitated a near-uniform strain distribution in the rebars- of column $(2612 \mu \varepsilon)$, beam $(2608 \mu \varepsilon)$ and joint panel $(2263 \mu \varepsilon)$ (Table 42) thus, resulting in superior performance over other joint reinforcing schemes. 
9. Comparing joint specimens reinforced with medium- and high- strength filler-modules coupled with CFRP gussets, the latter exhibited $\sim 41 \%, 25 \%$, and $64 \%$ in strength, ductility, and energy dissipation capacities. The superior performance of the high-strength filler-module is attributed to the enhanced compression force (strut) resistance offered by the filler-module beyond the elastic zone. The higher the strength of filler-module, the longer the plateau between points B and C, i.e., more prolonged inelastic zone.

10. The shape of the filler-modules did not significantly alter the behavior of joints in terms of strength, ductility, and energy dissipation capacities (<9\% variation).

11. Dowel reinforcement in filler-modules increased the strength and energy dissipation of joint specimens by $\sim 47 \%$ when compared to the specimen without the dowel reinforcement. This behavior reveals that the presence of dowels ensured efficient composite action between the beam, column, and joint through shear transfer, thus resulting in a superior structural capacity.

12. Among the joint reinforcing schemes using PSL filler-modules, the specimen reinforced with PSL wedge filler-modules, dowels, and GFRP wrap exhibited superior performance (section 6.4.1.4). The superior performance reveals the stiffness compatibility between FRP wraps and the underlying substrate is essential.

\subsection{Recommendations for Future Research}

The following recommendations are made for future research to expand the scope of this study:

1. Joints of a building frame are formed by the intersection of beams, columns, and slab in two or more directions. Therefore, to understand the behavior of a real structural scenario, future research on the current approach with filler-modules and FRP wraps/gussets must be directed towards evaluating the performance of joints with additional stiffness contributions from the slab, and transverse beams to establish joint limit states and to devise appropriate design methods.

2. Under seismic actions, exterior joints are subjected to varying axial load and bidirectional lateral load reversals. Therefore, the response of exterior joints reinforced with filler- 
modules and FRP wraps/gussets under varying axial load and bidirectional lateral load reversals must be evaluated.

3. Varying a/d (distance from CG of joint to effective depth) ratios for both column and beam stubs meeting at a joint must be evaluated to investigate the influence of the current approach under different failure modes.

4. Due to the limited availability of experimental data on joints under many independent parameters, machine learning tools must be employed to train, evaluate and develop strength prediction models after generating additional test data in a strategic sense with an understanding developed from current research studies.

5. To optimize the joint performance, finite element analysis studies on joint inelastic behavior must be performed by incorporating material nonlinearity (post-cracking behavior of concrete joint or element) to arrive at the optimized shape of filler-modules and optimized fiber orientation of composite wraps/gussets as a function of the substrate strength and bond strengths of glue lines of the substrate, filler-modules and wrap or gusset. 


\section{References}

1. P. Low, "The natural disasters of 2018 in figures," www.munichre.com, 01 August 2019. [Online]. Available: https://www.munichre.com/topics-online/en/climate-change-and-naturaldisasters/natural-disasters/the-natural-disasters-of-2018-in-figures.html. [Accessed 10 December 2019].

2. S. Hallegatte, A. Vogt-Schilb, M. Banglore and J. Rozenberg, "Unbreakable: Building the Resilience of the Poor in the Face of Natural Disasters," The World Bank Group, Washington, DC, 2017.

3. California Governor's Office of Emergency Services, "2018 State of California Hazard Mitigation Plan," Mather, CA, 2018.

4. United States Geological Survey (USGS), "Forecasting California's Earthquakes - What Can We Expect in the Next 30 Years?," 2008.

5. C. A. Kircher, H. A. Seligson, J. Bouabid and G. C. Morrow, "When the Big One Strikes Again

- Estimated Losses due to a Repeat of the 1906 San Francisco Earthquake," Earthquake Spectra, vol. 22, no. 52, April 2006.

6. K. D. Palmer, "Nonductile Concrete Frames," STRUCTURE magazine, pp. 24-26, November 2019.

7. C. Clyde, C. P. Pantelides and L. D. Reaveley, "Performance-Based Evaluation of Exteriro Reinnforced Concrete Building Joints for Seismic Excitation," Pacific Earthquake Engineering Research Center, Berkeley, CA, 2000.

8. J. P. Moehle and S. A. Mahin, "Observations on the Behavior of Reinforced Concrete Buildings During Earthquakes," American Concrete Institute, 1991.

9. G. C. Lee and C.-H. Loh, "The Chi-Chi, Taiwan Earthquake of September 21, 1999: Reconnaissance Report," Multidisciplinary Center for Earthquake Engineering Research, University at Buffalo, State University of New York, Buffalo, NY, 2000.

10. H. Sezen, K. J. Elwood, A. S. Whittaker, K. M. Mosalam, J. W. Wallace and J. F. Stanton, "Structural Engineering Reconnaissance of theAugust 17, 1999, Kocaeli (Izmit), Turkey, Earthquake," Pacific Earthquake Engineering Research Center, University of California, Berkeley, CA, 2000. 
11. B. Li, Z. Wang, K. M. Mosalam and H. Xie, "Wenchuan Earthquake Field Reconnaissance on Reinforced Concrete Framed Buildings With and Without Masonry Infill Walls," Beijing, China, 2008.

12. W. Y. Kam and S. Pampanin, "Revisiting performance-based seismic design in the aftermath of the Christchurch 2010-2011: raising the bar to meet societal expectations.," Lisbon, Portugal, 2012.

13. C. Sim, E. Villalobos, J. P. Smith, P. Rojas, S. Pujol, A. Puranam and L. A. Laughery, "Performance of Low-rise Reinforced Concrete Building in the 2016 Ecuador Earthquake," Purdue University Research Repository, 2017.

14. E. E. R. Institute, "EERI Concrete Buildings Damaged in Earthquakes - A Collection of Case Studies," concretecoalition.org, [Online]. Available: http://db.concretecoalition.org/building/132. [Accessed 10 December 2019].

15. G.-J. Ha, C.-G. Cho, H.-W. Kang and L. Feo, "Seismic improvement of RC beam-column joints using hexagonal CFRP bars combined with CFRP sheets," Composite Structures, vol. 95, pp. 464-470, 2013.

16. M. W. Halling, K. C. Womack and R. M. Moyle, "Retrofit of Existing Concrete Beam_Column Joints using Advanced Carbon-Fiber Composites," Utah State University, Logan, Utah, 1998.

17. Parvin and P. Granata, "Investigation on the effects of fiber composites at concrete joints," Composites: Part B, vol. 31, pp. 499-509, 2000.

18. J. Gergely, C. P. Pantelides and L. D. Reaveley, "Shear Strengthening of RCT-Joints Using CFRP Composites," Journal of Composites for Construction, vol. 4, no. 2, pp. 56-64, 2000.

19. F. Faleschini, J. Gonzalez-Libreros, M. A. Zanini, L. Hofer, L. Sneed and C. Pellegrino, "Repair of severely-damaged RC exterior beam-column joints with FRP and FRCM composites," Composite Structures, vol. 207, pp. 352-363, 2019.

20. Beres, S. El-Borgi, R. N. White and P. Gergely, "Experimental Results of Repaired and Retrofitted Beam-Column Joint Tests in Lightly Reinforced Concrete Frame Buildings," National Center for Earthquake Engineering Research, Buffalo, NY, 1992.

21. Arzeytoon, A. Hosseini and A. Goudarzi, "Seismic Rehabilitation of Exterior RC BeamColumn Joints Using Steel Plates, Angles, and Posttensioning Rods," Journal of Performance of Constructed Facilities, 2014. 
22. G. Genesio, A. Sharma, R. Eligehausen, S. Pampanin and G. R. Reddy, "Development of seismic retrofit technique of RC frame using fully fastened haunch elements: static to dynamic testing," in 14th Symposium on Earthquake Engineering, Roorkee, India, 2010.

23. G. Genesio, "Seismic Assessement of RC Exterior Beam-Column Joints and Retrofit with Haunches Using Post-Installed Anchors," Universität Stuttgart, 2012.

24. Sharma, R. Eligehausen and J. Hofmann, "Numerical Modeling of Joints Retrofitted with Haunch Retrofit Solution," ACI Structural Journal, vol. 111, no. 4, pp. 861-872, 2014.

25. Y.-C. Wang and M.-G. Lee, "Rehabilitation of nonductile beam-column joint using concrete jacketing," in 13th World Conference on Earthquake Engineering , Vancouver, B.C., Canada, 2004.

26. S. Misir and S. Kahraman, "Strengthening of non-seismically detailed reinforced concrete beam-column joints using SIFCON blocks," Sadhana, Indian Academy of Sciences, vol. 38, no. 1, pp. 69-88, 2013.

27. P. Chaimahawan and A. Pimanmas, "Nonlinear FEM Analysis of RC Beam-Column Joint Strengthened by Cast In-Situ Joint Expansion," Journal of Advanced Concrete Technology, vol. 7, no. 3, pp. 307-326, 2009.

28. Pimanmas and P. Chaimahawan, "Shear Strength of beam-cloumn joint with enlarged area," Engineering Structures, vol. 32, pp. 2529-2545, 2010.

29. Li, E. S.-s. Lam, Y.-k. Cheng, B. Wu and Y.-y. Wang, "Strengthening of non-seismically designed beam-cloumn joints by ferrocement jackets with chamfers," Earthquakes and Structures, vol. 8, no. 5, pp. 1017-1038, 2015.

30. J. Shafaei, A. Hosseini and M. S. Marefat, "Seismic retrofit of external RC beam-column joints by joint enlargement using prestressed steel angles," Engineering Structures , vol. 81, pp. 265$288,2014$.

31. M. N. Hadi and T. M. Tran, "Retrofitting nonseismically detailed exterior beam-column joints using concrete covers togther with CFRP jacket," Construction and Building Materials, vol. 63, pp. 161-173, 2014.

32. M. N. Hadi and T. M. Tran, "Seismic rehabilitation of reinforced concrete beam-cloumn joints by bonding with concrete covers and wrapping with FRP composites," Materials and Structures, vol. 49, pp. 467-485, 2016. 
33. H. V. GangaRao and P. K. Majjigapu, "Durable, fire resistant, energy absorbing and costeffective strengthening systems for structural joints and members". US Patent 9,611,667, 4 April 2017.

34. H. V. GangaRao and P. K. Majjigapu, "Durable, fire resistant, energy absorbing and costeffective strengthening systems for structural joints and members". US Patent 10,100,542, 16 October 2018.

35. H. V. GangaRao and P. K. Majjigapu, "Durable, fire resistant, energy absorbing and costeffective strengthening systems for structural joints and members". US Patent 16,133,337 (pending), 2019.

36. K. Baldridge, "Disaster Resilience: A Study of San Francisco's Soft-Story Building Problem," The Urban Lawyer, vol. 44, no. 2, pp. 465-492, 2012.

37. Applied Technology Council, "San Francisco’s Earthquake Risk," Department of Building Inspection, 2009.

38. D. Bonowitz, "THE DILEMMA OF EXISTING BUILDINGS: PRIVATE PROPERTY, PUBLIC RISK," San Francisco Planning + Urban Research Association, 2009.

39. M. Saatcioglu, D. Mitchell, R. Tinawi, N. J. Gardner, A. G. Gillies, A. Ghobarah, D. L. Anderson and D. Lau, "The August 17, 1999, Kocaeli (Turkey) earthquake - Damage to structures," Canadian Journal of Civil Engineering, vol. 28, pp. 715-737, 2001.

40. P. Pantelides, J. Hansen, J. Nadauld and L. D. Reaveley, "Assessment of Reinforced Concrete Building Exterior Joints with Substandard Details," PEER Report, University of California, Berkeley, CA, 2002.

41. J. P. Moehle and S. A. Mahin, "Observations on the Behavior of Reinforced Concrete Buildings During Earthquakes," in Earthquake-Resistant Concrete Structures-Inelastic Response and Design, SP-127, 1991.

42. J. P. Moehle, W. Ghannoum and Y. Bozorgnia, "Collapse of lightly confined reinforced concrete frames during earthquakes," in Advances in Earthquake Engineering for Urban Risk Reduction, Istanbul, Turkey, 2006.

43. N. Robins-Early, "Huffington Post," 17 April 2016. [Online]. Available: https://www.huffpost.com/entry/ecuador-earthquake-photos_n_5713d4efe4b0018f9cba5126. [Accessed 5 November 2019]. 
44. Y. Kitagawa and H. Hiraishi, "Overview of the 1995 Hygro-Ken Nanbu Earthquake and Proposals for Earthquake Mitigation Measures," Journal of Japanese Association for Earthquake Engineering, vol. 4, no. 3, 2004.

45. M. Bruneau, "Building damage from the Marmara, Turkey earthquake of August 17, 1999," Journal of Seismology, vol. 6, pp. 357-377, 2002.

46. T. Paulay and M. Priestley, Seismic design of reinforced concrete and masonry buildings, John Wiley \& Sons, Inc., 1992.

47. S. Sasmal, "Performance Evaluation and Strengthening of Deficient Beam-Column Subassemblages under Cyclic Loading," Universitaet Stuttgart, 2009.

48. Bousselham, "State of Research on Seismic Retrofit of RC Beam-Column Joints with Externally Bonded FRP," ASCE JOURNAL OF COMPOSITES FOR CONSTRUCTION, pp. 49-61, 2010.

49. R. Park and T. Paulay, Reinforced Concrete Structure, John Wiley \& Sons, Inc., 1975.

50. S. Hakuto, R. Park and H. Tanaka, "Seismic Load Tests on Interior and Exterior Beam-Column Joints with Substandard Reinforcing Details," ACI Structural Journal, pp. 11-25, 2000.

51. M. Priestley, "Displacement-Based Seismic Assessment of Reinforced Concrete Buildings," Journal of Earthquake Engineering, pp. 157-192, 1997.

52. S. Pampanin and C. Christopoulos, "Non-invasive Retrofit of Existing RC Frames Designed for Gravity Loads only," in Concrete Structures in Seismic Regions: fib 2003 Symposium, Athens, Greece, 2003.

53. S.-J. Hwang and H.-J. Lee, "Analytical Model for Predicting Shear Strengths of Exterior," ACI Structural Journal, pp. 846-858, 1999.

54. . U. Akguzel, "Seismic Performance of FRP Retrofitted Exterior RC Beam-Column Joints under Varying Axial and Bidirectional Loading," Ph.D. Thesis, University of Cantebury, Christchurch, New Zealand, 2011.

55. Ghobarah and T. El-Amoury, "Seismic Rehabilitation of Deficient Exterior Concrete Frame Joints," Journal of Composites for Construction, vol. 9, pp. 408-416, 2005.

56. O. J. Jirsa and J. L. G. Marques, "A study of hooked bar anchorages in beam-column joints," Final Report - Reinforced Concrete Research Council, Project 33, University of Texas , Austin, Texas, 1972. 
57. E. Parker and P. J. M. Bullmann, "Shear strength within reinforced concrete beam-column joints," The Structural Engineer, vol. 75, no. 4, pp. 53-57, 1997.

58. Clyde, C. P. Pantelides and L. D. Reaveley, "Performance-Based Evaluation of Exterior Reinforced Concrete Building Joints for Seismic Excitation," PEER Report, University of California, Berkeley, CA, 2000.

59. R. Scott, I. Feltham and R. Whittle, "Reinforced concrete beam-column connections and BS 8110," The Structural Engineer, vol. 72, no. 4, pp. 55-60, 1994.

60. V. G. Haach, A. L. H. D. C. E. Debs and M. K. E. Debs, "Evaluation of the influence of the column axial load on the behavior of monotonically loaded RC exterior beam-column joints through numerical simulations," Engineering Structures, vol. 30, no. 4, pp. 965-975, 2008.

61. P. Sangjoon, "Experimental and Analytical Studies on Old Reinforced Concrete Buildings with Seismically Vulnerable Beam-Column Joints," UC Berkeley Electronic Theses and Dissertations, Berkeley, 2010.

62. J. Kim and J. M. LaFave, "Key influence parameters for the joint shear behaviour of reinforced concrete (RC) beam-column connections," Engineering Structures, vol. 29, pp. 2523-2539, 2007.

63. Nilsson, "Reinforced concrete corners and joints subjected to bending moment," National Swedish Institute for Building Research, Stockholm, Sweden, 1973.

64. H. P. J. Taylor, "The behaviour of in situ concrete beam-column joints," Cement and Concrete Association, London, 1974.

65. N. 3101:1995, "The Design of Concrete Structures," Standards New Zealand, Wellington, NZ, 1995.

66. M. J. Priestley, F. Seible, G. M. Calvi and G. M. Calvi, Seismic Design and Retrofit of Bridges, John Wiley \& Sons, 1996.

67. Prota, A. Naani, G. Manfredi and E. Cosenza, "Selective upgrade of beam-column joints with composites," in International Conference on FRP Composites in Civil Engineering, Vol. I, Elsevier Science, New York, 2001.

68. H. F. Wong, "Shear Strength and Seismic Performance of Non-Seismically Designed RC Beam-Column Joints.," PhD Thesis, University of Science and Technology, Hong Kong, 2005. 
69. W. Kam, P. Quintana Gallo, U. Akgüzel and S. Pampanin, "Influence of slab on the seismic response of sub-standard detailed exterior reinforced concrete beam column joints," in 9th U.S. National and 10th Canadian Conference on Earthquake Engineering: Reaching Beyond Borders, Toronto, Canada, 2010.

70. Hertanto, "Seismic assessment of pre-1970s reinforced concrete structures," MS Thesis, University of Canterbury, Christchurch, NZ, 2005.

71. T.-H. Chen, "Development of a low invasive seismic retrofit solution for under-designed frame systems based on a metallic haunch," MS Thesis, University of Canterbury, Christchurch, NZ, 2006.

72. Genesio, "Seismic Assessment of RC Exterior Beam-Column Joints and Retrofit with Haunches Using Post-Installed Anchors," Ph.D. Thesis, University of Stuttgart, Italy, 2012.

73. M. Calvi, G. Magenes and S. Pampanin, "Relevance of beam-column damage and collapse in RC frame assessment," Journal of Earthquake Engineering, vol. 6, no. S1, pp. 75-100, 2002.

74. S. Pampanin, G. Calvi and M. Moratti, "Seismic Behaviour of R.C. Beam-Column Joints Design for Gravity Loads," in 12th European Conference on Earthquake Engineering, London, 2002.

75. M. A. Mahajan, "Evaluation of shear strength and effective width of reinforced concrete exterior beam-column joints," PhD Thesis, IIT Madras, Chennai, India, 2009.

76. S. Park and K. M. Mosalam, "Experimental Investigation of Nonductile RC Corner BeamColumn Joints with Floor Slabs," Journal of Structural Engineering, vol. 139, no. 1, pp. 1-14, 2013.

77. J.-S. Jeon, A. Shafieezadeh and R. DesRoches, "Statistical models for shear strength of RC beam-column joints using machine-learning techniques," Earthquake Engineering \& Structural Dynamics, vol. 43, pp. 2075-2095, 2014.

78. T. Palucka and B. Bensaude-Vincent, "History of Recent Science \& Technology," October 2002.

[Online].

Available: https://authors.library.caltech.edu/5456/1/hrst.mit.edu/hrs/materials/public/composites/Comp osites_Overview.htm. [Accessed 17 September 2019].

79. R. Garcia, I. Hajirasouliha, K. Pilakoutas and M. Guadagnini, "Seismic Strengthening of Deficient RC Buildings Using Externally Bonded FRPs," in ECEE 14th, Orhid, 2010. 
80. K. Galal, "FRP Retrofitting of Bridges," in The Second International Conference on Infrastructure Management, Assessment and Rehabilitation Techniques, United Arab Emirates, 2016.

81. M. Ludovico, A. Prota, G. Manfredi and E. Cosenza, "Seismic strengthening of an underdesigned RC structure with FRP," Earthquake Engineering \& Structural Dynamics, vol. 37, no. 1, pp. 141-162, 2008.

82. GangaRao and P. V. Vijay, "Bending Behavior of Concrete Beams Wrapped with Carbon Fabric," Journal of Structural Engineering, vol. 124, no. 1, 1998.

83. G. Teng, G. M. Chen, J. F. Chen and O. A. Rosenboom, "Behavior of RC Beams Shear Strengthened with Bonded or Unbonded FRP wraps," Journal of Composites for Construction, vol. 13, no. 5, 2009.

84. I. Mohammad and B. J. Al-Sulayfani, "Torsional Strengthening of RC Beams with CFRP Wrap," Tikrit Journal of Engineering Sciences, vol. 20, no. 3, pp. 1-9, 2013.

85. Olivova and J. Bilcik, "Strengthening of Concrete Columns with CFRP," Slovak Journal of Civil Engineering, pp. 1-9, 2009.

86. Amir, A. Naseer and O. Azam, "Strengthening of Existing Building Column Using FRP Wrap \& GI Wire Mesh," International Journal of Science \& Engineering Research, vol. 4, no. 5, pp. 211-216, 2013.

87. S. A. Guralnick and L. Gunawan, "Strengthening of Reinforced Concrete Bridge Columns with FRP wrap," Practice PEriodical on Structural Design and Construction, vol. 11, no. 4, 2006.

88. C. G. Karayannis and G. M. Sirkelis, "Seismic Resistance of Reinforced Concrete Beam_Column," Earthquake Engineering \& Structural Dynamics, vol. 37, no. 5, pp. 769-790, 2008.

89. M. Elsouri and M. H. Harajli, "Repair and FRP Strengthening of Earthquake-Damaged RC Shallow Beam-Column Joints," Advances in Structural Engineering, vol. 18, no. 2, pp. 237249, 2016.

90. D. Vecchio, M. D. Ludovico, A. Prota and G. Manfredi, "Modelling beam-column joints and FRP strengthening in the seismic performance assessment of RC existing frames," Composite Structures, vol. 142, pp. 107-116, 2016. 
91. H. Harajli, K. A. Soudki and T. Kudsi, "Strengthening of Interior Slab-Column Connections Using a Combination of FRP Sheets and Steel Bolts," Journal of Composites for Construction, vol. 10 , no. $5,2006$.

92. J. G. Teng, L. Lam, W. Chan and J. Wang, "Retrofitting of Deficient RC Cantilever Slabs Using GFRP Strips," Journal of Composites for Construction, vol. 4, no. 2, 2000.

93. P. Garner, "Strengthening reinforced concrete slabs using a combination of CFRP and UHPC," University of New Mexico, 2011.

94. K. Soudki, "2- Using fibre reinforced polymer (FRP) composites to extend the service life of corroded concrete structures," Service Life Estimation and Extension of Civil Engineering Structures, pp. 75-95, 2011.

95. S. Pantazopoulou, J. F. Bonacci, S. A. Sheikh and M. D. Thomas, "Repair of CorrosionDamaged Columns with FRP Wraps," Journal of Composites for Construction, vol. 5, no. 1, 2001.

96. R. Sen, G. Mullins, K. Suh and D. Winters, "SP-230-64 FRP Application in Underwater Repair of Corroded Piles," ACI.

97. D. A. Roy, U. K. Sharma and P. Bhargava, "Strengthening heat damaged reinforced concrete beams using glass fiber-reinforced polymer (GFRP) laminates," in Applications of Structural Fire Engineering, Dubrovnik, Croatia, 2015.

98. A. Bisby, J. F. Chen, S. Q. Li, T. J. Stratford, N. Cueva and K. Crossking, "Strengthening firedamaged concrete by confinement with fibre-reinforced polymer wraps," Engineering Structures, vol. 33, no. 12, pp. 3381-3391, 2011.

99. U. Demir, G. Unal, A. F. Sert, R. O. Calis and A. Ilki, "Influence of FRP Repair on the Axial Behavior of Fire Damaged Concrete," in SMAR 2019 - Fifth Conference on Smart Monitoring, Assessment and Rehabilitation of Civil Structures, 2019.

100. J. L. kasan, "On the repair of impact-damaged prestressed concrete bridge girders," University of Pittsburgh, 2012.

101. ElSafty, M. K. Graeff and S. Fallaha, "Behavior of Laterally Damaged Prestressed Concrete Bridge Girders Repaired with CFRP Laminates Under Static and Fatigue Loading," International Journal of Concrete Structures and Materials, vol. 8, no. 1, pp. 43-59, 2014. 
102. J. Gangi, "Analytical modeling of the repair of impact-damaged prestressed concrete bridge girders," Virginia Polytechnic Institute and State University, Blacksburg, Virginia, 2015.

103. S. Mosallam, "Strength and ductility of reinforced concrete moment frame connections strengthened with quasi-isotropic laminates," Journal of Composites, Part B, vol. 31, pp. 481497, 2000.

104. Pantelides, C. Clyde and L. Reaeley, "Rehabilitation of R/C building joints with FRP composites," in 12th World Conference on Earthquake Engineering, 2000.

105. Granata and A. Parvin, "An experimental study on kevlar strengthening of beam-column connections," Journal of Composite Structures, vol. 53, no. 2, pp. 163-171, 2001.

106. Ghobarah and A. Said, "Shear strengthening of beam-column joints," Engineering Structures, vol. 24, no. 7, pp. 881-888, 2002.

107. P. Antonopoulos and T. C. Triantafillou, "Experimental Investigation of FRP-Strengthened RC Beam-Column Joints," ASCE Journal of Composites for Construction, pp. 39-49, 2003.

108. S. S. Mahini and H. R. Ronagh, "A new method for improving ductility in existing RC ordinary moment resisting frames using FRPs," Asian Journal of Civil Engineering (Building and Housing), vol. 8, no. 6, pp. 581-595, 2007.

109. Kaya, C. Yalcin, A. Parvin and S. Altay, "Retrofitting of Reinforced Concrete BeamColumn Joints by Composites - Part I: Experimental Study," ACI Structural Journal, pp. 1729, 2019.

110. E. Ilia and D. Mostofinejad, "Seismic retrofit of reinforced concrete strong beam-weakcolumn joints using EBROG method combined with CFRP anchorage system," Engineering Structures, pp. 300-319, 2019.

111. S. Hamad and F. G. Ibrahim, "Effect of FRP Confinement on Bond Strength of Hooked Bars: Normal-Strength Concrete Structures," ASCE Journal of Composites for Construction, pp. 279-291, 2009.

112. J. Gergely, C. P. Pantelides and L. D. Reaveley, "Shear Strengthening of RCT-Joints using CFRP Composites," Journal of Composites for Construction, pp. 56-64, 2000.

113. K. Le-Trung, K. Lee, J. Lee, D. H. Lee and S. Woo, "Experimental study of RC beamcolumn joints strengthened using CFRP composites," Composites: Part B, pp. 76-85, 2010. 
114. M. N. S. Hadi and T. M. Tran, "Seismic rehabilitation of reinforced concrete beam-column joints by bonding with concrete covers and wrapping with FRP composites," Materials and Structures, pp. 467-485, 2016.

115. "Trus Joist Beam, Header and Column Specifier's Guide," June 2019. [Online]. Available: https://www.weyerhaeuser.com/application/files/2715/6259/8623/TJ-9000.pdf. [Accessed 23 December 2019].

116. "Engineered Syntactic Systems," 2019. [Online]. Available: https://esyntactic.com/wpcontent/uploads/2019/08/SF-Data-Sheet-2019.pdf. [Accessed 23 December 2019].

117. "SikaWrap Hex-100 G," November 2018. [Online]. Available: https://usa.sika.com/dms/getdocument.get/2ff9c6e4-0cd2-48ef-958ea827bfd39b01/sikawrap_hex-100g.pdf. [Accessed 23 December 2019].

118. "SikaWrap Hex-103 C," March 2019. [Online]. Available: https://usa.sika.com/dms/getdocument.get/976b0a5e-9133-4fb1-b1312f1fee871a96/sikawrap_hex-103c.pdf. [Accessed 23 December 2019].

119. "omega.com," OMEGA, [Online]. Available: https://www.omega.com/en-us/sensors-andsensing-equipment/displacement/displacement-sensors/displacementtransducers/ld320/p/LD320-25?msclkid=07514684e8d610cda9741814ca77e4f8\&utm_source=bing\&utm_medium=cpc\&u tm_campaign=Omega-PLA-US-BNG-Sensors_And_Sensin. [Accessed 23 December 2019].

120. "Intertechnology Inc.," Intertechnology Inc., [Online]. Available: https://intertechnology.com/products/Vishay-System-7000-StrainSmart-Data-Systemp46117712. [Accessed 23 December 2019].

121. S. Pantazopoulou and J. Bonacci, "Consideration of Questions about Beam-Column Joints," ACI Structural Journal, vol. 89, no. 1, pp. 27-36, 1993.

122. H. Nilson, Design of Reinforced Concrete Structures, McGraw-Hill, 1997.

123. Li, E. S.-s. Lam, Y.-k. Cheng, B. Wu and Y.-y. Wang, "Strengthening of non-seismically designed beam-column joints by ferrocement jackets with chamfers," Earthquakes and structures, vol. 8, no. 5, pp. 1017-1038, 2015.

124. E. Englekirk, Seismic Design of Reinforced and Precast Concrete Buildings, John Wiley \& Sons, Inc., 2003. 
125. Pimanmas and P. Chaimahawan, "Cyclic Shear Resistance of Expanded Beam-Column Joint," Procedia Engineering, vol. 14, pp. 1292-1299, 2011. 


\section{Appendix-A: Beam-Column Joints Reinforced with Concrete/Syntactic Foam Filler-Modules and CFRP Wraps}

This section provides additional test data generated through experimental evaluation of beamcolumn joints reinforced with concrete/syntactic foam filler-modules with/out FRP wraps under monotonic loads.

\section{A.1 Specimen JI-1}

A.1.1 Strain in steel rebar

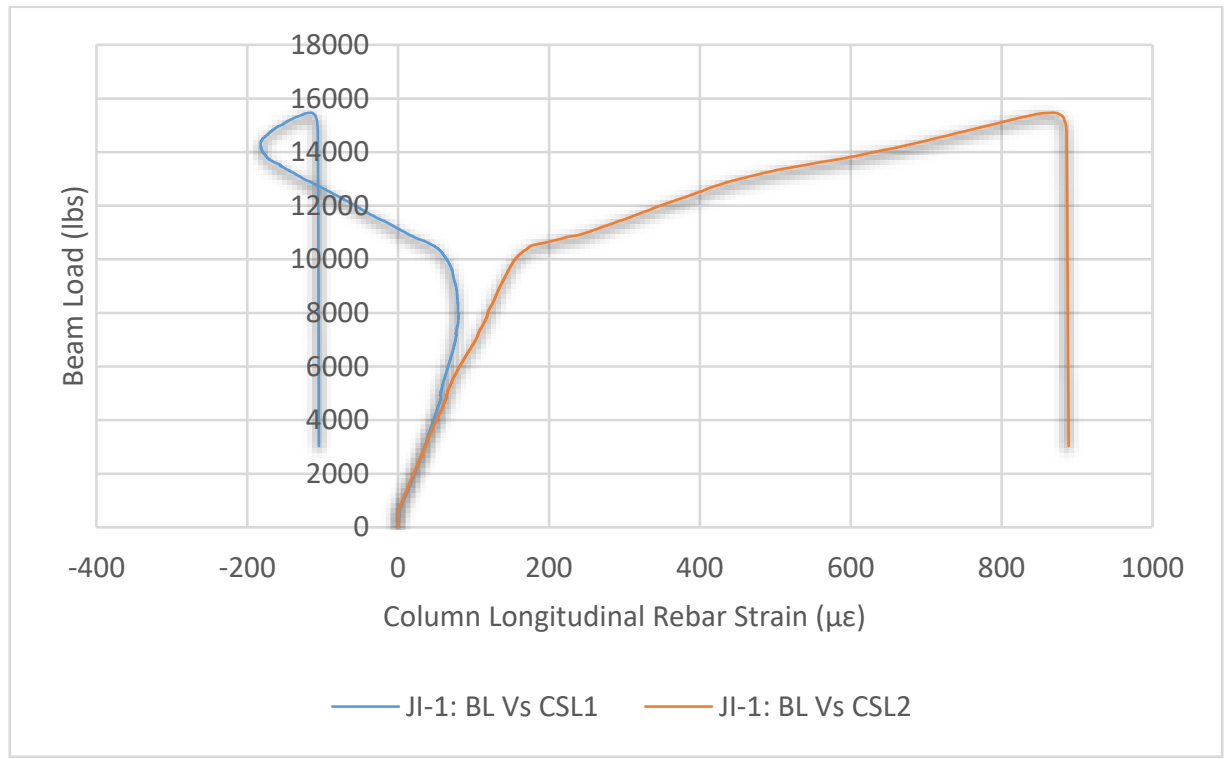

Figure A.1. Beam load vs. column longitudinal rebar strain 


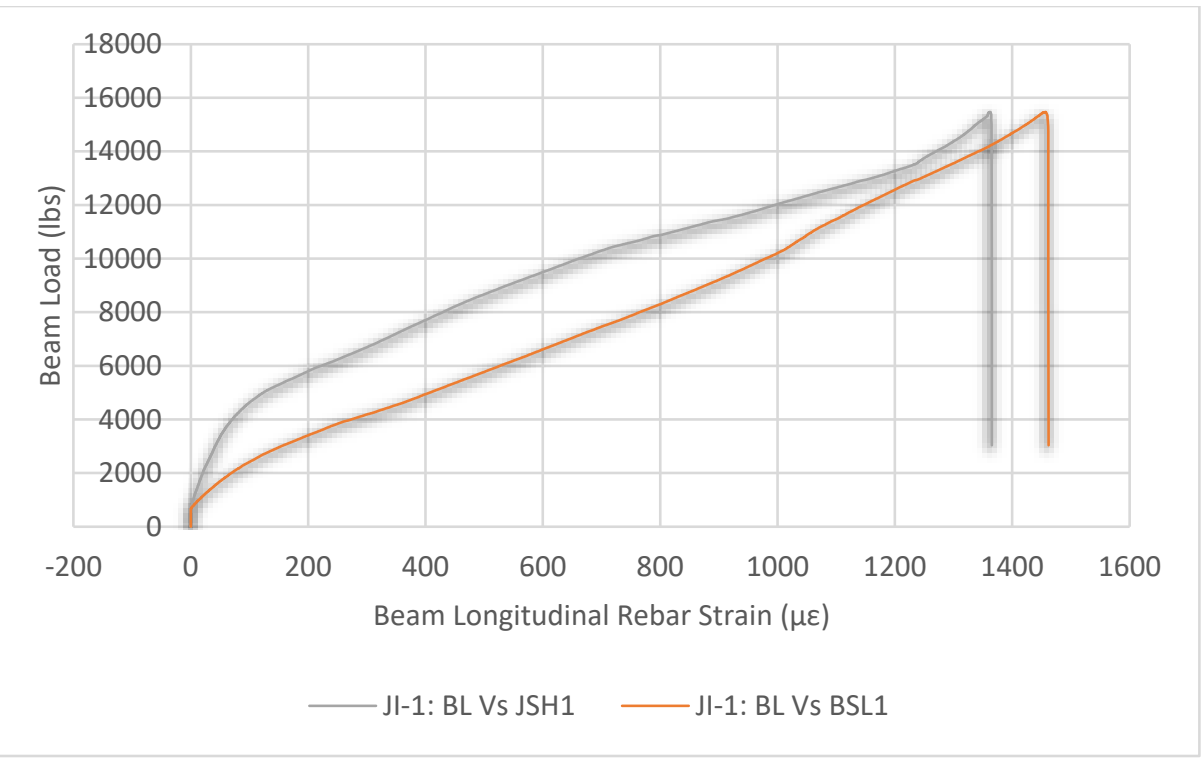

Figure A.2. Beam load vs. beam longitudinal rebar strain

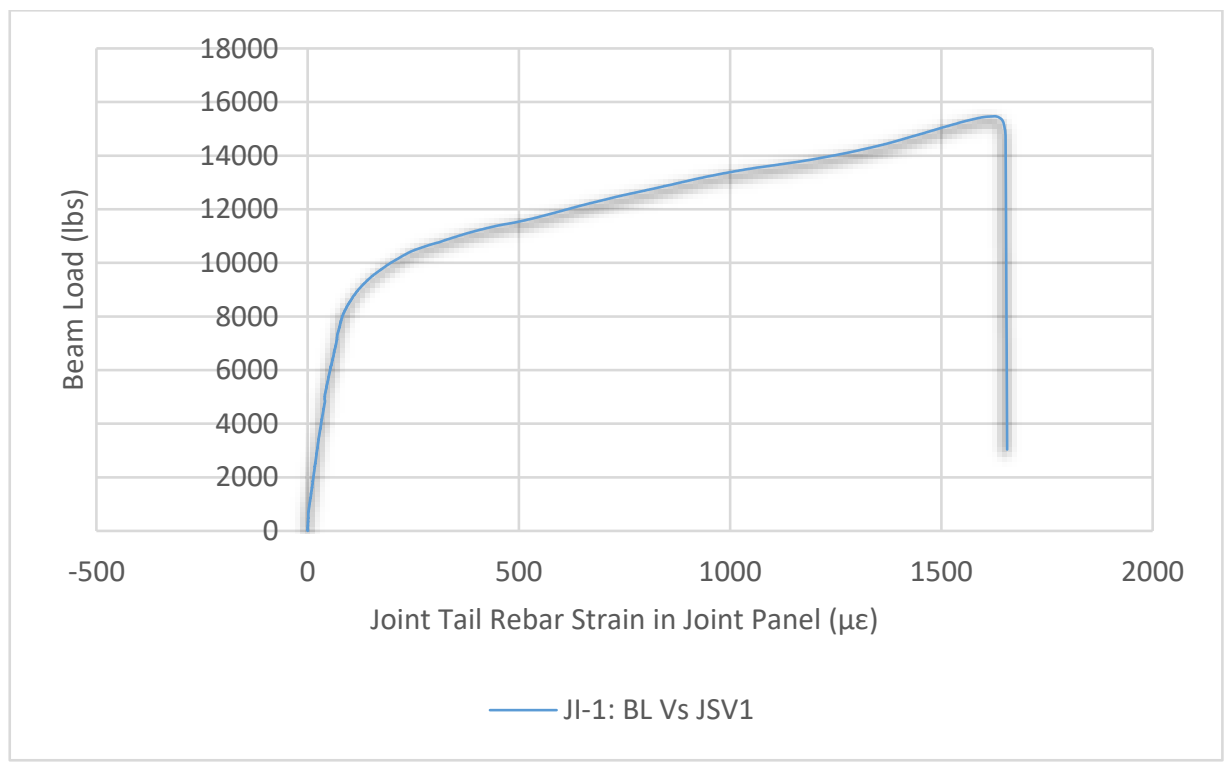

Figure A.3. Beam load vs. joint tail rebar strain 


\section{A.2 Specimen JI-2}

\section{A.2.1 Strain in steel rebar}

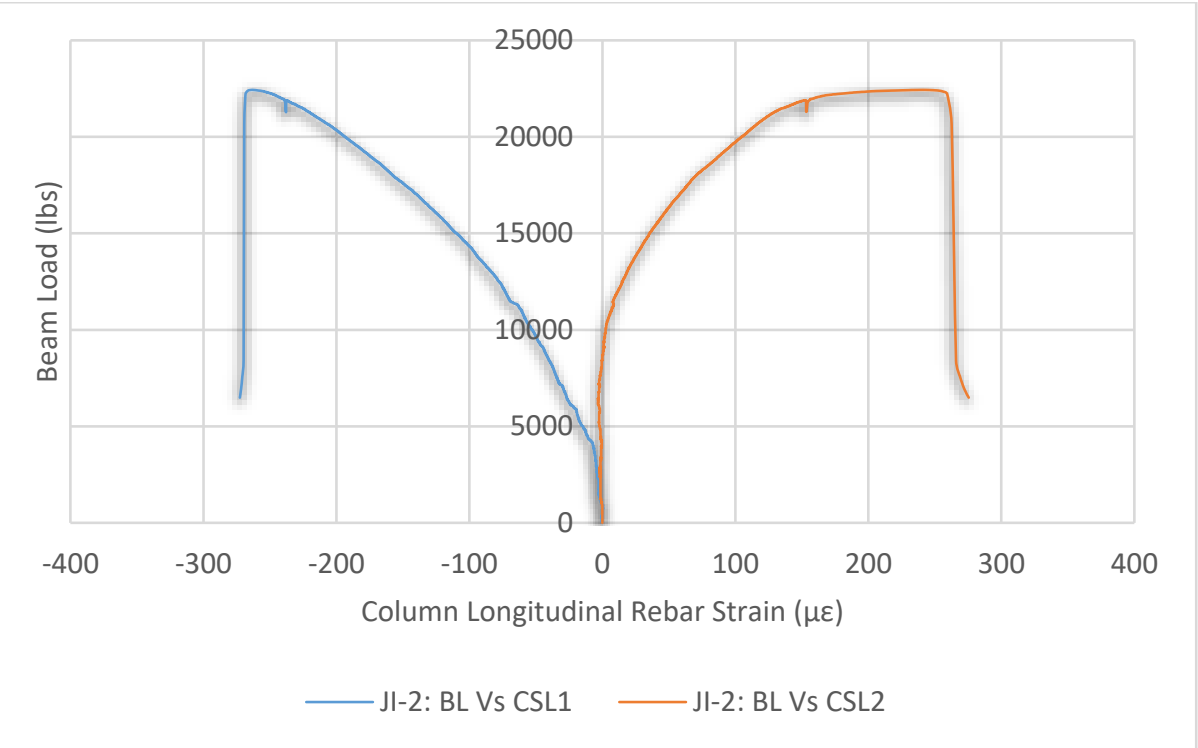

Figure A.4. Beam load vs. column longitudinal rebar strain

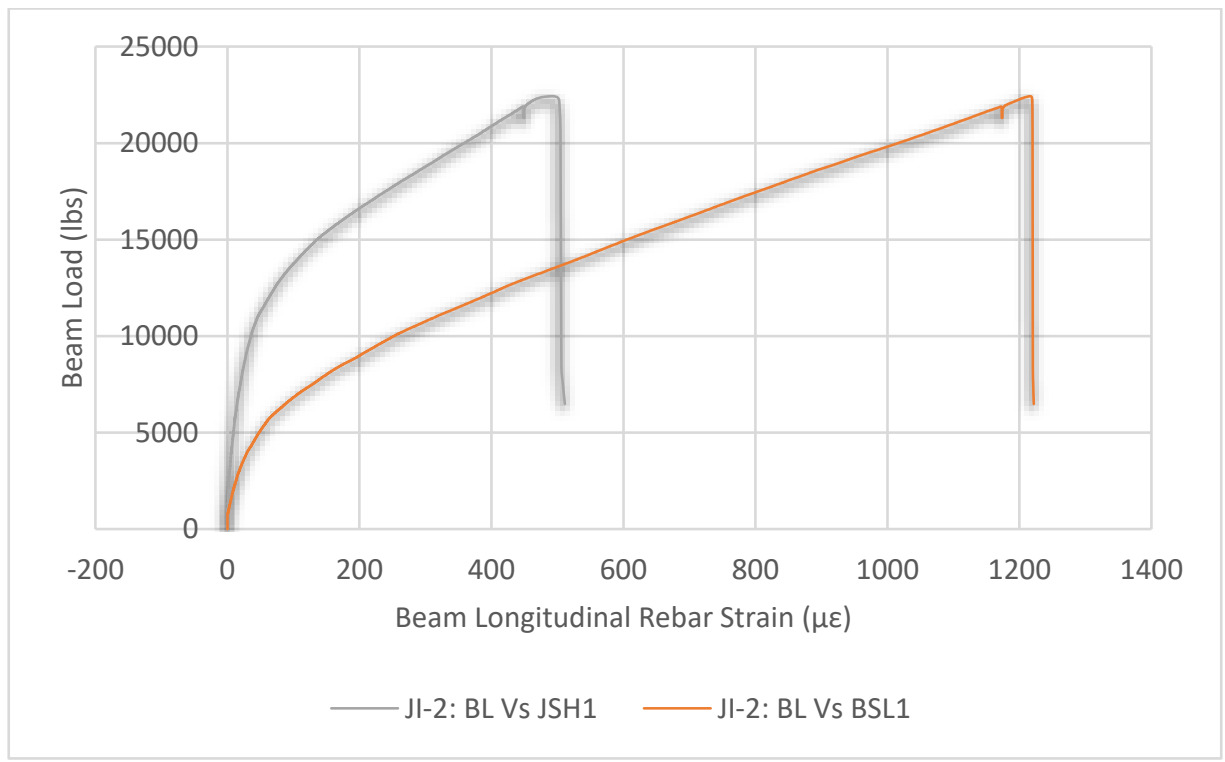

Figure A.5. Beam load vs. beam longitudinal rebar strain 


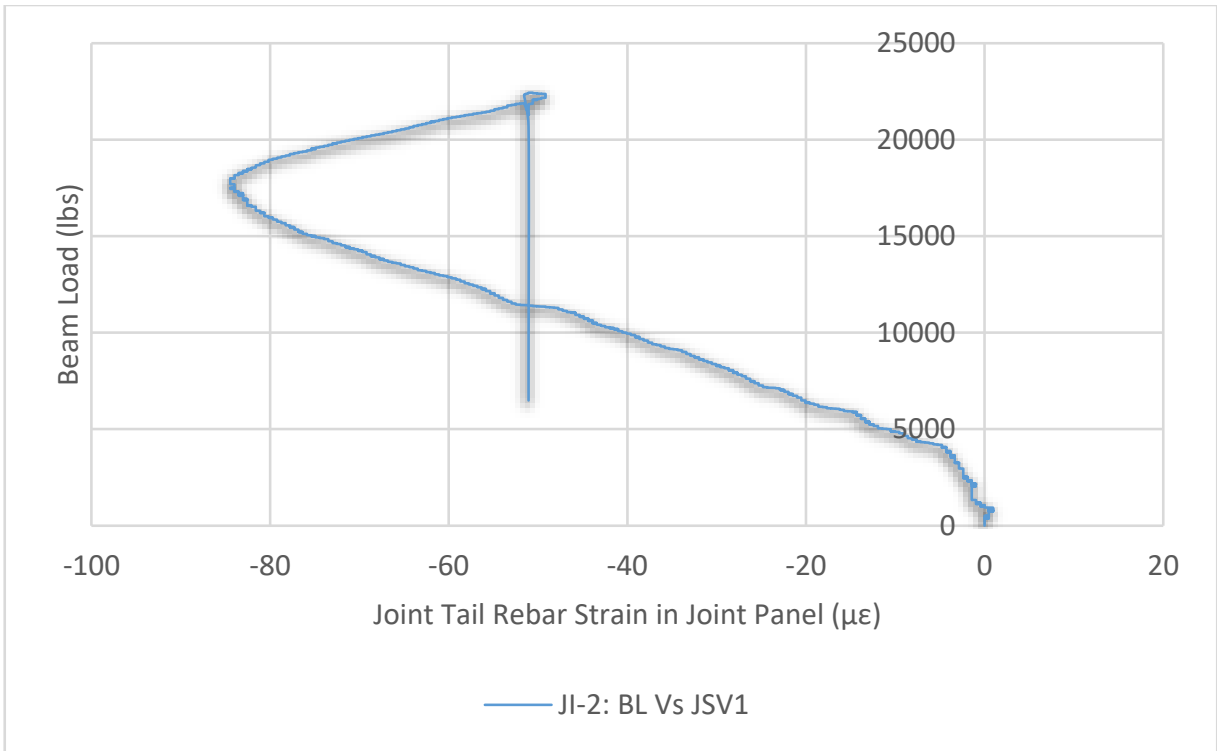

Figure A.6. Beam load vs. joint tail rebar strain

\section{A.2.2 Strain in concrete}

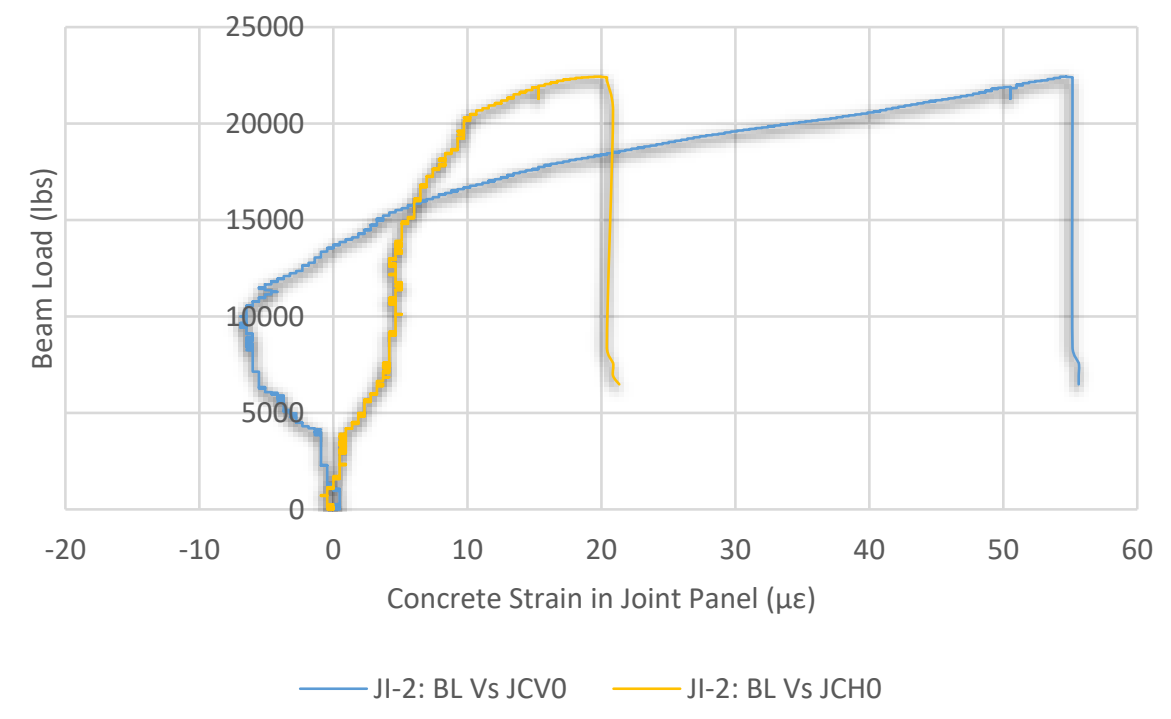

Figure A.7. Beam load vs. concrete strain in joint panel 


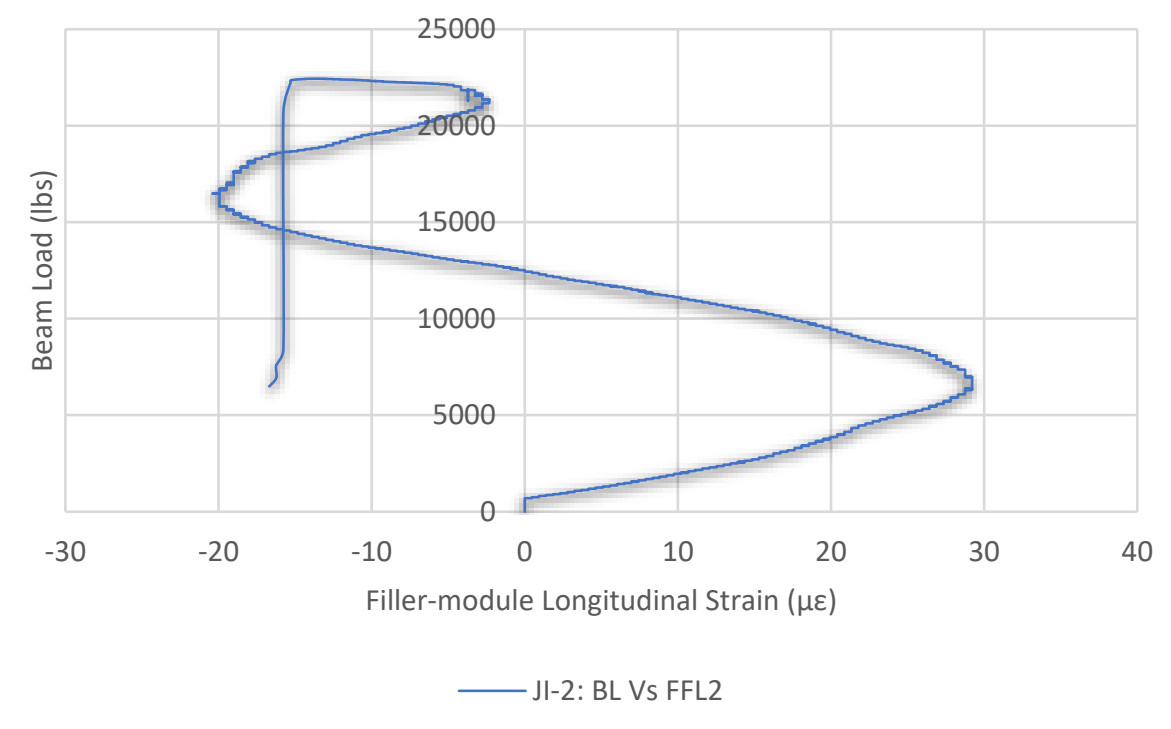

Figure A.8. Beam load vs. filler-module longitudinal strain 


\section{A.3 Specimen JI-3}

\section{A.3.1 Strain in steel rebar}

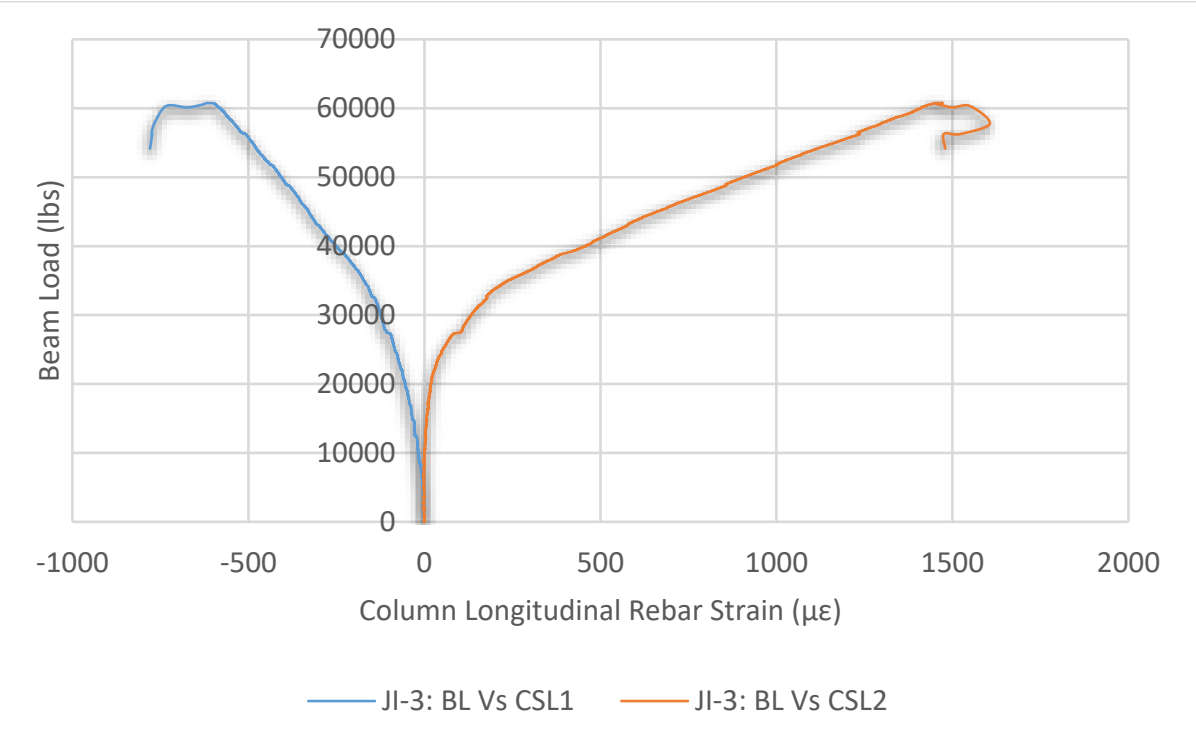

Figure A.9. Beam load vs. column longitudinal rebar strain

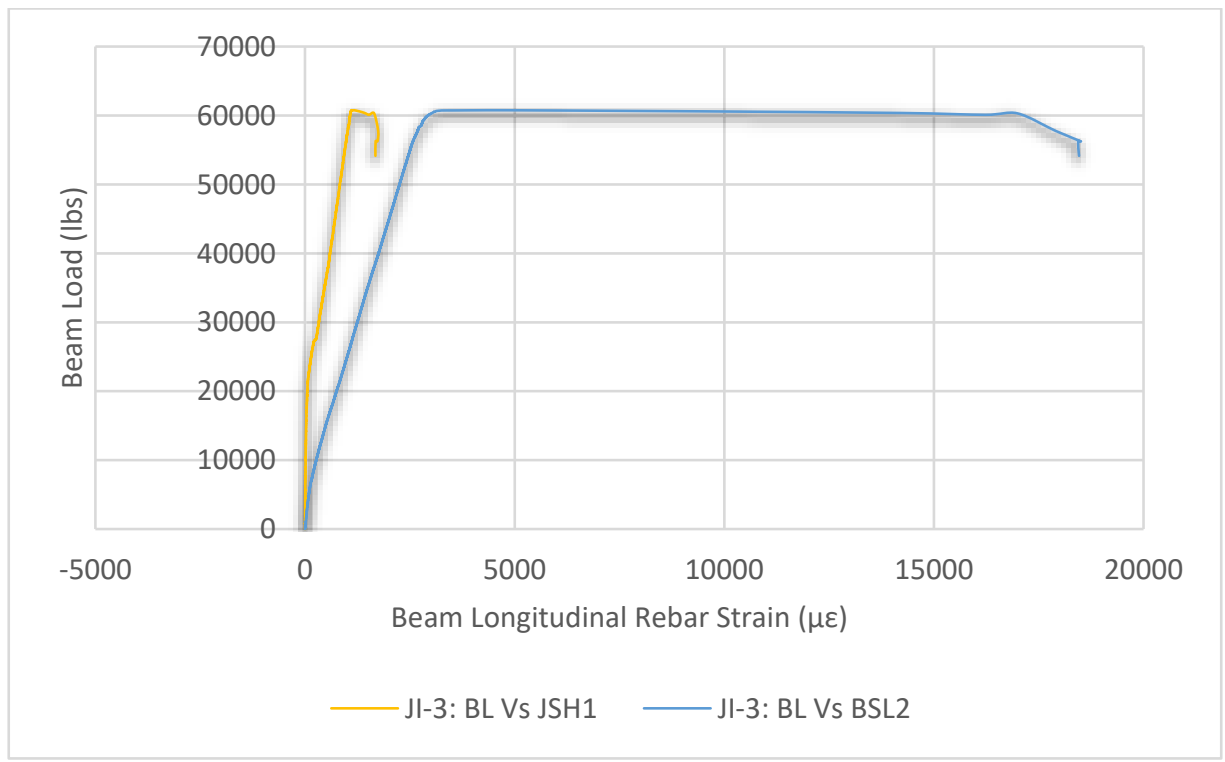

Figure A.10. Beam load vs. beam longitudinal rebar strain 


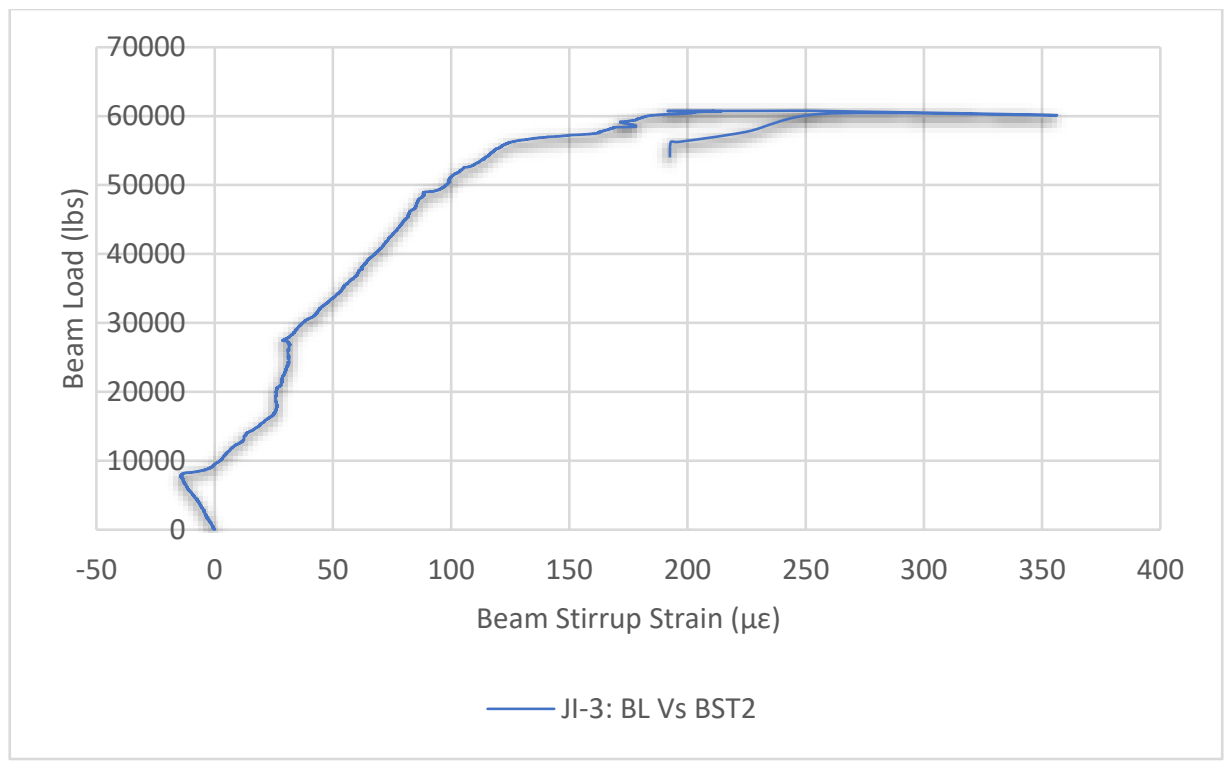

Figure A.11. Beam load vs. beam stirrup strain

\section{A.3.2 Strain in FRP}

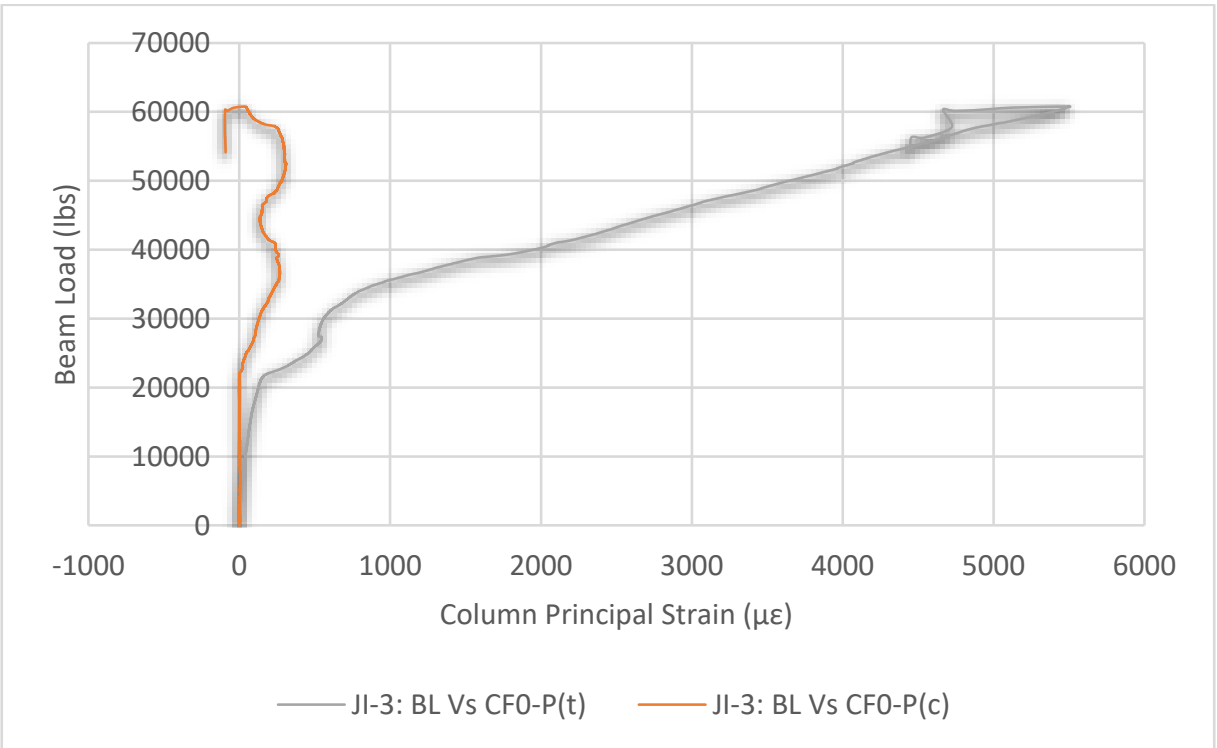

Figure A.12. Beam load vs. column principal strain 


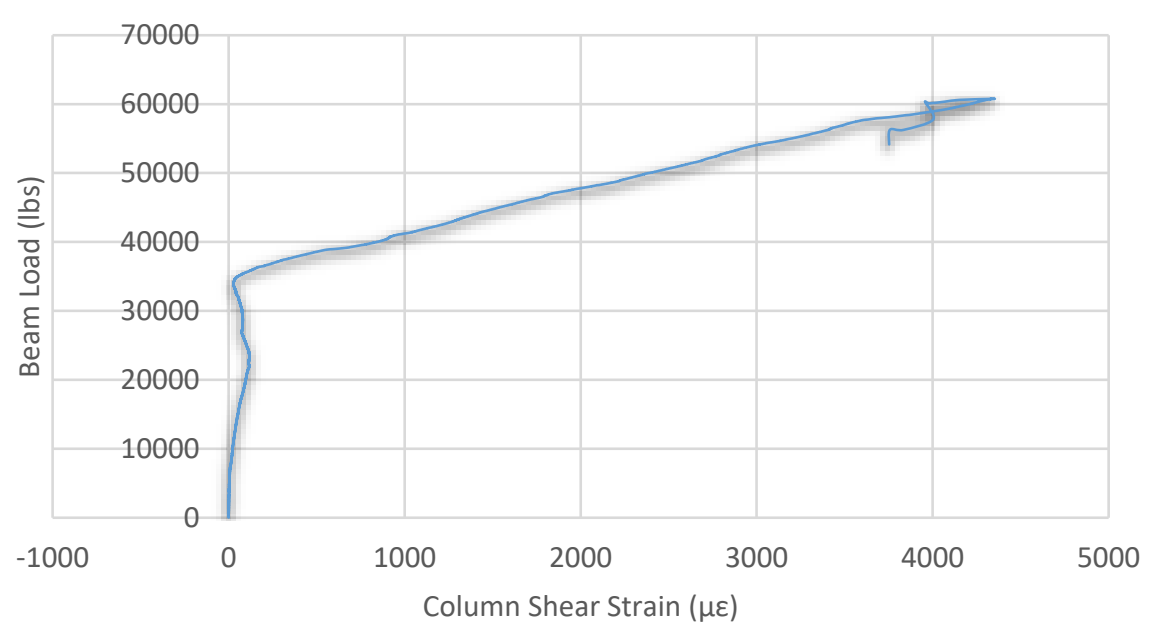

— JI-3: BL Vs CFO- $\gamma$

Figure A.13. Beam load vs. column shear strain

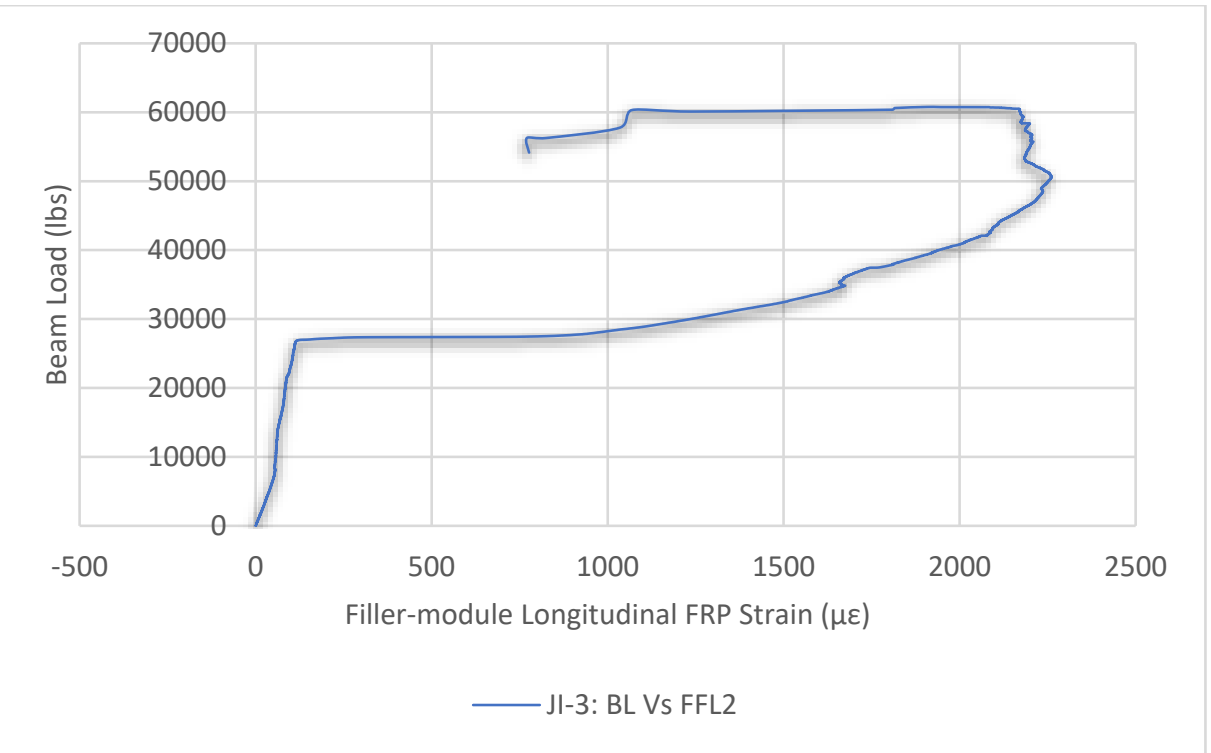

Figure A.14. Beam load vs. filler-module longitudinal strain 


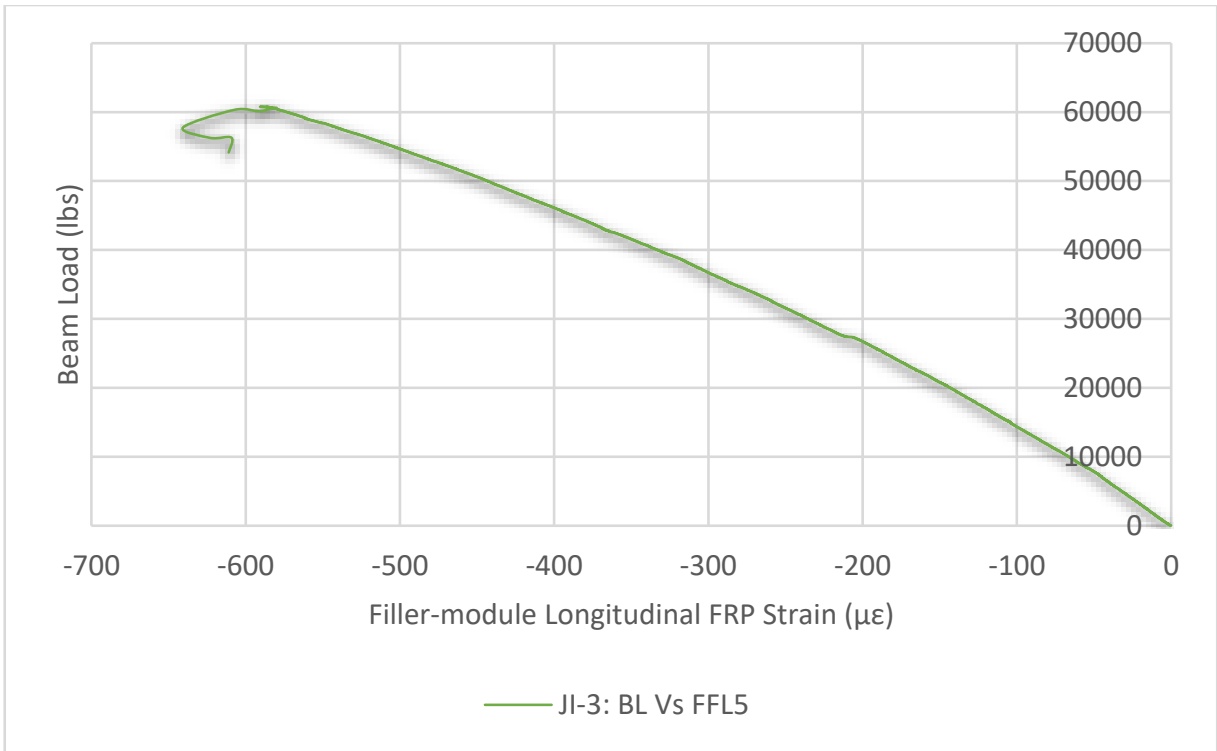

Figure A.15. Beam load vs. filler-module longitudinal strain 


\section{A.4 Specimen JI-4}

\section{A.4.1 Strain in steel rebar}

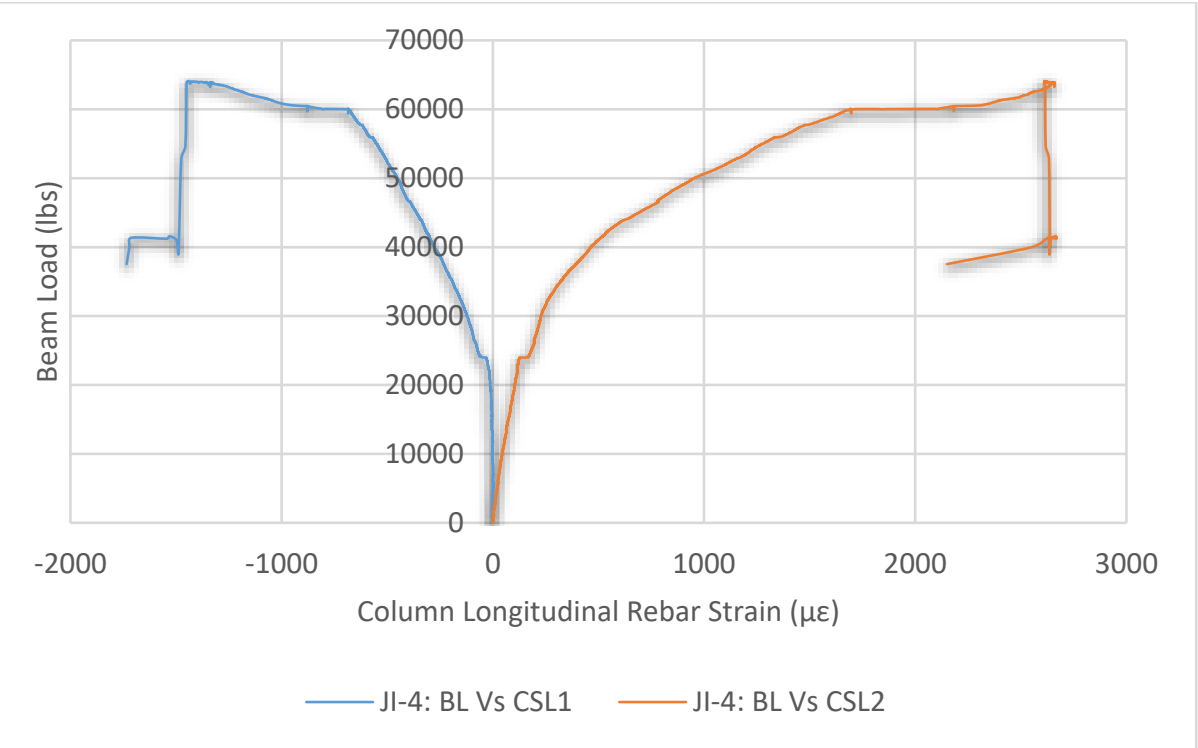

Figure A.16. Beam load vs. column longitudinal rebar strain

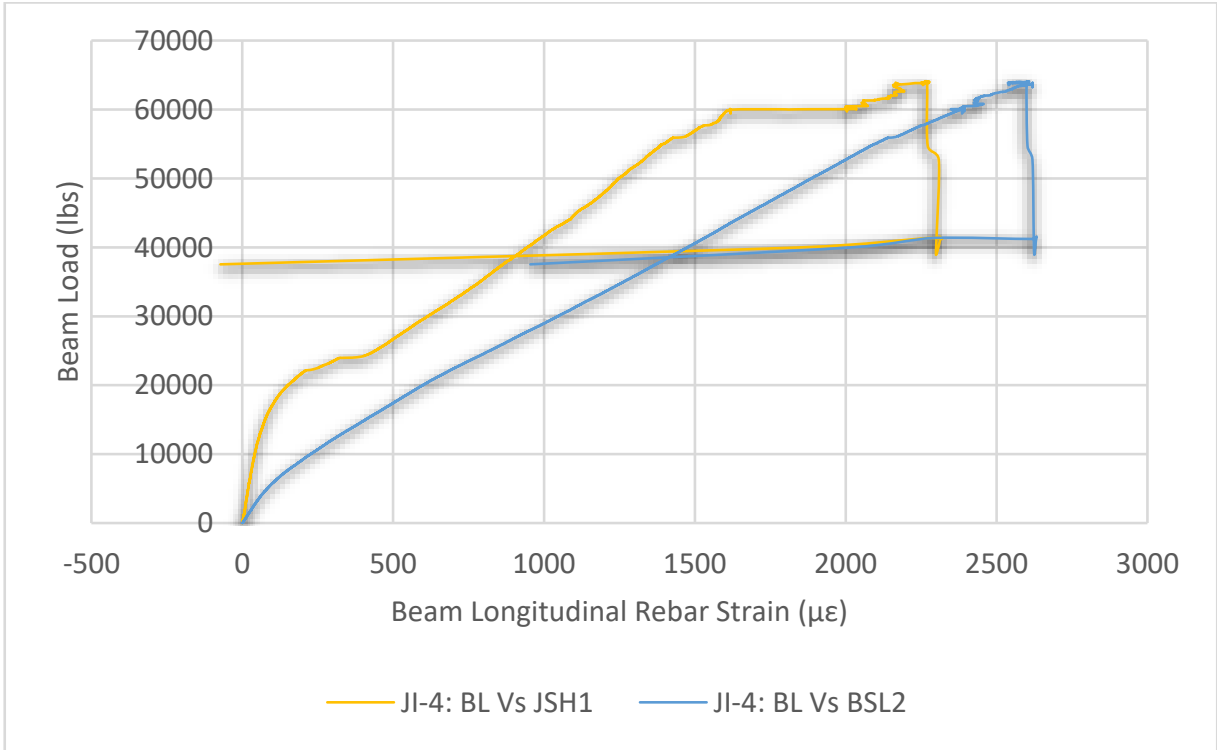

Figure A.17. Beam load vs. beam longitudinal rebar strain 


\section{A.4.2 Strain in FRP}

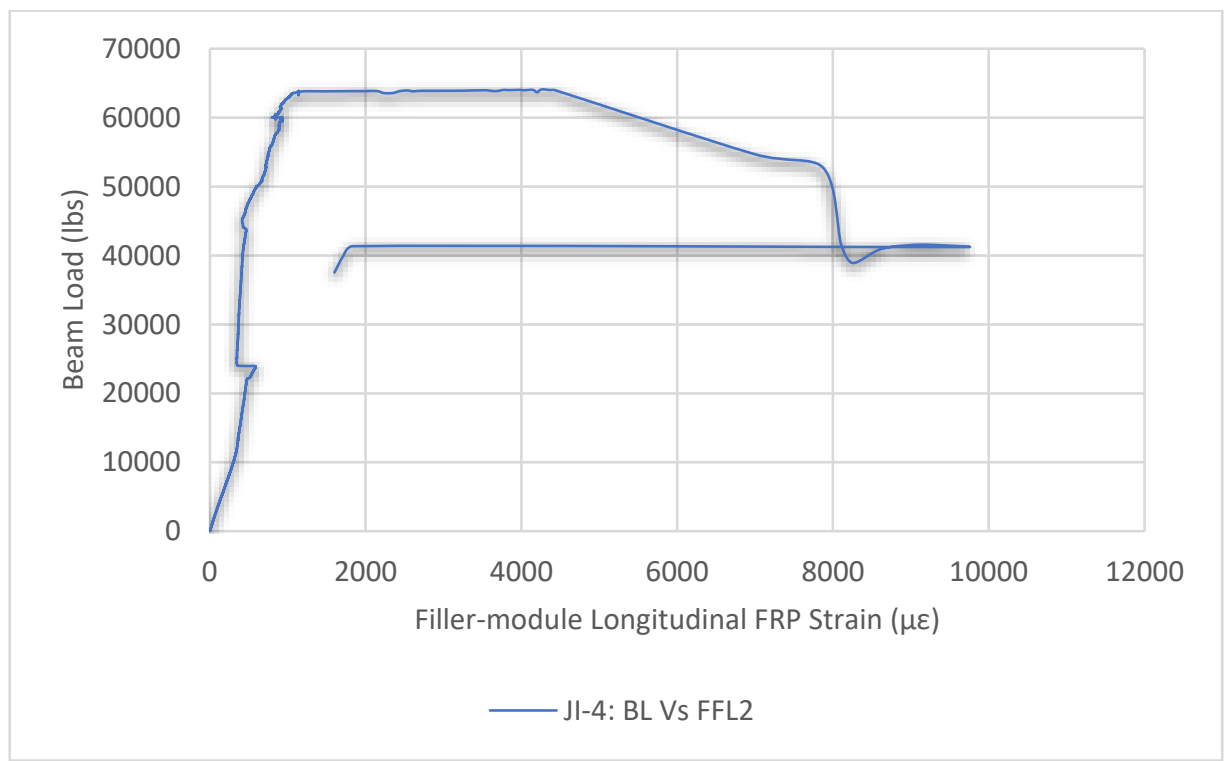

Figure A.18. Beam load vs. filler-module longitudinal strain

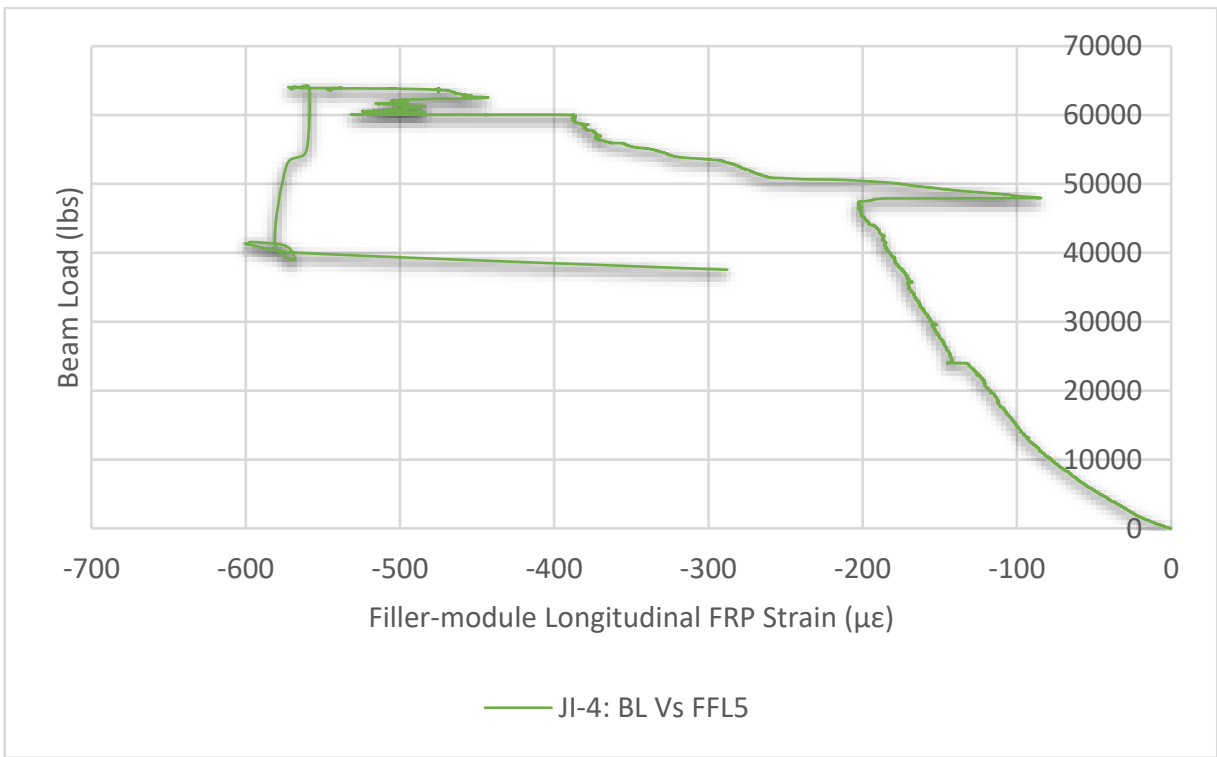

Figure A.19. Beam load vs. filler-module longitudinal strain 


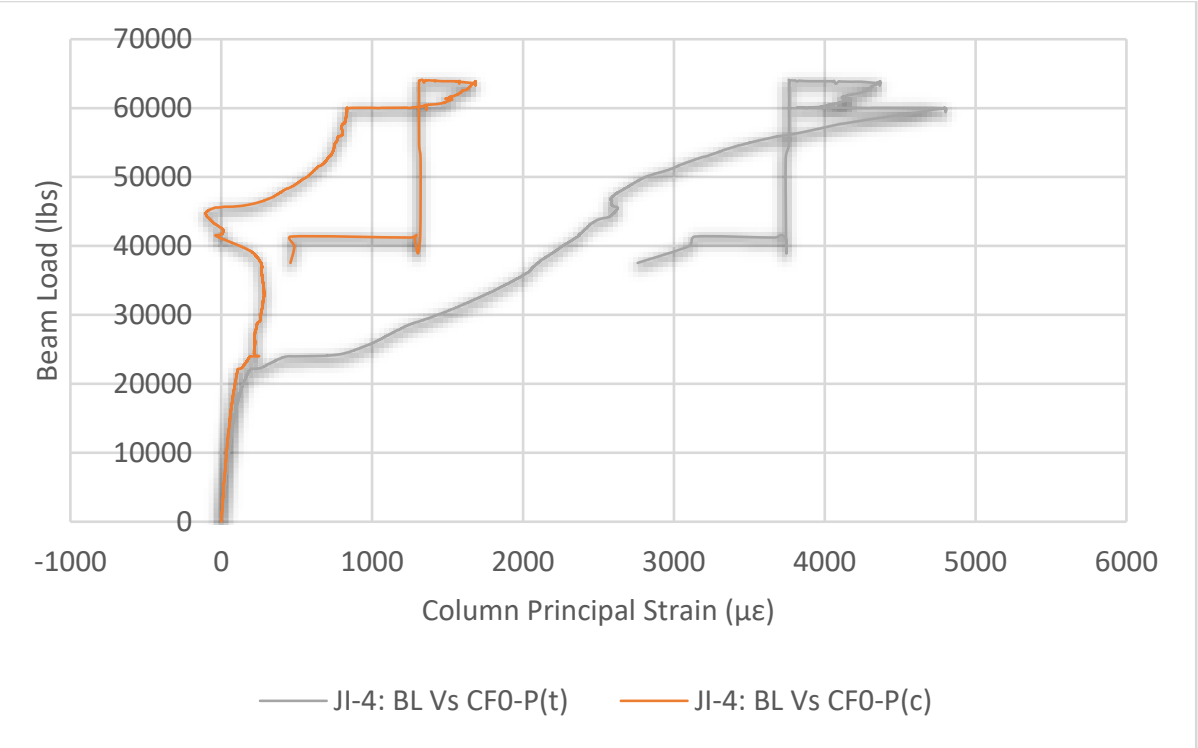

Figure A.20. Beam load vs. column principal strain

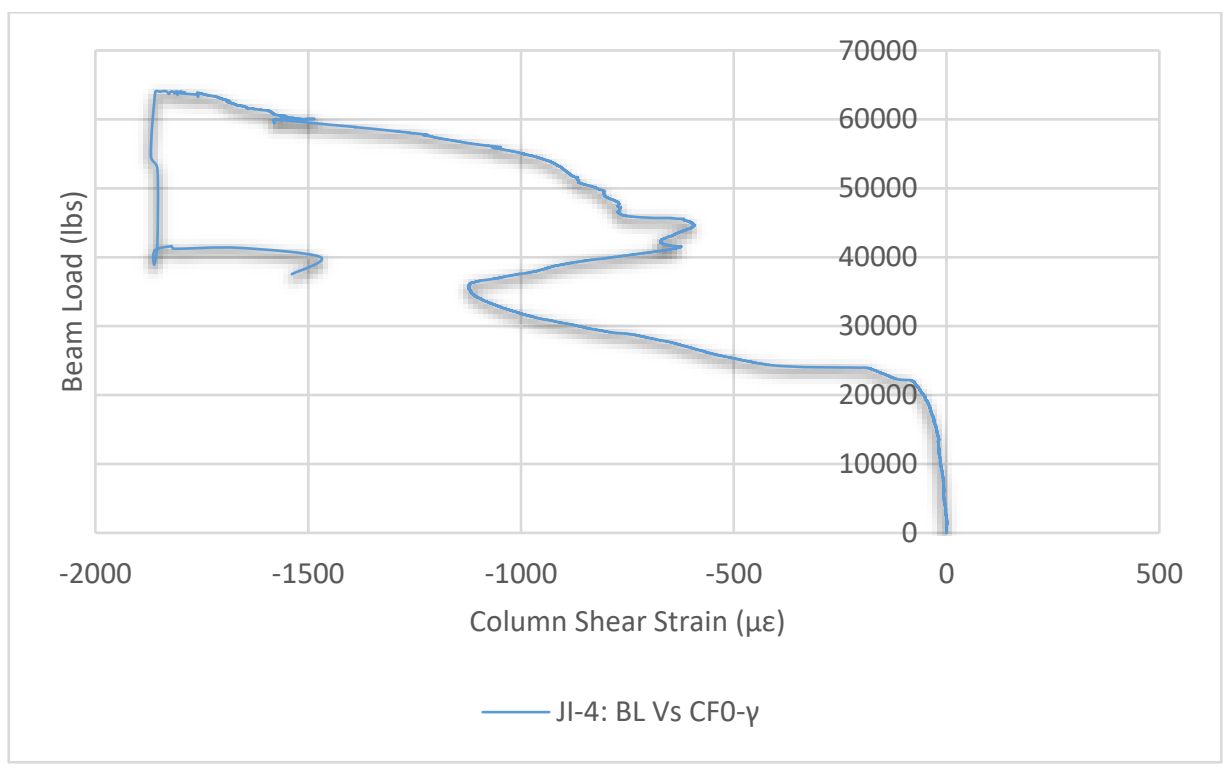

Figure A.21. Beam load vs. column shear strain 


\section{Appendix-B: Beam-Column Joints Reinforced with Concrete Filler-Modules and CFRP Gussets}

This section provides additional test data generated through experimental evaluation of beamcolumn joints reinforced with concrete filler-modules with CFRP gussets under monotonic loads.

\section{B.1 Specimen JI-5}

B.1.1 Strain in steel rebar

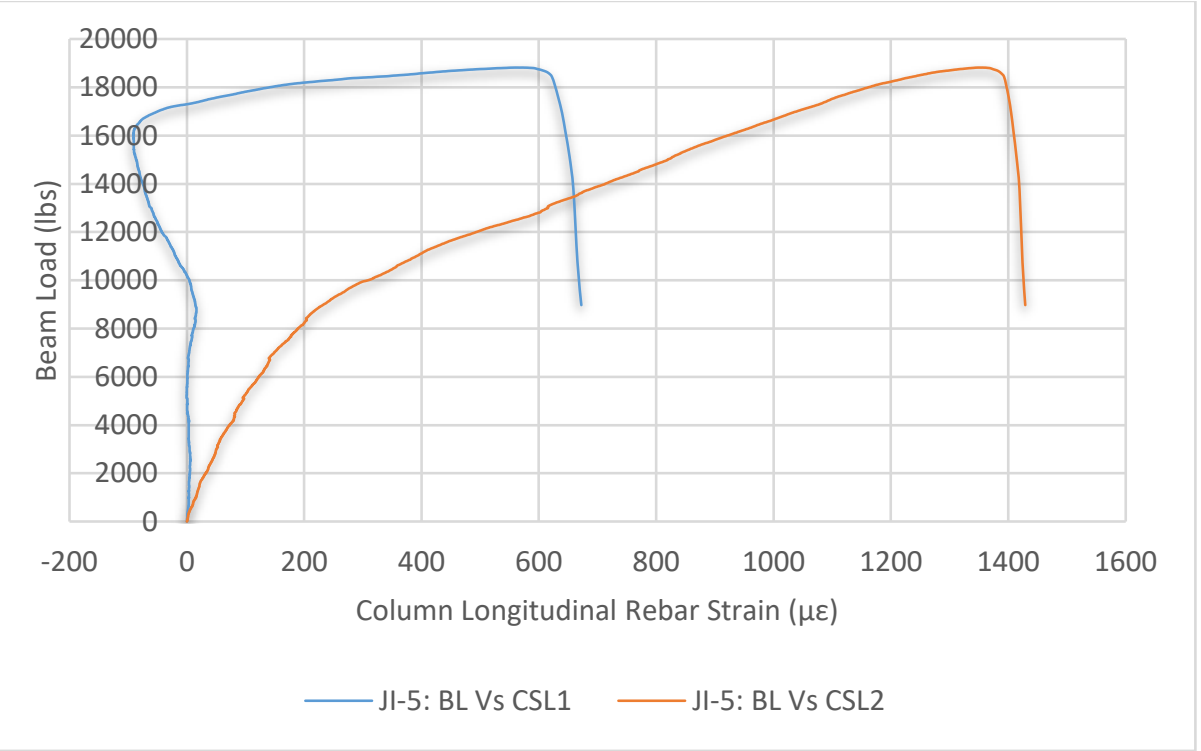

Figure B.1. Beam load vs. column longitudinal rebar strain 


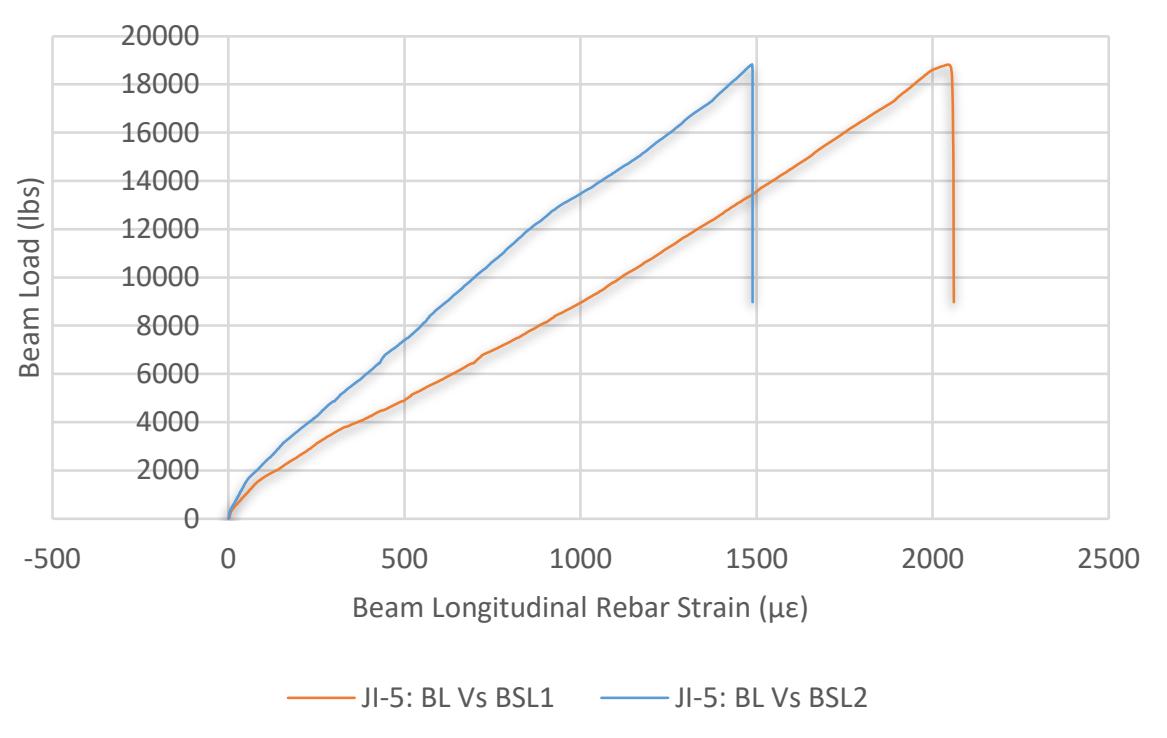

Figure B.2. Beam load vs. beam longitudinal rebar strain

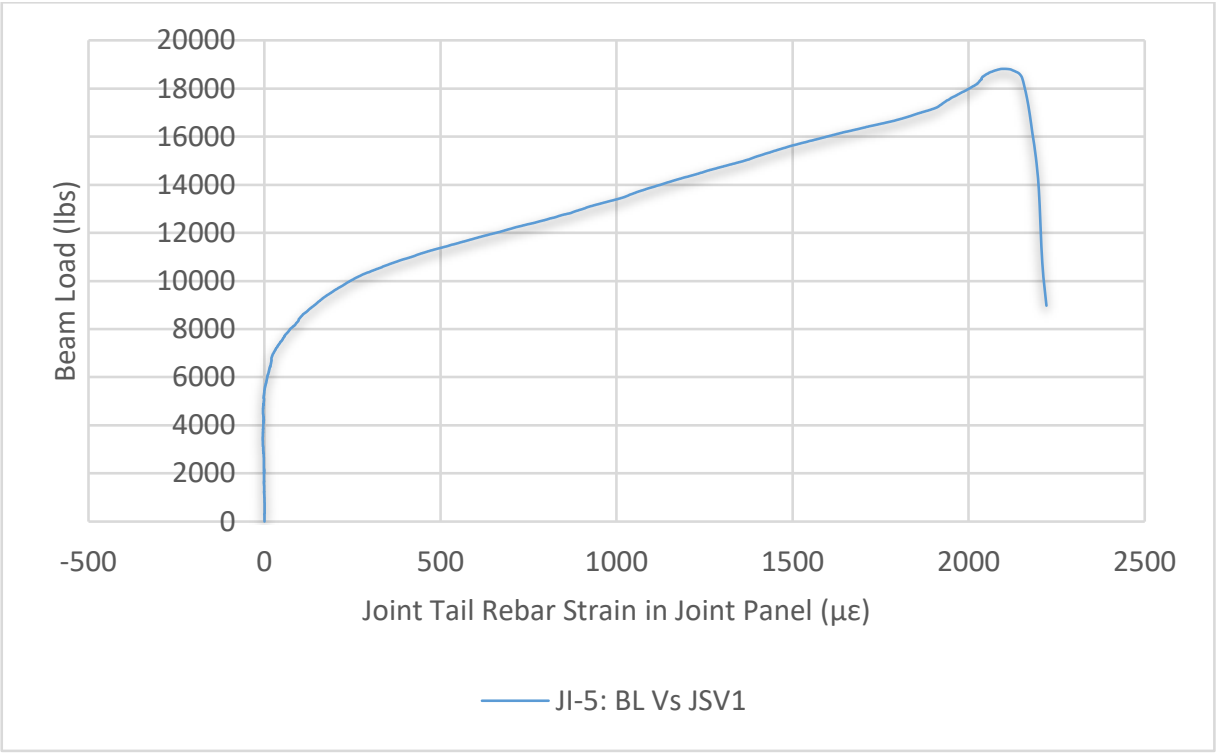

Figure B.3. Beam load vs. joint tail rebar strain 


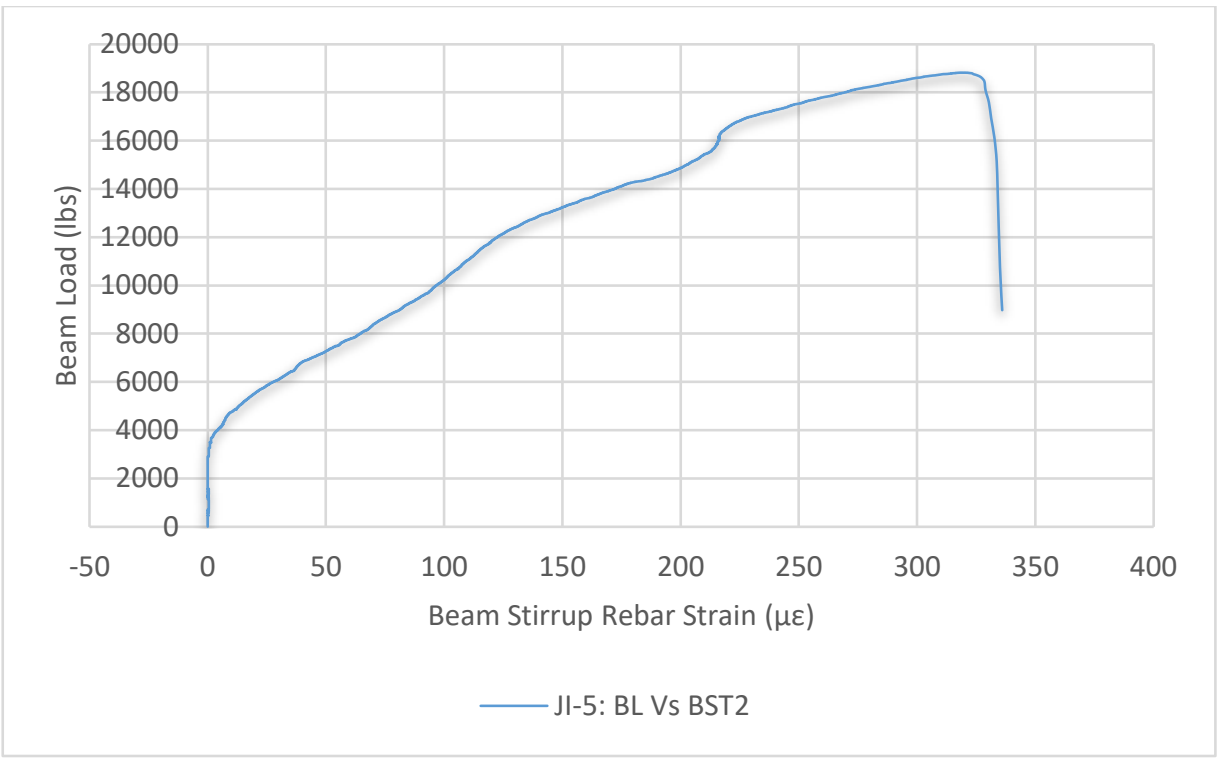

Figure B.4. Beam load vs. beam stirrup strain

\section{B.1.2 Strain in concrete}

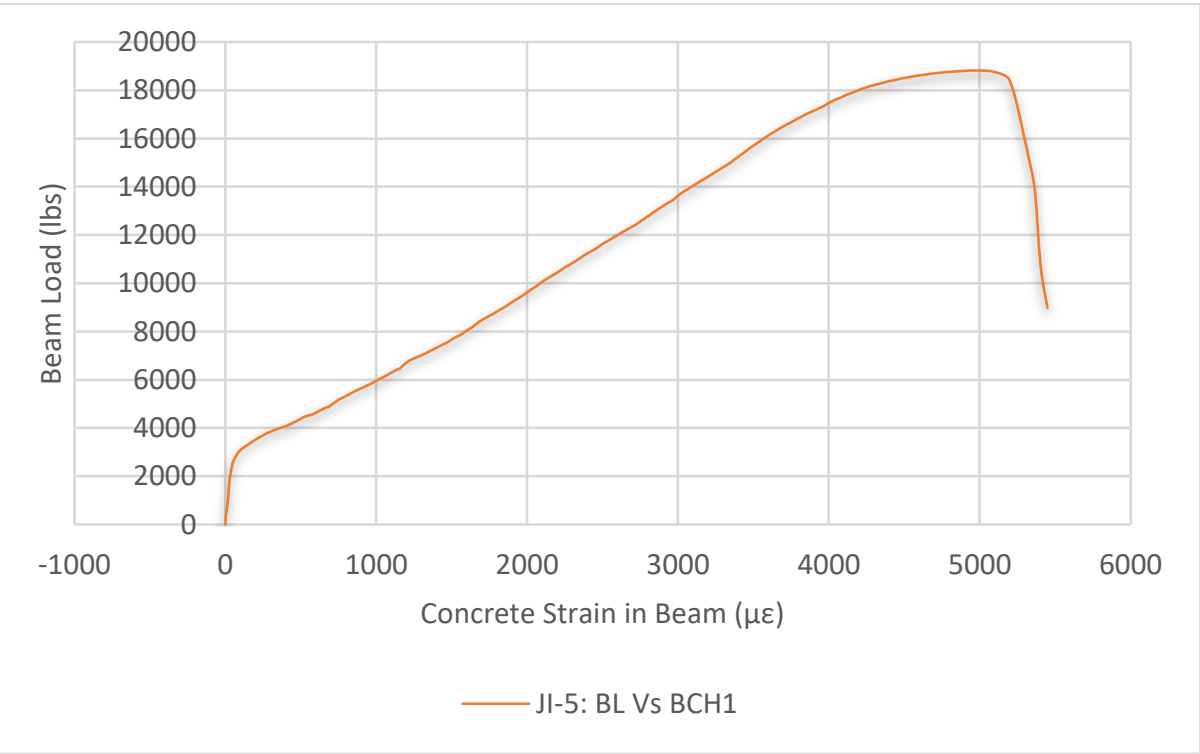

Figure B.5. Beam load vs. concrete strain in beam 


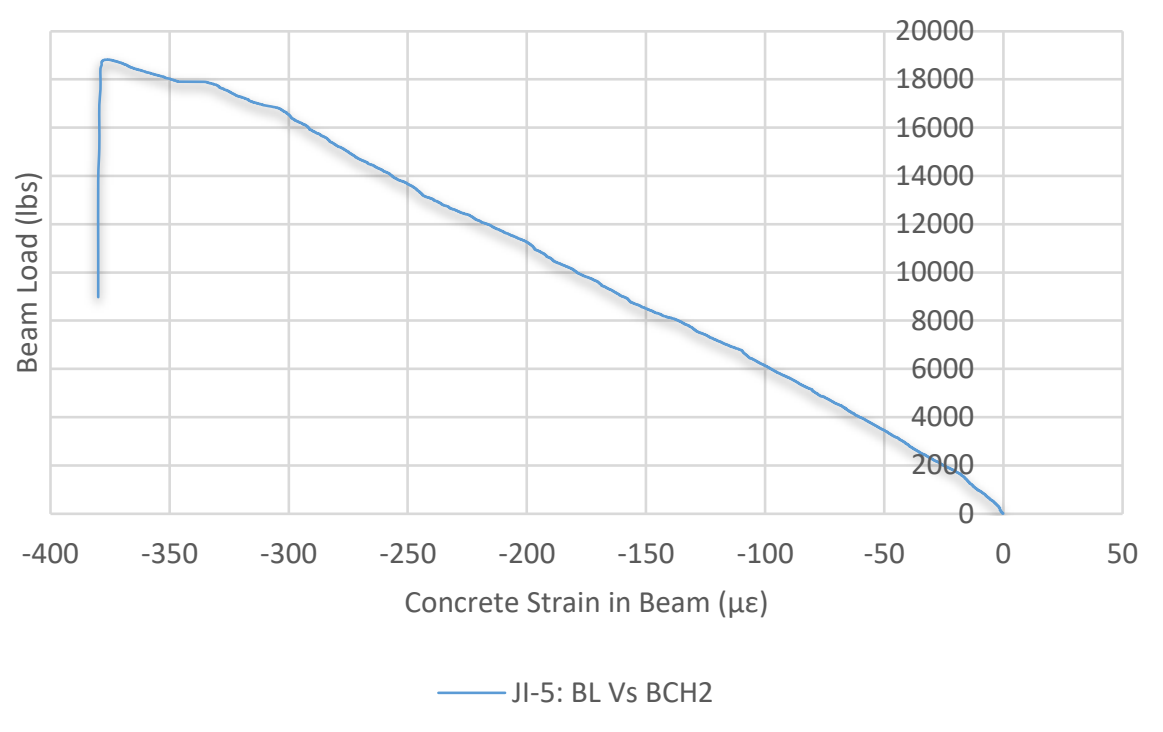

Figure B.6. Beam load vs. concrete strain in beam

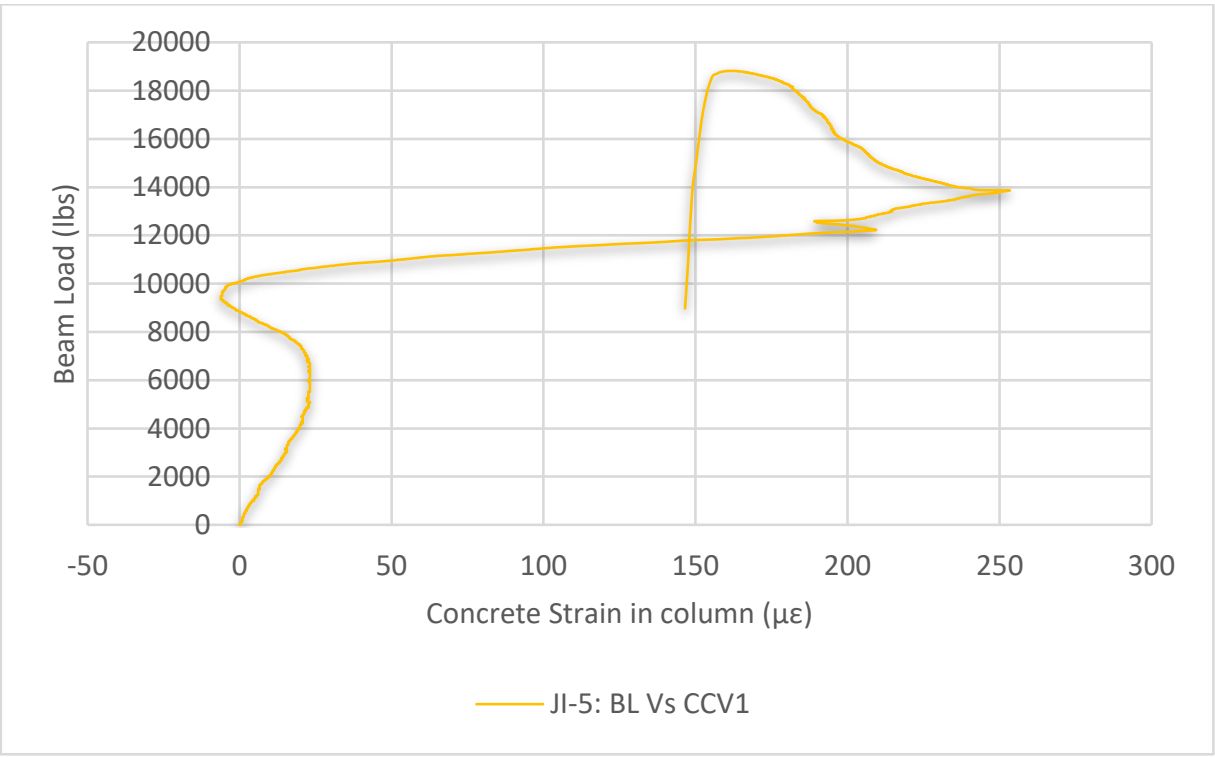

Figure B.7. Beam load vs. concrete strain in column 


\section{B.2 Specimen JI-6}

\section{B.2.1 Strain in steel rebar}

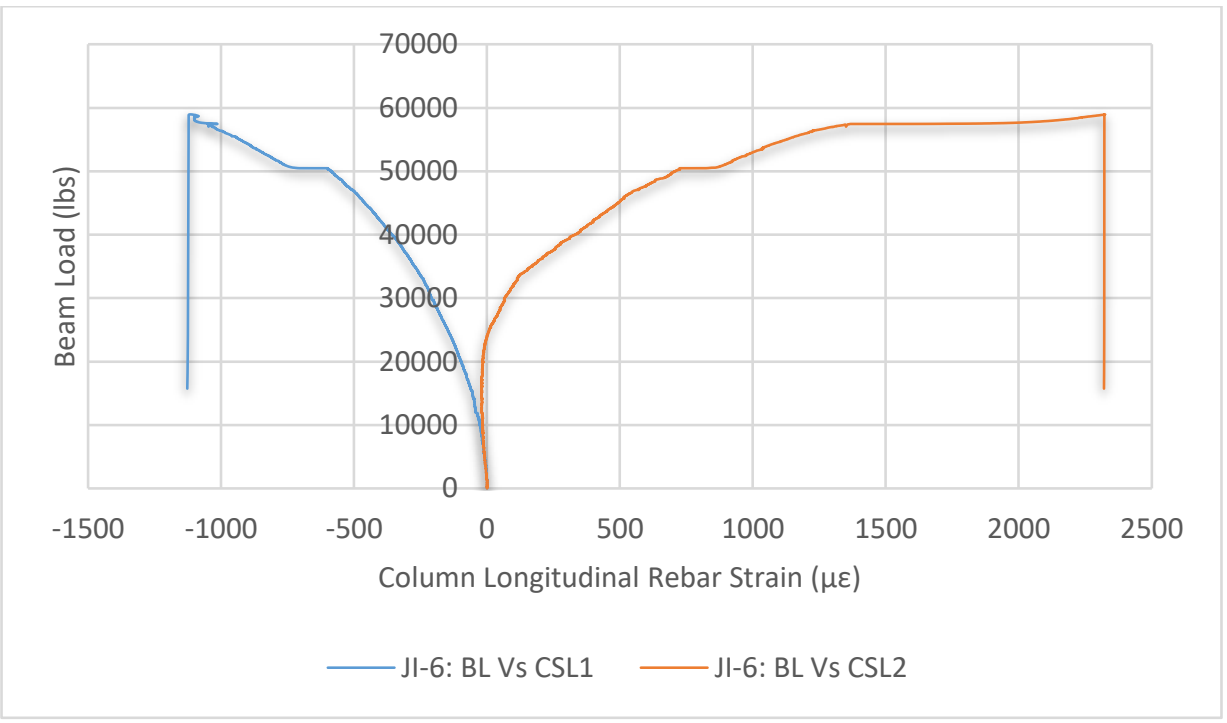

Figure B.8. Beam load vs. column longitudinal rebar strain

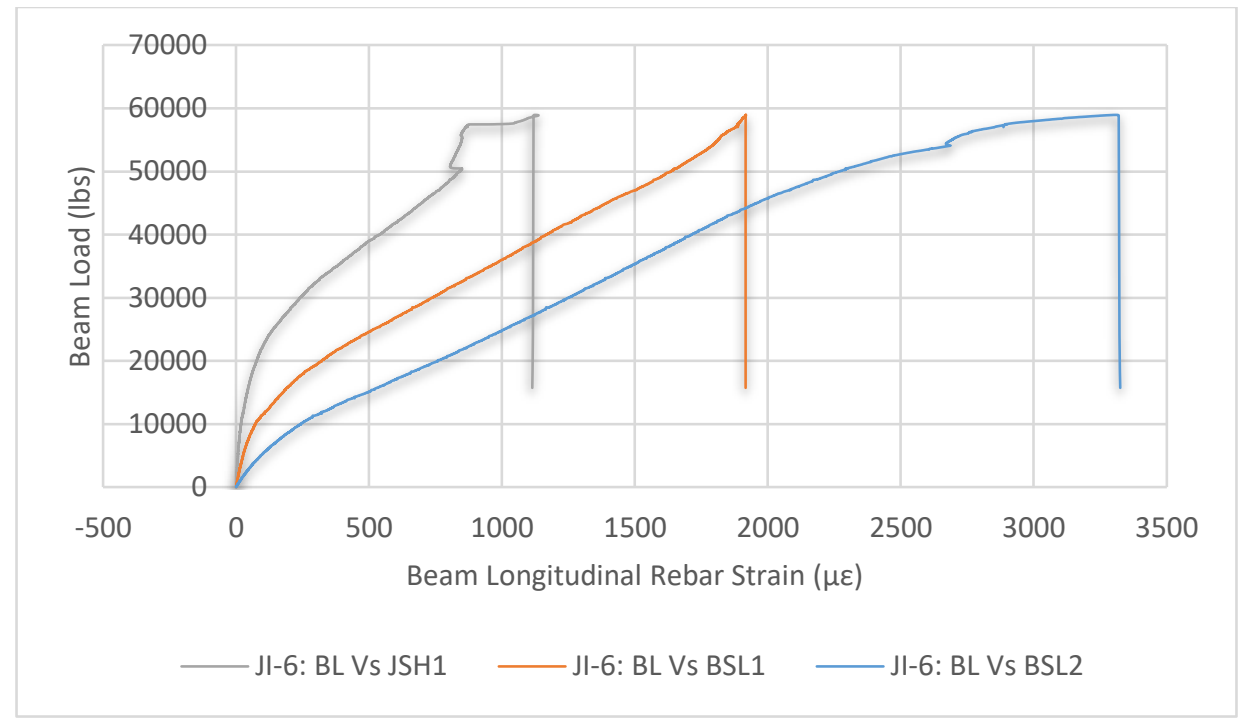

Figure B.9. Beam load vs. beam longitudinal rebar strain 


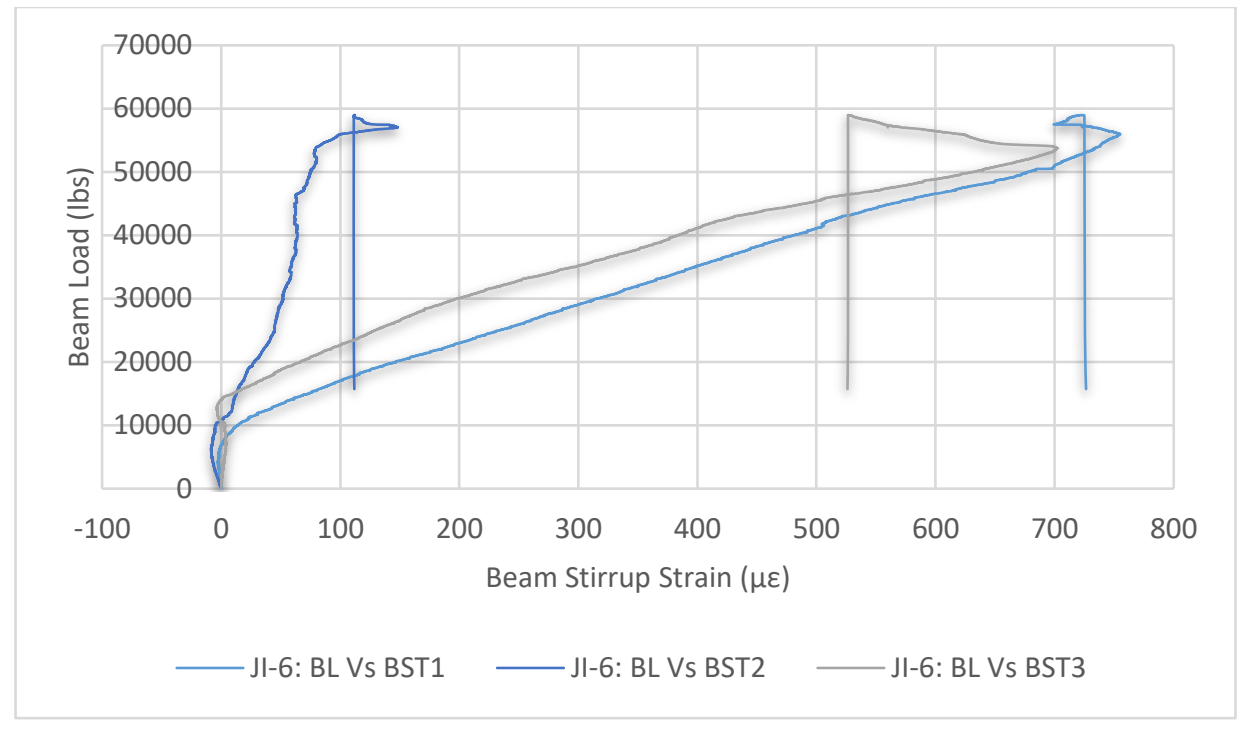

Figure B.10. Beam load vs. beam stirrup strain 


\section{B.2.2 Strain in FRP}

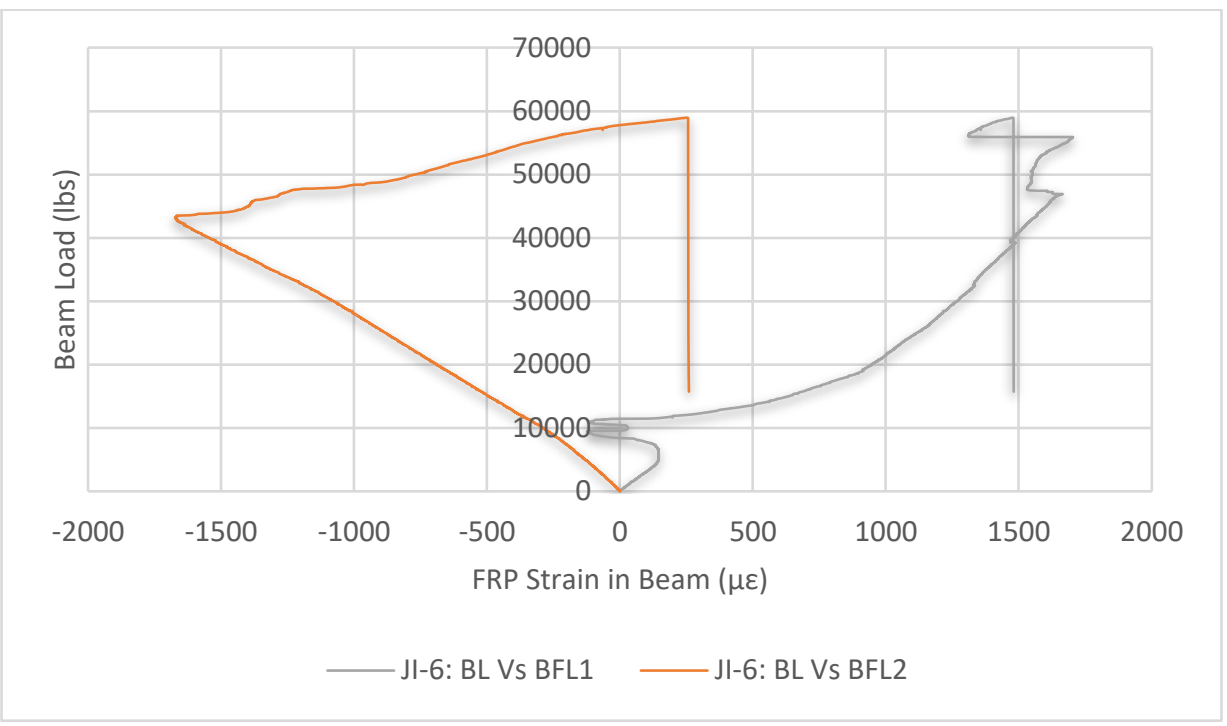

Figure B.11. Beam load vs. FRP strain in beam

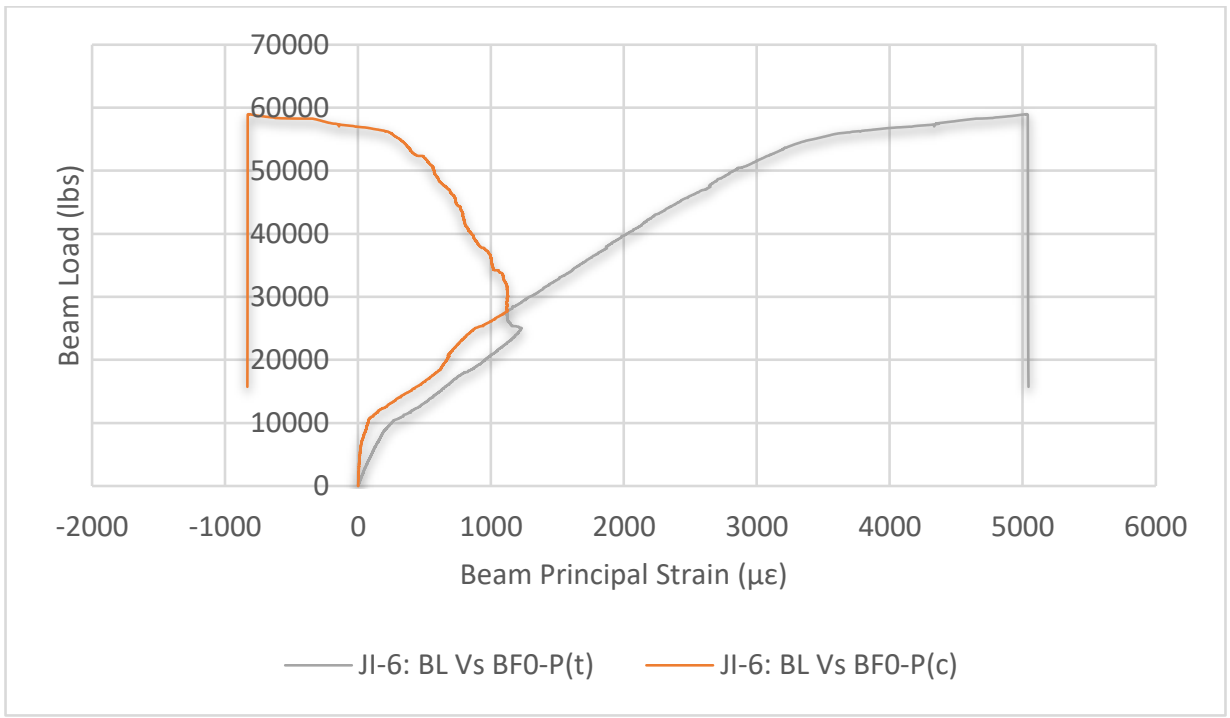

Figure B.12. Beam load vs. beam principal strain 


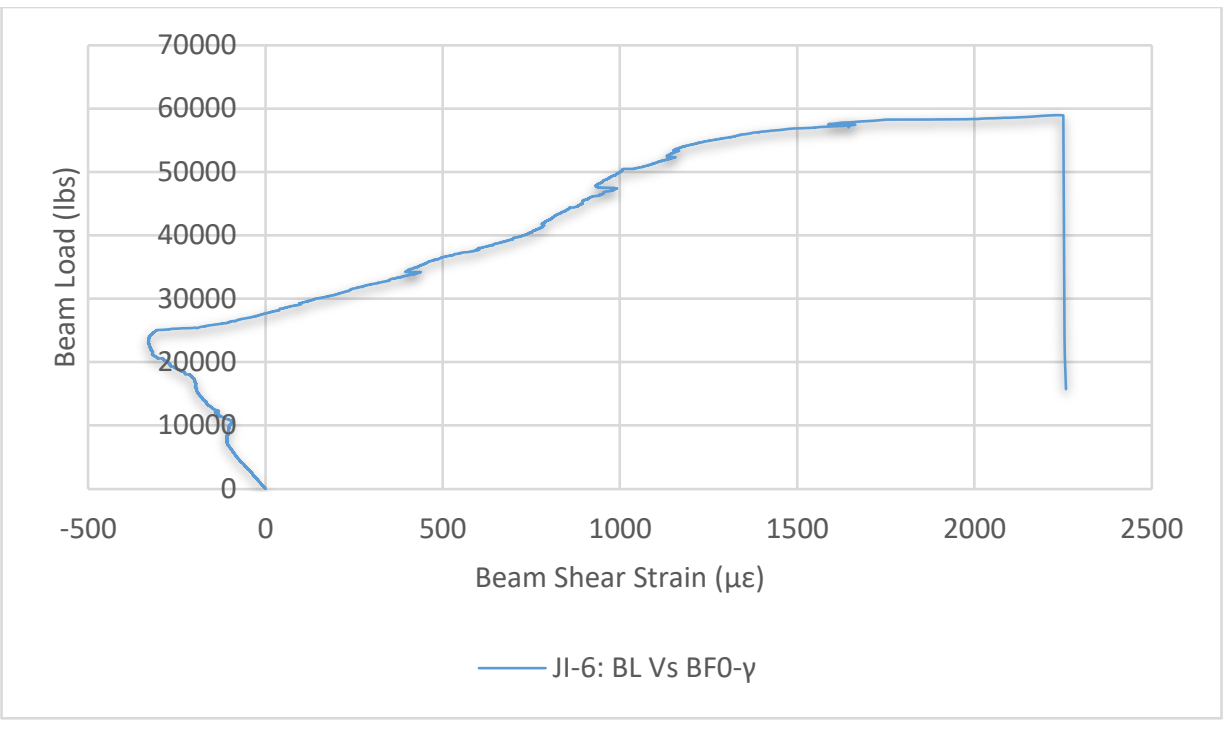

Figure B.13. Beam load vs. beam shear strain

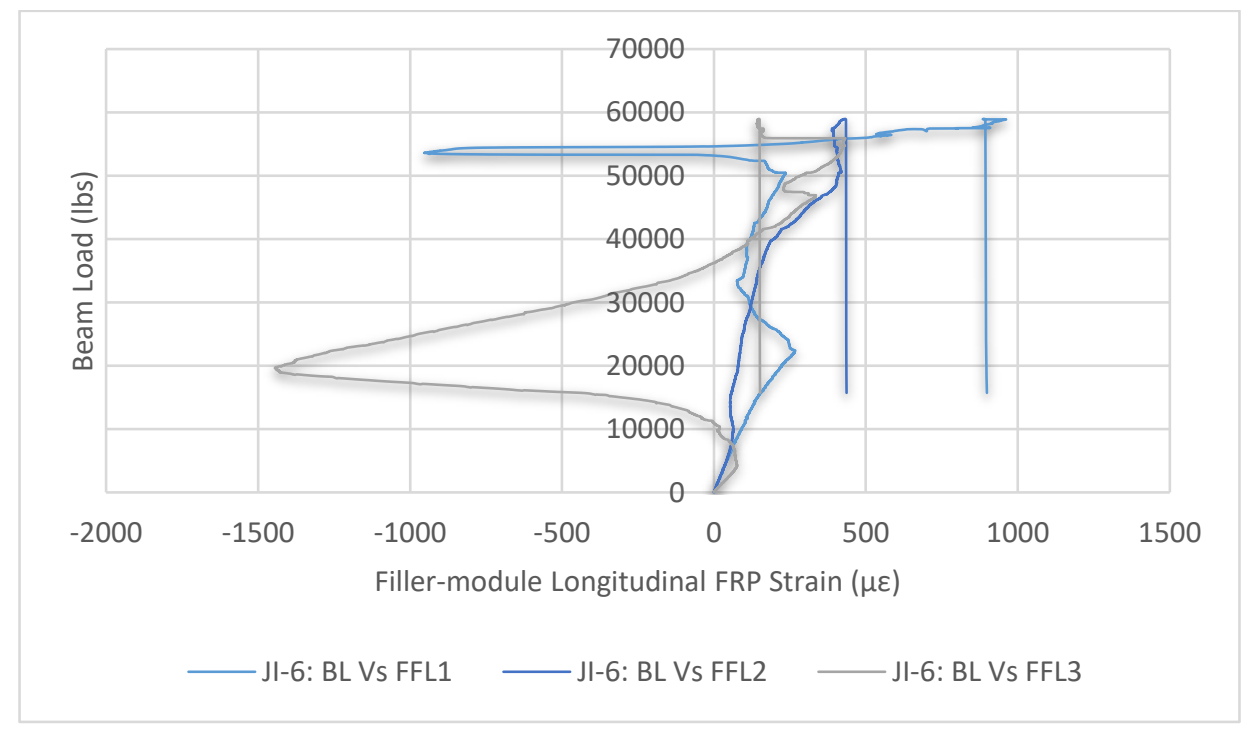

Figure B.14. Beam load vs. filler-module longitudinal strain 


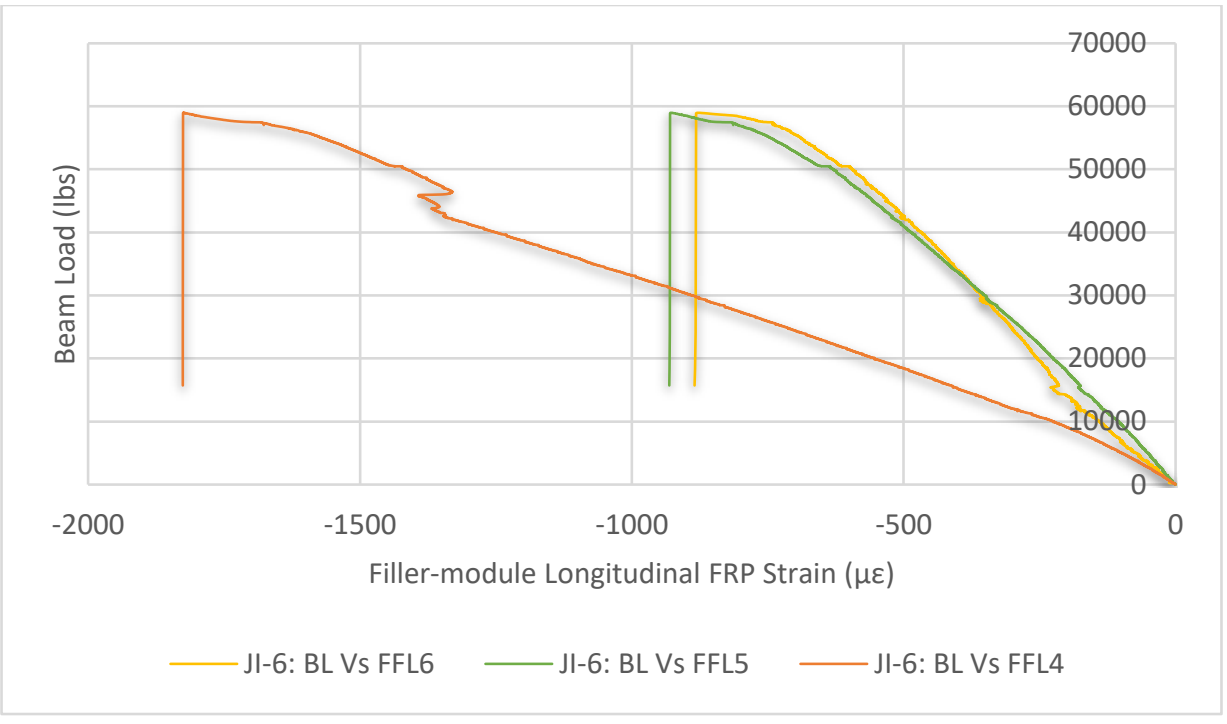

Figure B.15. Beam load vs. filler-module longitudinal strain

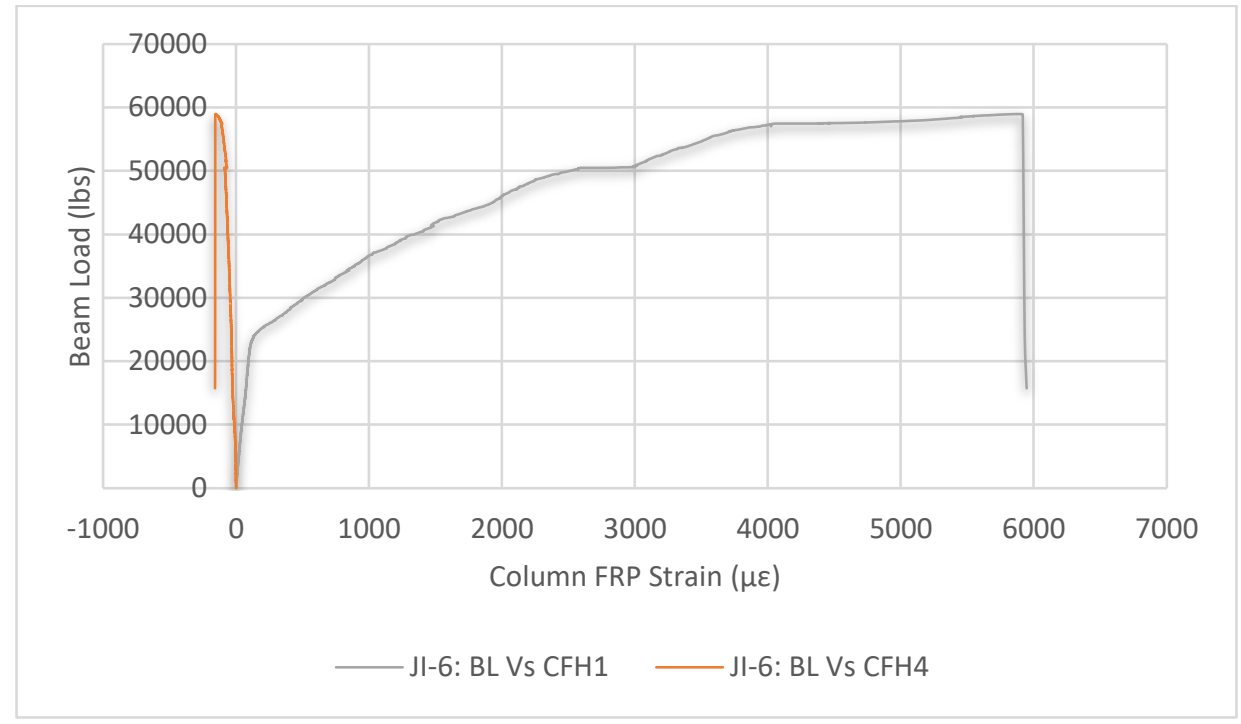

Figure B.16. Beam load vs. column FRP strain 


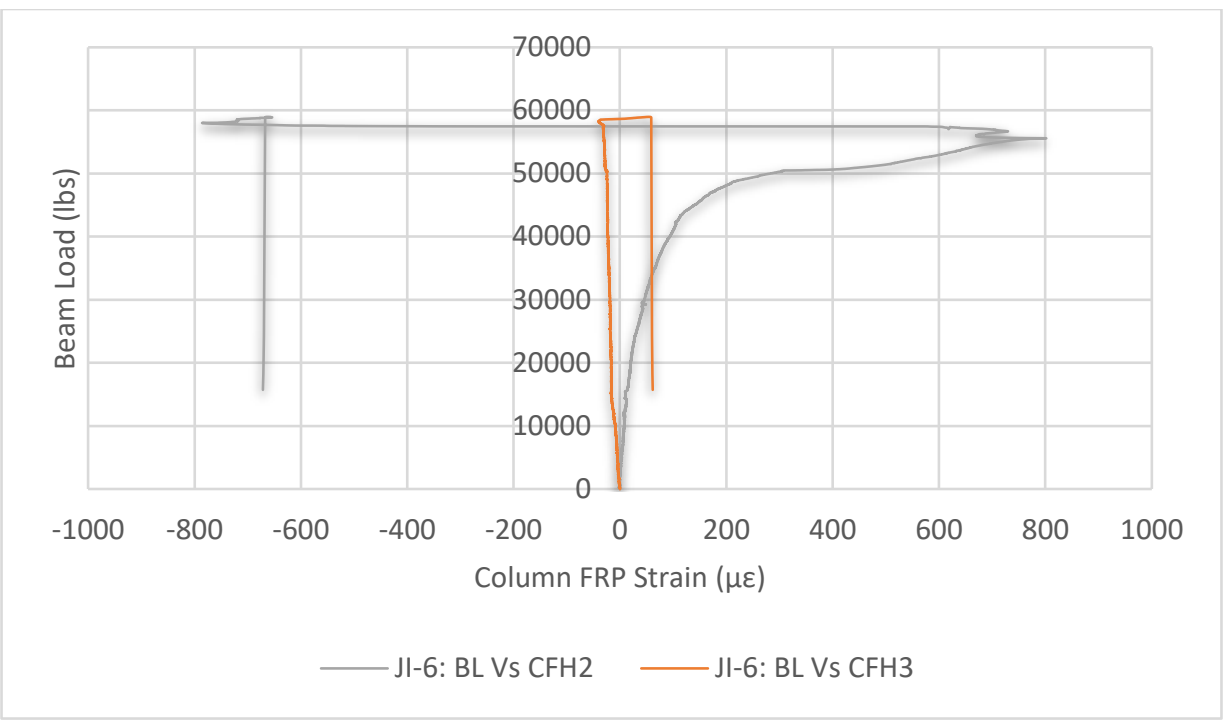

Figure B.17. Beam load vs. column rebar FRP strain

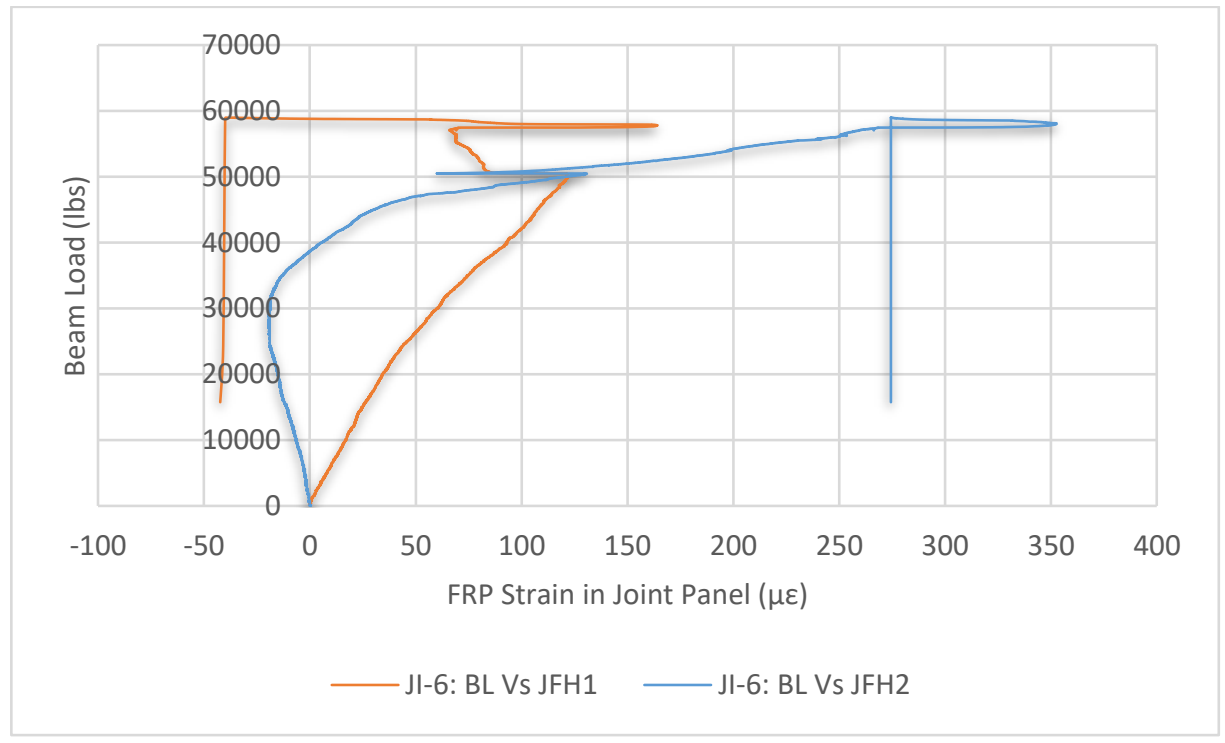

Figure B.18. Beam load vs. FRP strain in joint panel 


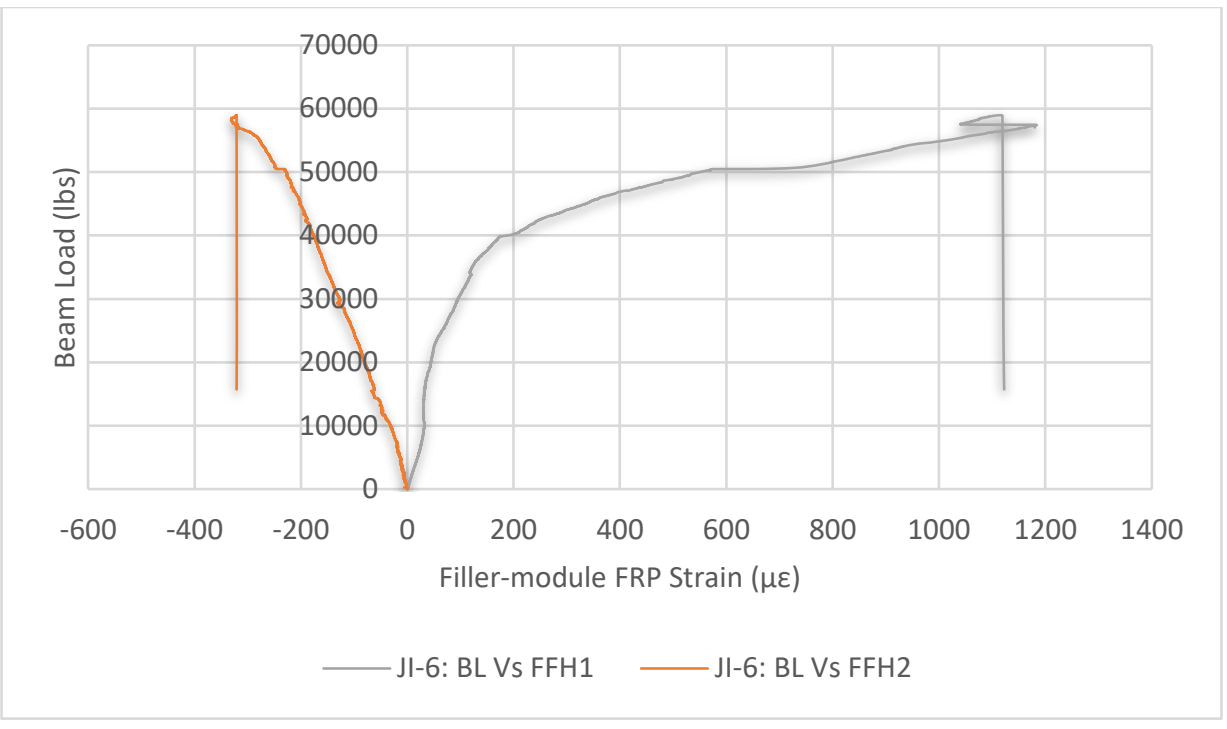

Figure B.19. Beam load vs. filler-module FRP strain

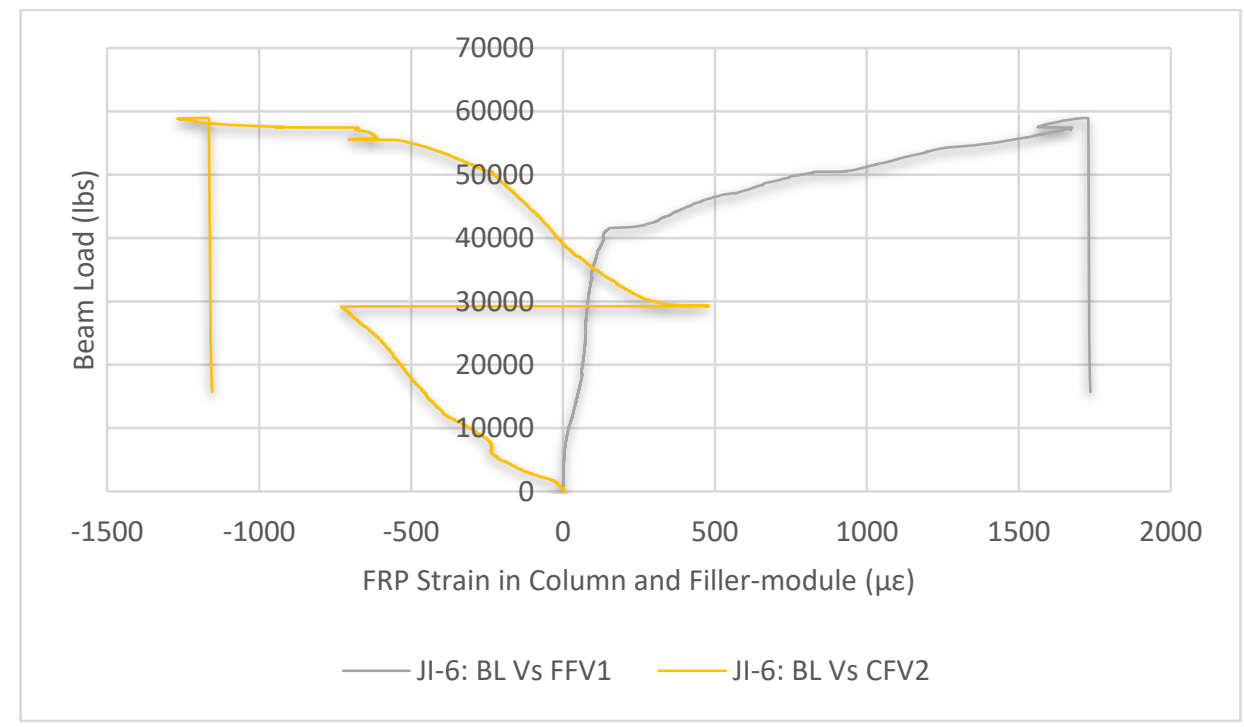

Figure B.20. Beam load vs. FRP strain in column and filler-module 


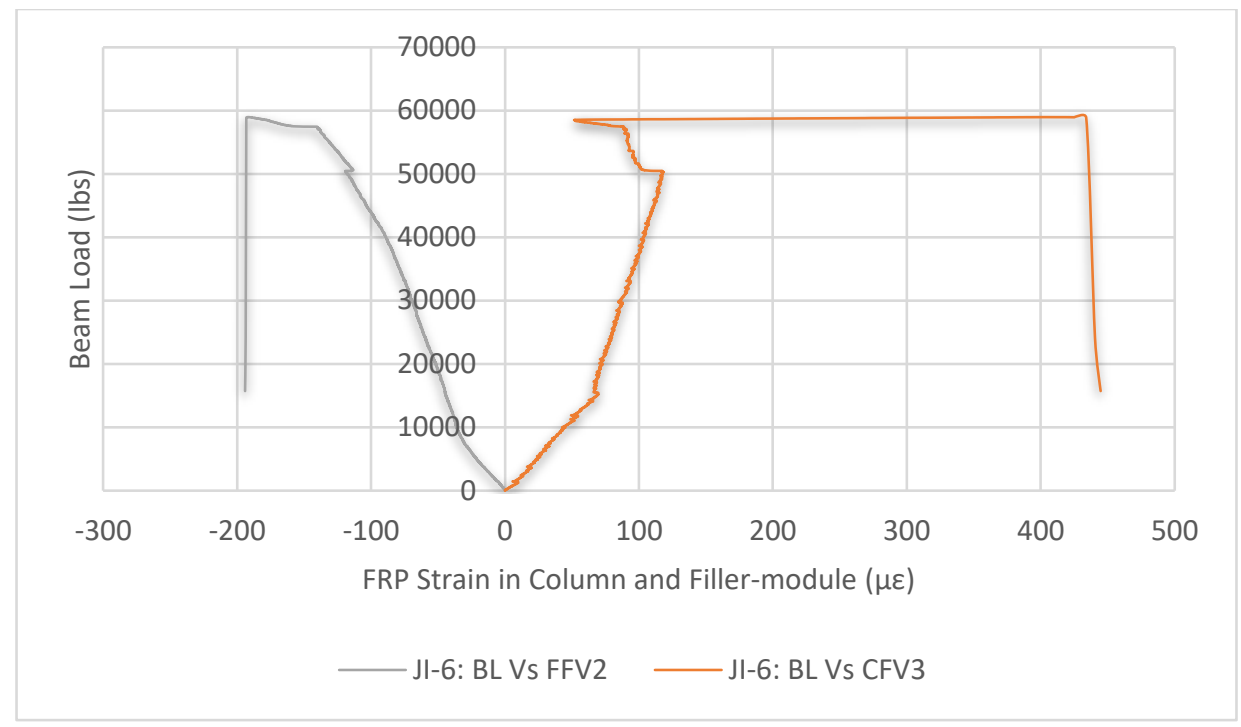

Figure B.21. Beam load vs. FRP strain in column and filler-module 


\section{B.3 Specimen JI-7}

\section{B.3.1 Strain in steel rebar}

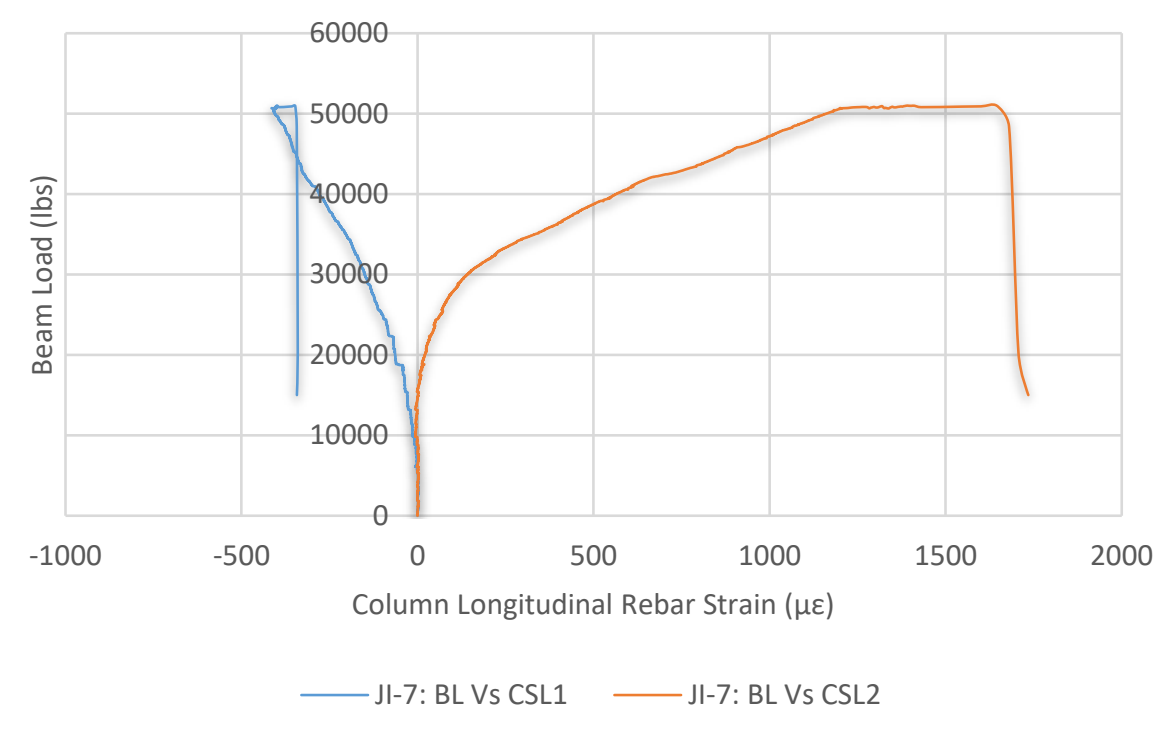

Figure B.22. Beam load vs. column longitudinal rebar strain

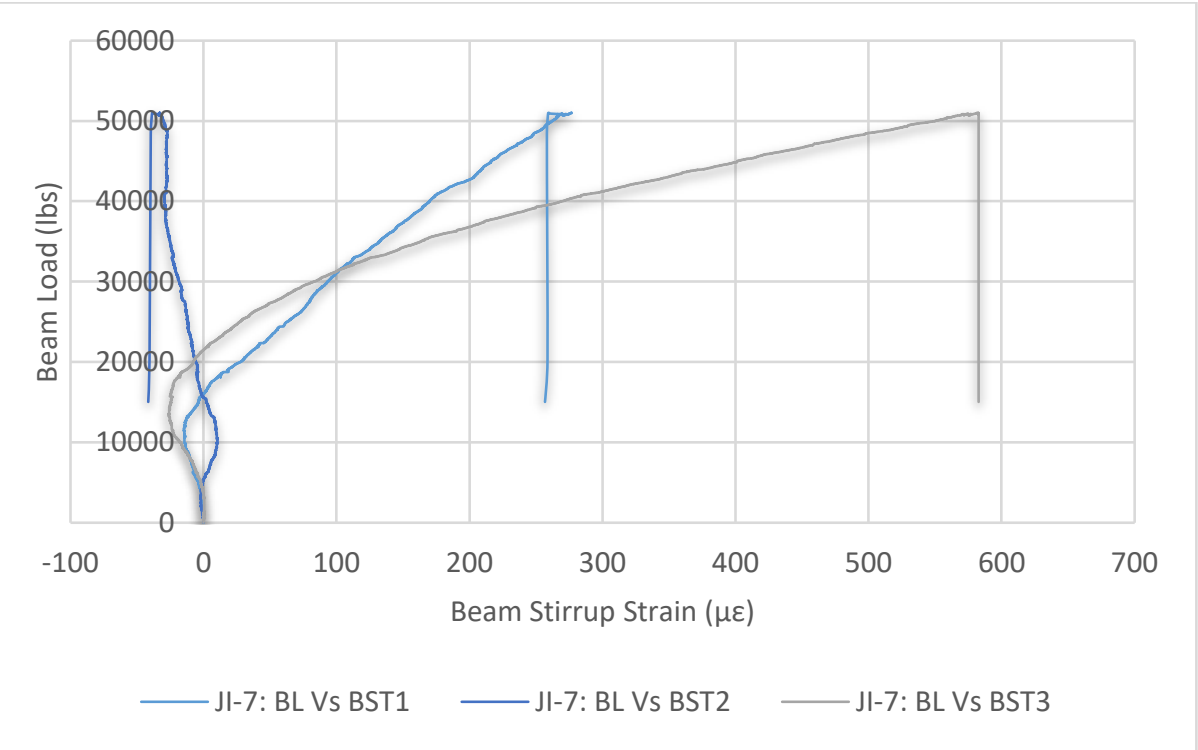

Figure B.23. Beam load vs. beam stirrup strain 


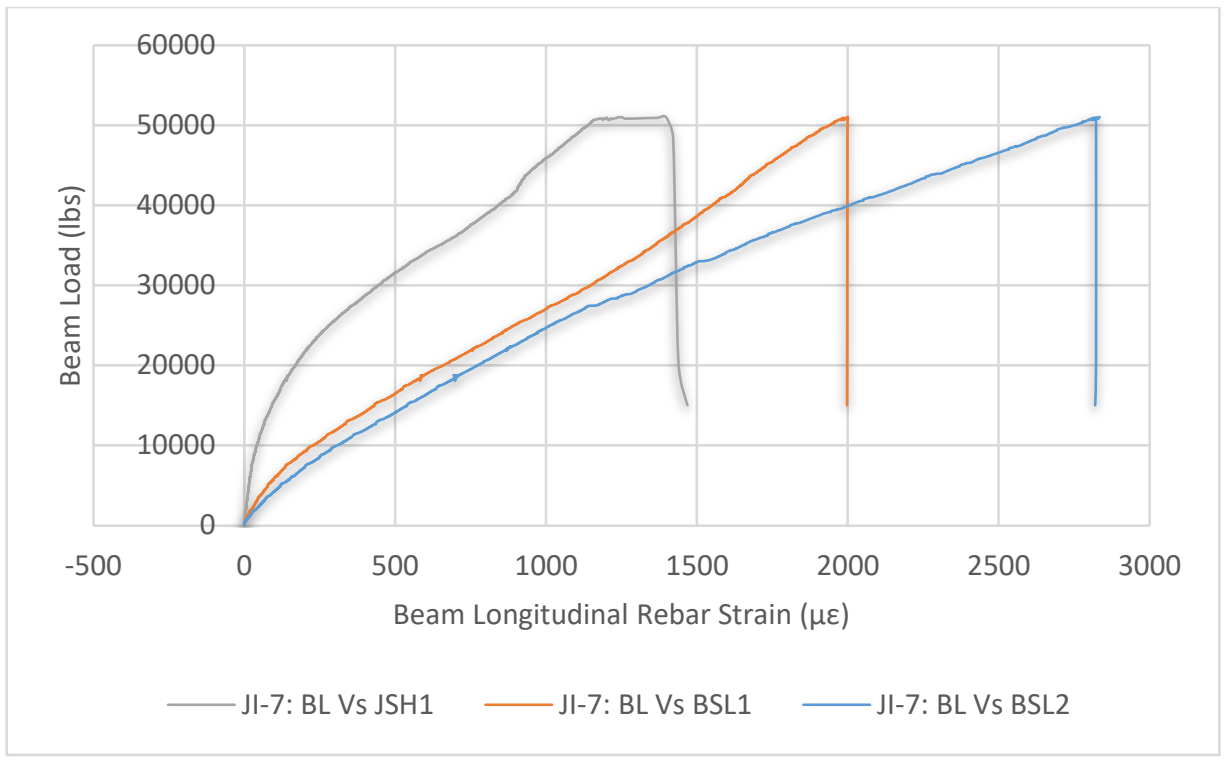

Figure B.24. Beam load vs. beam longitudinal rebar strain 


\section{B.3.2 Strain in FRP}

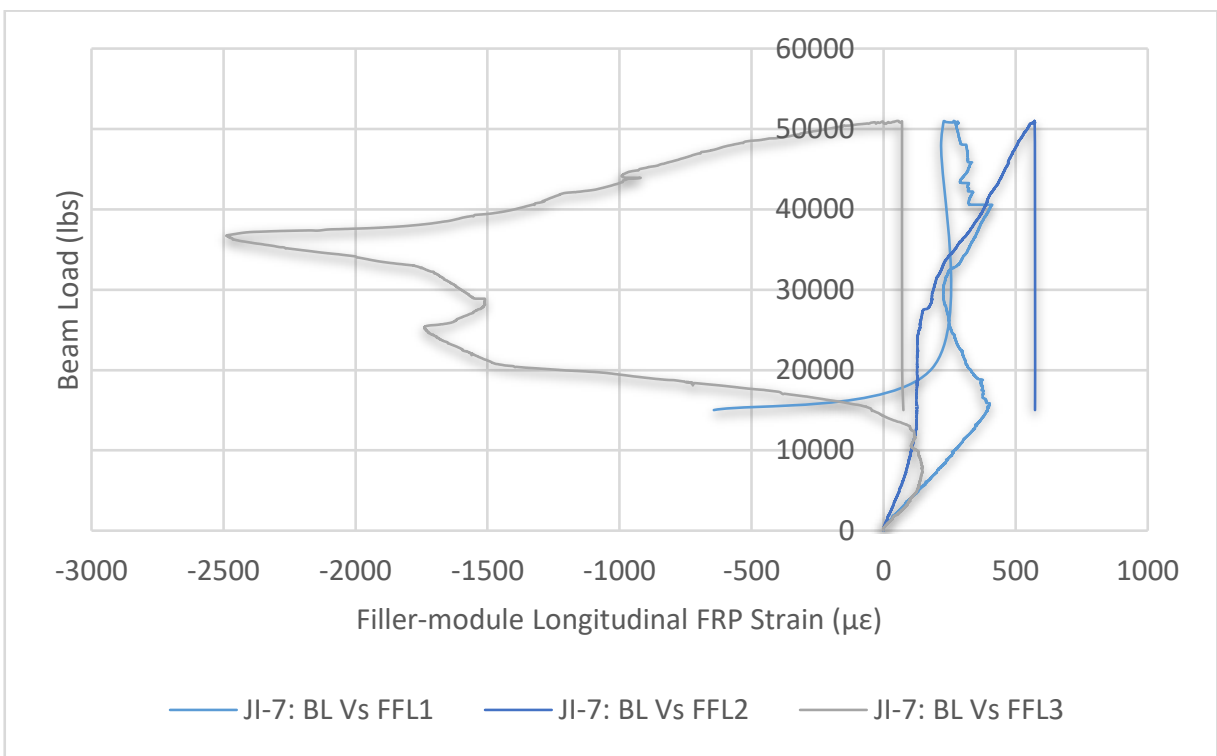

Figure B.25. Beam load vs. filler-module longitudinal strain

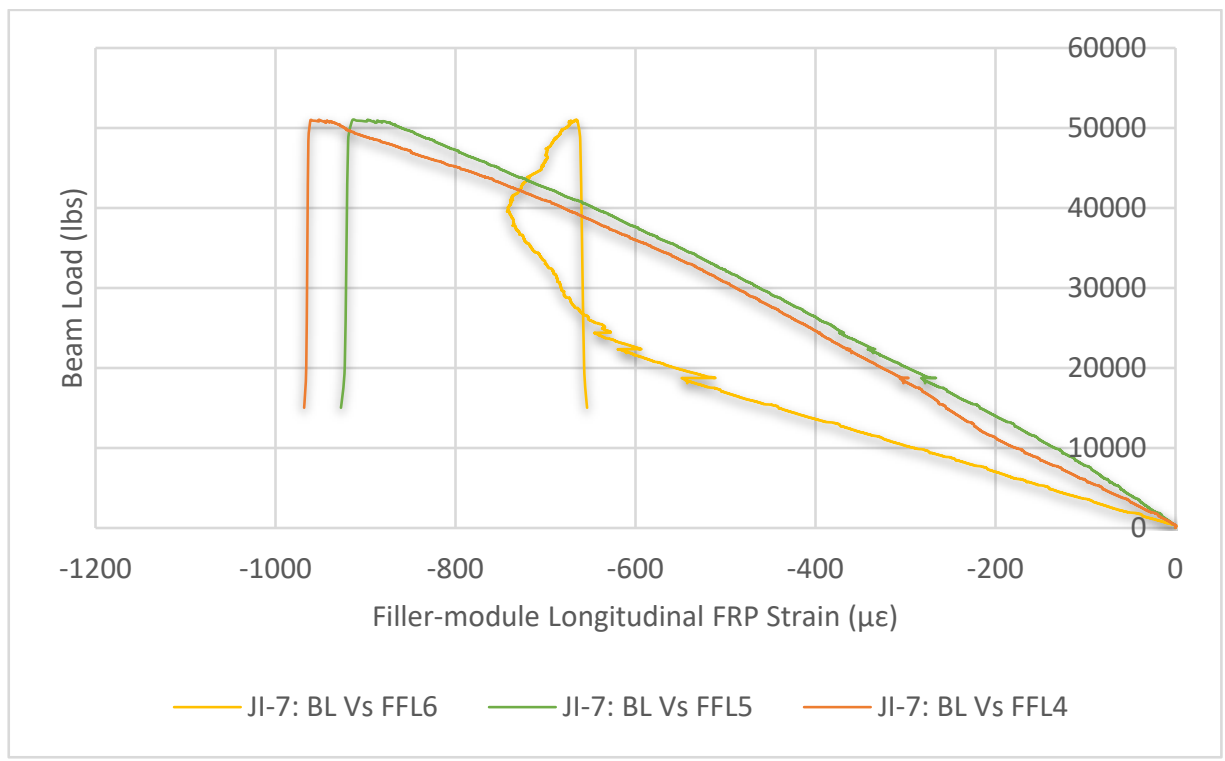

Figure B.26. Beam load vs. filler-module longitudinal strain 


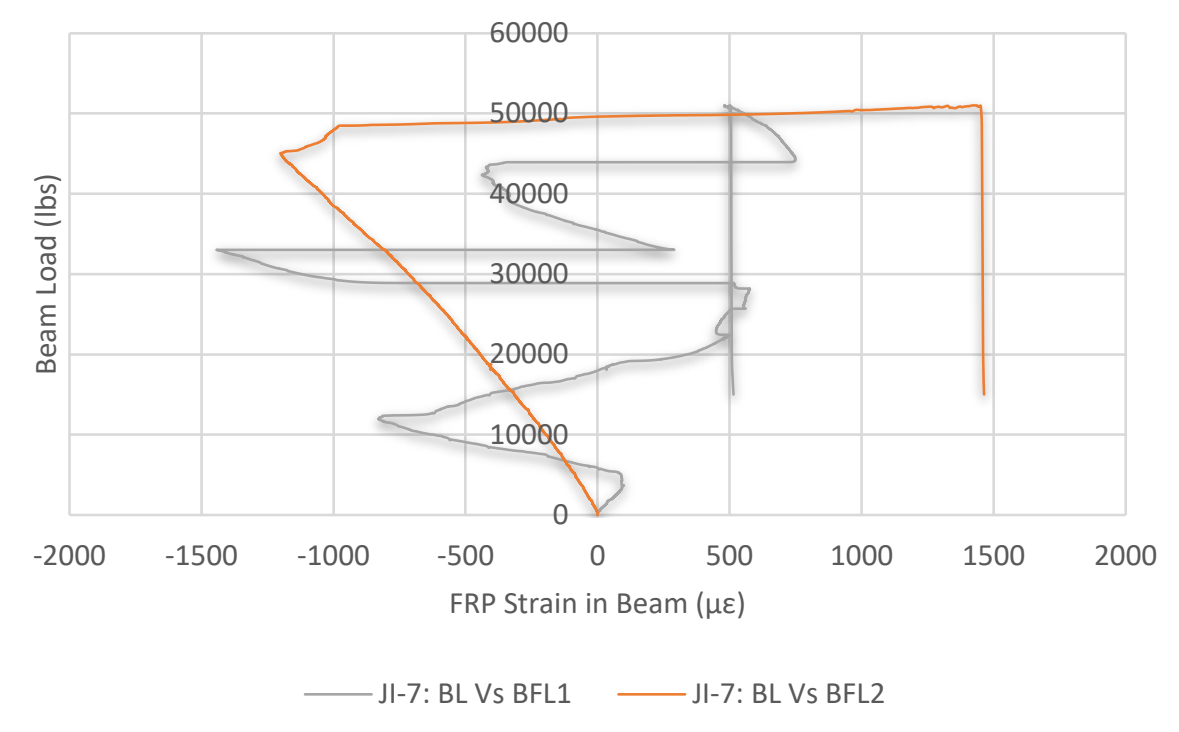

Figure B.27. Beam load vs. FRP strain in beam

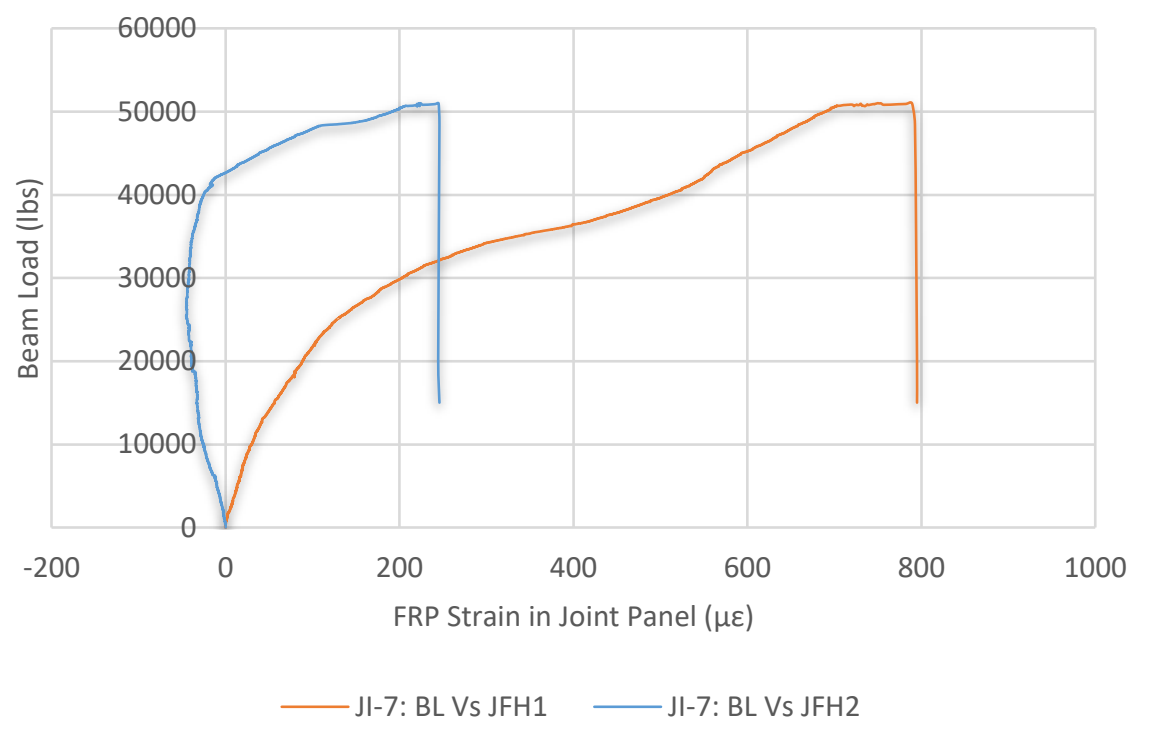

Figure B.28. Beam load vs. FRP strain in joint panel 


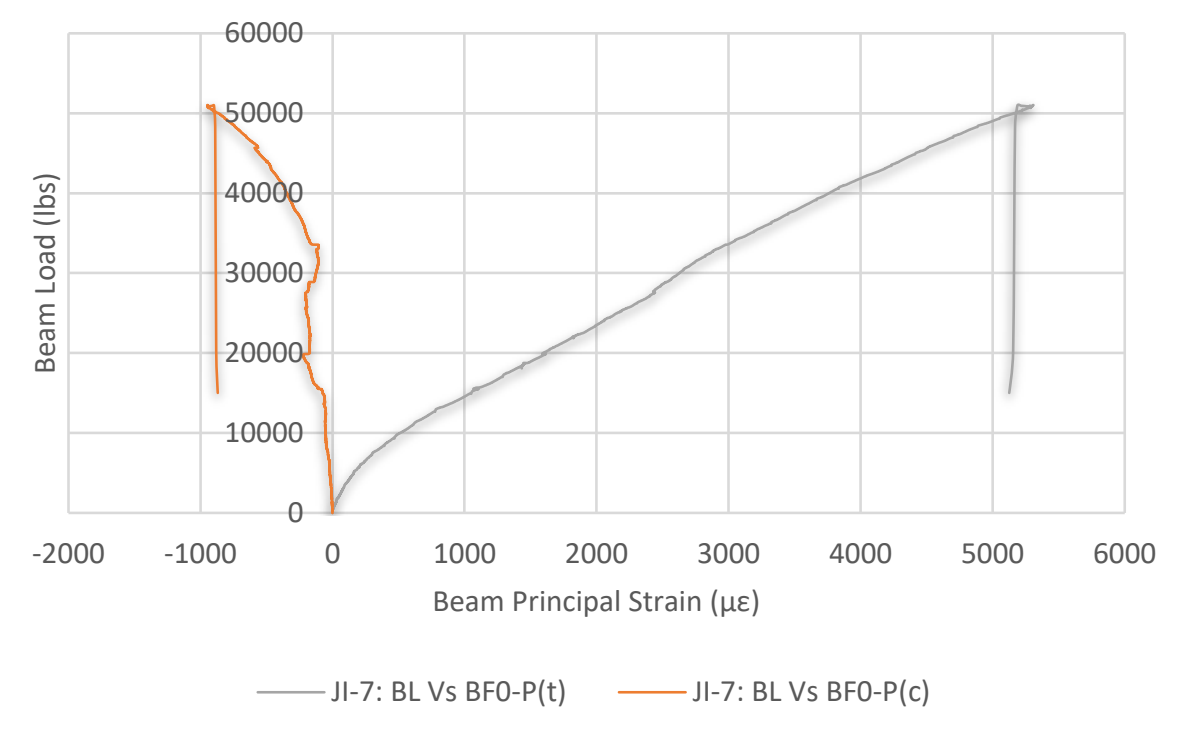

Figure B.29. Beam load vs. beam principal strain

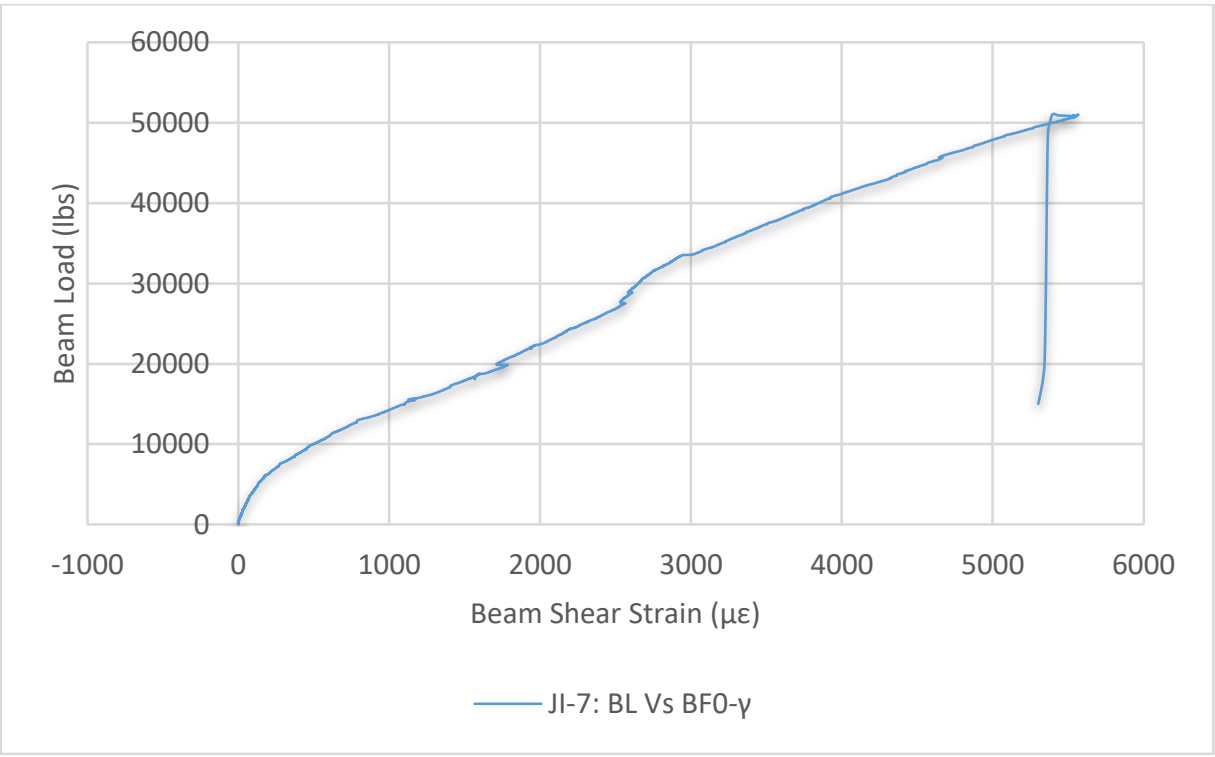

Figure B.30. Beam load vs. beam shear strain 


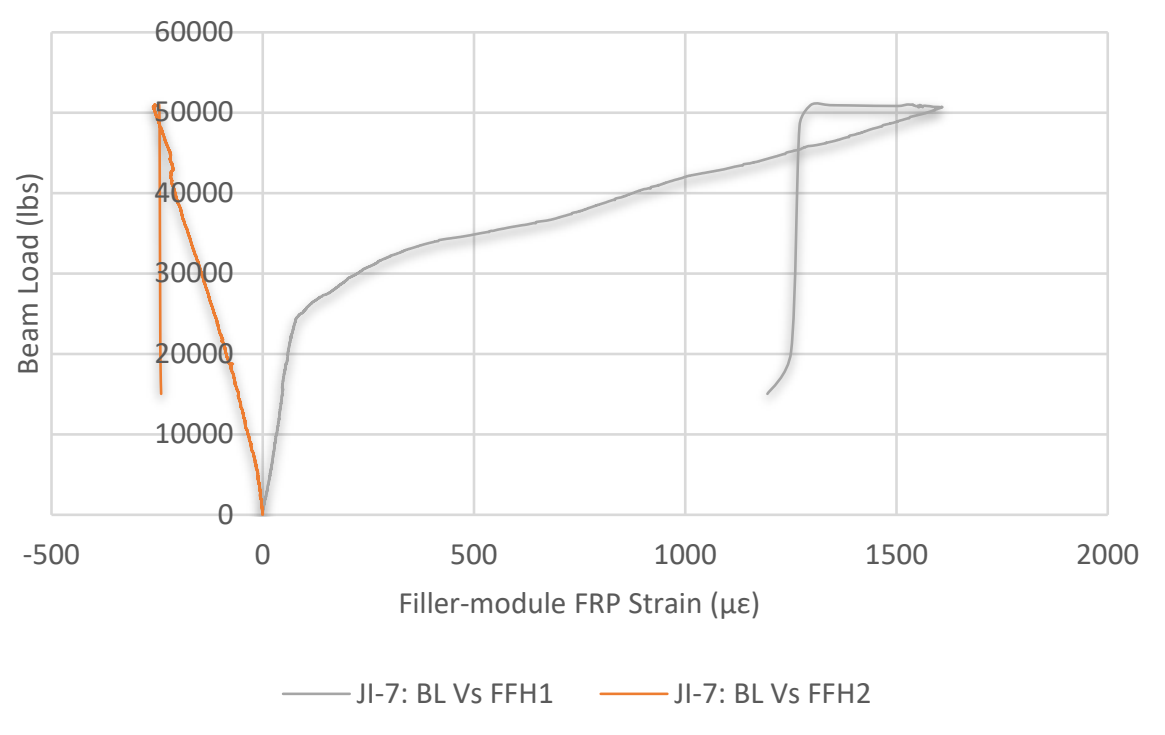

Figure B.31. Beam load vs. filler-module FRP strain

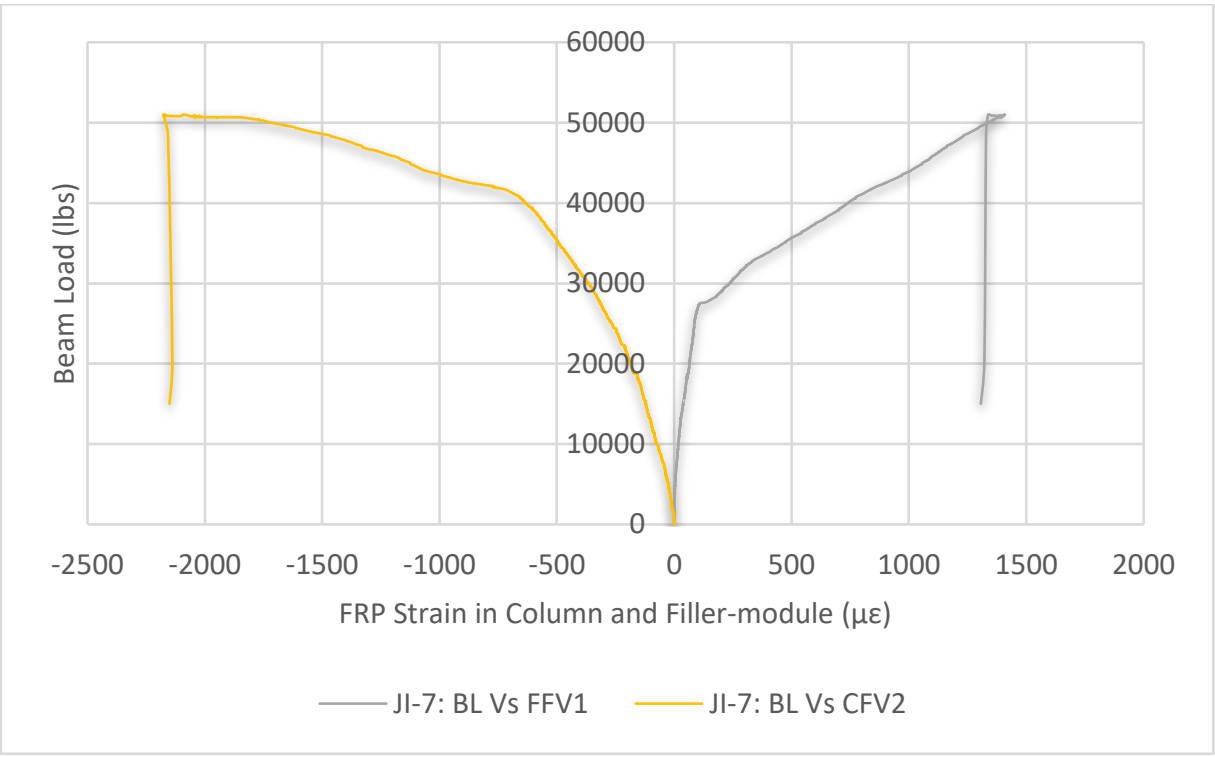

Figure B.32. Beam load vs. FRP strain in column and filler-module 


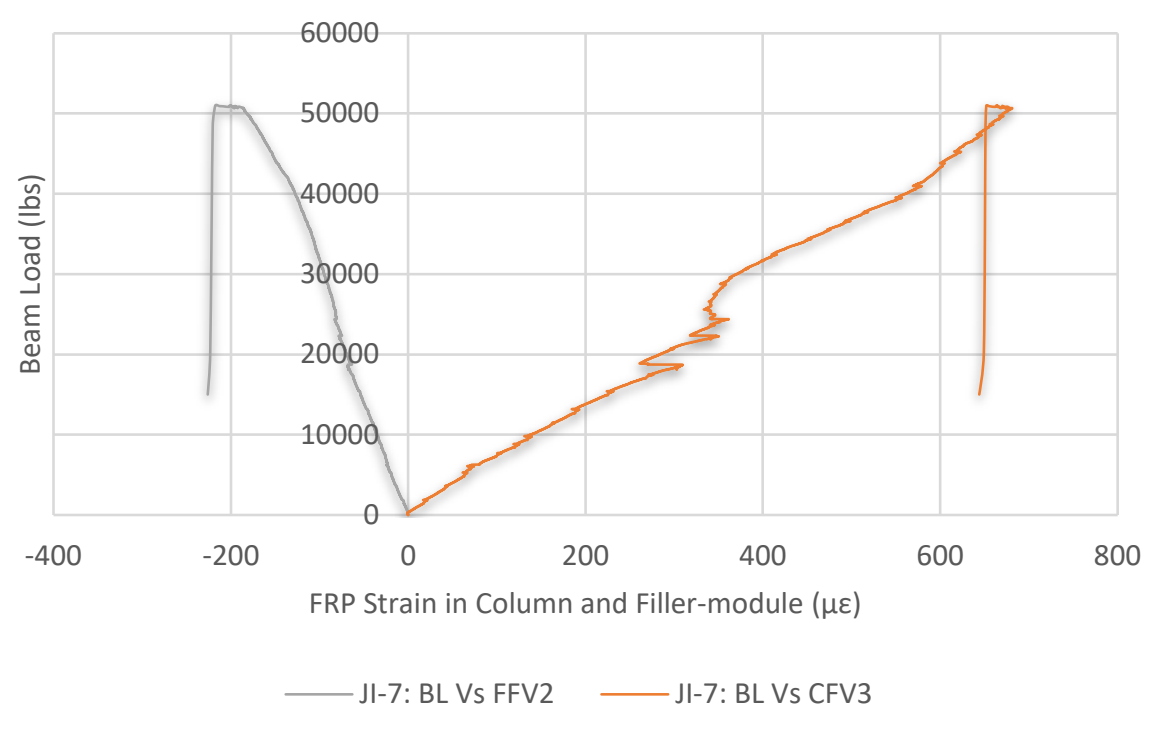

Figure B.33. Beam load vs. FRP strain in column and filler-module

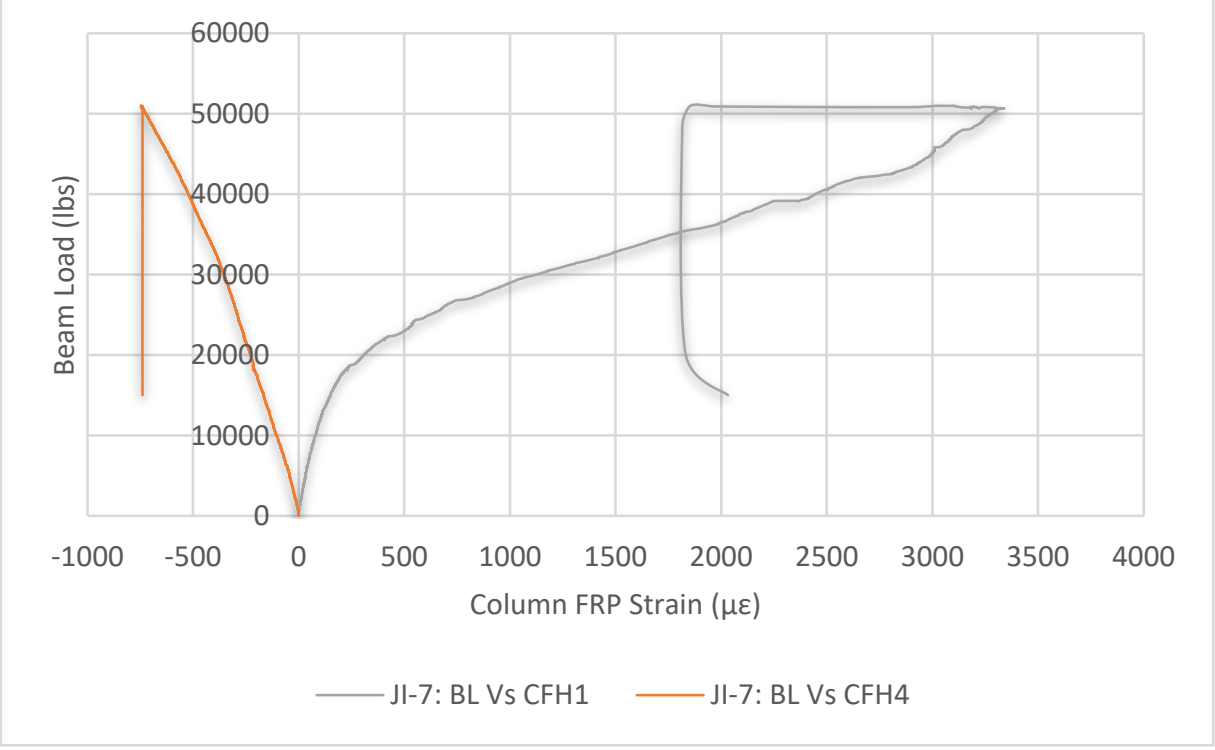

Figure B.34. Beam load vs. column FRP strain 


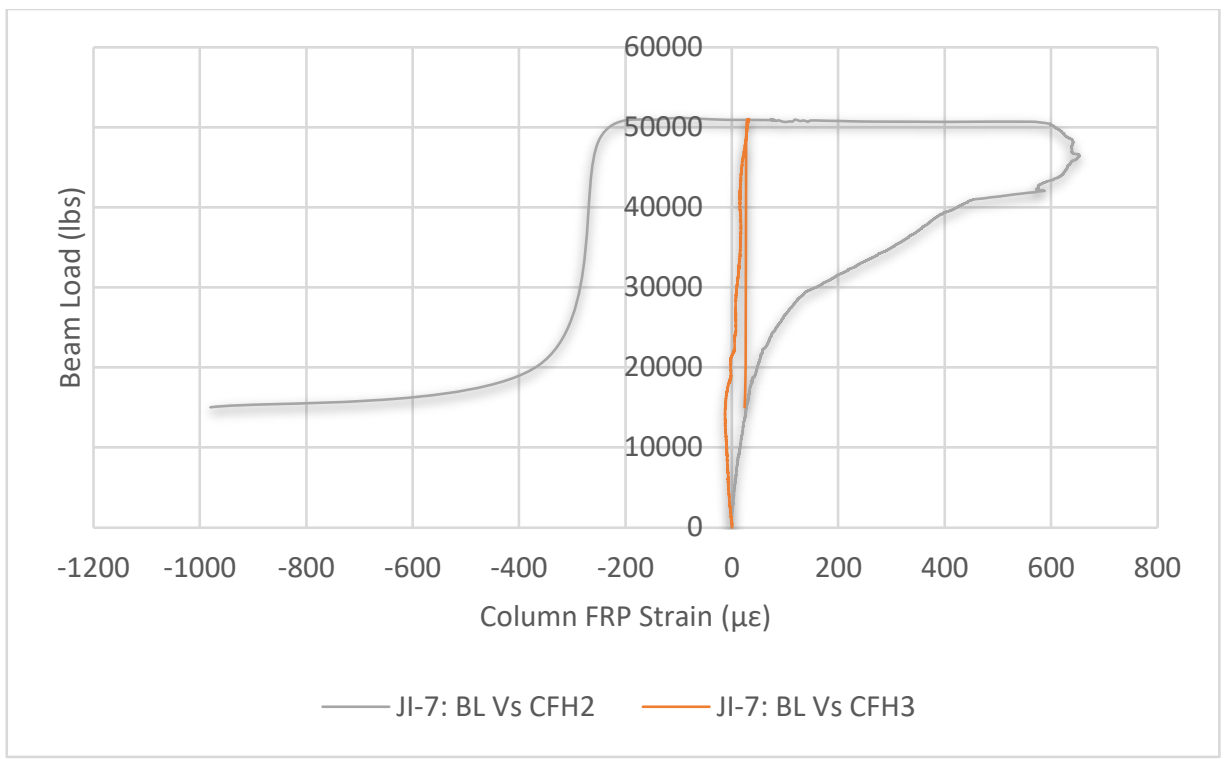

Figure B.35. Beam load vs. column FRP strain 


\section{B.4 Specimen JI-8}

\section{B.4.1 Strain in steel rebar}

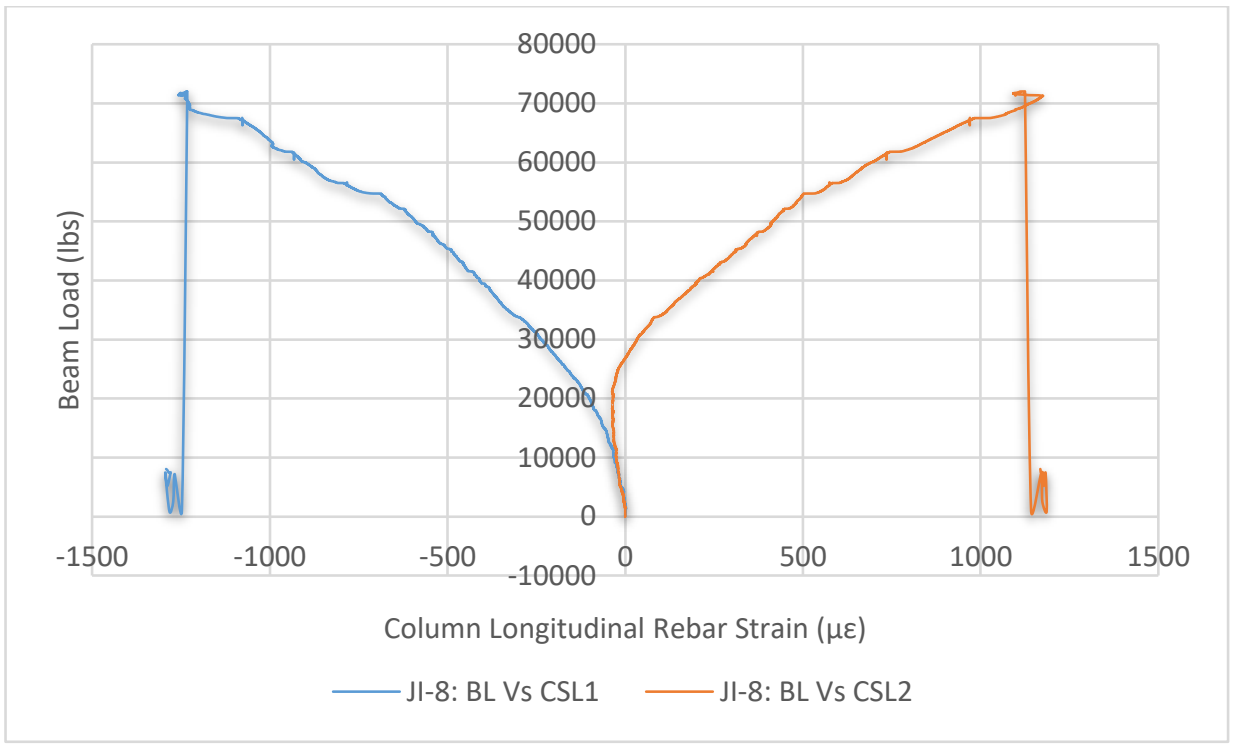

Figure B.36. Beam load vs. column longitudinal rebar strain

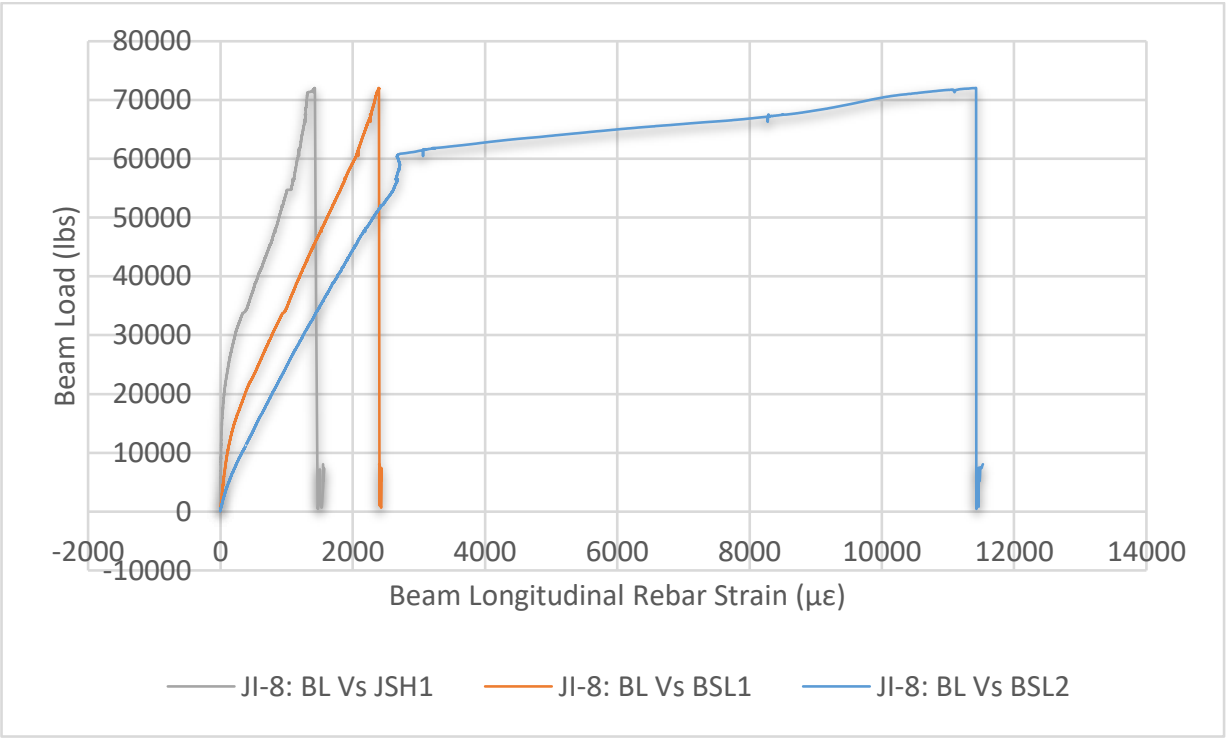

Figure B.37. Beam load vs. beam longitudinal rebar strain 


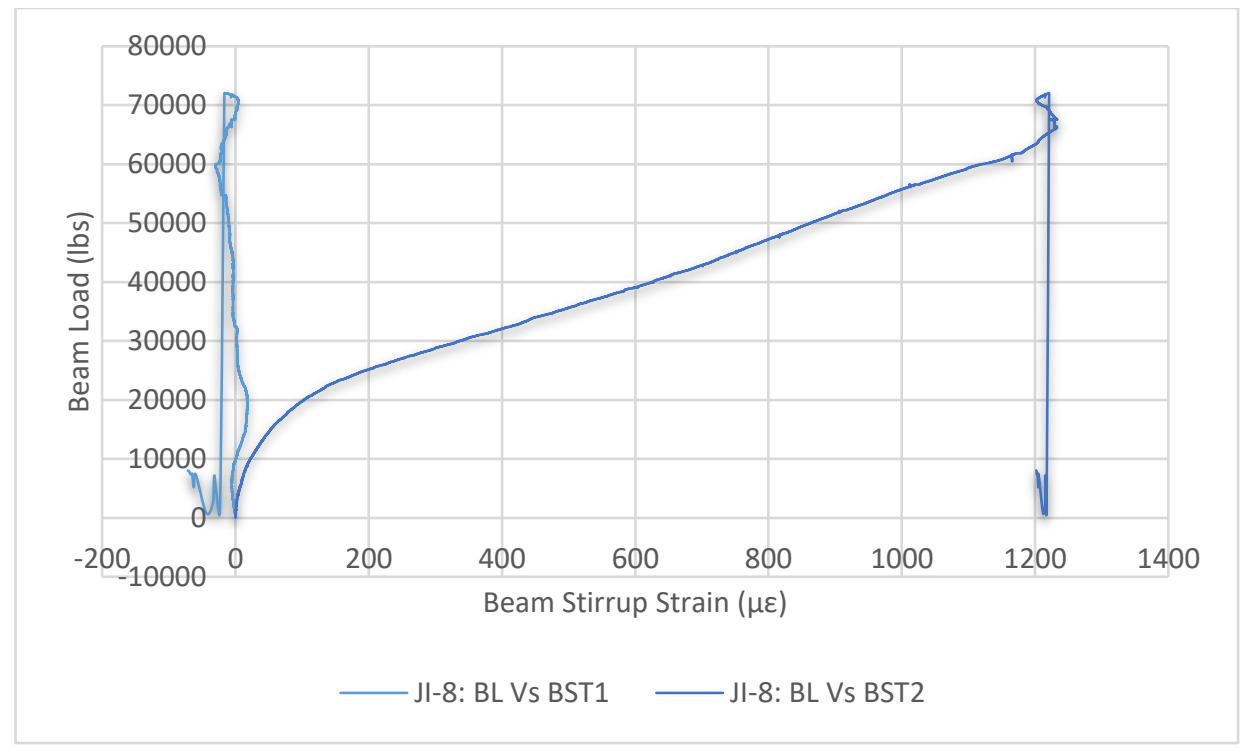

Figure B.38. Beam load vs. beam stirrup strain 


\section{B.4.2 Strain in FRP}

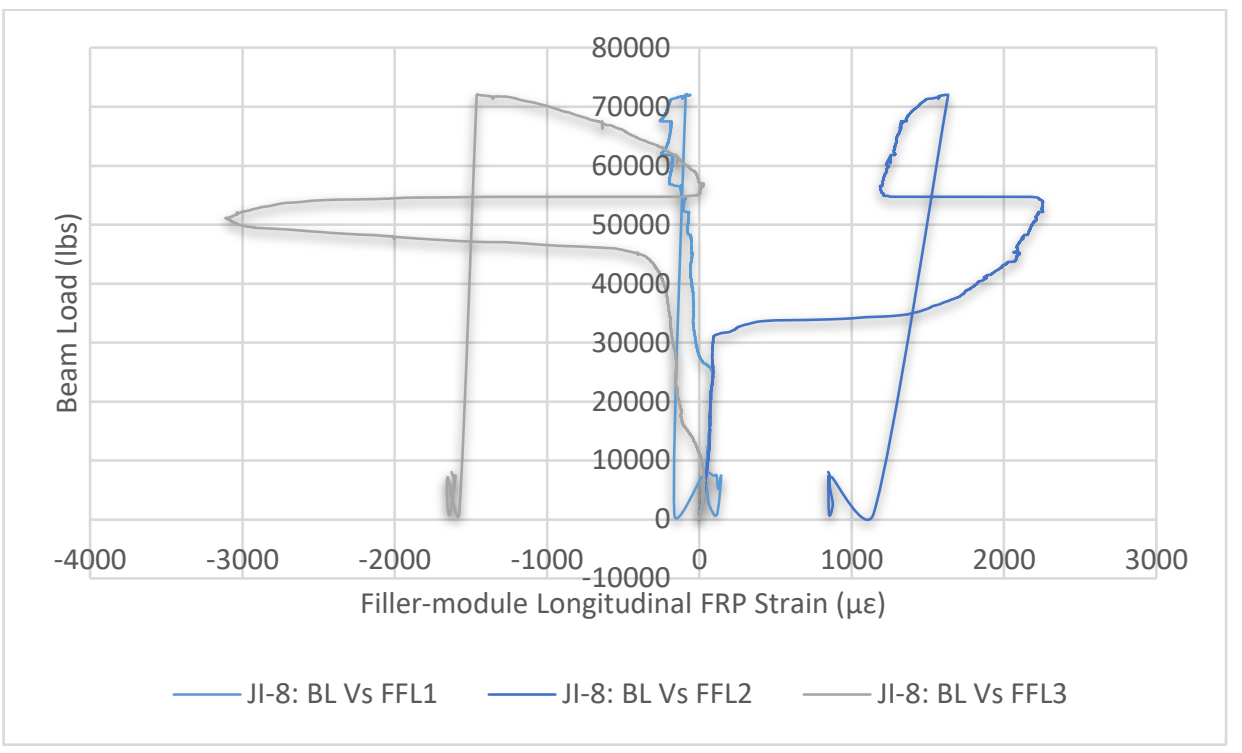

Figure B.39. Beam load vs. filler-module longitudinal strain

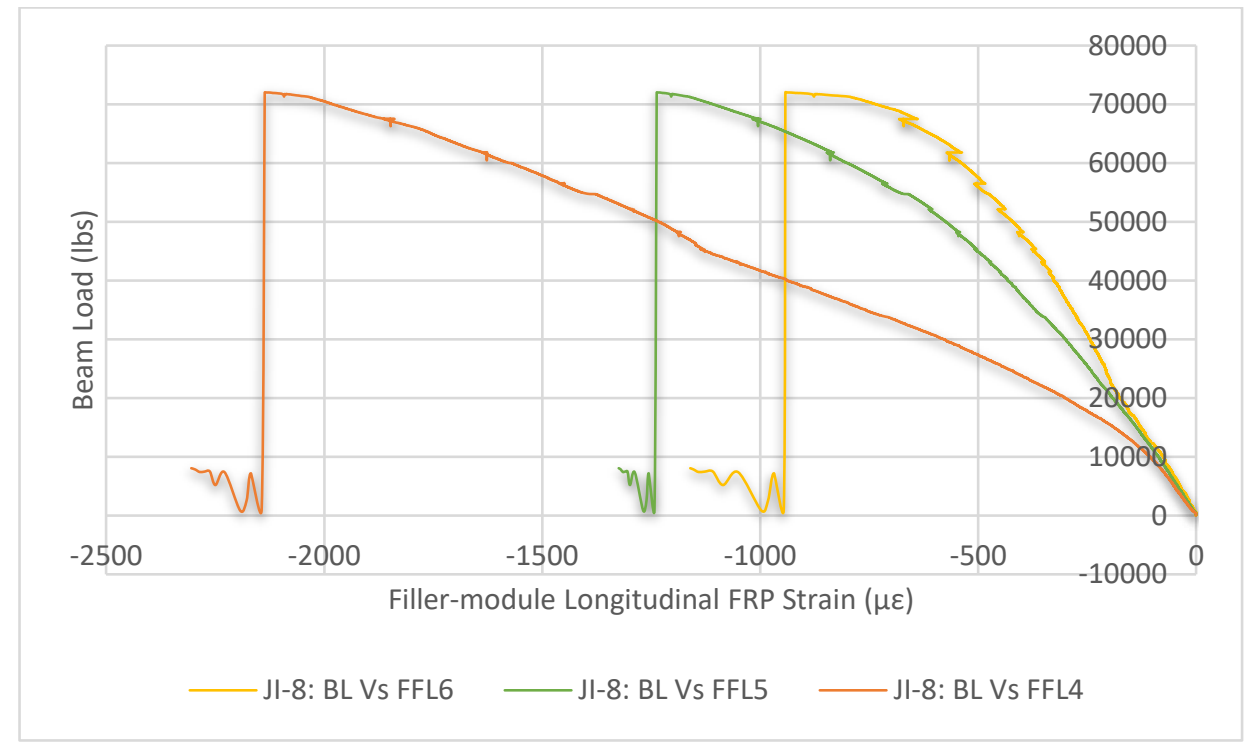

Figure B.40. Beam load vs. filler-module longitudinal strain 


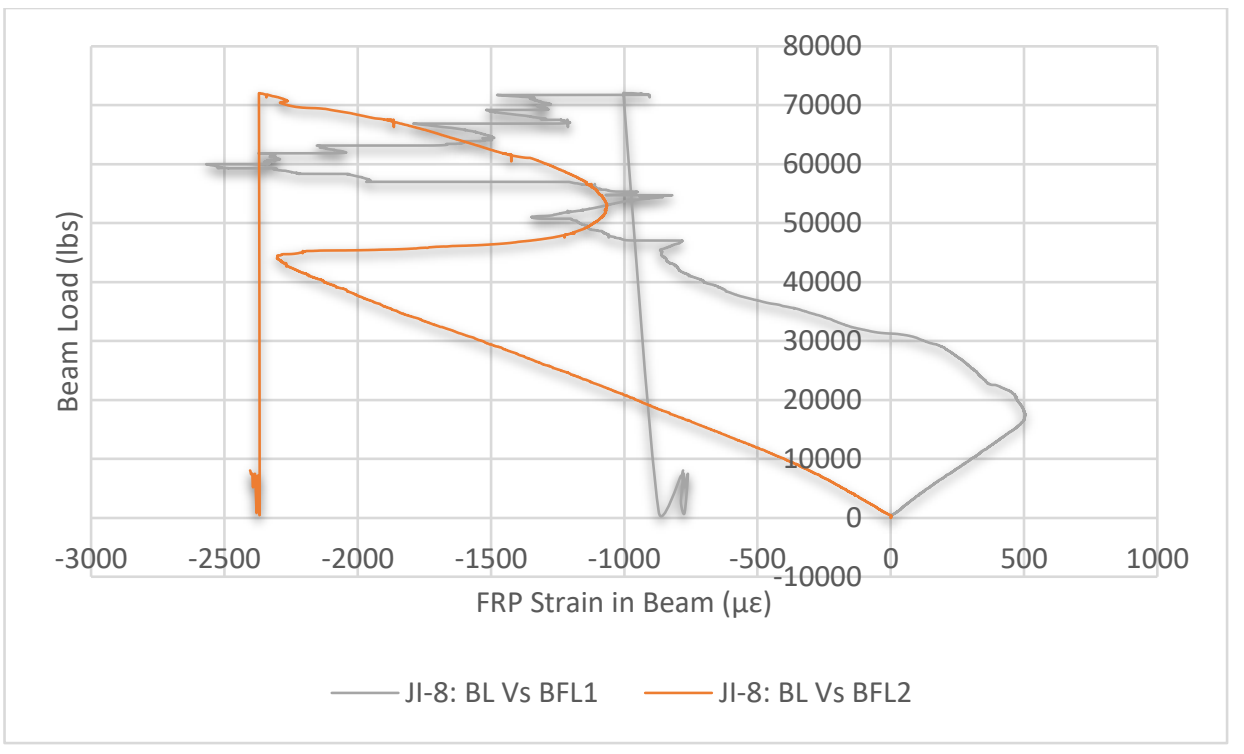

Figure B.41. Beam load vs. FRP strain in beam

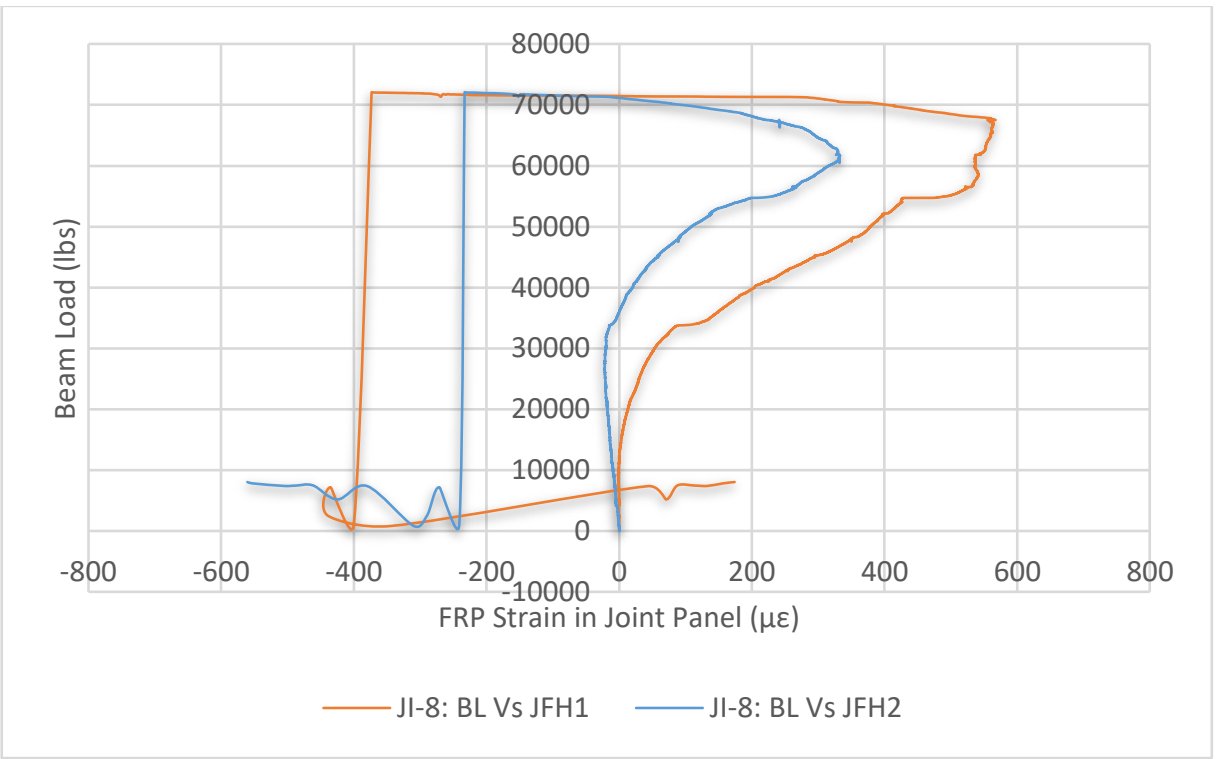

Figure B.42. Beam load vs. FRP strain in joint panel 


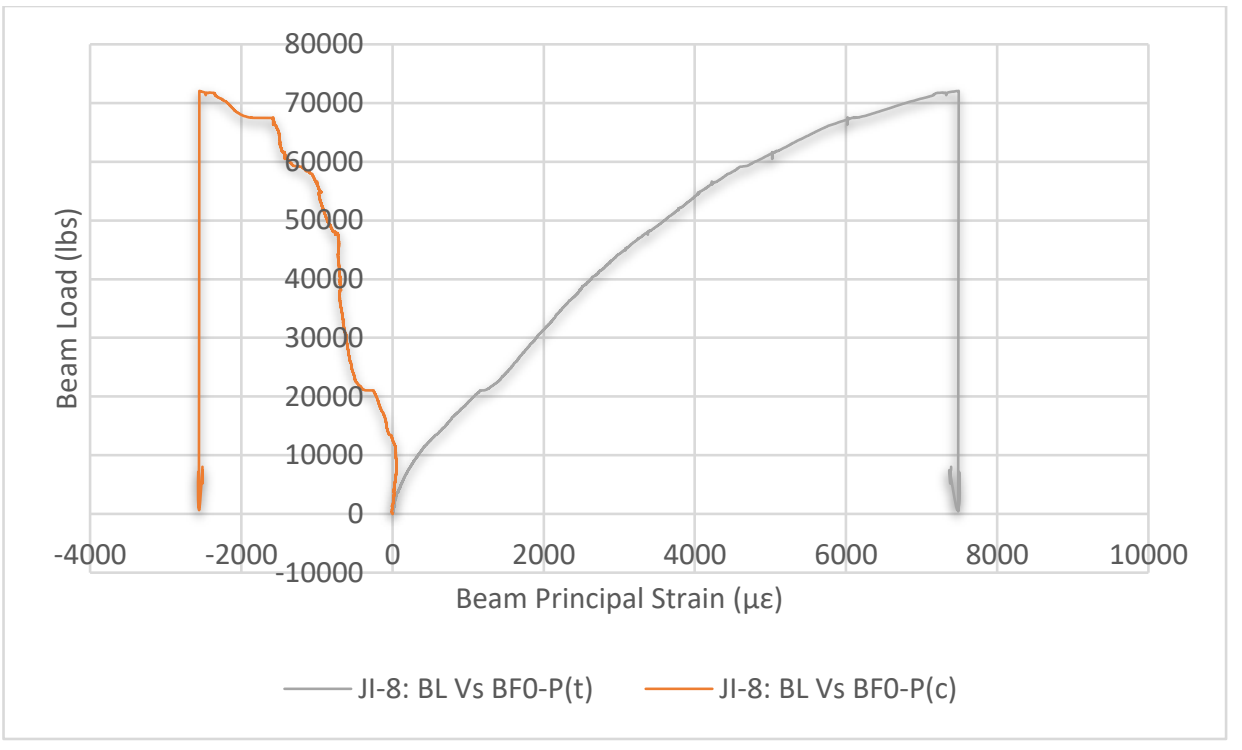

Figure B.43. Beam load vs. beam principal strain

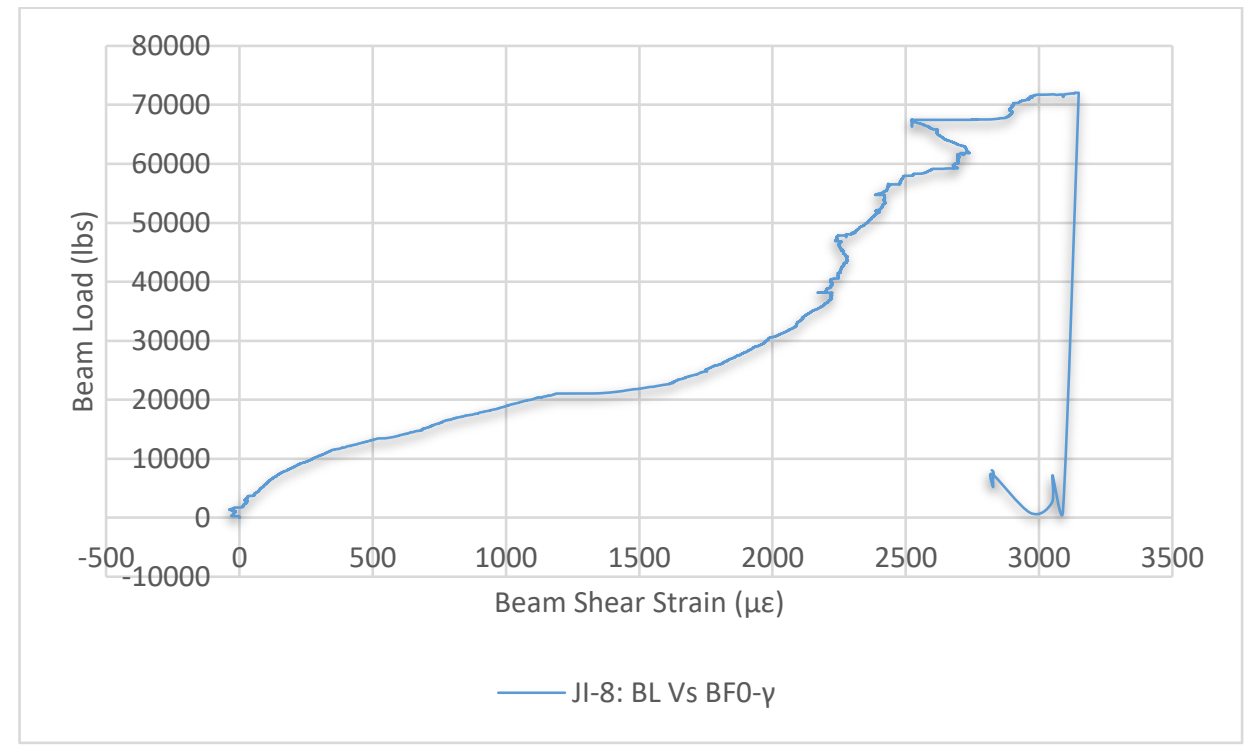

Figure B.44. Beam load vs. beam shear strain 


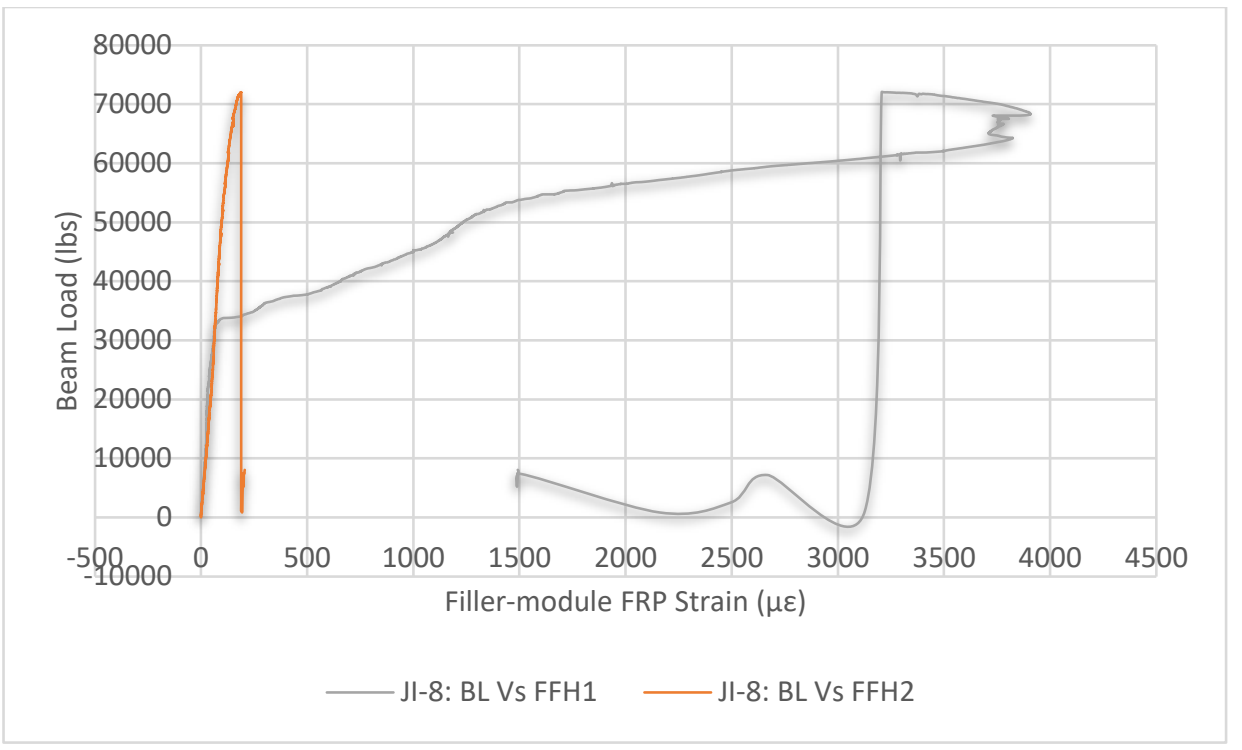

Figure B.45. Beam load vs. filler-module FRP strain

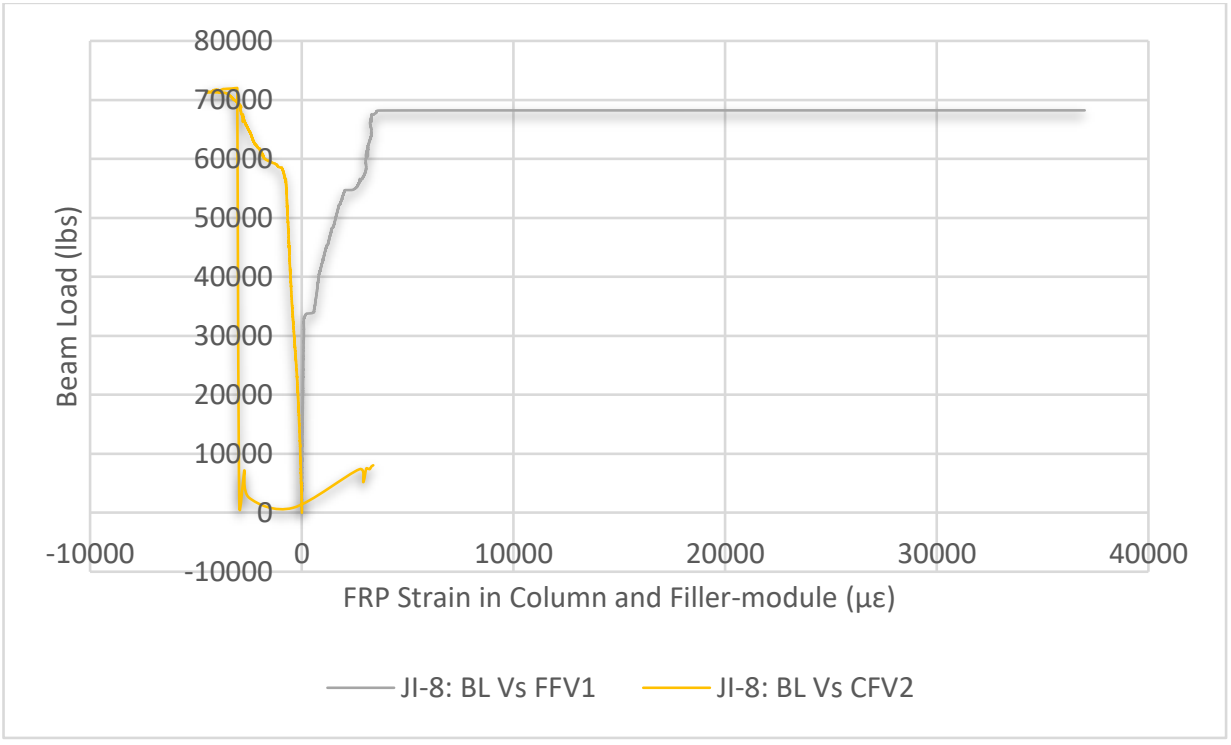

Figure B.46. Beam load vs. FRP strain in column and filler-module 


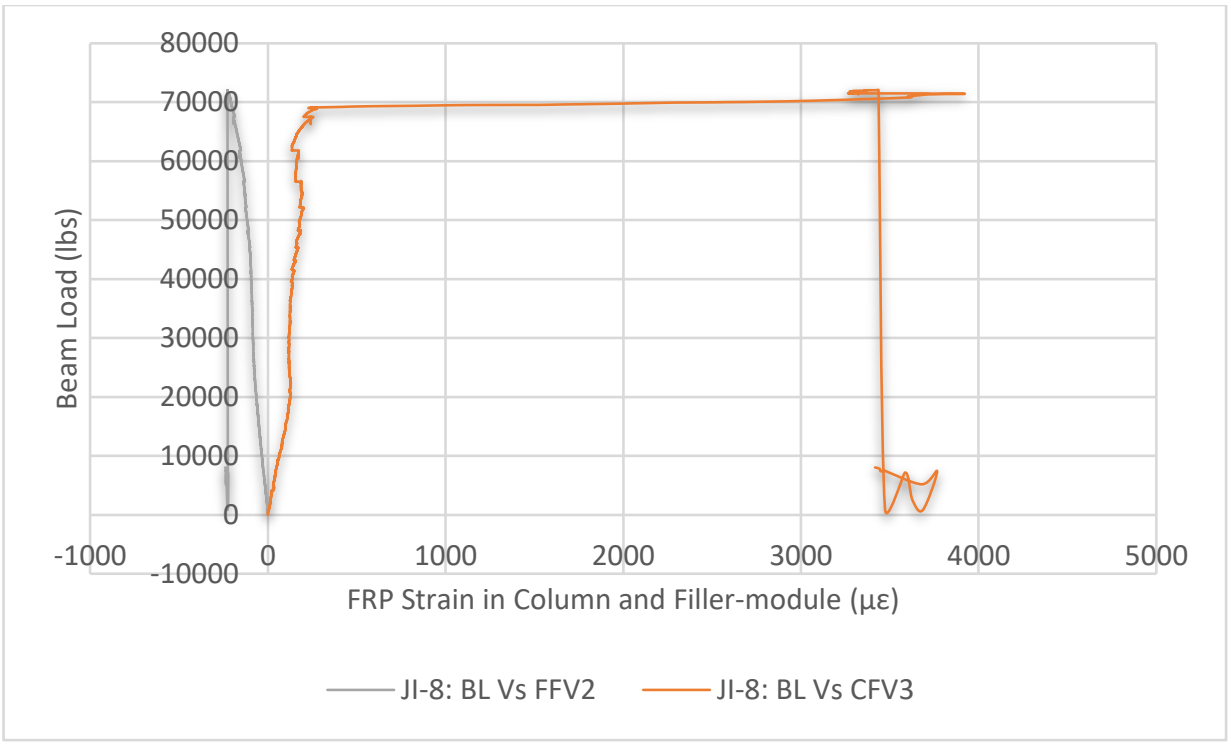

Figure B.47. Beam load vs. FRP strain in column and filler-module

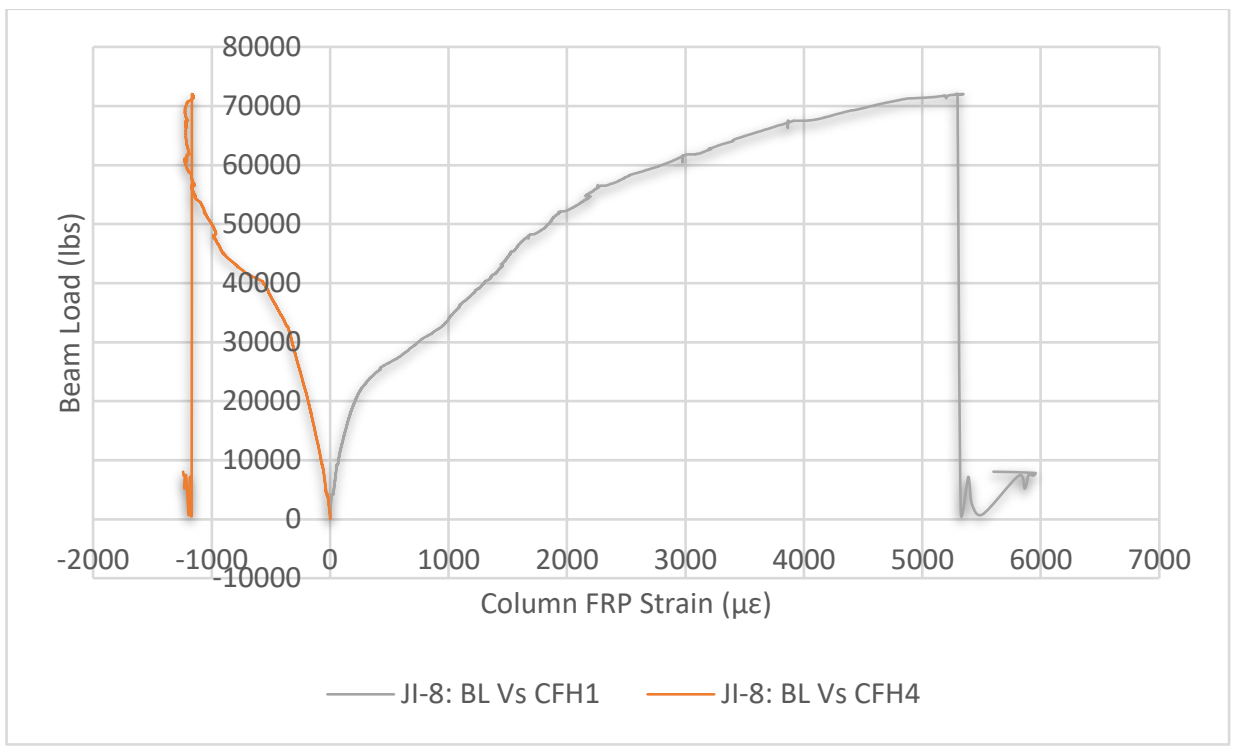

Figure B.48. Beam load vs. column FRP strain 


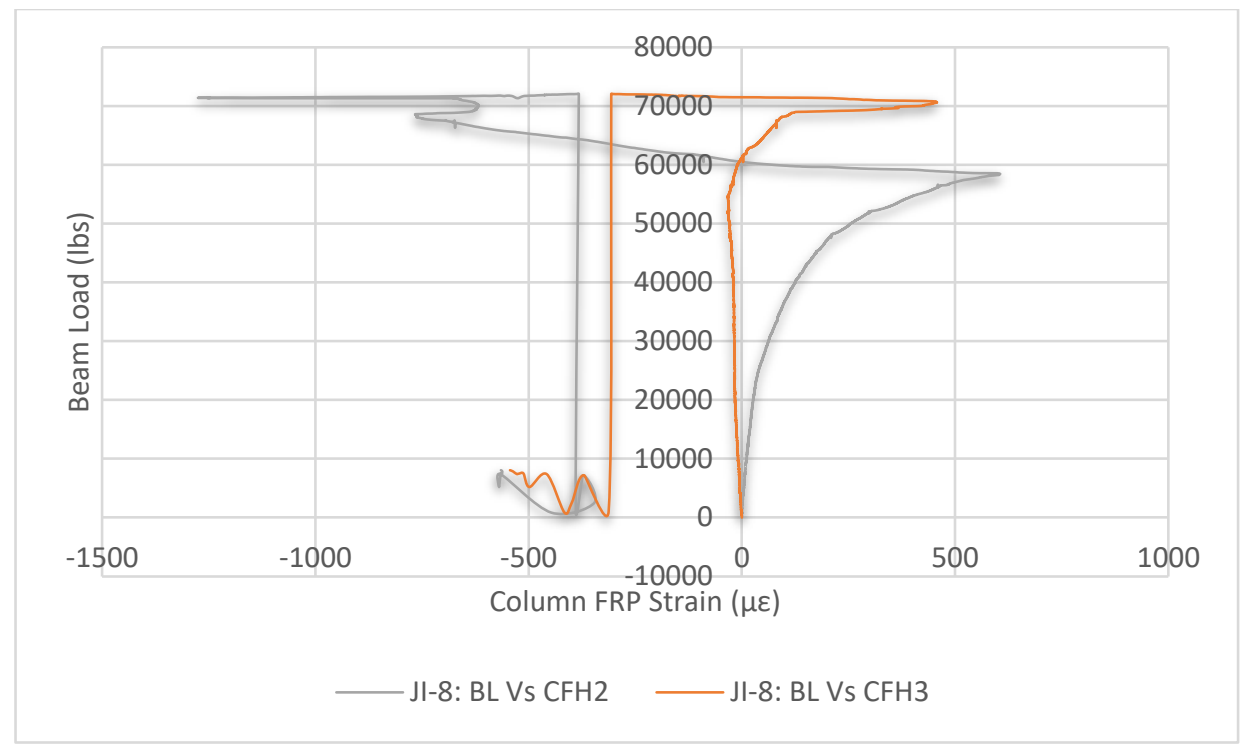

Figure B.49. Beam load vs. column FRP strain 


\section{Appendix-C: Beam-Column Joints Reinforced with Concrete Filler-Modules and GFRP Wraps}

This section provides additional test data generated through experimental evaluation of beamcolumn joints reinforced with concrete filler-modules with GFRP wraps under monotonic loads.

\section{C.1 Specimen JIII-1}

C.1.1 Strain in steel rebar

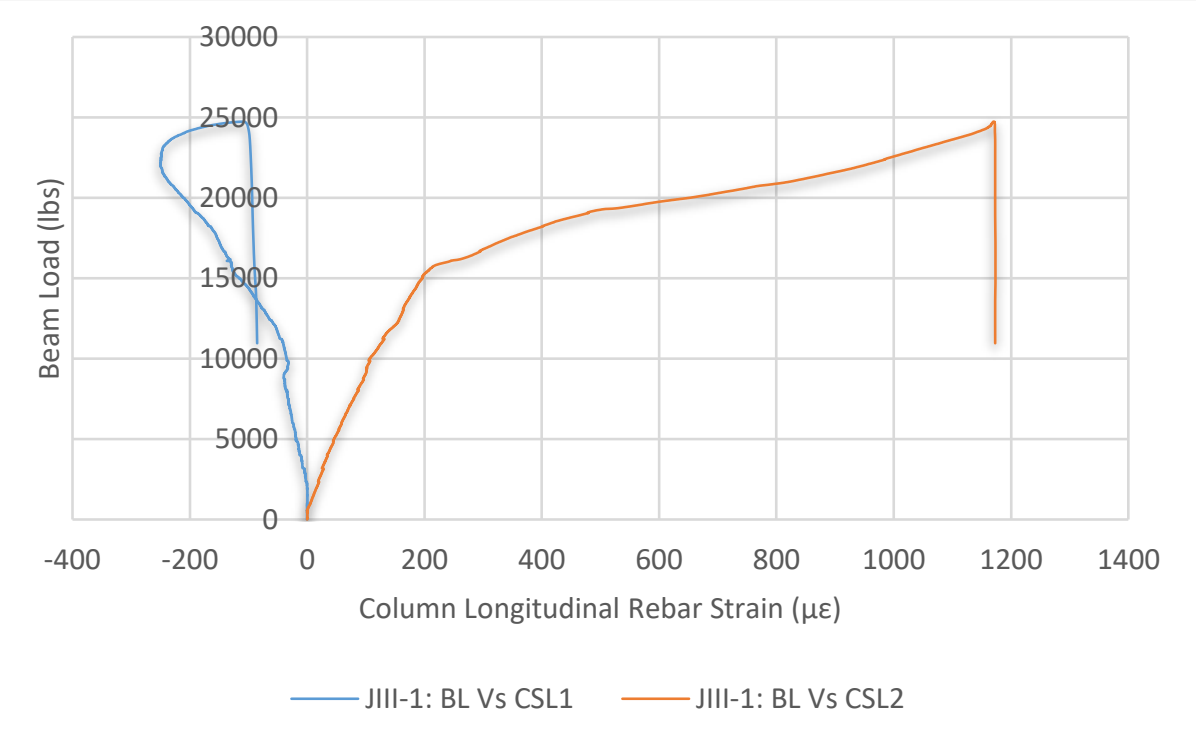

Figure C.1. Beam load vs. column longitudinal rebar strain 


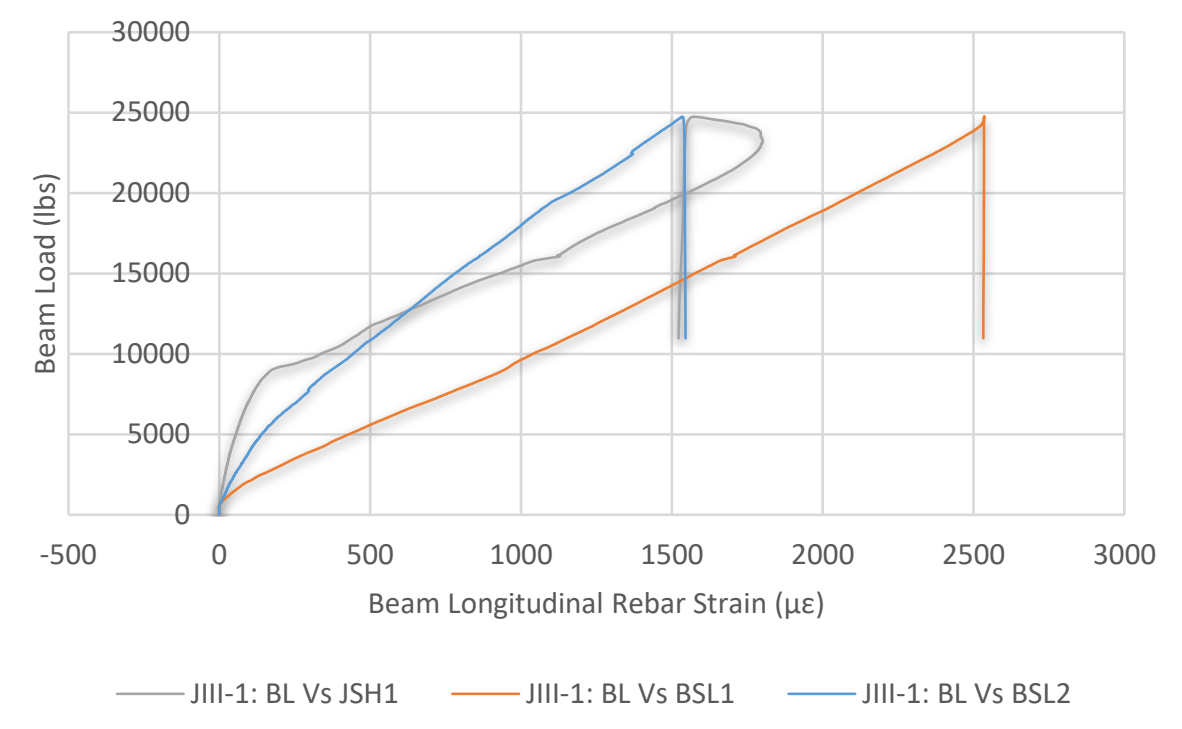

Figure C.2. Beam load vs. beam longitudinal rebar strain

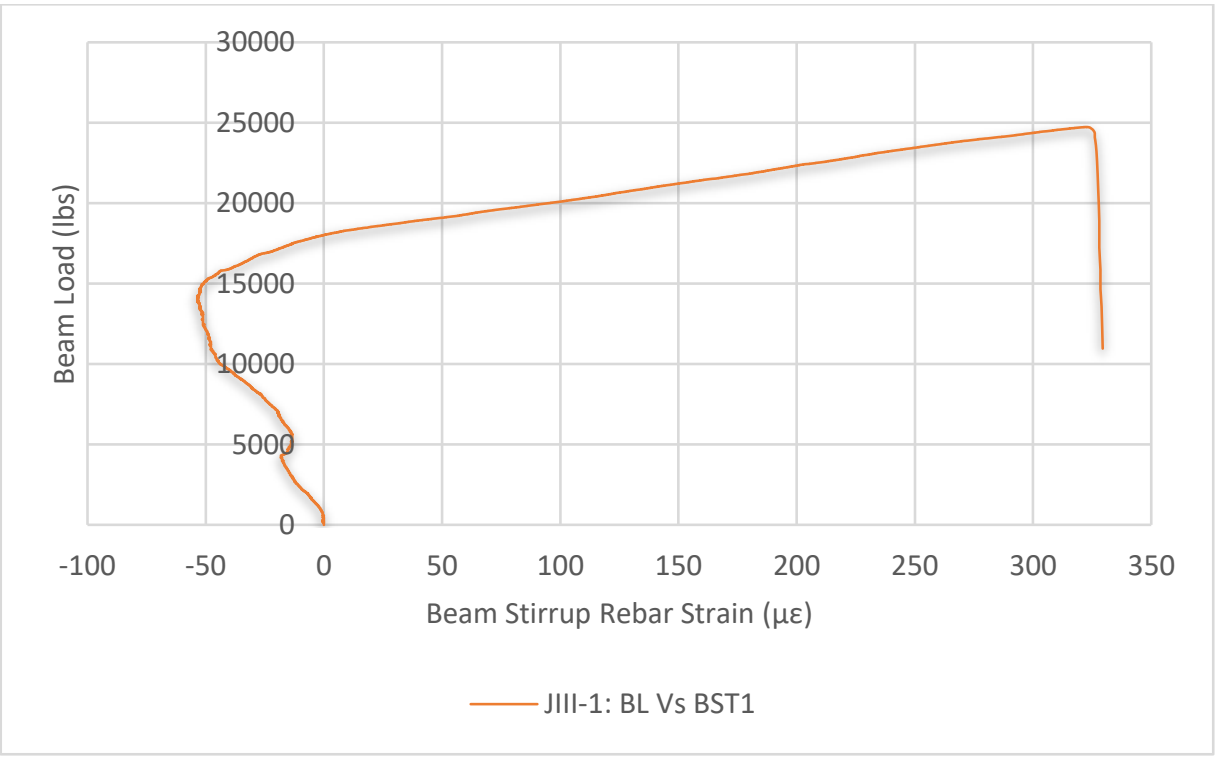

Figure C.3. Beam load vs. beam stirrup strain 


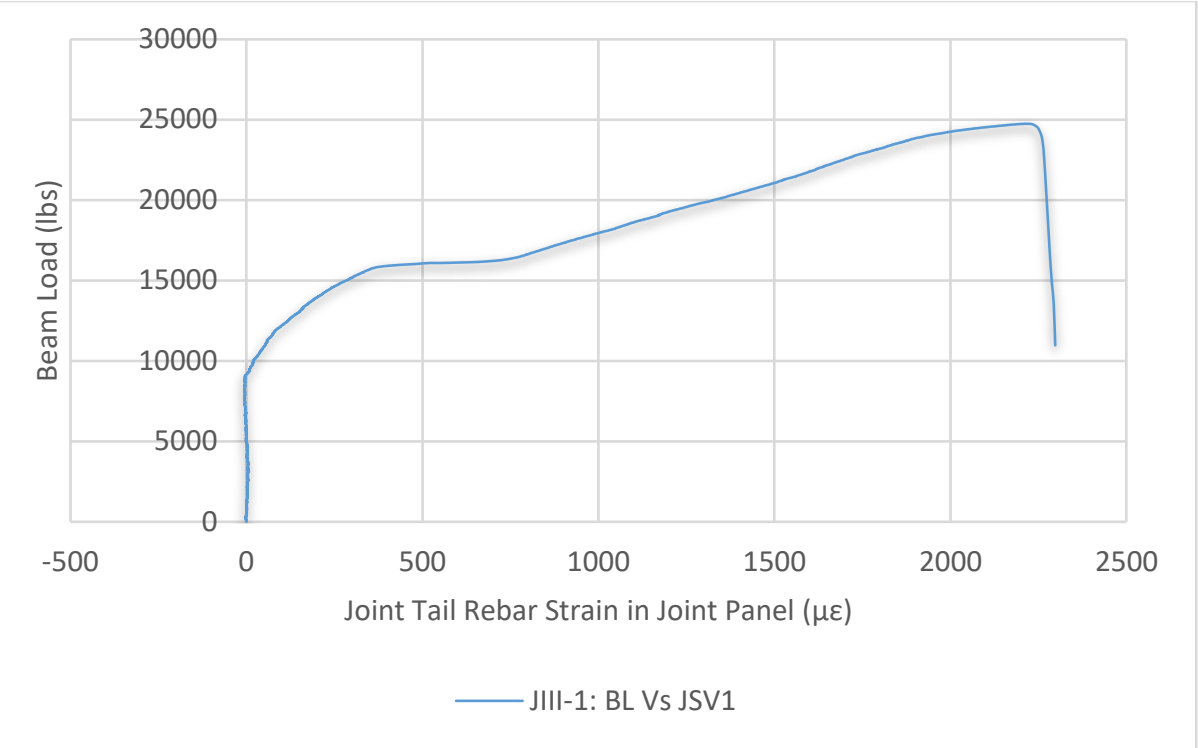

Figure C.4. Beam load vs. joint tail rebar strain 


\section{C.1.2 Strain in concrete}

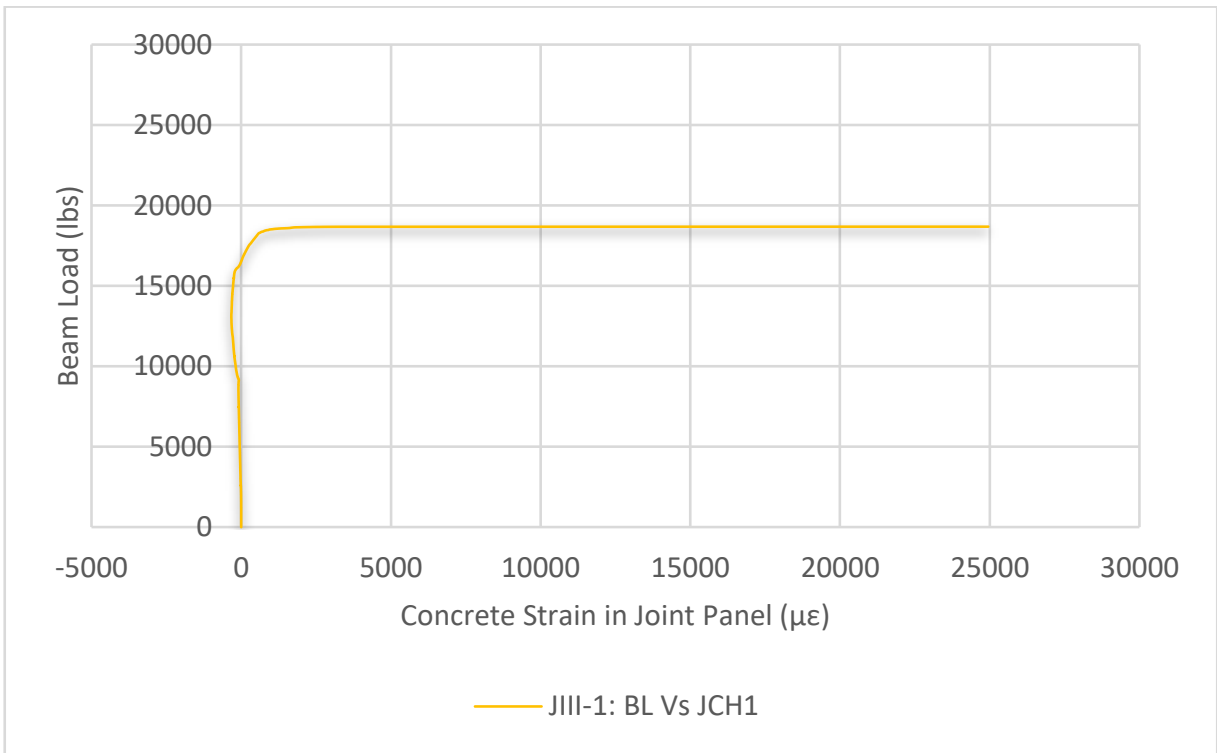

Figure C.5. Beam load vs. concrete strain in the joint panel

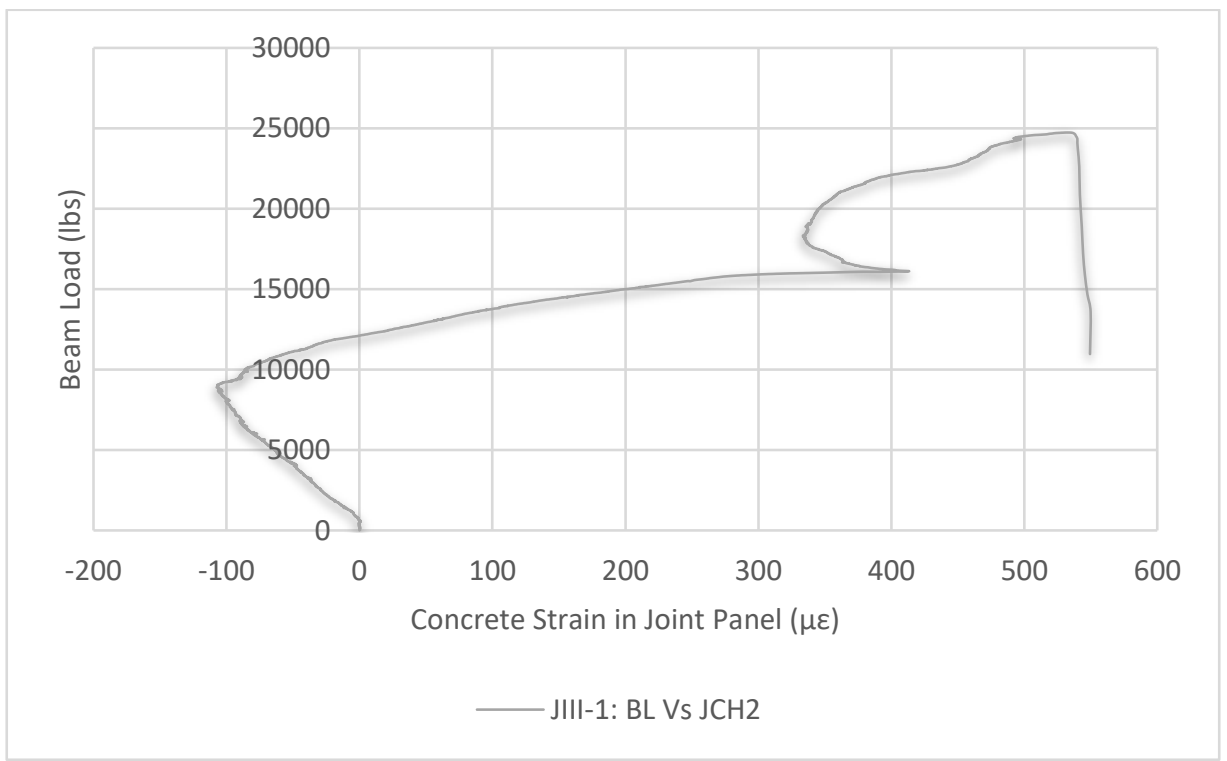

Figure C.6. Beam load vs. concrete strain in the joint panel 


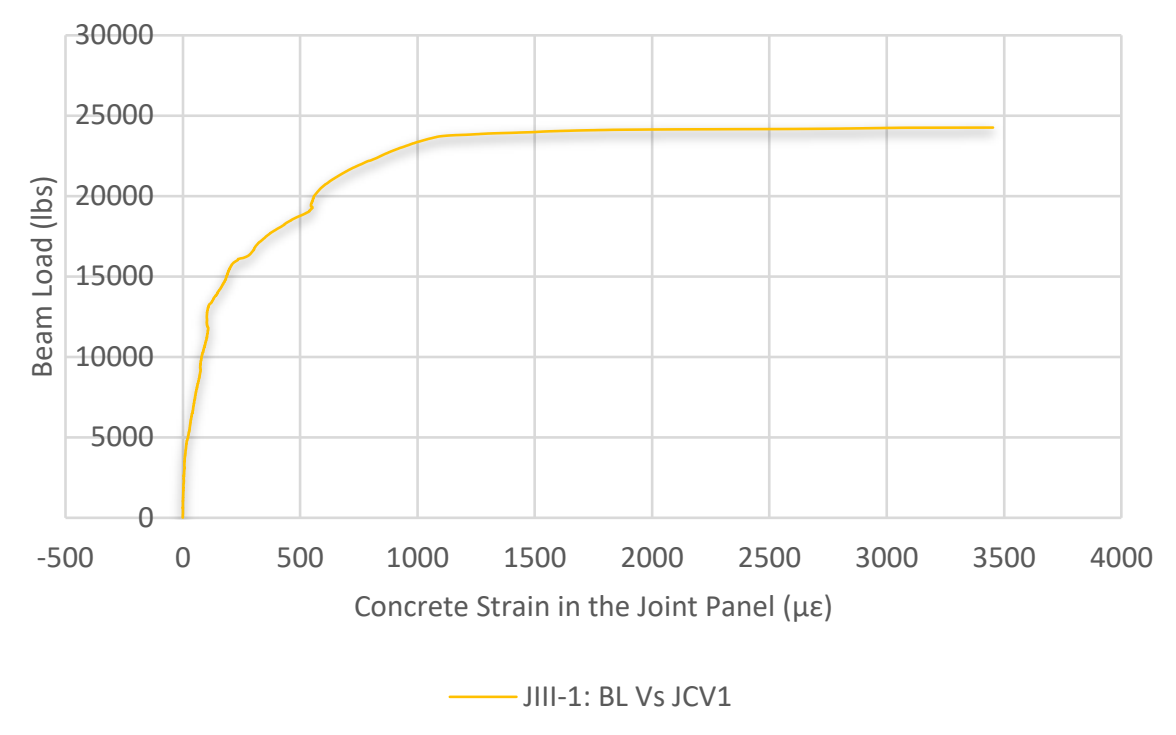

Figure C.7. Beam load vs. concrete strain in the joint panel

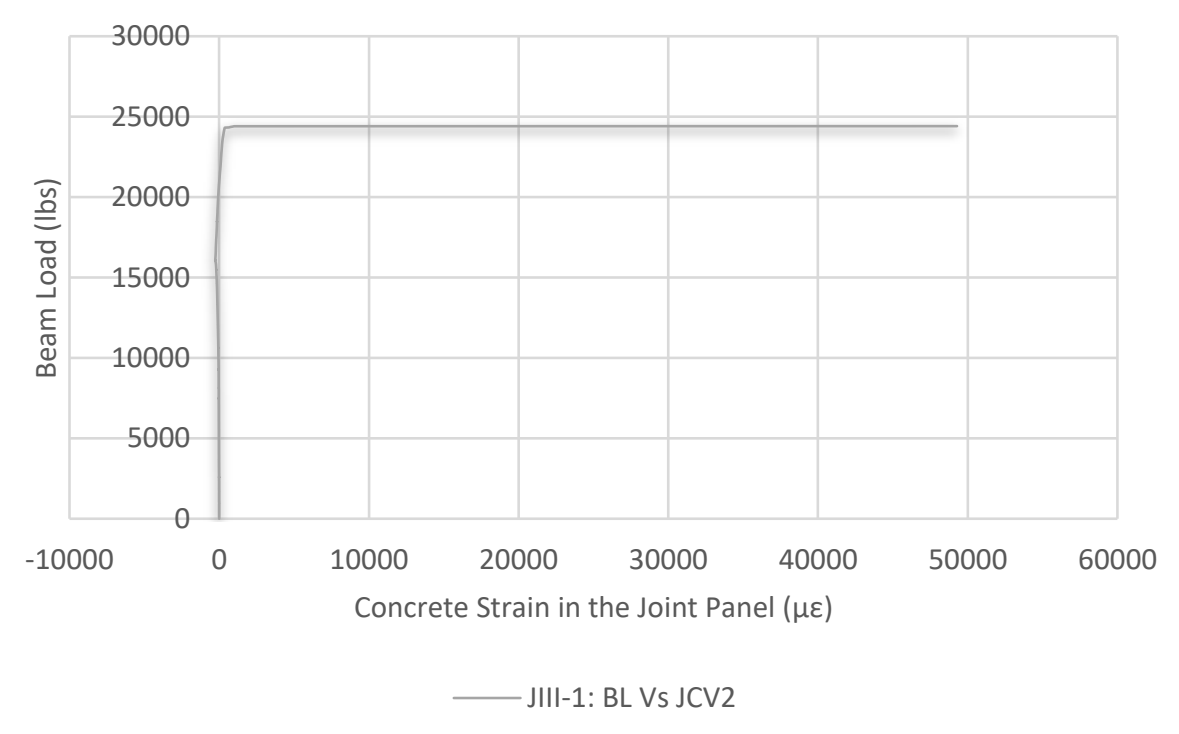

Figure C.8. Beam load vs. column strain in the joint panel 


\section{C.2 Specimen JIII-2}

\section{C.2.1 Strain in steel rebar}

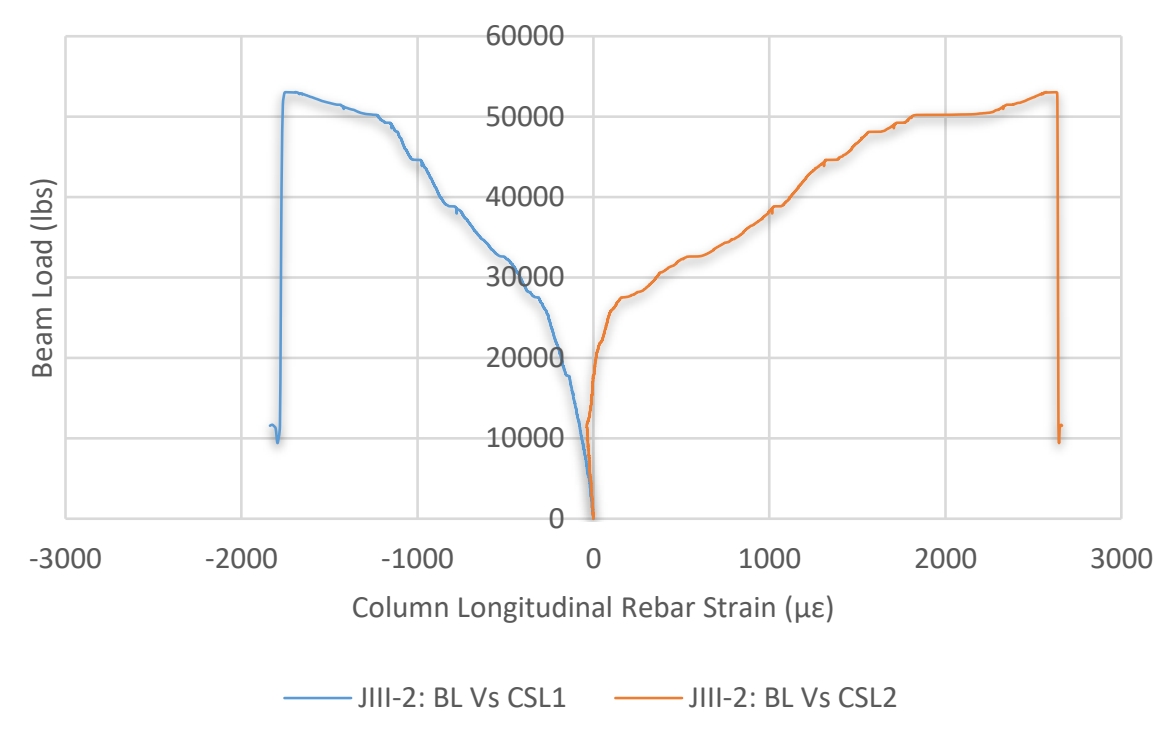

Figure C.9. Beam load vs. column longitudinal rebar strain

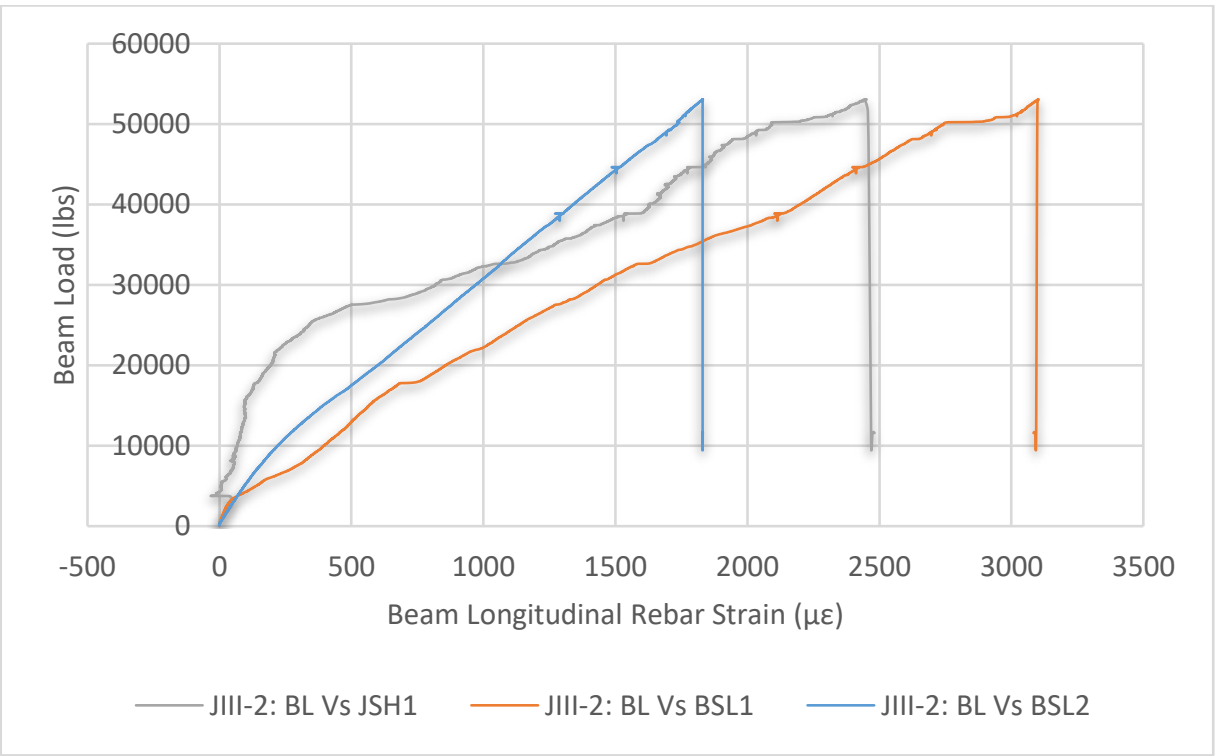

Figure C.10. Beam load vs. beam longitudinal rebar strain 


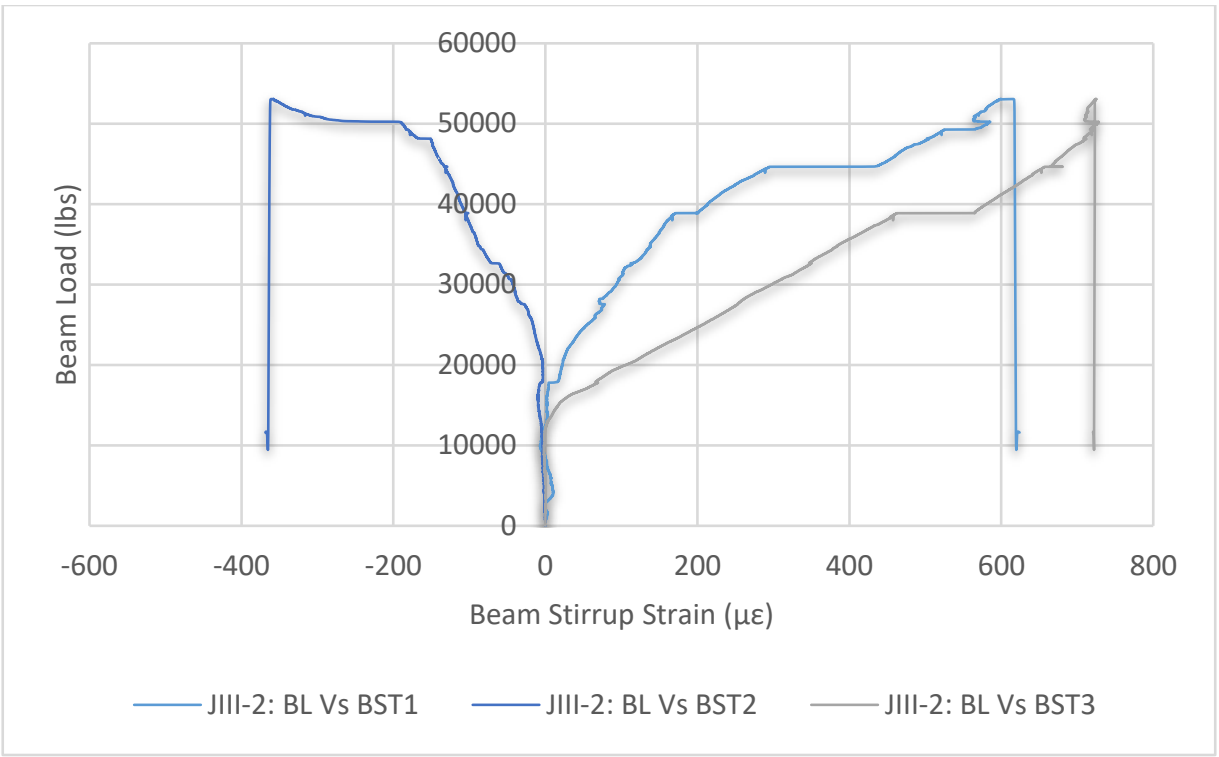

Figure C.11. Beam load vs. beam stirrup strain 


\section{C.2.2 Strain in FRP}

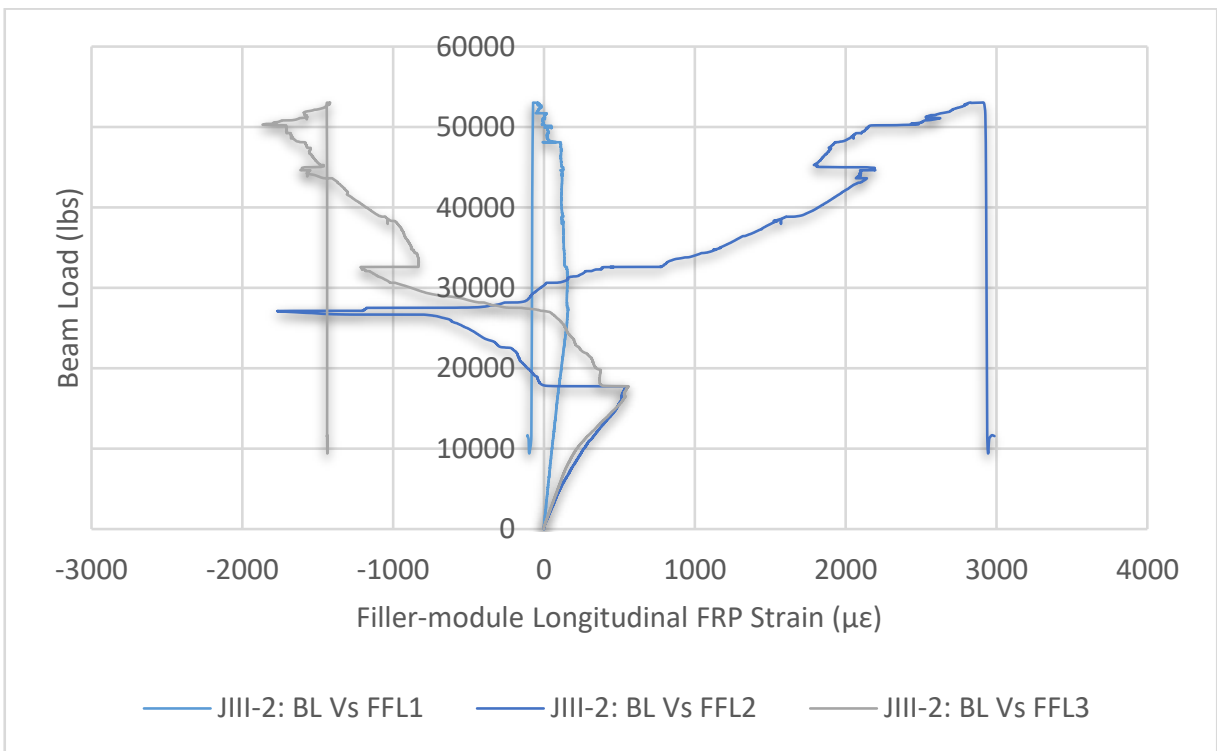

Figure C.12. Beam load vs. filler-module longitudinal strain

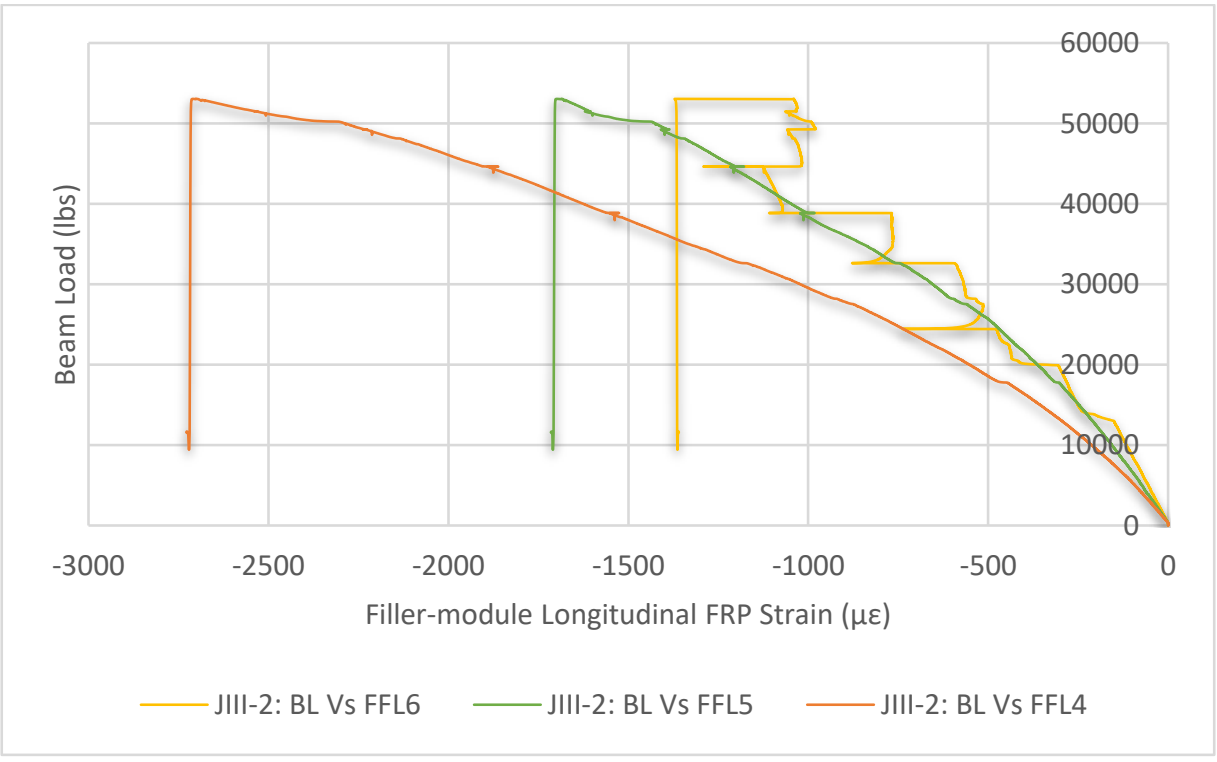

Figure C.13. Beam load vs. filler-module longitudinal strain 


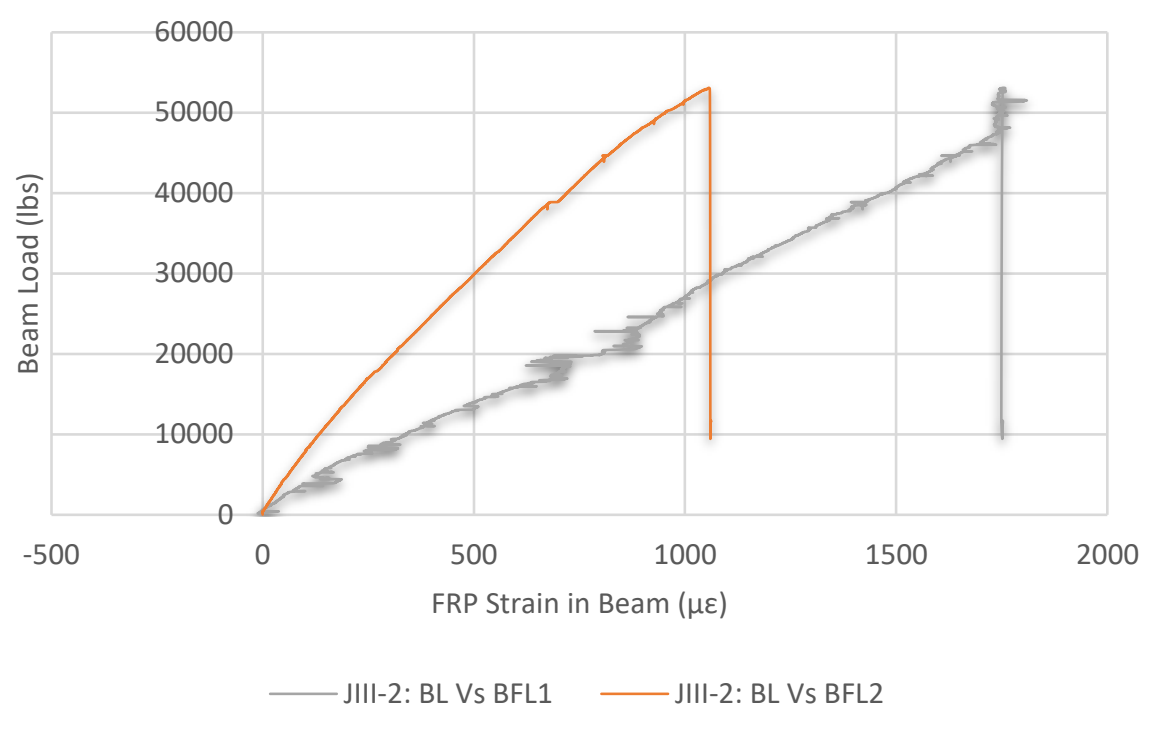

Figure C.14. Beam load vs. FRP strain in the beam

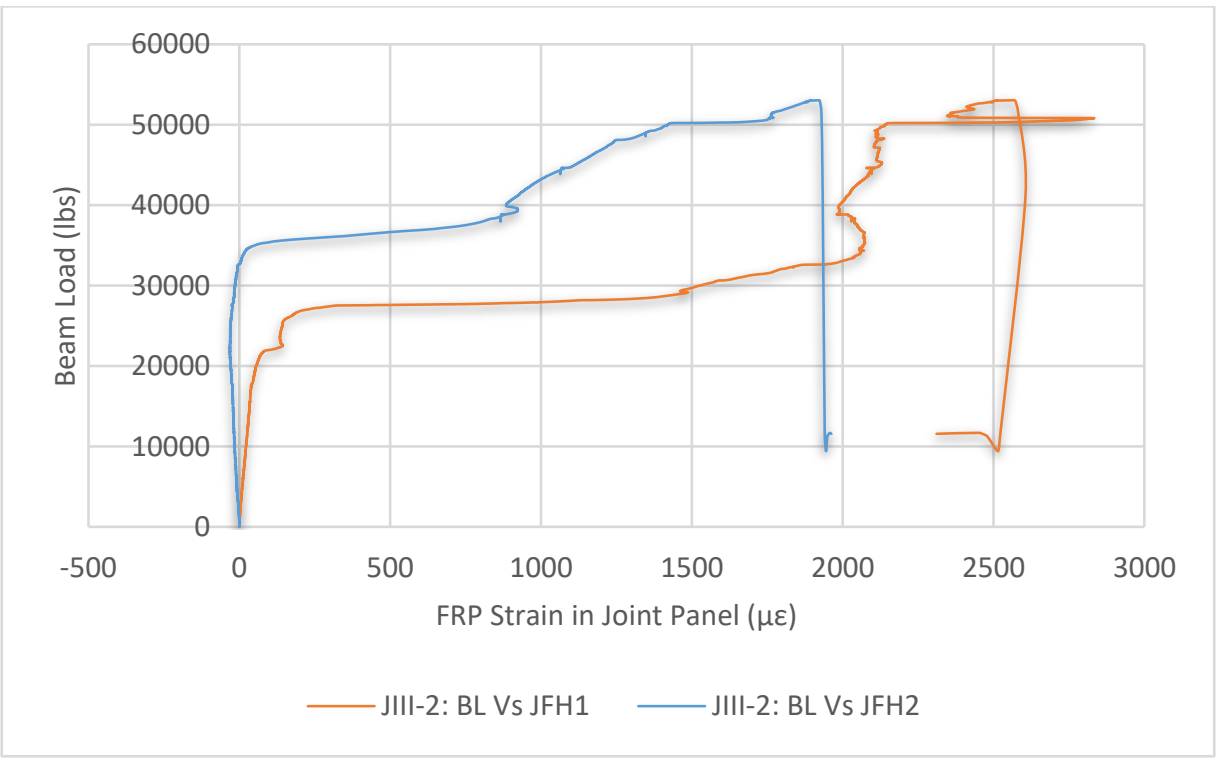

Figure C.15. Beam load vs. FRP strain in the joint panel 


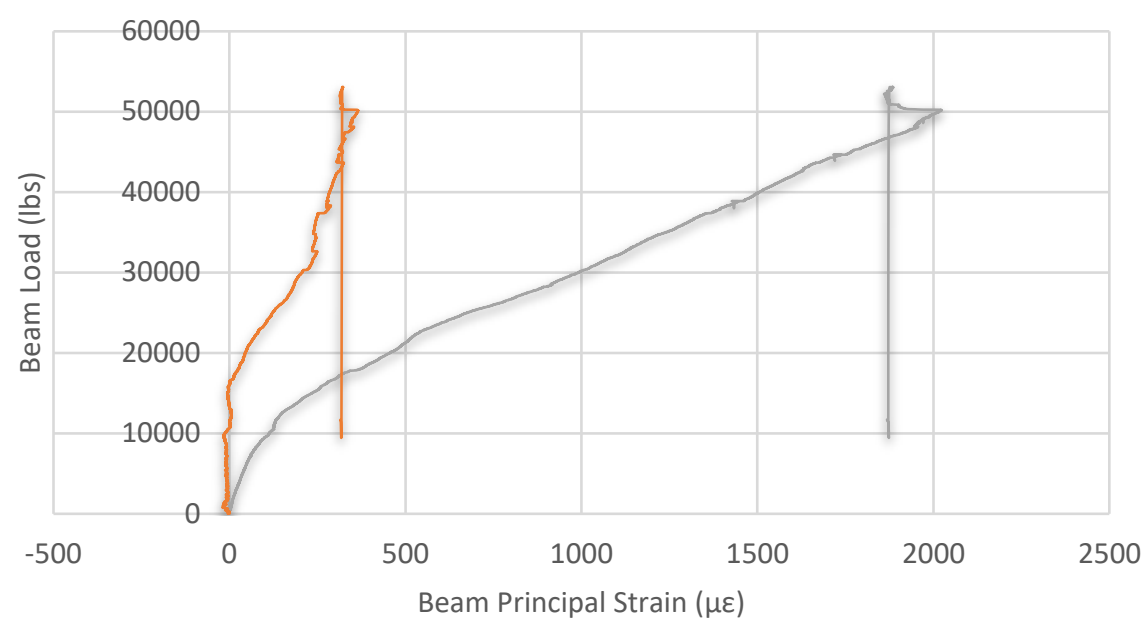

JIII-2: BL Vs BFO-P(t) _ JIII-2: BL Vs BFO-P(c)

Figure C.16. Beam load vs. beam principal strain

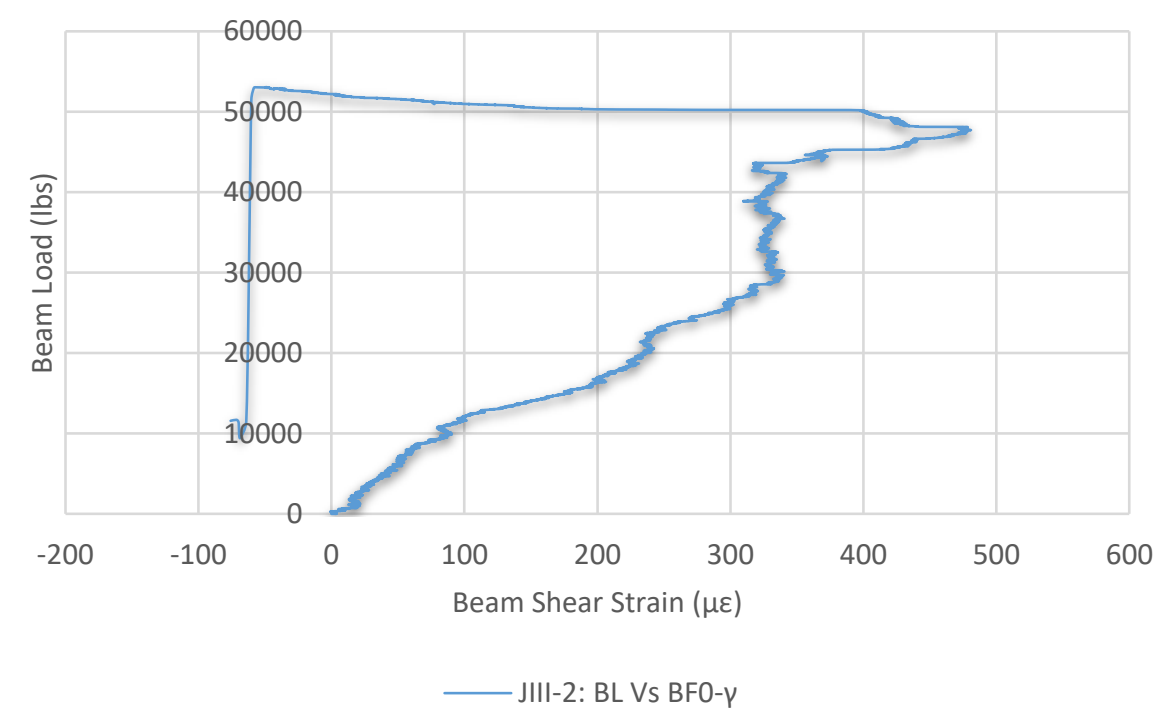

Figure C.17. Beam load vs. beam shear strain 


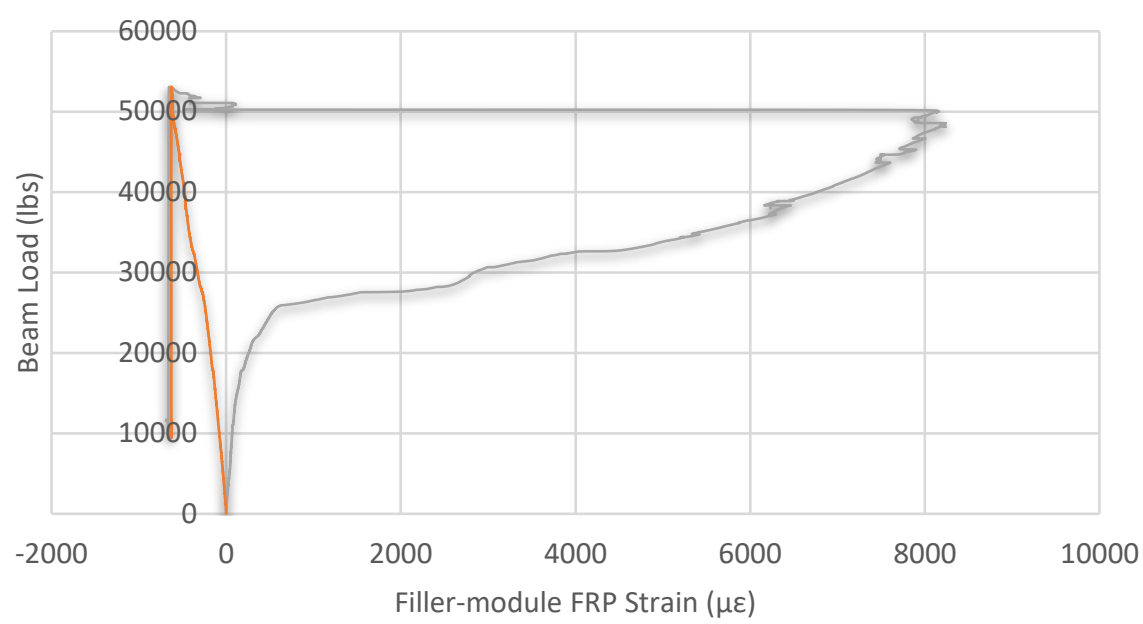

JIII-2: BL Vs FFH1 — JIII-2: BL Vs FFH2

Figure C.18. Beam load vs. filler-module FRP strain

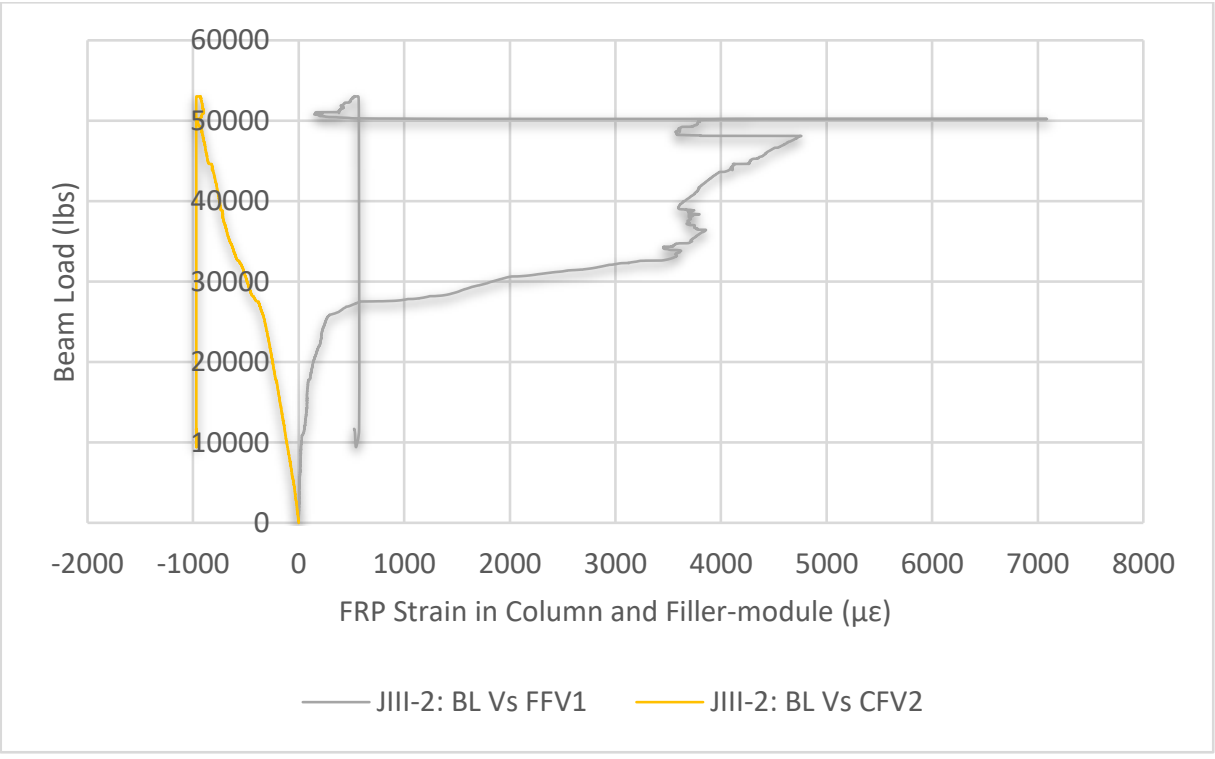

Figure C.19. Beam load vs. FRP strain in column and filler-module 


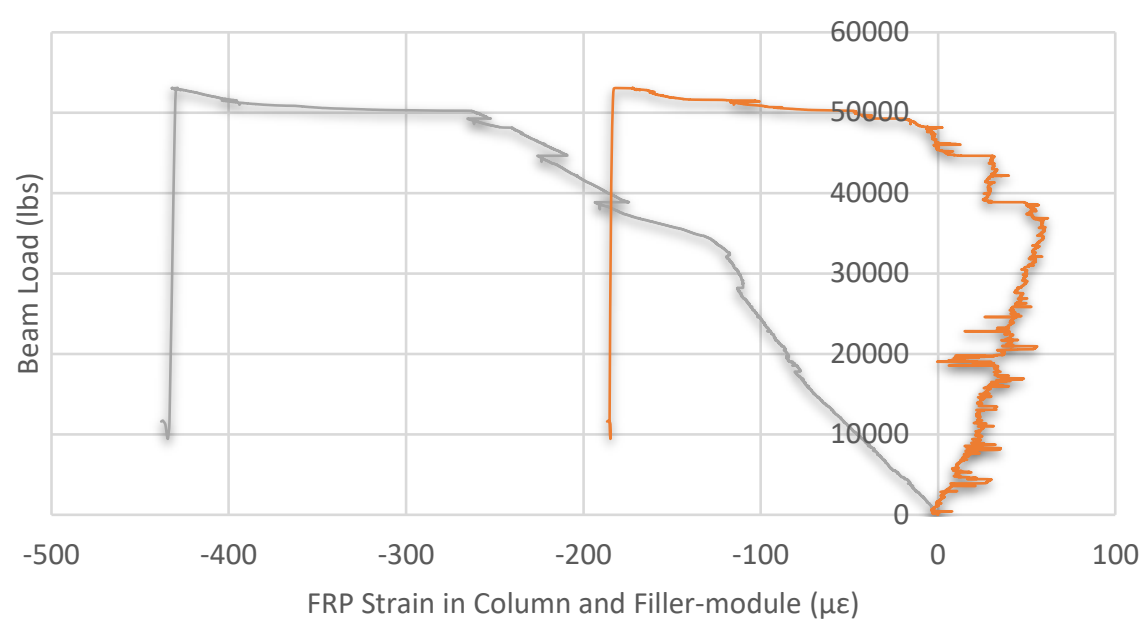

JIII-2: BL Vs FFV2 — JIII-2: BL Vs CFV3

Figure C.20. Beam load vs. FRP strain in column and filler-module

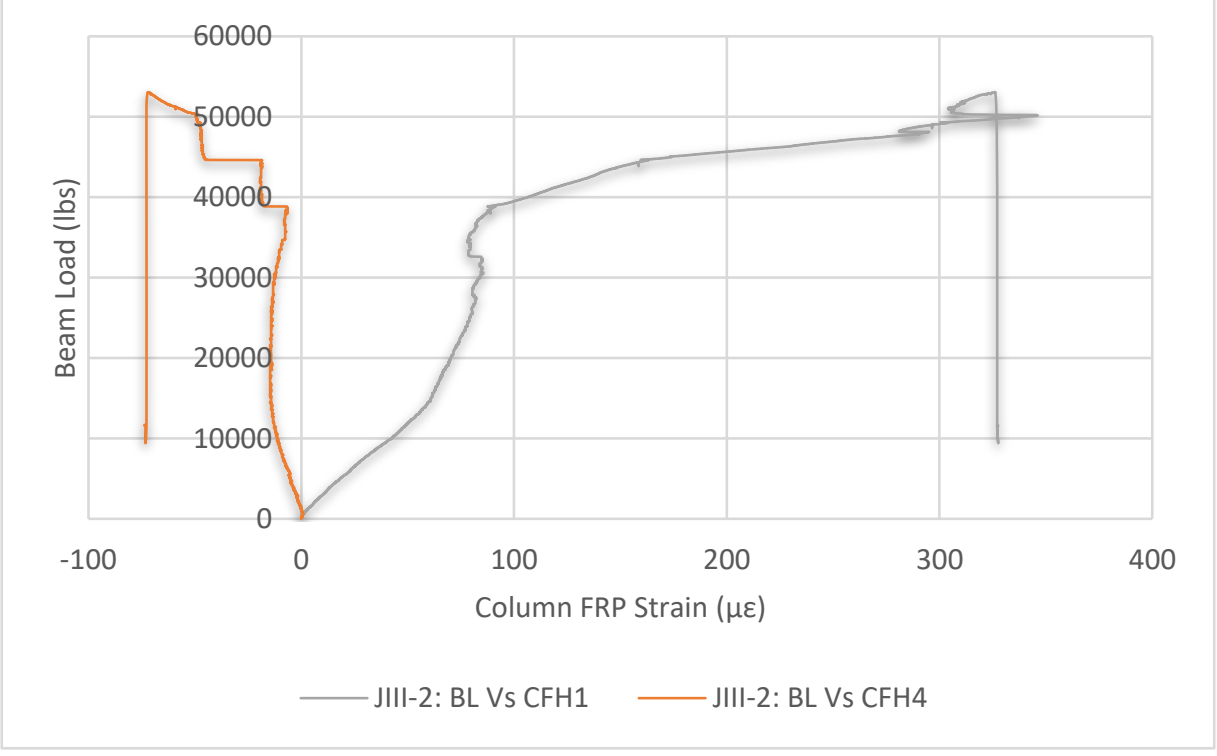

Figure C.21. Beam load vs. column FRP strain 


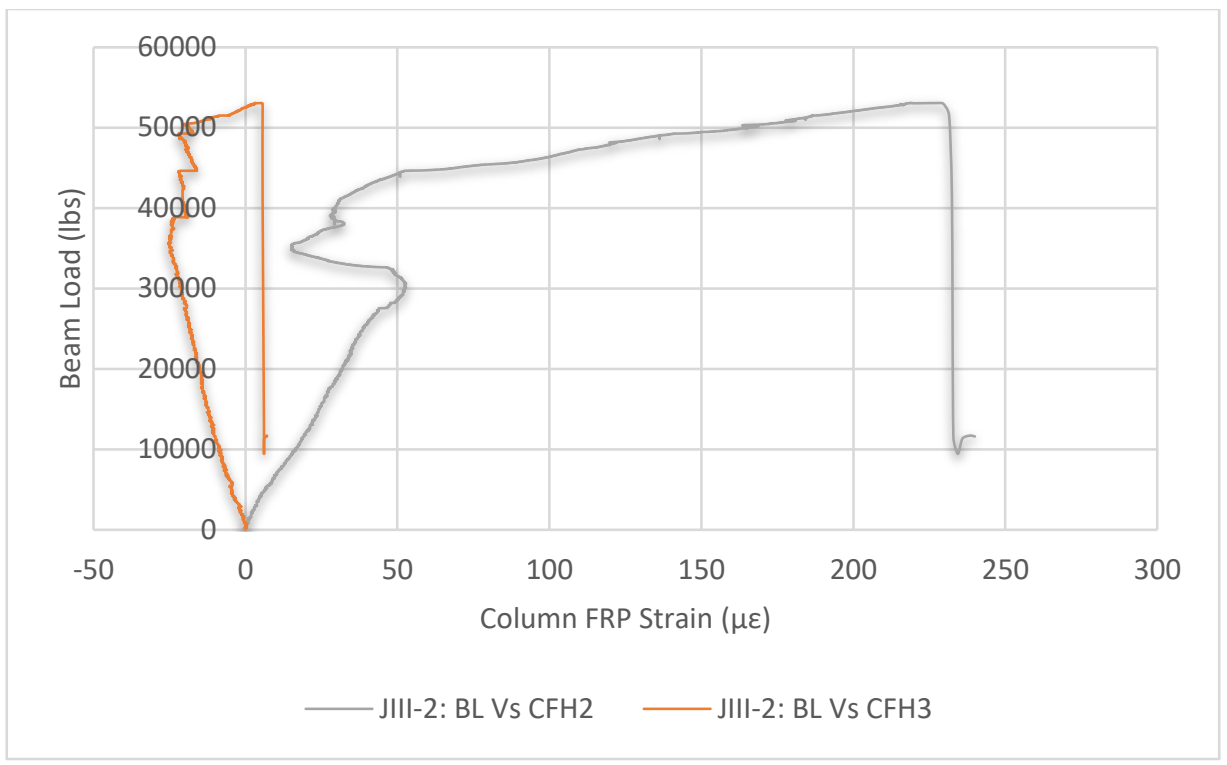

Figure C.22. Beam load vs. column FRP strain 


\section{C.3 Specimen JIII-3}

\section{C.3.1 Strain in steel rebar}

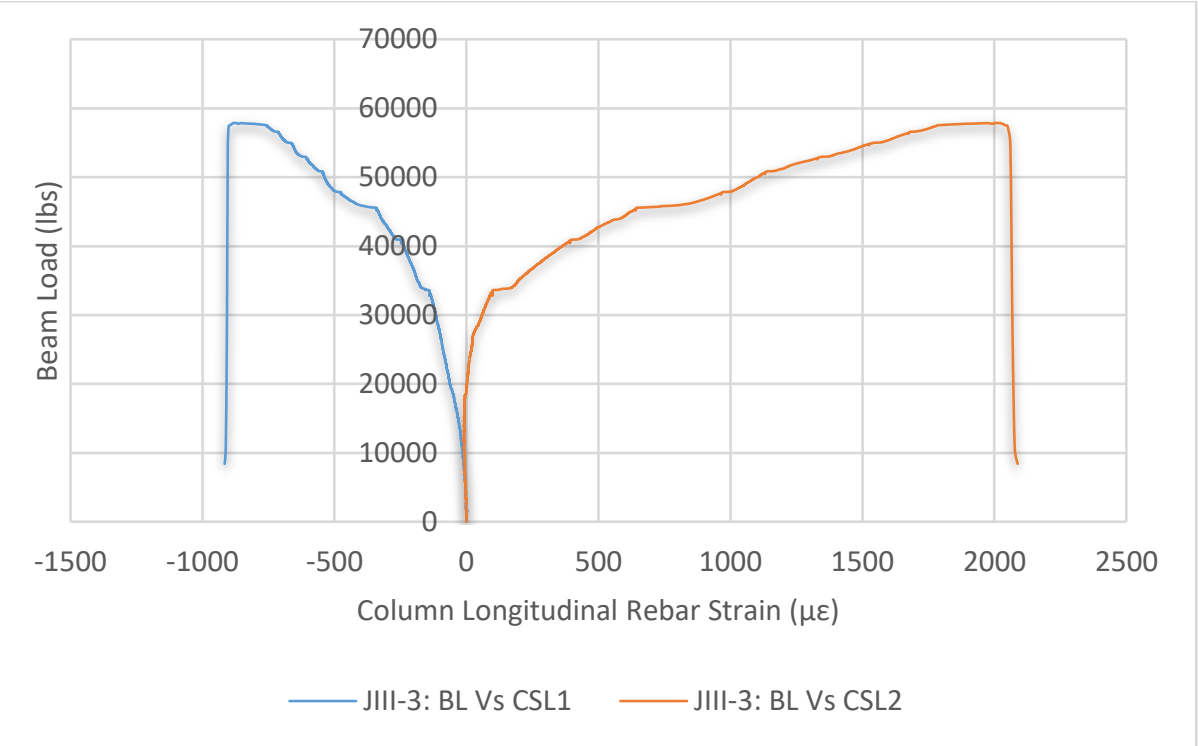

Figure C.23. Beam load vs. column longitudinal rebar strain

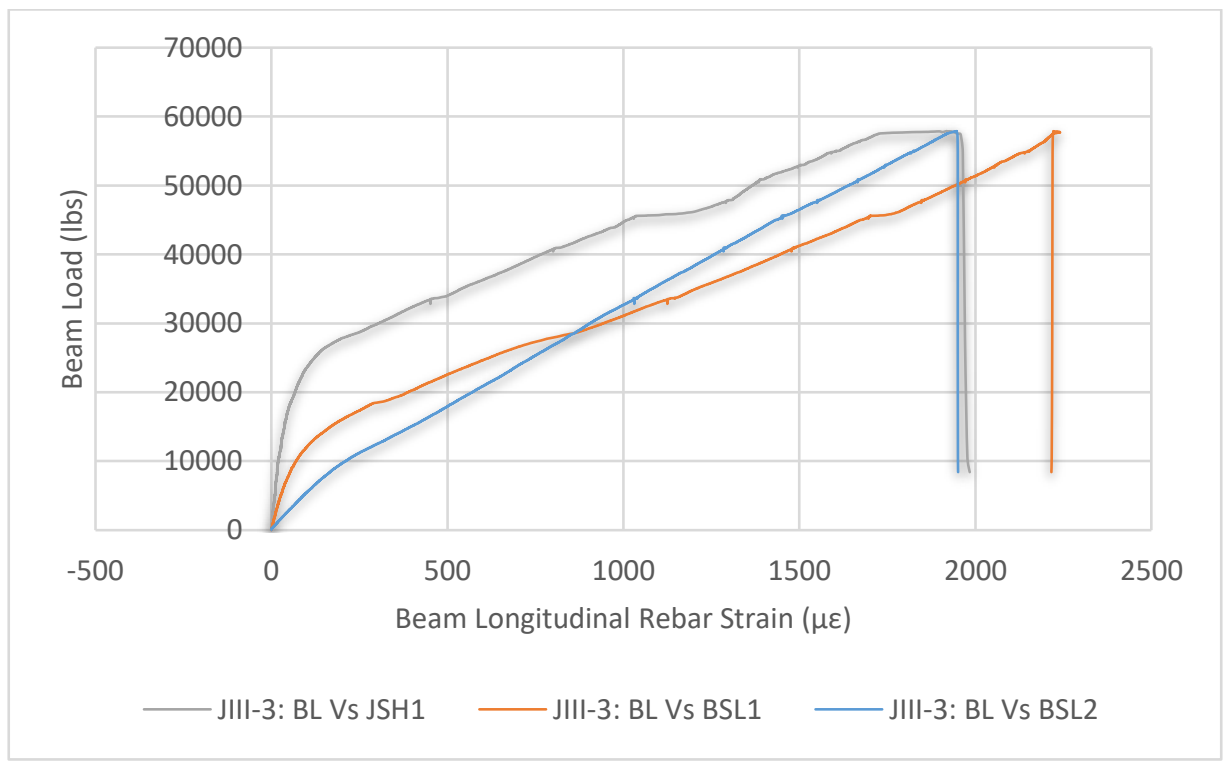

Figure C.24. Beam load vs. beam longitudinal rebar strain 


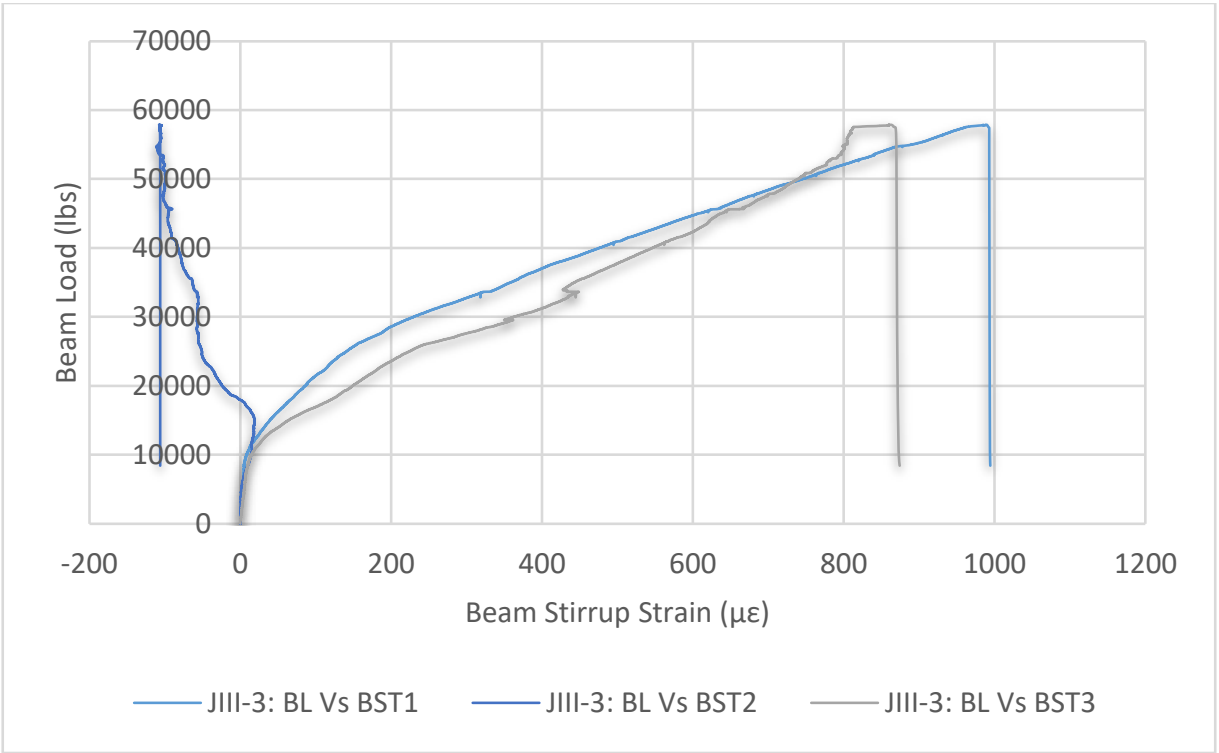

Figure C.25. Beam load vs. beam stirrup strain 


\section{C.3.2 Strain in FRP}

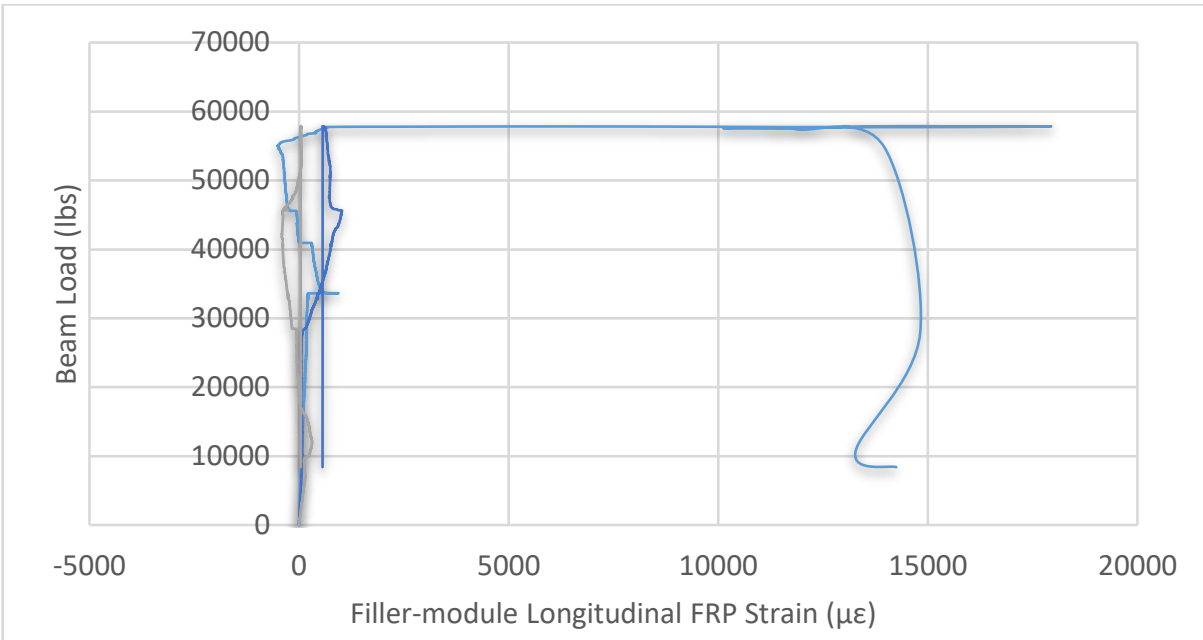

—III-3: BL Vs FFL1 — JIII-3: BL Vs FFL2 — JIII-3: BL Vs FFL3

Figure C.26. Beam load vs. filler-module longitudinal strain

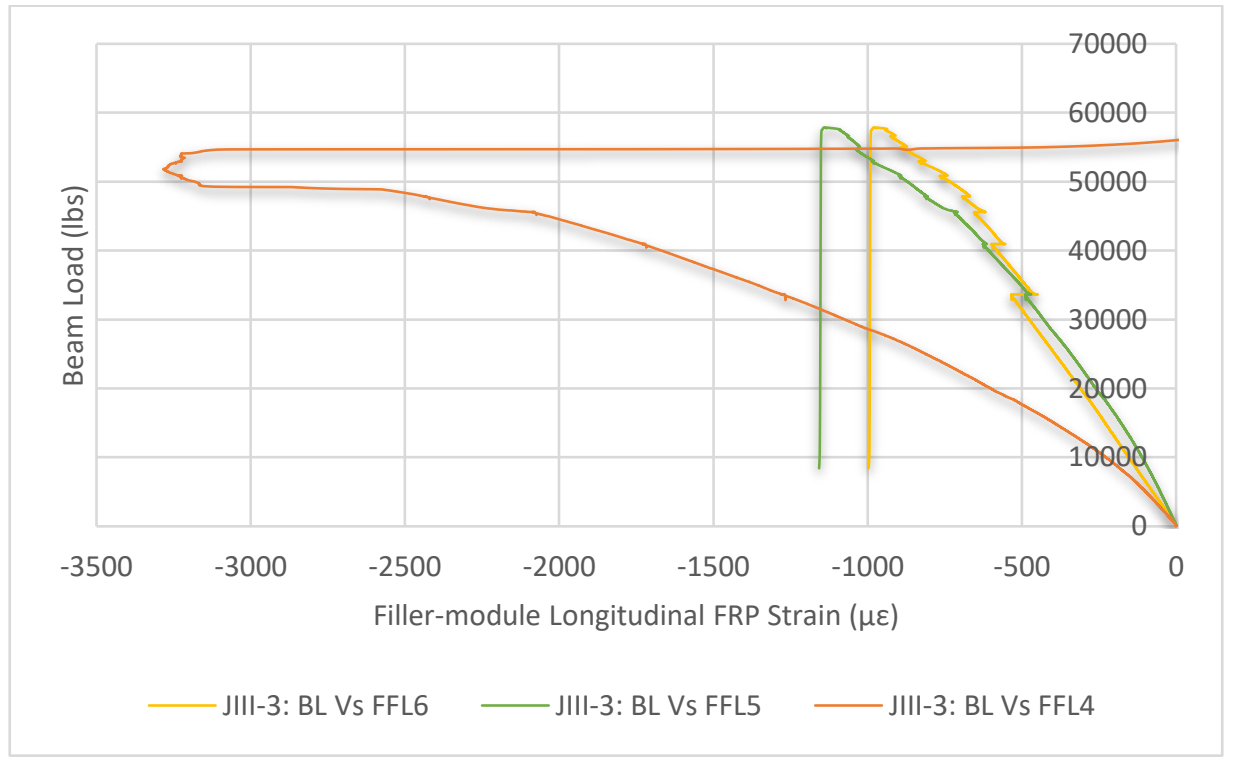

Figure C.27. Beam load vs. filler-module longitudinal strain 


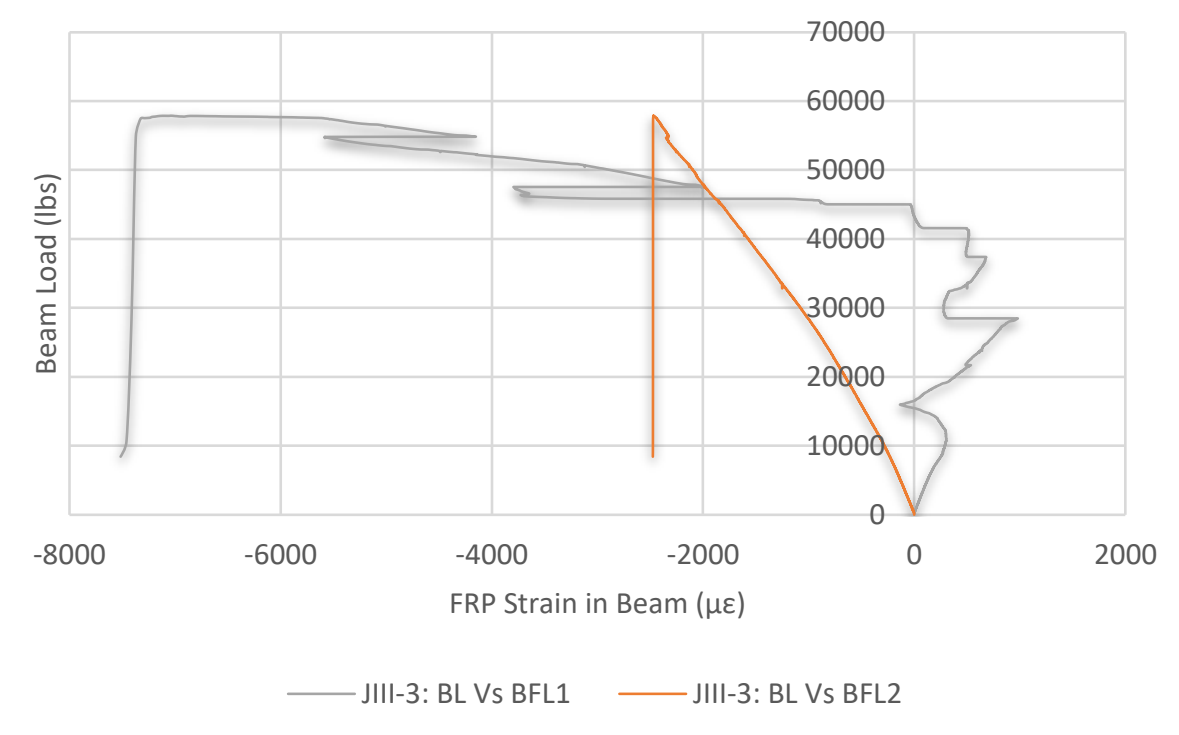

Figure C.28. Beam load vs. FRP strain in the beam

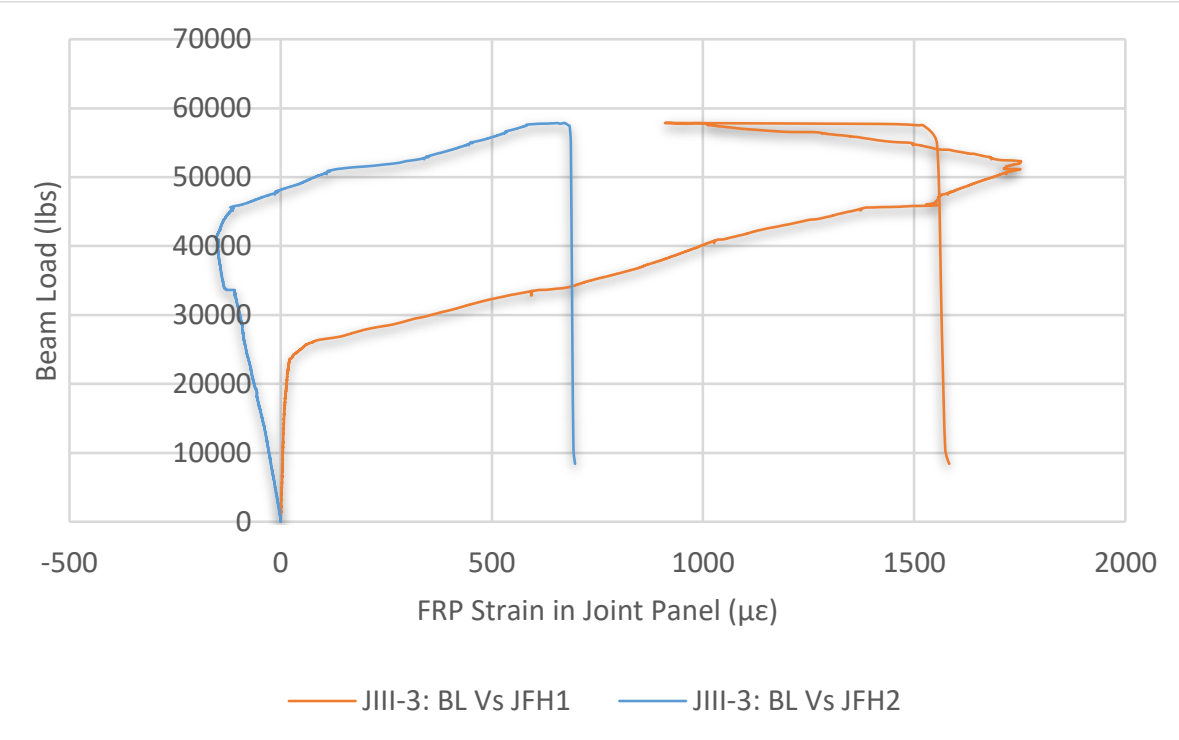

Figure C.29. Beam load vs. FRP strain in the joint panel 


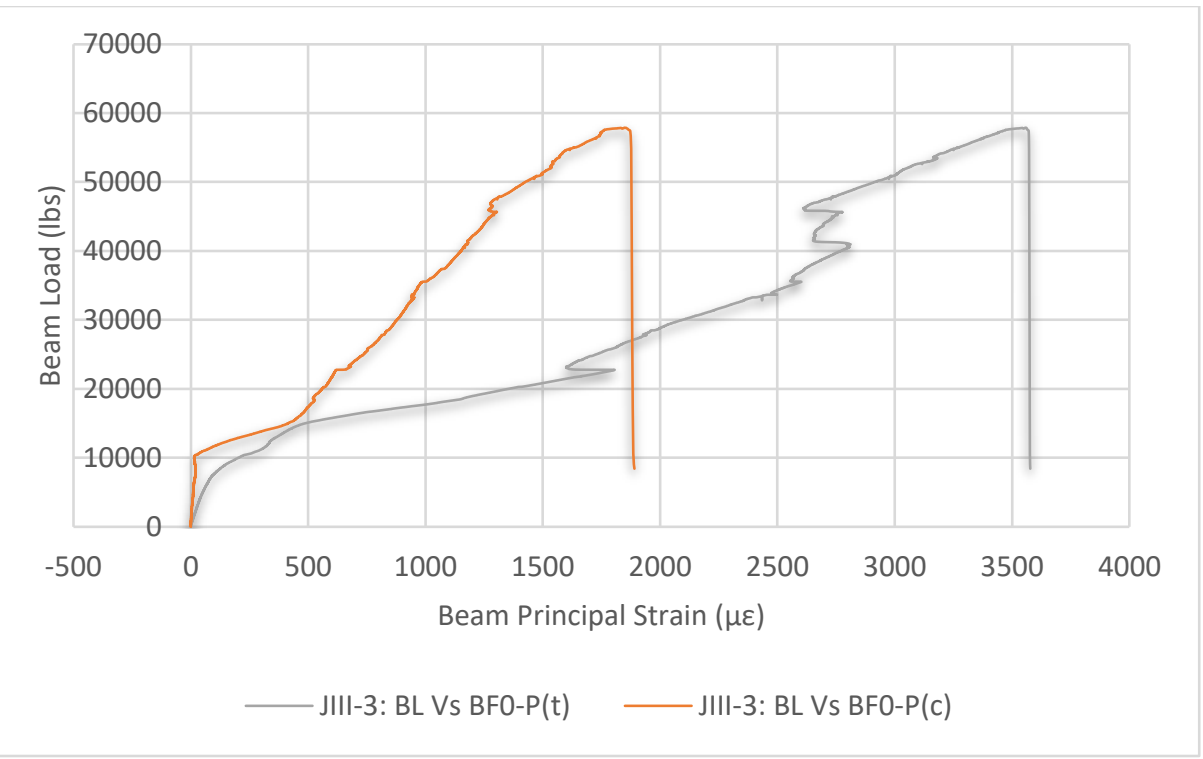

Figure C.30. Beam load vs. beam principal strain

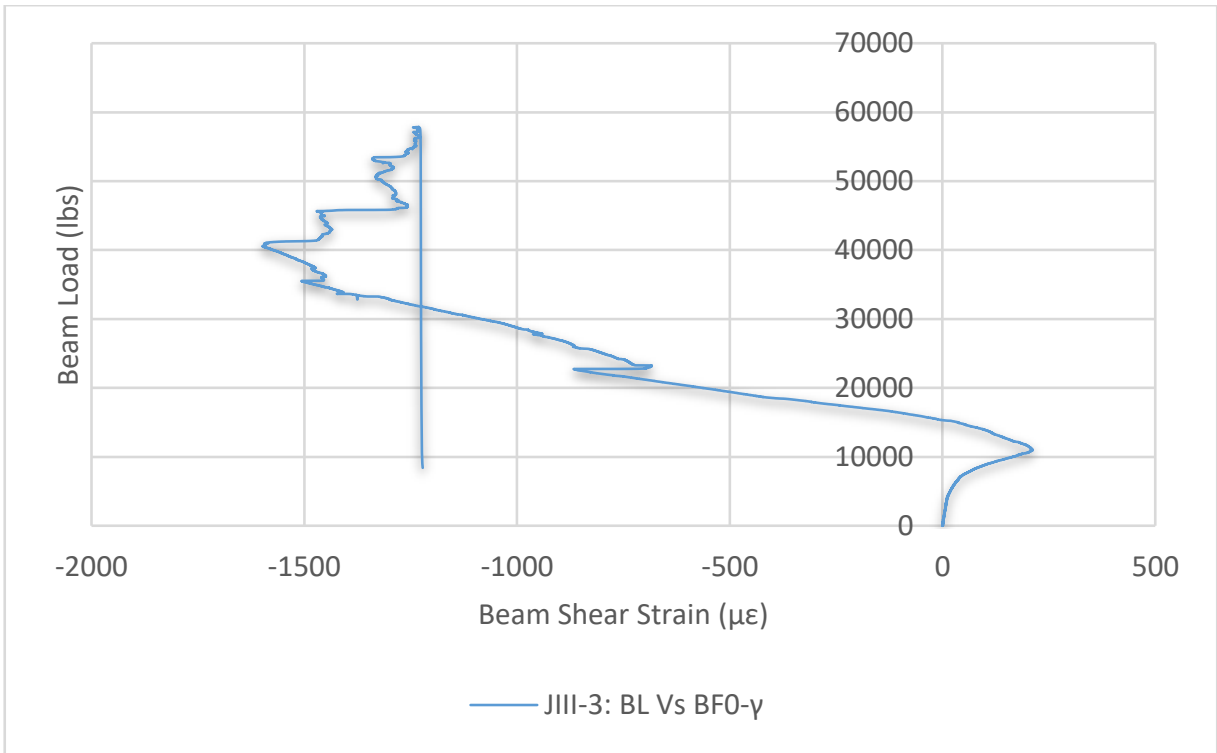

Figure C.31. Beam load vs. beam shear strain 


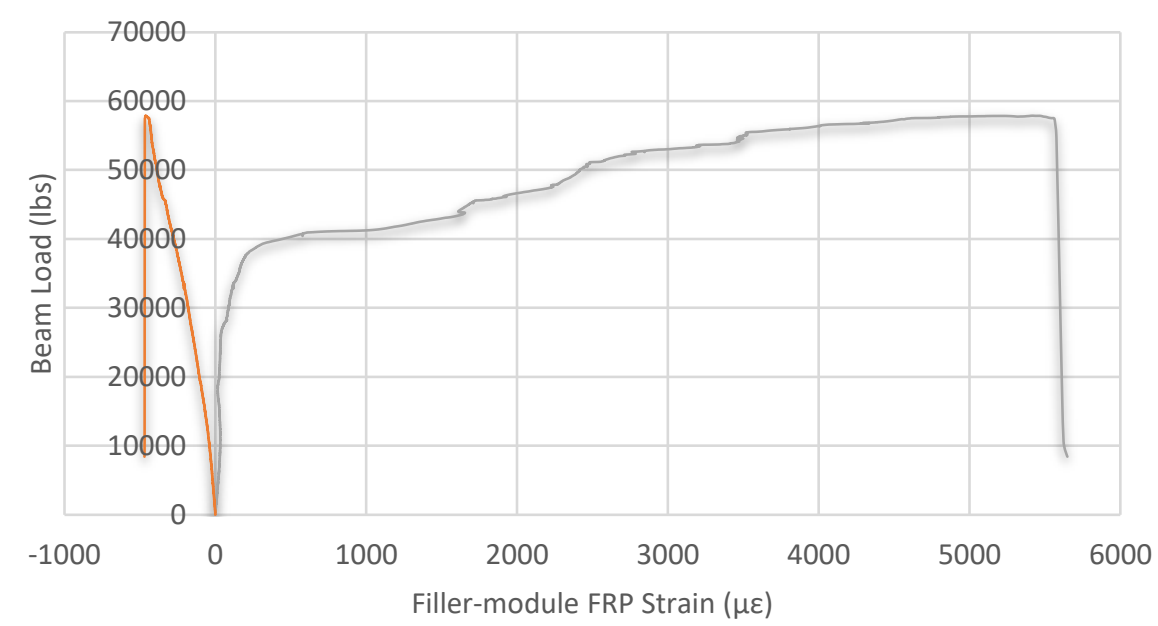

—JII-3: BL Vs FFH1 — JIII-3: BL Vs FFH2

Figure C.32. Beam load vs. filler-module FRP strain

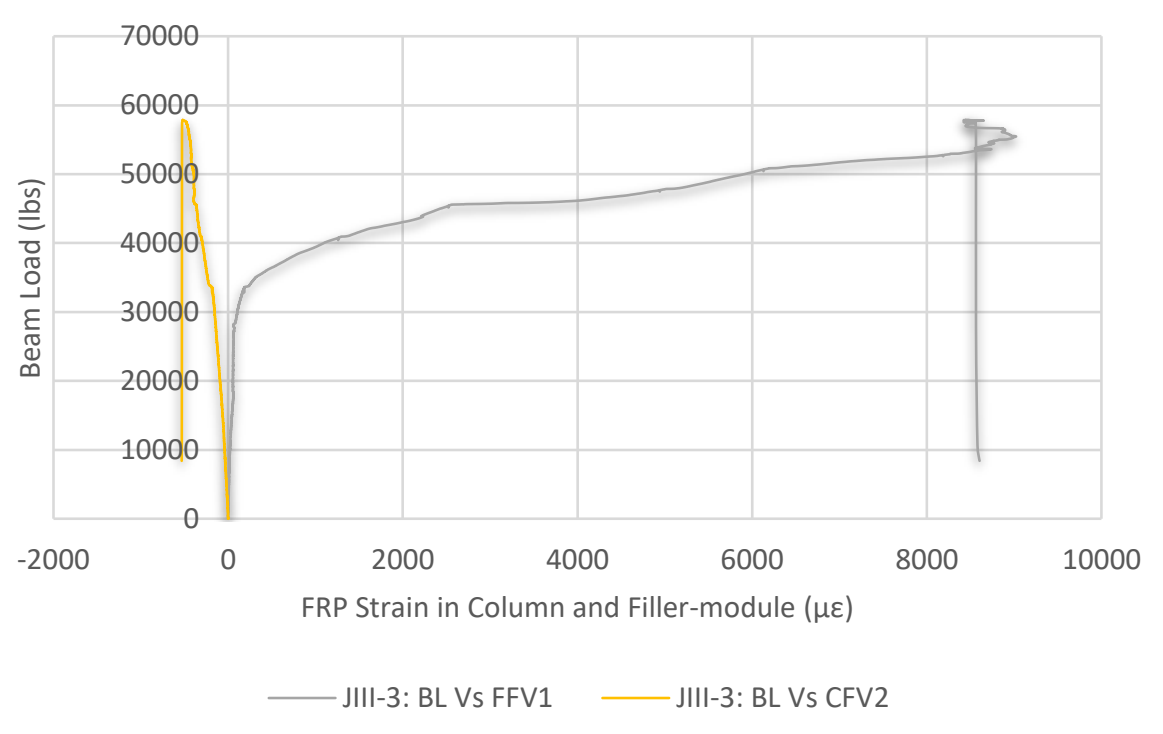

Figure C.33. Beam load vs. FRP strain in column and filler-module 


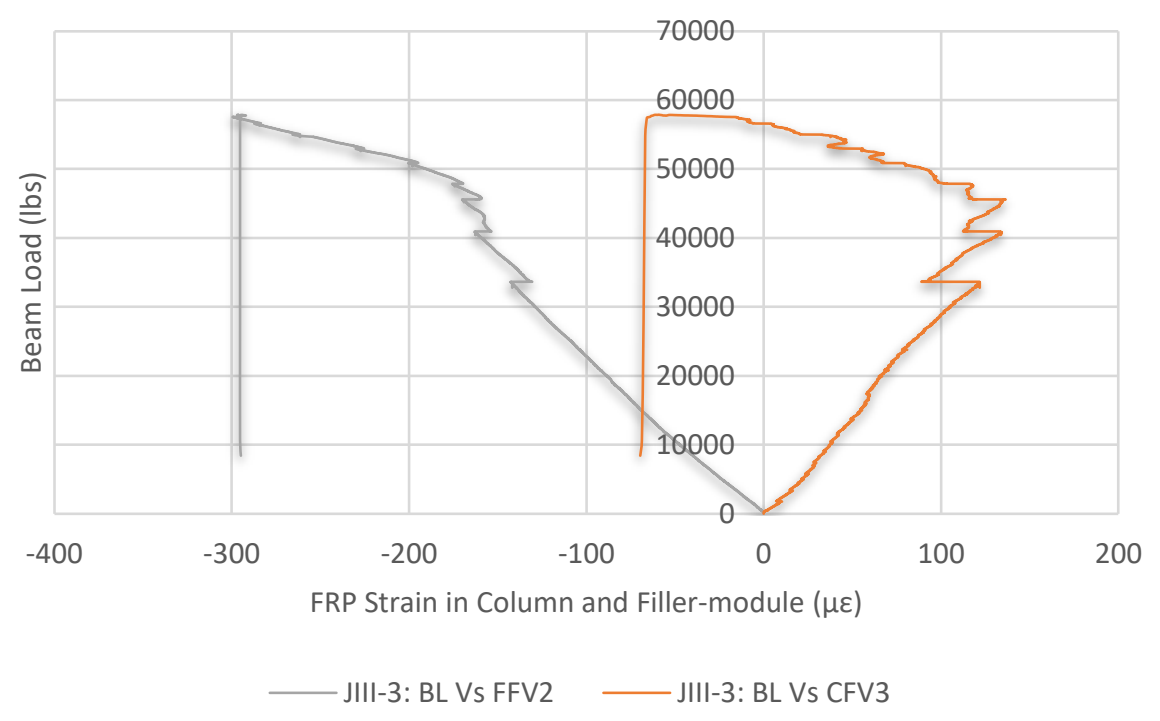

Figure C.34. Beam load vs. FRP strain in column and filler-module

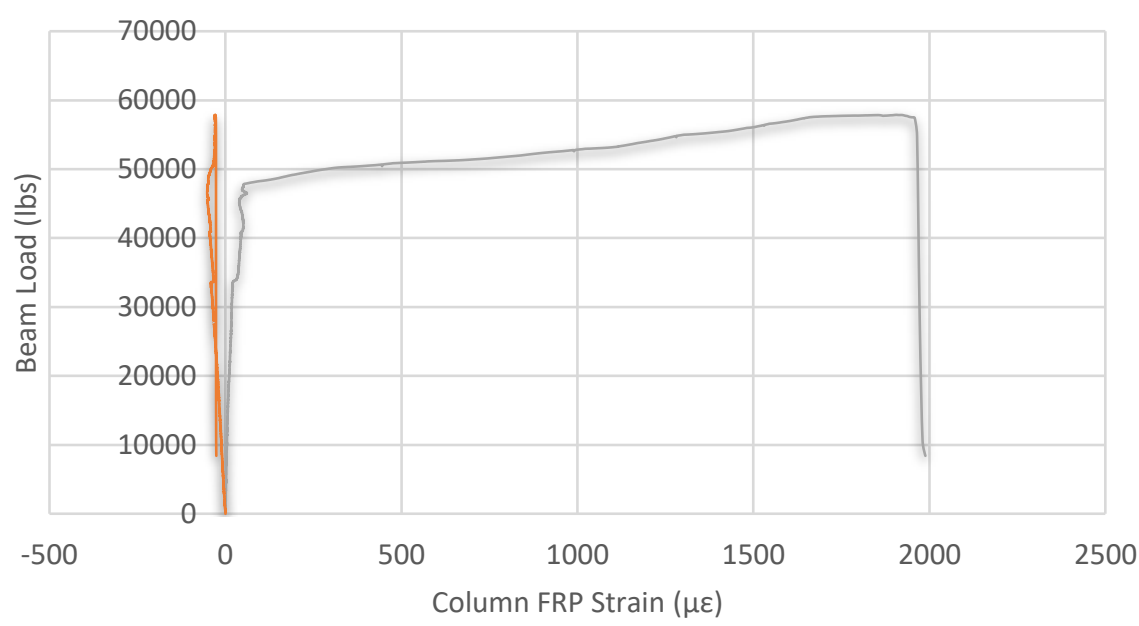

JIII-3: BL Vs CFH2 —JIII-3: BL Vs CFH3

Figure C.35. Beam load vs. column FRP strain 


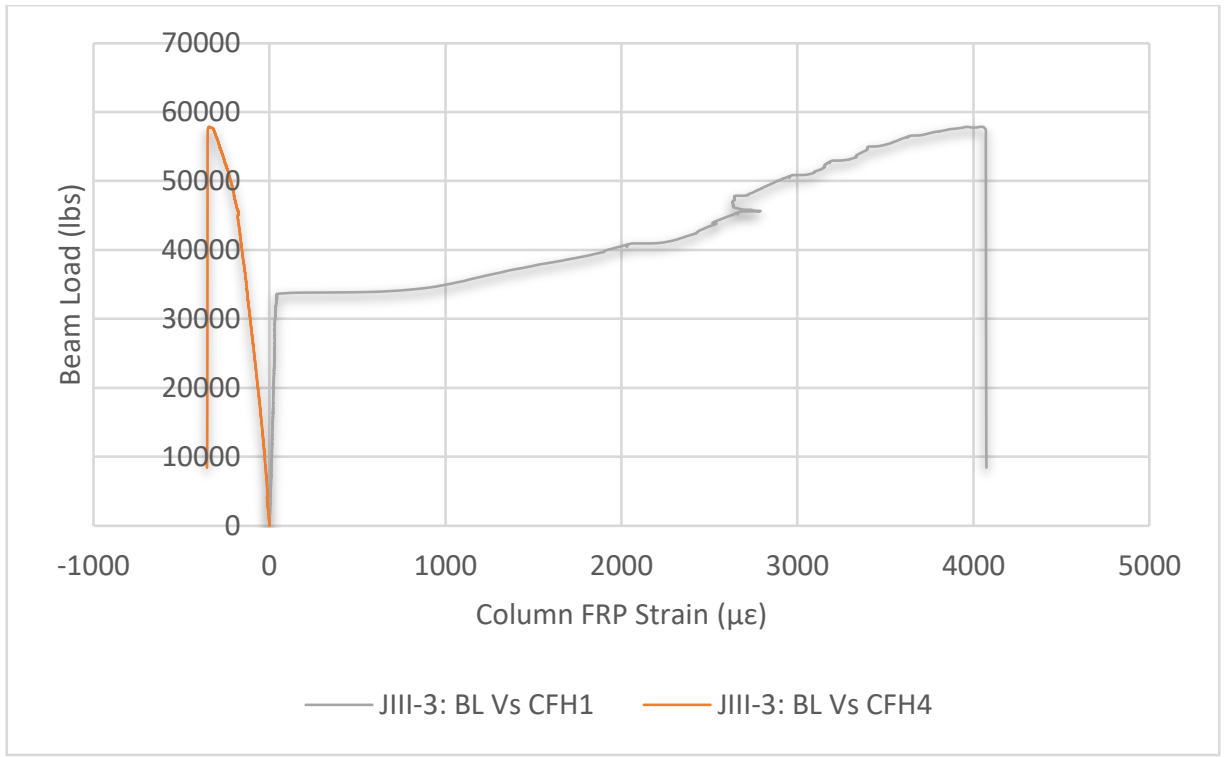

Figure C.36. Beam load vs. column FRP strain 


\section{C.4 Specimen JIII-4}

\section{C.4.1 Strain in steel rebar}

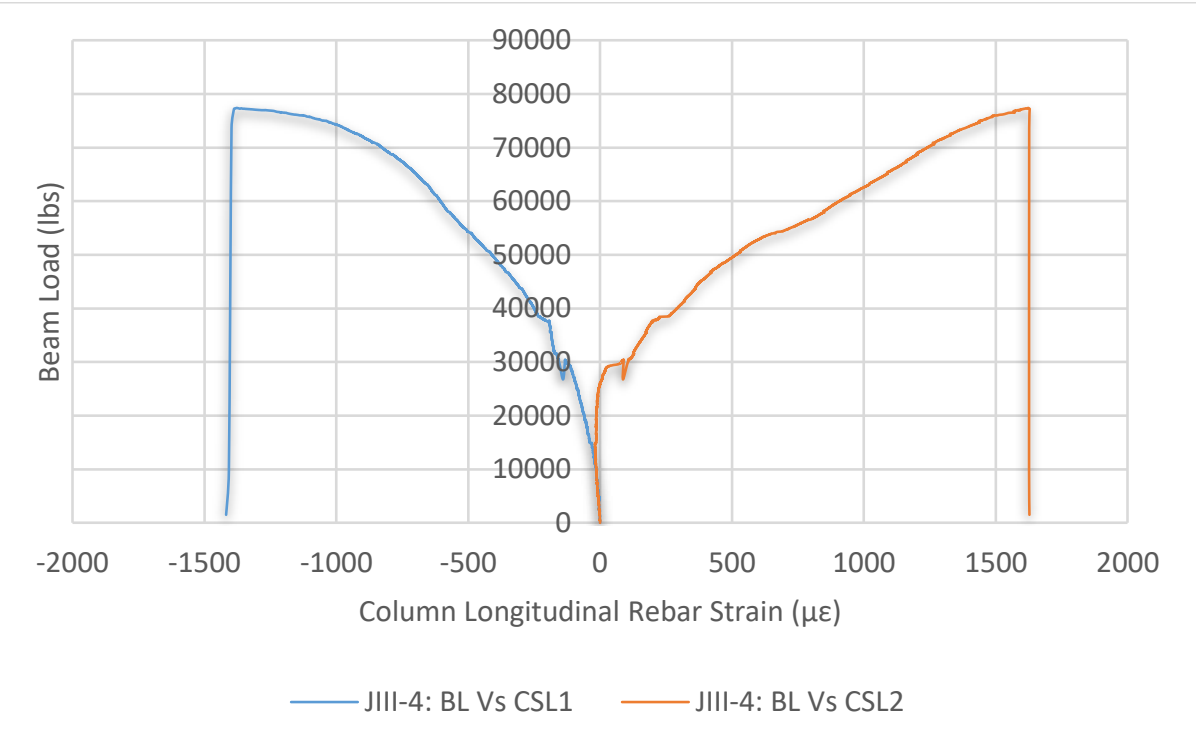

Figure C.37. Beam load vs. column longitudinal rebar strain

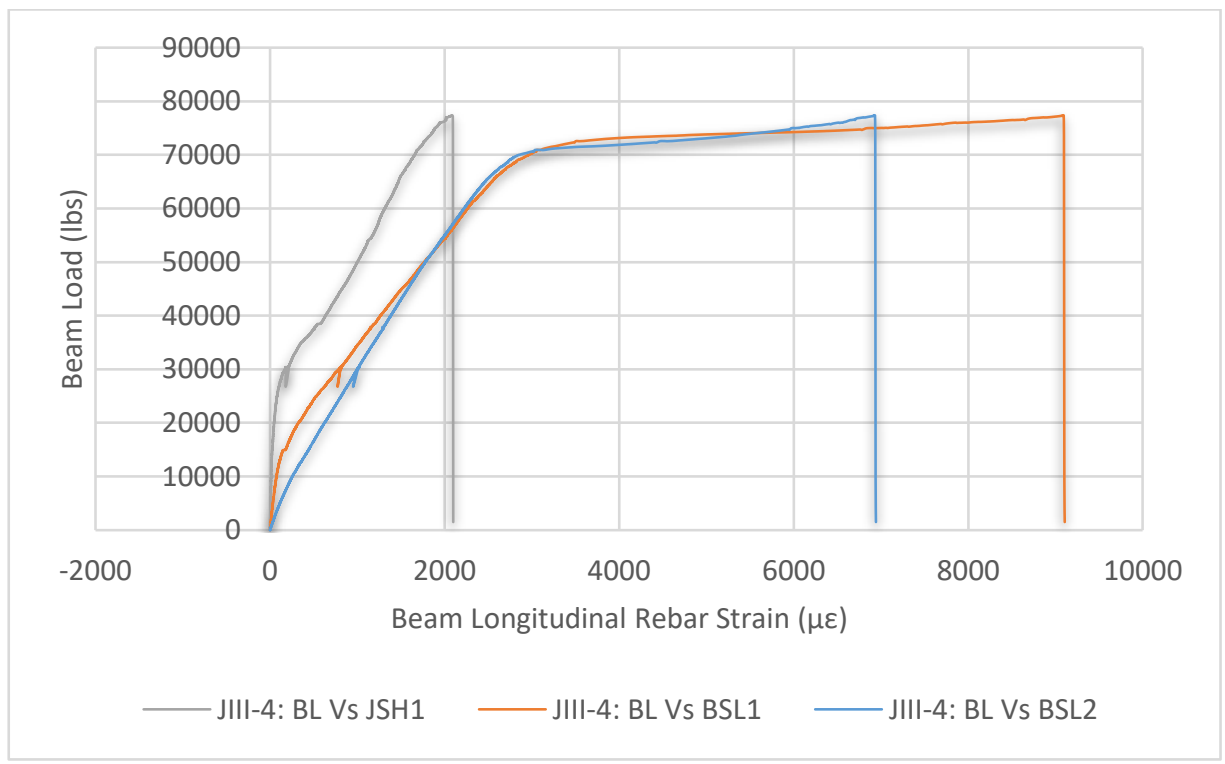

Figure C.38. Beam load vs. beam longitudinal rebar strain 


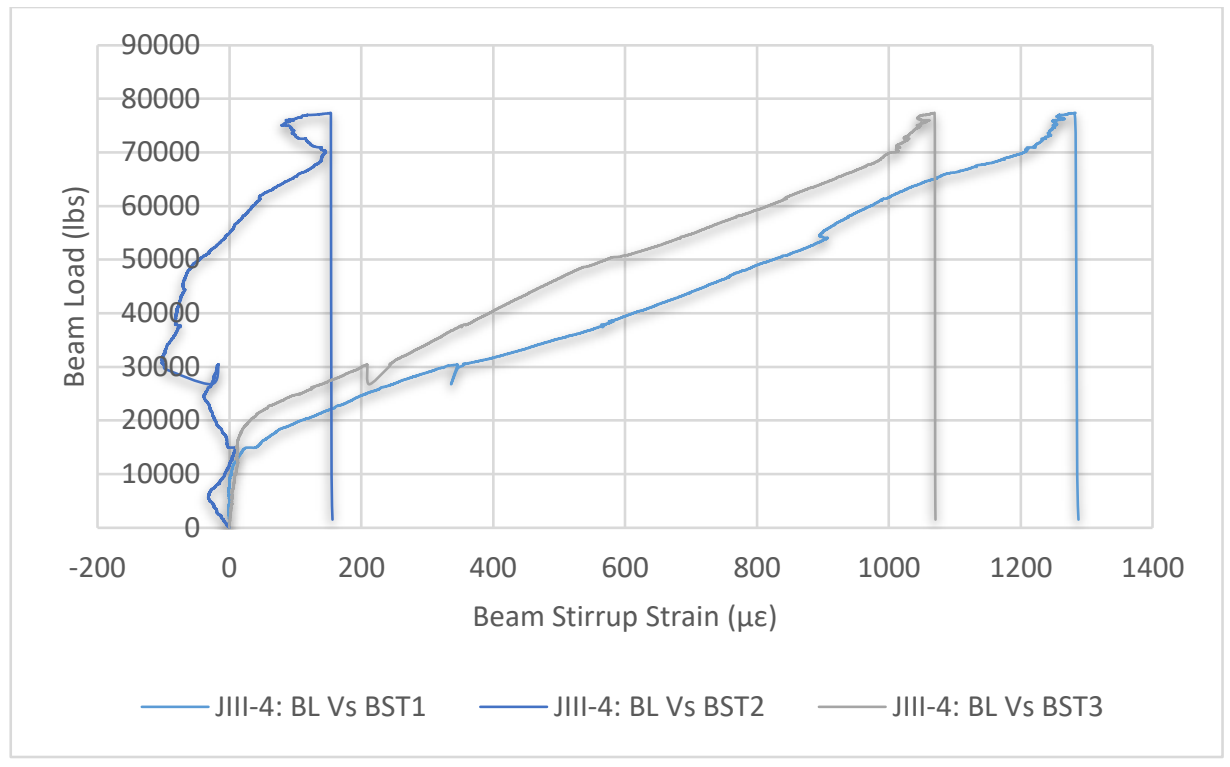

Figure C.39. Beam load vs. beam stirrup strain 


\section{C.4.2 Strain in FRP}

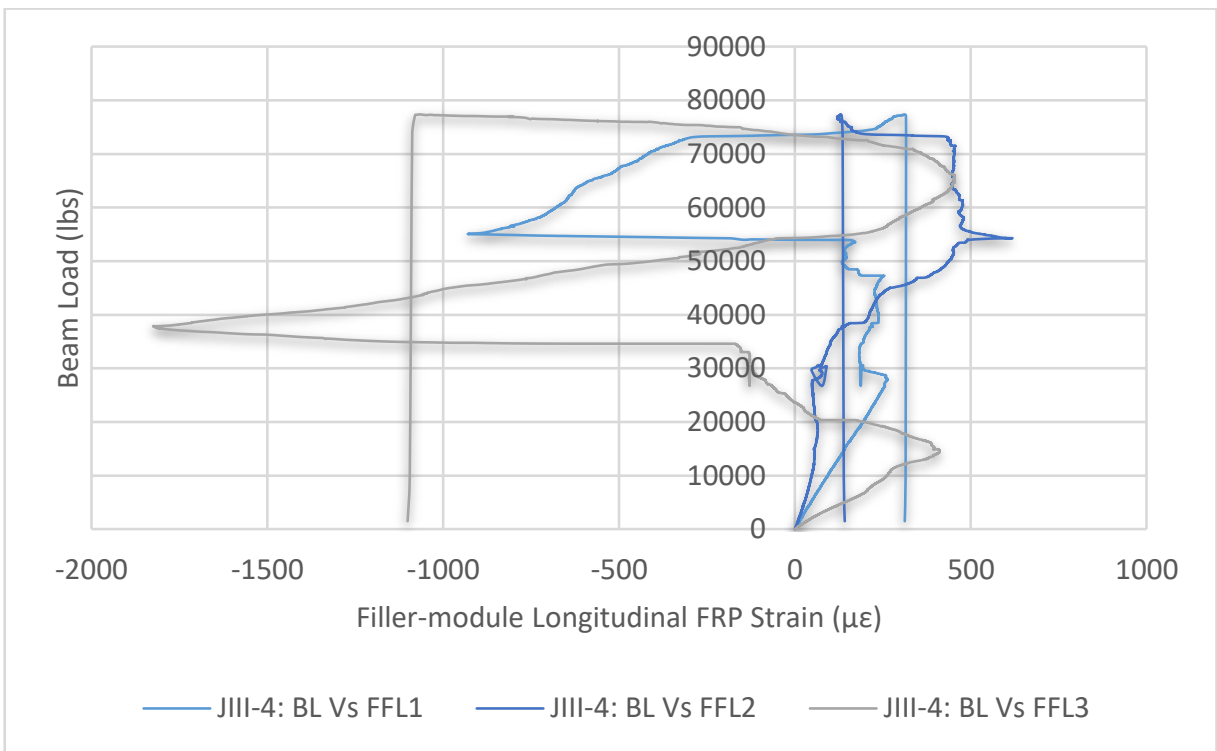

Figure C.40. Beam load vs. filler-module longitudinal strain

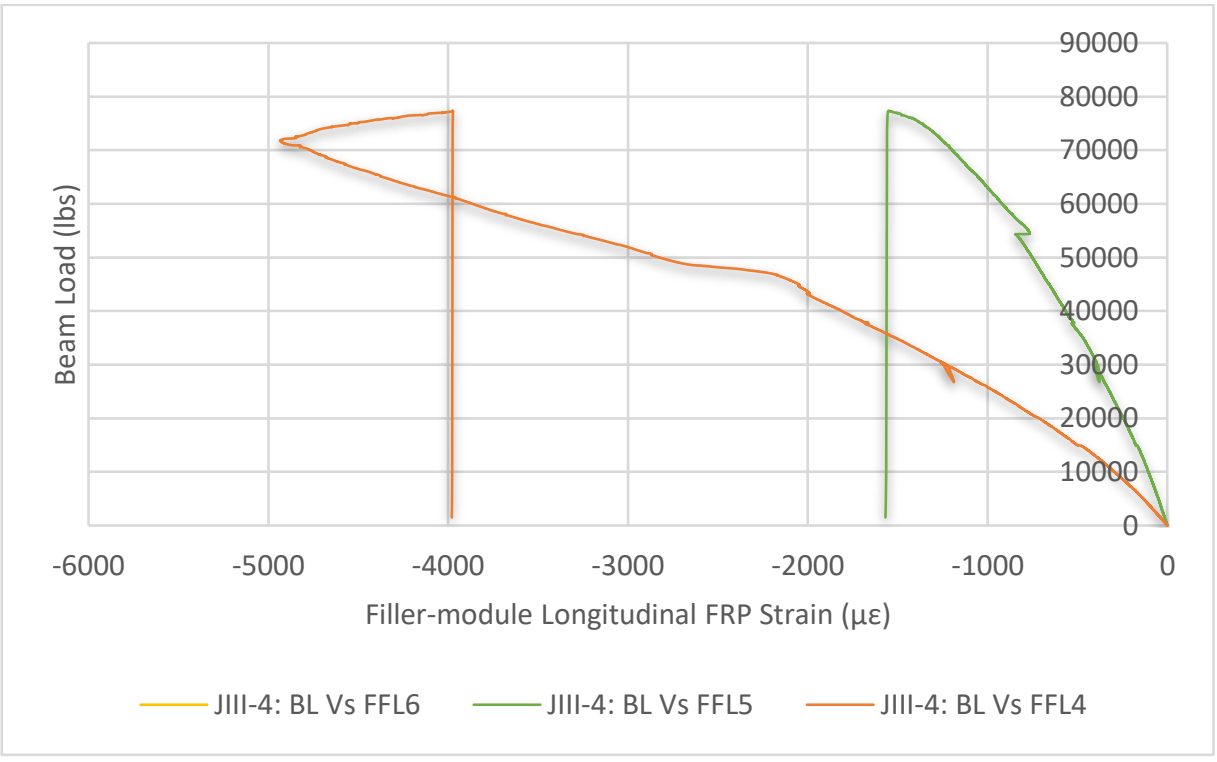

Figure C.41. Beam load vs. filler-module longitudinal strain 


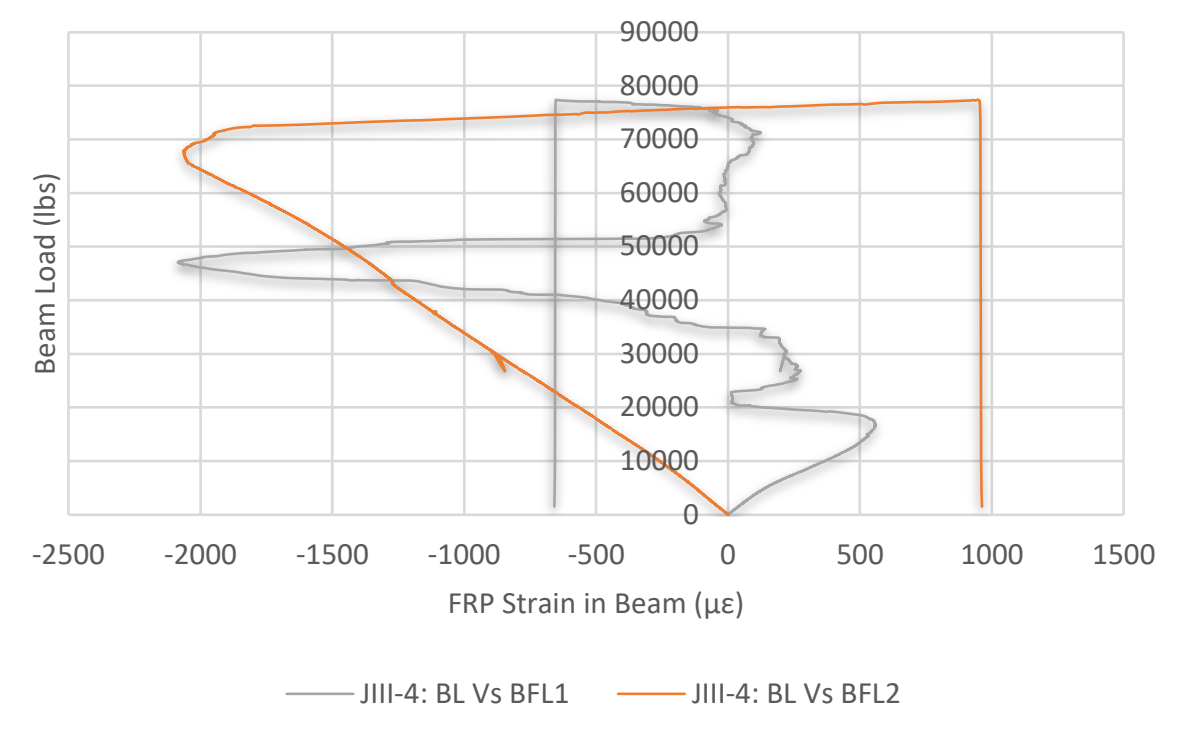

Figure C.42. Beam load vs. FRP strain in the beam

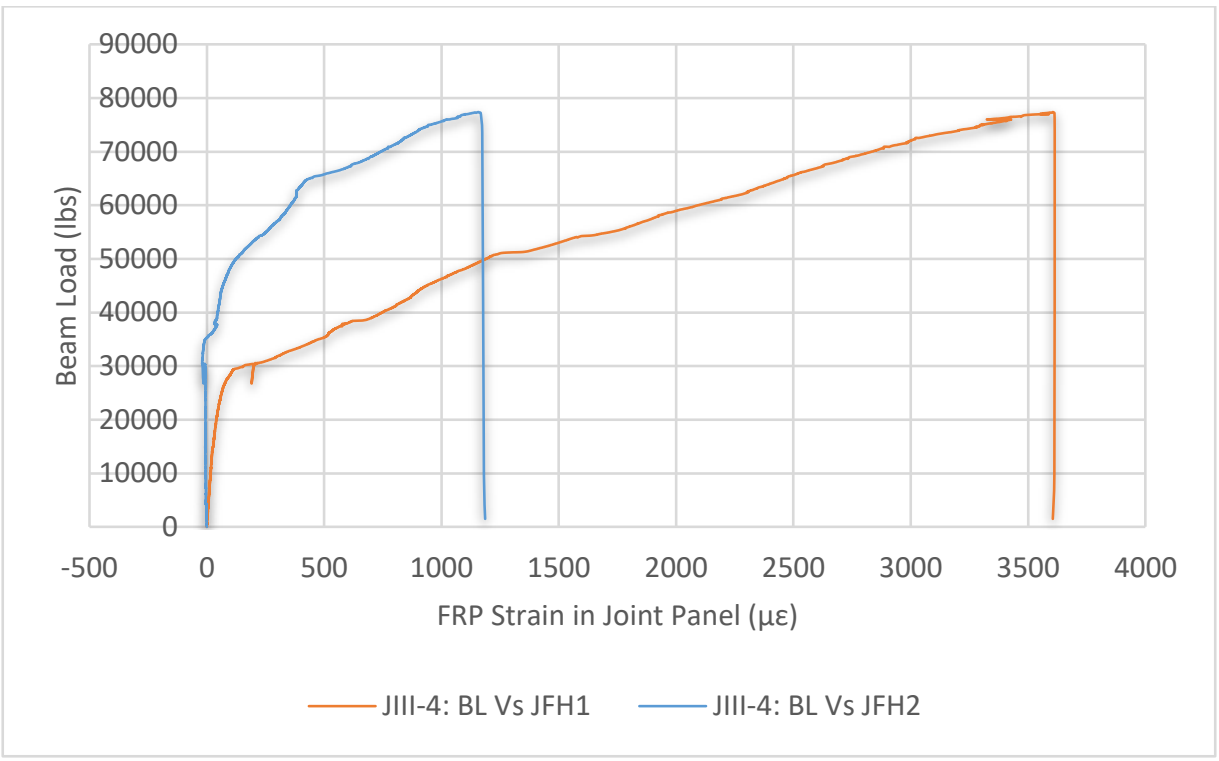

Figure C.43. Beam load vs. FRP strain in the joint panel 


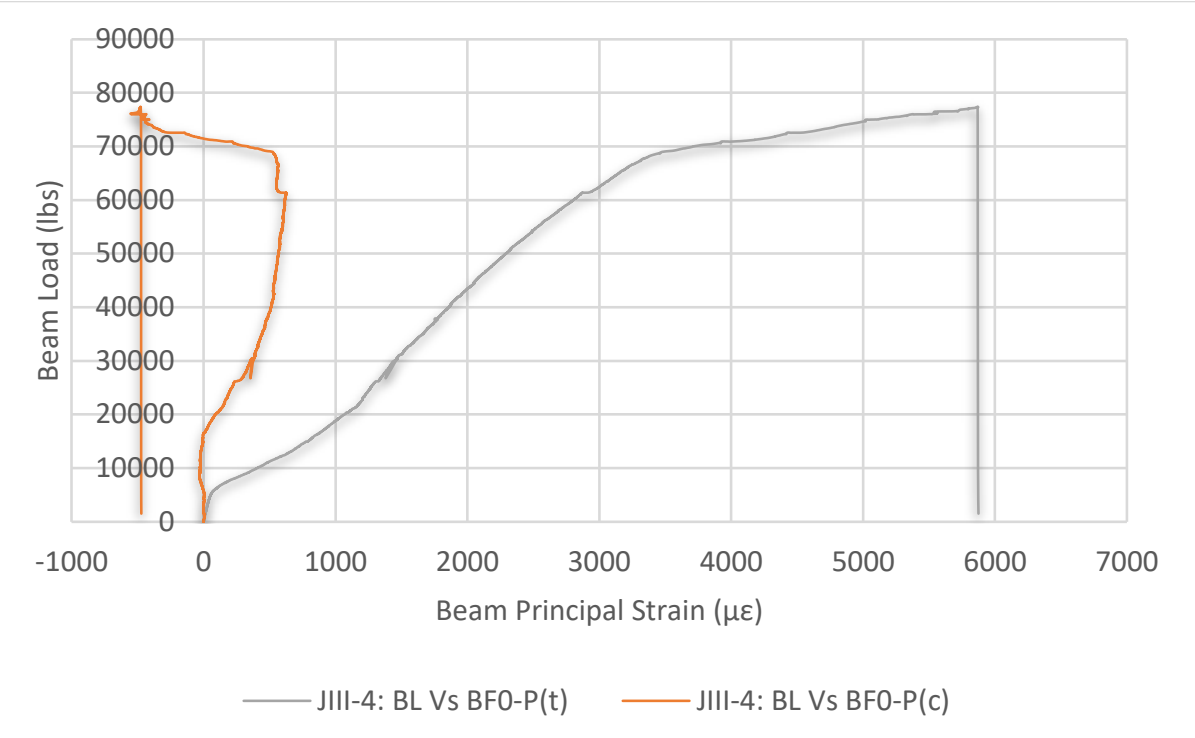

Figure C.44. Beam load vs. beam principal strain

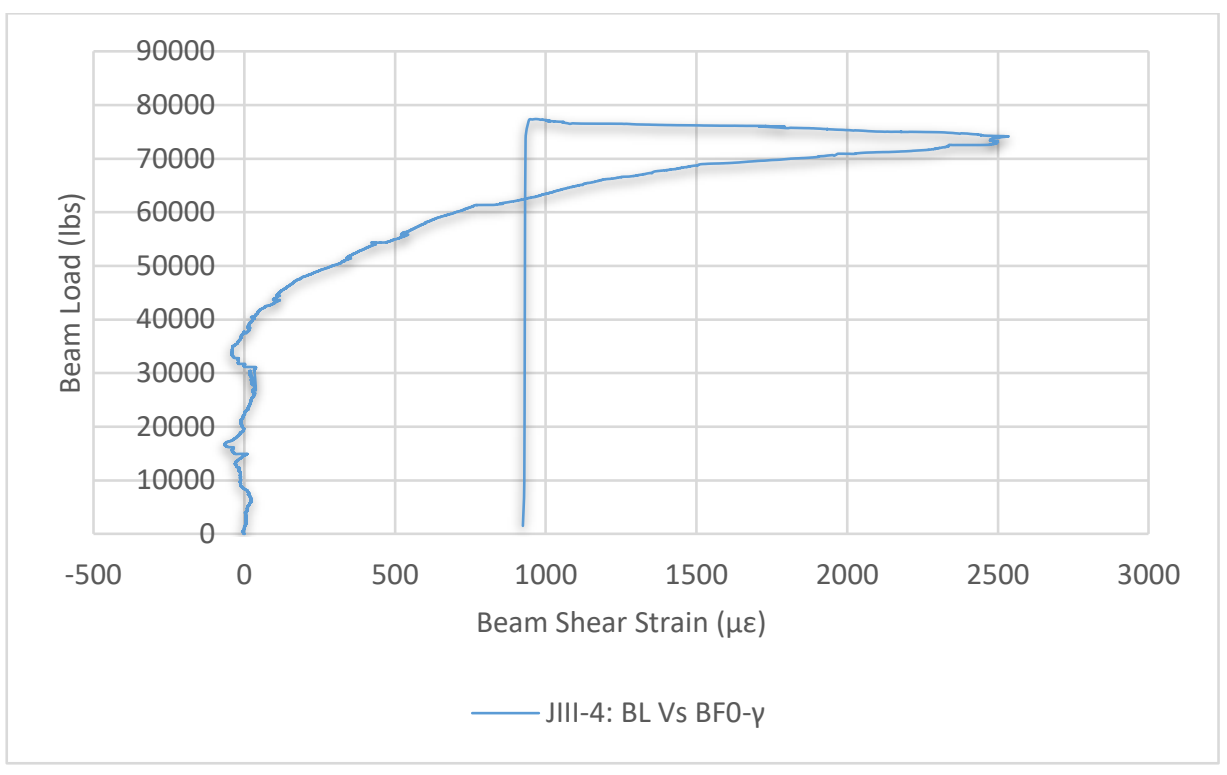

Figure C.45. Beam load vs. beam shear strain 


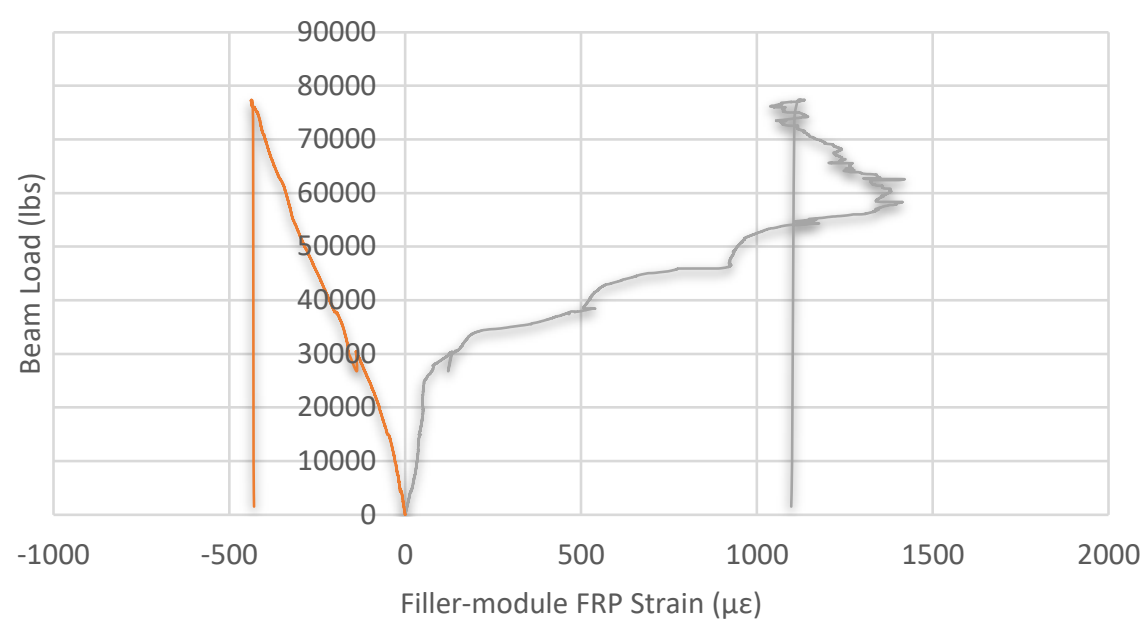

JIII-4: BL Vs FFH1 _ JIII-4: BL Vs FFH2

Figure C.46. Beam load vs. filler-module FRP strain

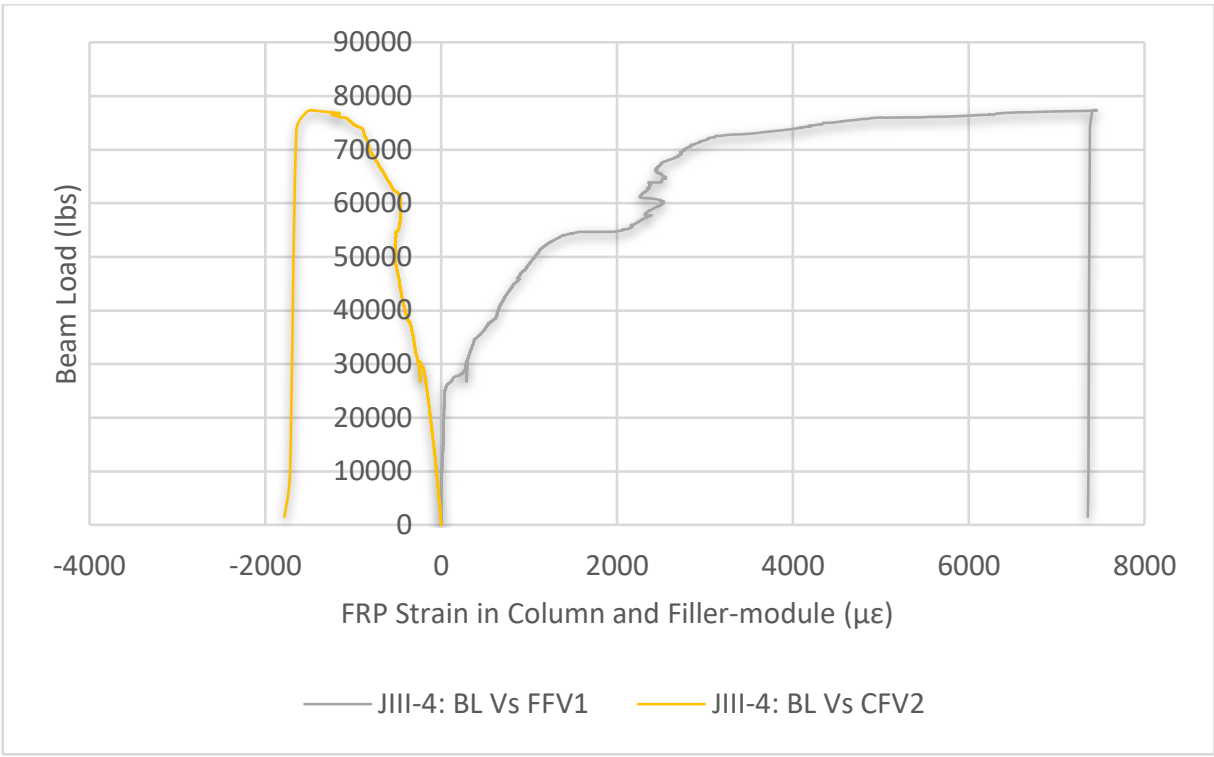

Figure C.47. Beam load vs. FRP strain in column and filler-module 


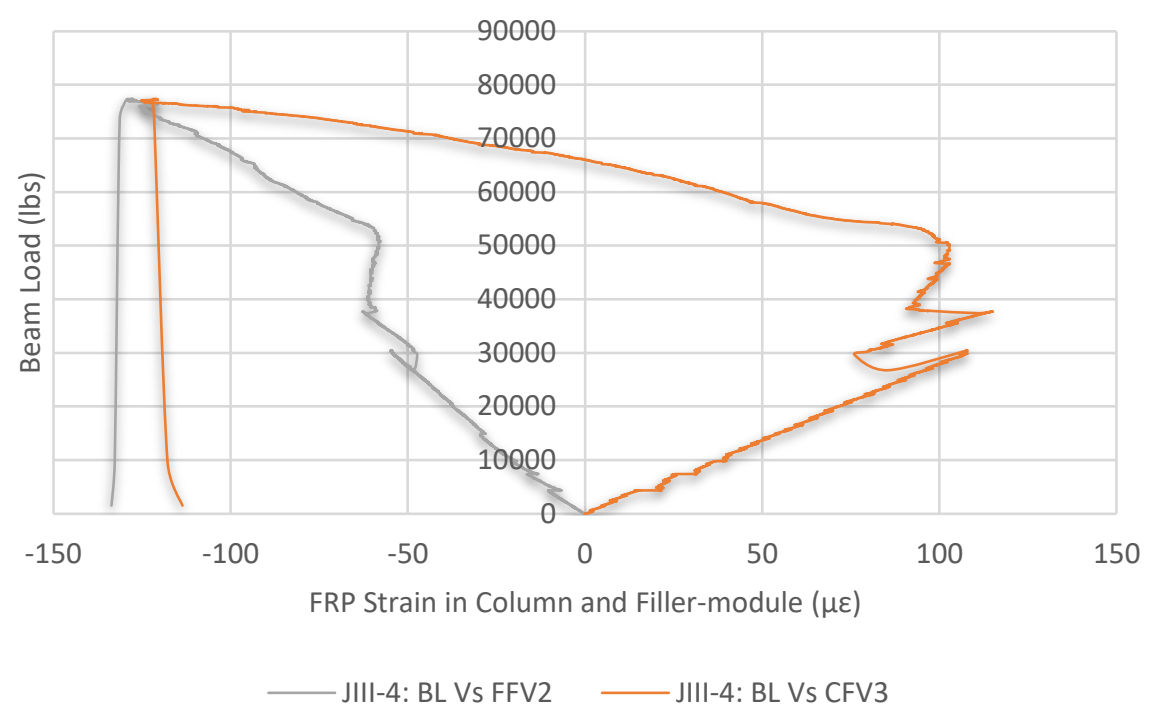

Figure C.48. Beam load vs. FRP strain in column and filler-module

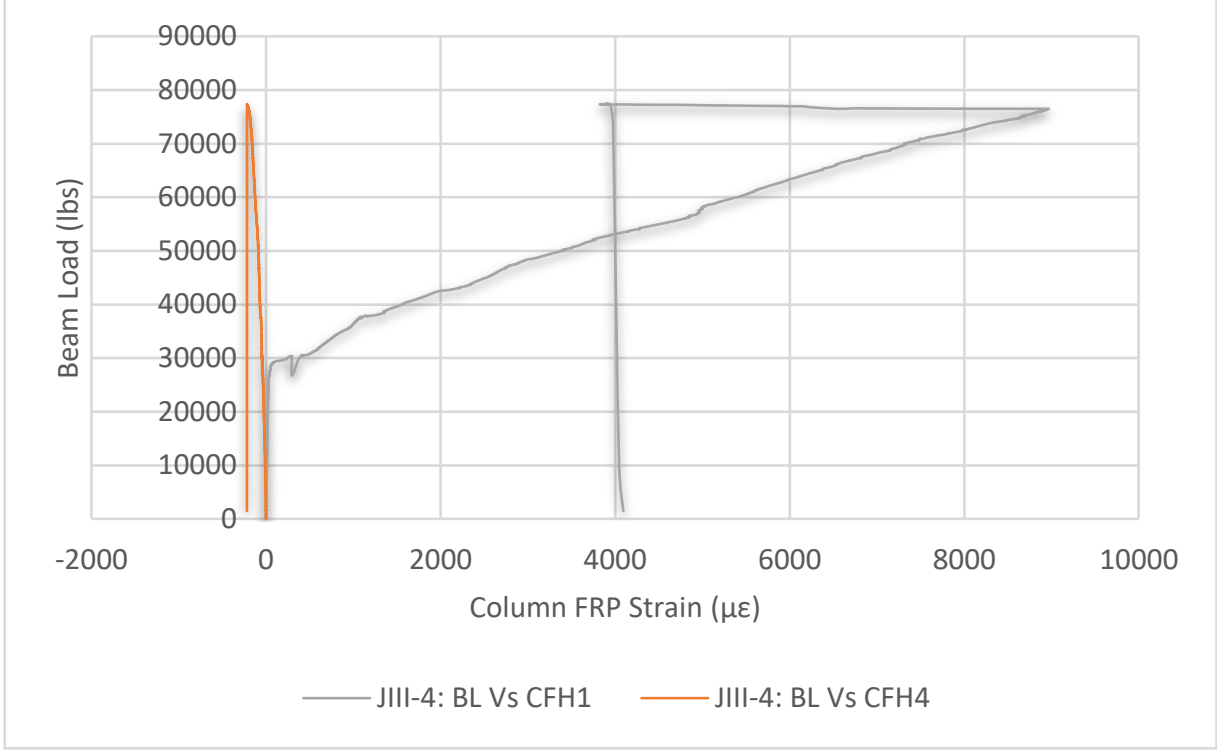

Figure C.49. Beam load vs. column FRP strain 


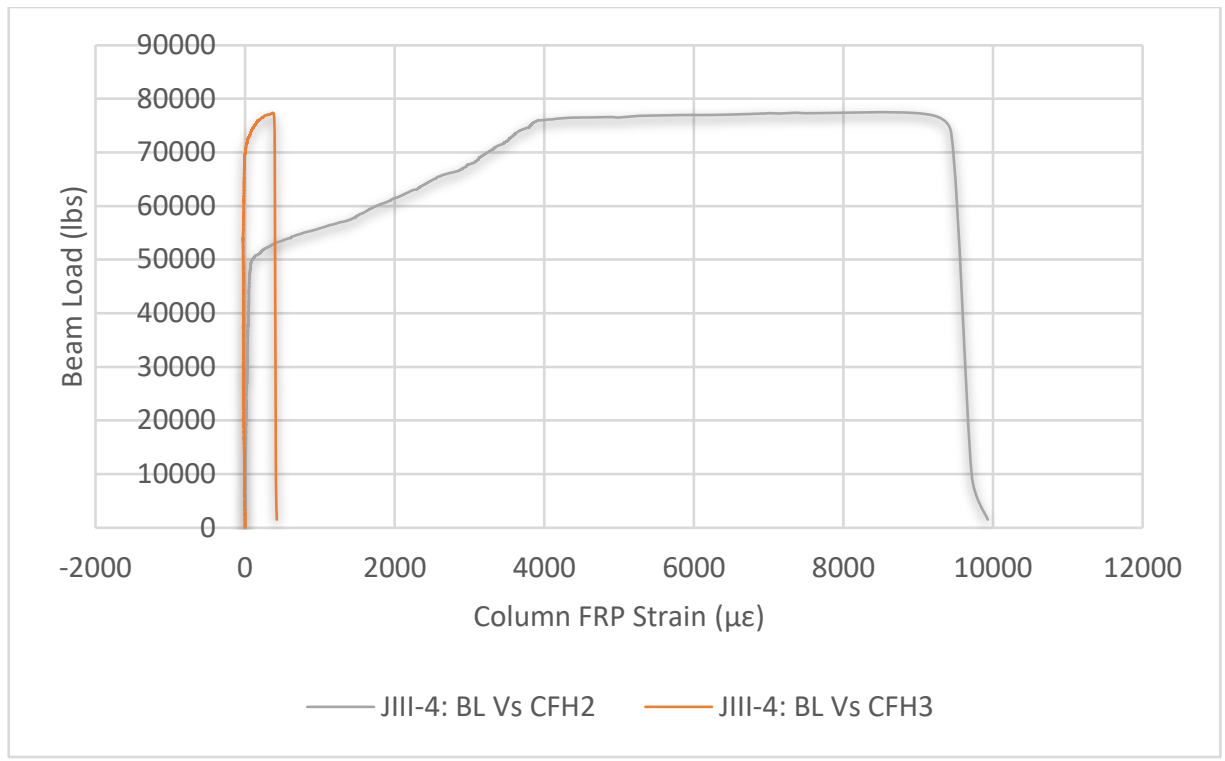

Figure C.50. Beam load vs. column FRP strain 


\section{Appendix-D: Beam-Column Joints Reinforced with Engineered Wood Filler-Modules and FRP Wraps}

This section provides additional test data generated through experimental evaluation of beamcolumn joints reinforced with engineered wood (PSL) filler-modules with FRP wraps under monotonic loads.

\section{D.1 Specimen JII-1}

D.1.1 Strain in steel rebar

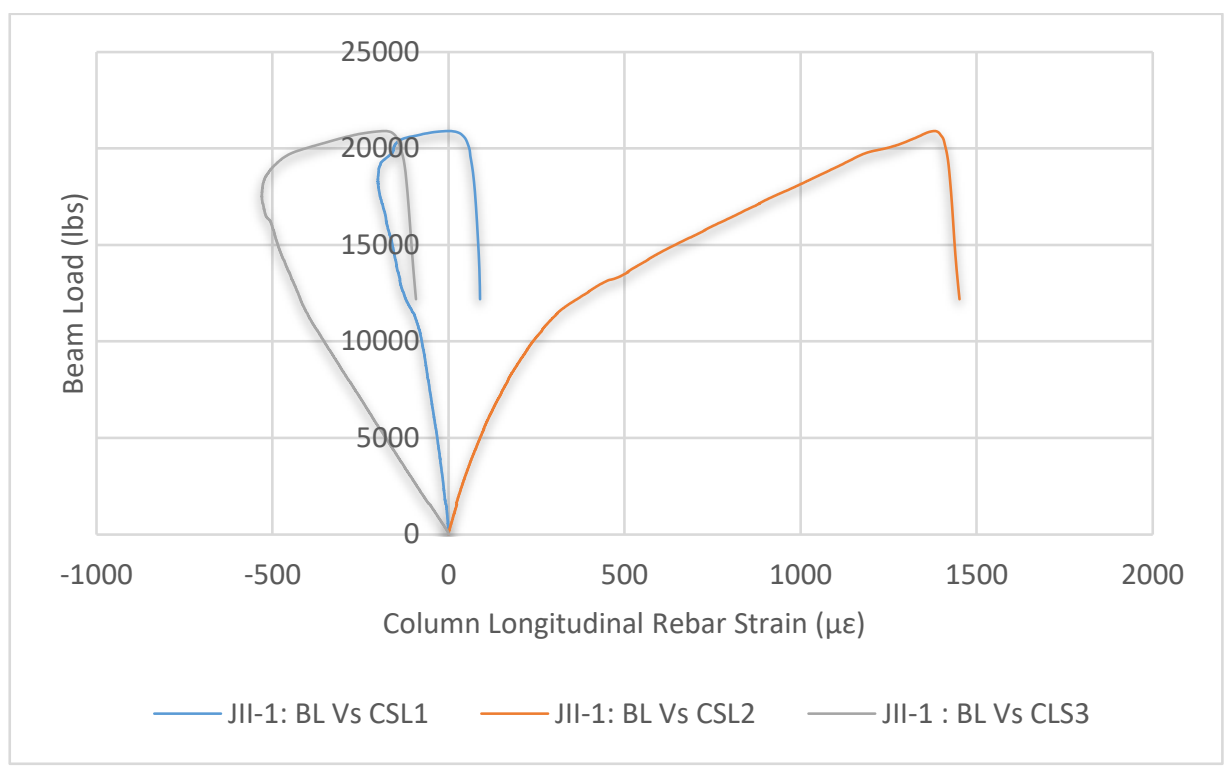

Figure D.1. Beam load vs. column longitudinal rebar strain 


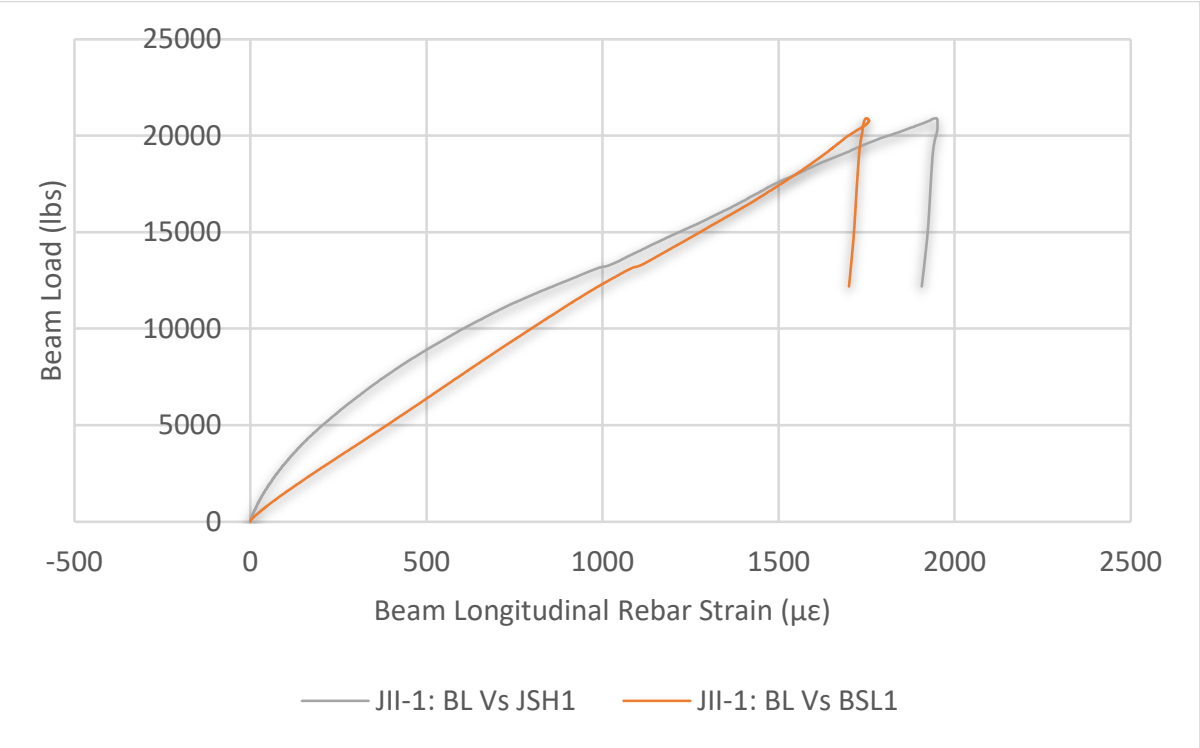

Figure D.2. Beam load vs. beam longitudinal rebar strain

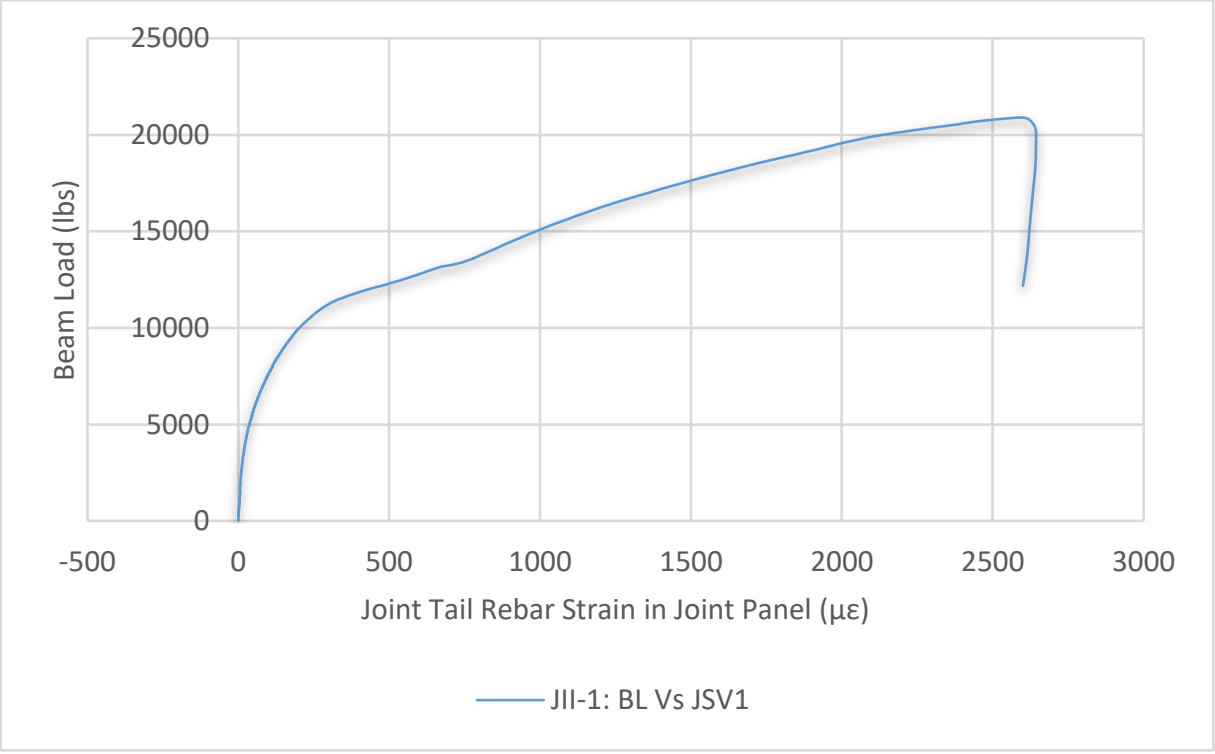

Figure D.3. Beam load vs. joint tail rebar strain 


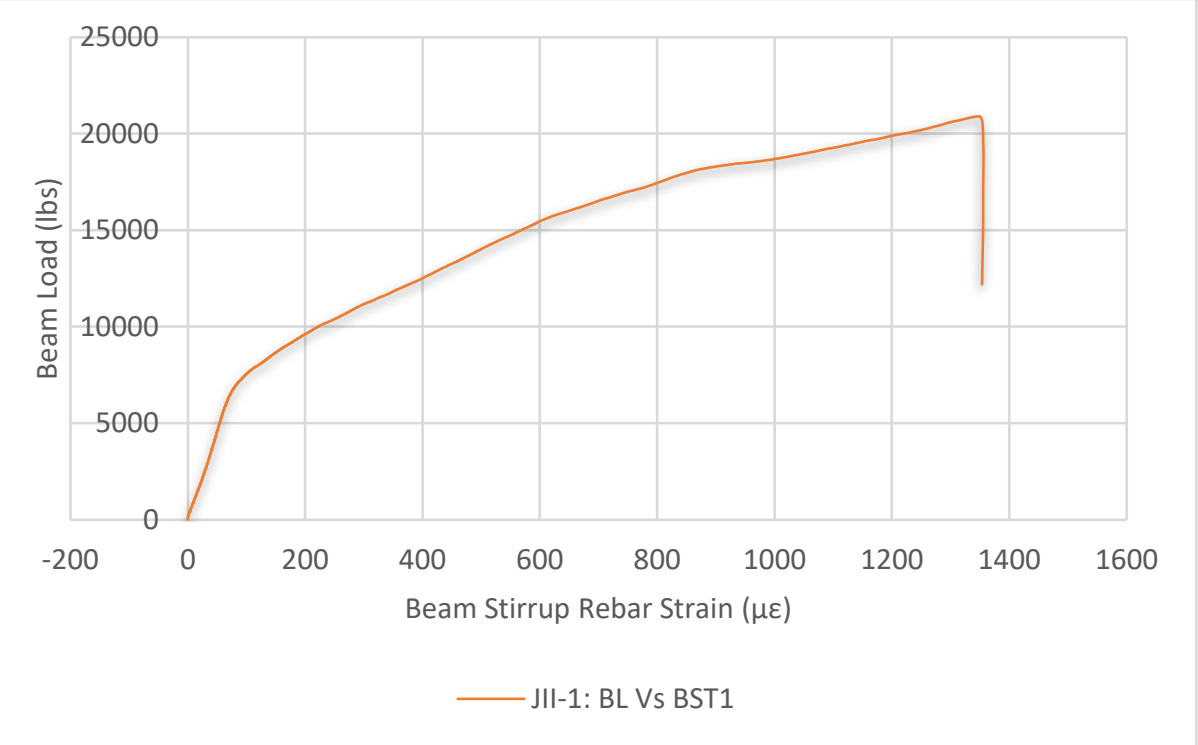

Figure D.4. Beam load vs. beam stirrup strain 


\section{D.1.2 Strain in concrete}

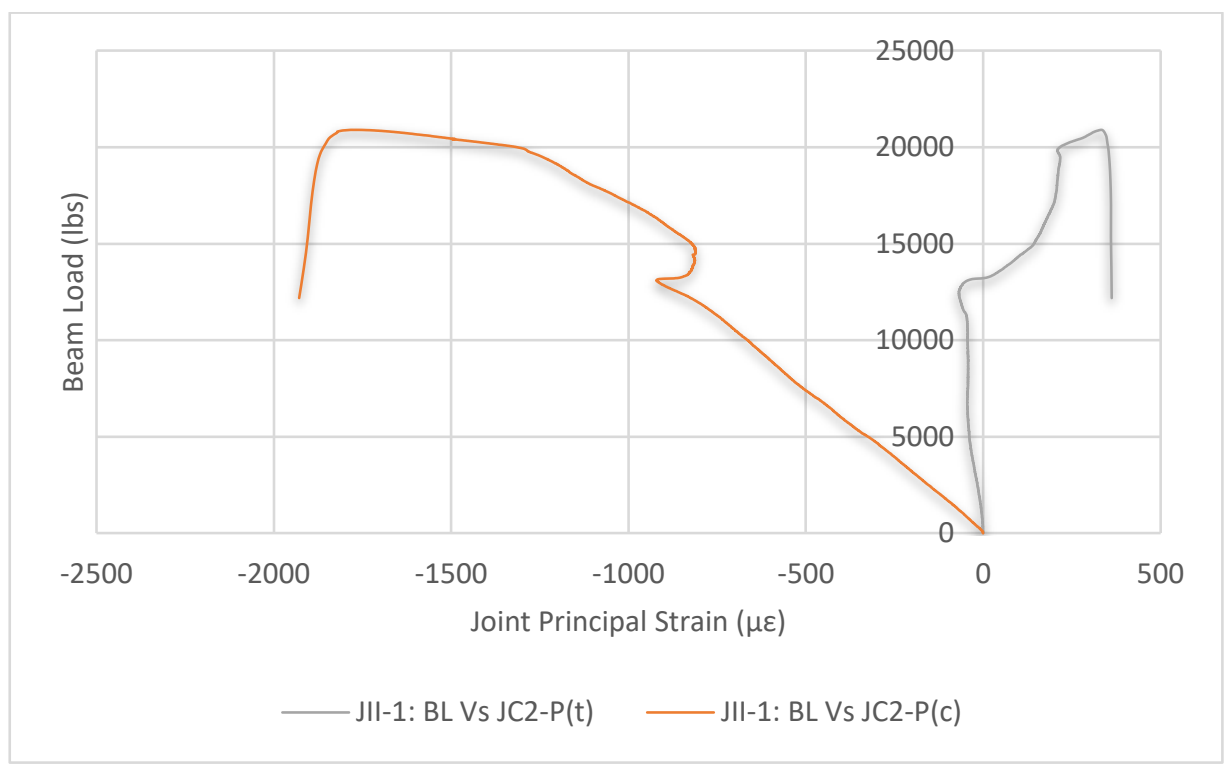

Figure D.5. Beam load vs. joint principal strain

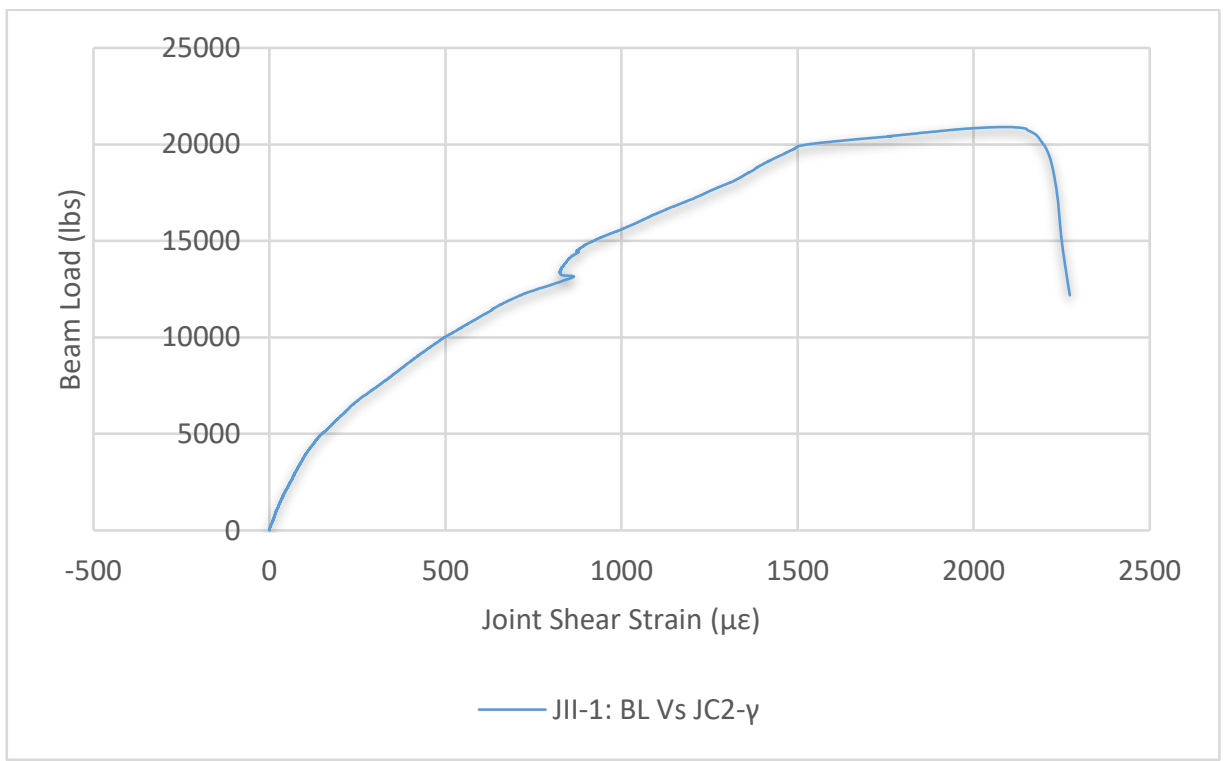

Figure D.6. Beam load vs. joint shear strain 


\section{D.3 Specimen JII-3}

\section{D.3.1 Strain in steel rebar}

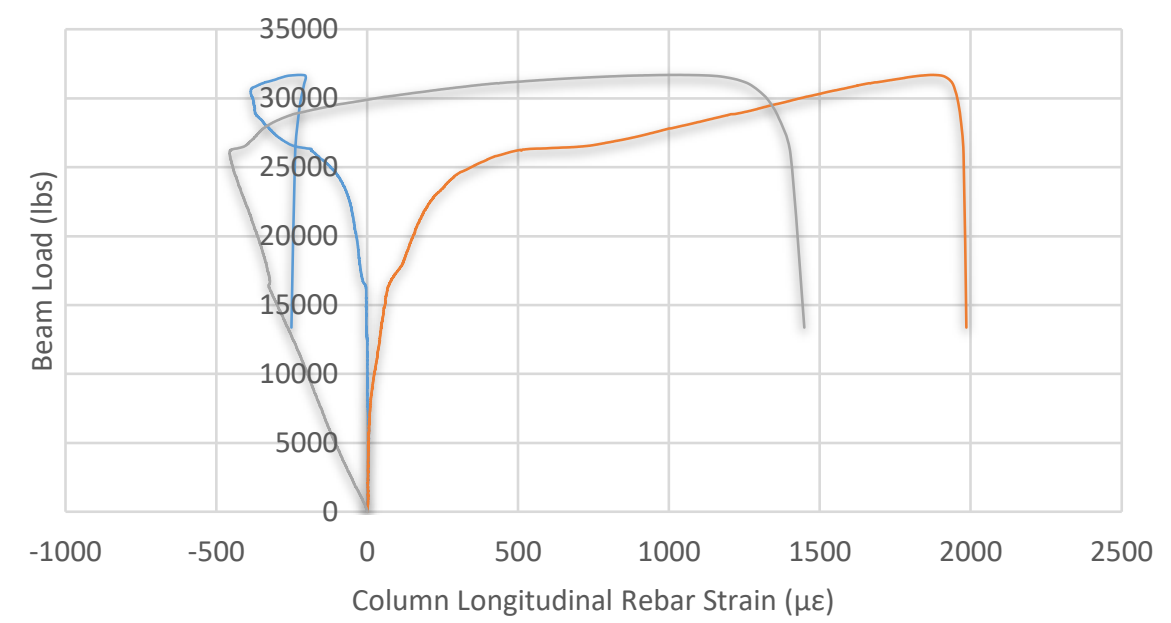

JII-3: BL Vs CSL1 — JII-3: BL Vs CSL2 — JI-3: BL Vs CLS3

Figure D.7. Beam load vs. column longitudinal rebar strain

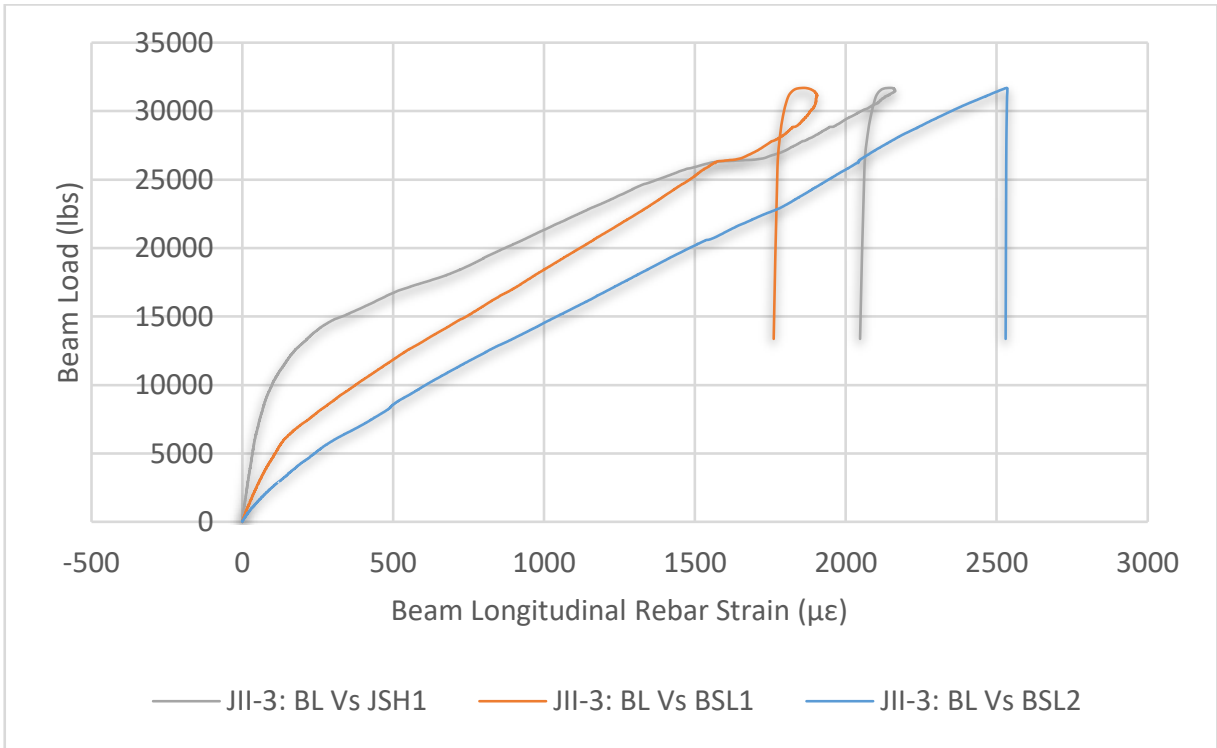

Figure D.8. Beam load vs. beam longitudinal rebar strain 


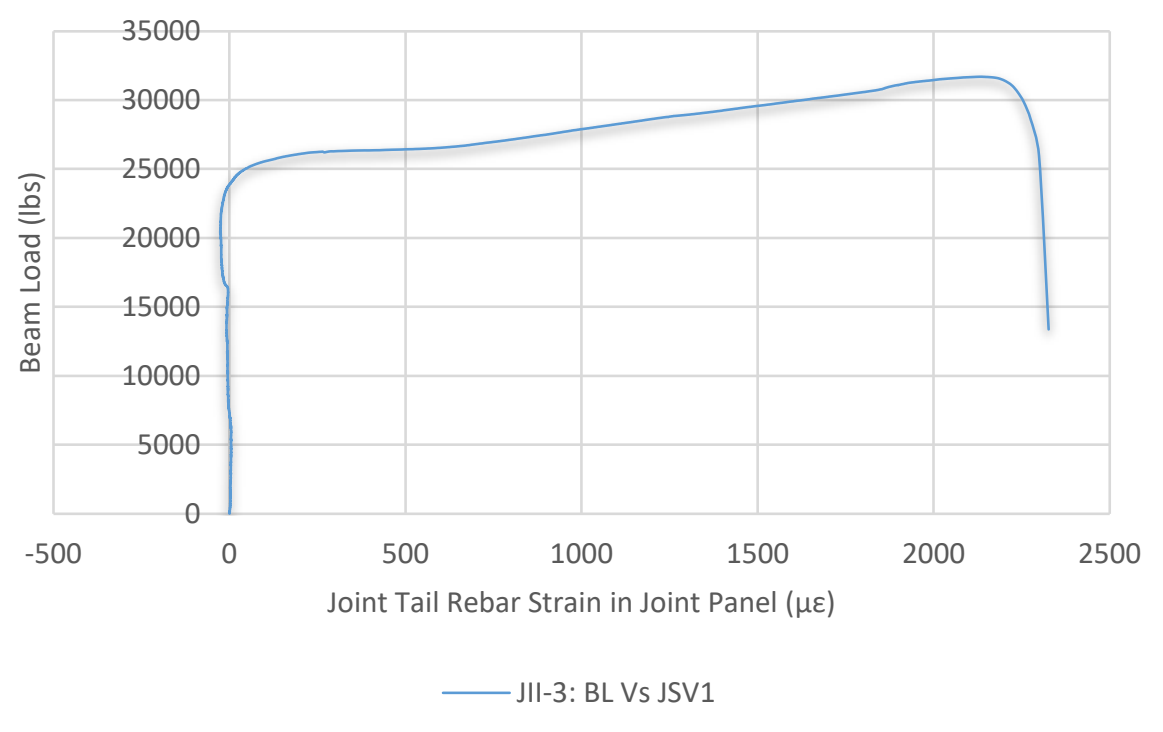

Figure D.9. Beam load vs. joint tail rebar strain

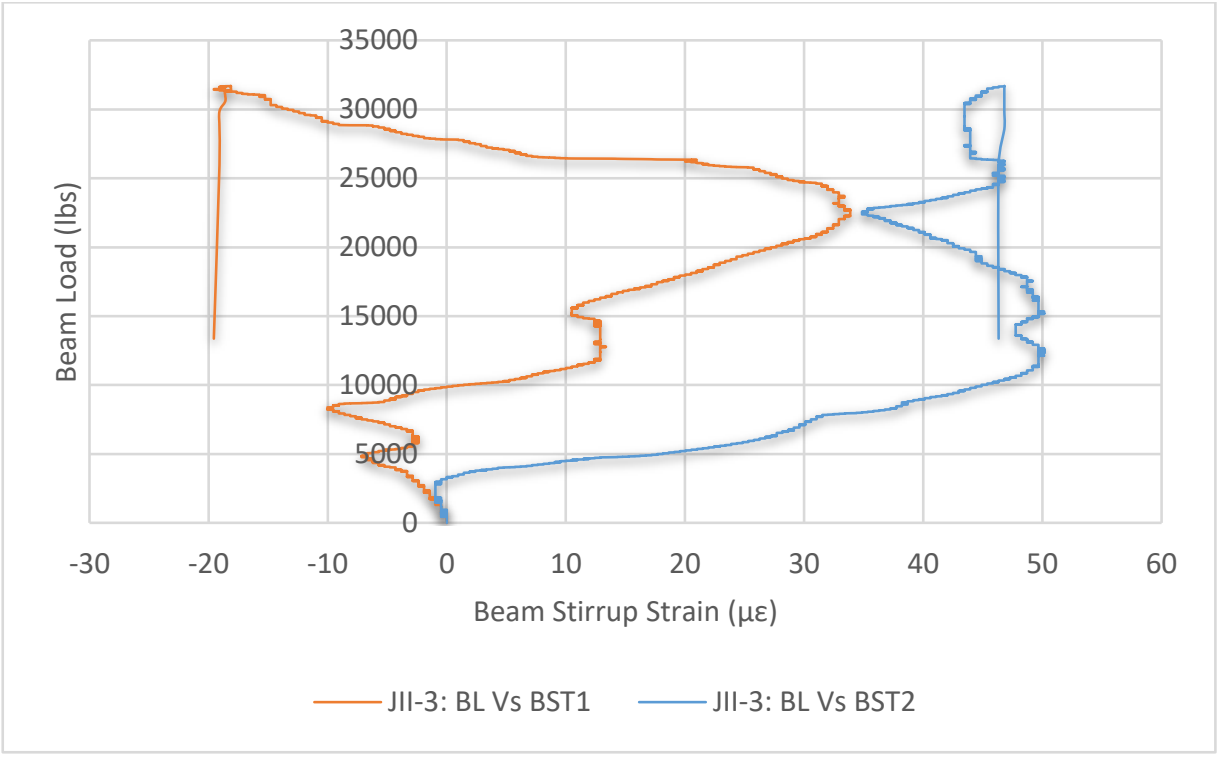

Figure D.10. Beam load vs. beam stirrup strain 


\section{D.3.2 Strain in concrete}

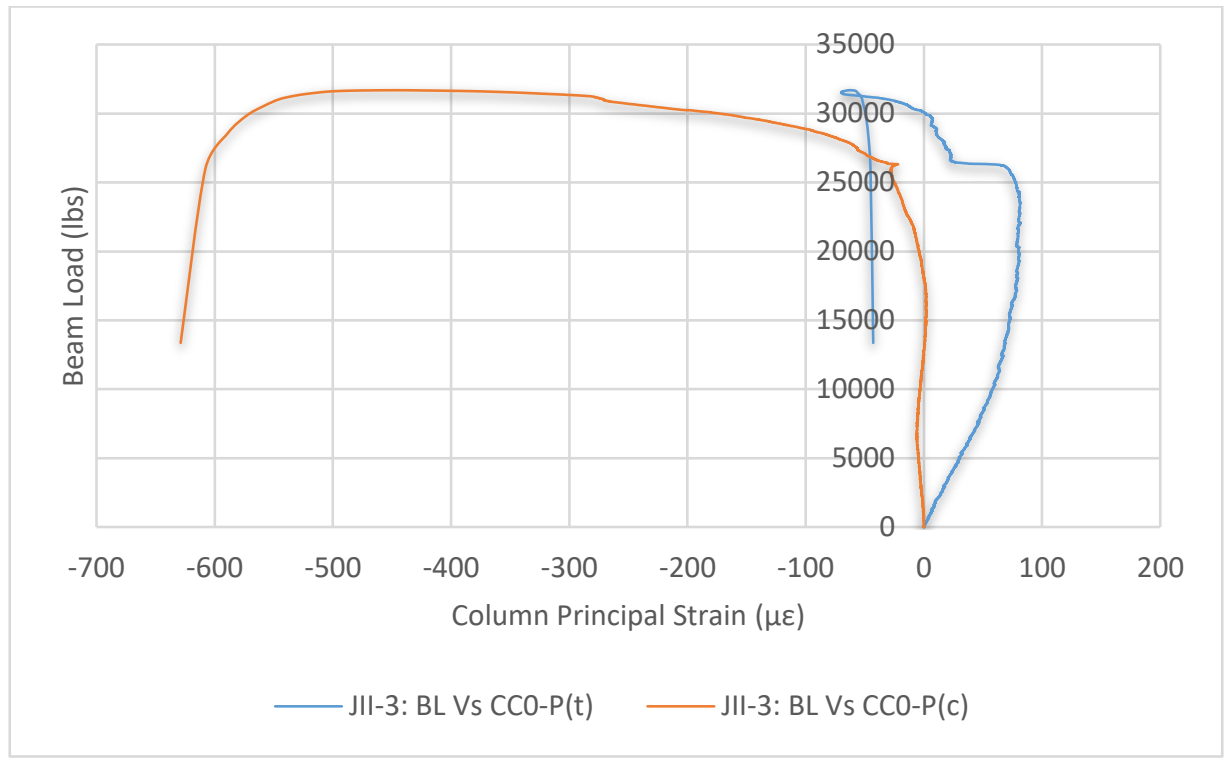

Figure D.11. Beam load vs. column principal strain

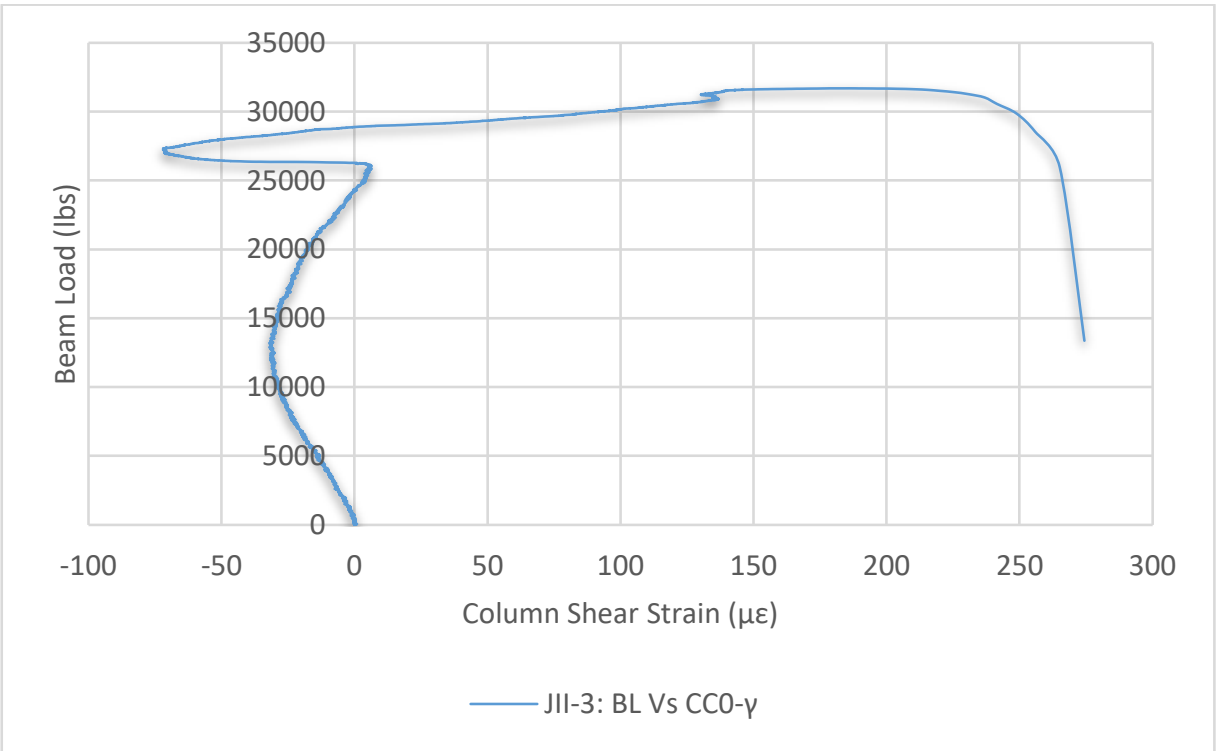

Figure D.12. Beam load vs. column shear strain 


\section{D.4 Specimen JII-4}

\section{D.4.1 Strain in steel rebar}

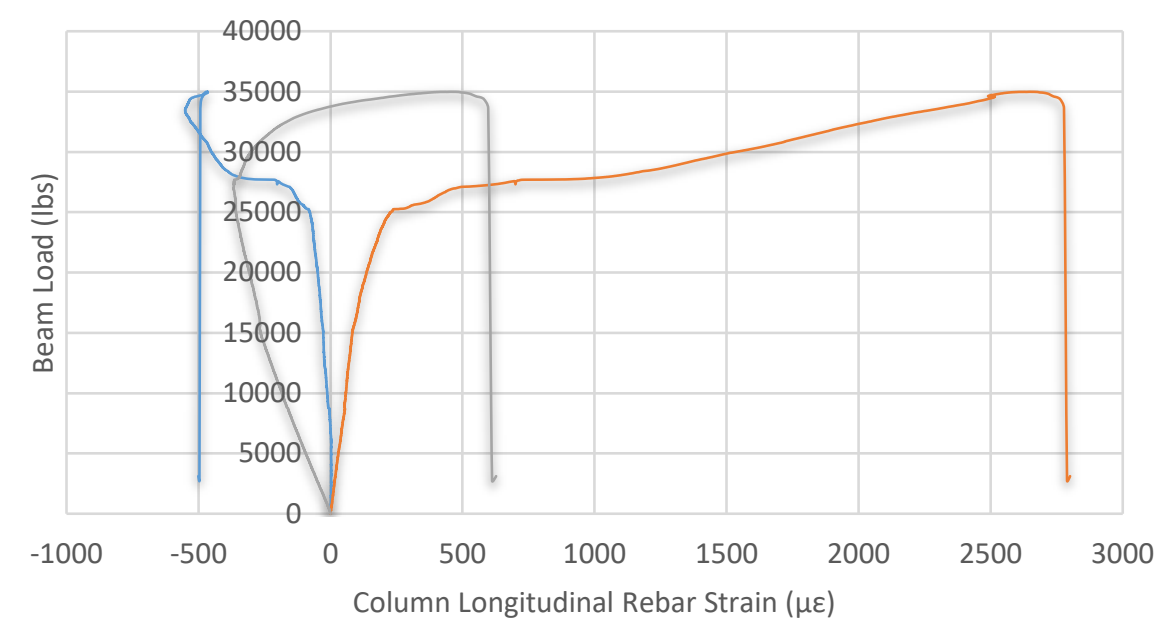

JII-4: BL Vs CSL1 — JII-4: BL Vs CSL2 — JI-4: BL Vs CLS3

Figure D.13. Beam load vs. column longitudinal rebar strain

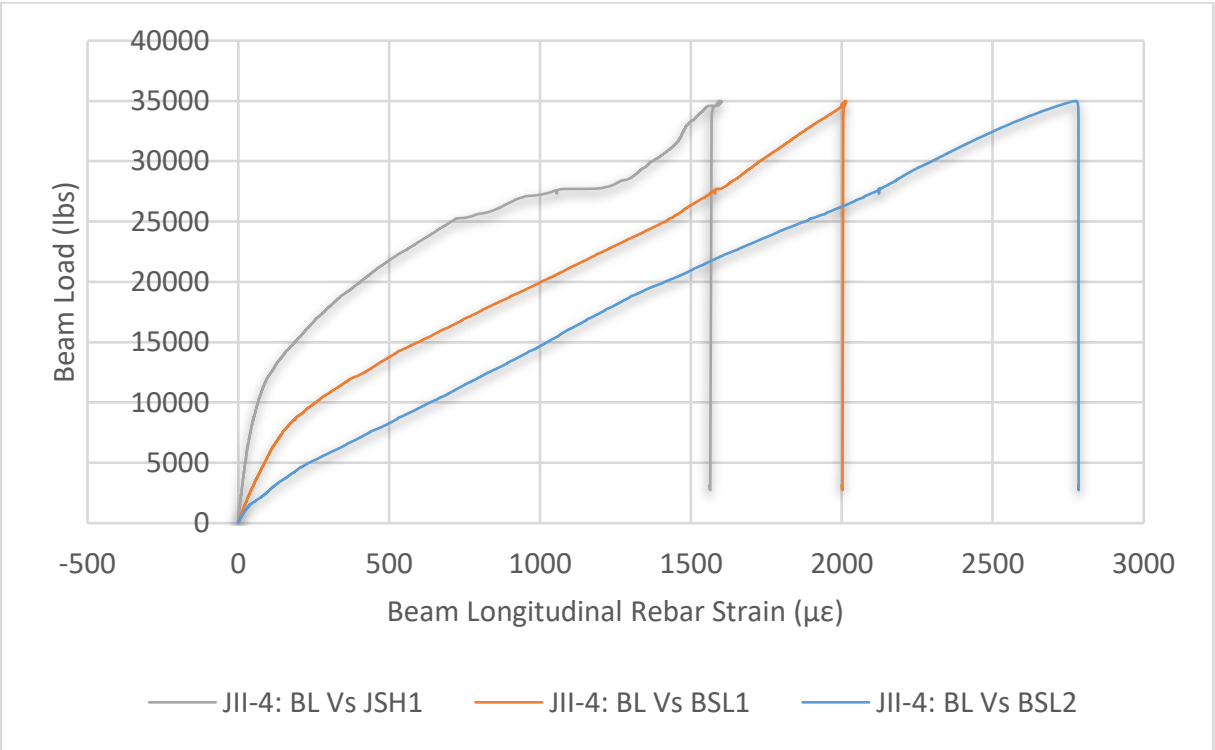

Figure D.14. Beam load vs. beam longitudinal rebar strain 


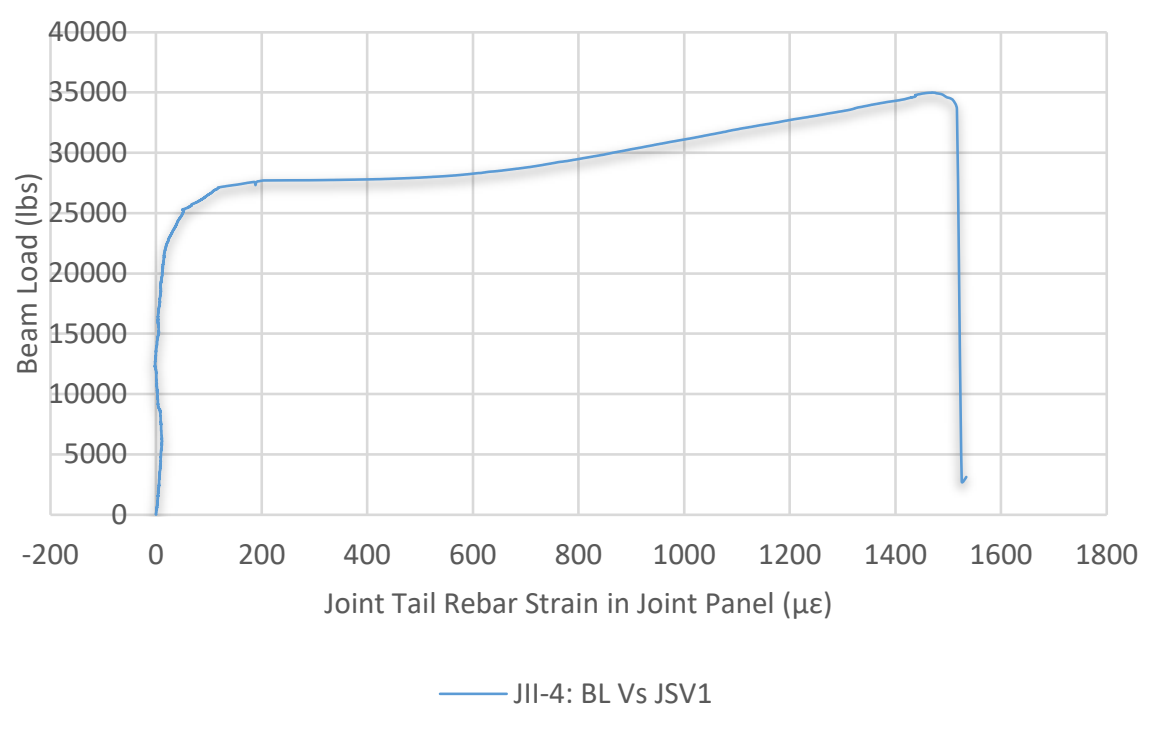

Figure D.15. Beam load vs. joint tail rebar strain

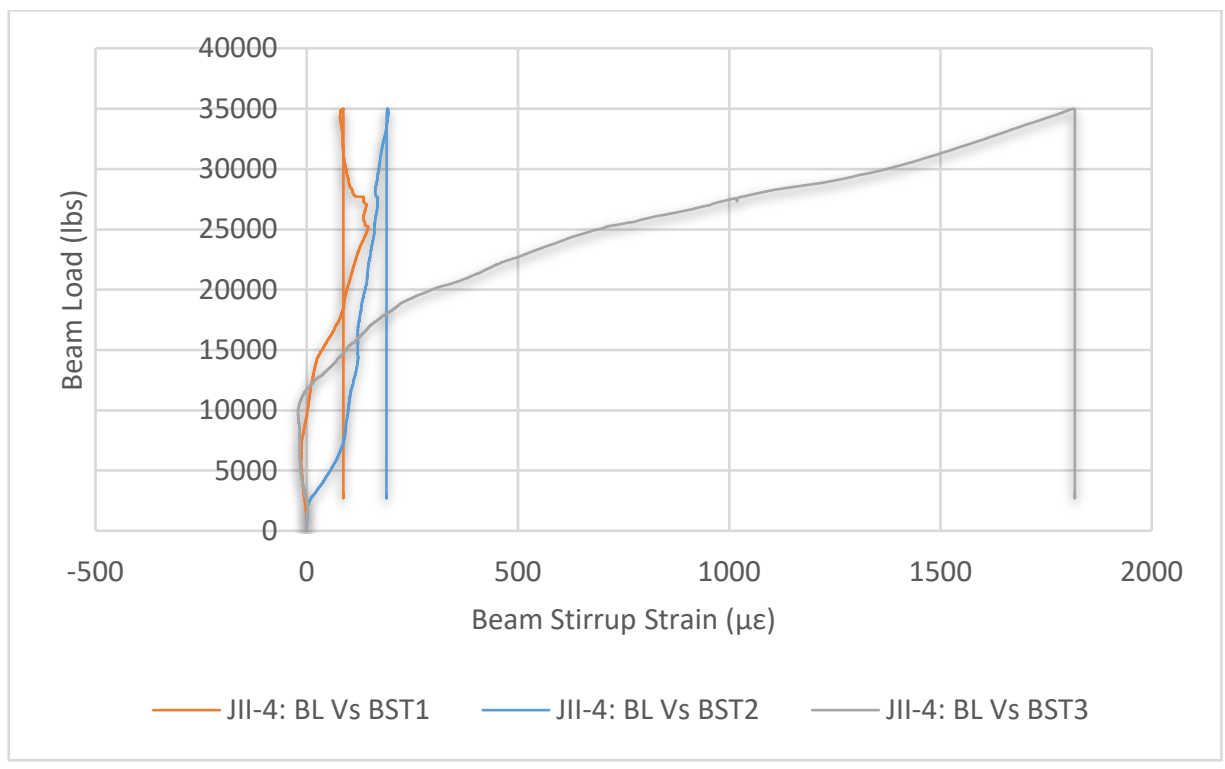

Figure D.16. Beam load vs. beam stirrup strain 


\section{D.4.2 Strain in concrete}

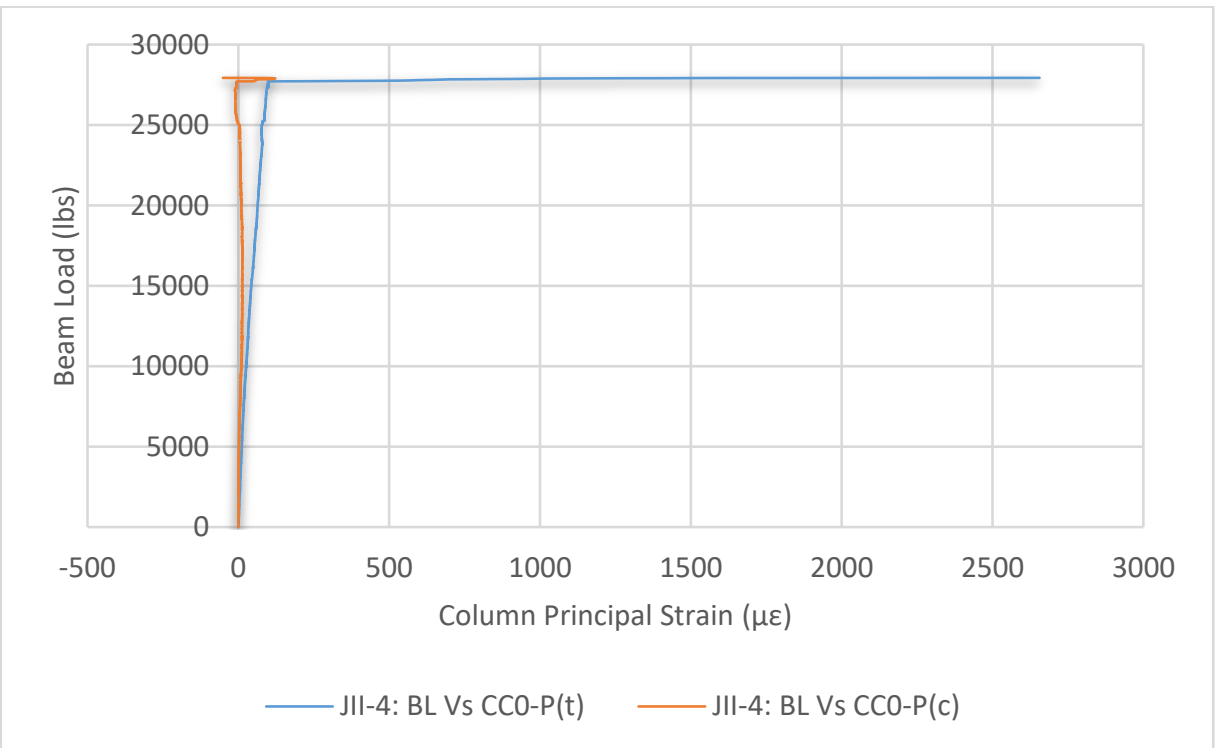

Figure D.17. Beam load vs. column principal strain

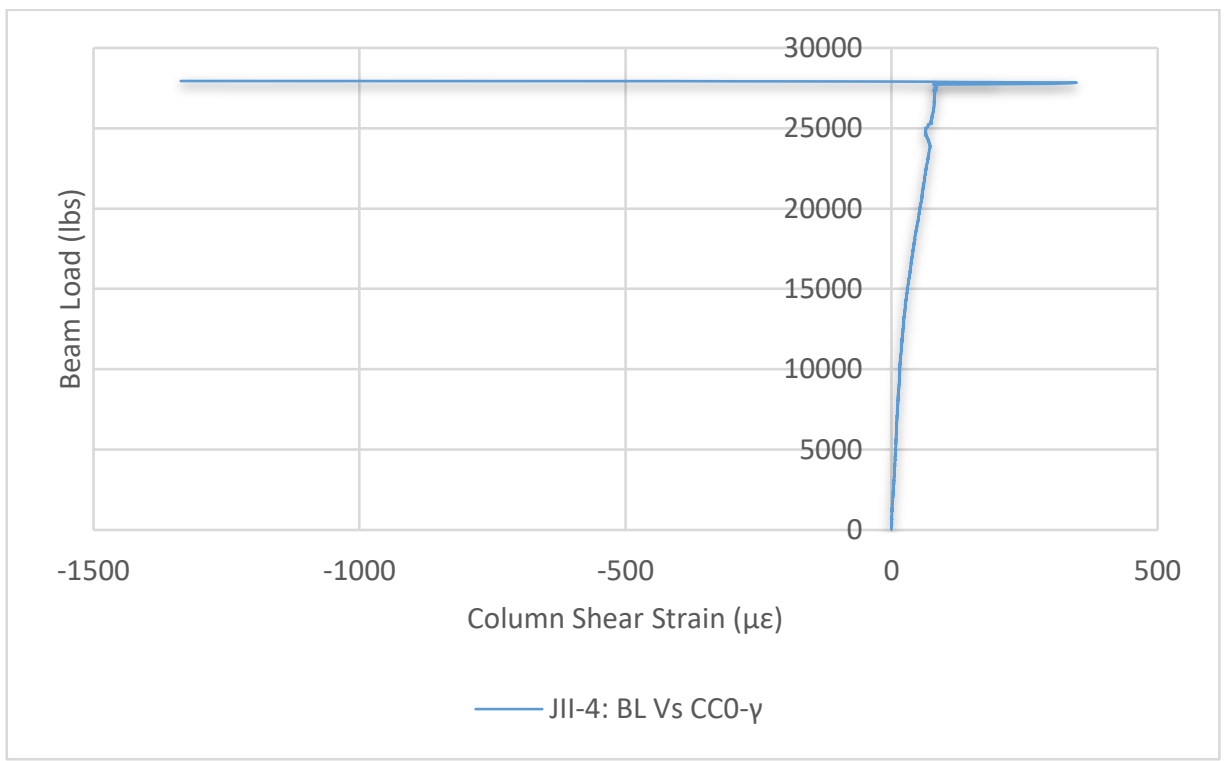

Figure D.18. Beam load vs. column shear strain 


\section{D.5 Specimen JII-5}

\section{D.5.1 Strain in steel rebar}

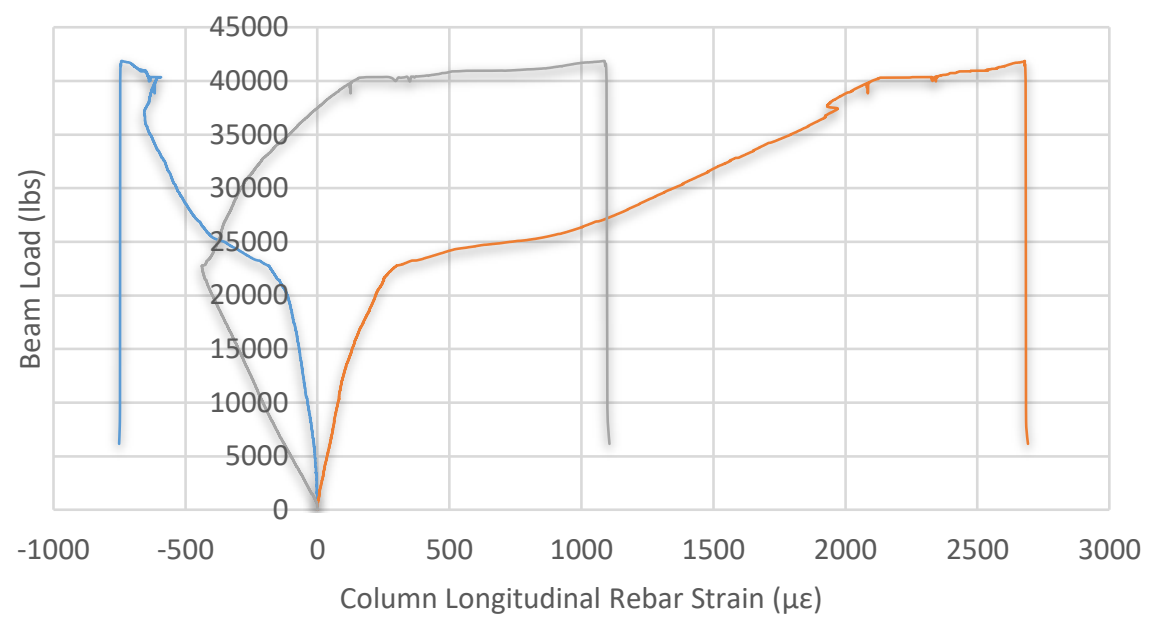

JII-5: BL Vs CSL1_—JI-5: BL Vs CSL2 — JII-5: BL Vs CLS3

Figure D.19. Beam load vs. column longitudinal rebar strain

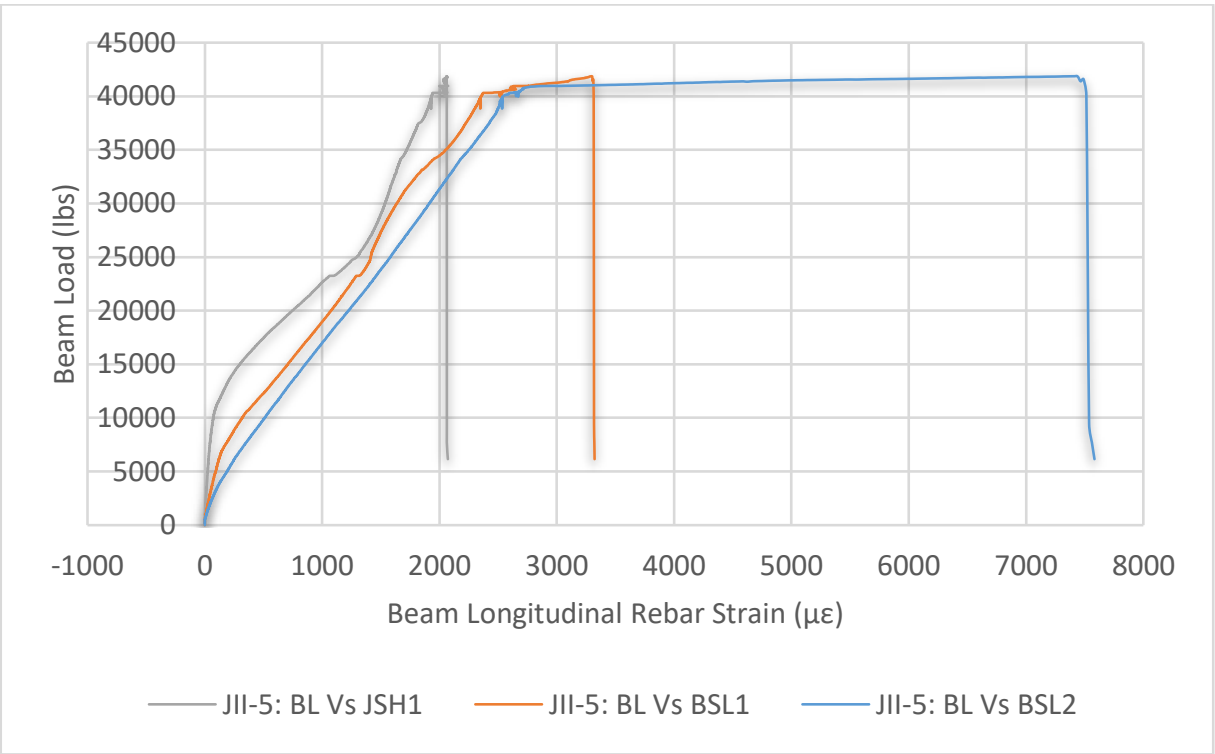

Figure D.20. Beam load vs. beam longitudinal rebar strain 


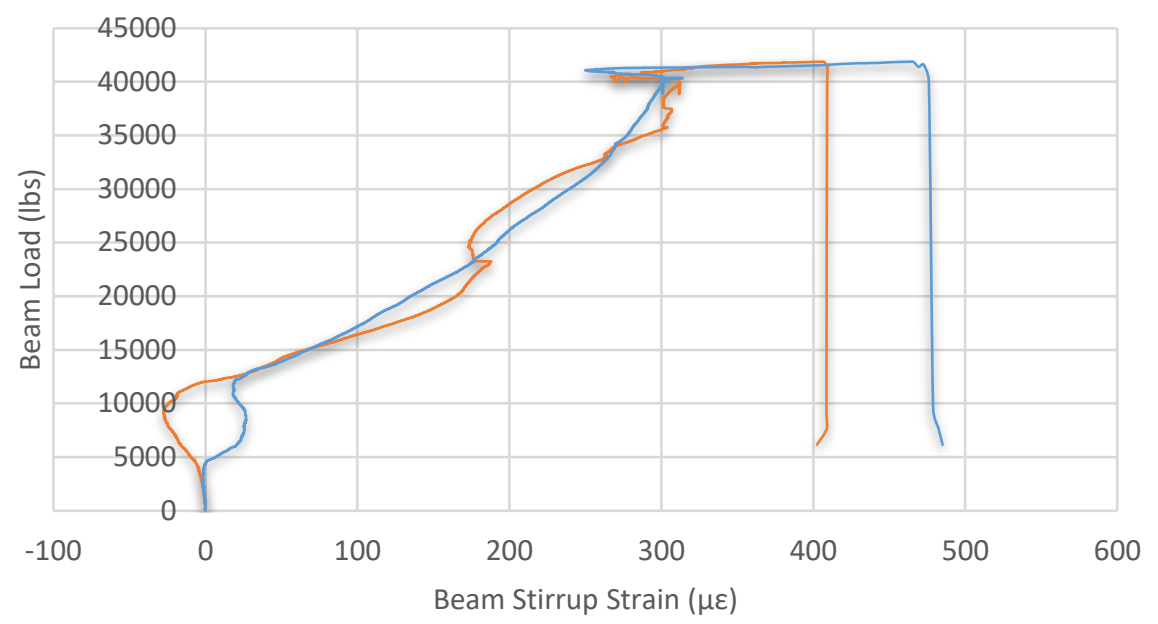

— JII-5: BL Vs BST1 — JII-5: BL Vs BST2

Figure D.21. Beam load vs. beam stirrup strain

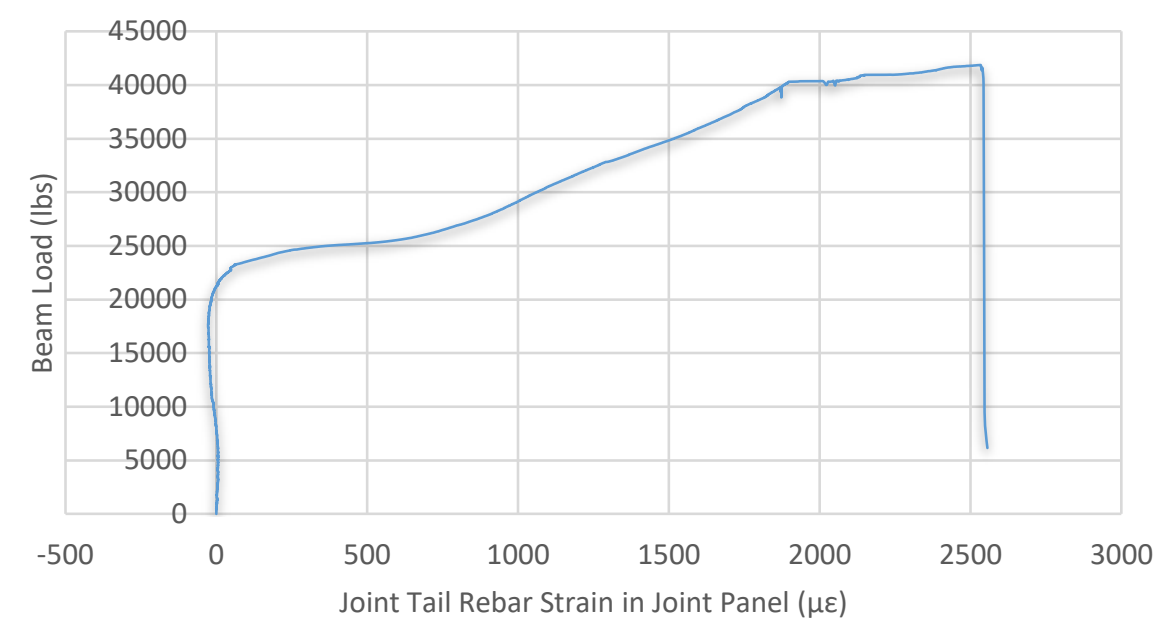

JII-5: BL Vs JSV1

Figure D.22. Beam load vs. joint tail rebar strain 


\section{D.5.2 Strain in FRP}

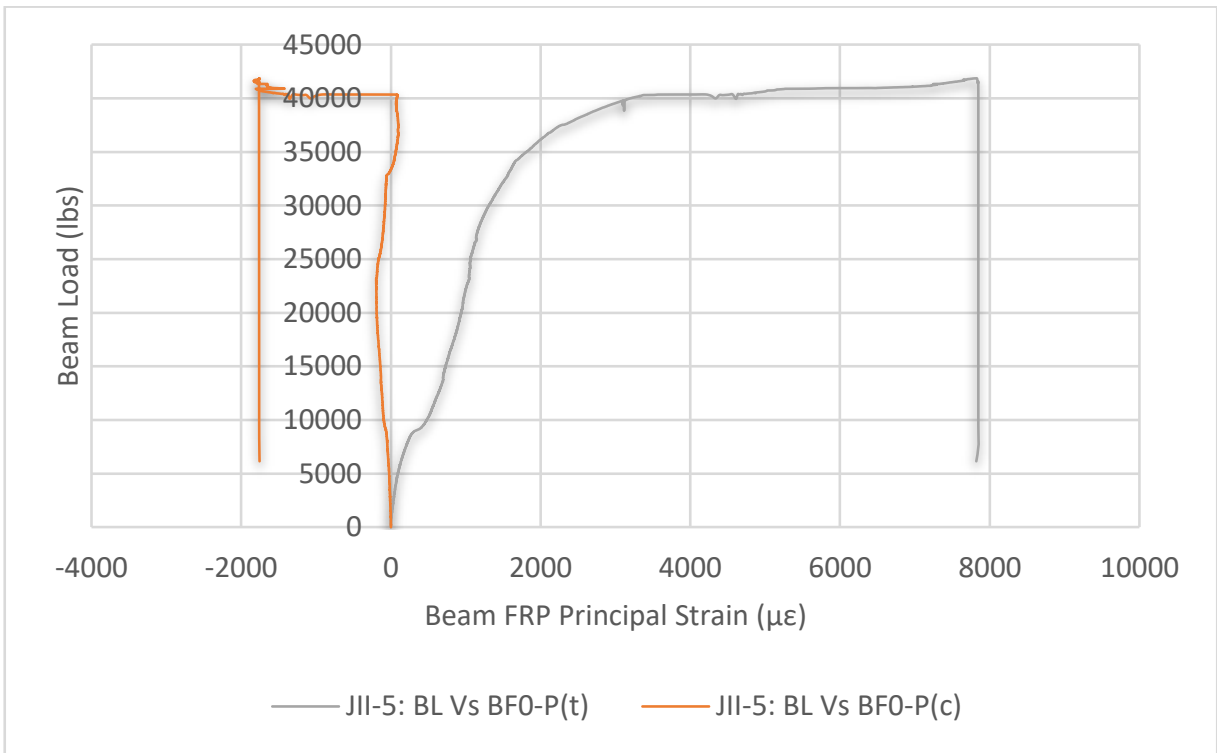

Figure D.23. Beam load vs. beam principal strain

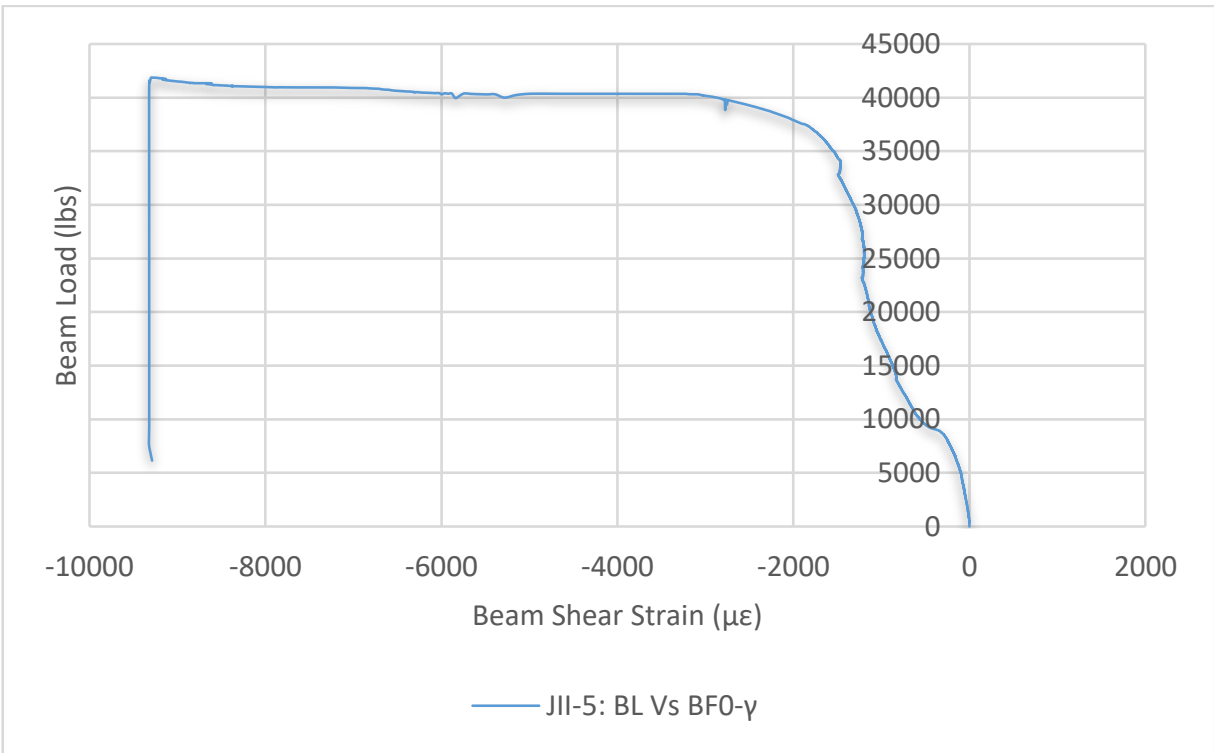

Figure D.24. Beam load vs. beam shear strain 


\section{D.6 Specimen JII-6}

\section{D.6.1 Strain in steel rebar}

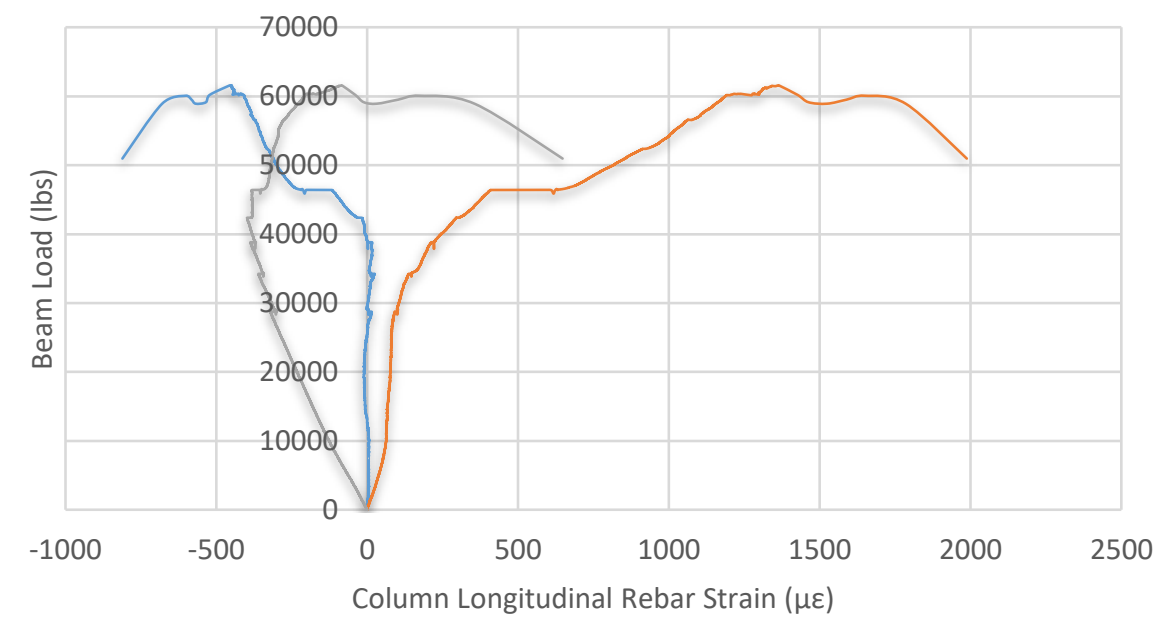

JII-6: BL Vs CSL1 — JII-6: BL Vs CSL2 _ JII-6: BL Vs CLS3

Figure D.25. Beam load vs. column longitudinal rebar strain

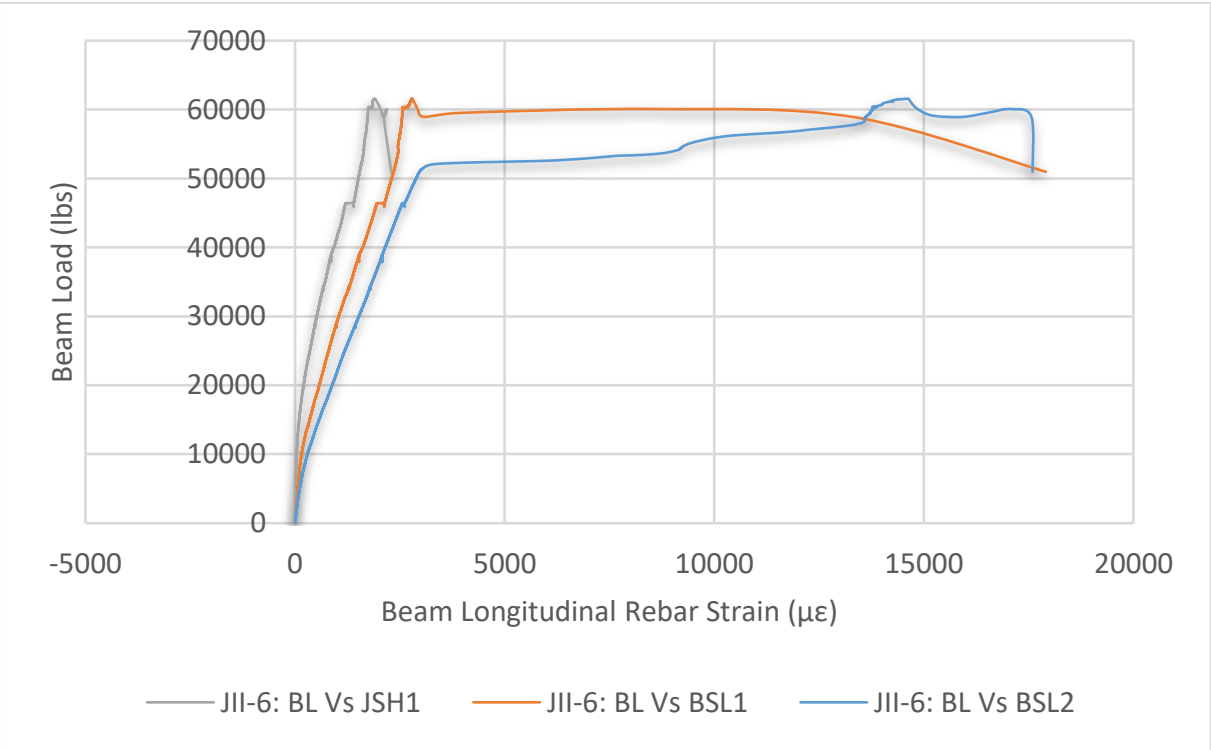

Figure D.26. Beam load vs. beam longitudinal rebar strain 


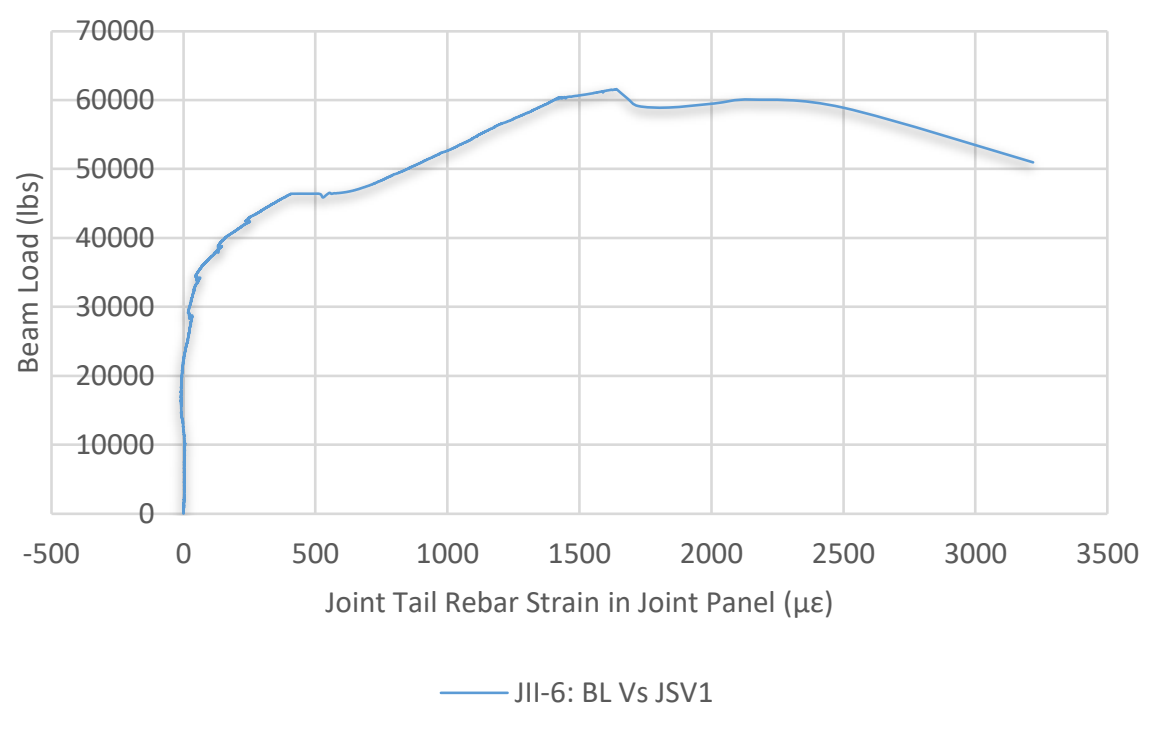

Figure D.27. Beam load vs. joint tail rebar strain

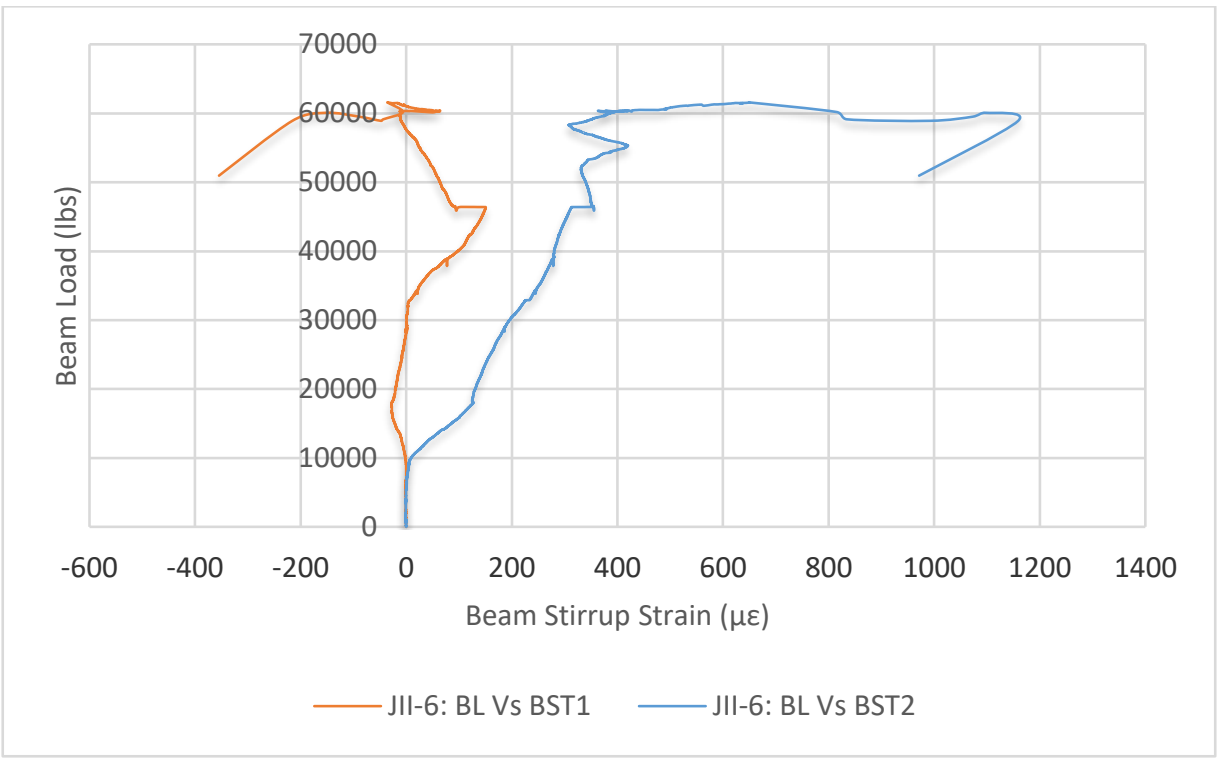

Figure D.28. Beam load vs. beam stirrup strain 


\section{D.6.2 Strain in FRP}

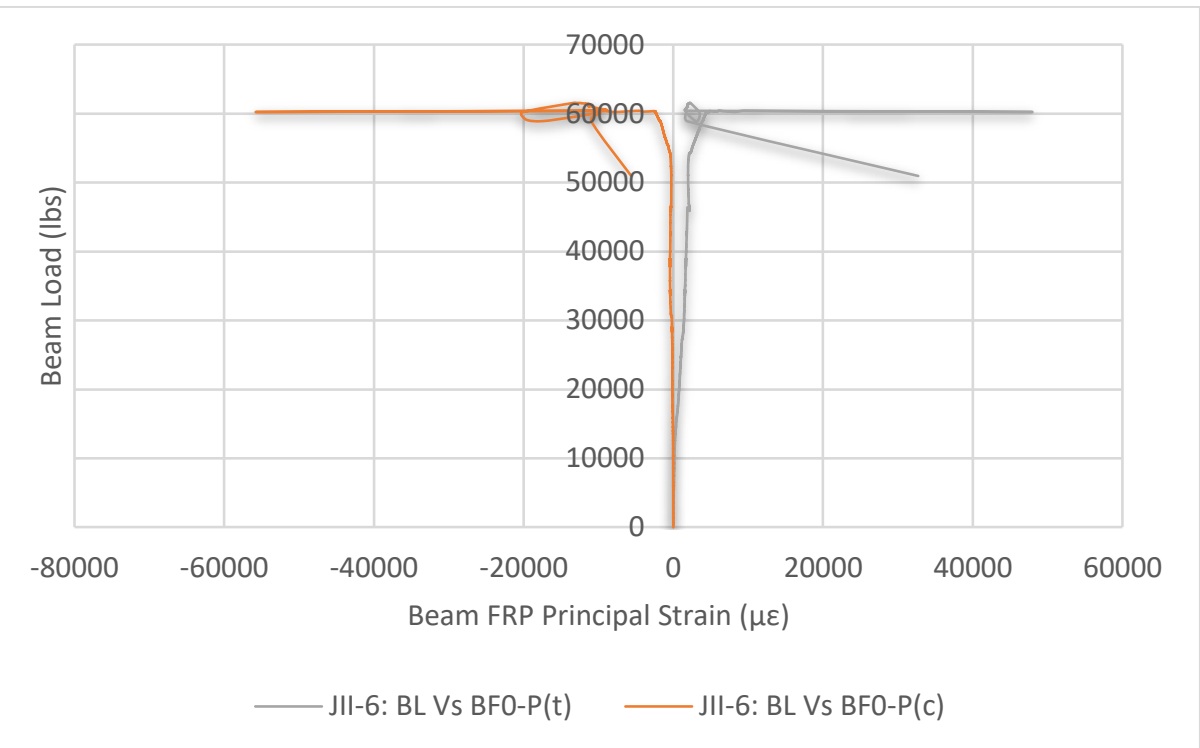

Figure D.29. Beam load vs. beam principal strain

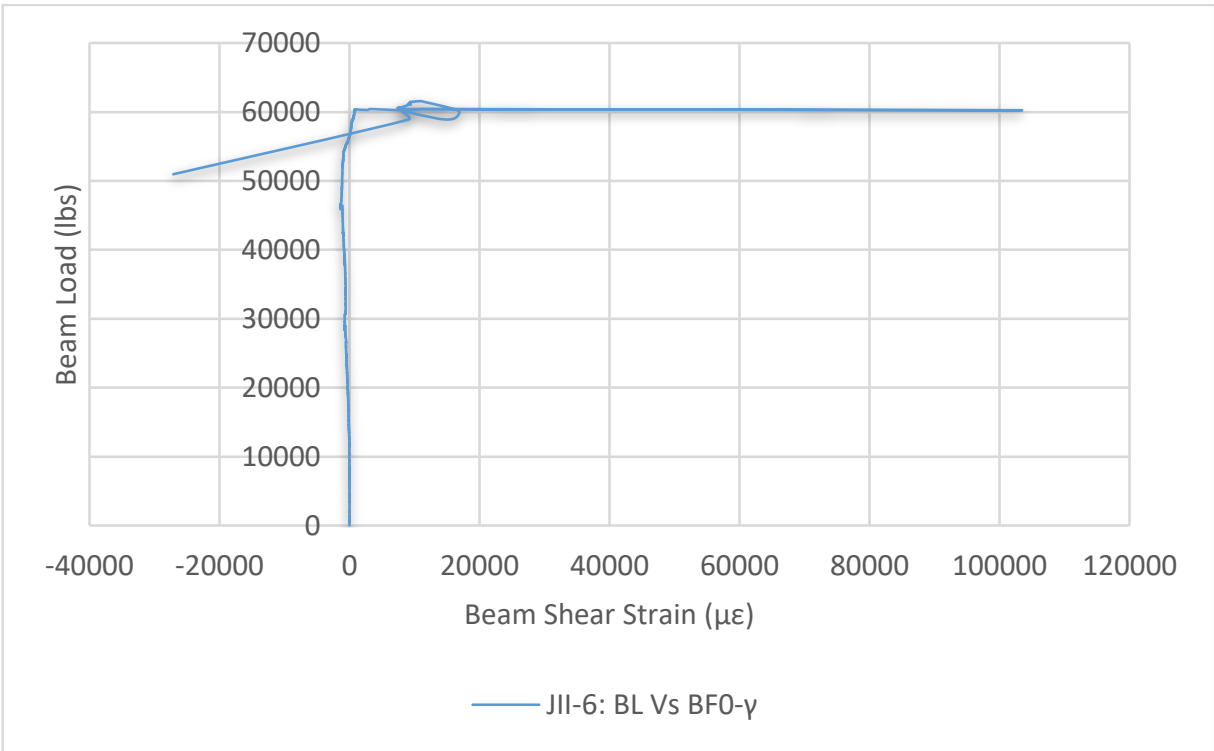

Figure D.30. Beam load vs. beam shear strain 


\section{D.7 Specimen JII-7}

\section{D.7.1 Strain in steel rebar}

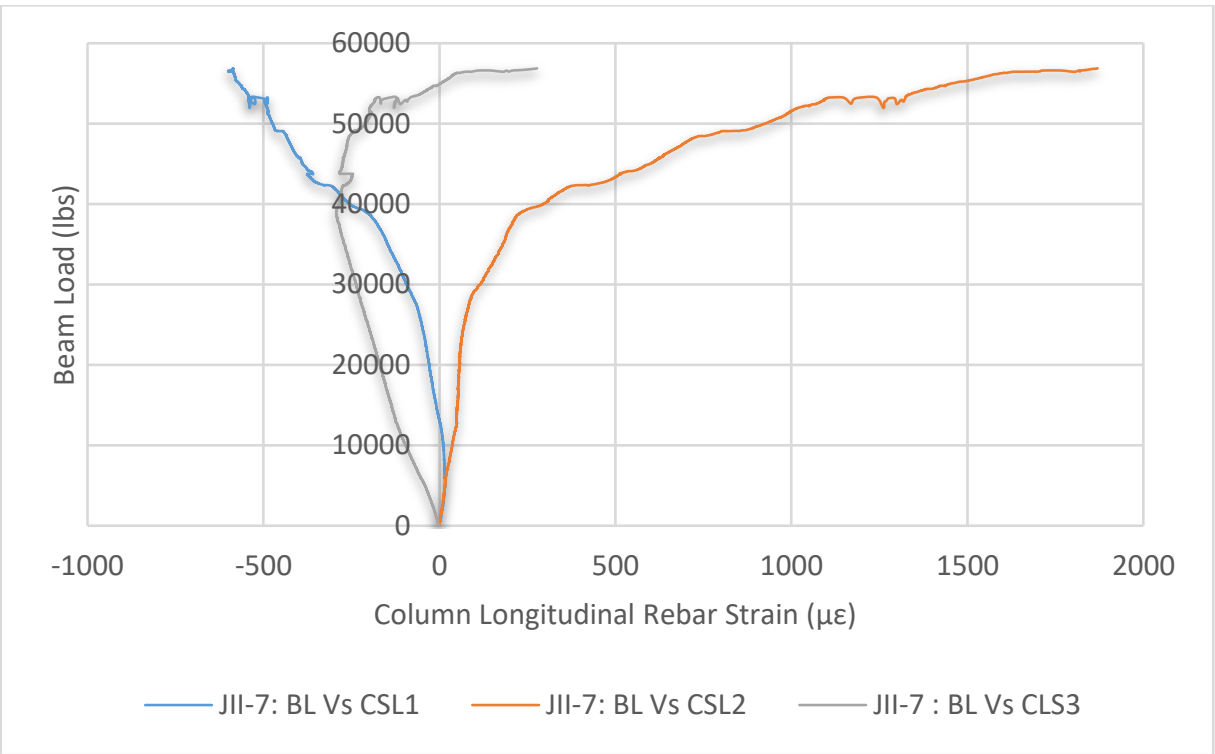

Figure D.31. Beam load vs. column longitudinal rebar strain

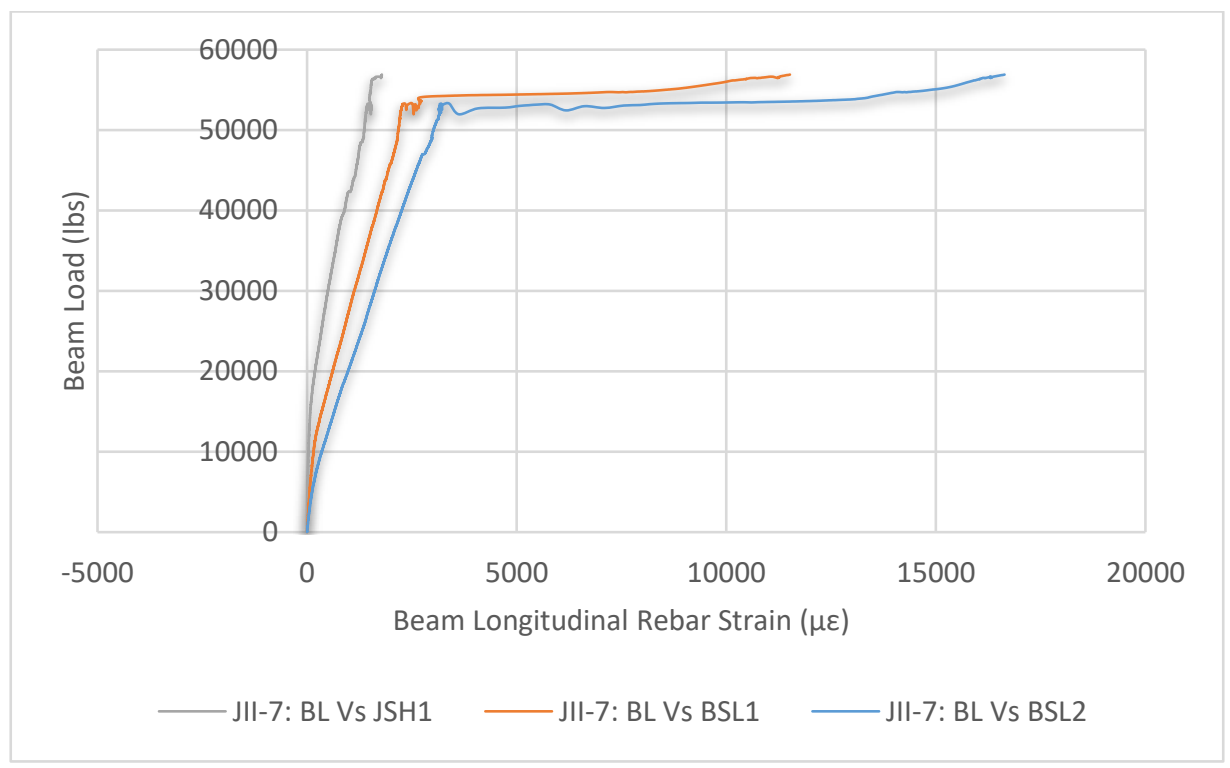

Figure D.32. Beam load vs. beam longitudinal rebar strain 


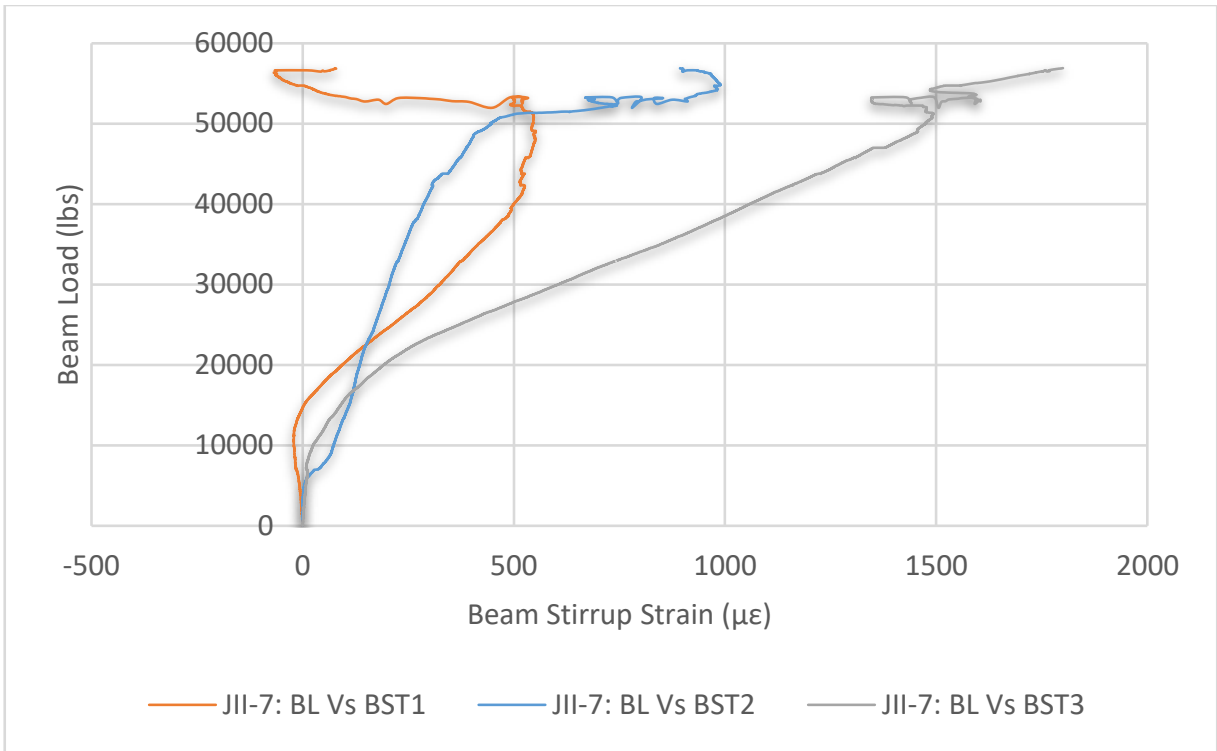

Figure D.33. Beam load vs. beam stirrup strain 


\section{D.8 Specimen JII-8}

\section{D.8.1 Strain in steel rebar}

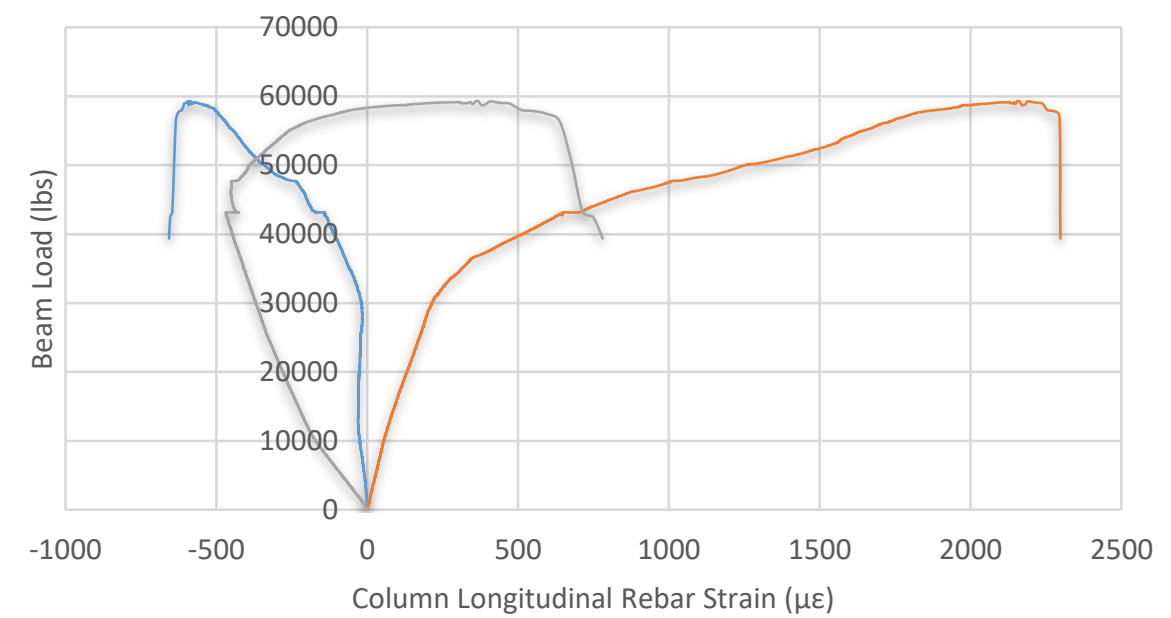

-JII-8: BL Vs CSL1 — JII-8: BL Vs CSL2 JII-8: BL Vs CLS3

Figure D.34. Beam load vs. column longitudinal rebar strain

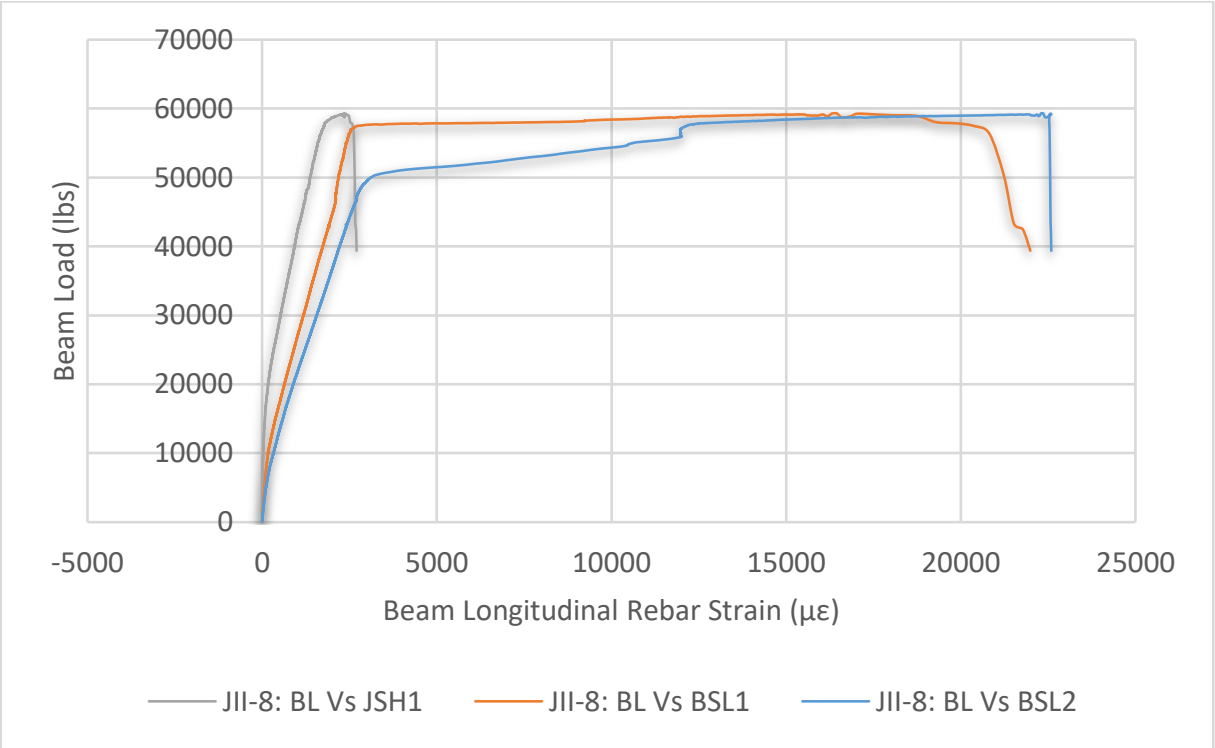

Figure D.35. Beam load vs. beam longitudinal rebar strain 


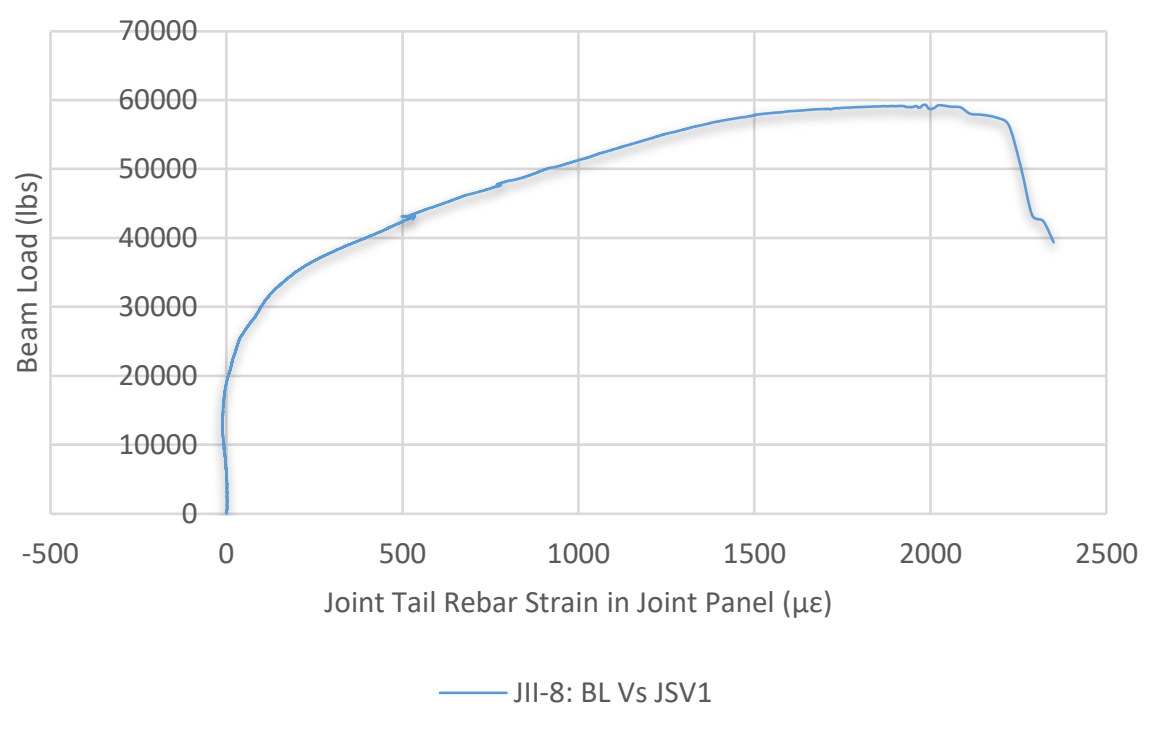

Figure D.36. Beam load vs. joint tail rebar strain

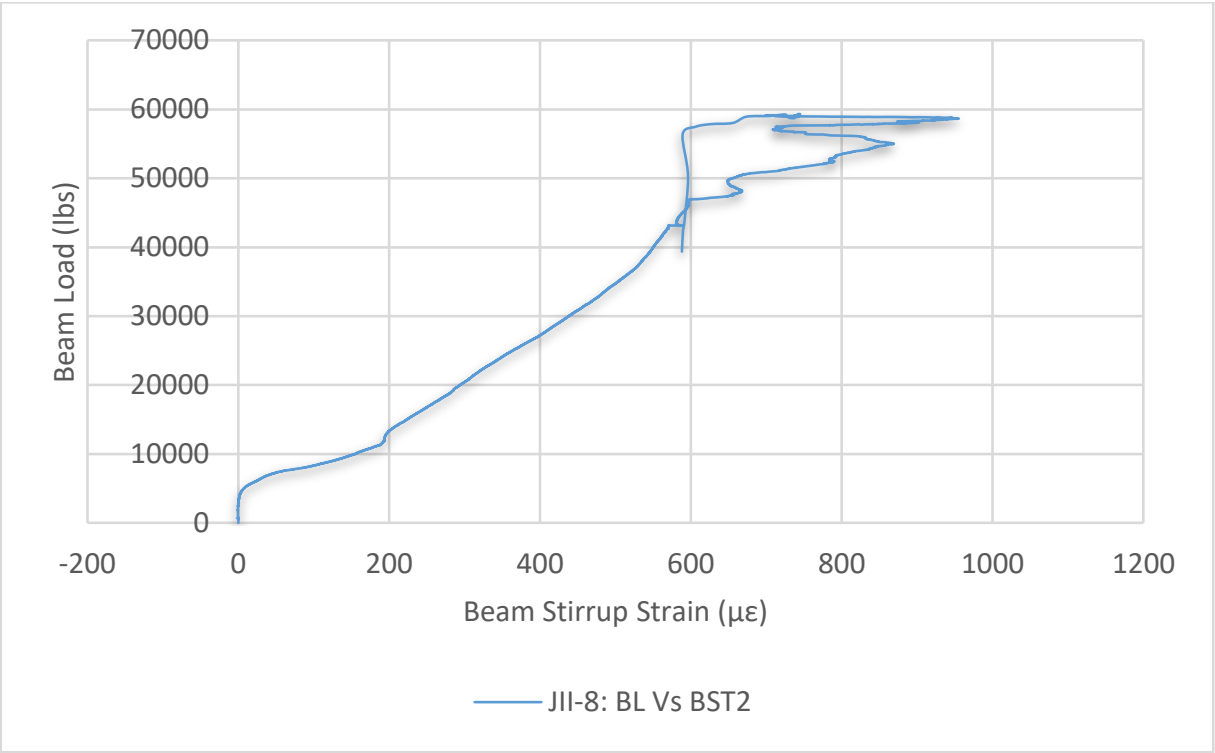

Figure D.37. Beam load vs. beam stirrup strain 


\section{D.8.2 Strain in FRP}

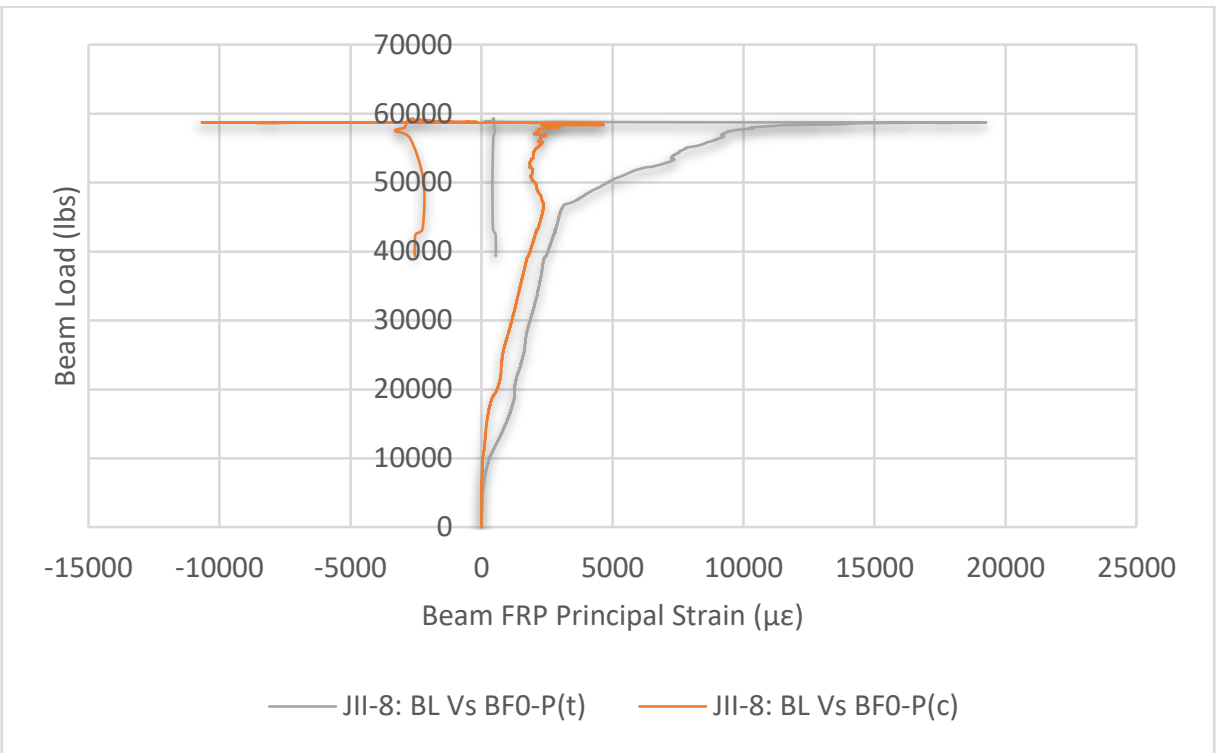

Figure D.38. Beam load vs. beam principal strain

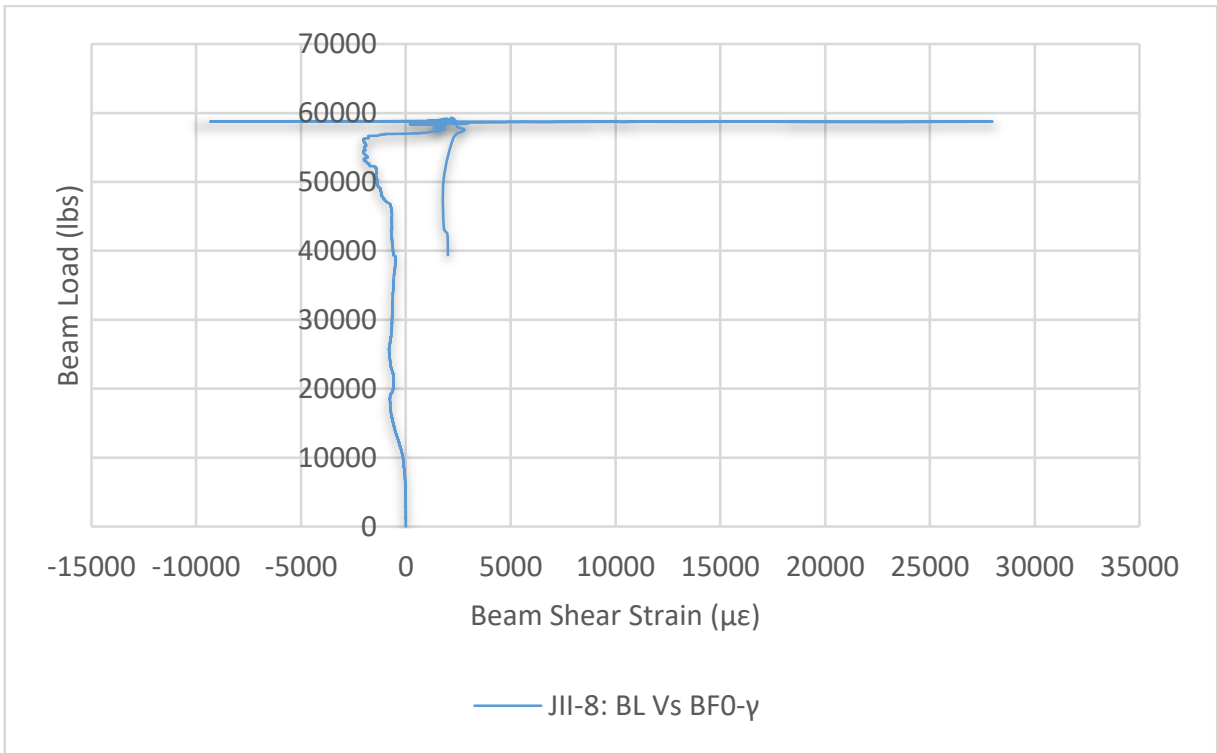

Figure D.39. Beam load vs. beam shear strain 


\section{Appendix-E：FRP Wrapped Concrete Cylinders}

This section provides additional stress vs. strain plots generated through experimental evaluation of FRP wrapped concrete (4in. $x$ 8in.) cylinders under axial-compression and split-tension tests.

\section{E.1 Axial-Compression Test}
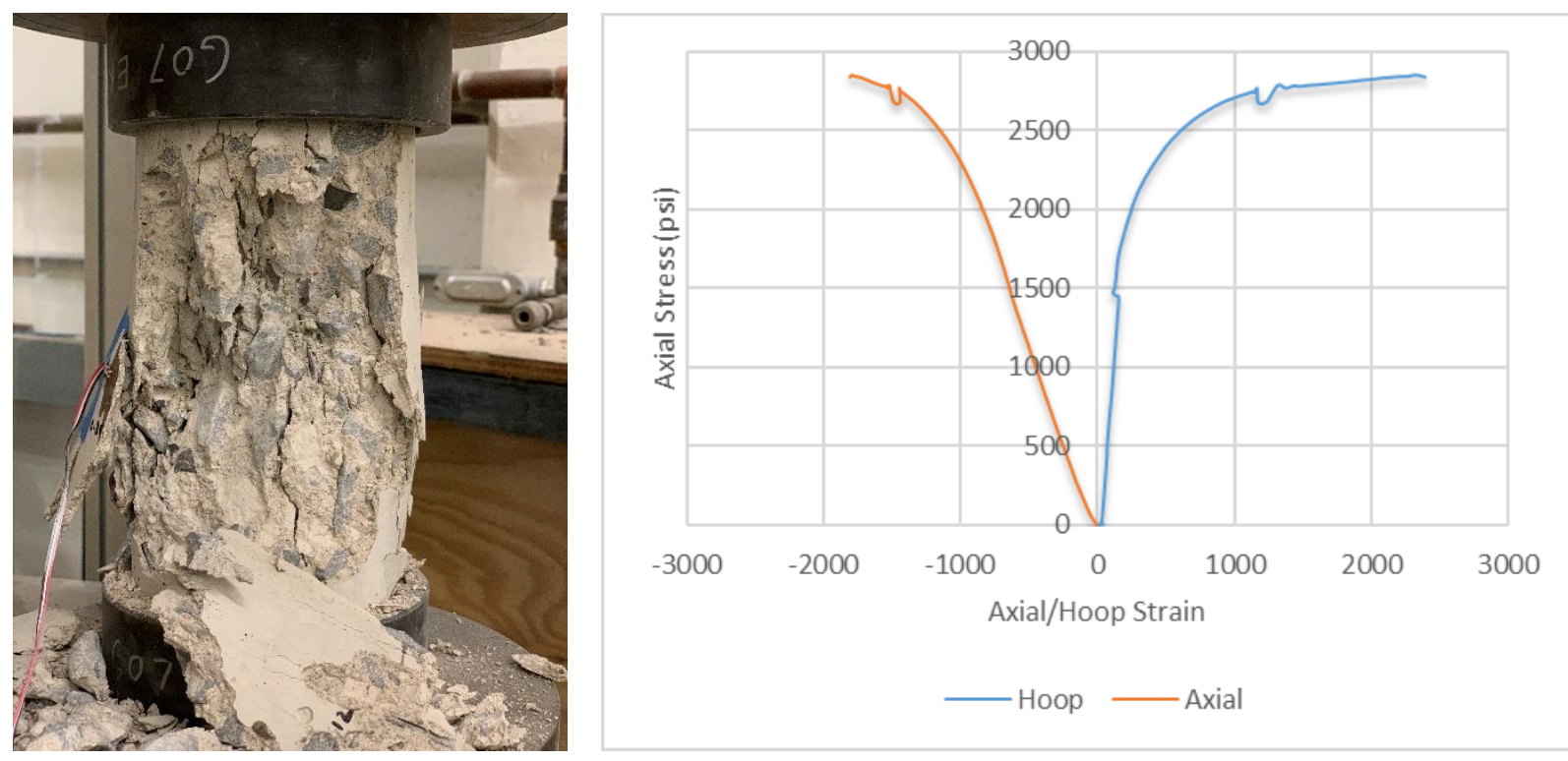

Figure E.1. Stress vs. strain behavior of JI-CS
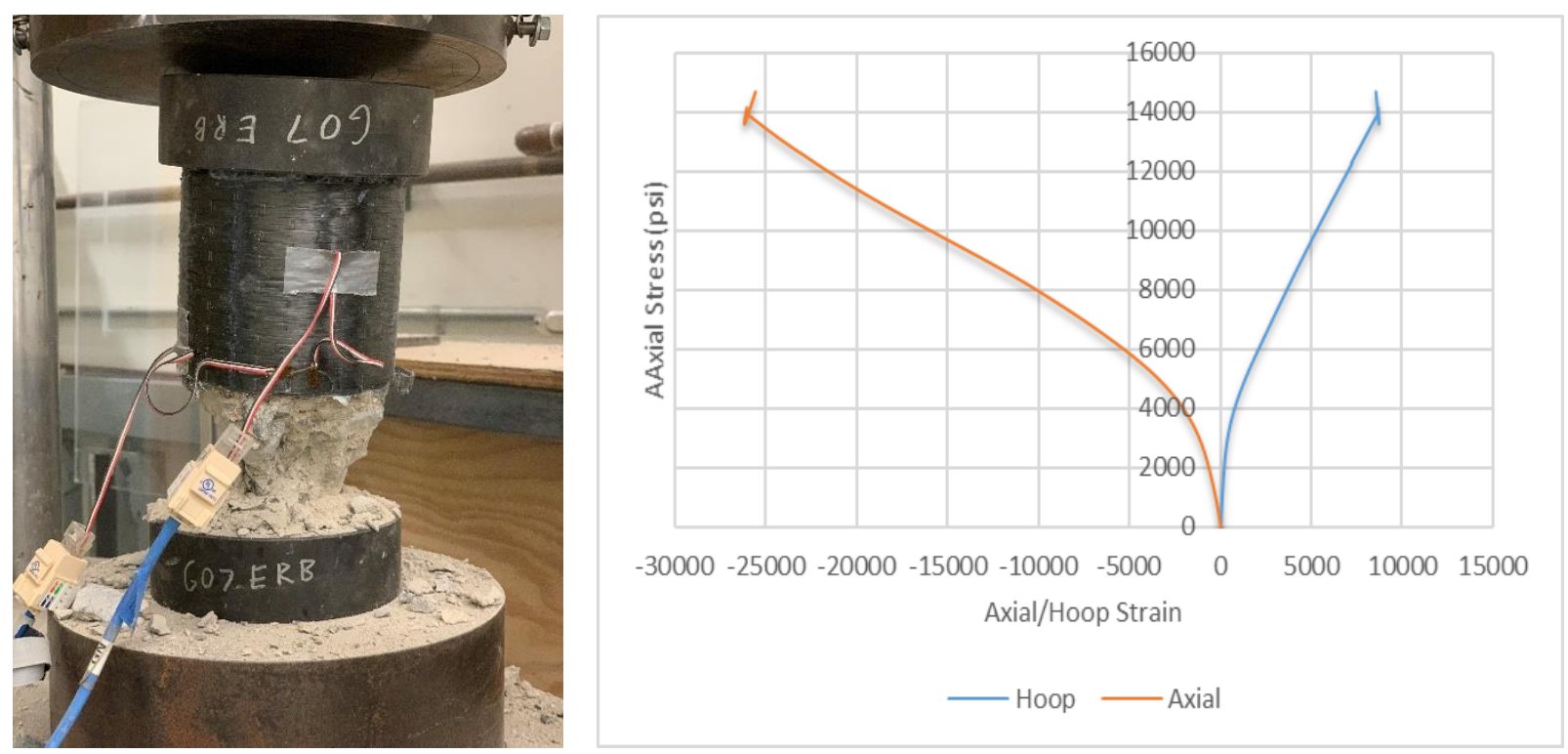

Figure E.2. Stress vs. strain behavior of JI-S1 

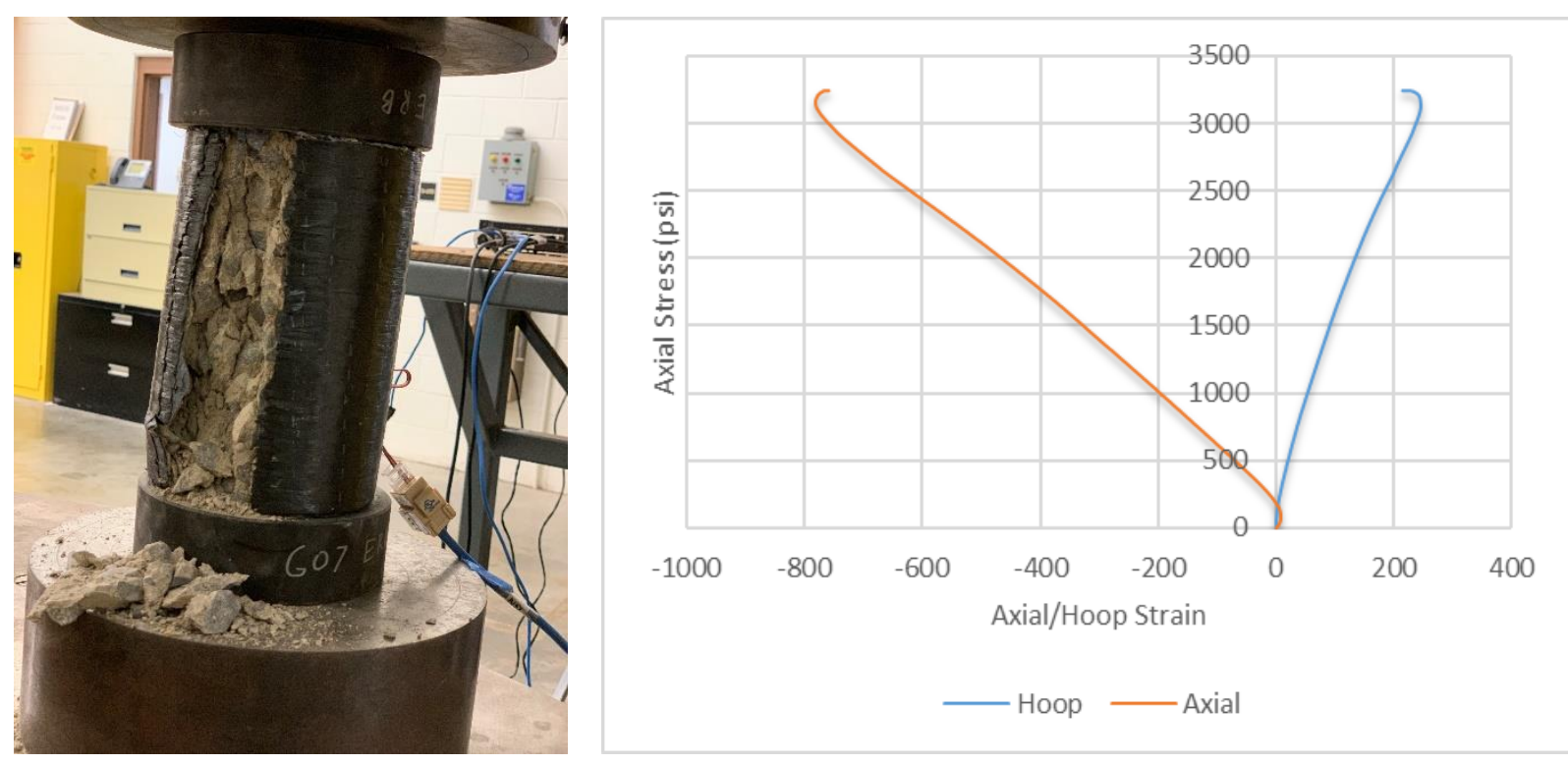

Figure E.3. Stress vs. strain behavior of JI-S2
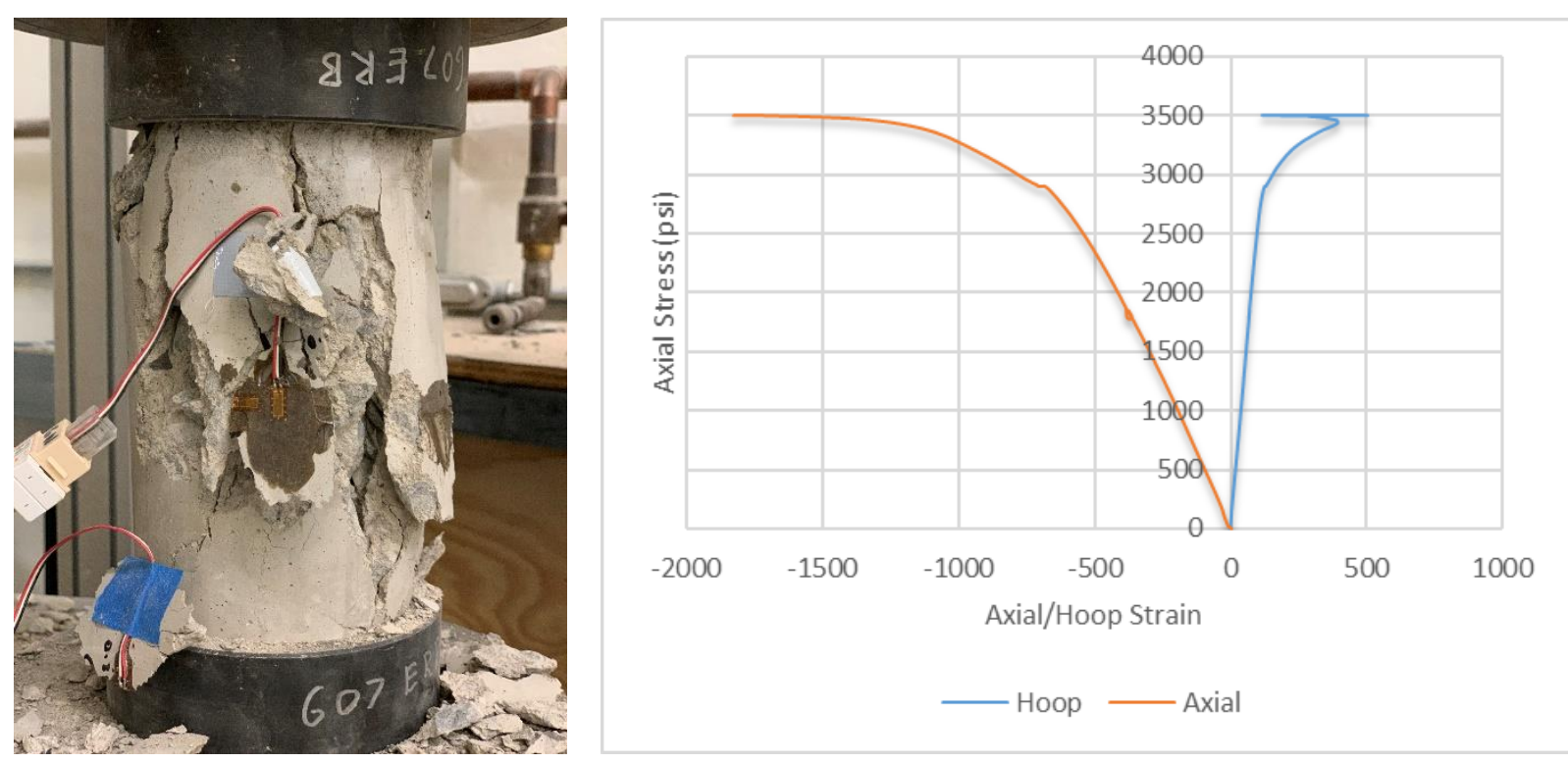

Figure E.4. Stress vs. strain behavior of JII-CS 

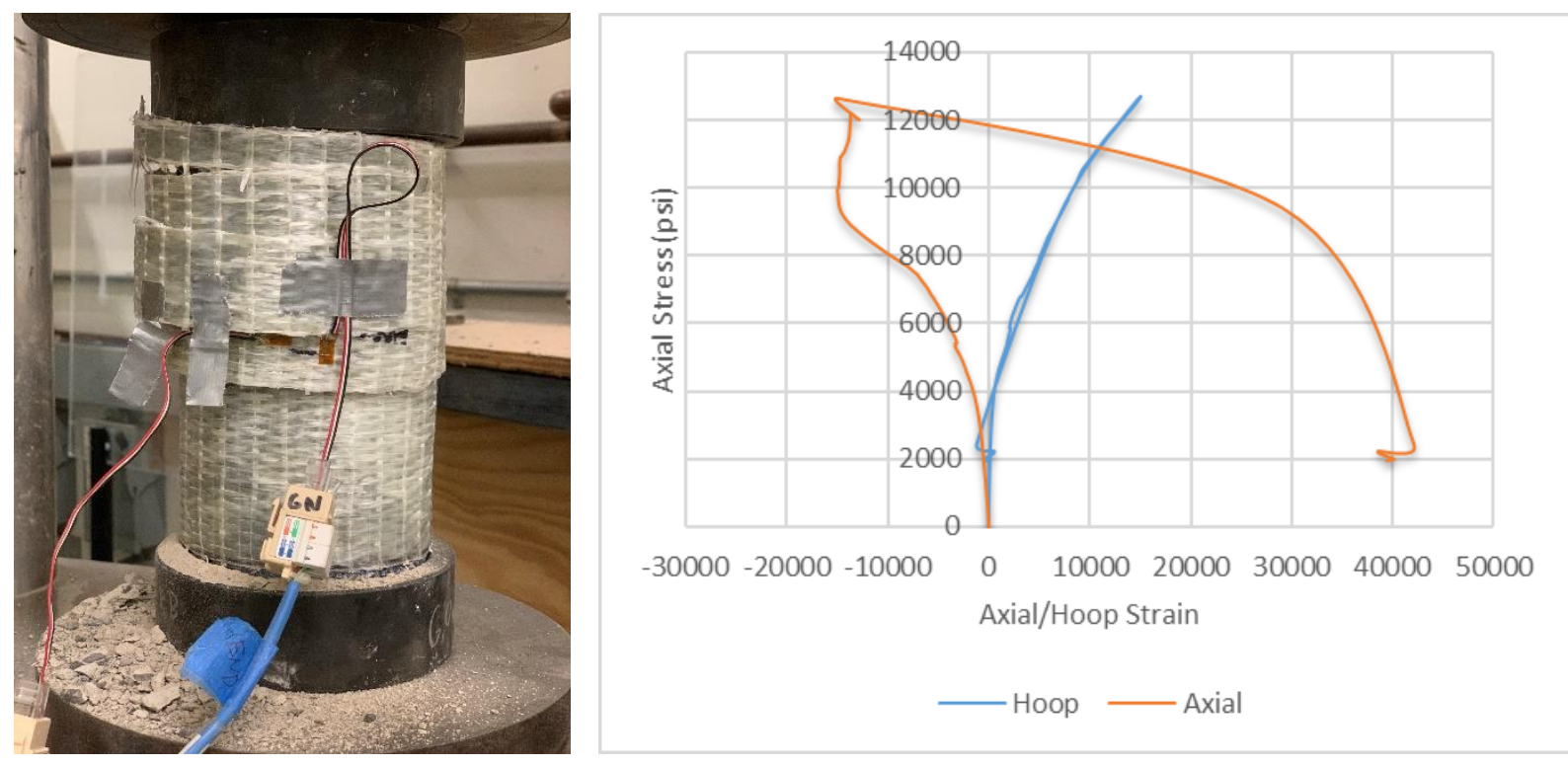

Figure E.5. Stress vs. strain behavior of JII-S1
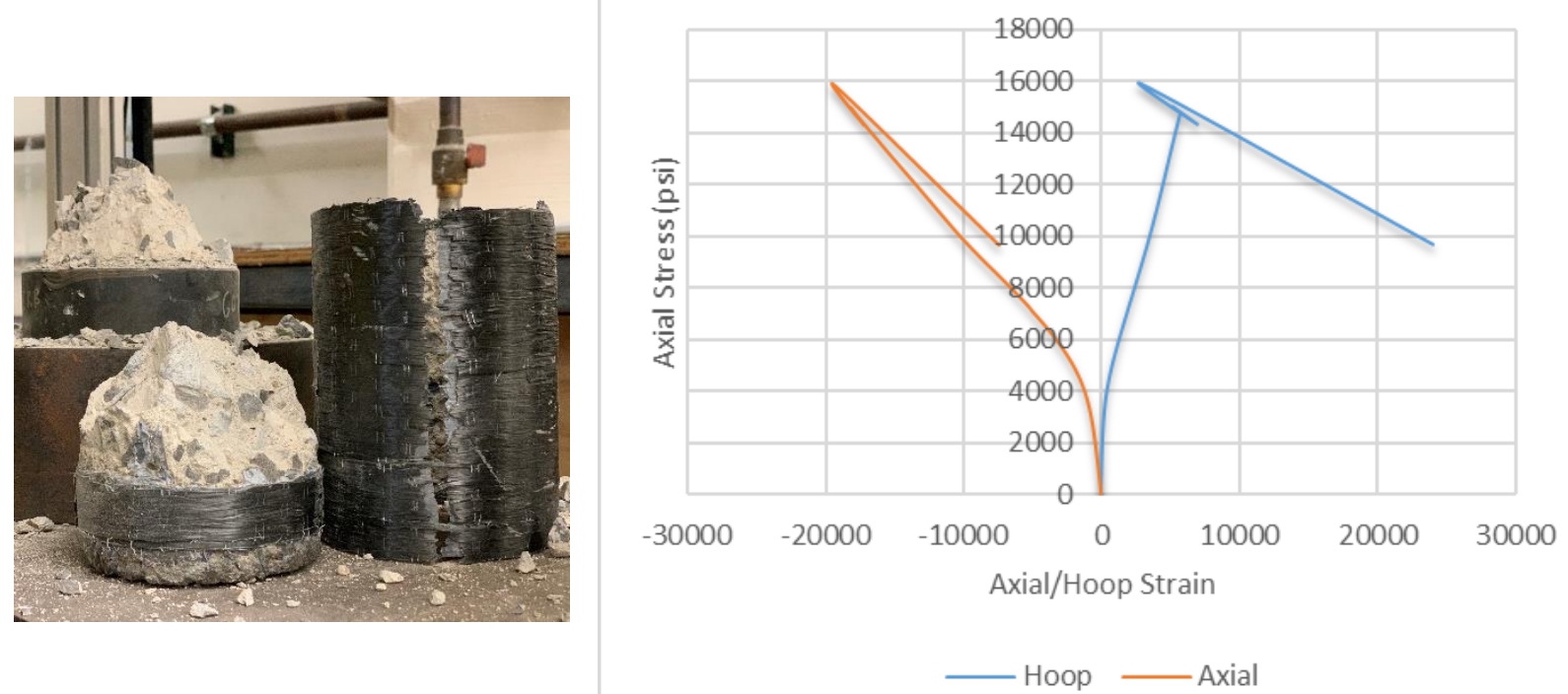

Figure E.6. Stress vs. strain behavior of JII-S2 


\section{E.2 Split-Tension Test}
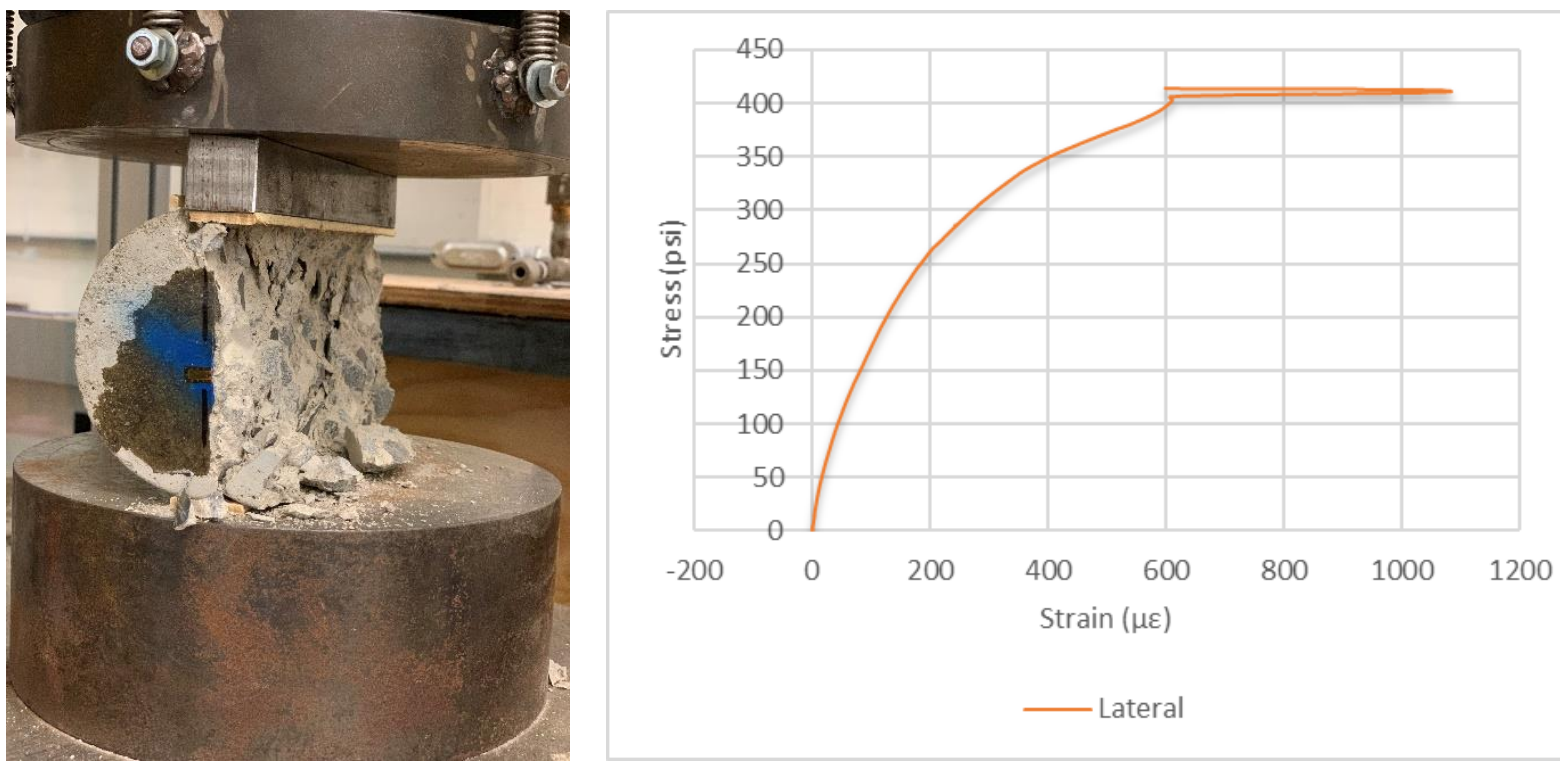

Figure E.7. Stress vs. strain behavior of JI-CS
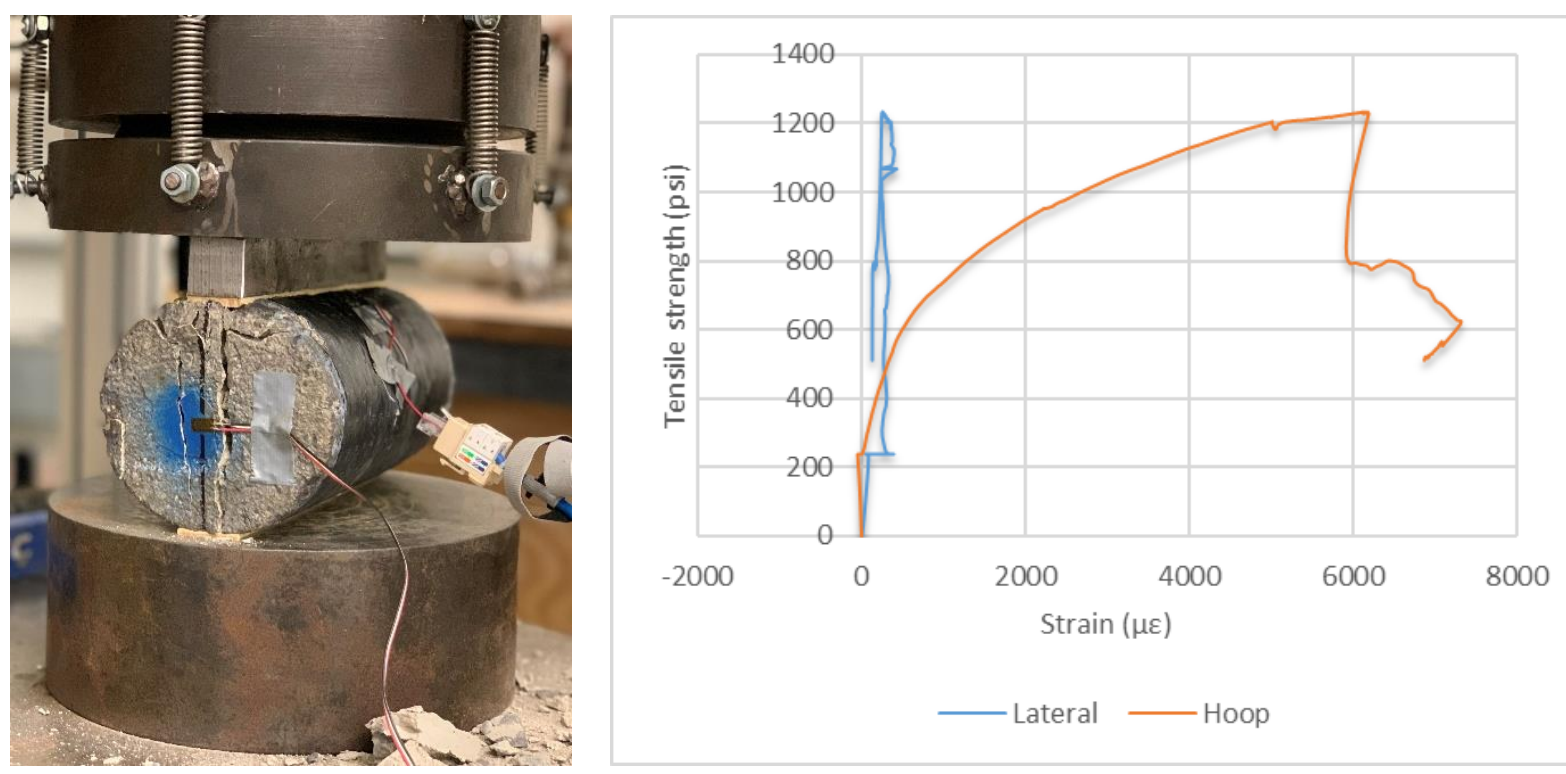

Lateral - Hoop

Figure E.8. Stress vs. strain behavior of JI-S1 

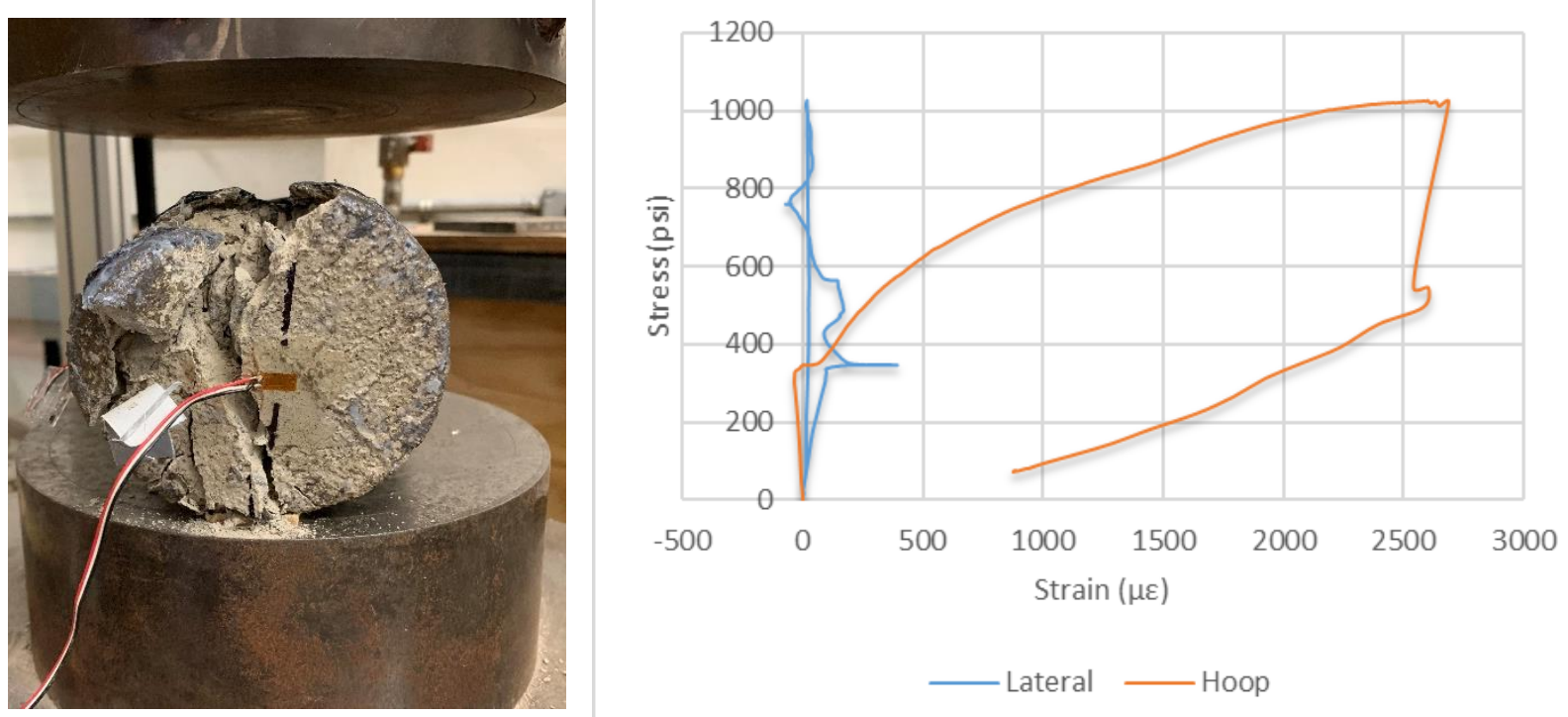

Figure E.9. Stress vs. strain behavior of JI-S2
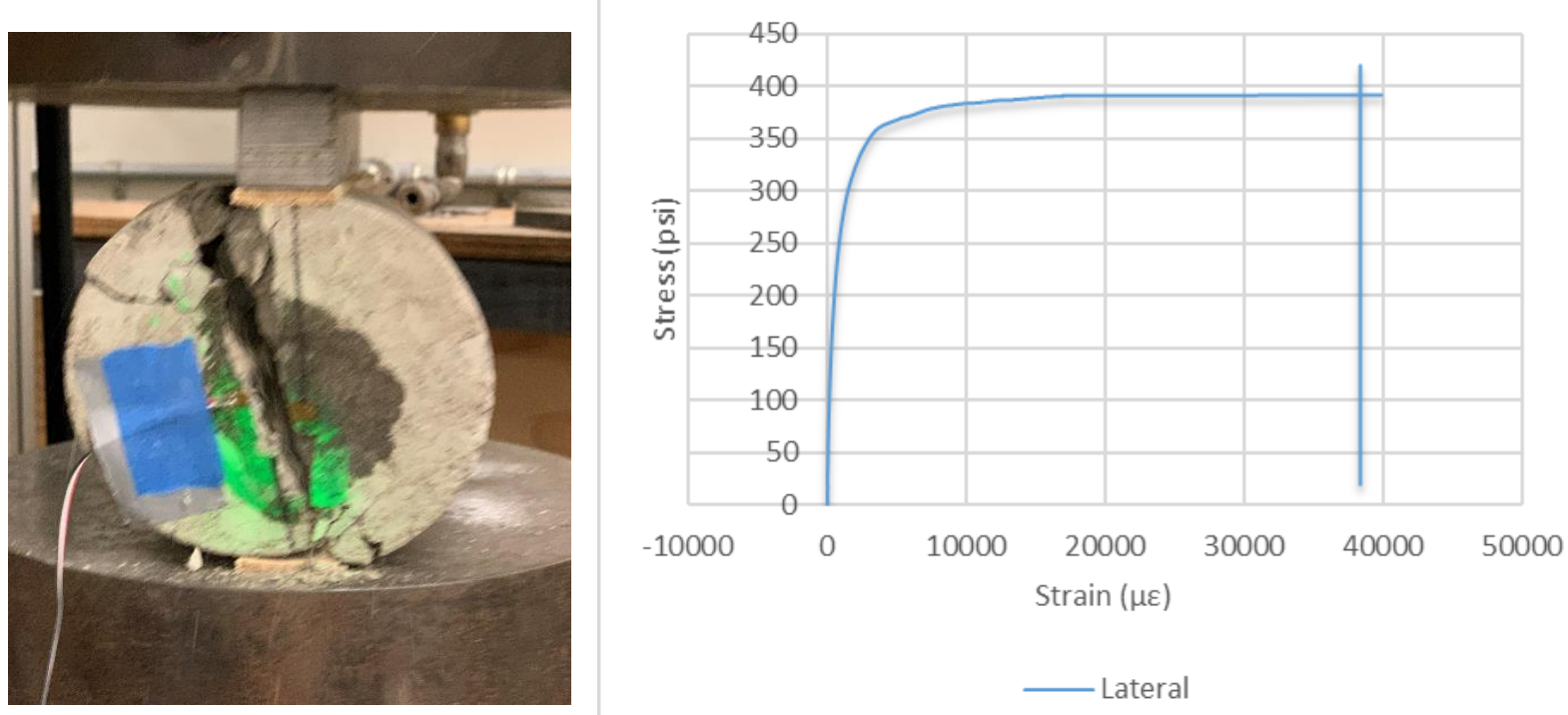

Lateral

Figure E.10. Stress vs. strain behavior of JII-CS 

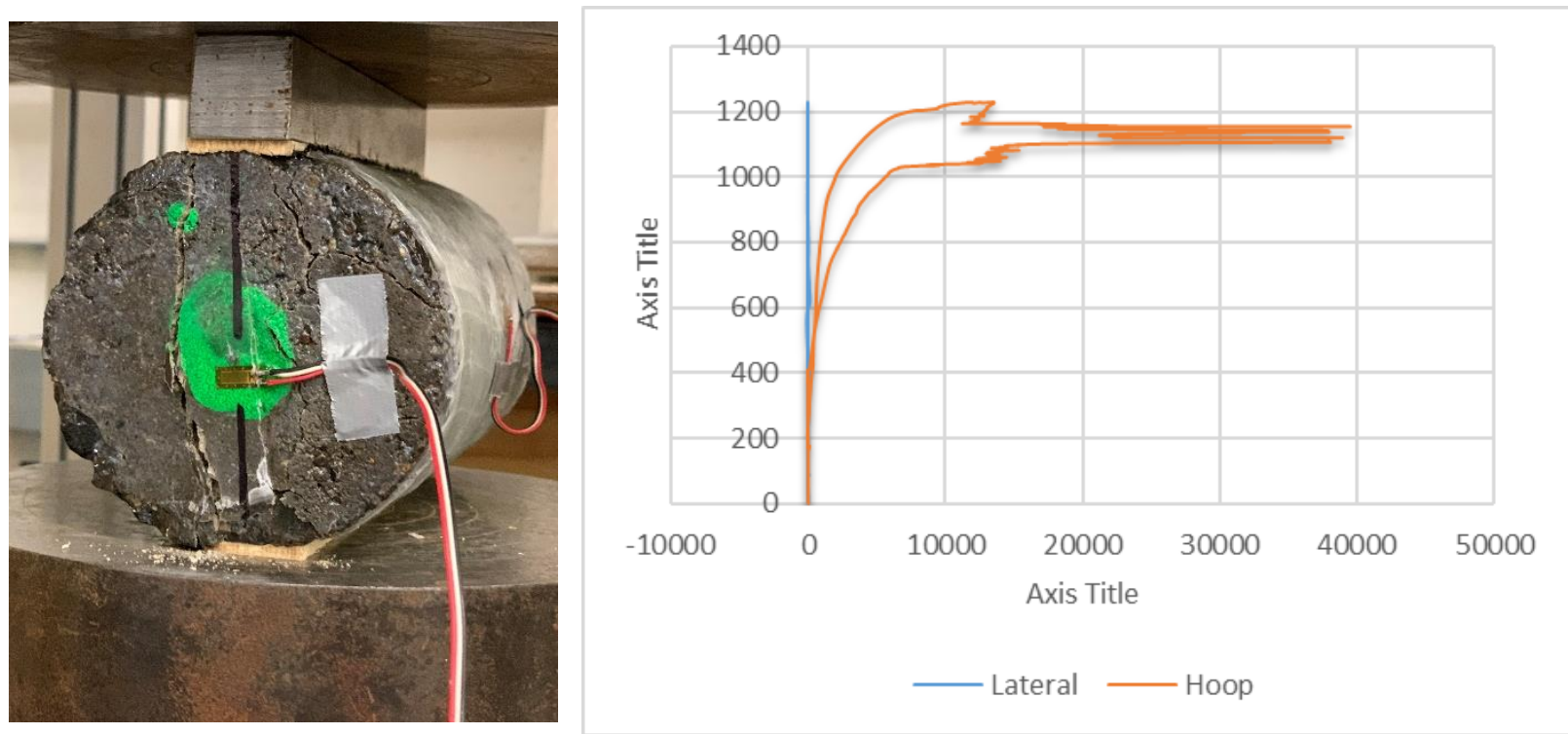

Figure E.11. Stress vs. strain behavior of JII-S1
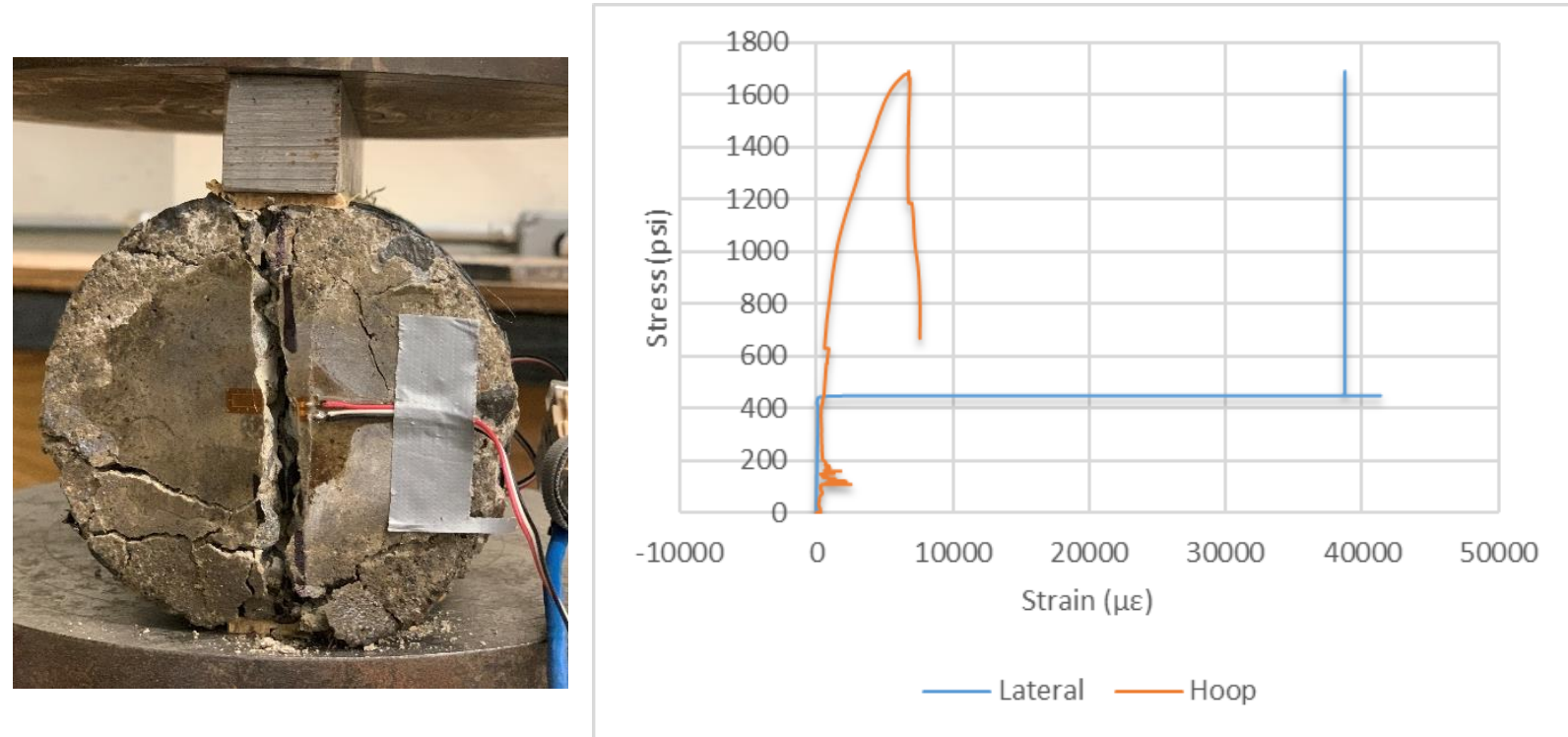

Figure E.12. Stress vs. strain behavior of JII-S2

Note:

1. J-I S-1: 2 layers of Sika103C unidirectional carbon wrap with 3 in. overlap.

2. J-I S-2: 2 layers of Sika103C unidirectional carbon wrap with 0.75 in. gap between the ends.

3. J-II S-1: 2 layers of Sika103C unidirectional carbon wrap with no overlap and no gap.

4. J-II S-2: 2 layers of Sika100G unidirectional glass wrap with no overlap and no gap.

5. Only one specimen was tested per category. 